Fall 1971

\title{
1971 Miracle Yearbook
}

\section{Cedarville College}

Follow this and additional works at: https://digitalcommons.cedarville.edu/yearbooks

Part of the Higher Education Commons, Organizational Communication Commons, and the Public Relations and Advertising Commons

\section{Recommended Citation}

Cedarville College, "1971 Miracle Yearbook" (1971). Yearbooks. 52.

https://digitalcommons.cedarville.edu/yearbooks/52

This Book is brought to you for free and open access by DigitalCommons@Cedarville, a service of the Centennial Library. It has been accepted for inclusion in Yearbooks by an authorized administrator of DigitalCommons@Cedarville. For more information, please contact digitalcommons@cedarville.edu. 


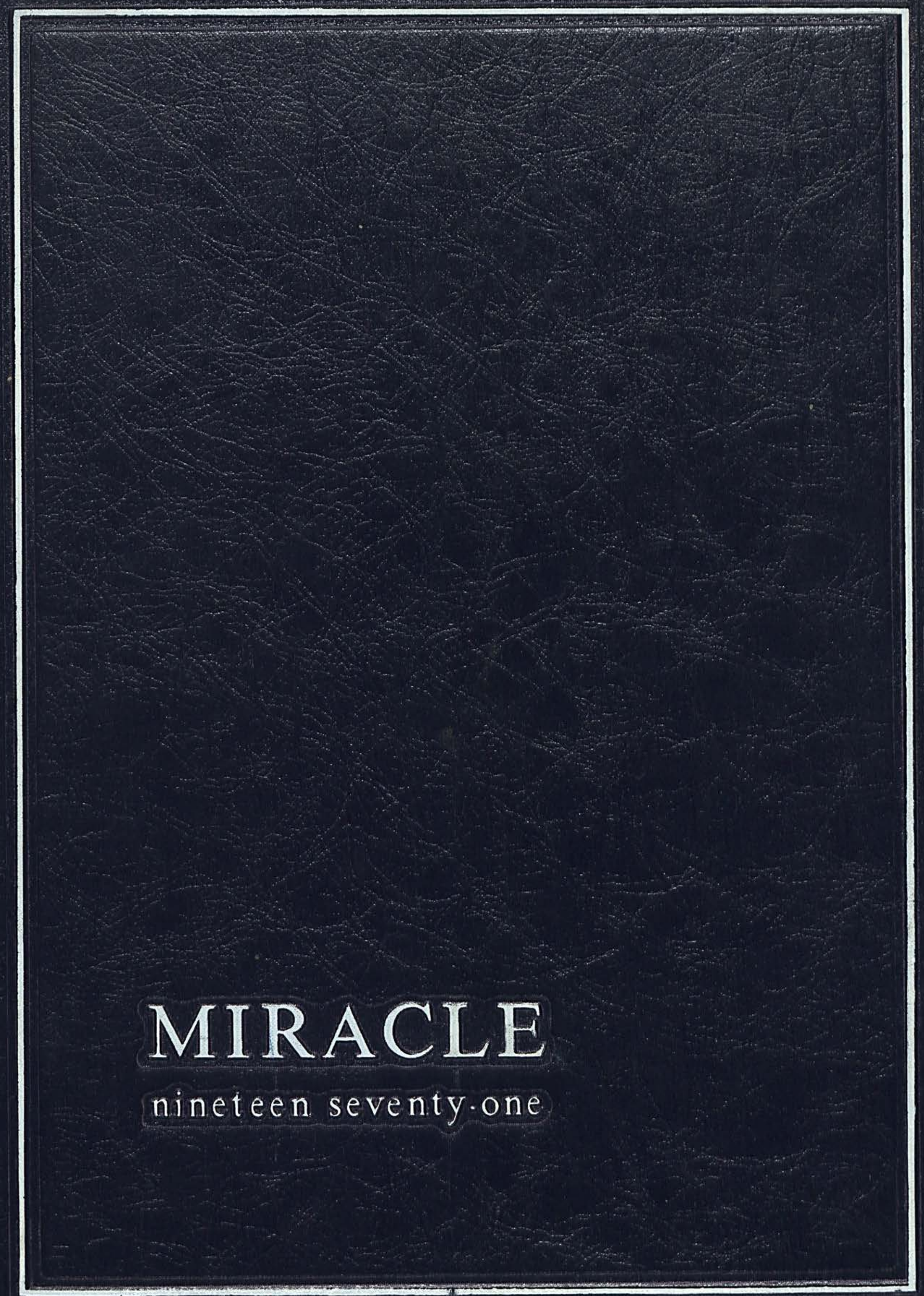




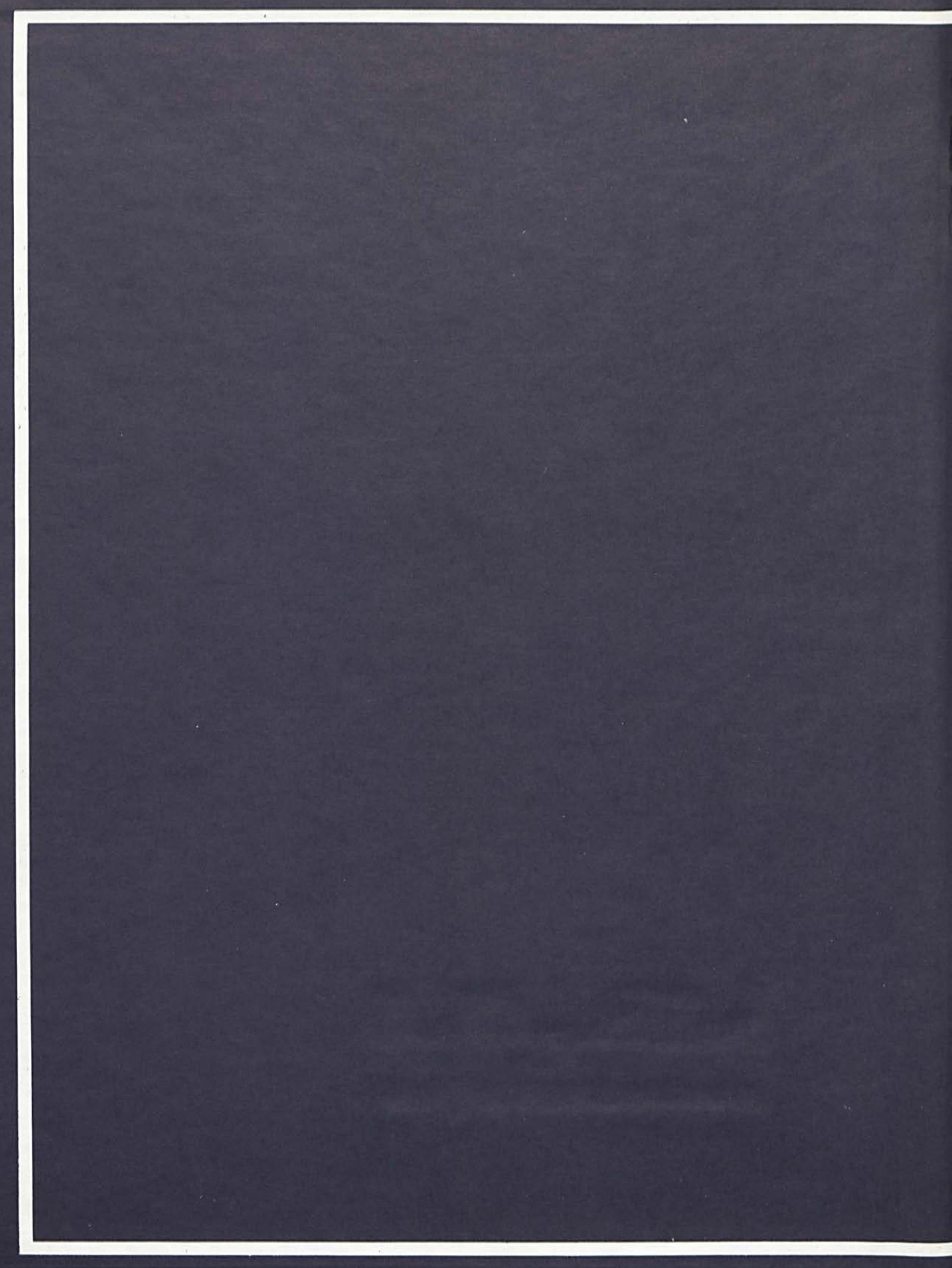




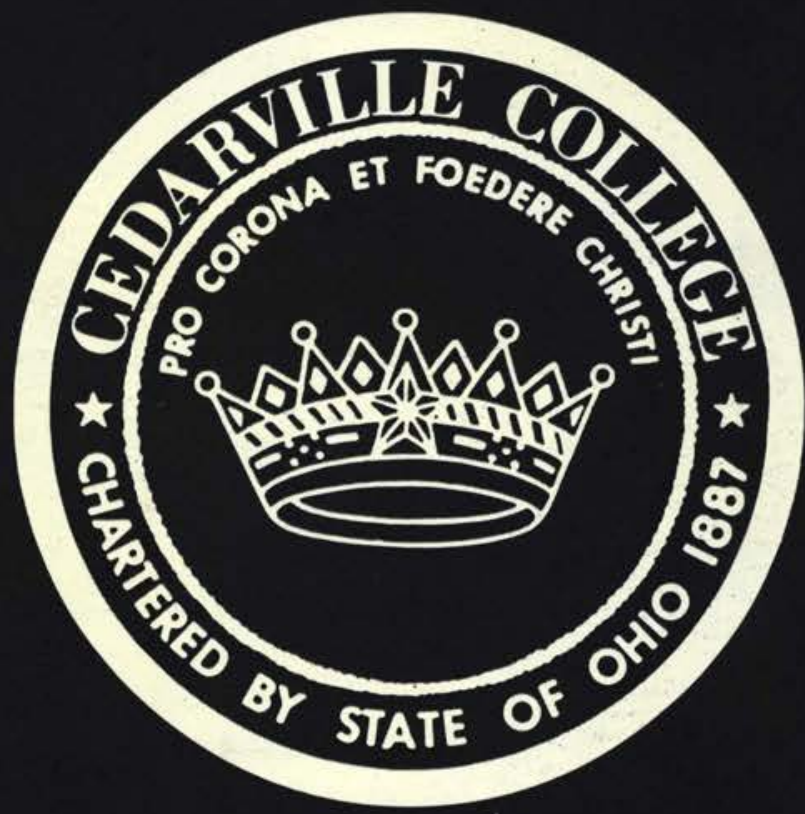


Editor: Carol Bierbaum Assistant Editor: Janet Hillery Photography Editor: Roy Kern Photography Assistant: Glen Huebner Yearbook Advisor: Dr. Donald Baumann Yearbook Representative: Charles Miller Publisher: American Yearbook Company 


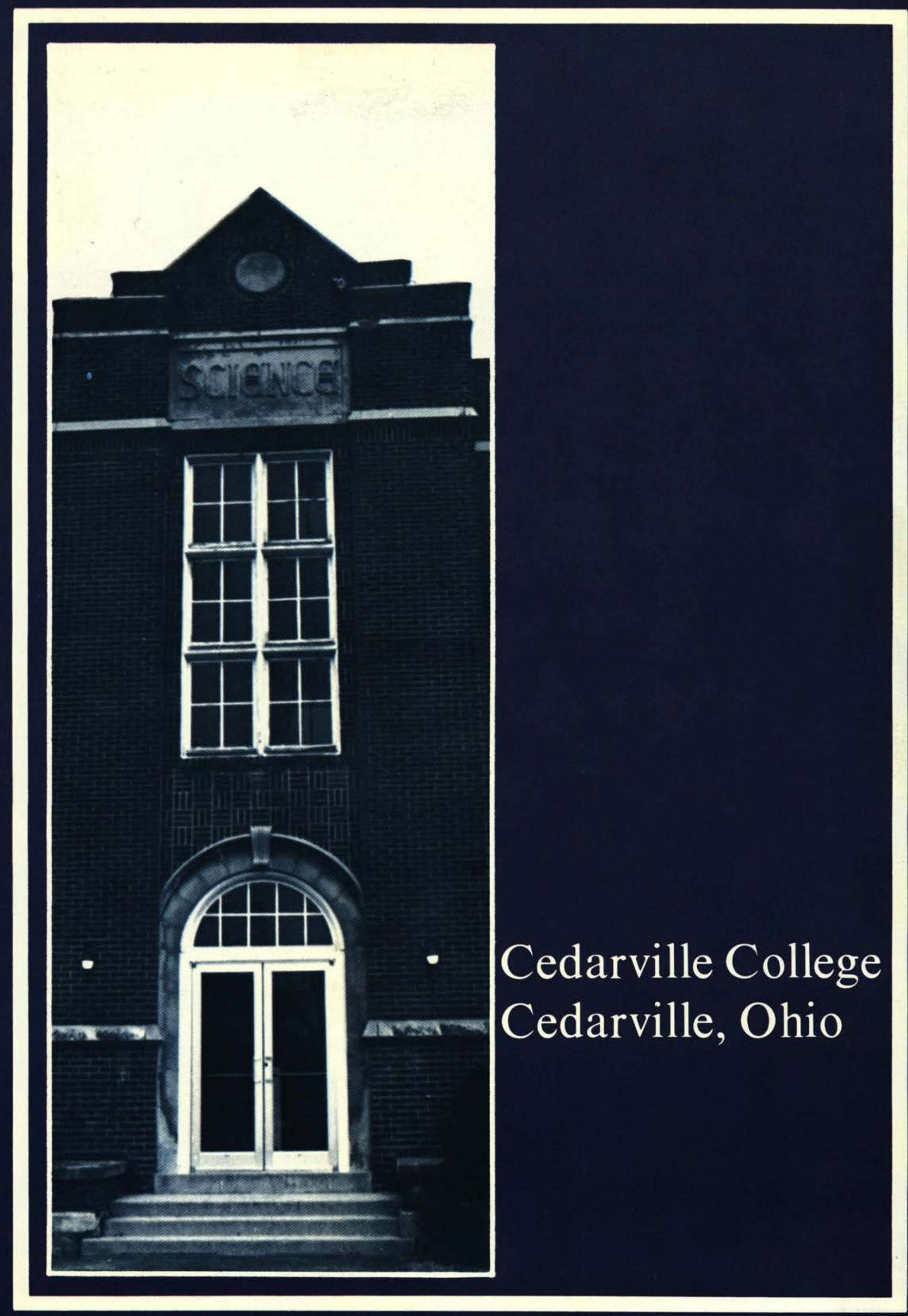


CEDAR VILLE COLLEGE -

a rich heritage -

Reformed Presbyterian Church and others

who cared

CEDAR VILLE COLLEGE -

State of Ohio charter,

January 26, 1887;

Biblical standards, not man's.

CEDAR VILLE COLLEGE -

"For the Word of God

and the testimony

of Jesus Christ

Unity of the spiritual

to the academic,

culture of the mind

nurtured by

growth of the spirit

in one's life,

a goal we pray

in every student's life.

CEDAR VILLE COLLEGE -

the Bible as guide

for all other texts,

marriage of education

to morality,

fulfillment of life

because of Christ,

our Saviour, our Lord.

CEDAR VILLE COLLEGE -

1953, transfer of operation

trustees of Baptist Bible

Institute of Cleveland,

the goal - growth of

an institute!

CEDAR VILLE COLLEGE -

changing faces

both external, internal,

but always progressing,

pressing onward

to a goal.

CEDAR VILLE COLLEGE transforming, as years pass by it, its foundation a constant -

Jesus Christ.

CEDAR VILLE COLLEGE -

a school approved

the General Association

of Regular Baptist Churches,

hold to the origin

of purpose by founders.

CEDAR VILLE COLLEGE -

led by God's chosen,

Dr. James T. Jeremiah,

since fifty-four,

enrollment increase,

tripling of size,

expanded facilities,

a faculty concerned

for the individual,

and objectives strengthened

God hath done marvelous works to the children of men,
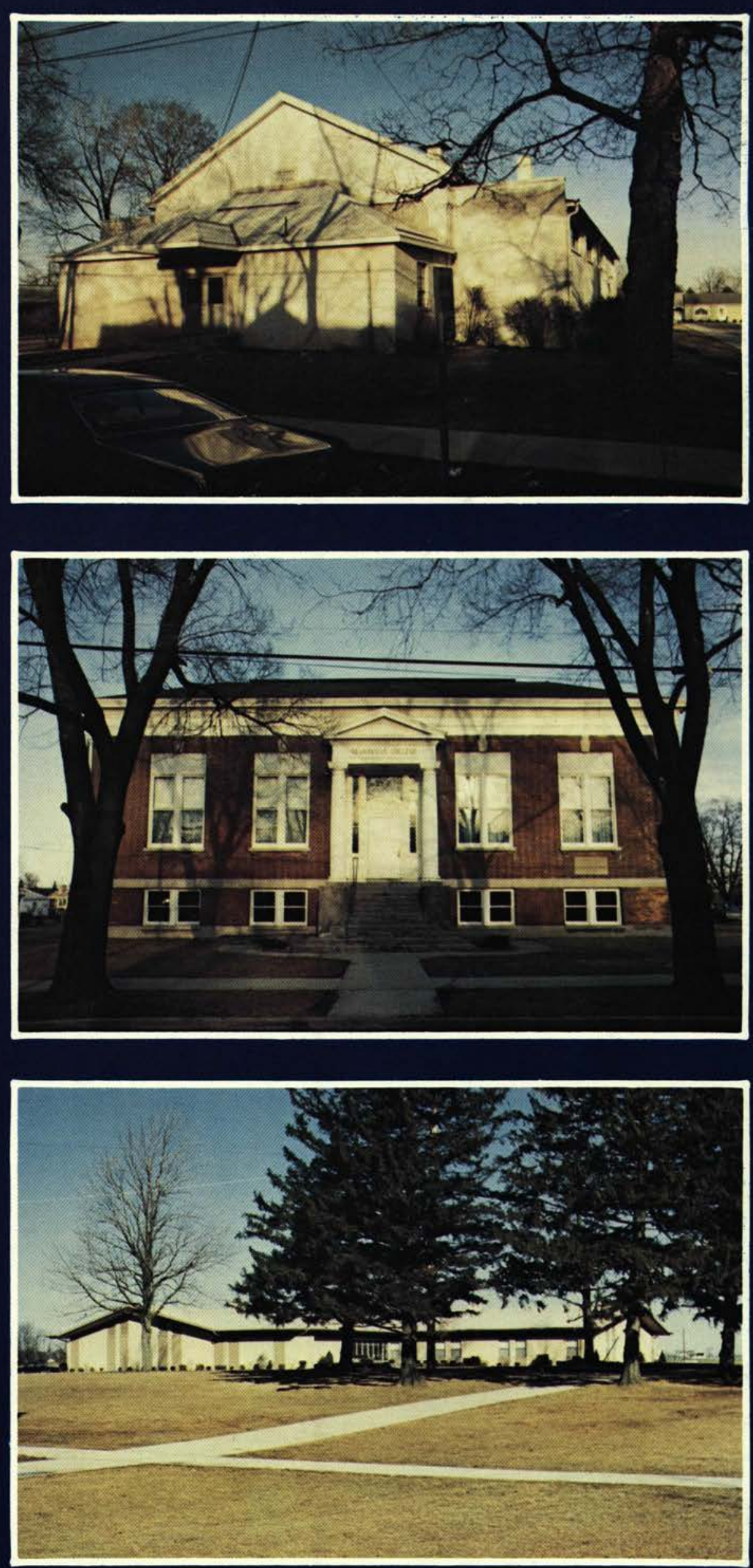

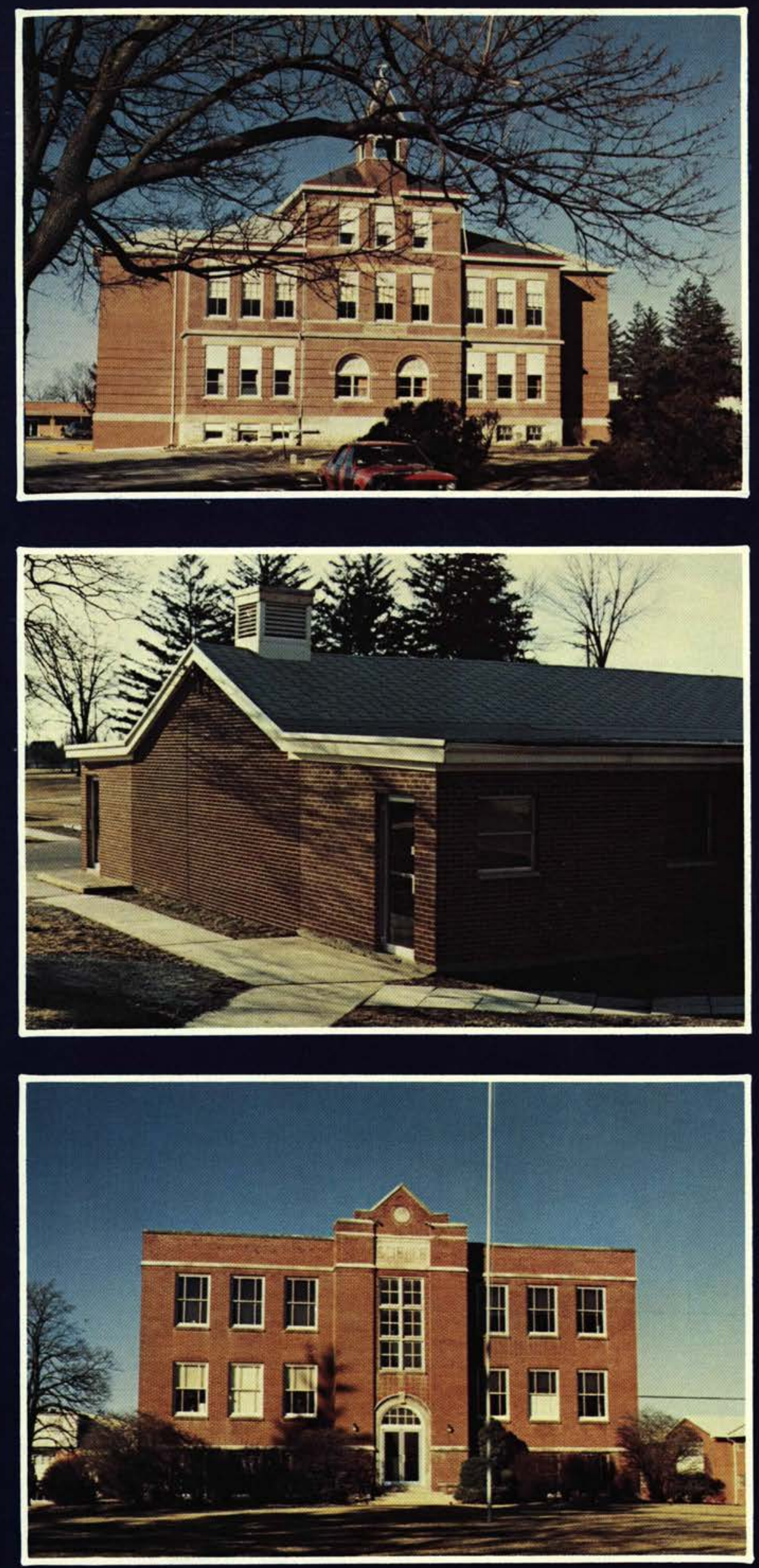

hath blessed,

hath rewarded faithfulness!

CEDARVILLE COLLEGE

1971, "Three to Grow On" program,

we all together stand,

"Endeavoring to keep

the spirit of unity

in the bond of peace,"

on the threshold

of new and higher goals,

visions, expectations

onward and upward

according only

to our measue of faith

for future generations!

ALFORD AUDITORIUM -

many things to many people:

to a freshman, the chapel;

to others ... a gymnasium past, a concert, vespers, artist-lecture, revival, meditation, drama production,

music lessons, oral readings,

talent night, and on

THE LIBRARY -

newest of our buildings,

ah, the potential that lies

therein, but only if taken

out and put to use

in the minds and lives of men

and women .... volumes of

books; periodicals and teaching aids; microfilms and other

audio-visual supplement

FINE ARTS

once a library, now a

composite of classrooms

for music, for art;

practice for band,

choir, choralaires, auditions;

even office and study.

OLD MAIN -

our link with the past,

its foundations and traditions,

remodeled, yet still

a landmark, whose walls

could speak

with age-old truth and

wisdom.

MILNER HALL -

once a chapel,

now a composite of bookstore,

practice rooms, and classroom.

SCIENCE BUILDING -

waiting for expansion

to better facilities,

continues to provide housing

of math, biology, physics,

chemistry, - the other sciences,

all working in agreement,

not opposition, to the

laws of God. 


\section{A New Beginning}
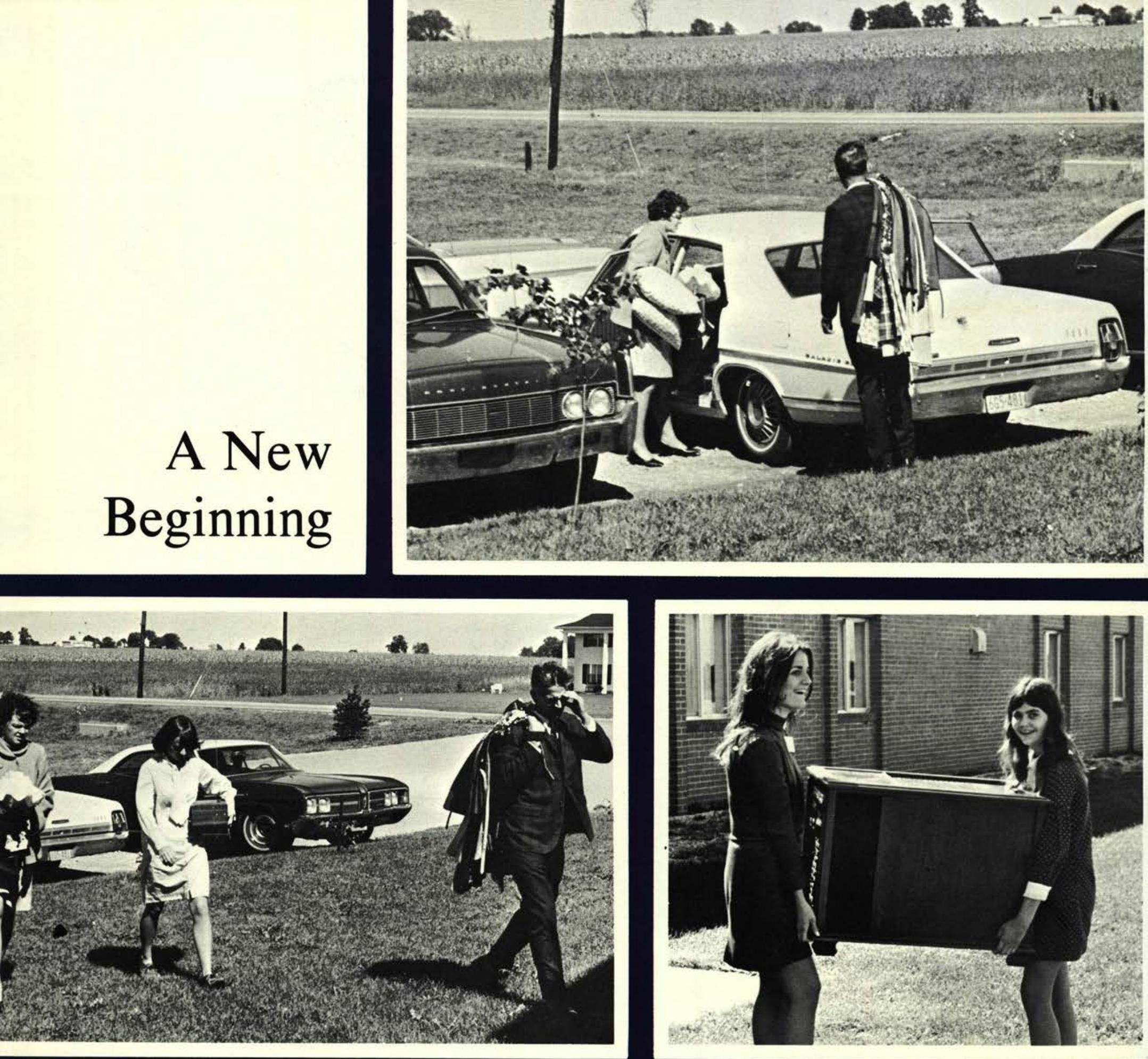

Anxious anticipation when entering the parking lot An endless caravan from the car to the room -

We didn't know we owned so much

Signing in at the lounge desk, Filling out cards, and pinning on name tags

This was the new beginning.

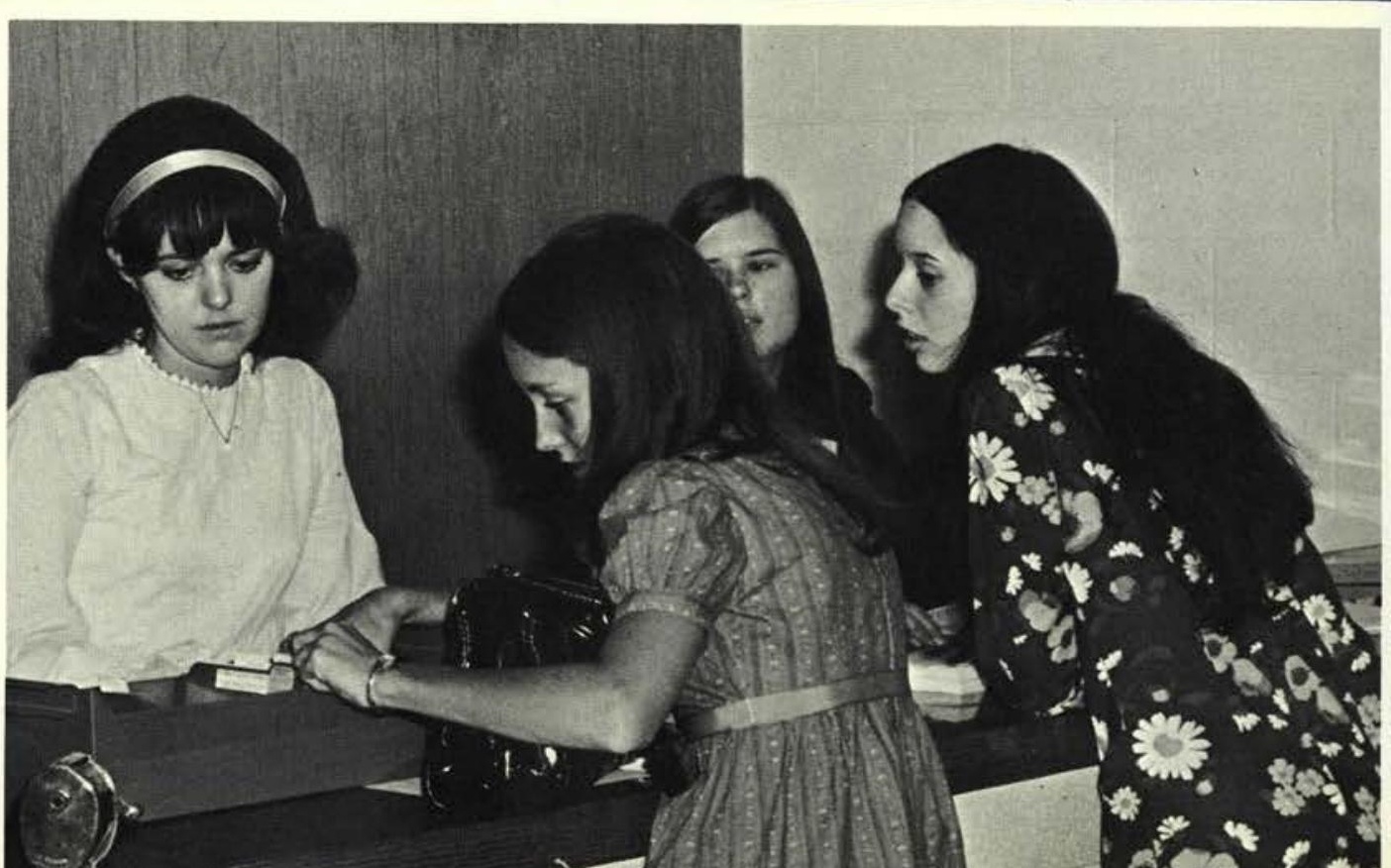



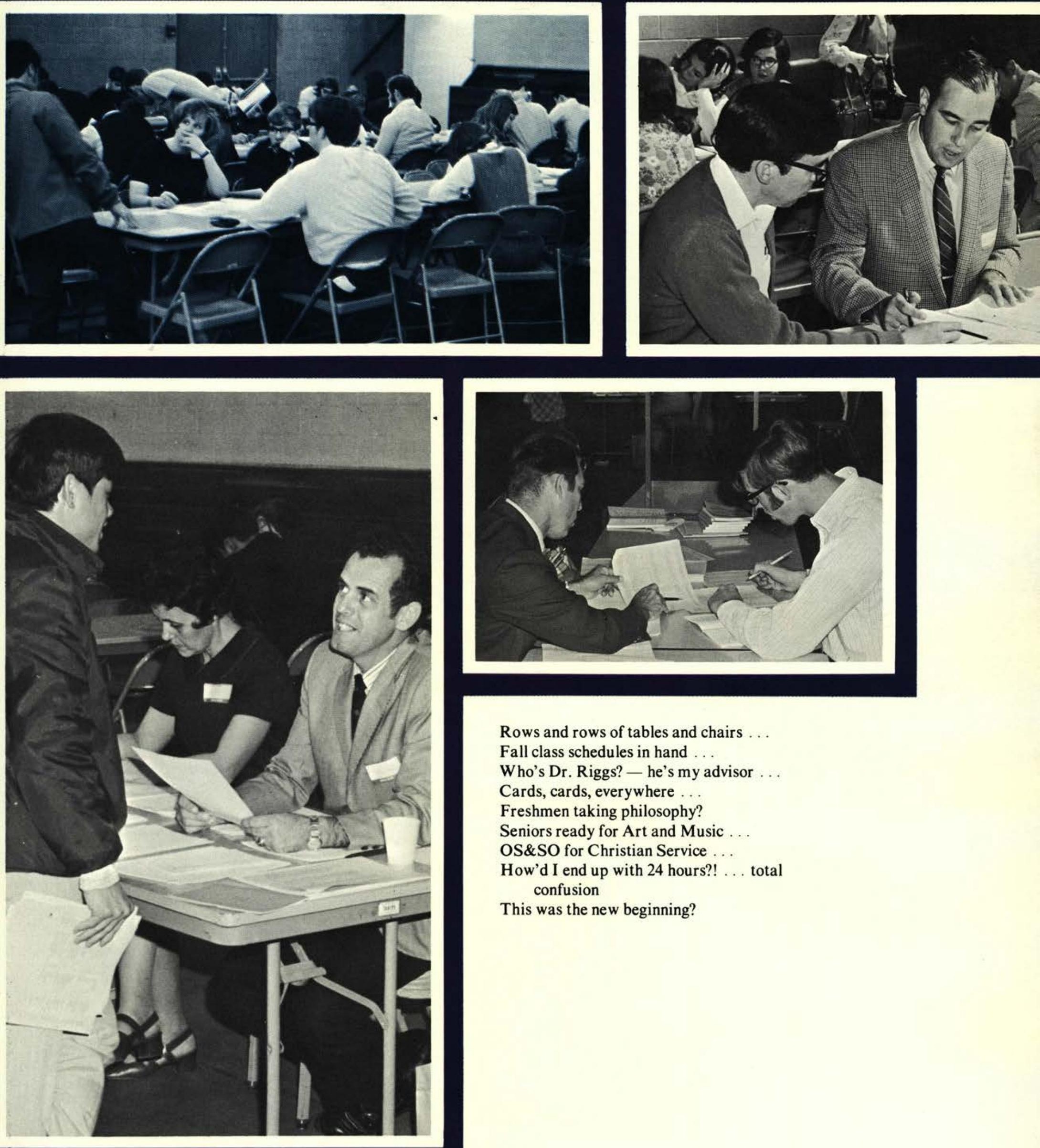

Rows and rows of tables and chairs ...

Fall class schedules in hand .. .

Who's Dr. Riggs? - he's my advisor . . .

Cards, cards, everywhere .

Freshmen taking philosophy?

Seniors ready for Art and Music . .

OS\&SO for Christian Service ... .

How'd I end up with 24 hours?! . . . total confusion

This was the new beginning? 

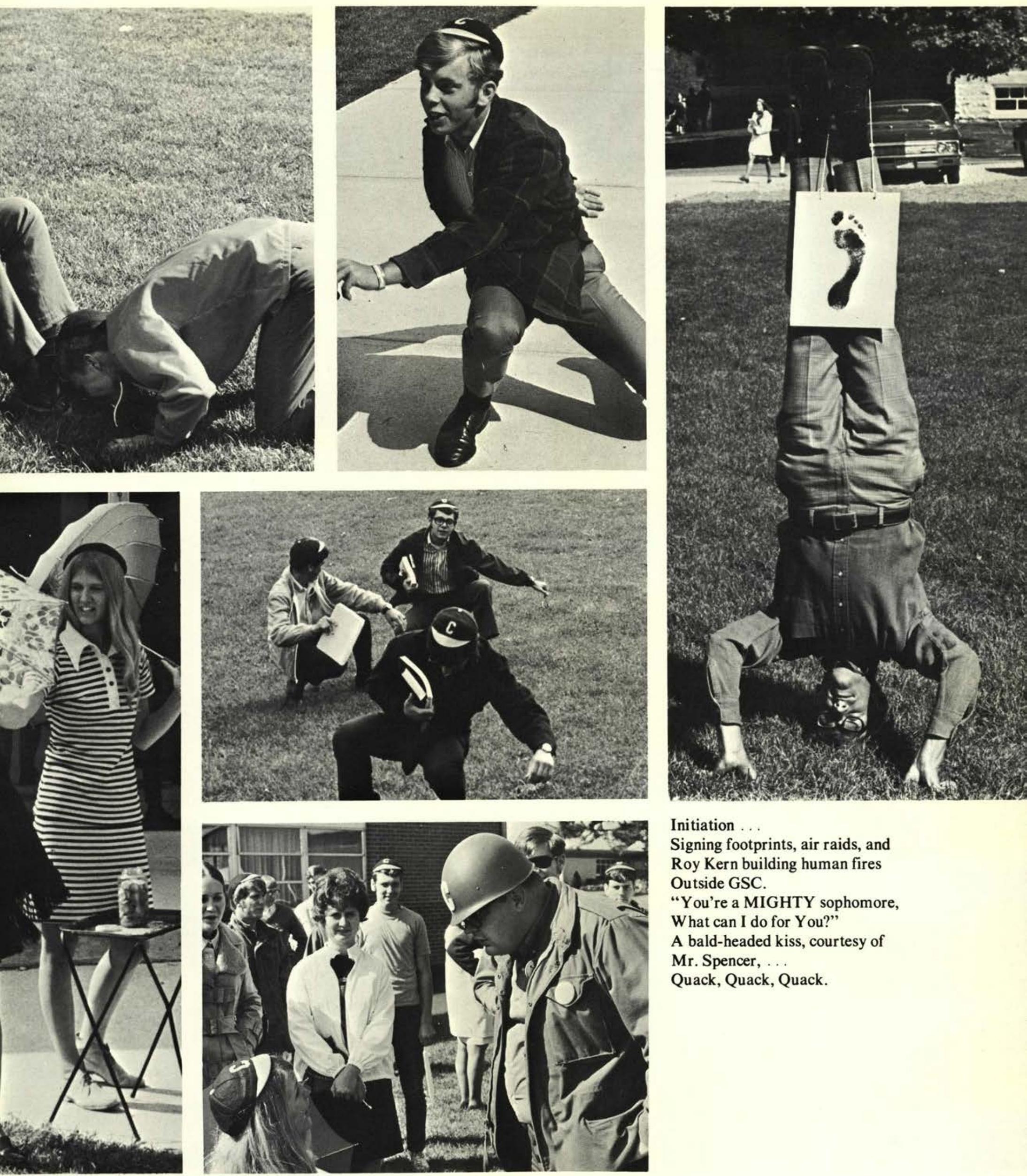

Initiation

Signing footprints, air raids, and

Roy Kern building human fires

Outside GSC

"You're a MIGHTY sophomore,

What can I do for You?"

A bald-headed kiss, courtesy of

Mr. Spencer,

Quack, Quack, Quack. 


\section{Court's In Session,}
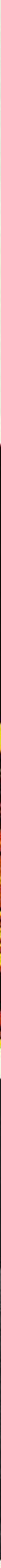

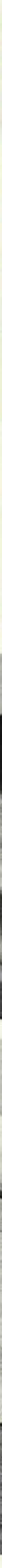

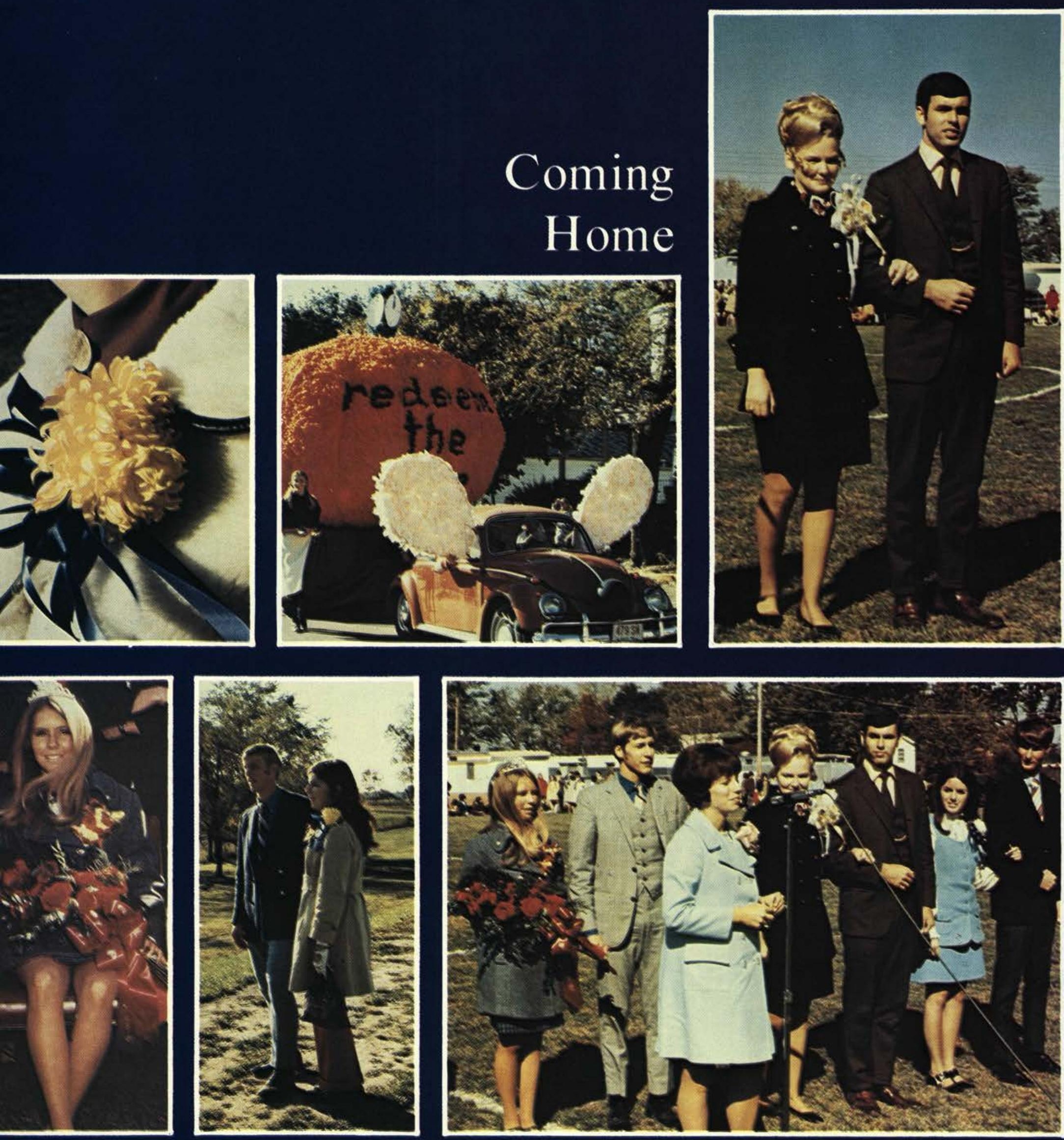

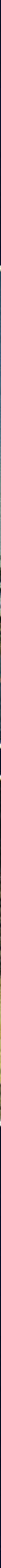

Busy nights - beautiful floats,

A precise march in a special parade,

The thrill of being an attendant

A yellow mum with a blue ribbon

The excitement of a soccer game,

Seeing old friends and a flood of memories

All part of coming home! 
Bonfires and cheerleaders,

A concerned coach -

Anxious players watching

Quick action on the field,

Attendants, queens, and coaches -

What a beautiful day for memories.
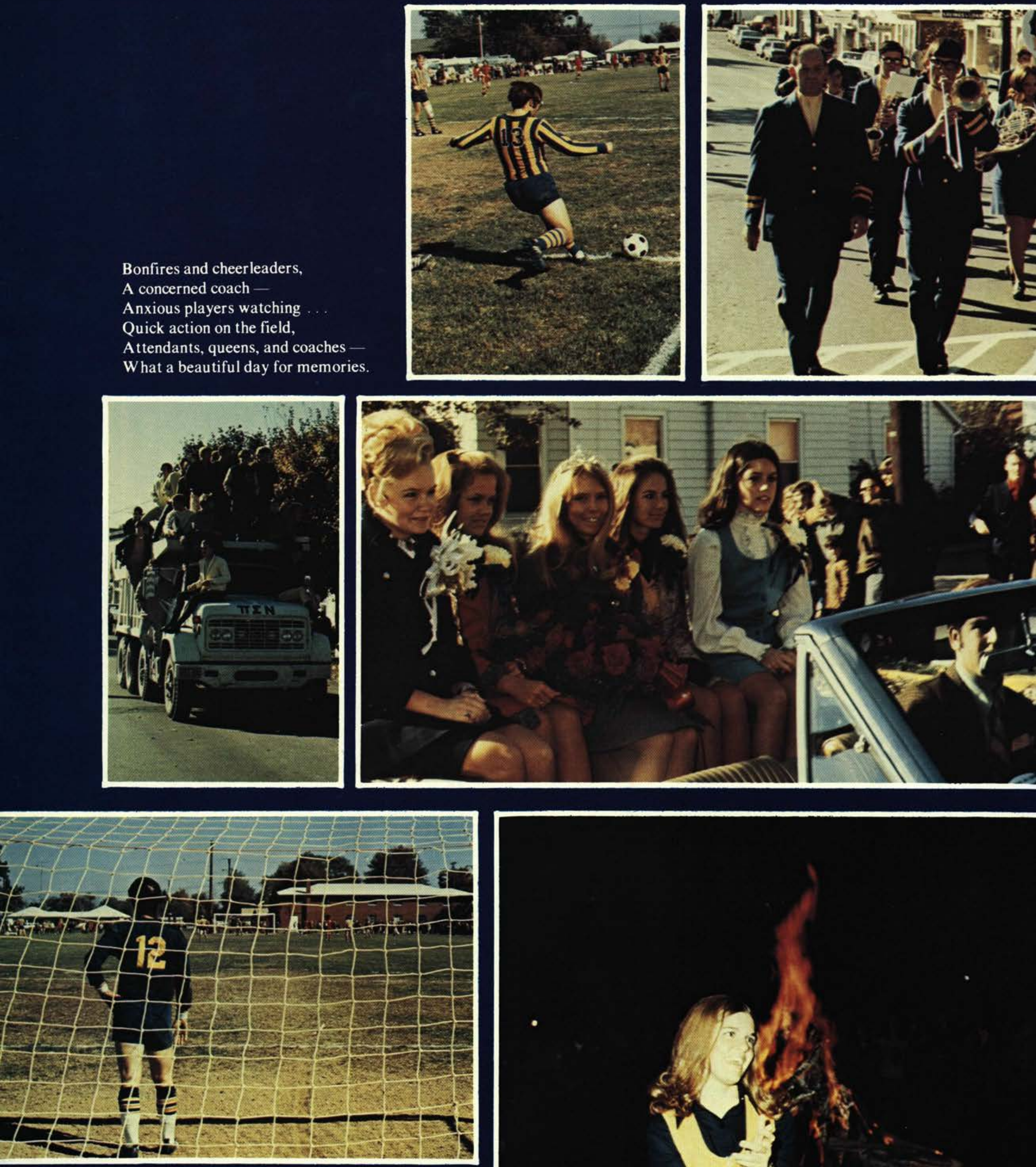


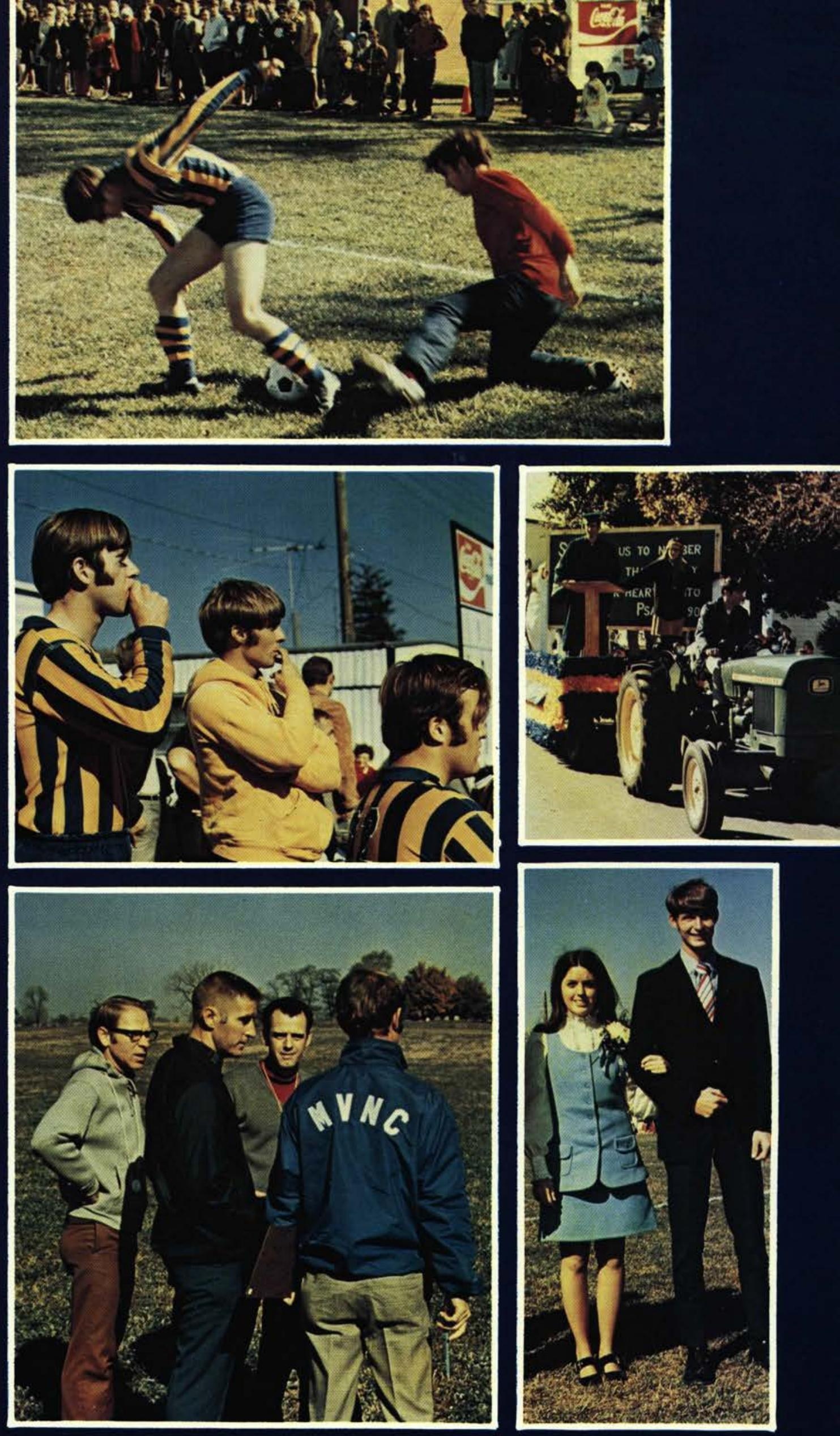



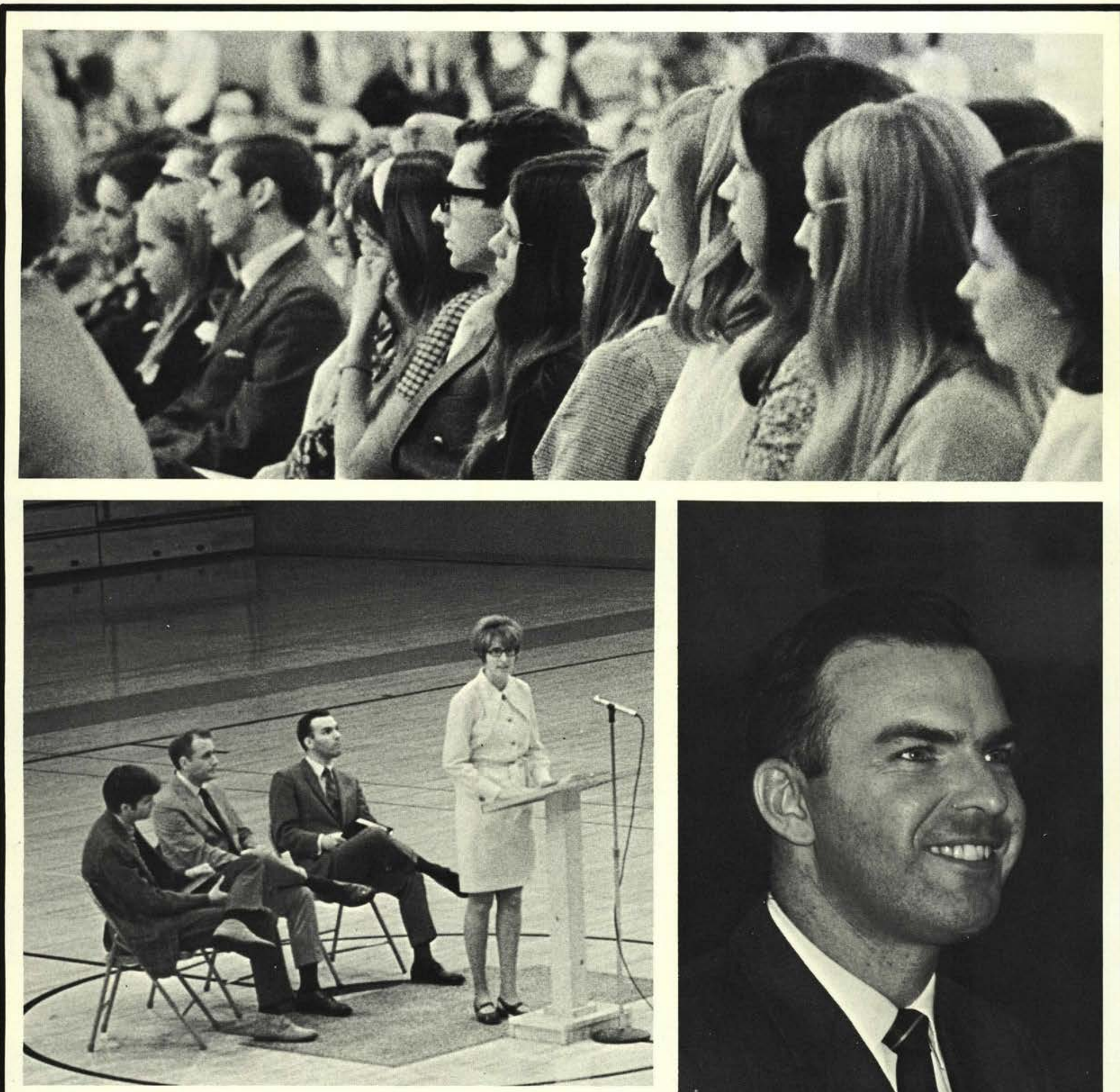

\section{A Time of Dedication}

Fall Bible Conference

Every morning - every night,

Looking and seeing, hearing -

"Ye are the Light of the world"

Messages from the moon

Presenting a challenge, accepting its responsibilities,

Achieving a goal ... 40 wrestlers for Christ Dr. Paul Tassel

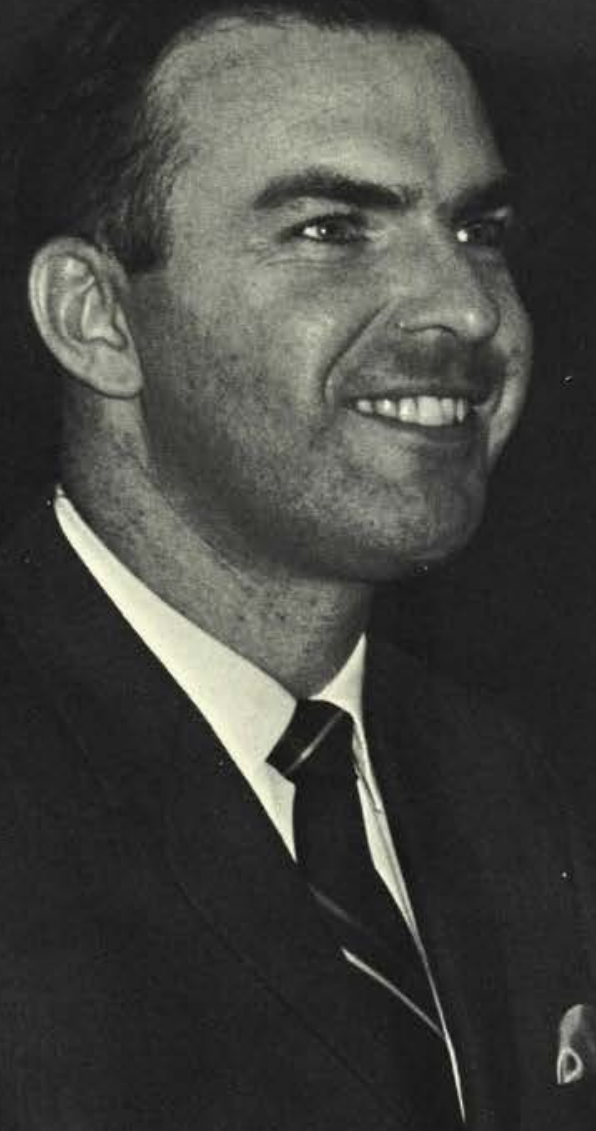




\section{Alfred Eric Street}

Saturday night

The first twenty minutes

We watched his fingers talking

To their guitar -

The rest of the time,

We listened to

What they were saying.
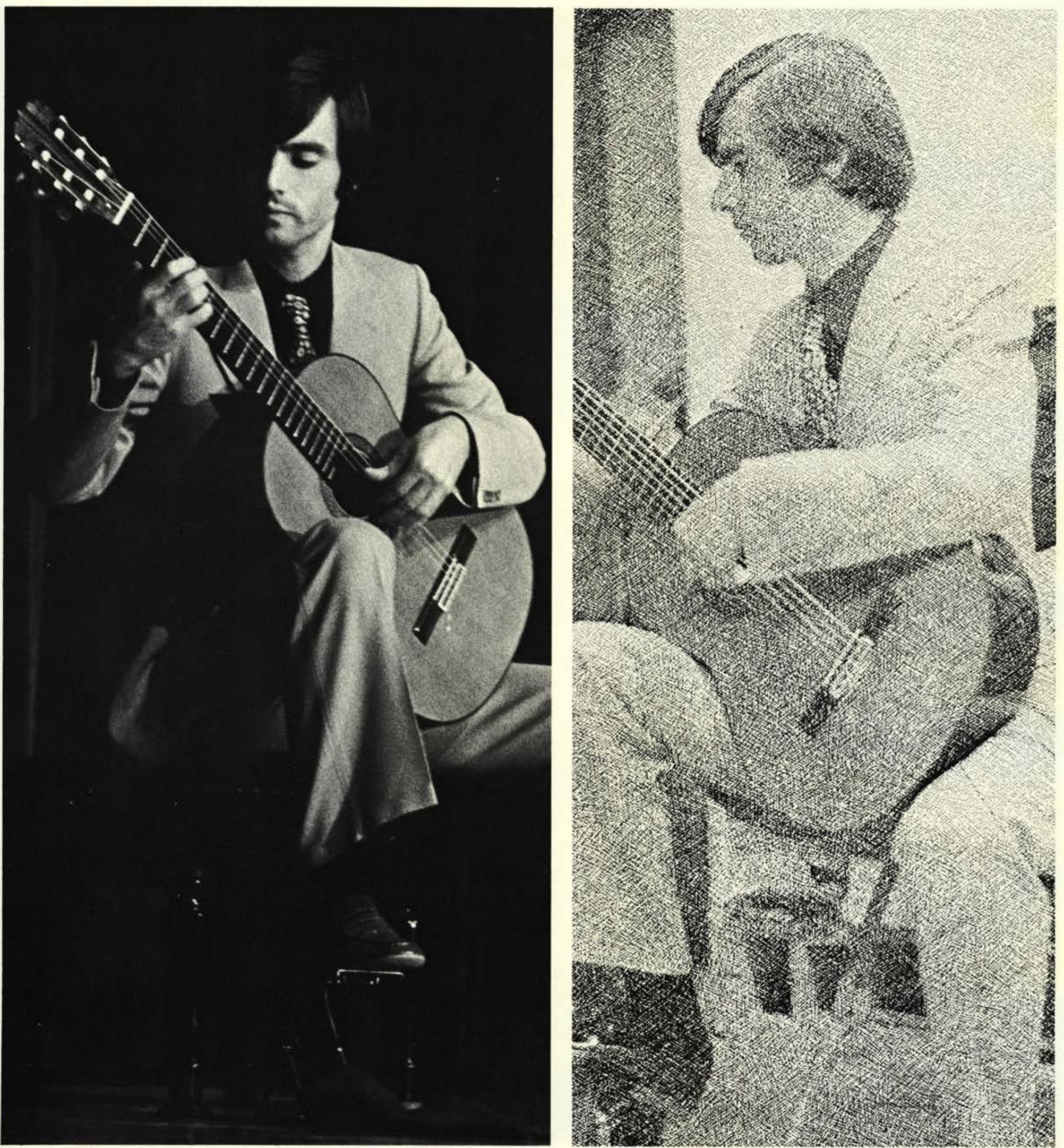

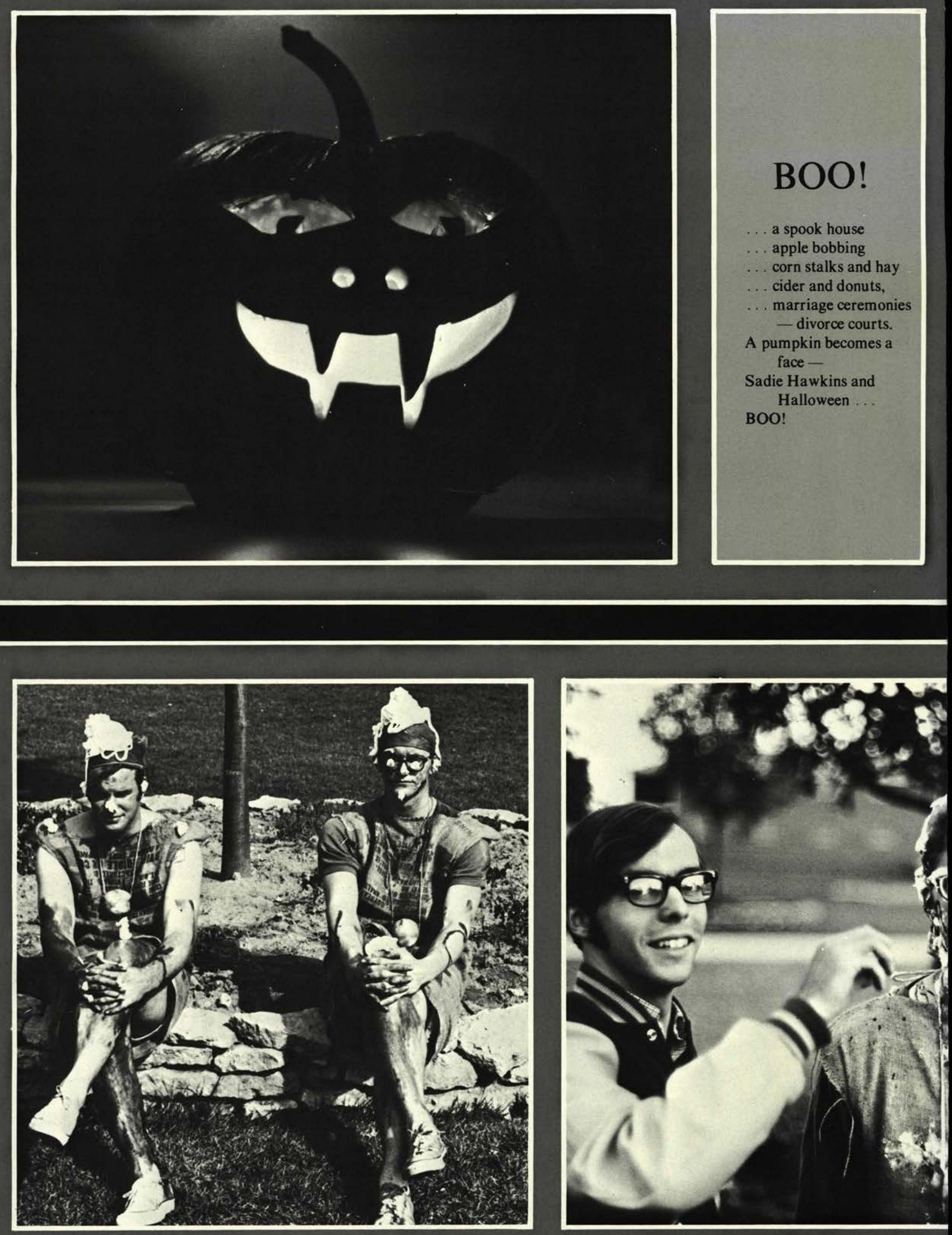


$$
\text { ㄴ. } y^{\frac{6}{2}}(5)
$$
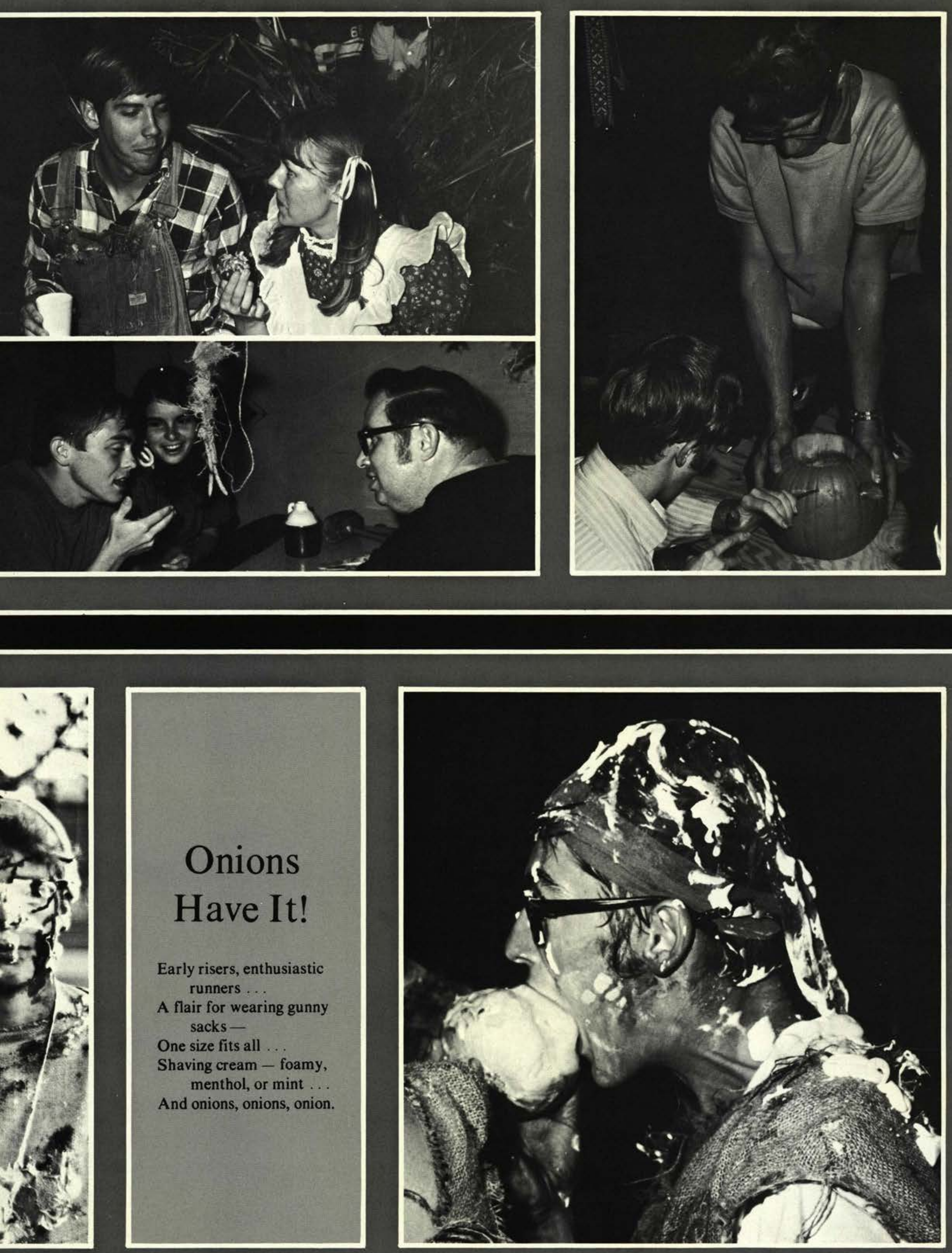


\title{
Village Players
}

\author{
present
}

Anne Frank
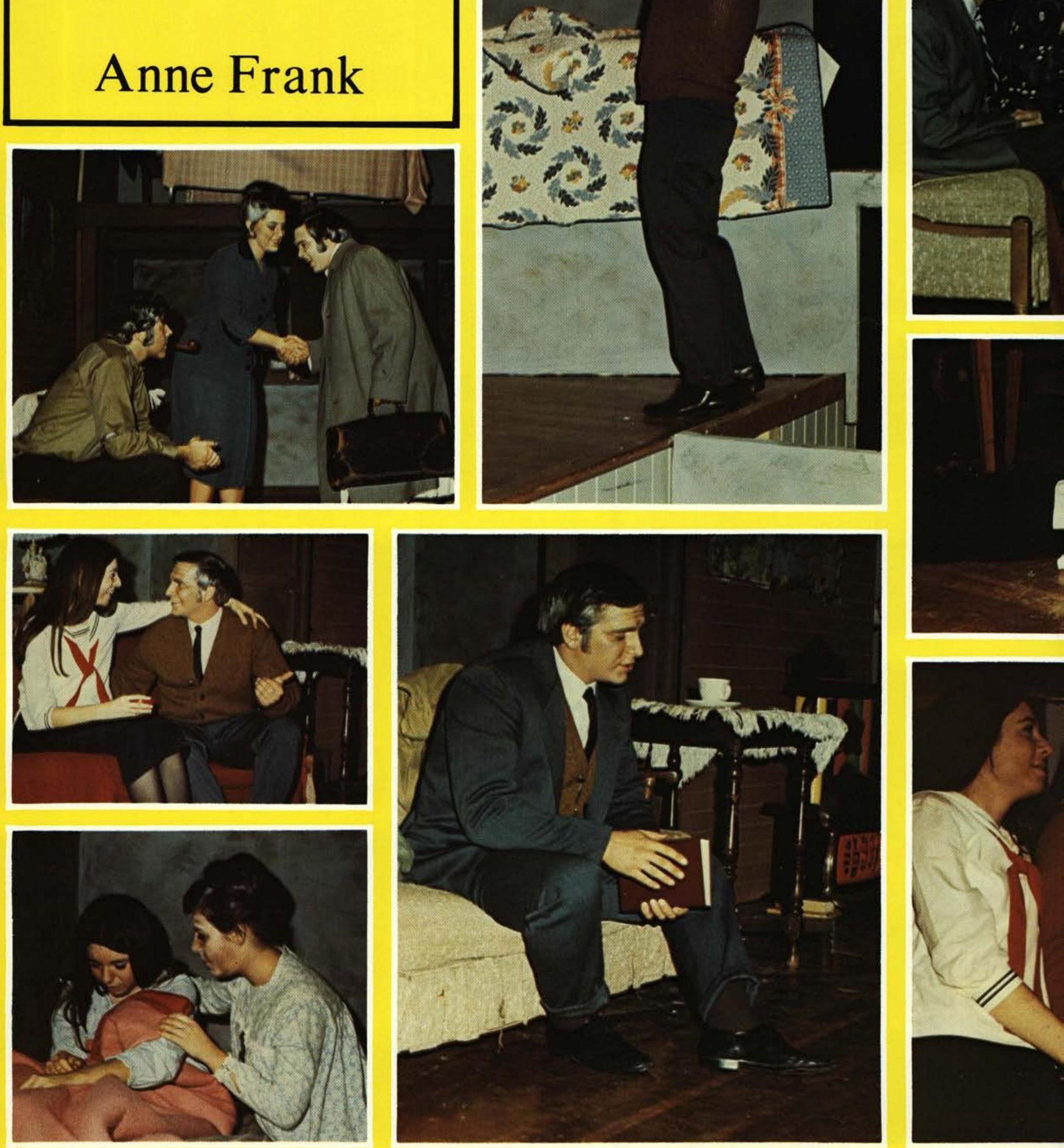

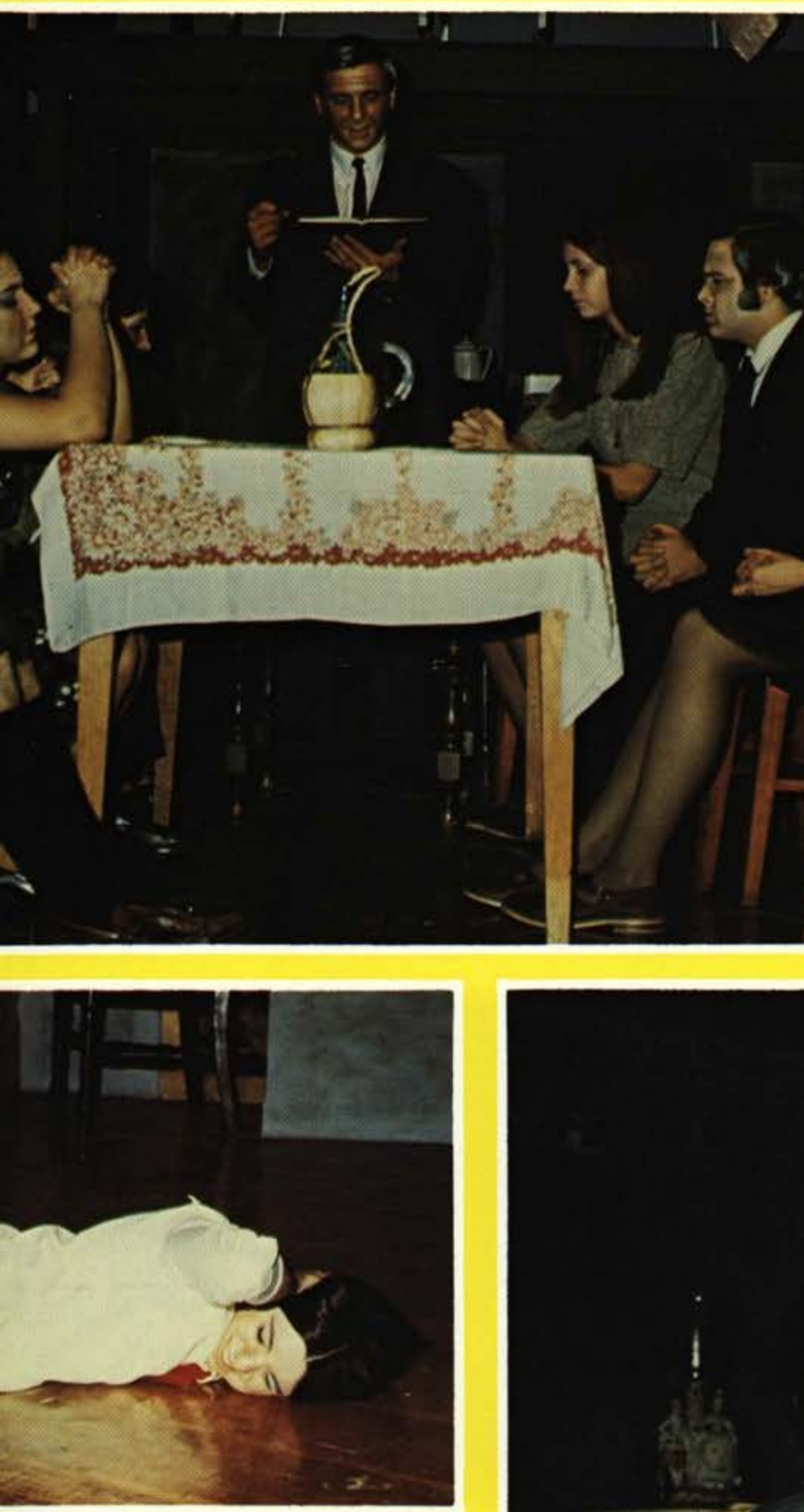

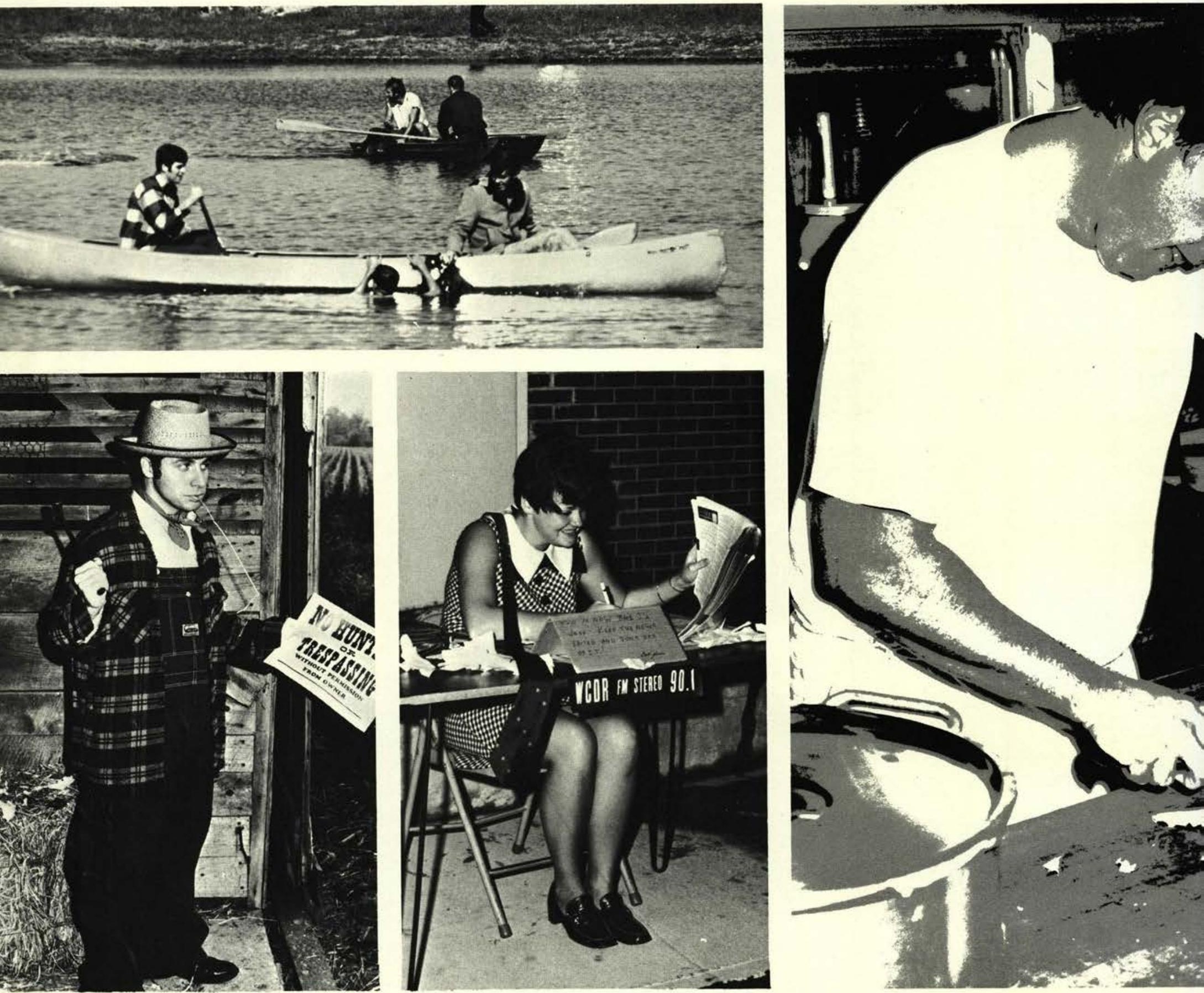

\section{THAT'S LIFELIFELF}



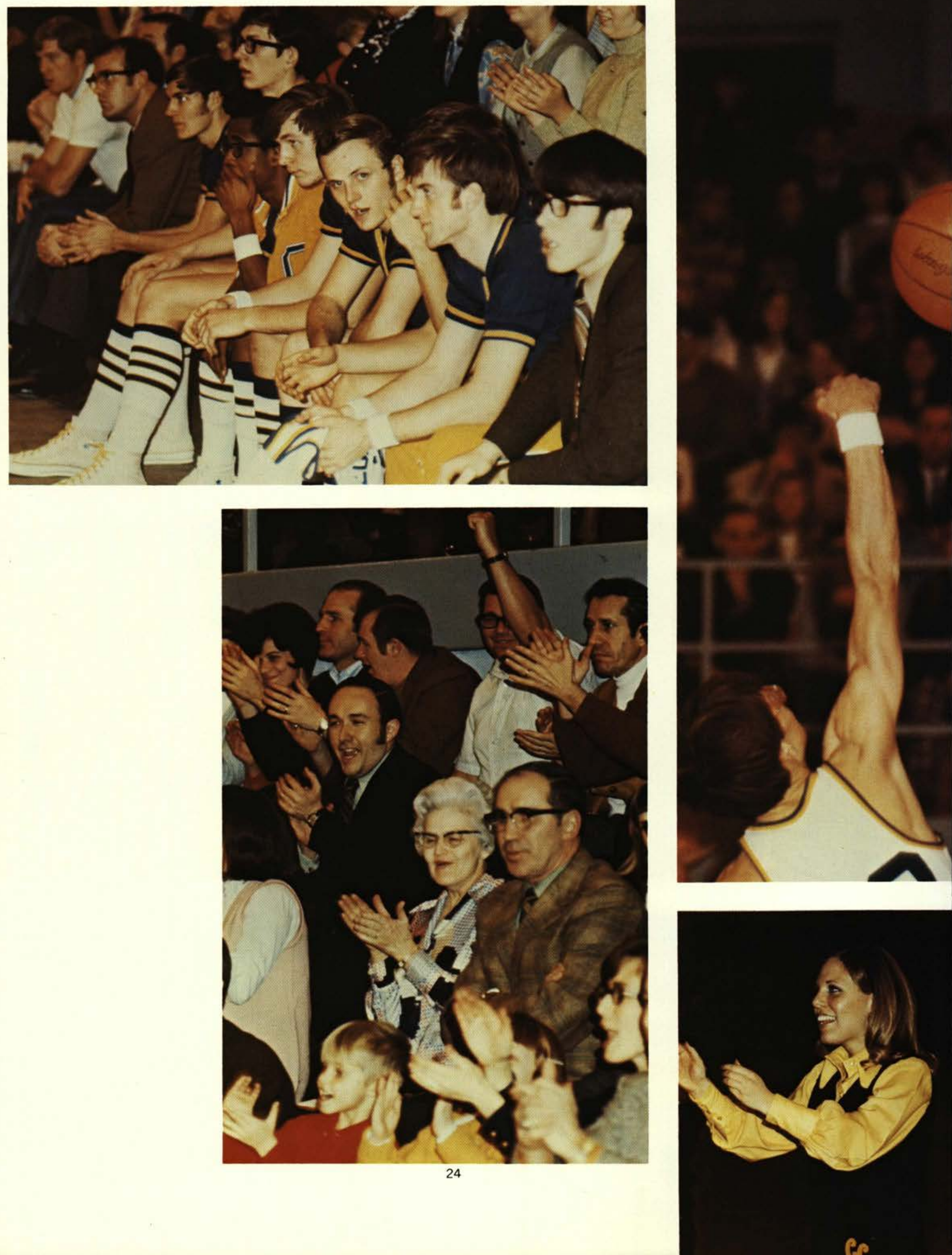


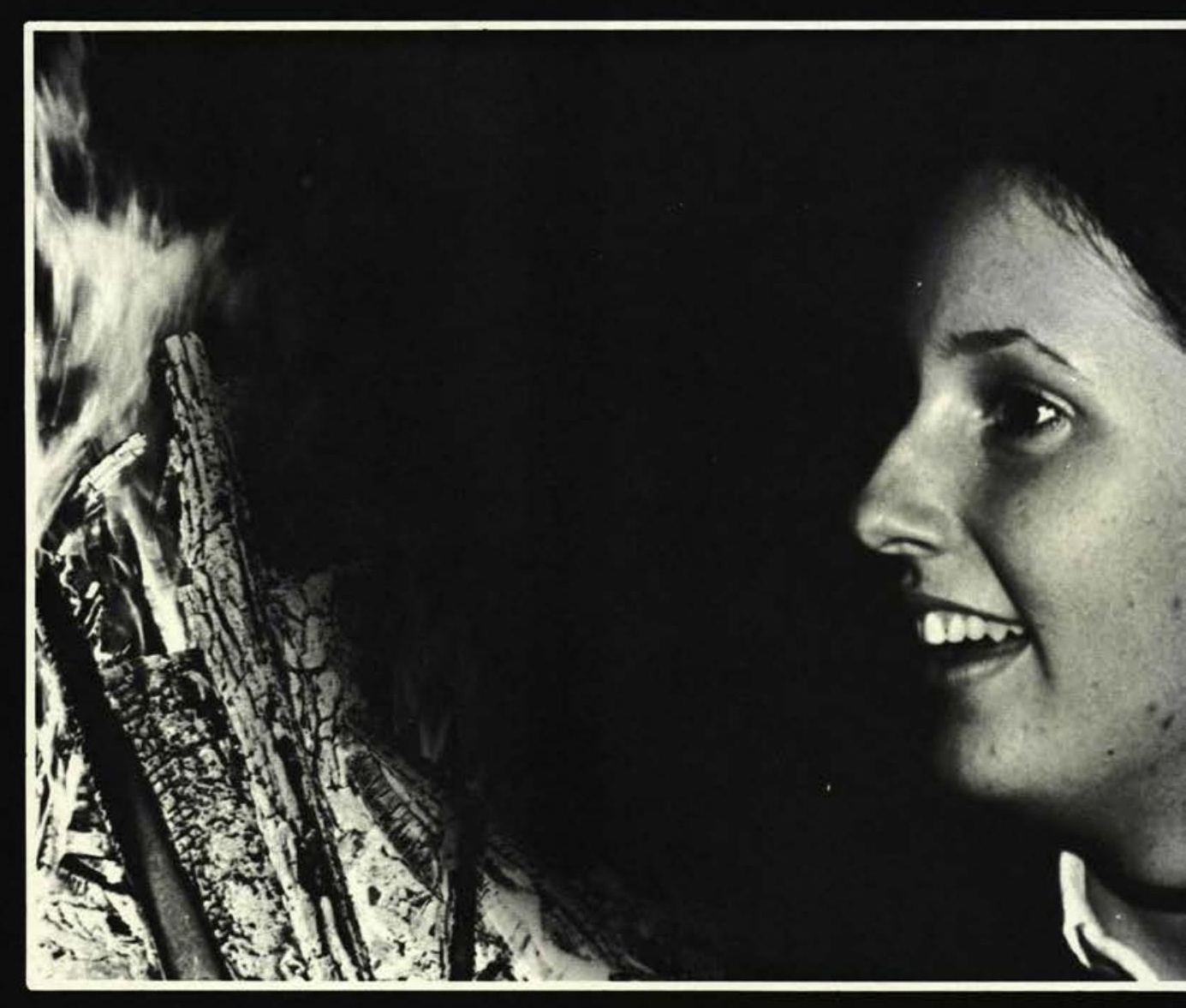

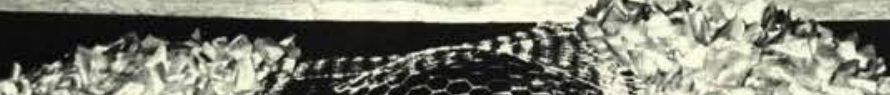

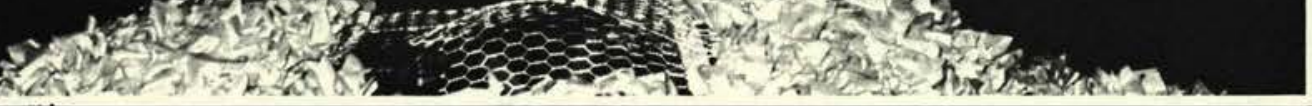
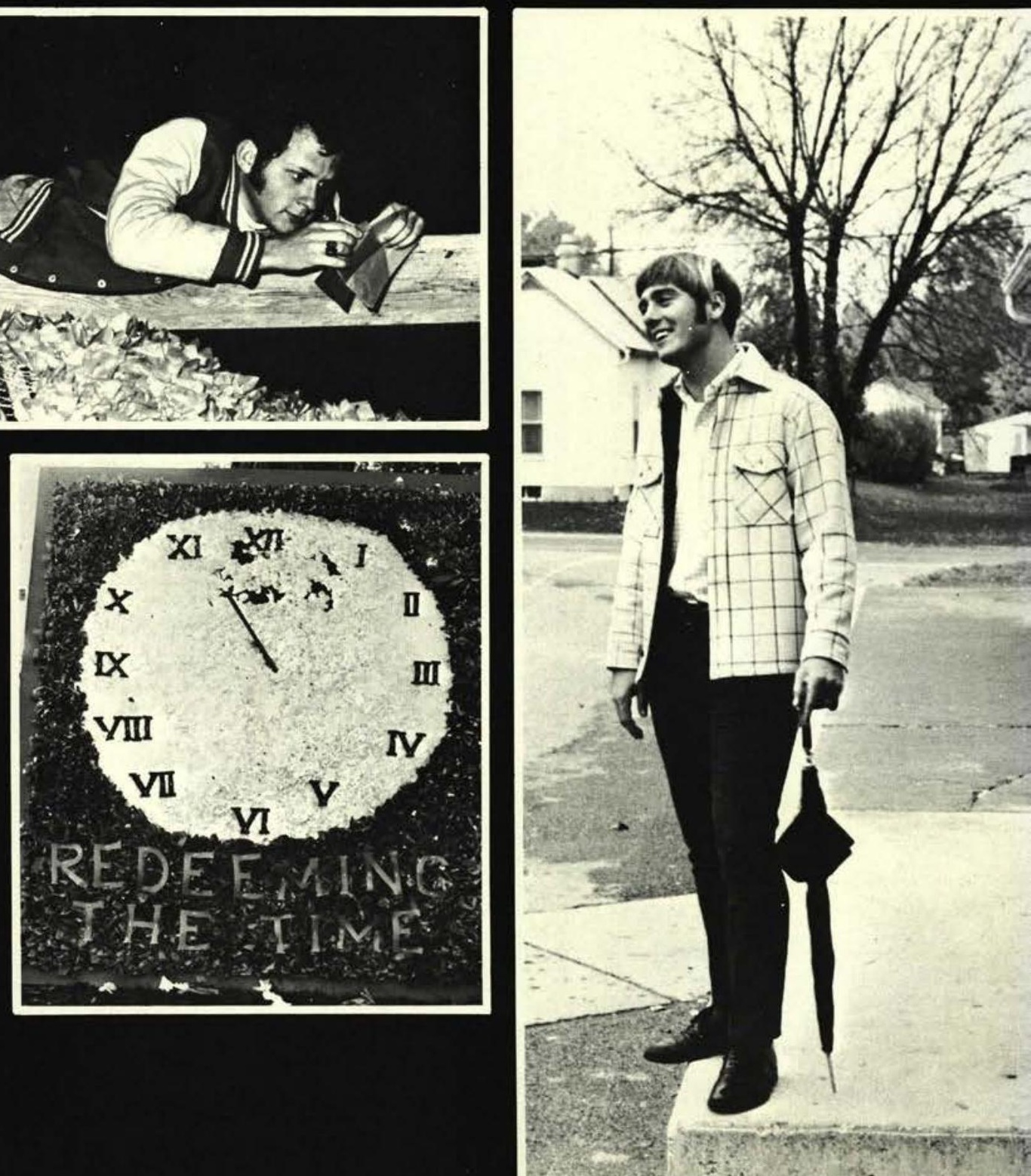


\section{A Face in the crowd? Don't be content to claim spectator rights Be a participant!}
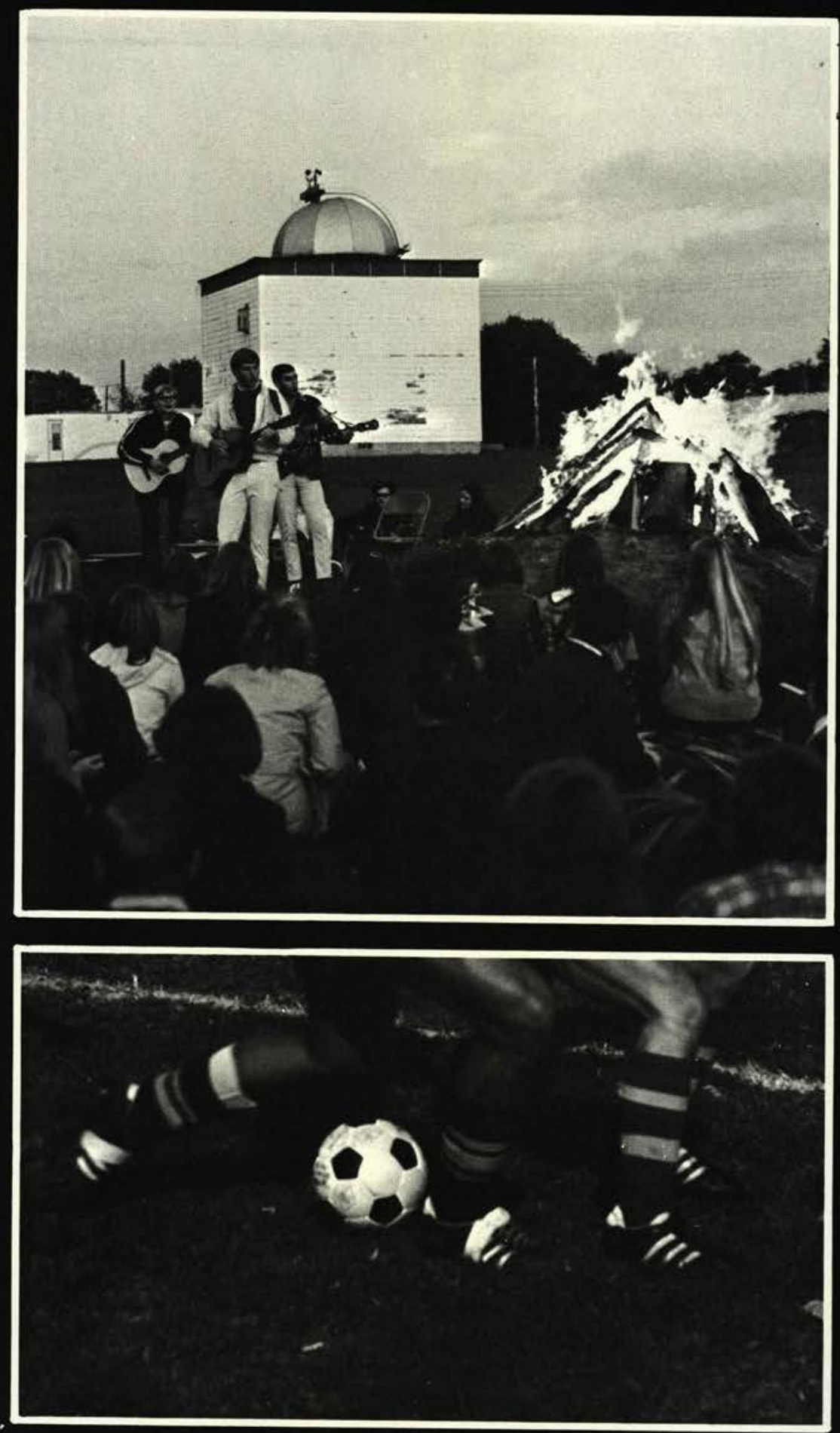

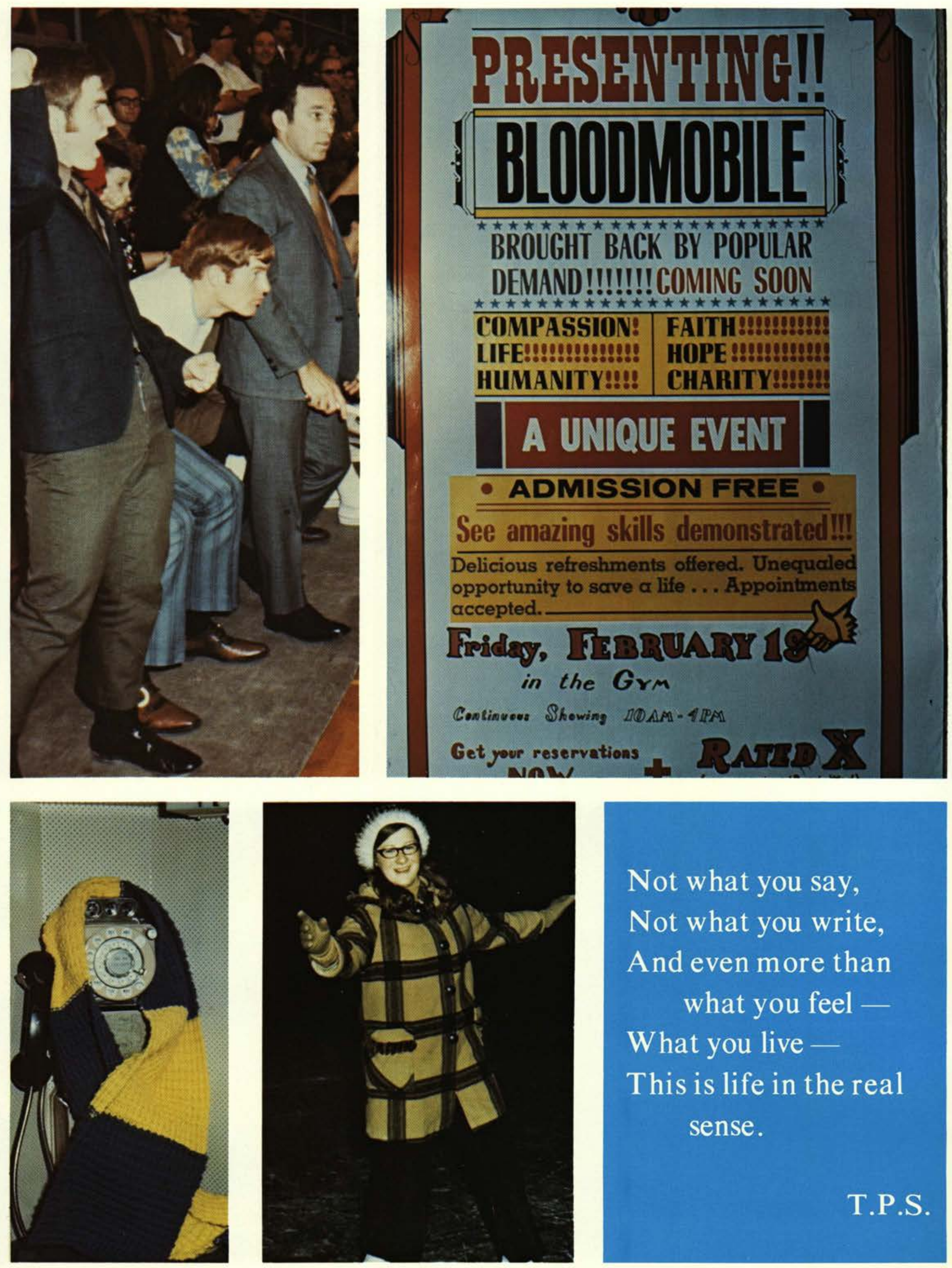

Not what you say,

Not what you write,

And even more than

what you feel -

What you live -

This is life in the real

sense.

T.P.S. 

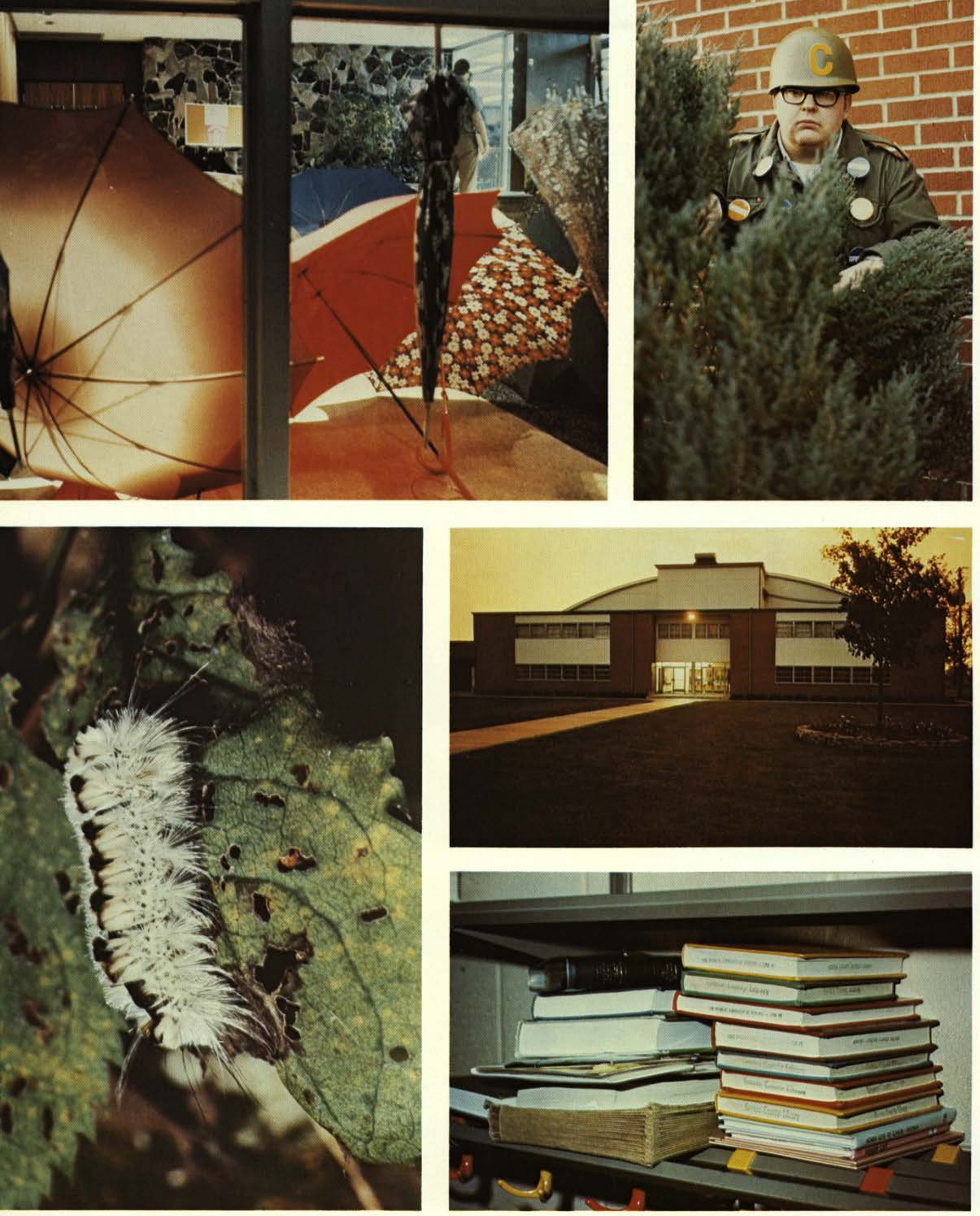


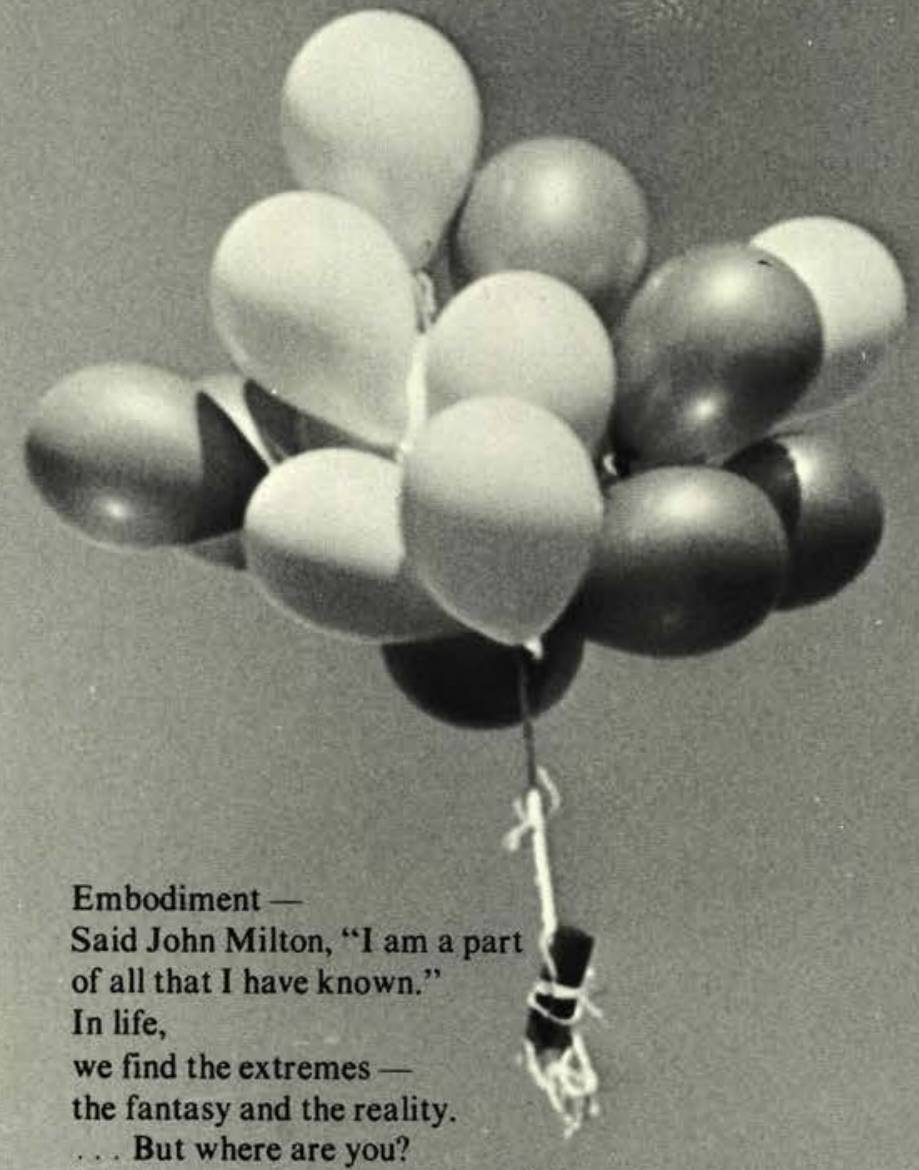

... But where are you?

The fantasy of balloons released,

floating, drifting, scattering

downward and outward ..

knoweth not where they may fall to

earth ...

unanchored, free,

without a pattern to follow

or course to run..

Man, from all walks of life, searches for

the unknown

in every corner of the earth,

searching for an answer

to what life really is.

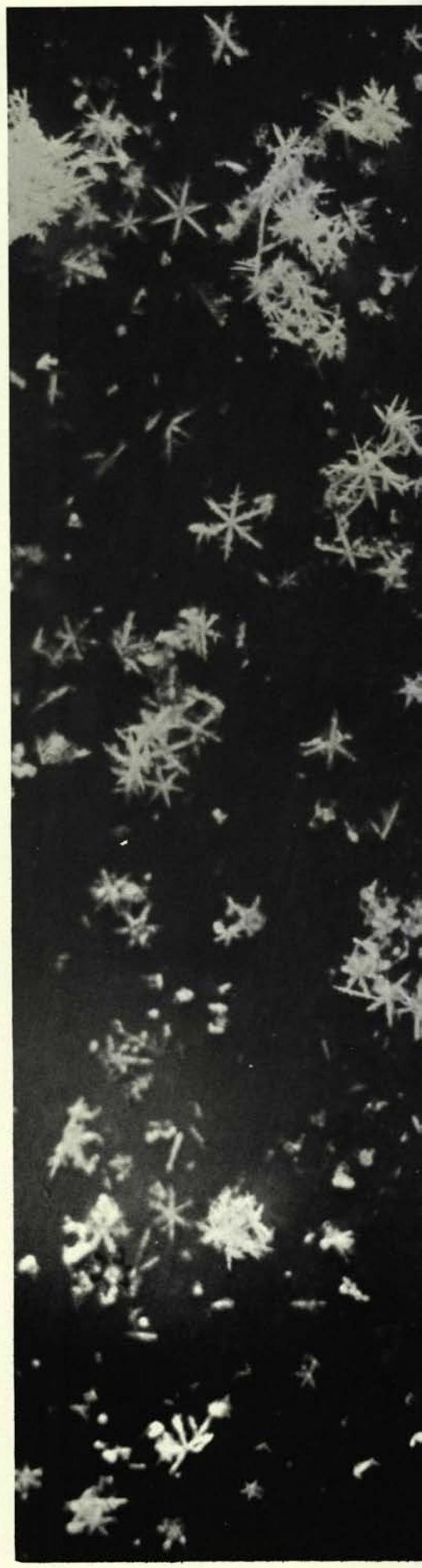



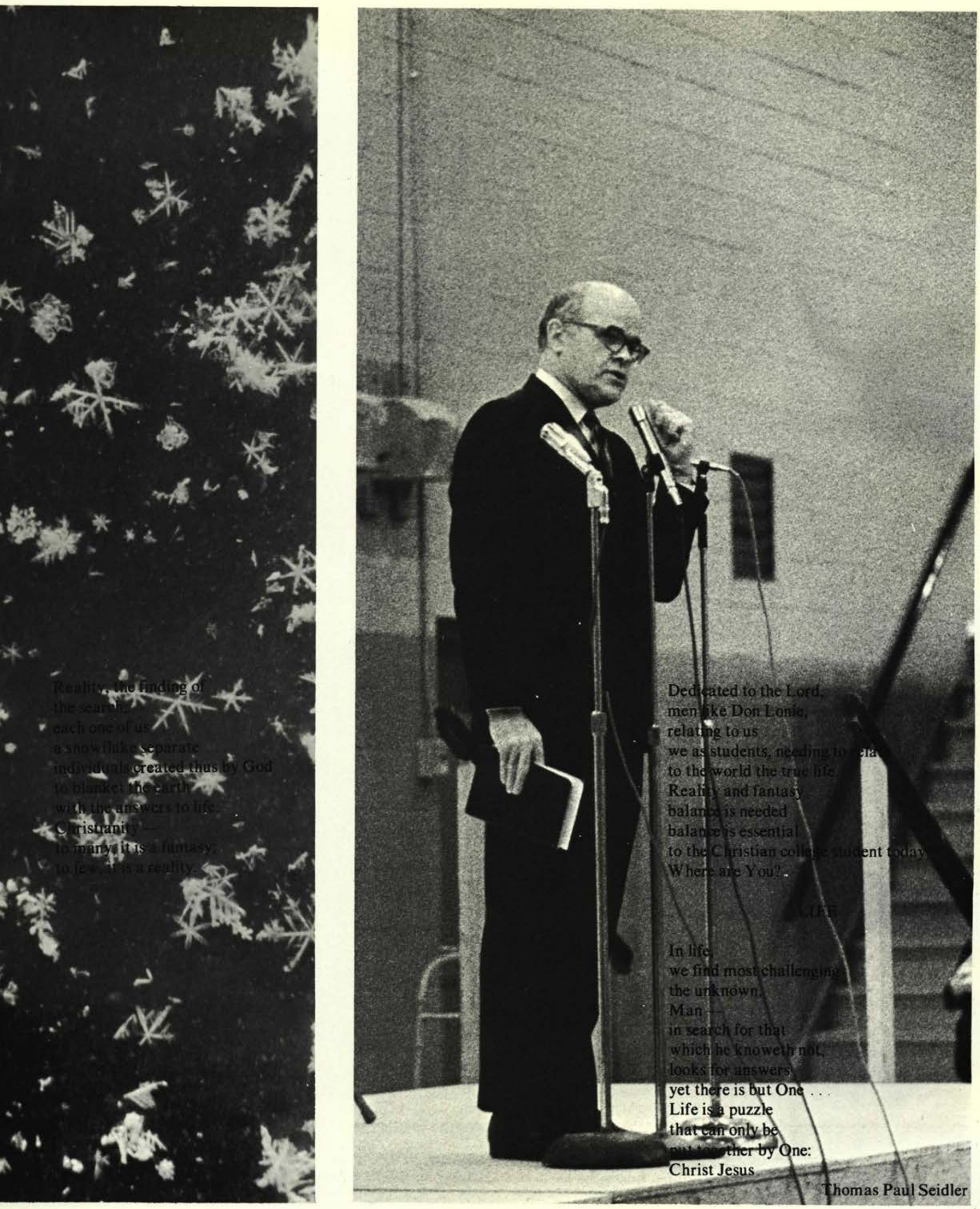


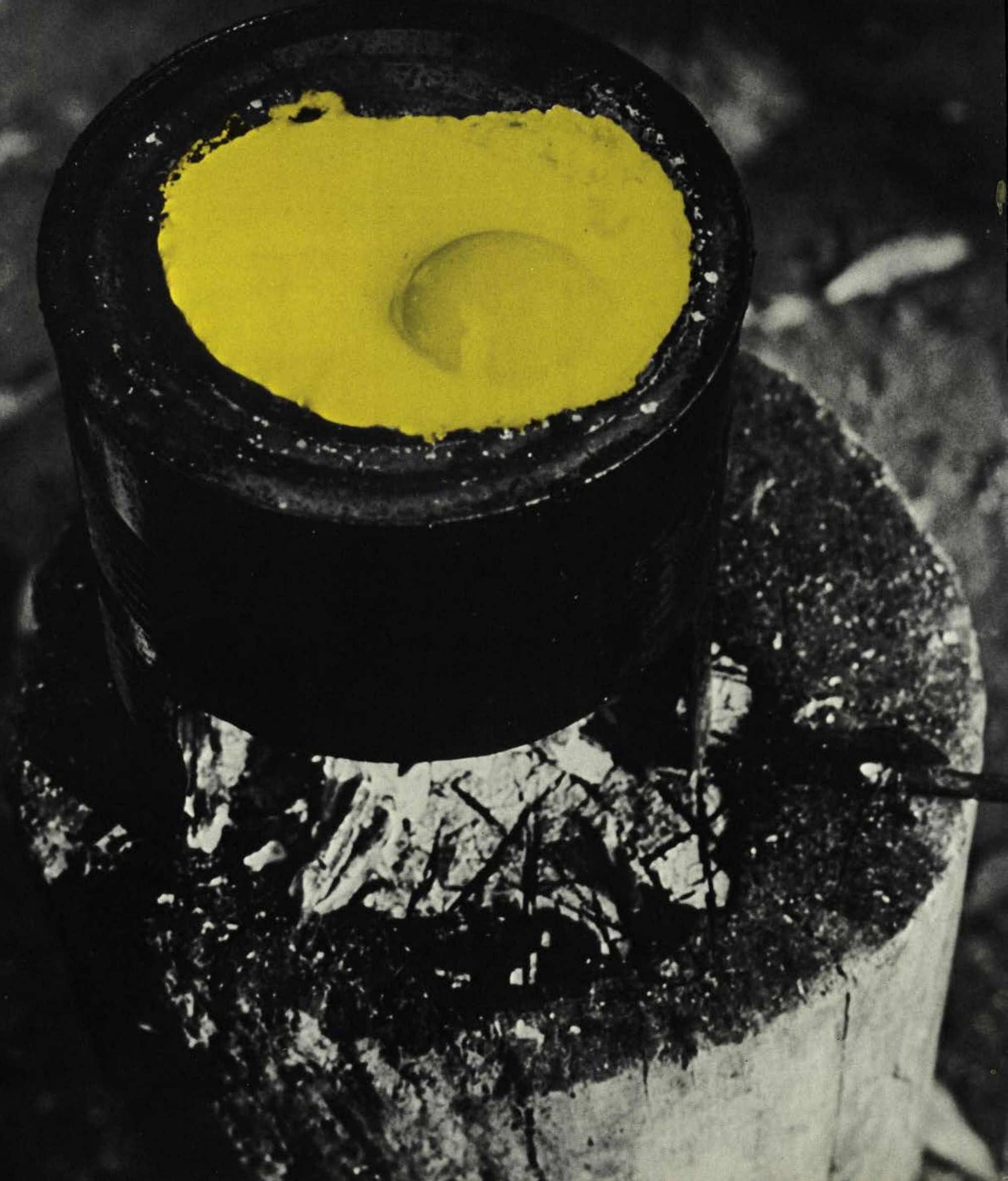


The Satisfaction of Giving

We came, we saw,

we fainted.

"It only hurts when I laugh."

147 pints, and

a first place trophy

The satisfaction of giving -

And no one had to say,

"thank you".

The Ice Follies

A cold night

A clear sk y

A blazing bonfire

A mug of hot chocolate,

And a donut or two

Cold cheeks

Bruised knees

Frozen toes

A numb nose

but it was fun.
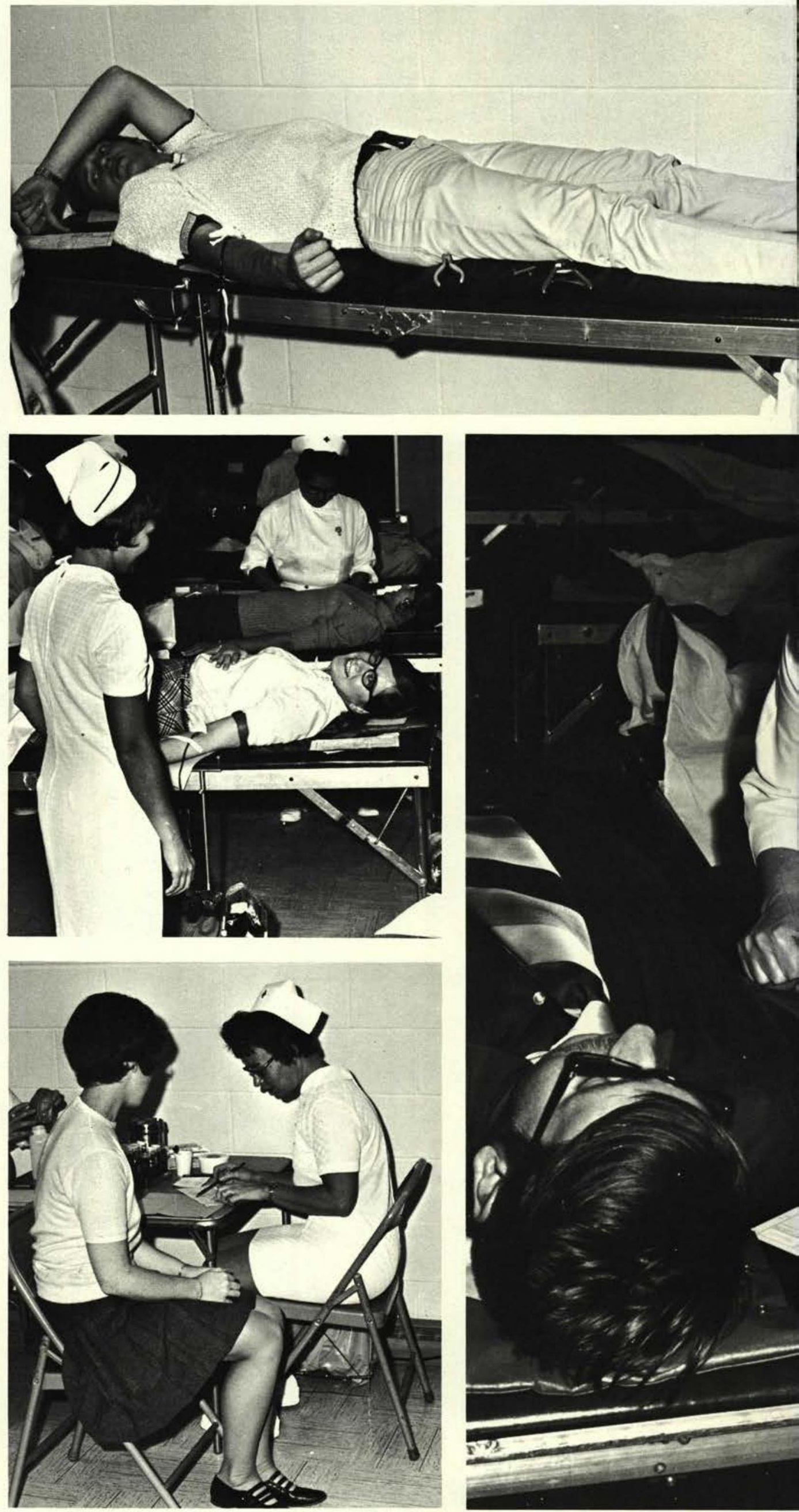

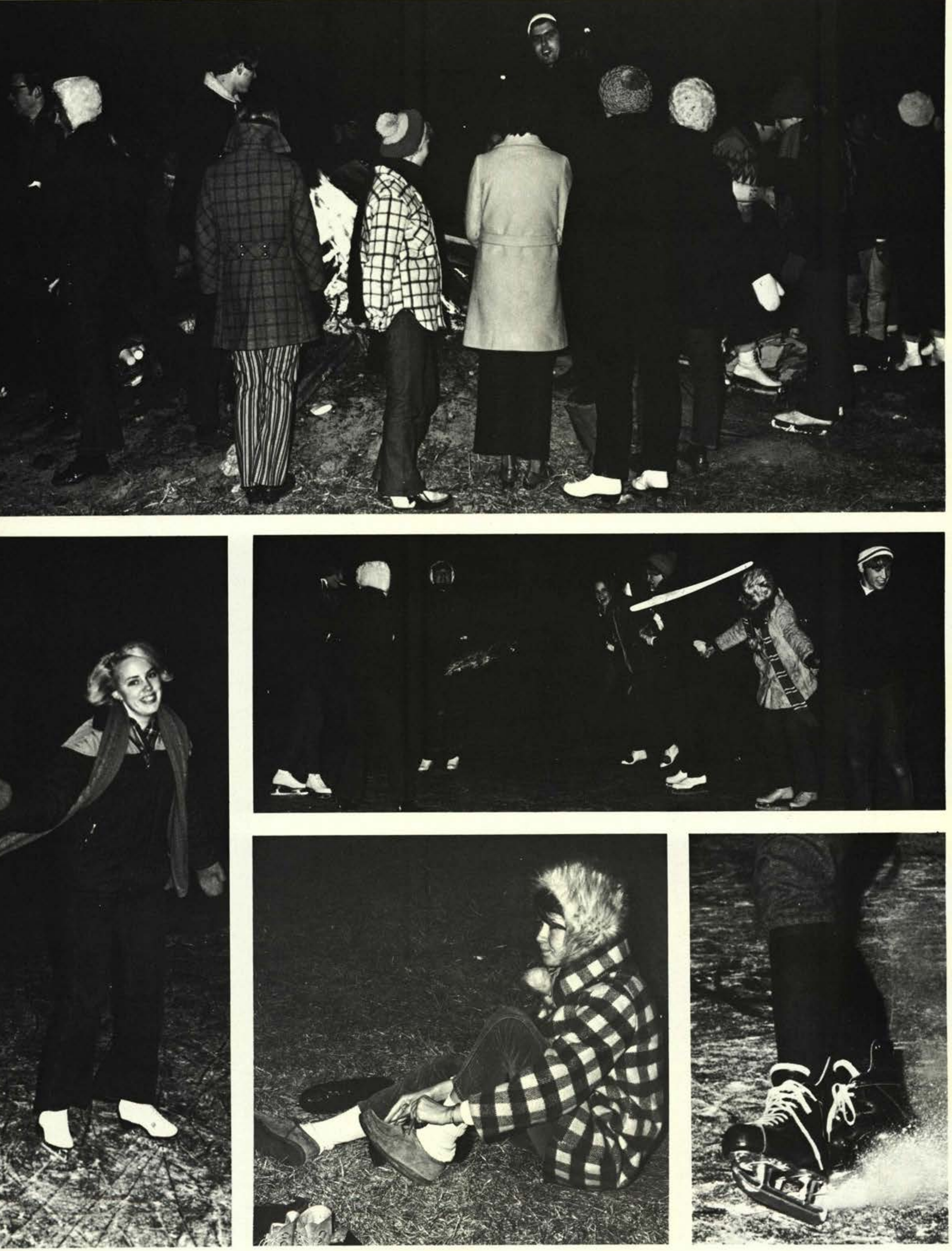


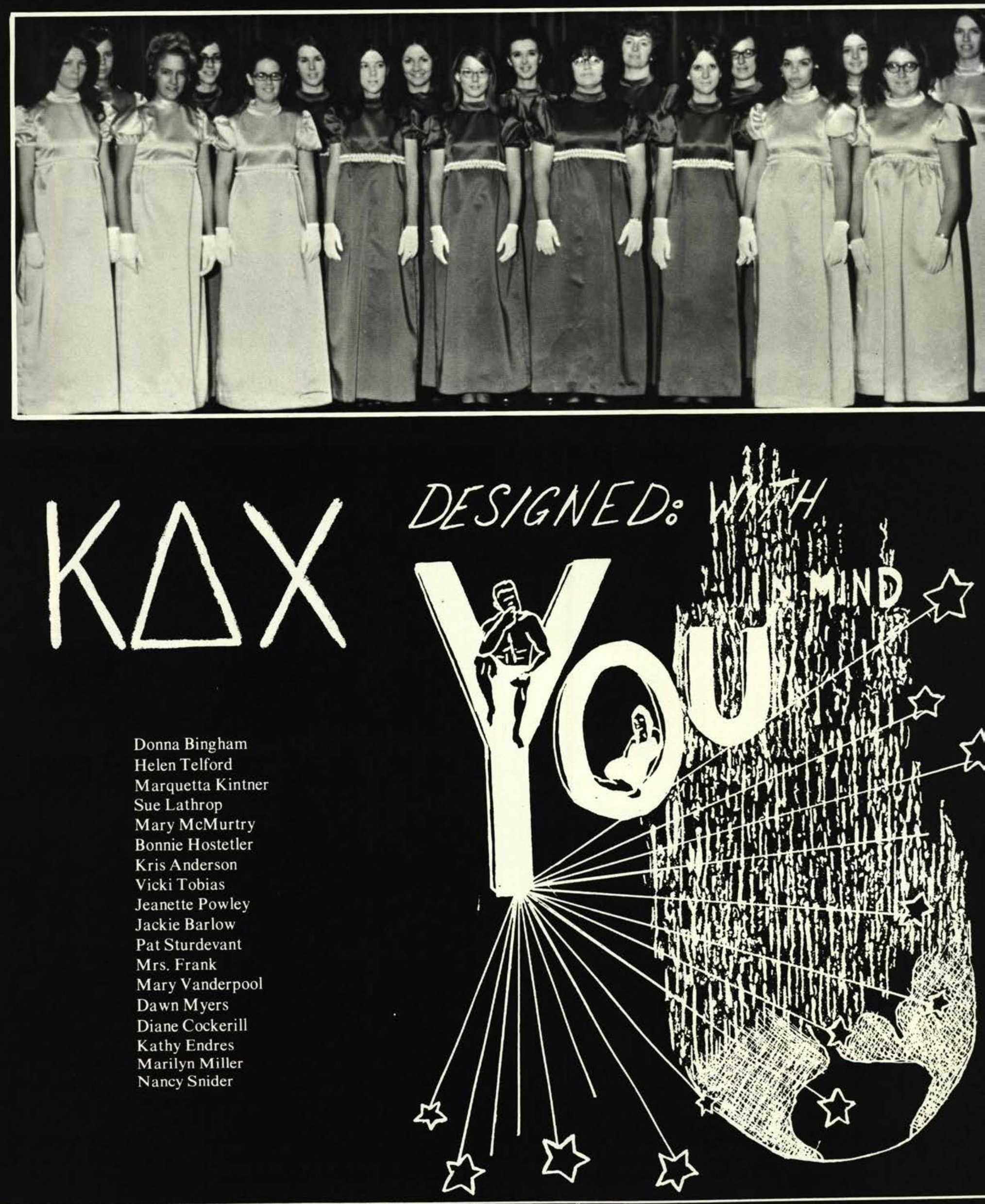



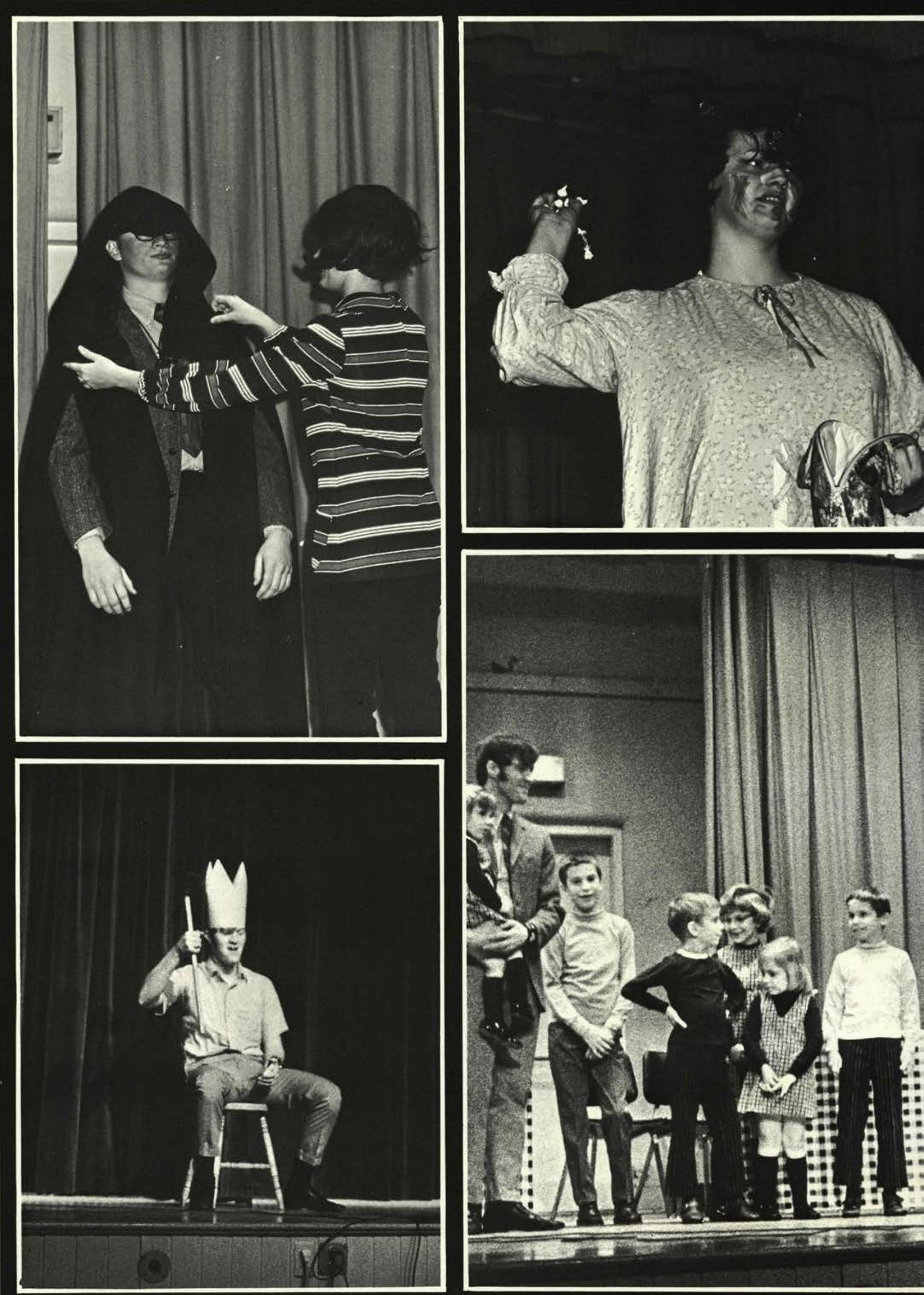

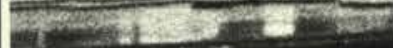




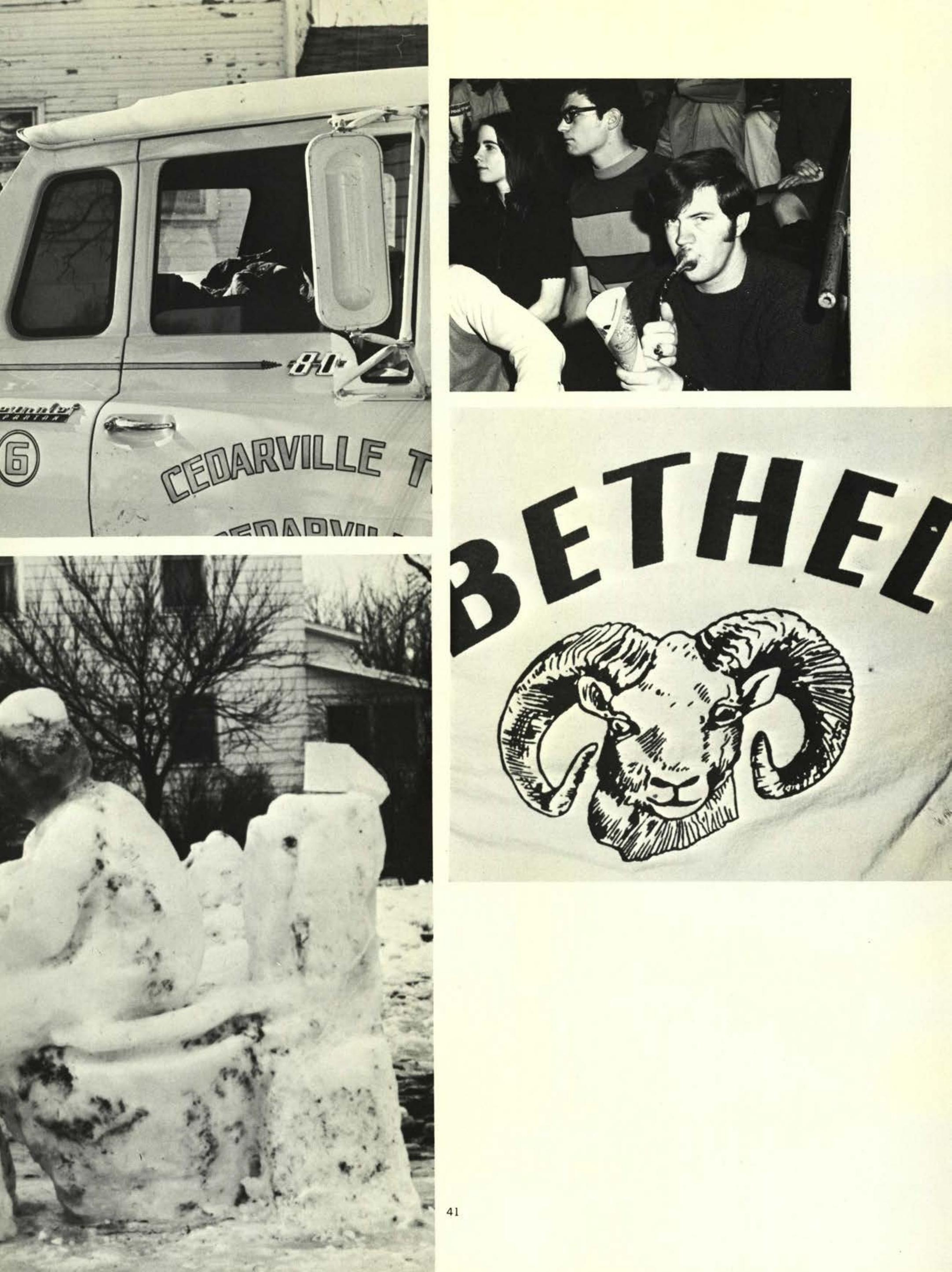



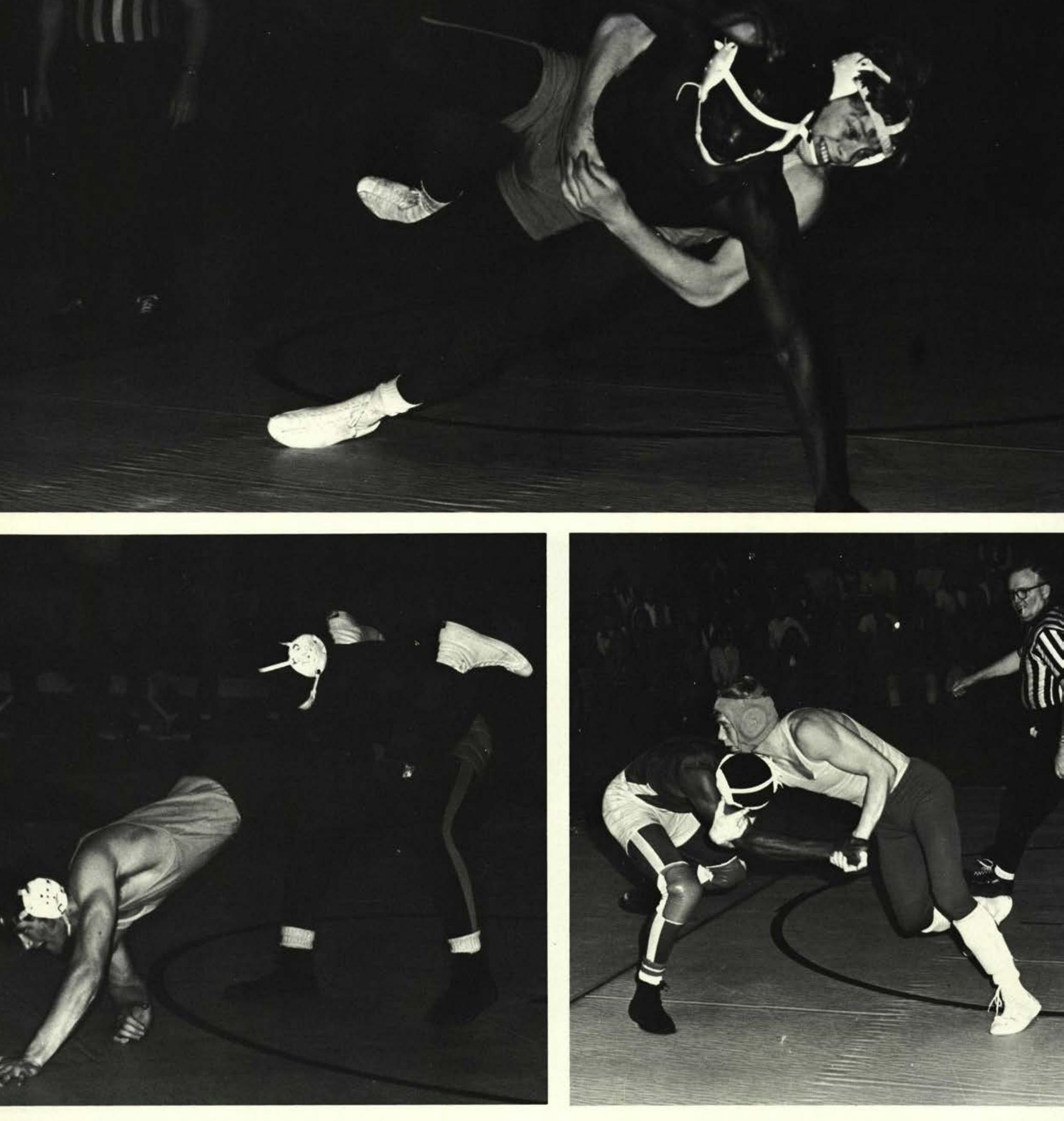

Our first year in the conference 

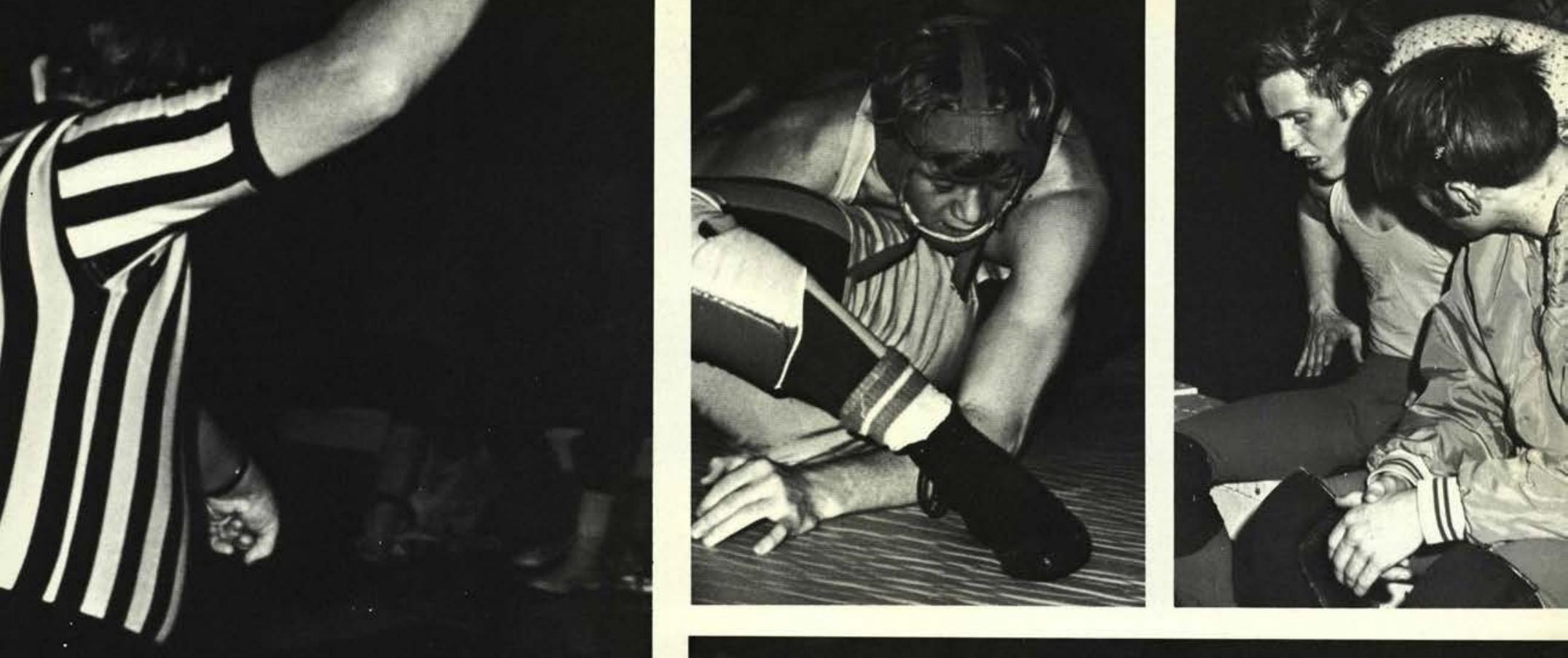

)
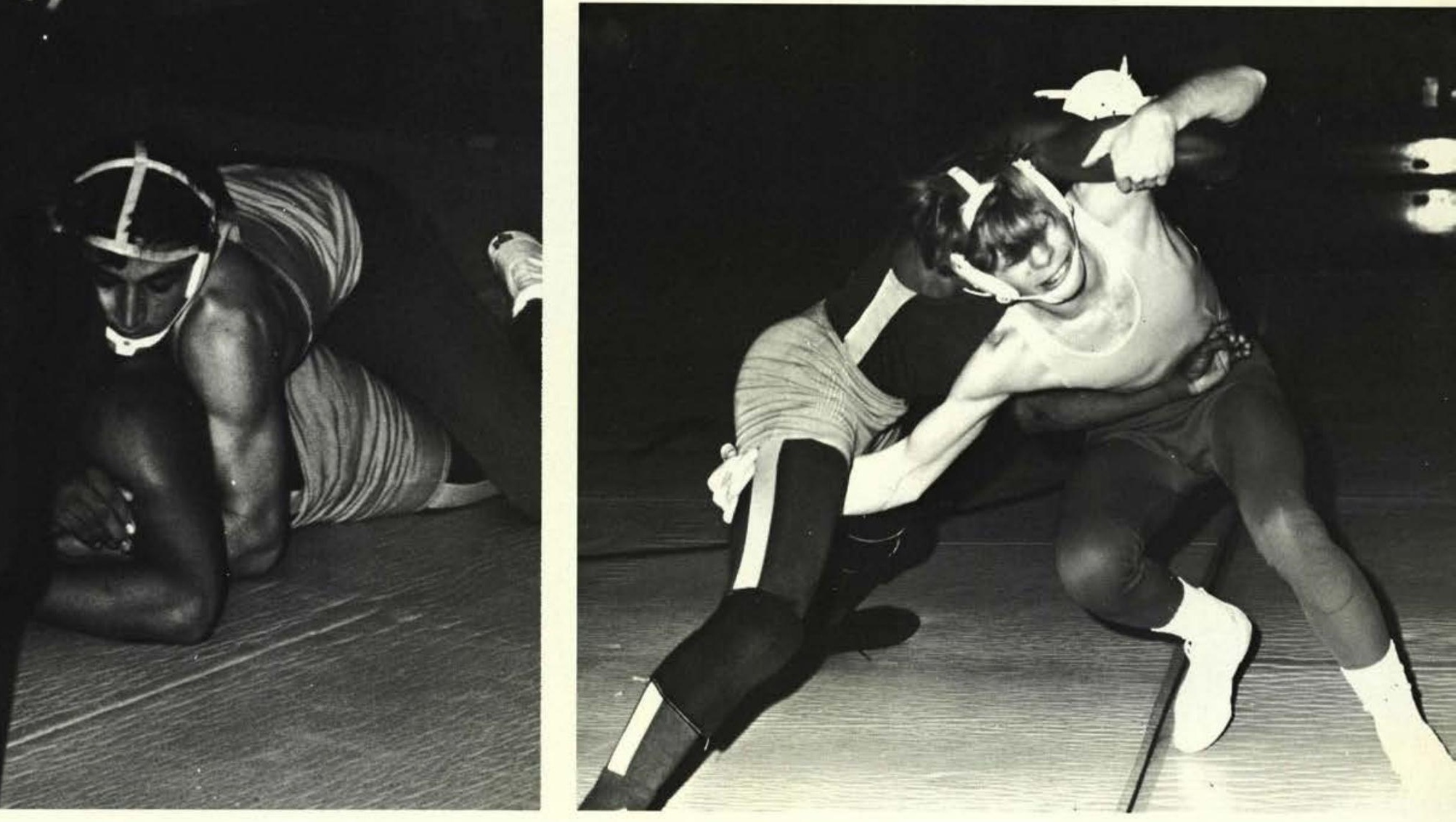

Prayer

Preparation

Mounting excitement

A new opponent

A good hold

A word from the coach

A call by the referee

A rough fight

Another win

The satisfaction of knowing

that you've done your best. 


\section{Duties, Diversions, Discussions . .}
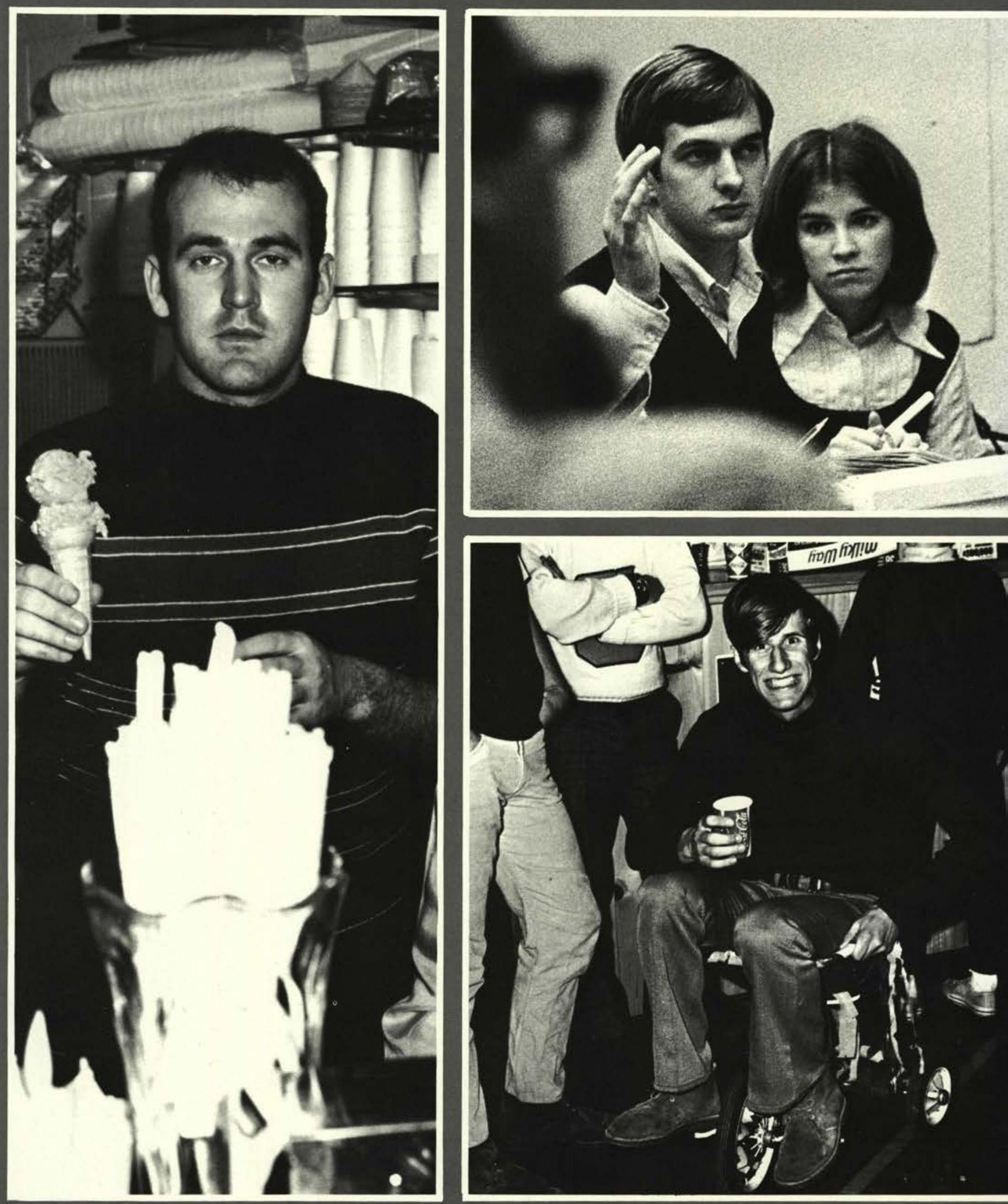
Development
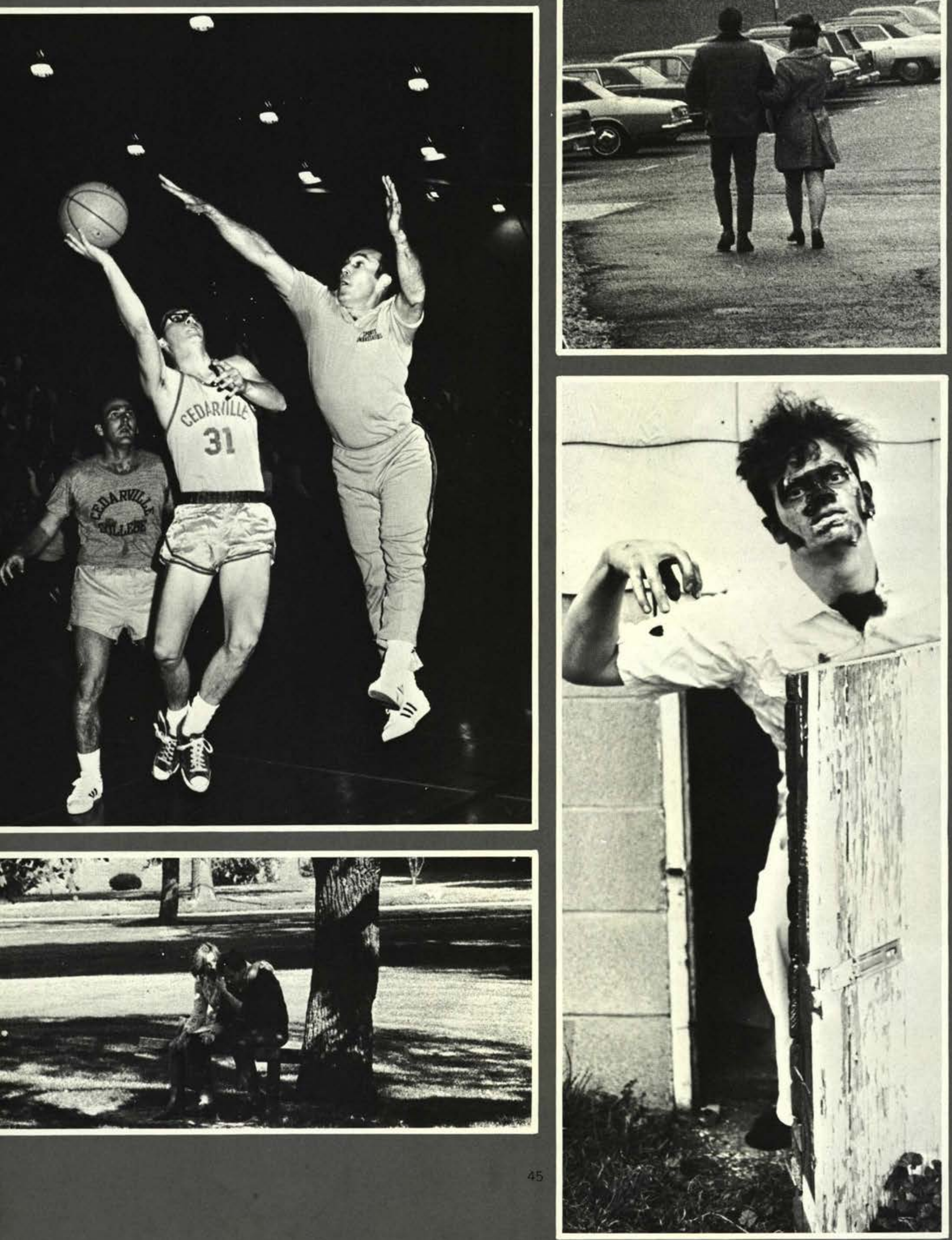

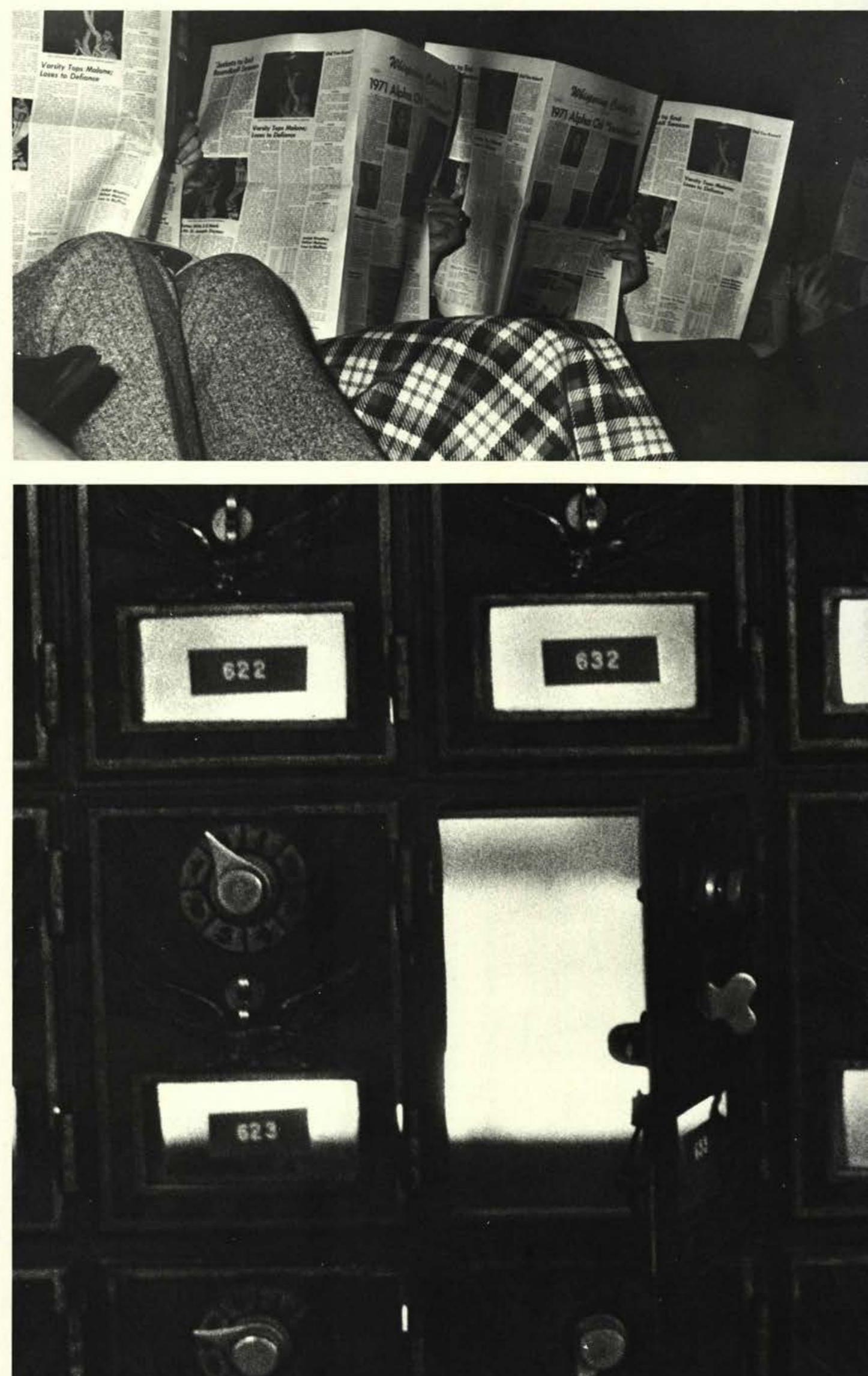


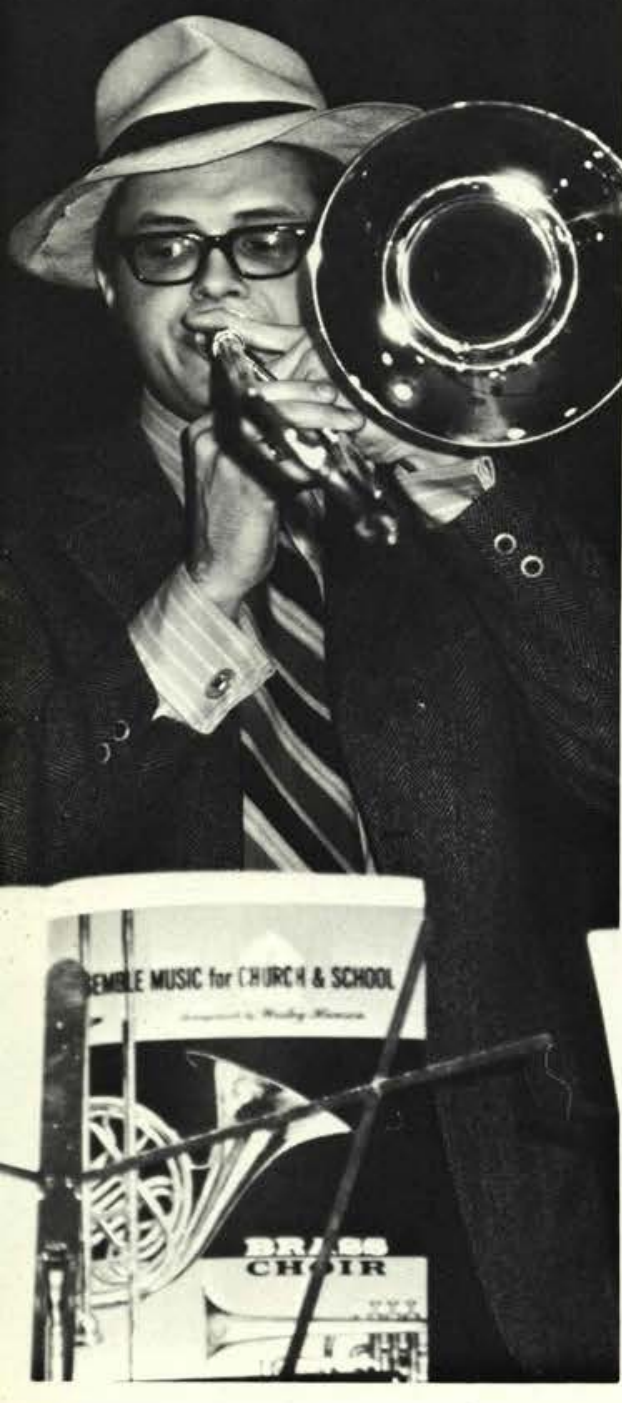

\section{Kaleidoscope of college life ...}
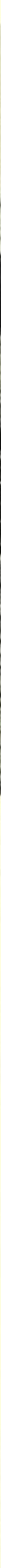

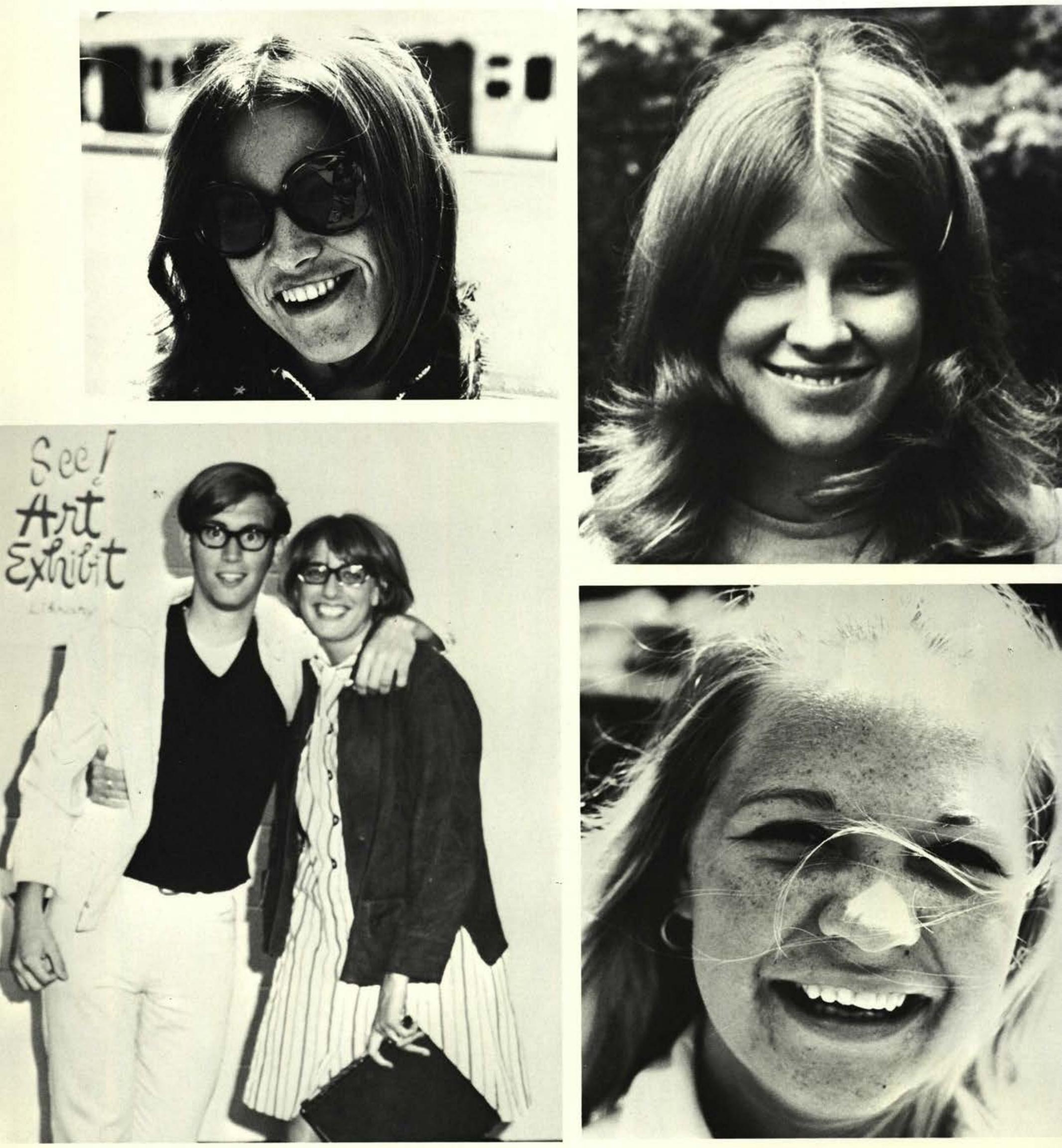

Smiles are contagious, if you are given one - share it with someone else. 


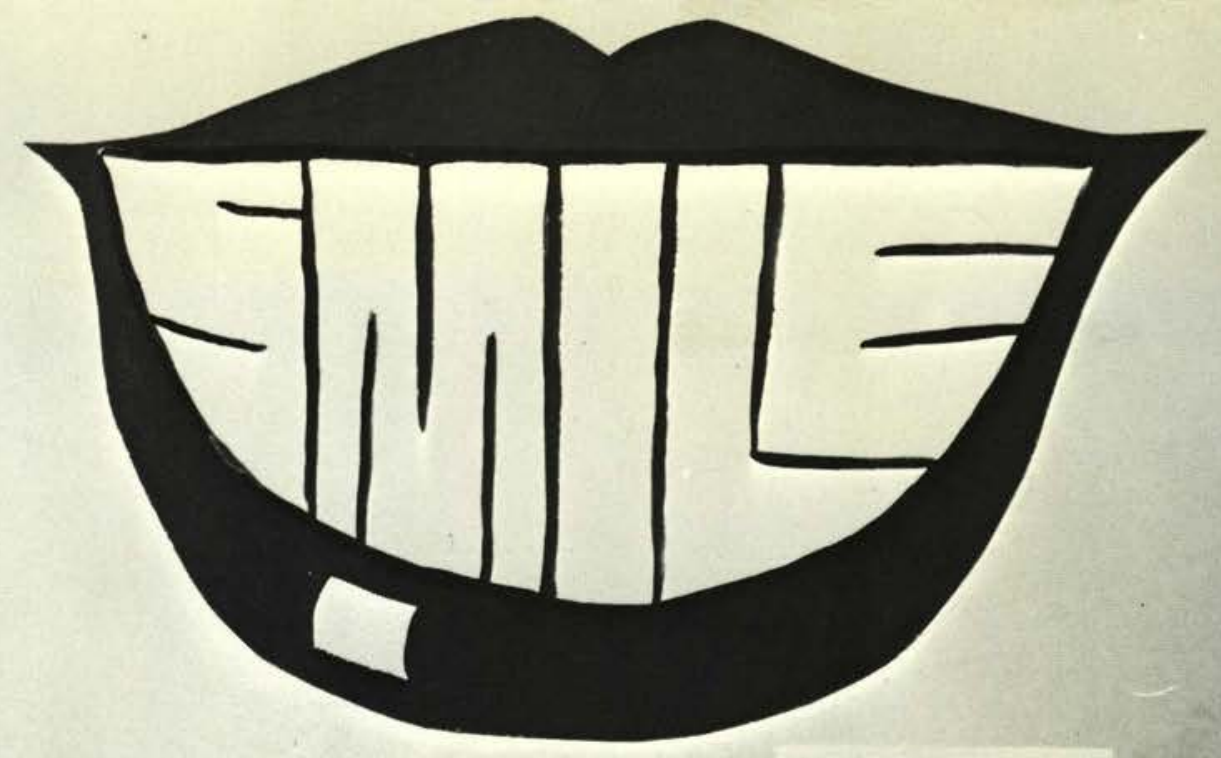

Word Form

by Mary Ans Butcher
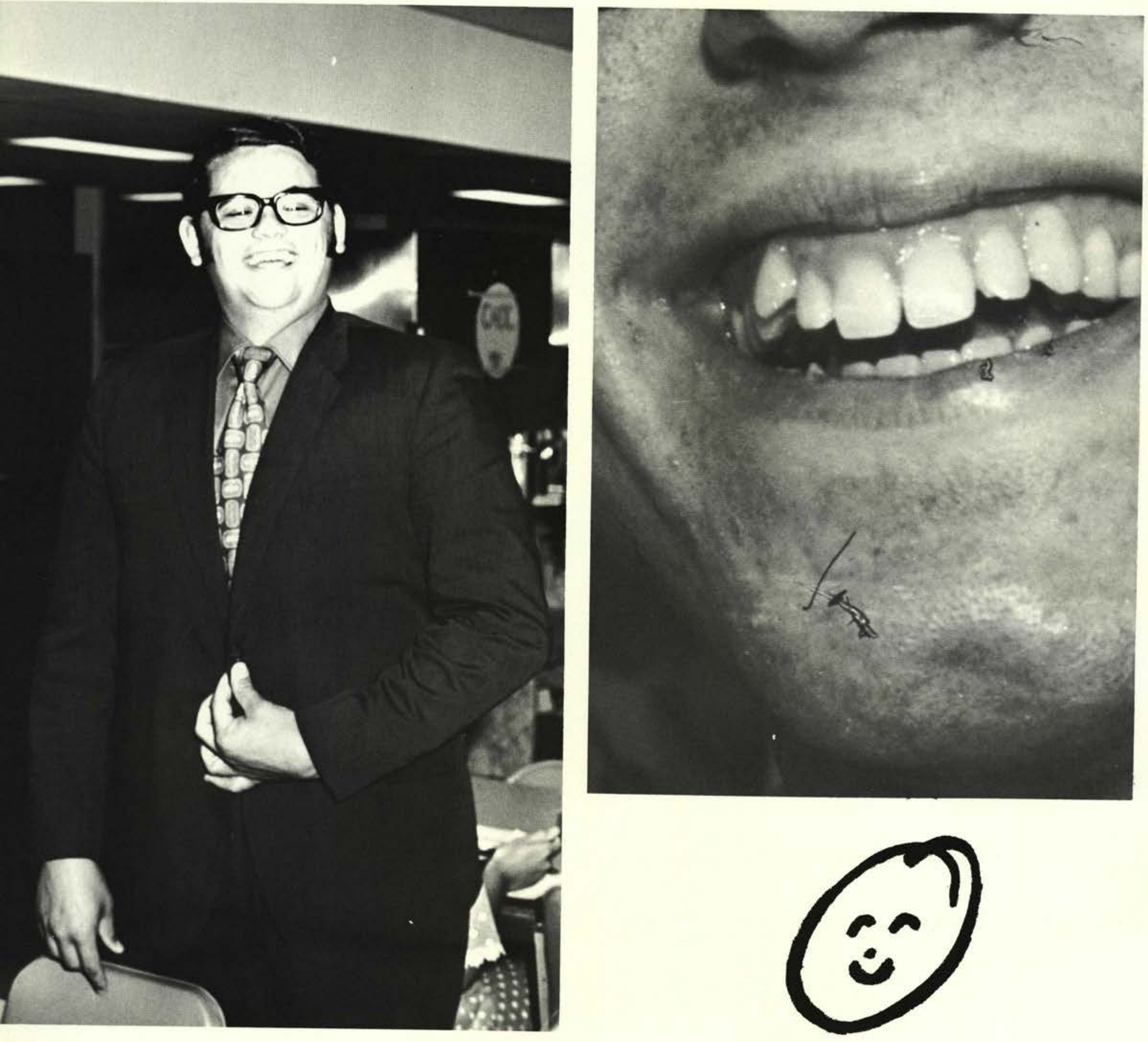


\section{Connie Bush}

\section{Paul Wheeler}
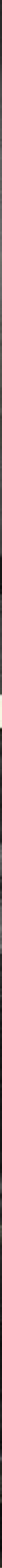

Dianne Kemp 


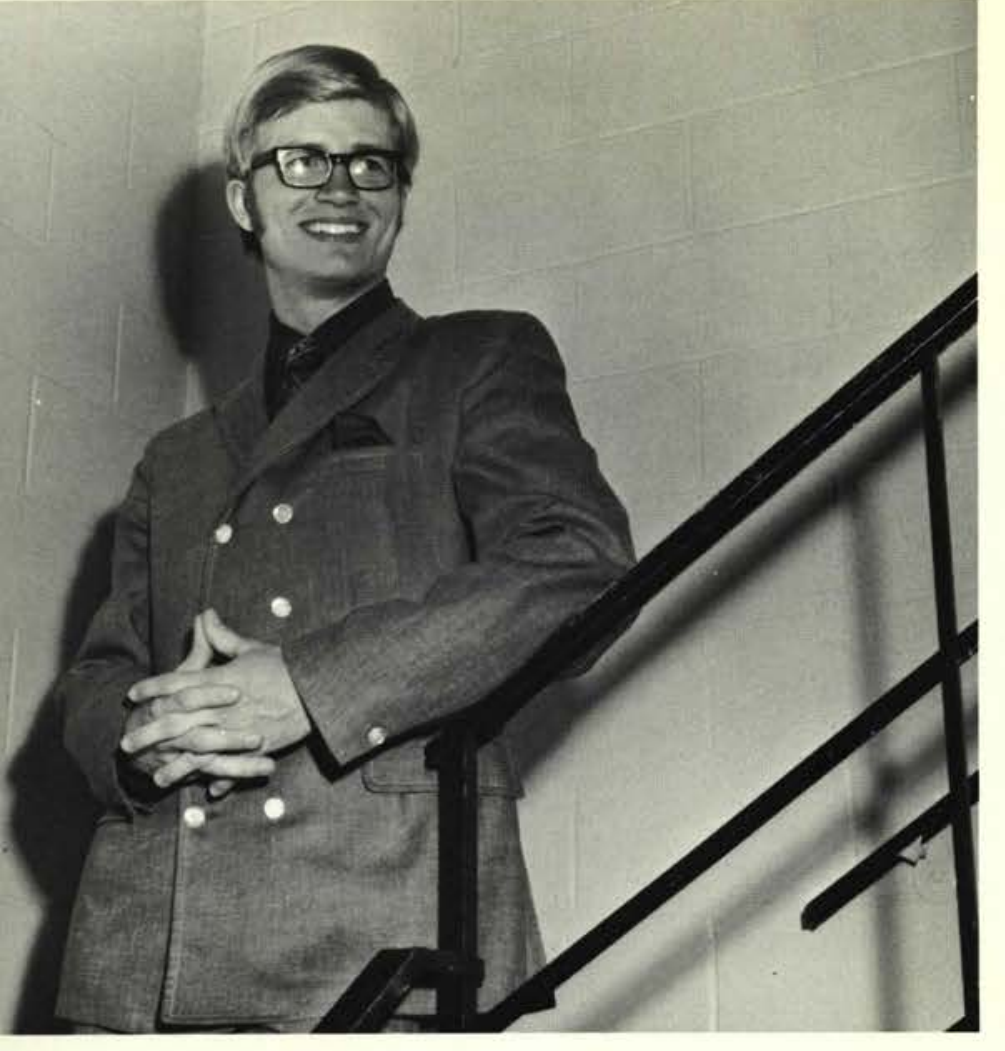

\section{Who's Who}

\section{Jeff Seeley}
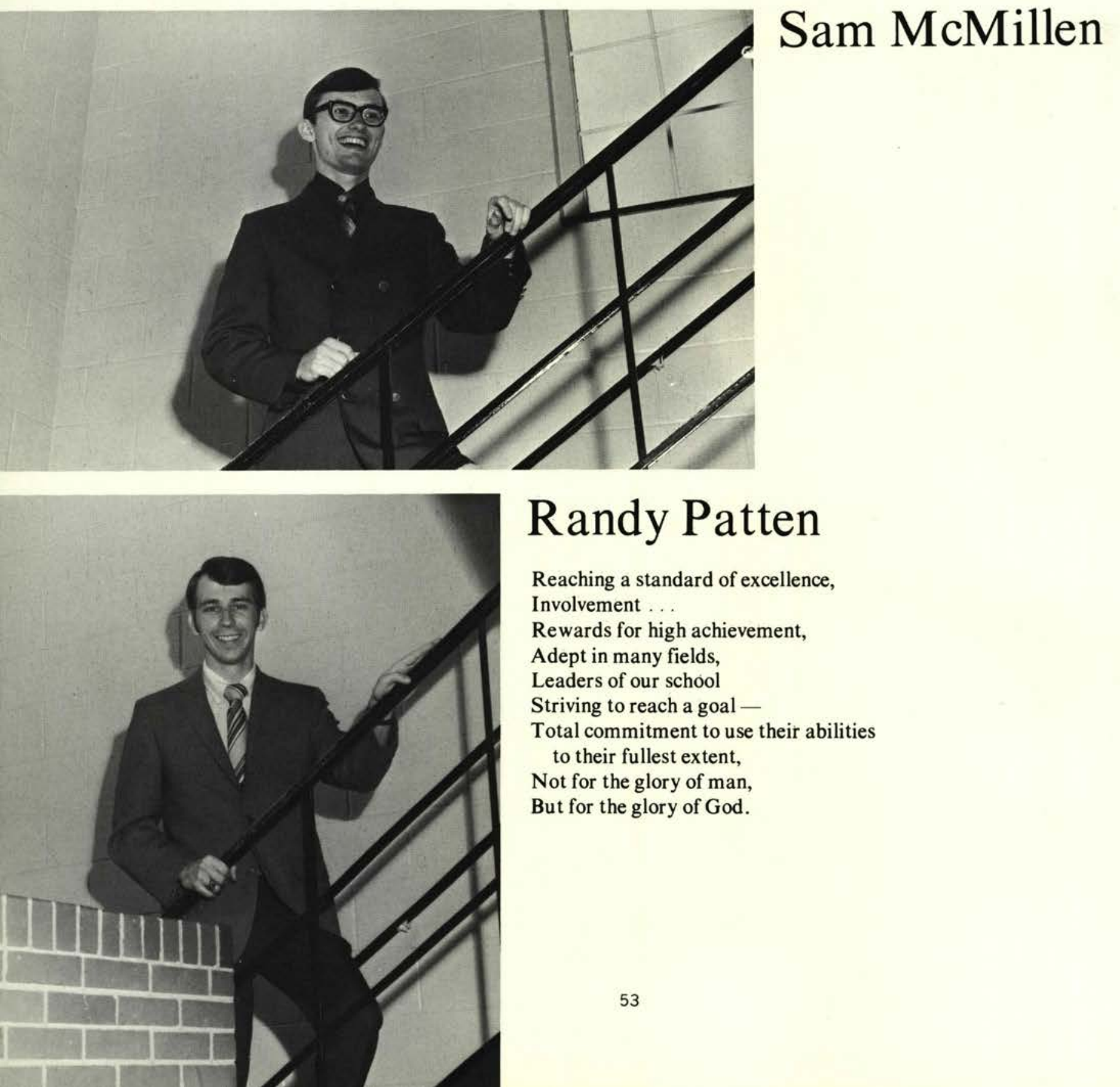

Randy Patten

Reaching a standard of excellence,

Involvement . .

Rewards for high achievement,

Adept in many fields,

Leaders of our school

Striving to reach a goal -

Total commitment to use their abilities to their fullest extent,

Not for the glory of man,

But for the glory of God. 

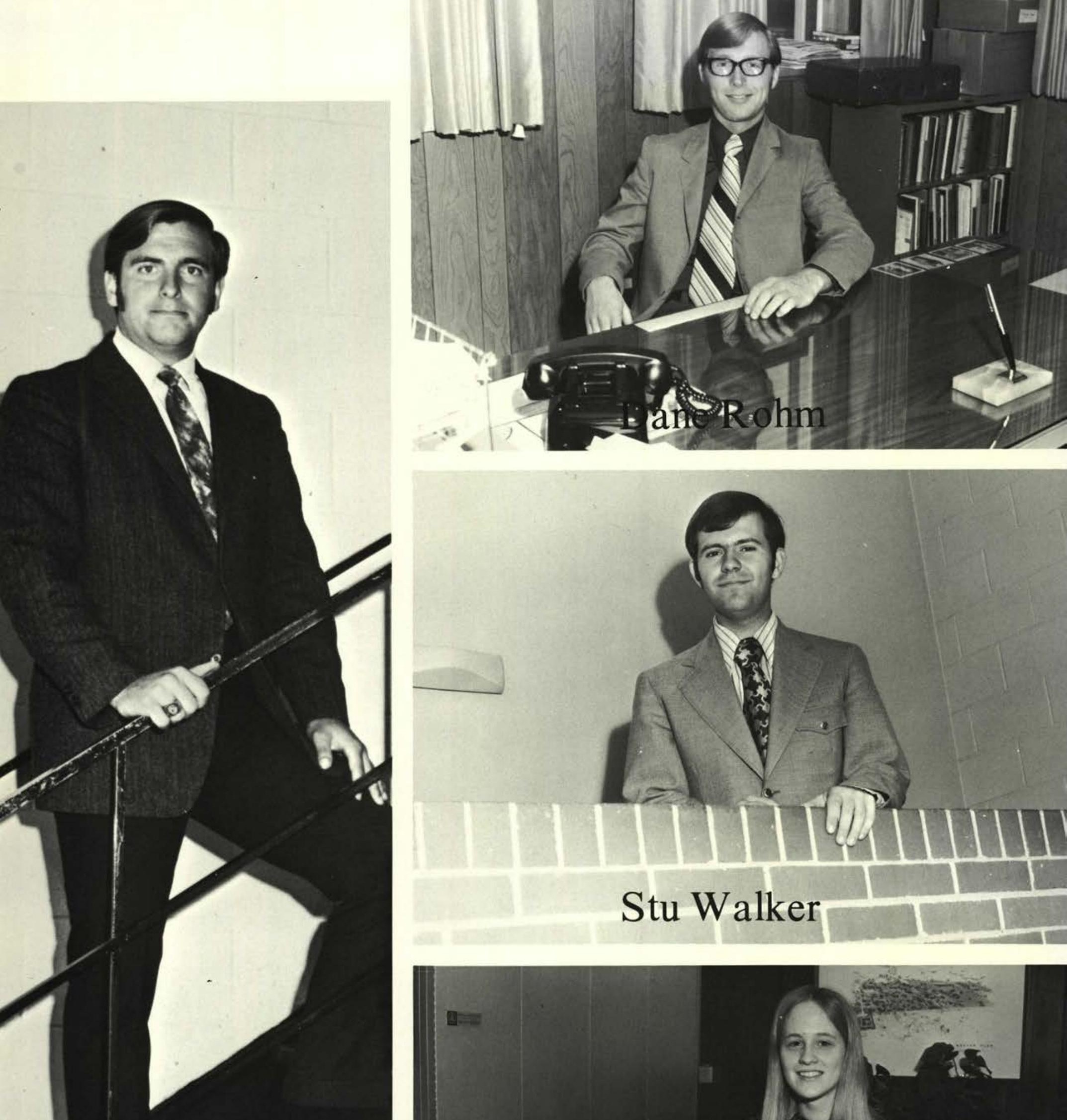

Stu Walker

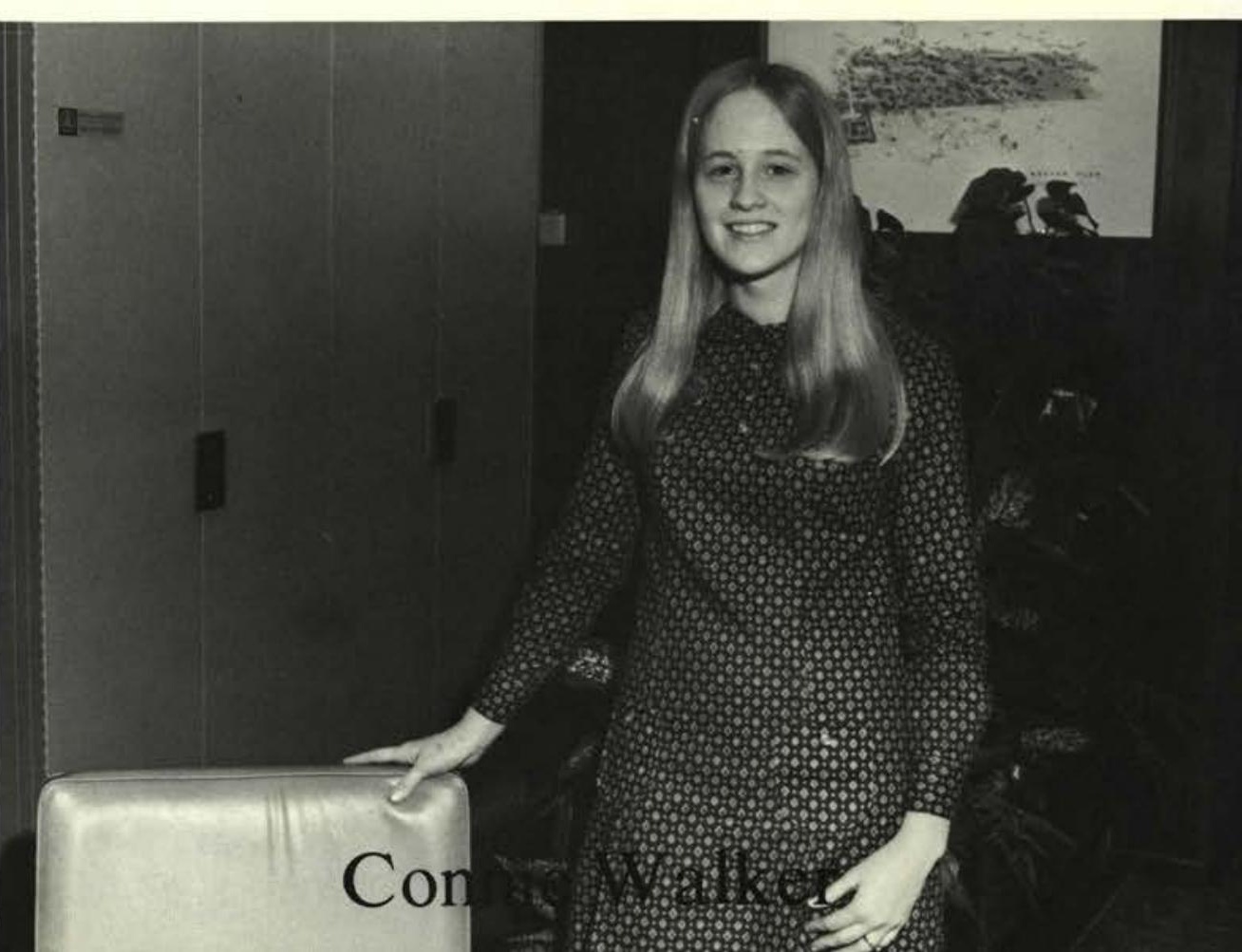




\title{
Snack Shop Charades
}

\author{
Composite of the Cedar-world \\ Role-playing ... \\ Self and others - \\ Ten cent pistachio-in-a-cup, \\ Stacking shakers of salt \\ Medium pepperoni and cheese; \\ "Your pizza will be done in 30 minutes," \\ Round tables, \\ First dates, ... \\ Friendships born! \\ Candlelight, music, and moods; \\ Checkered tablecloths, \\ Group parties, \\ Birthday cakes, \\ Chocolate shakes, \\ Fish and chips, \\ Over the books, \\ Thoughts shared, \\ Solos paired - \\ Charades of you and $\mathrm{I}$.
}
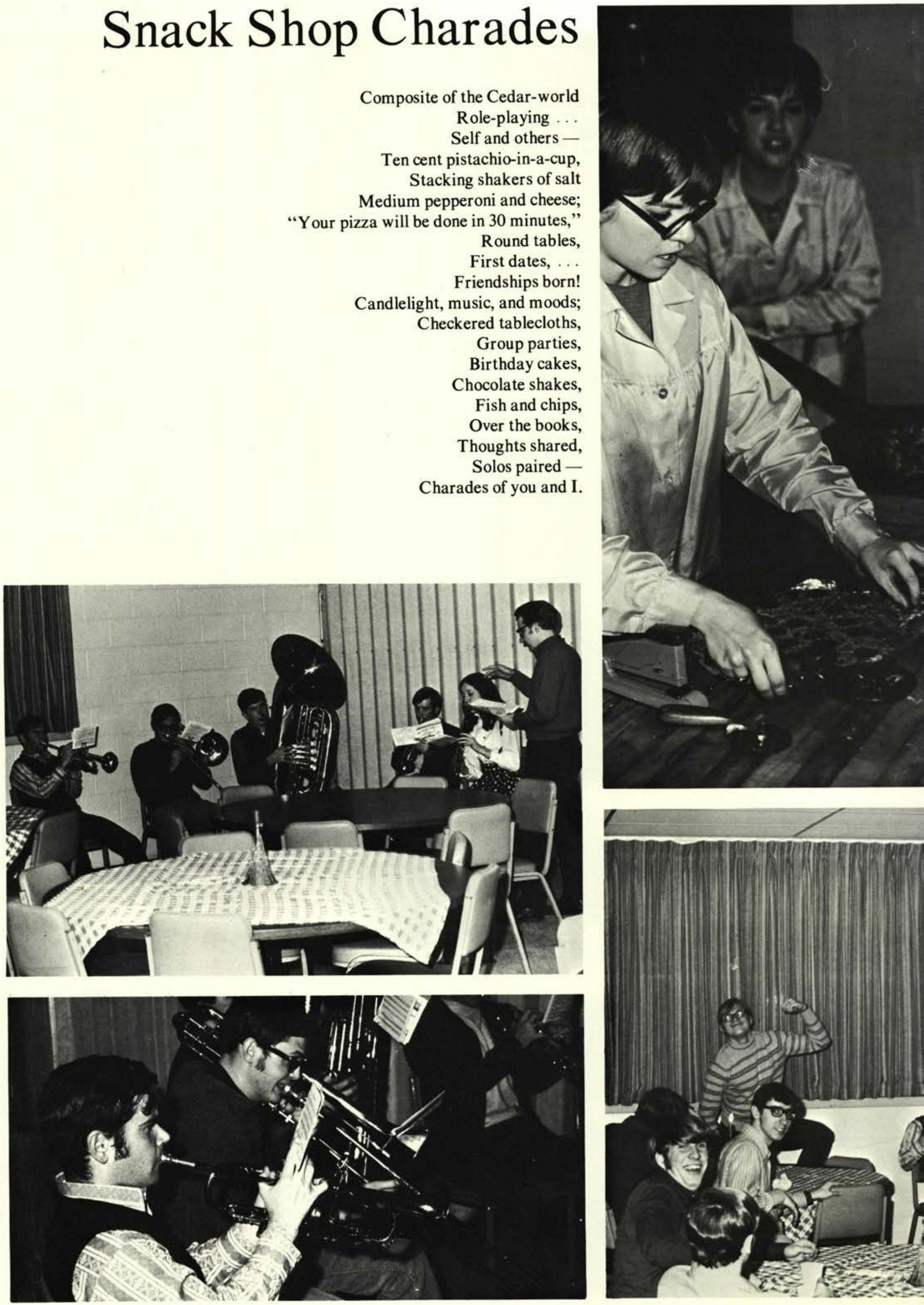

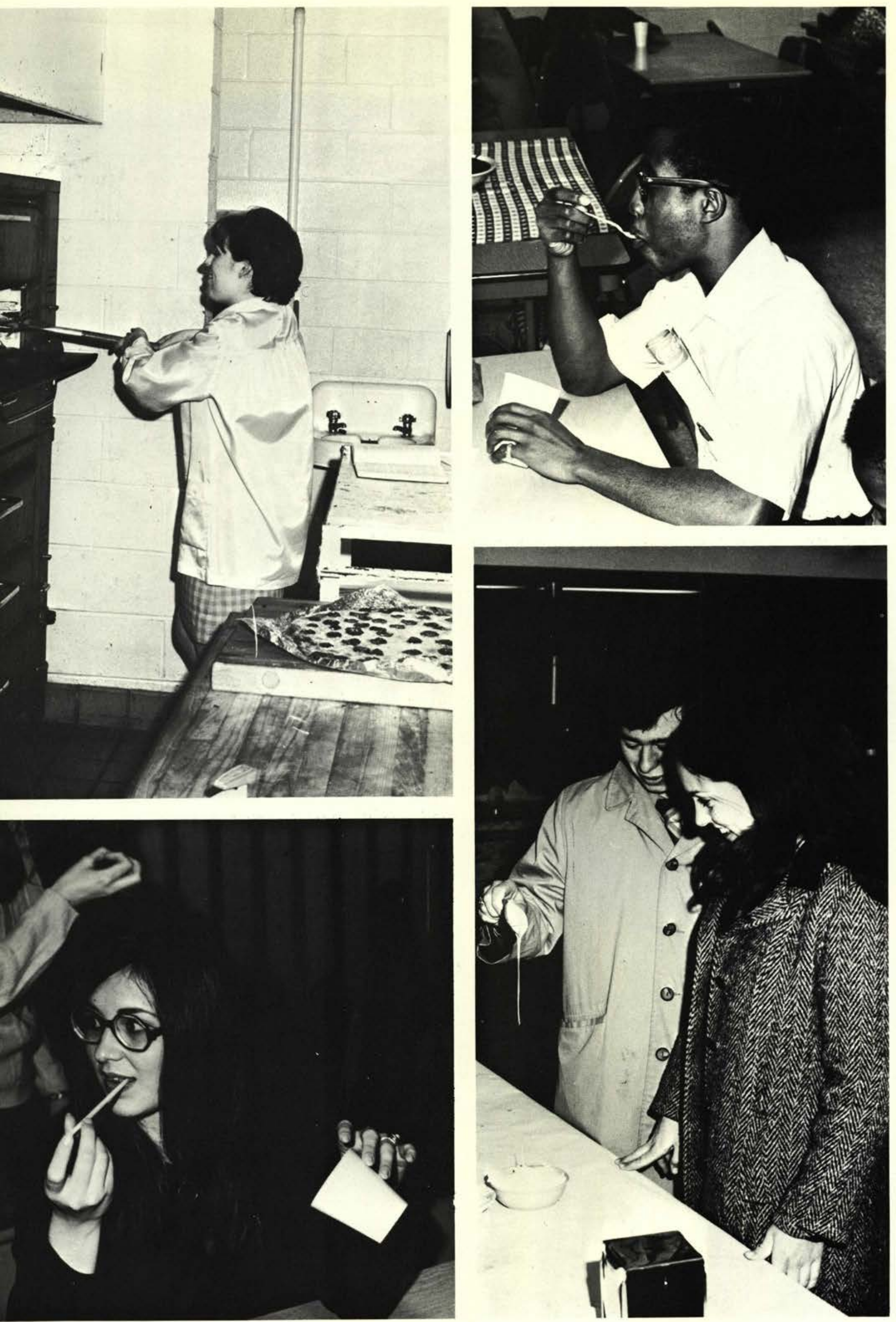

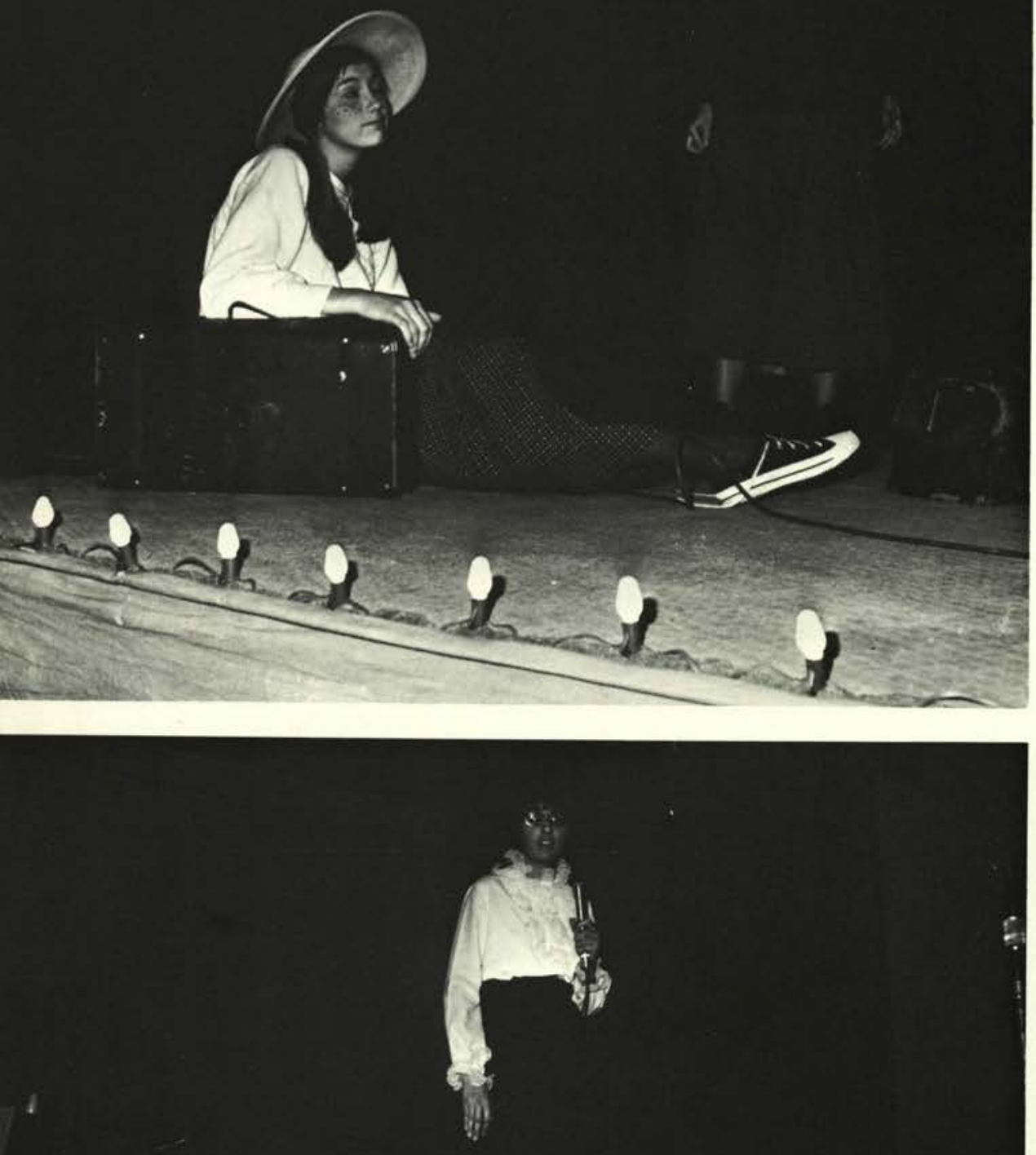

.000 .00

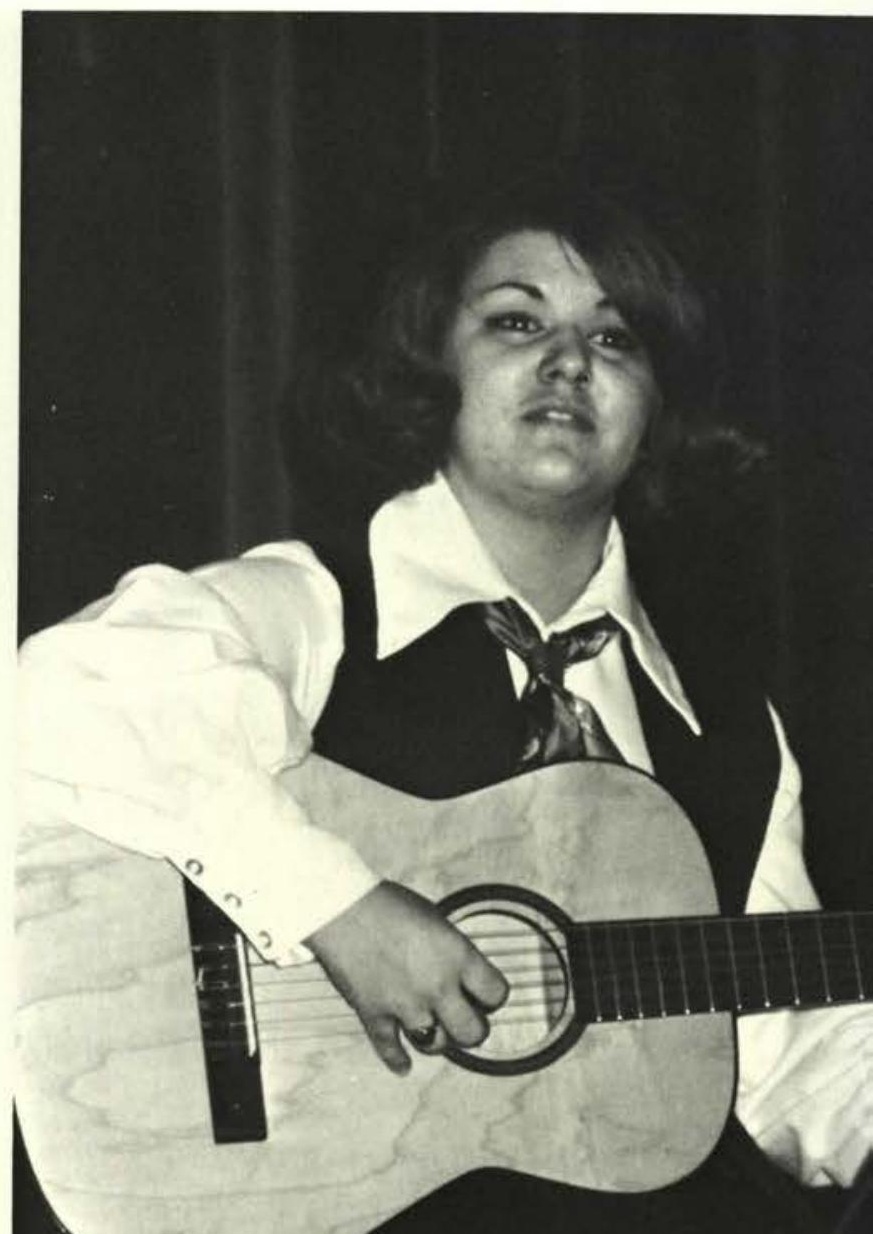

A Night Full of Talent 

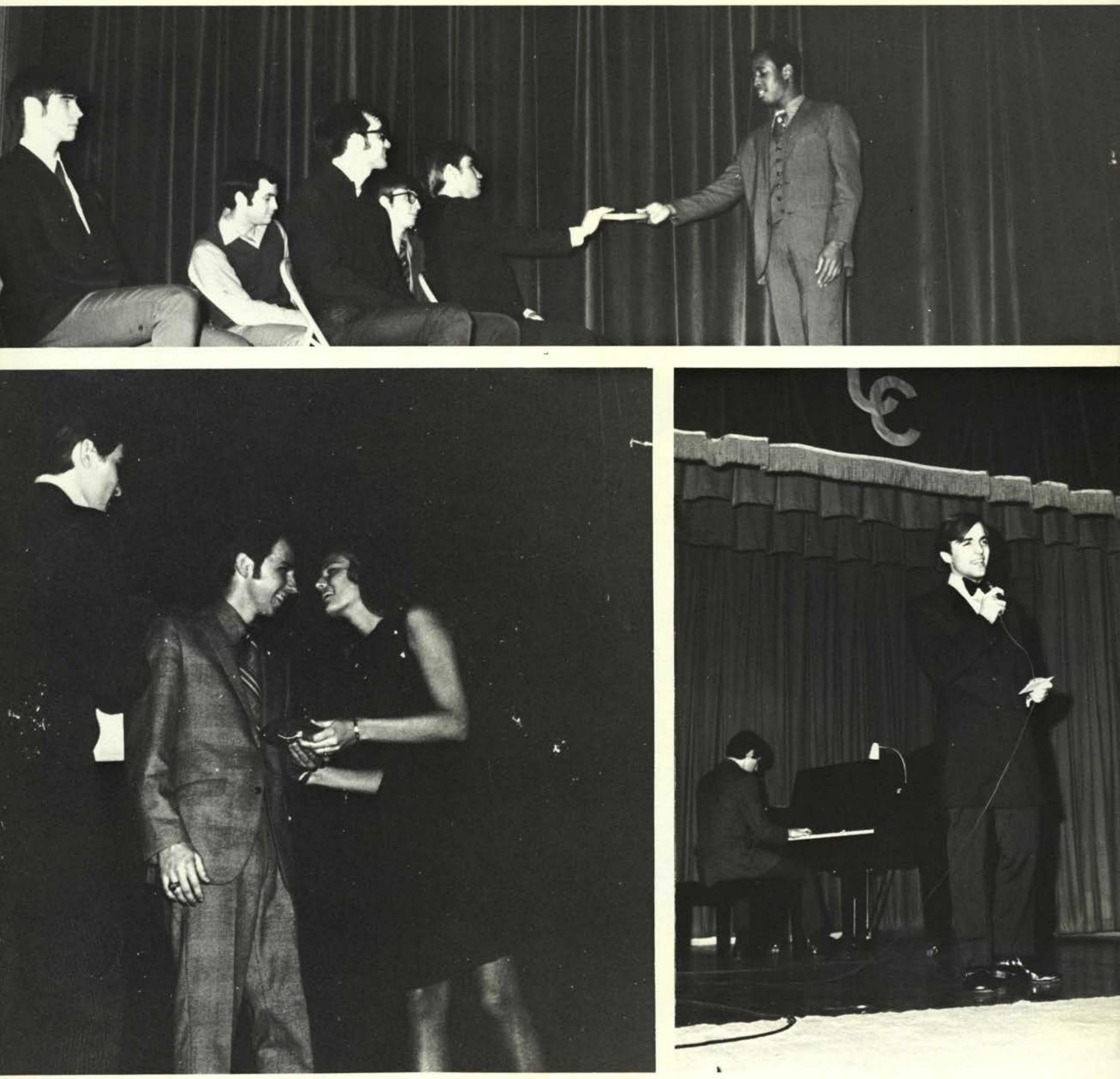

The hushed audience,

The spotlight,

The talented performers,

The applause,

The final votes,

The winner,

A brilliant display of

campus talent. 

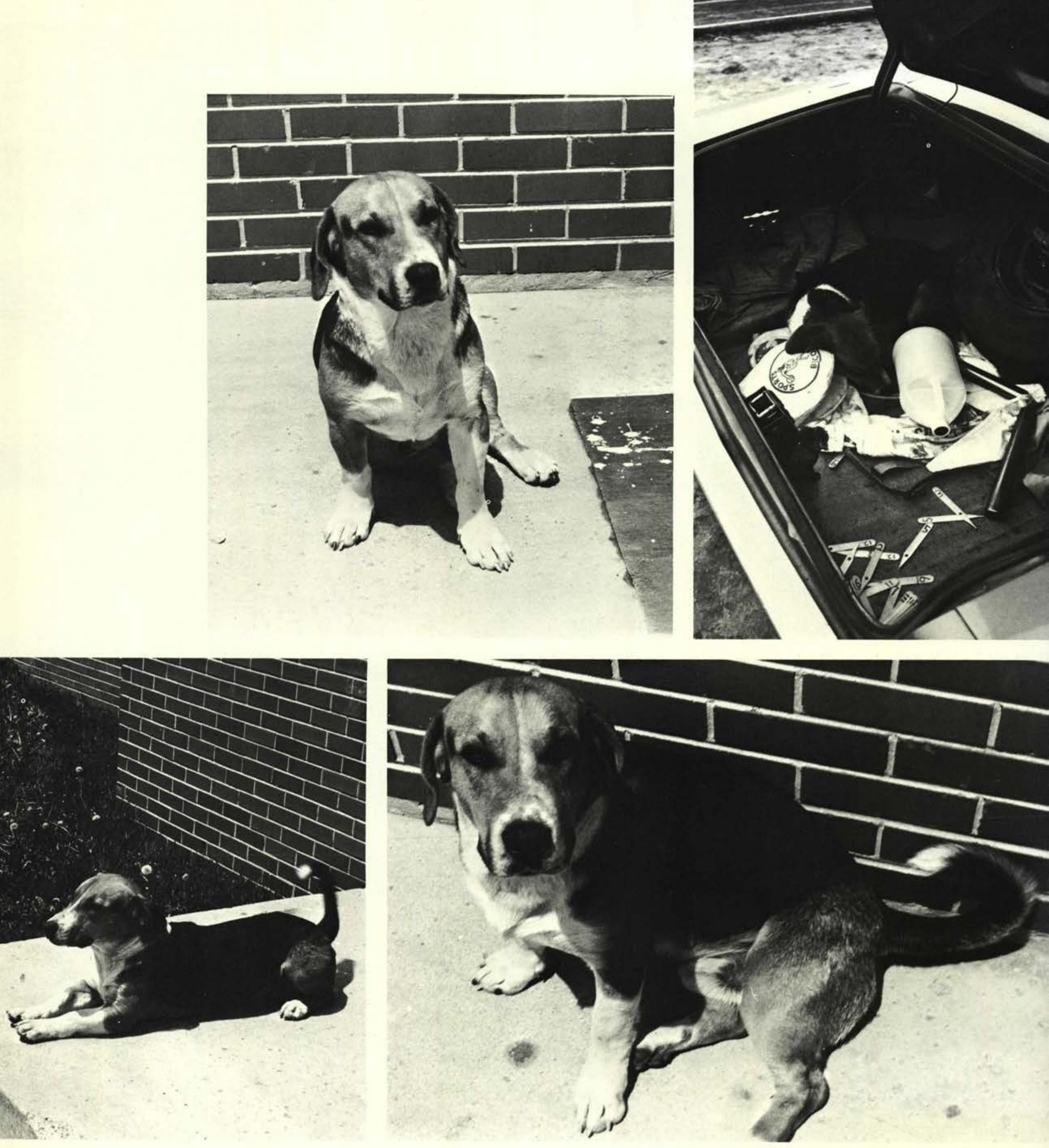

Due to circumstances beyond our has been cancelled: (by decree of 

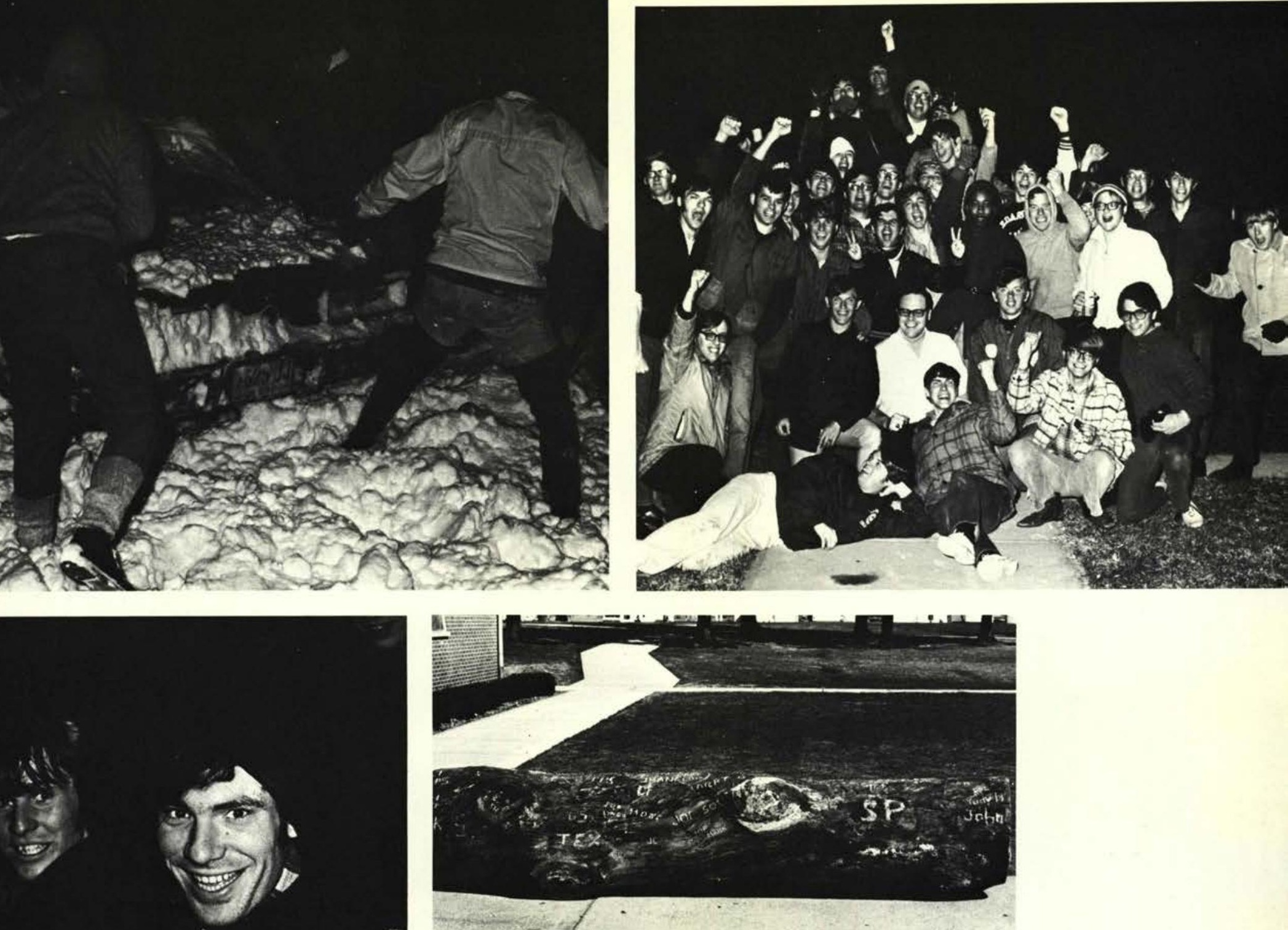

$37 a+4+3$ I 52

4: T. ELAPF TRM

M1. 1 .
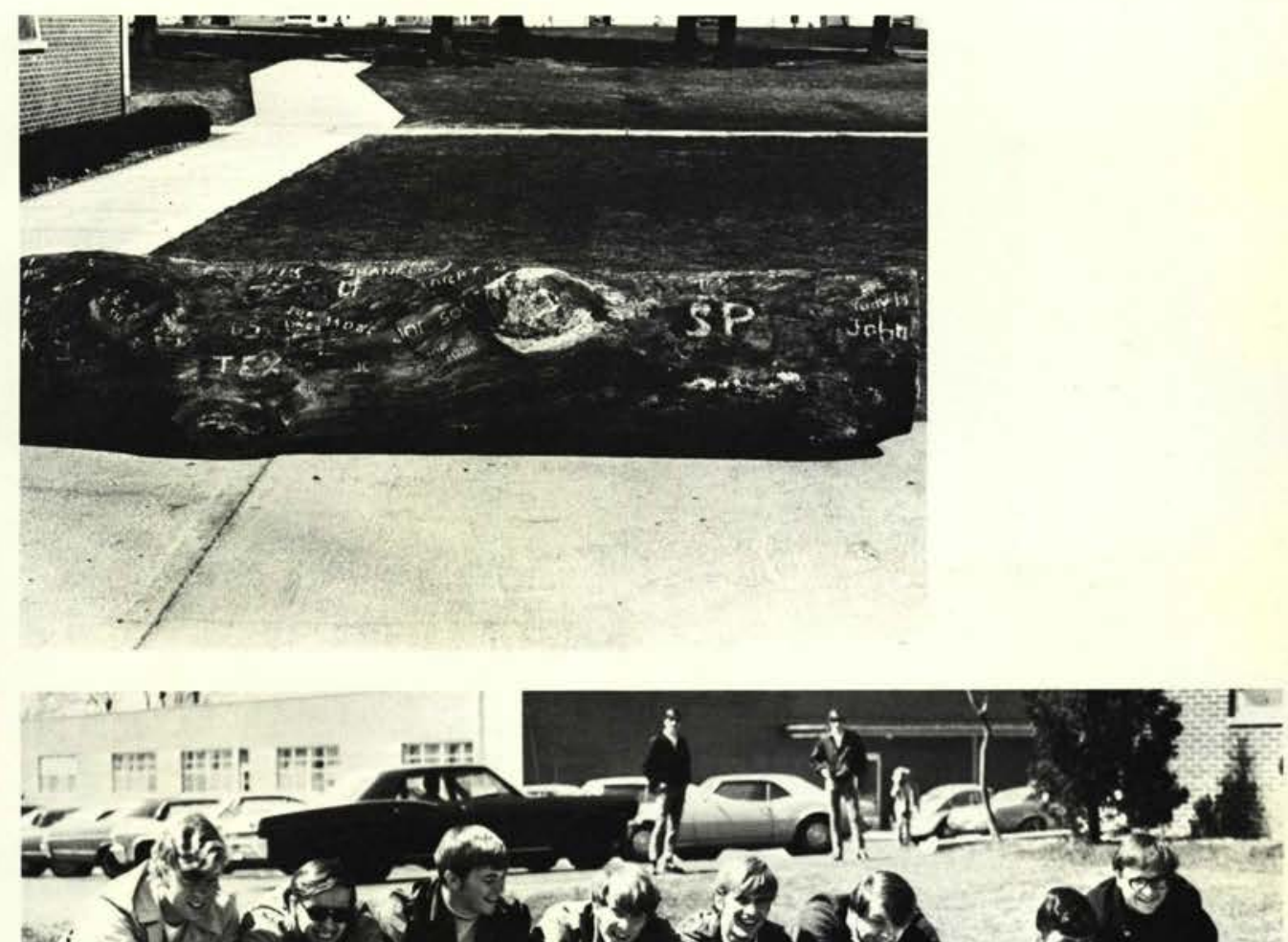

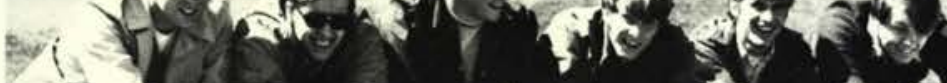

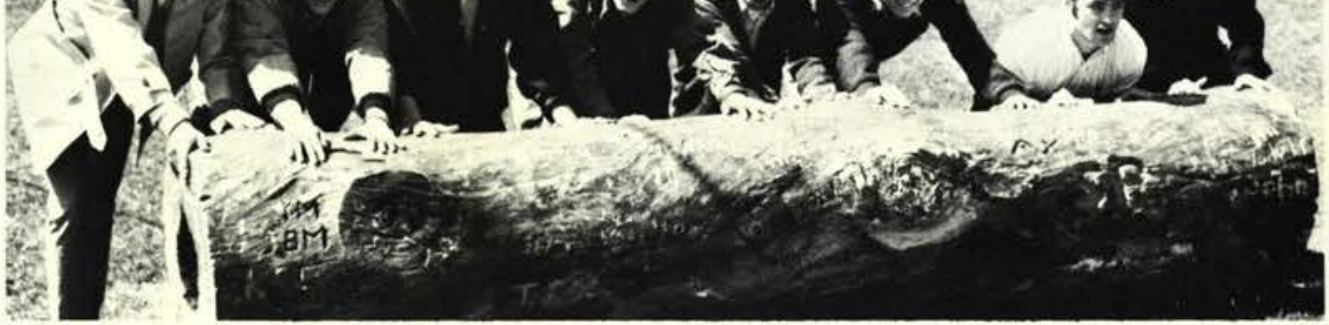

control, the copy for this page

Pharbus and men of Cedar Park.) 


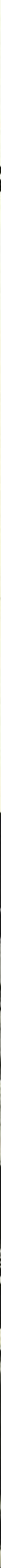


Lecture

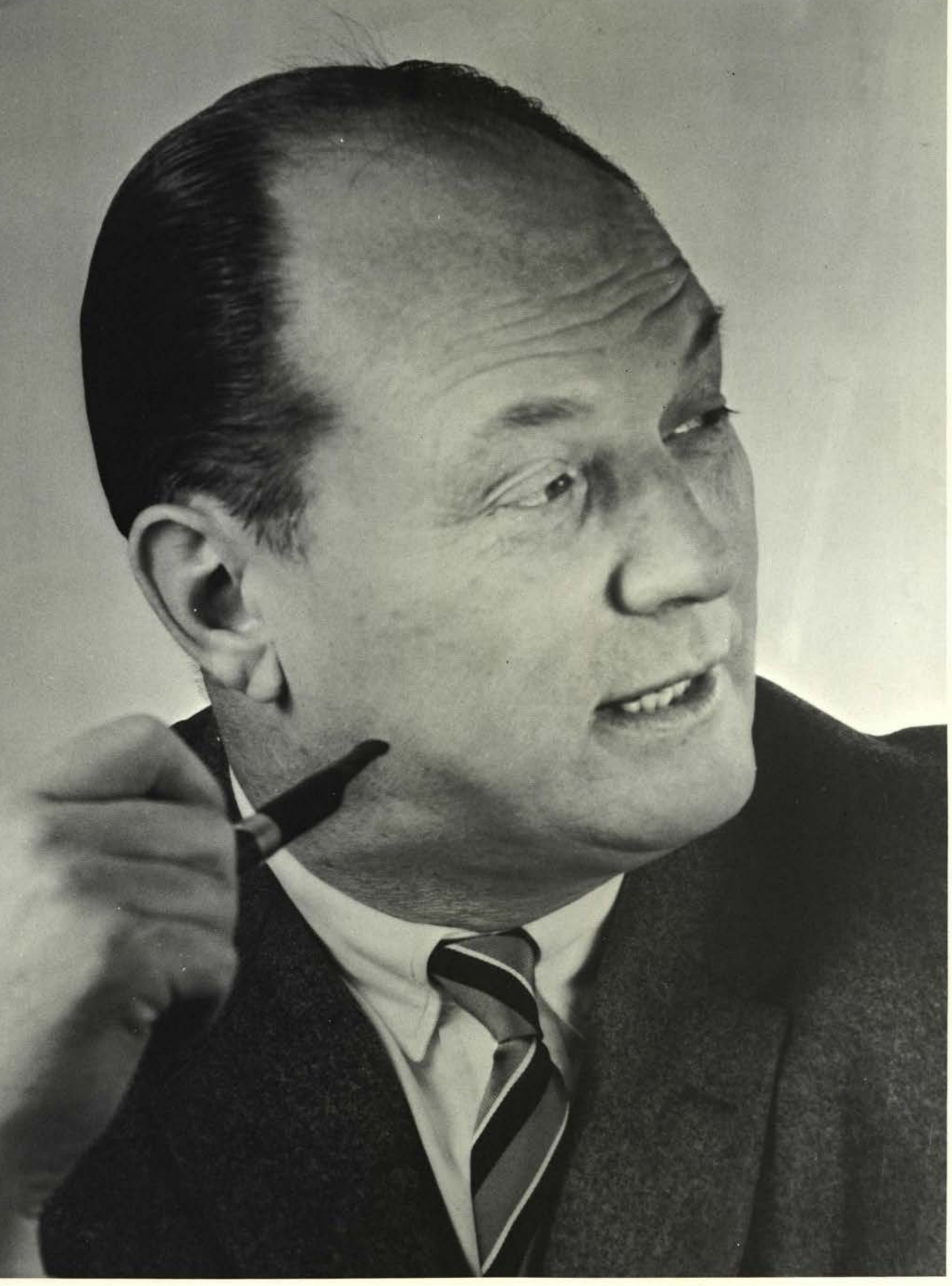




\section{Volumes}

\section{of the}

\section{Learned}

Concentration ... slippin' away ... I keep getting lost in the words and the desks gotta be too small for all the books and the aisles are just too narrow and how do I fill out this little brown card, please? Oh, well, the books help the grade ... Umm, lets study group ... we can tell who wears cleats and who wears tennies! And the music takes place in the corner and the padded chairs are the most comfortable and how does the Xerox machine work and stay awhile and watch the pipes burst. Oh quiet ... this is a library, not a zoo!
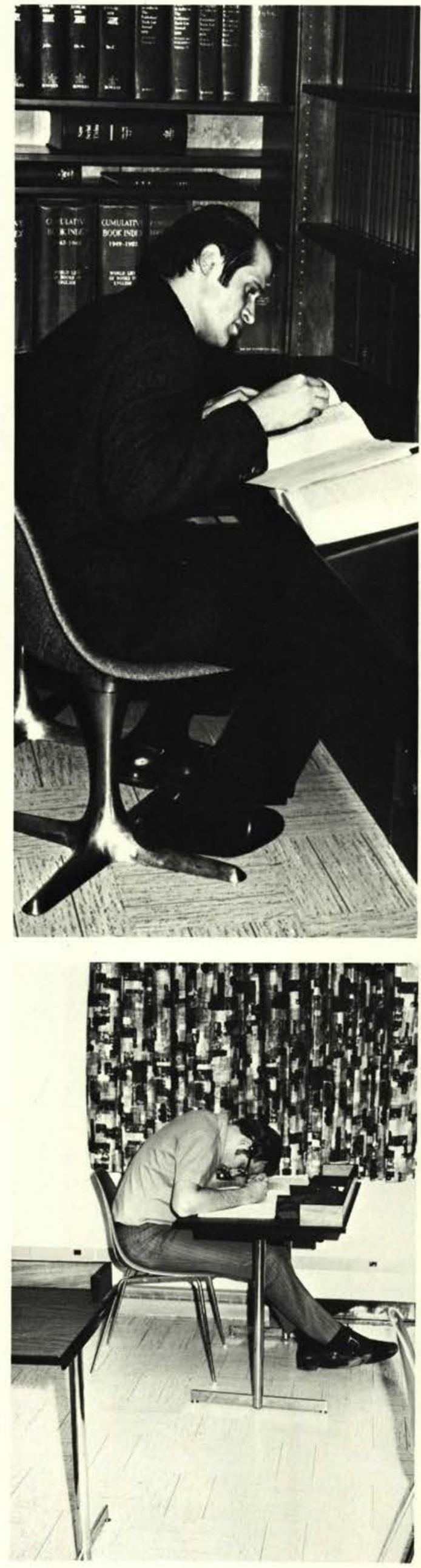
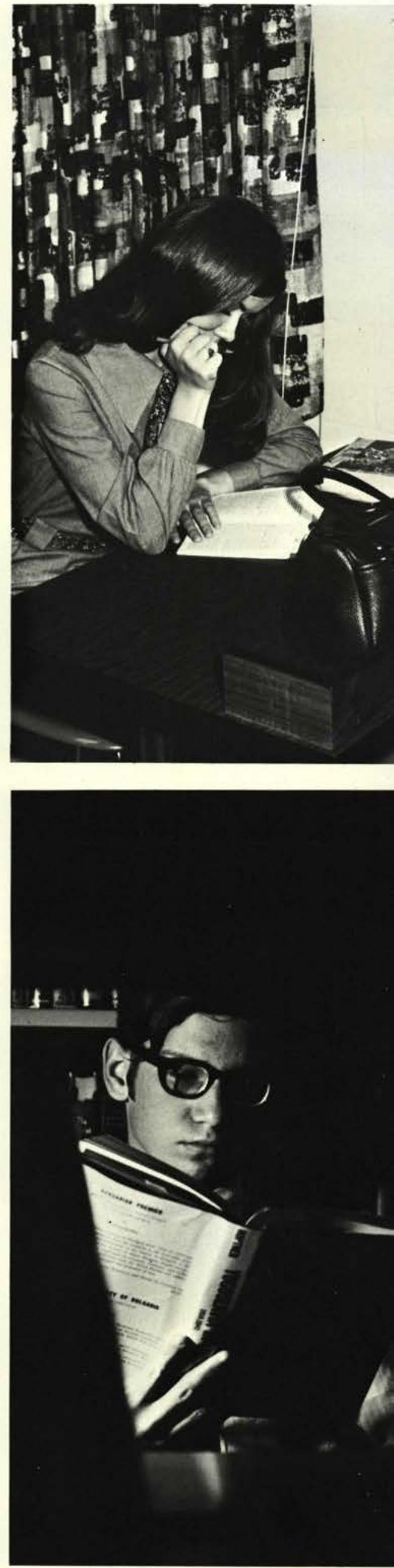

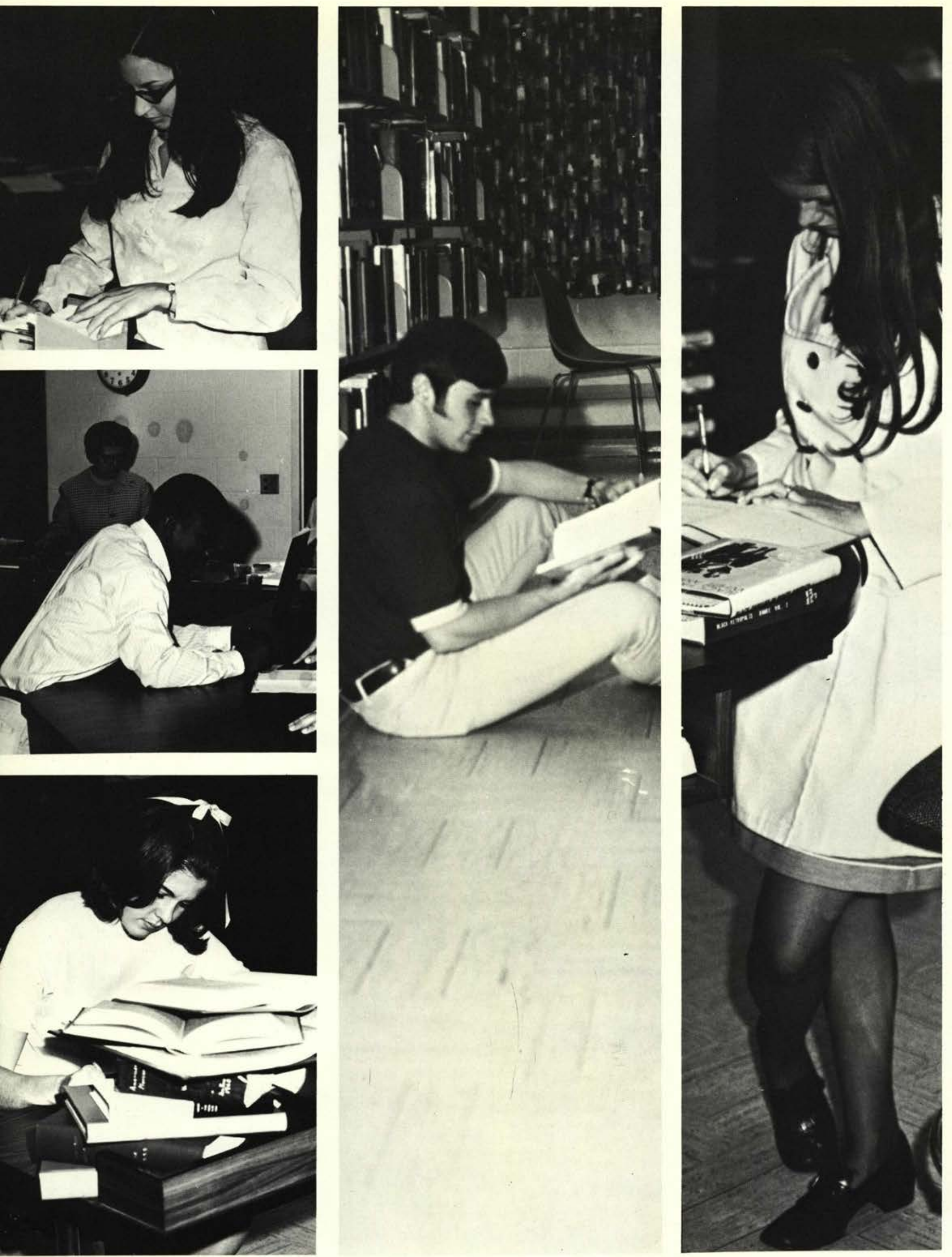
A car for Christian Service,

Advisory 12 planning,

Cedarville Baptist Fellowship -

Striving to do their part.

A great campus pastor -

Willing to help,

Giving encouragement,

Counselling.

A student body working -

Not only in school,

But in Christian Service,

Seeking to tell others,

Eager to share Christ.

Each one anxious to do his part

To serve out glorious God.

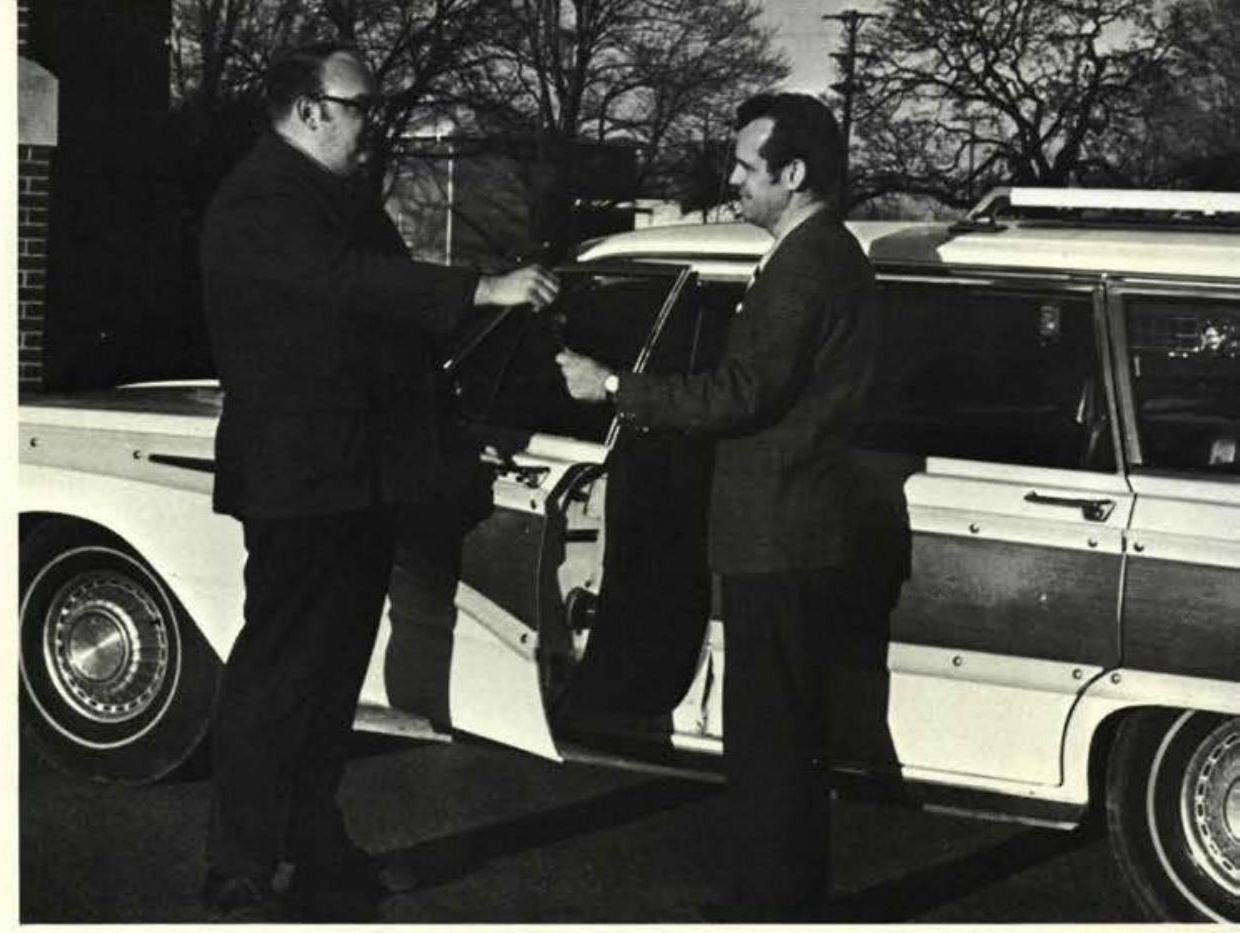

Our Goal:

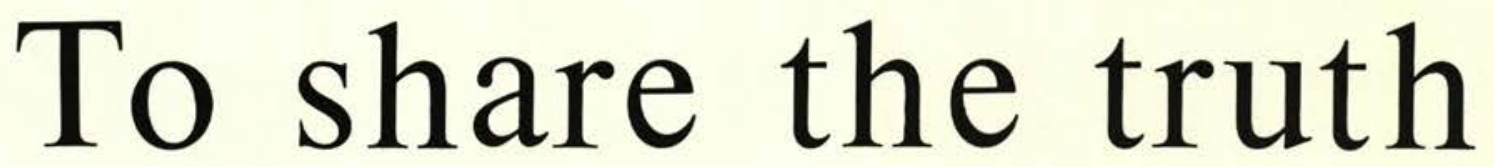

Opportunity: outreach and uplift;

A time of involvement.

Twenty chosen to represent Christ,

To share their love.

Pennsylvania, New York,

Joy of leading teens to the Lord,

Youth discussions,

Presentation of Christ and school.

Morning prayer together,

Hitting every Burger Chef,

Bearing our swords,

Communicating with others,

God using us.

Eleven great days locked in the memory of more than twenty;

The success lay in the other swordbearers at home,

Like Mr. Slabaugh,

Praying, praying daily,

Praying daily on their knees ...

Suzanne Hale

Kathy Strawn

Merle Hammock

Bernice Penegor

Linda Willms

Ellen Young

Mary Lou Lippert

Vicki Imbro
Dave Globig

Don Hendrickson

Tom Mudder

Al Konya

Tom Seidler

Mike Gallogly

Jim Griffith

Paul Radcliff

Chuck Rowe

\section{of \\ Jesus \\ Christ}

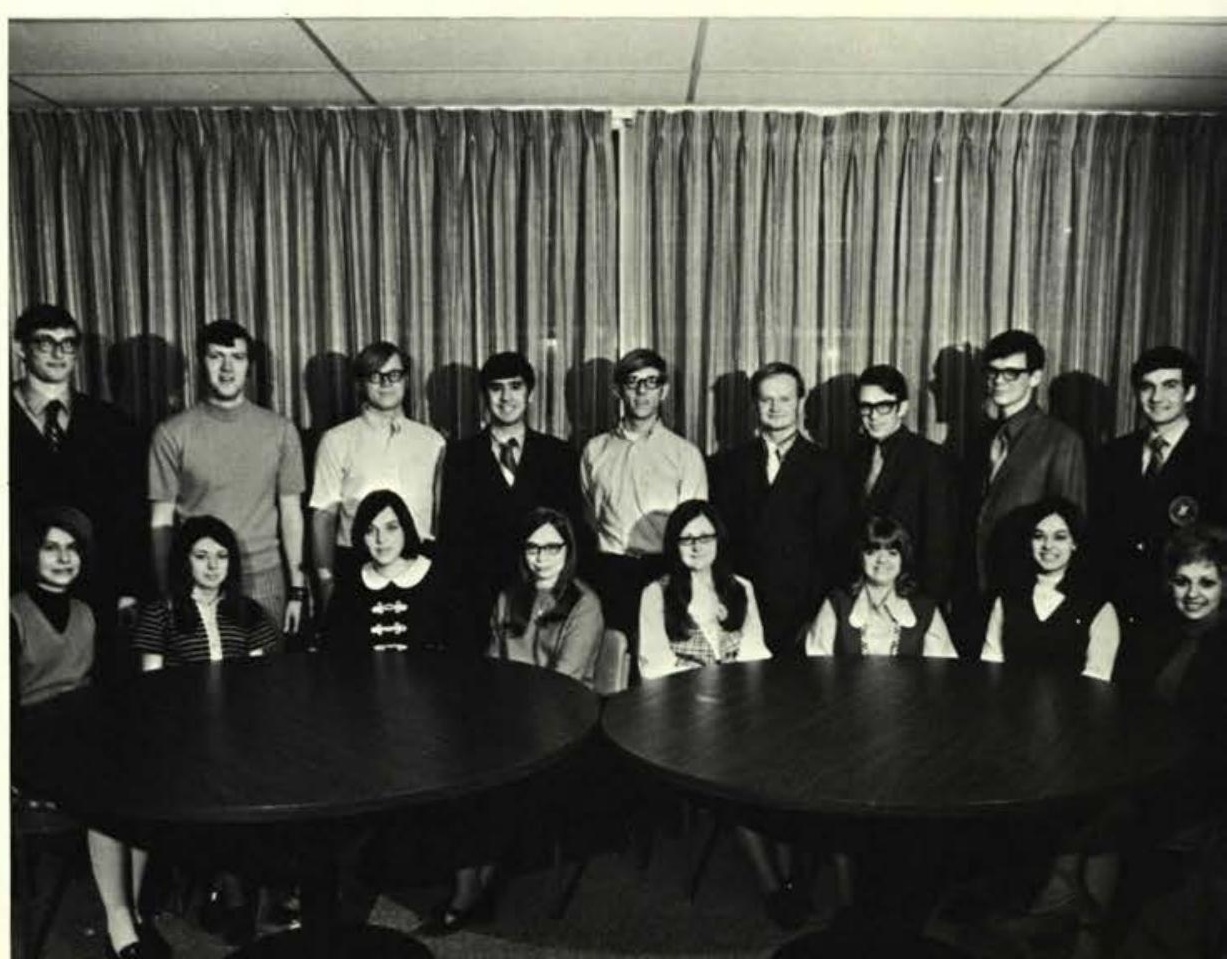




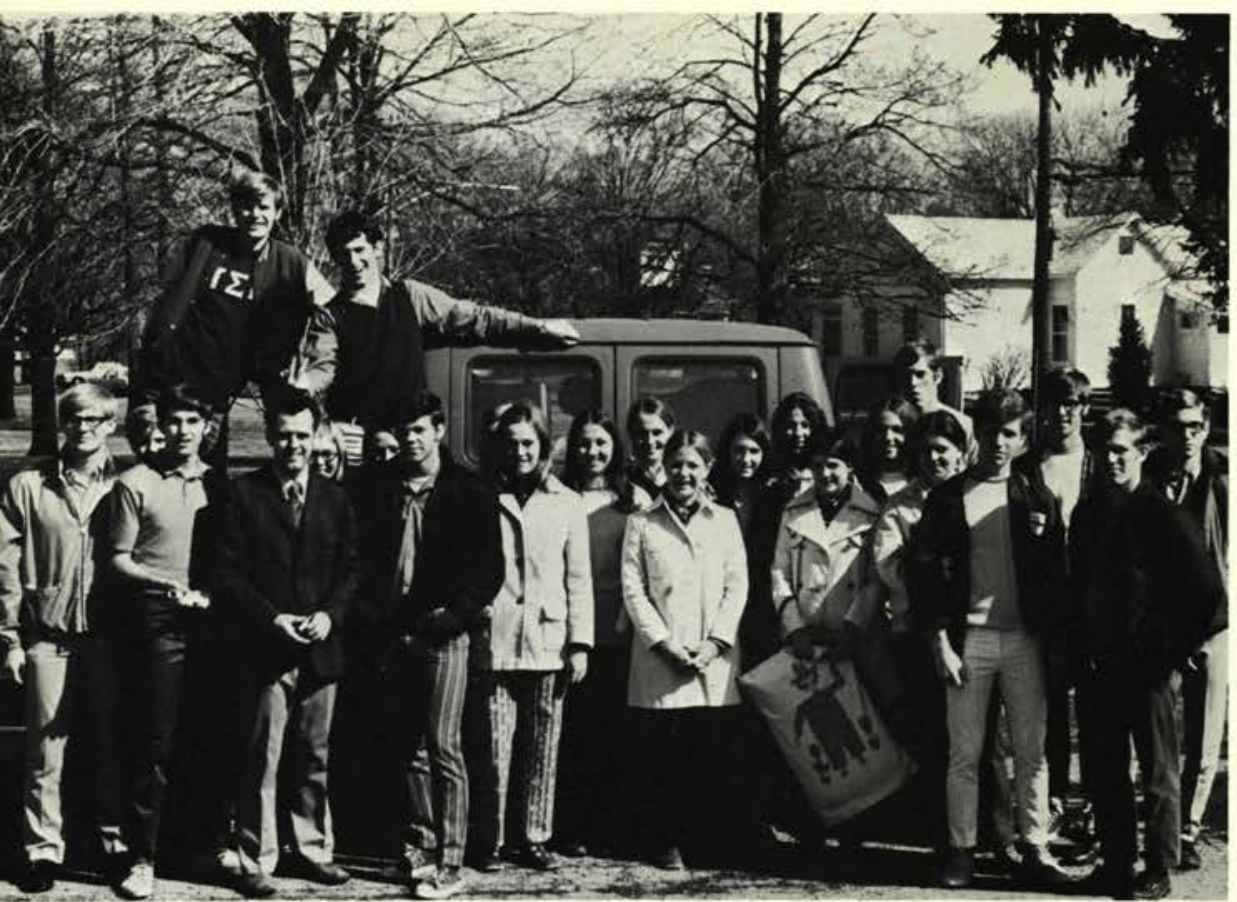

FRONT ROW:

Pete Lillback

Les Chambers

Bill Bacheller

Anne Bailey

Pat Emerson

Bob Pycraft

Holly Wenner

Shirley Rocher

Vicki Tobias

Dinah Arthur

Melody Crotty

Janet Young

Cindy Summers

Bev Kemp

Monica Bayne

Terry Clapp

Becky Kemble

Rich Hales

Steve Spencer

Jake Tipton

Dale Fogle

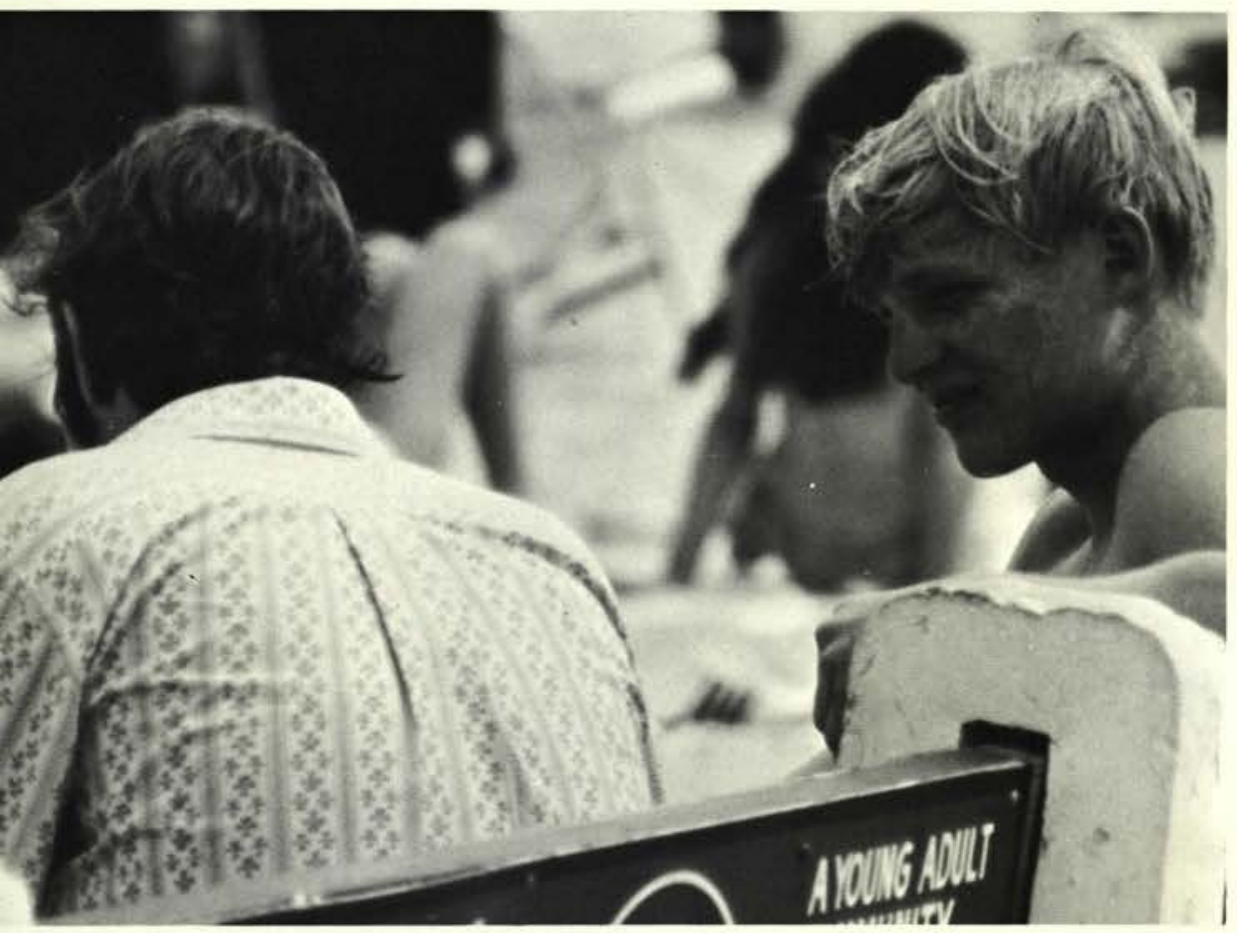

\section{Mission Field on the Beach}

Beach evangelism ...

Money needed . .

Anticipation ...

Prayerful anxiety ...

And the thought of a

L-O-N-G bus trip . .

The sharing, the

Talking, the hopes,

God using us

The sun

The ocean sprays ...

Sand between the toes ...

Sea shells by the sea

Shore.

Sea gulls..

The board walk

The trip home

And the rest,

A mission field on the beach. 


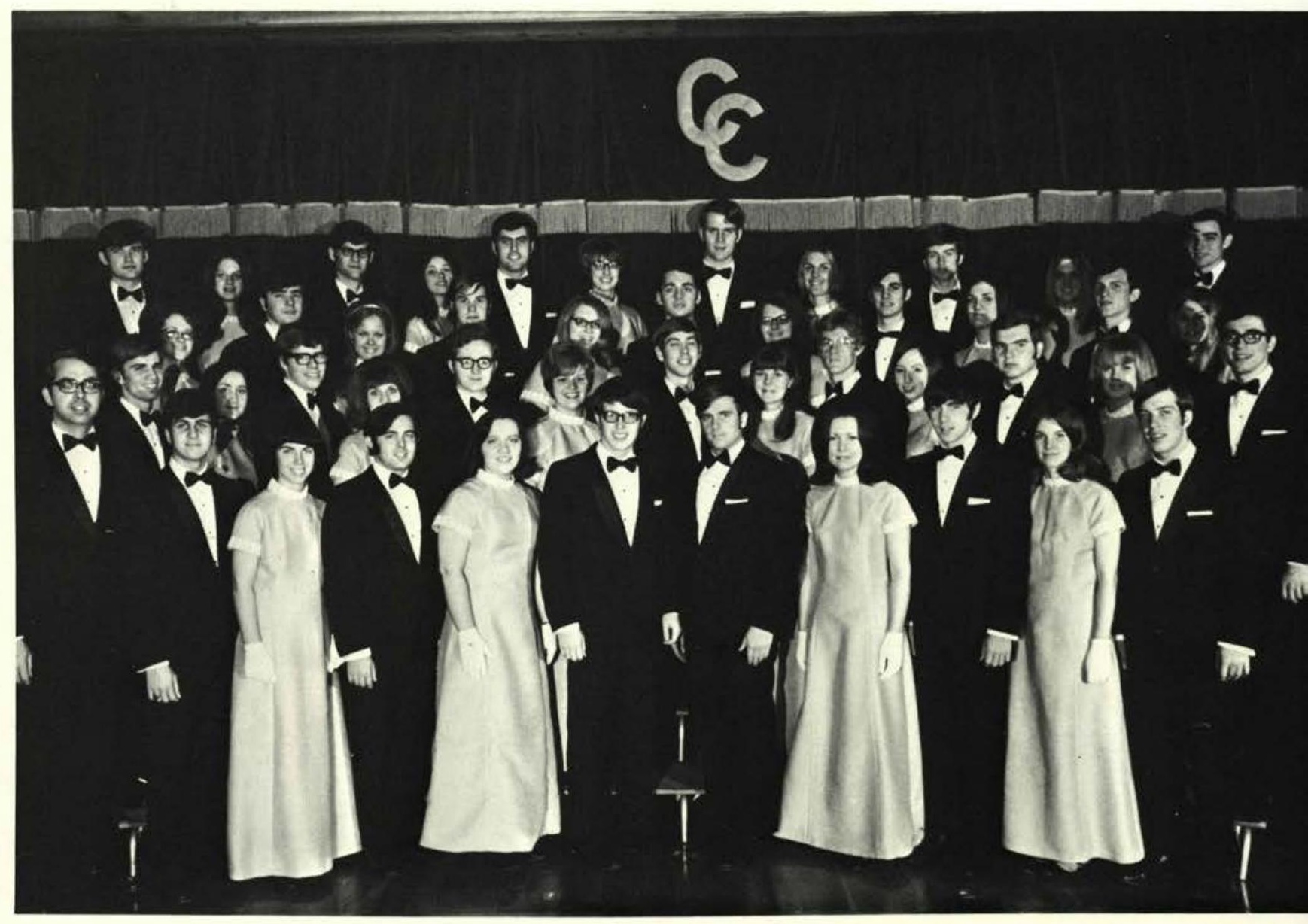

ROW 1:

Dan Bergen

Carol Morse

Don Harmon

Carolyn Lambert

Dan Estes

Dave Railsback

Jan Timpe

Steve McCallister

Paula Prater

Tim Hegg

ROW 2:

Mr. David Matson, Dir.

Yontz Miller

Sheila Rogers

Buddy McNiece

Connie Clark

Ron Coombs

Linda Betts

Dave Nelson

Becky Hull

Jerry Grayson

Becki Chandes

Charles Sheppard

Kathy Burkhard

Ron Bechtel

Sleeping breaks

New keyboards,

Crowded bus trips,

Large churches,

Stained glass windows,

A warm welcome,

And a purpose -

To glorify the Lord.
Marilyn Brown

Ron Meyers

Jan Beesley

Tom Strong

Ruth Coombs

Dave True

Bev Moore

David Durham

Debbie Durham

Lyle Rodman

Sue Scott

ROW 4:

Victor Lakes

Jacki Barraclough

Ron Spieth

Georgeanna Axiotis

Steve Lantz

Rita Gast

Lee Griffith

Marikay Schneider

Dan Henson

Jean Taylor

Steve Williams

The
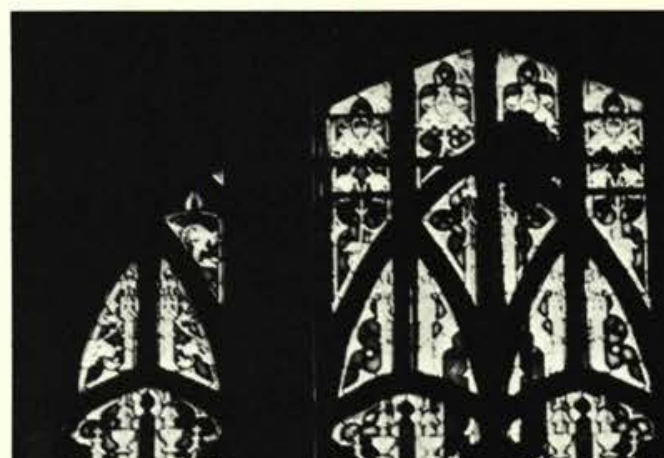

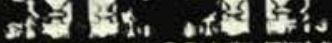

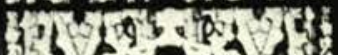

$33^{3} x^{3}$ a 32

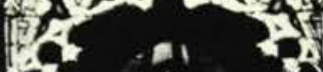

to

201020

in

E
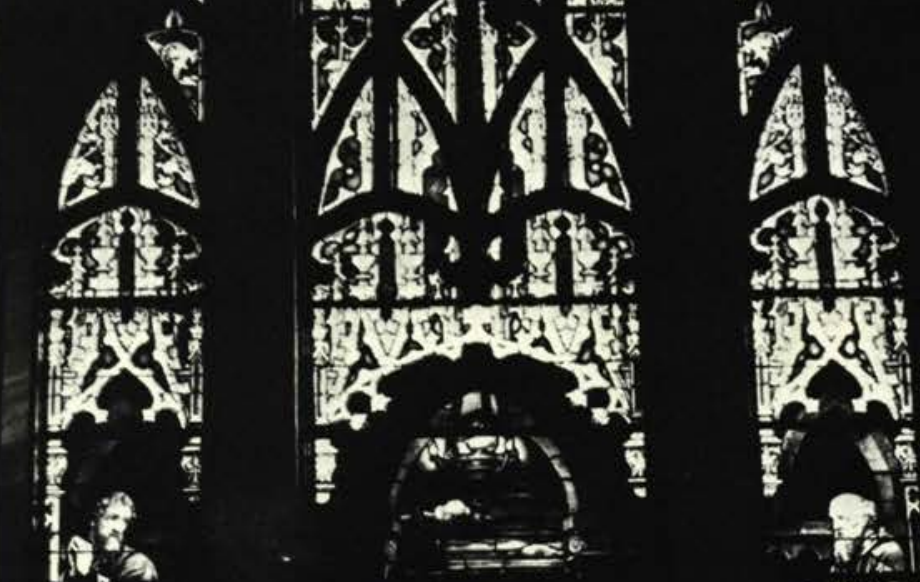

1) 1 . 45

Af 3 .

- $-\frac{1}{2}$

ती
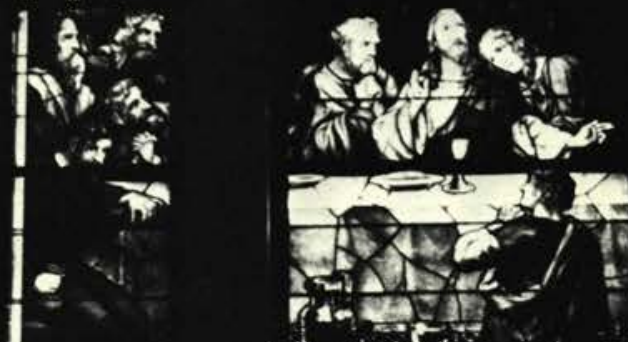

nidiste.

(1)

a $=\ldots+.14$

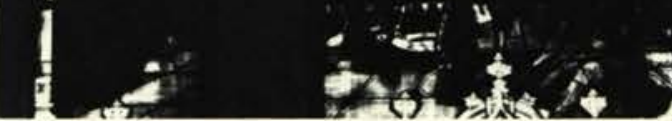

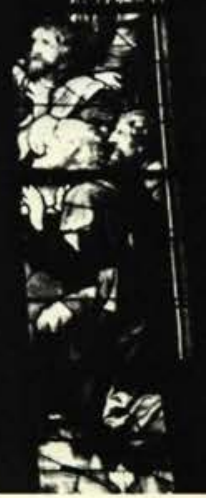




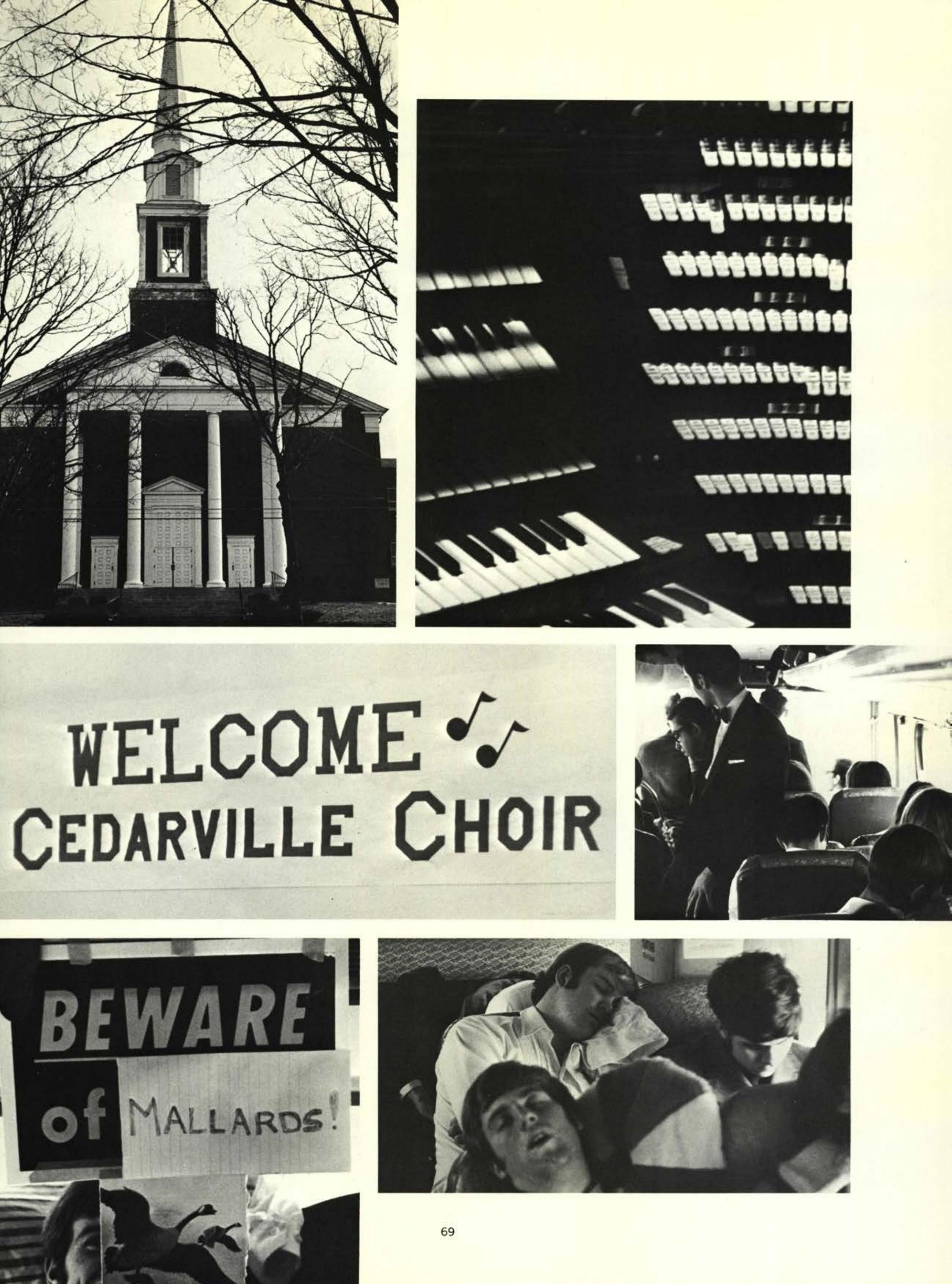



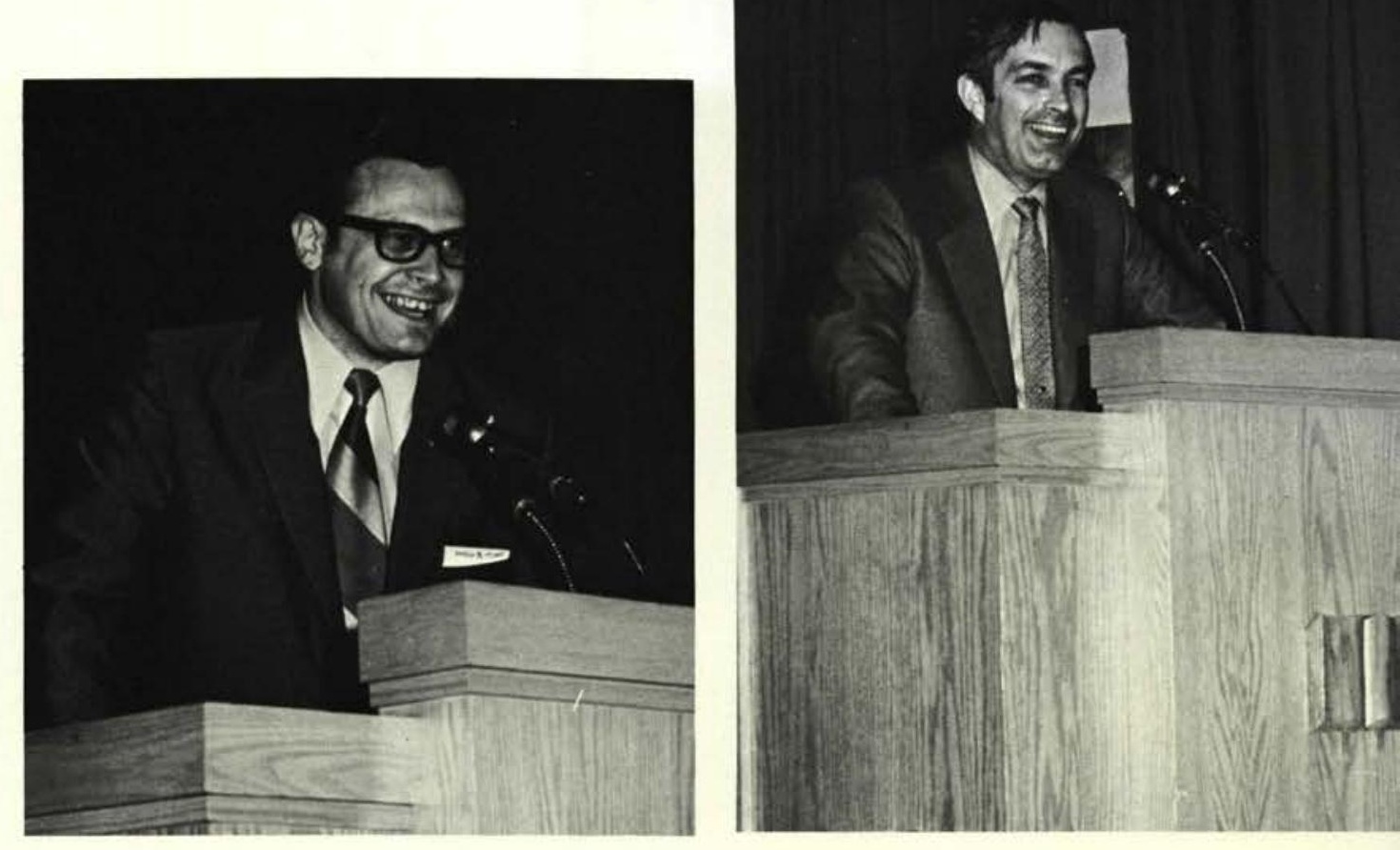

\section{"To make Christ known"}
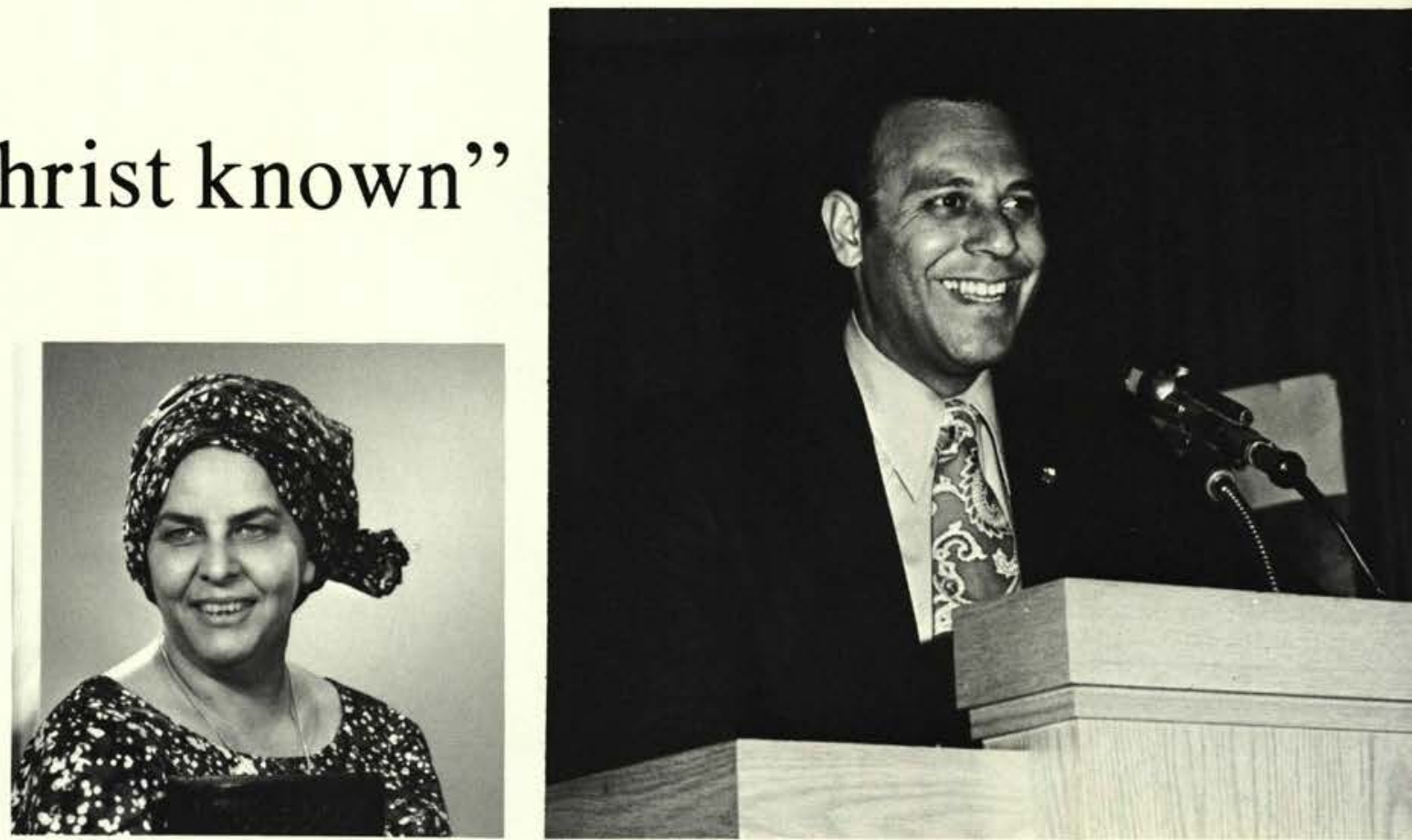

Missionary Conference,

Retreat from the cares,

Advance to our God,

A time of refreshment and growth; Spurlock, Kettenring, Armstrong, Adams, Toro-

We found they were more than just names, They're real people like you and me.

The comic side -

Coke on the head,

The serious side -

Rioting souls on our campuses,

Provision by God through a garage sale,

The changing of fields,
A single, faithful veteran to Niger, Call for reinforcements,

Deputation work,

Raising of money -

We heard them all.

A bible school in Jamacia,

A fainting soul in Australia,

Door to be opened ...

If only we'll push;

God speaking, hearts listening,

Challenge to win the teeming millions;

Challenge to save one, just one

Right around Cedarville ..

What did the conference mean to you? 

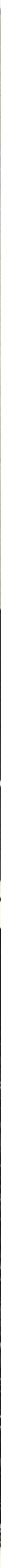

The many faces of education . . 


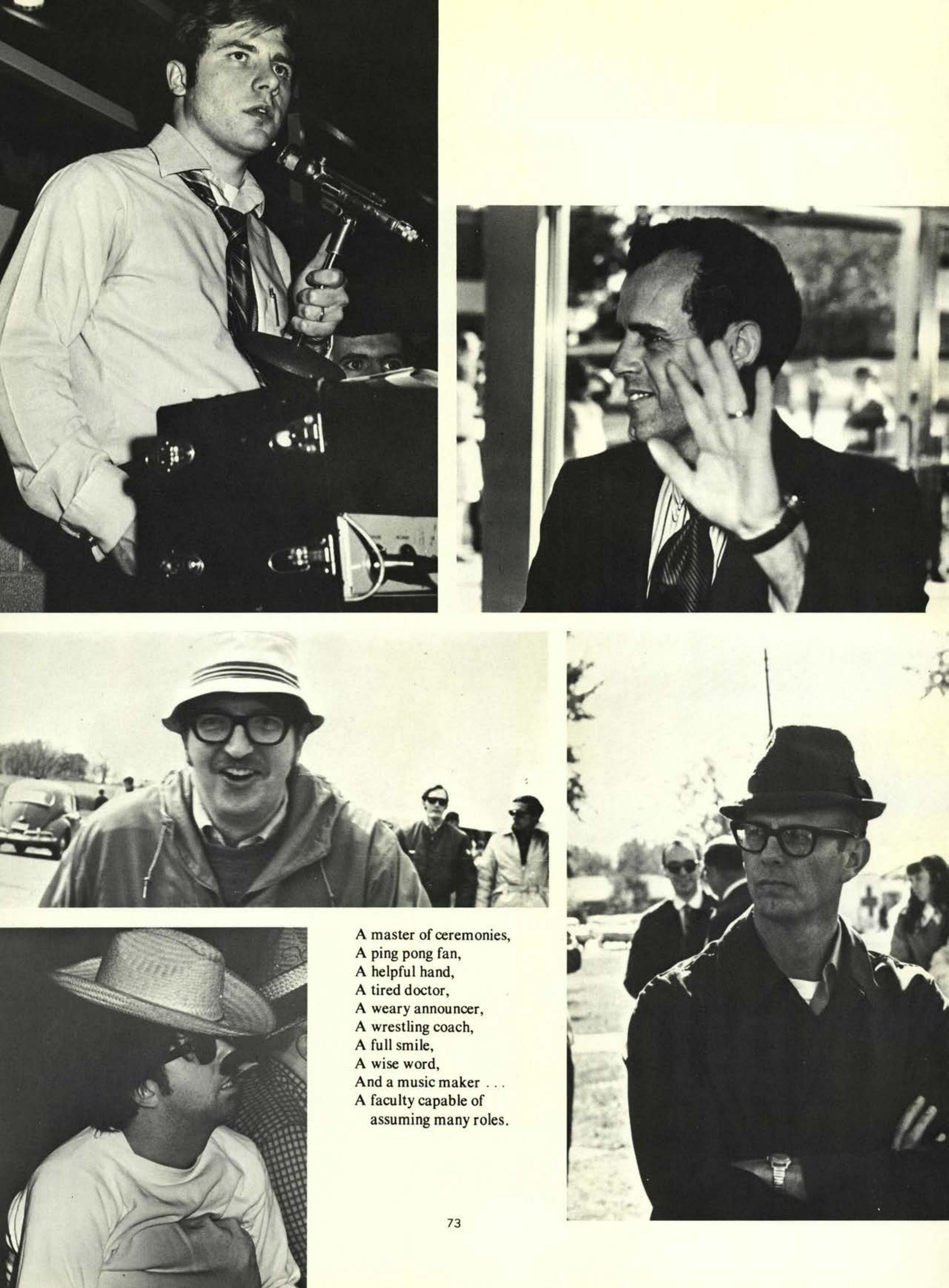




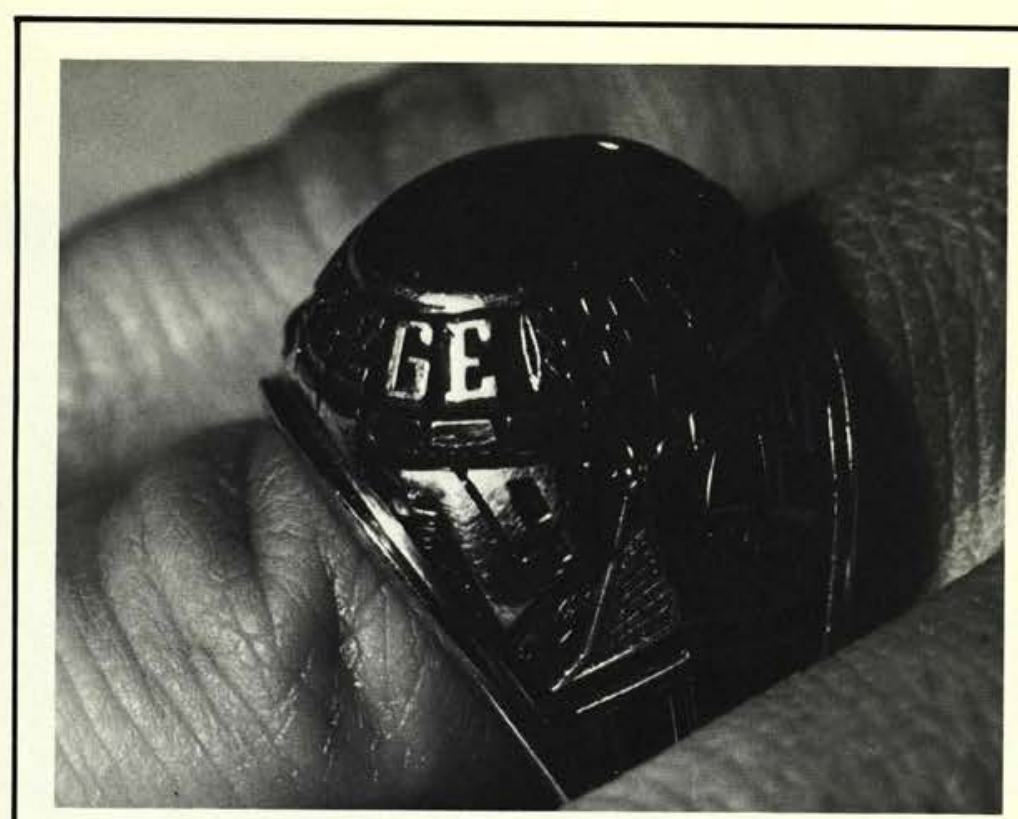

John Roberts Co. -

Class rings,

Bright rings,

Light rings,

Clean rings,

You ring,

My ring -

Classification.

One to go - anticipation.

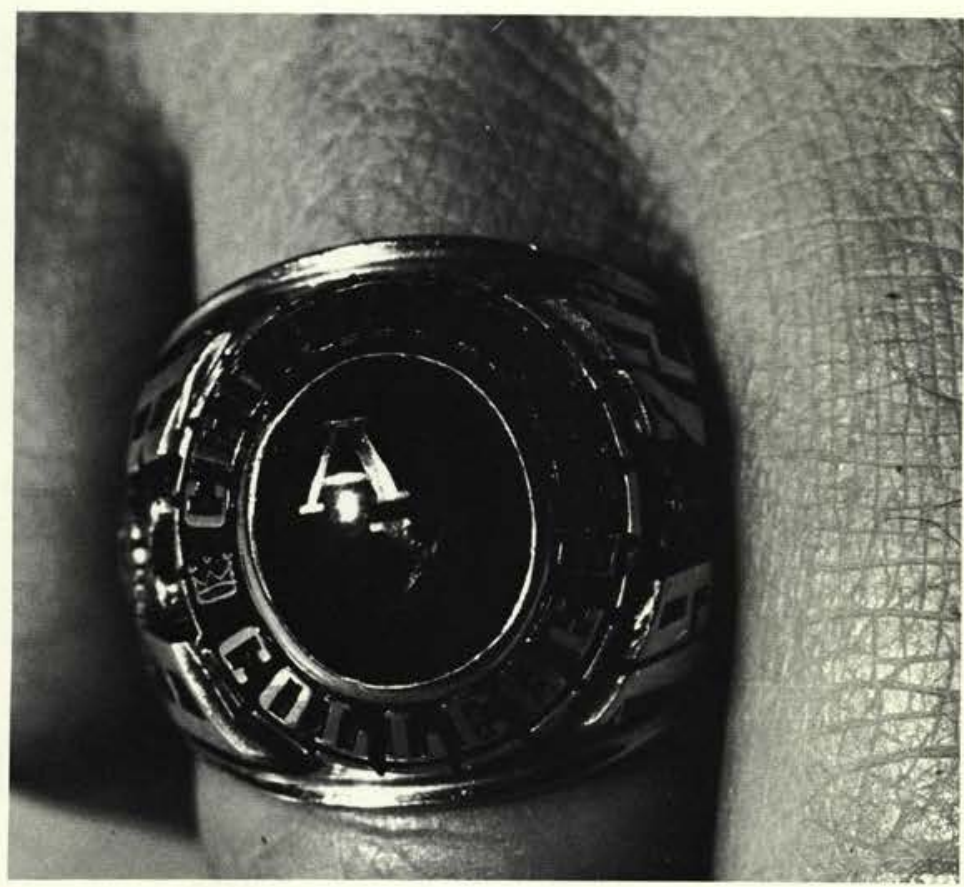

Junior rings.

A size too big,

A swollen knuckle,

"My favorite color!"

A symbol,

A unifying emblem for class-togetherness

A circle that encompasses

Every one of its wearers -

Male-female,

Math major-speech major

El ed-under grad.,

Blond hair-curly hair,

Studious-athlete ...

etc. and etc. and etc.

Junior rings.

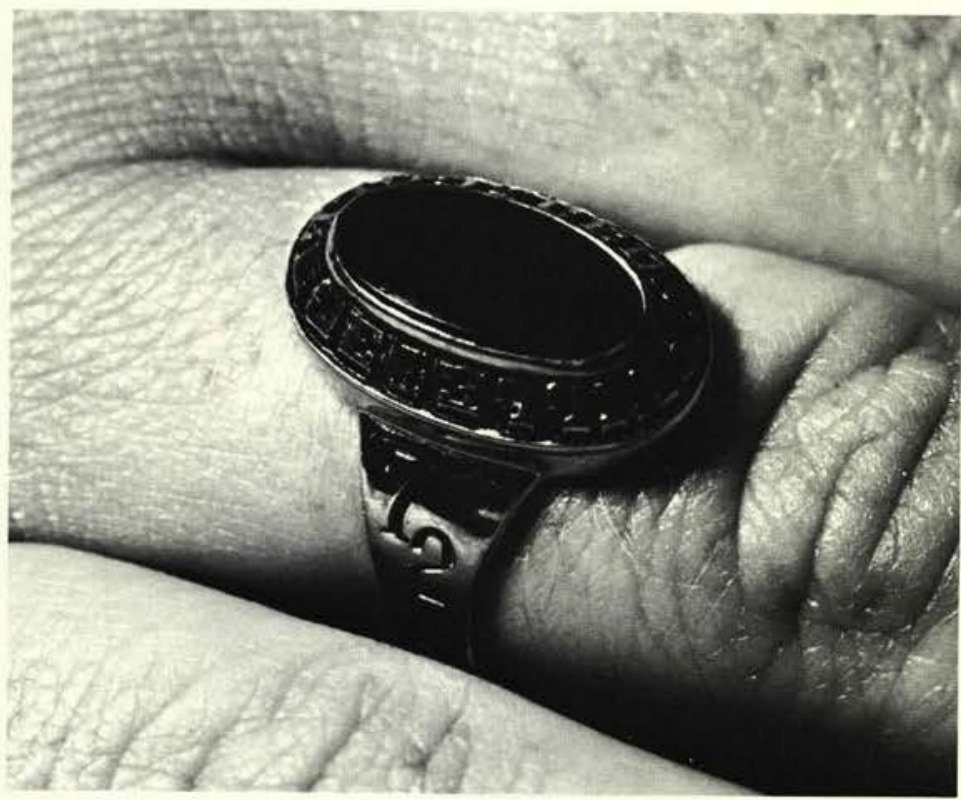


A great drive

By a great people;

Purpose: Two-fold -

One, glory to Christ;

And two, a project

To better our school -

Walk-a-thon.

Selfless individuals,

A bond of oneness,

Invocation around our proud flag.

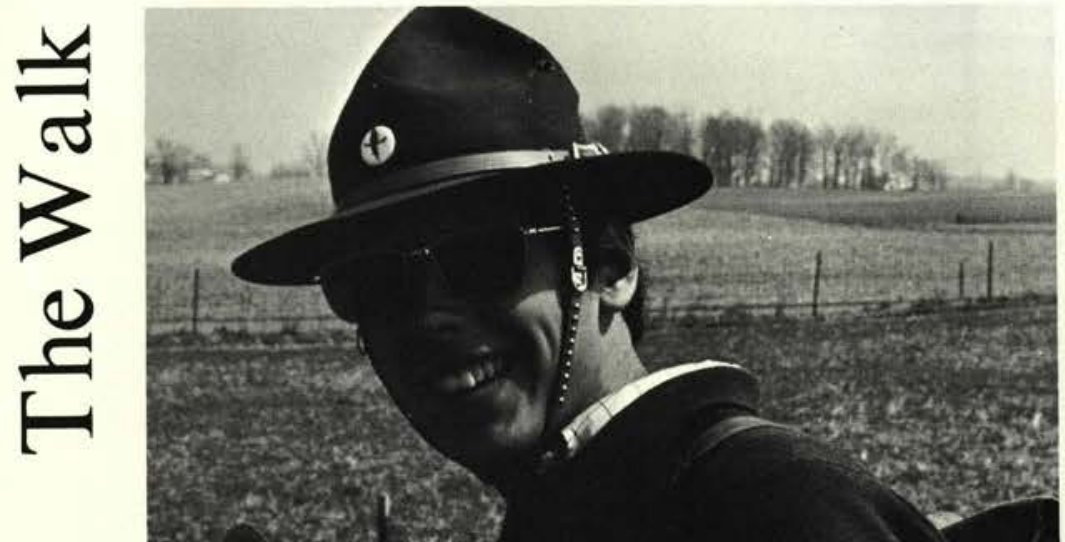

27.
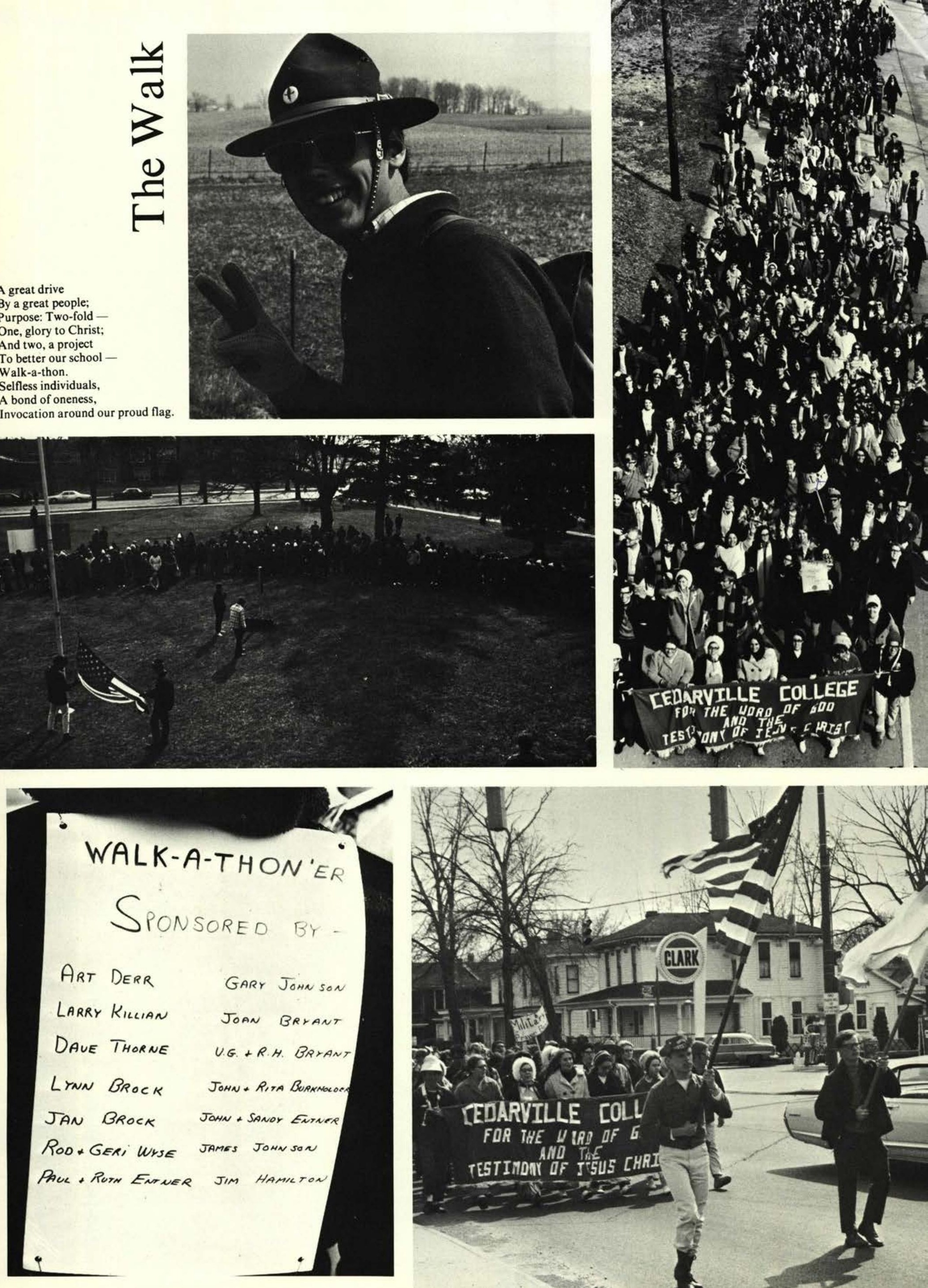


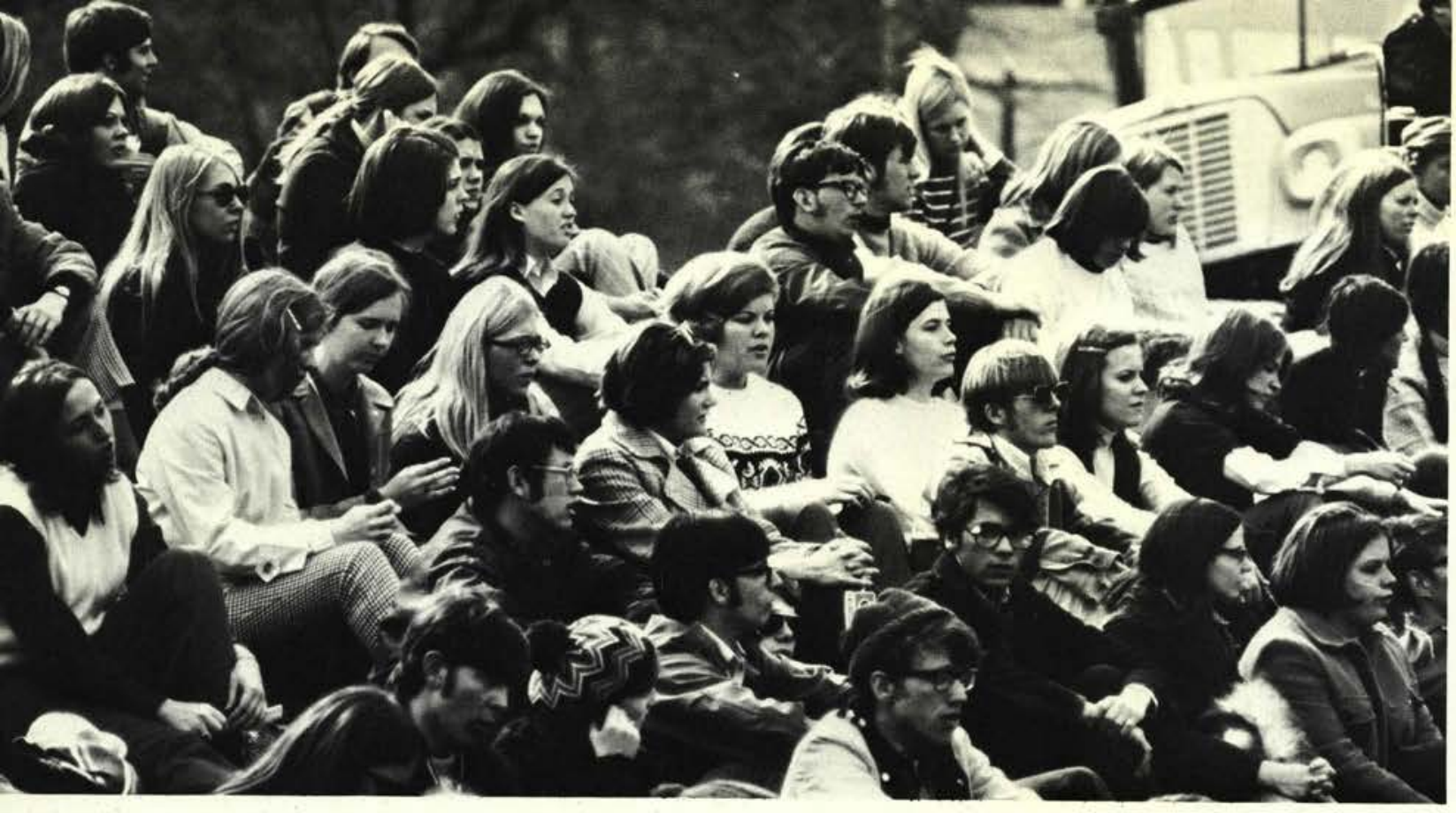

For the Word of God

And the testimony of Jesus Christ!"

Reporters and cameras.

One gargantuous throng

Of seven hundred strong,

But seven hundred faces,

Seven hundred persons

Devoted to a cause of good.

Singing, sharing, spelling

Jesus Christ;

Journey ended,

Really only begun

For futre dreams and aspirations,

Sucessful endeavor

In favour with God,

In favour with men,

A feeling of accomplishment -

Walk-a-thon.

TPS
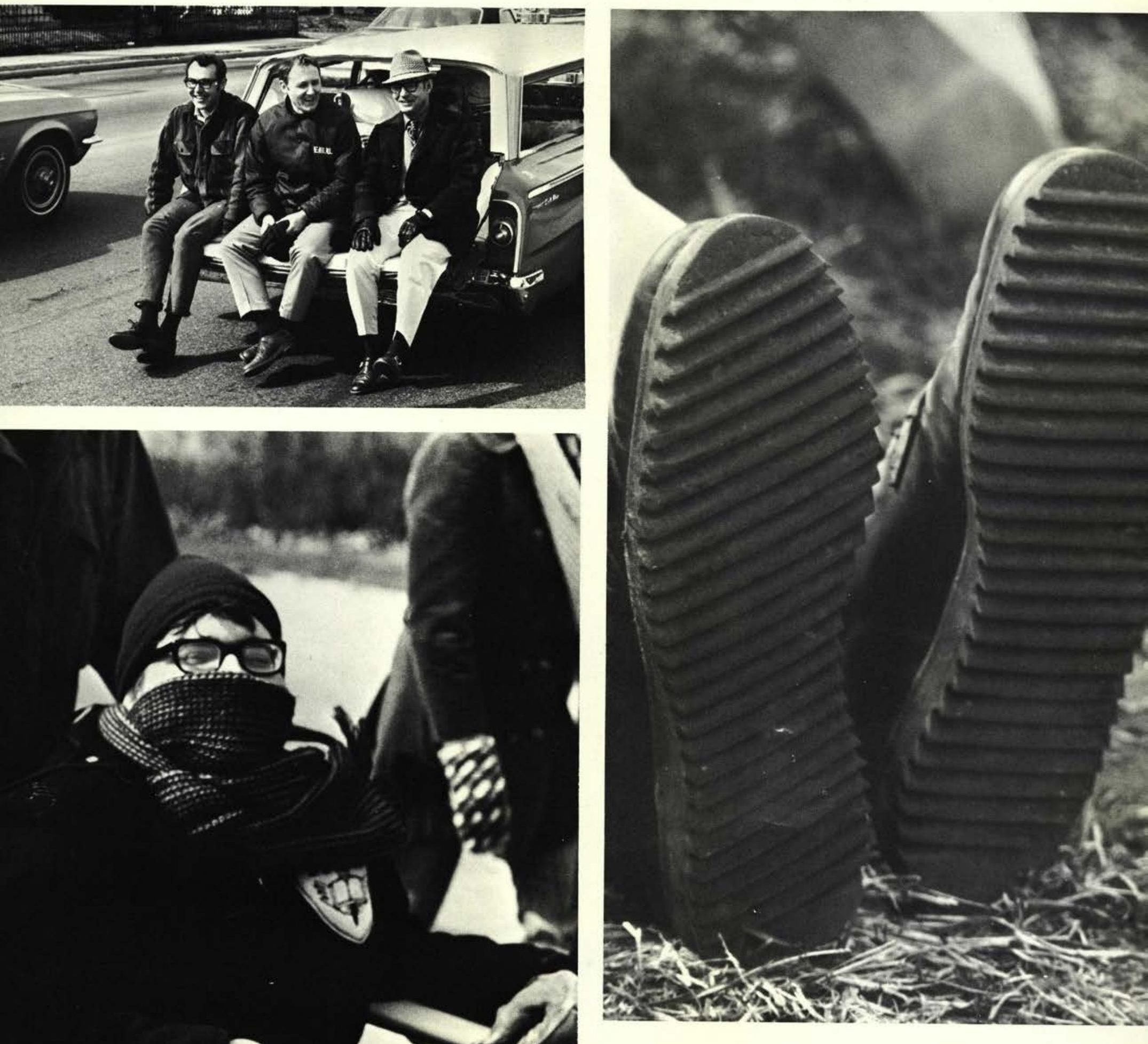


\section{Spring Sports}

A swing for the ville, refs

Sandtraps

keep yer eyes on the ball, team spirit,

run your best,

etc
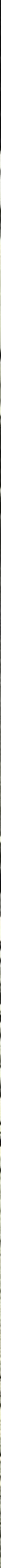


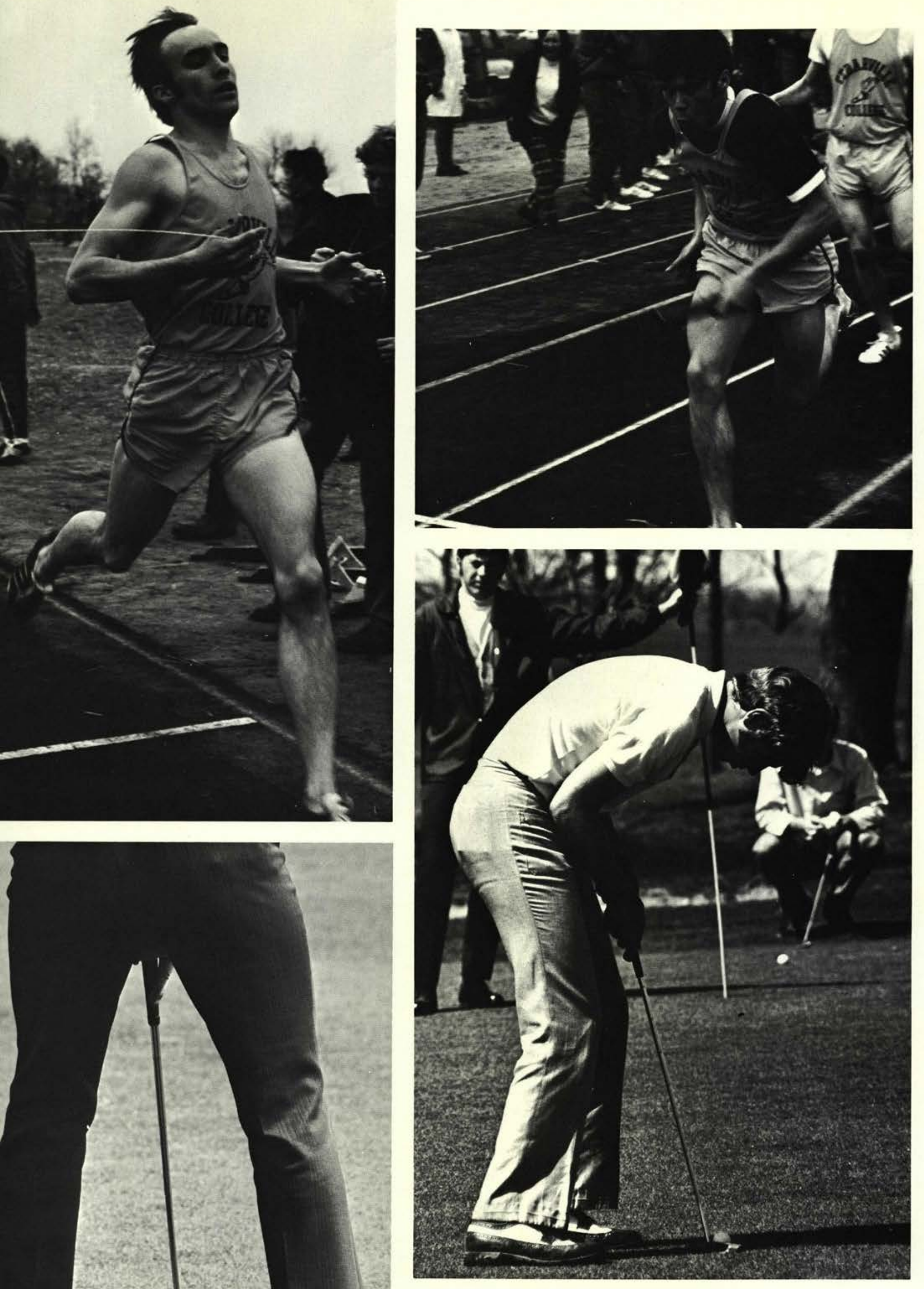




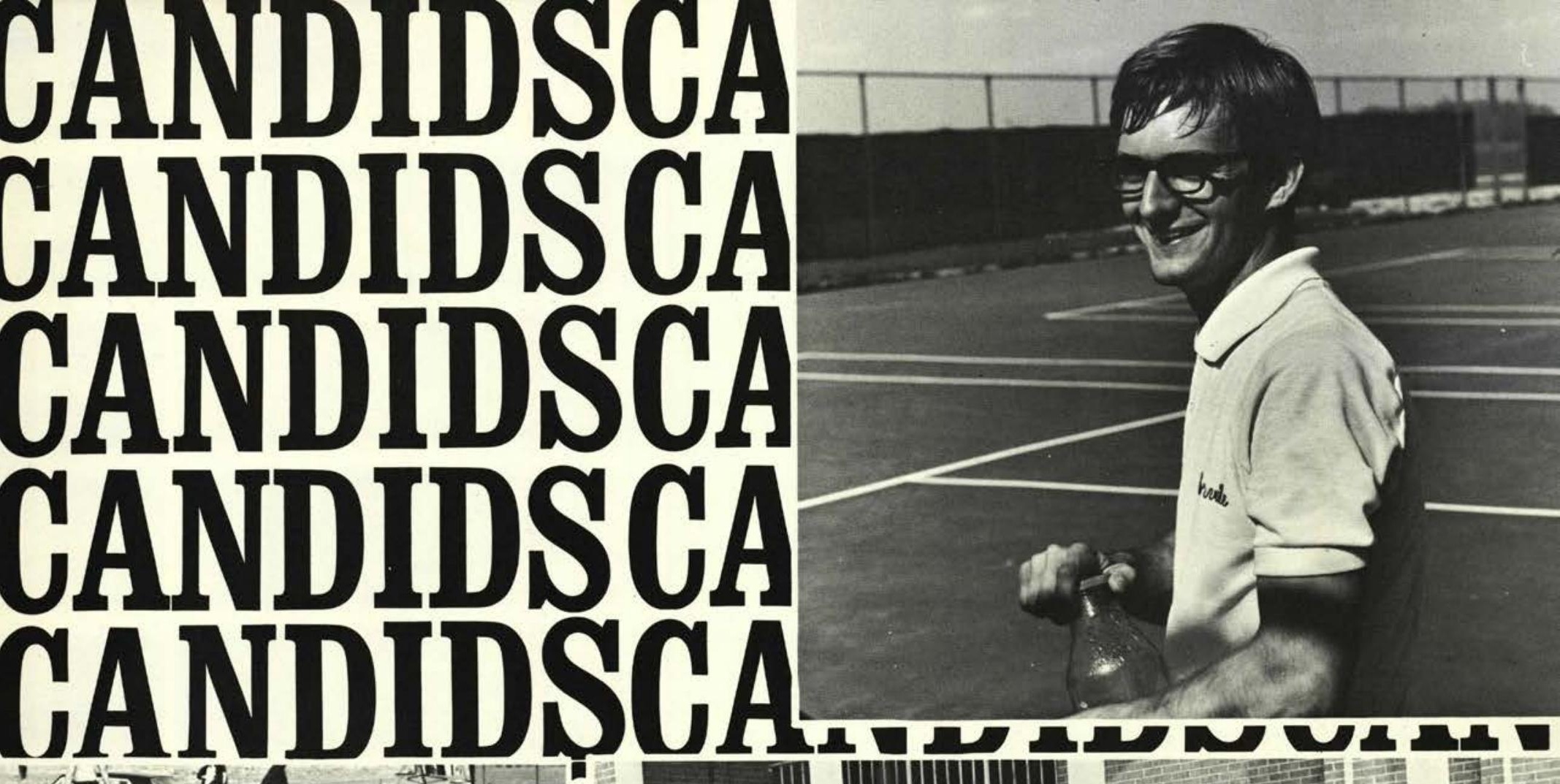

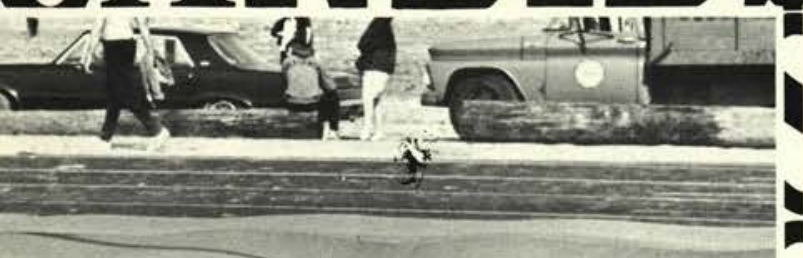
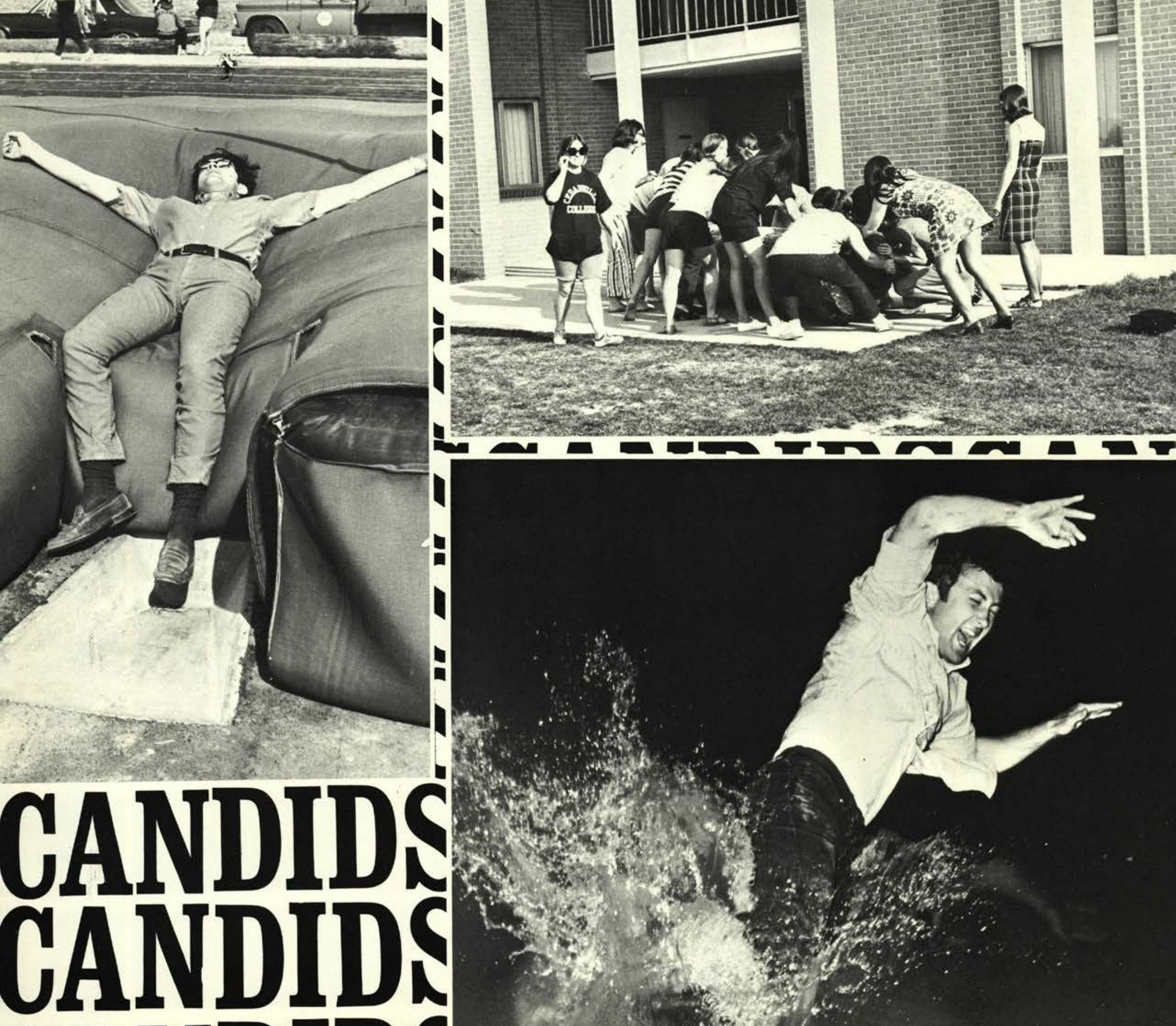
,

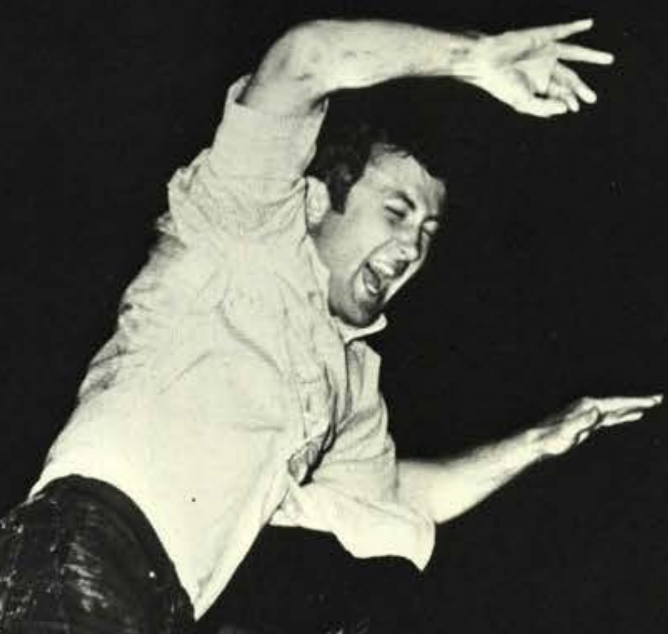

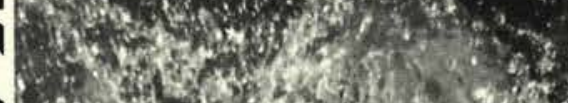

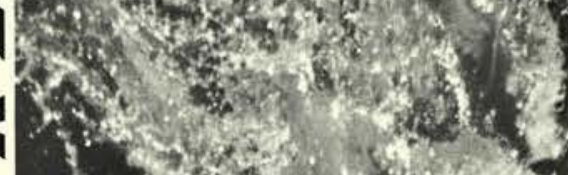
(6) 14 

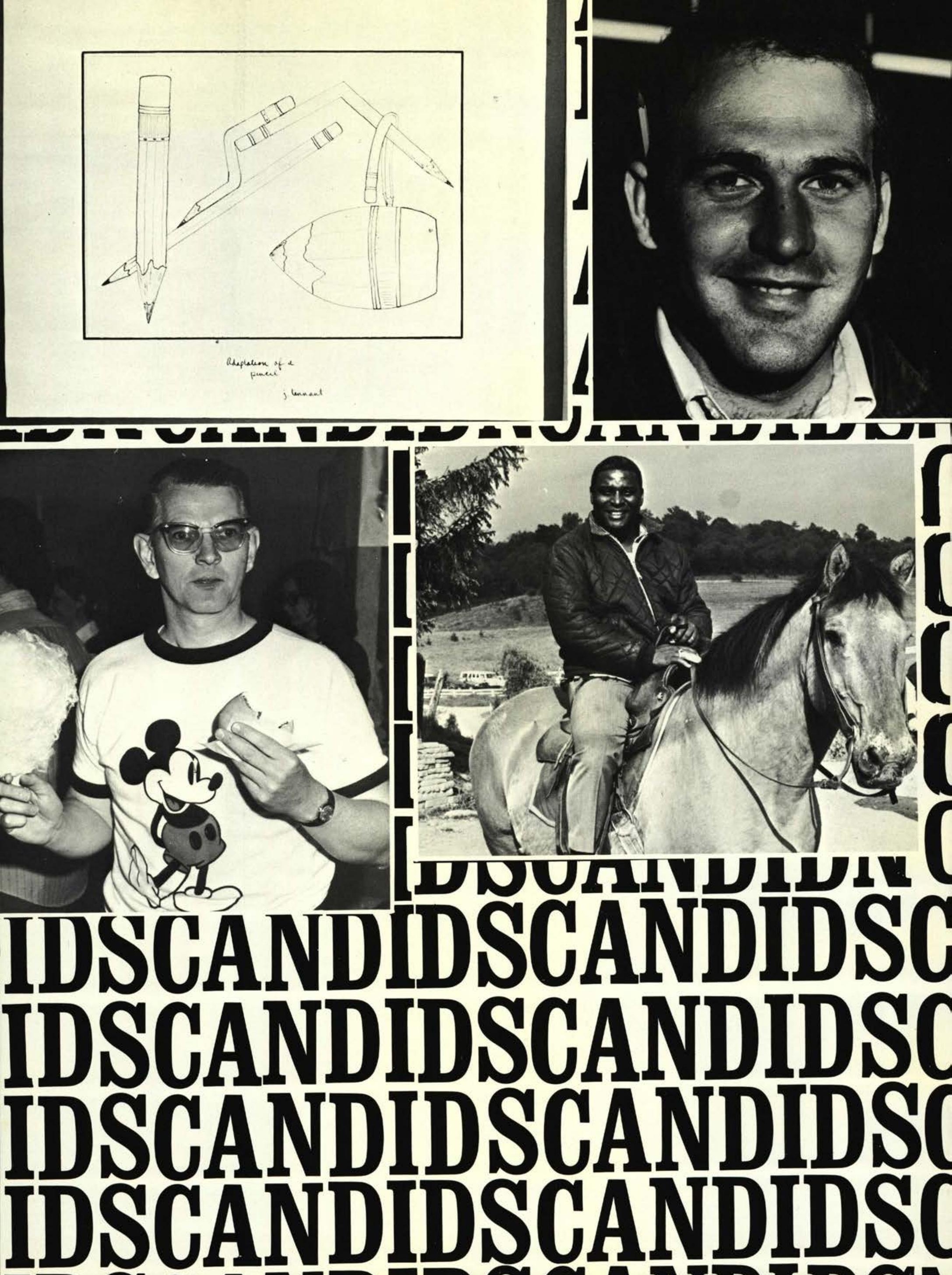

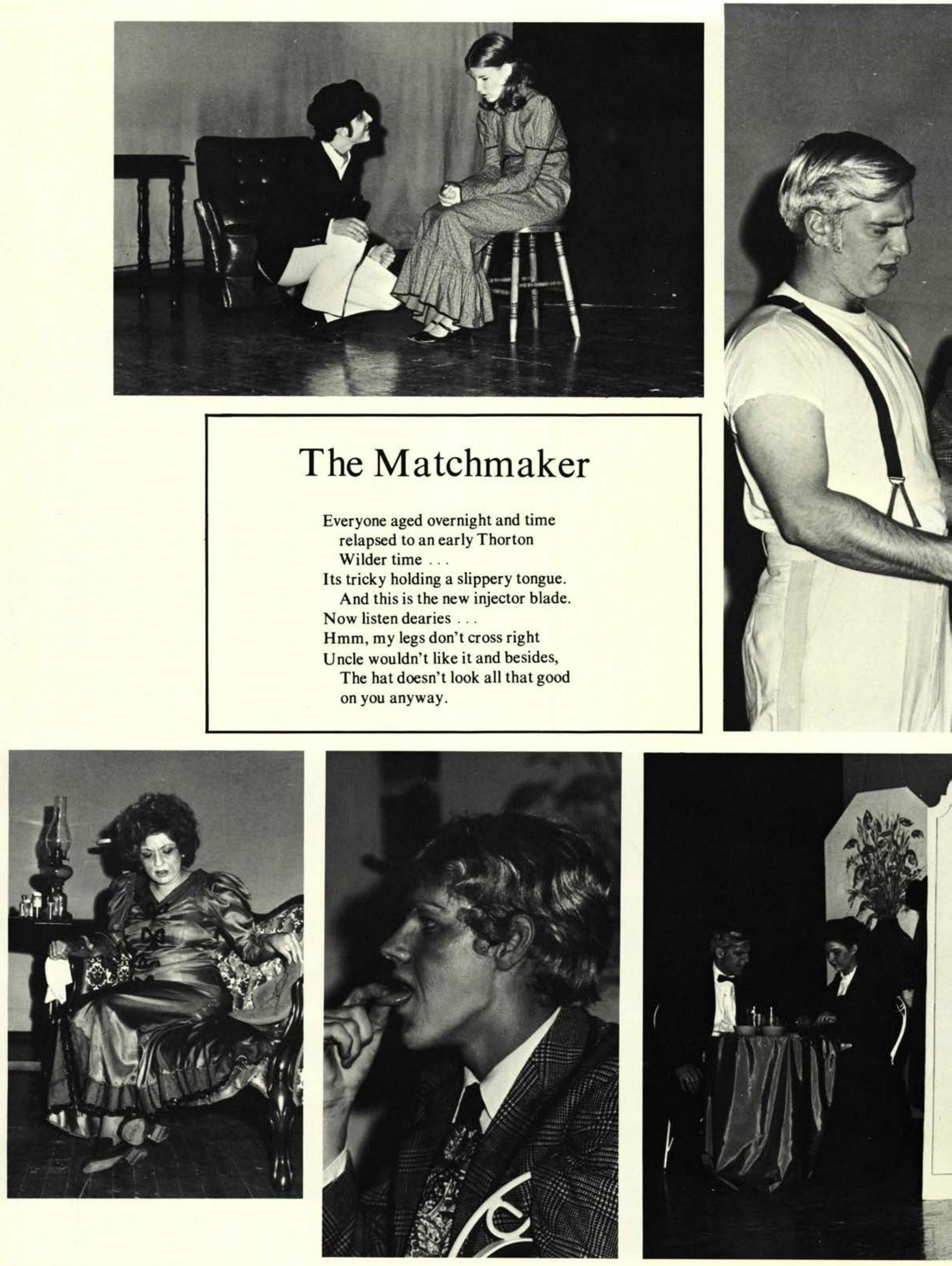

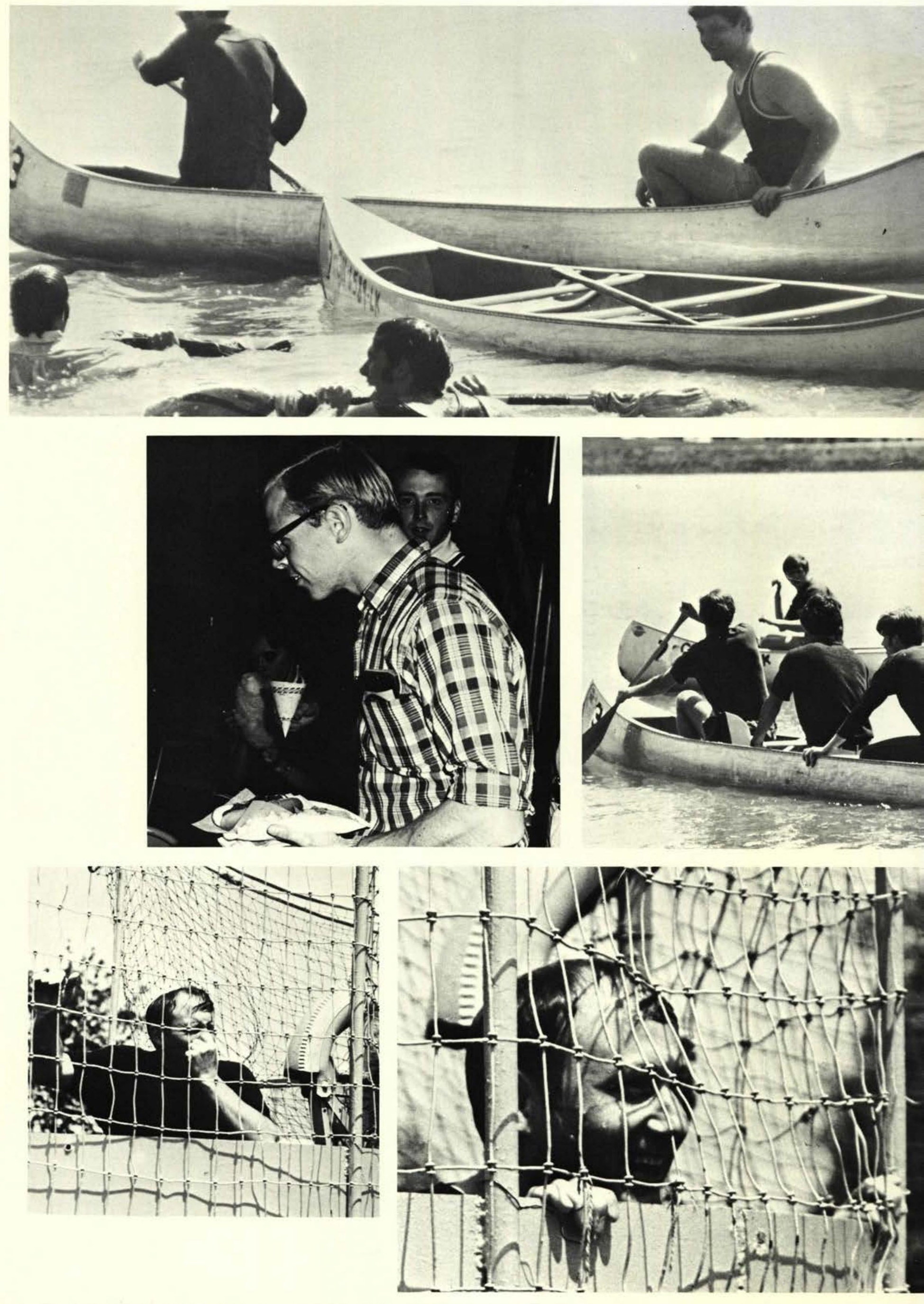

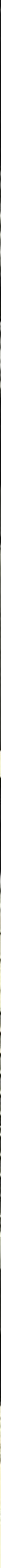


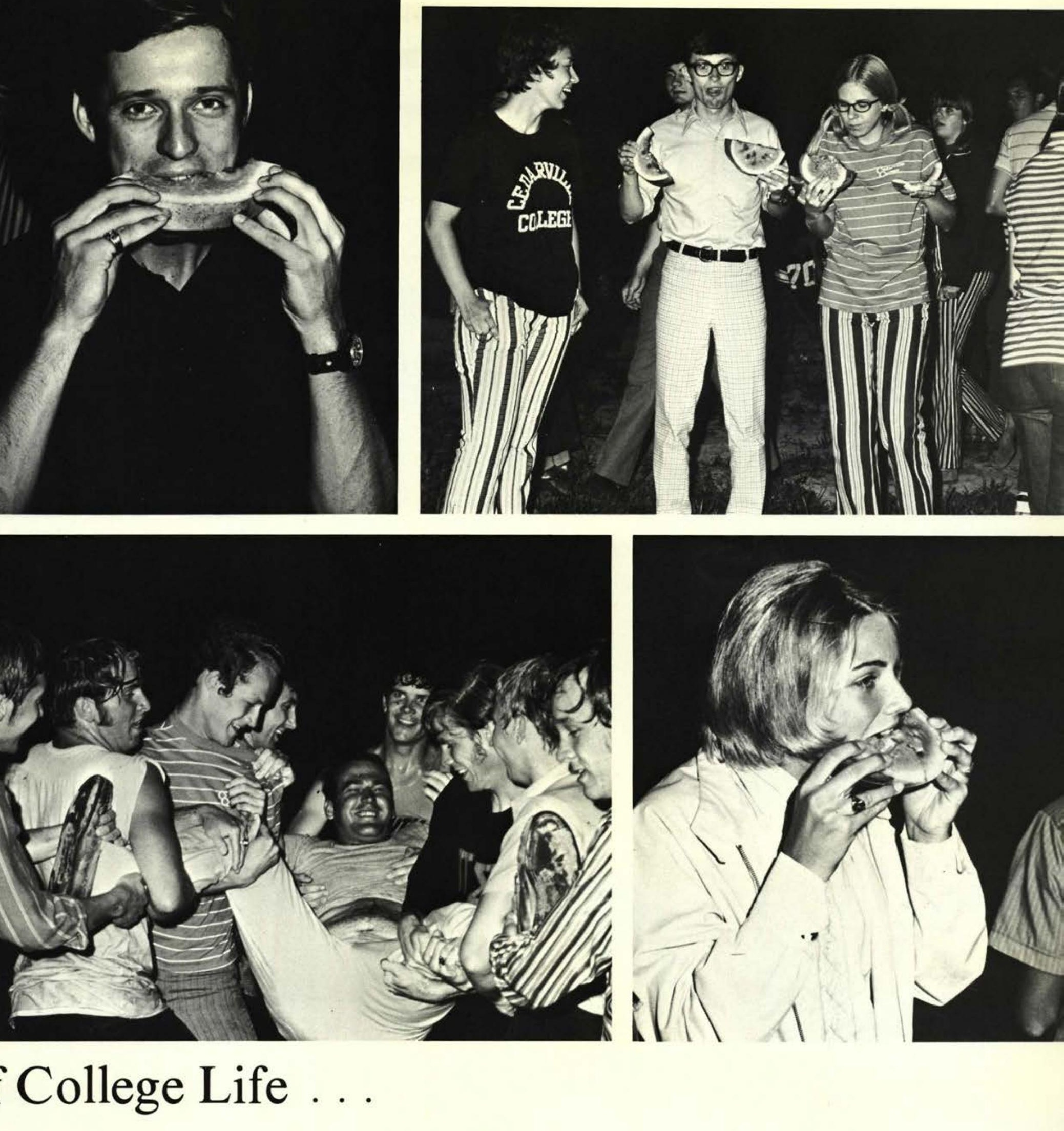




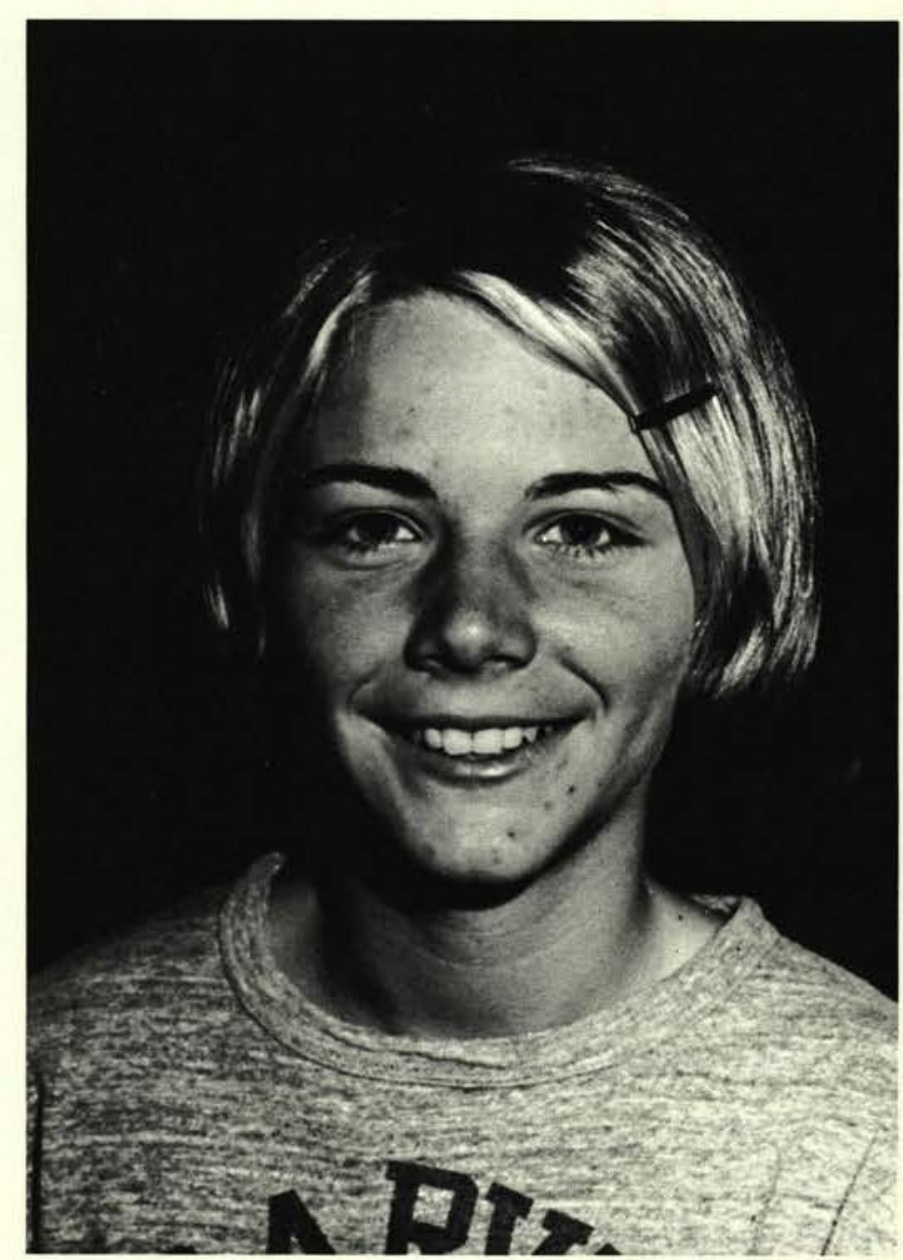

-

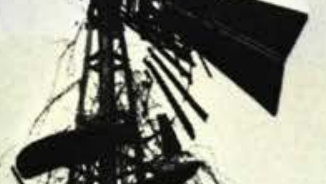

(3)
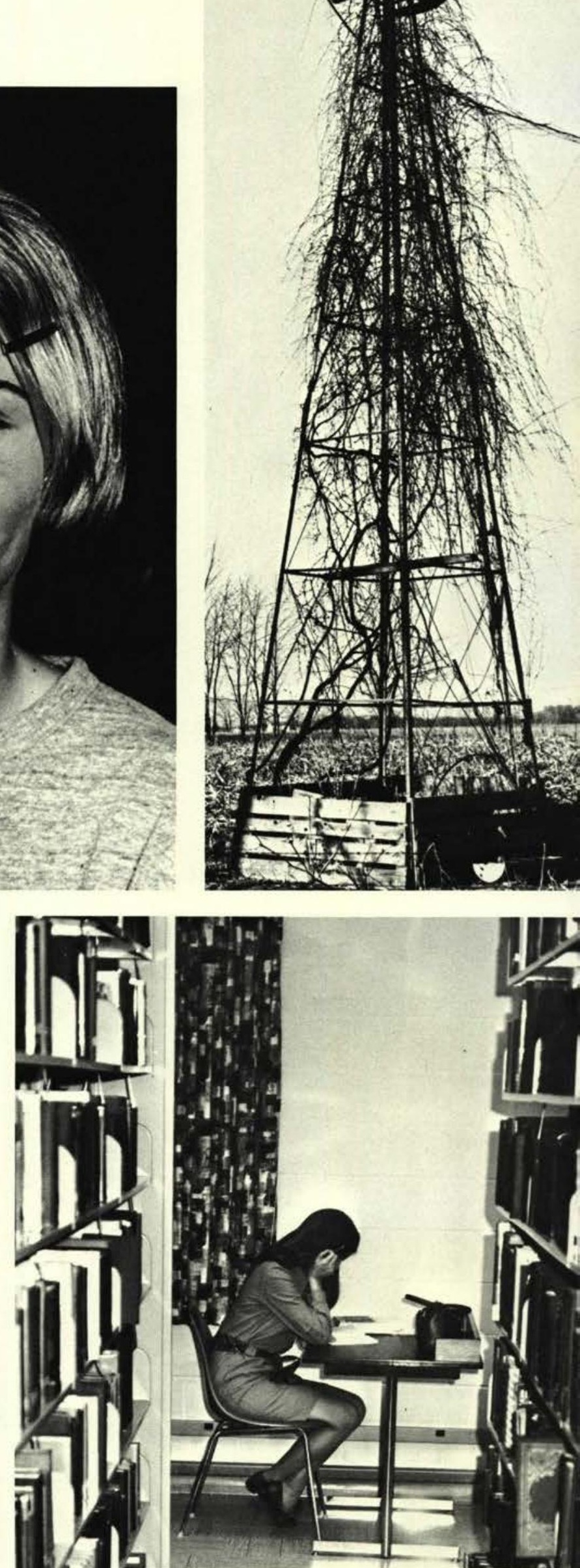

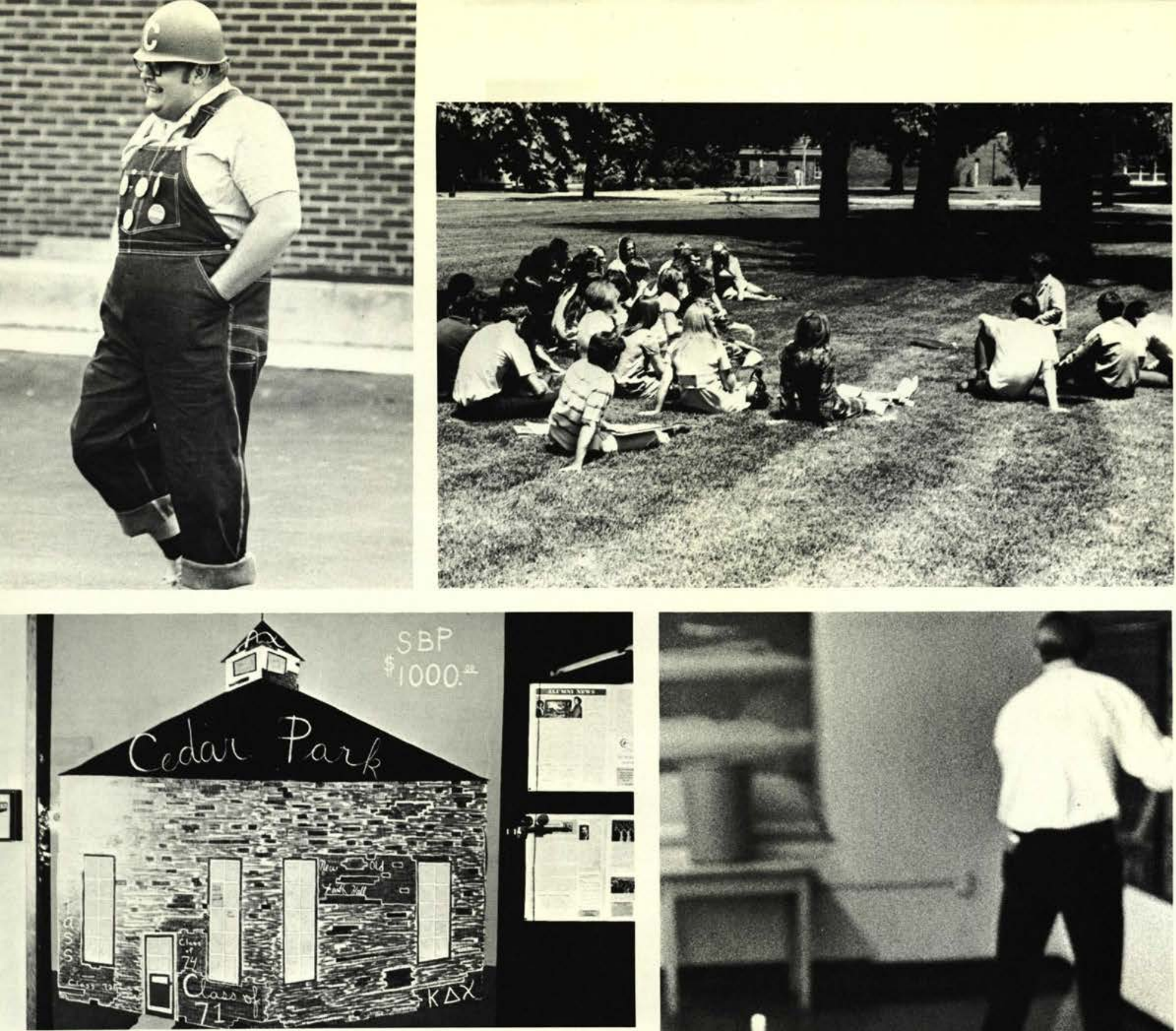

\section{Life should be}

lead as a cavalry

charge!

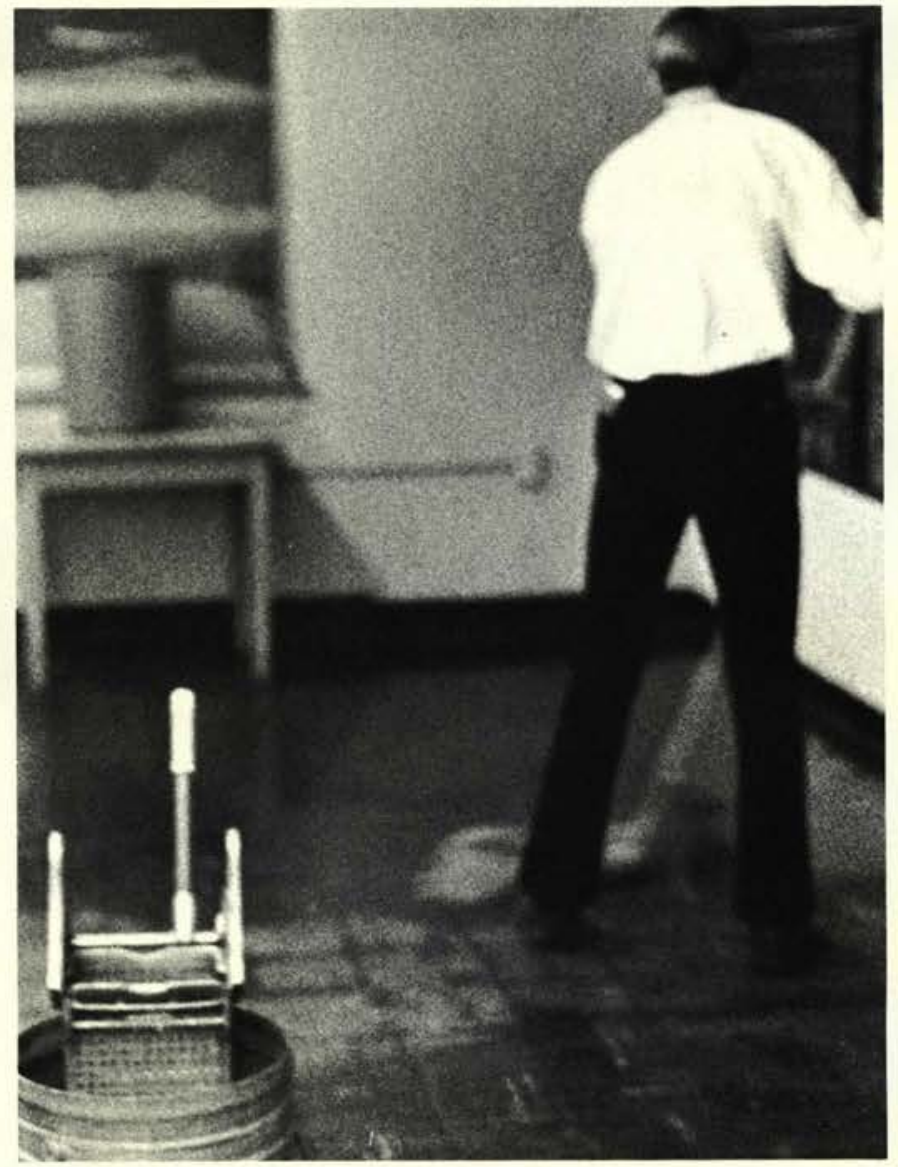




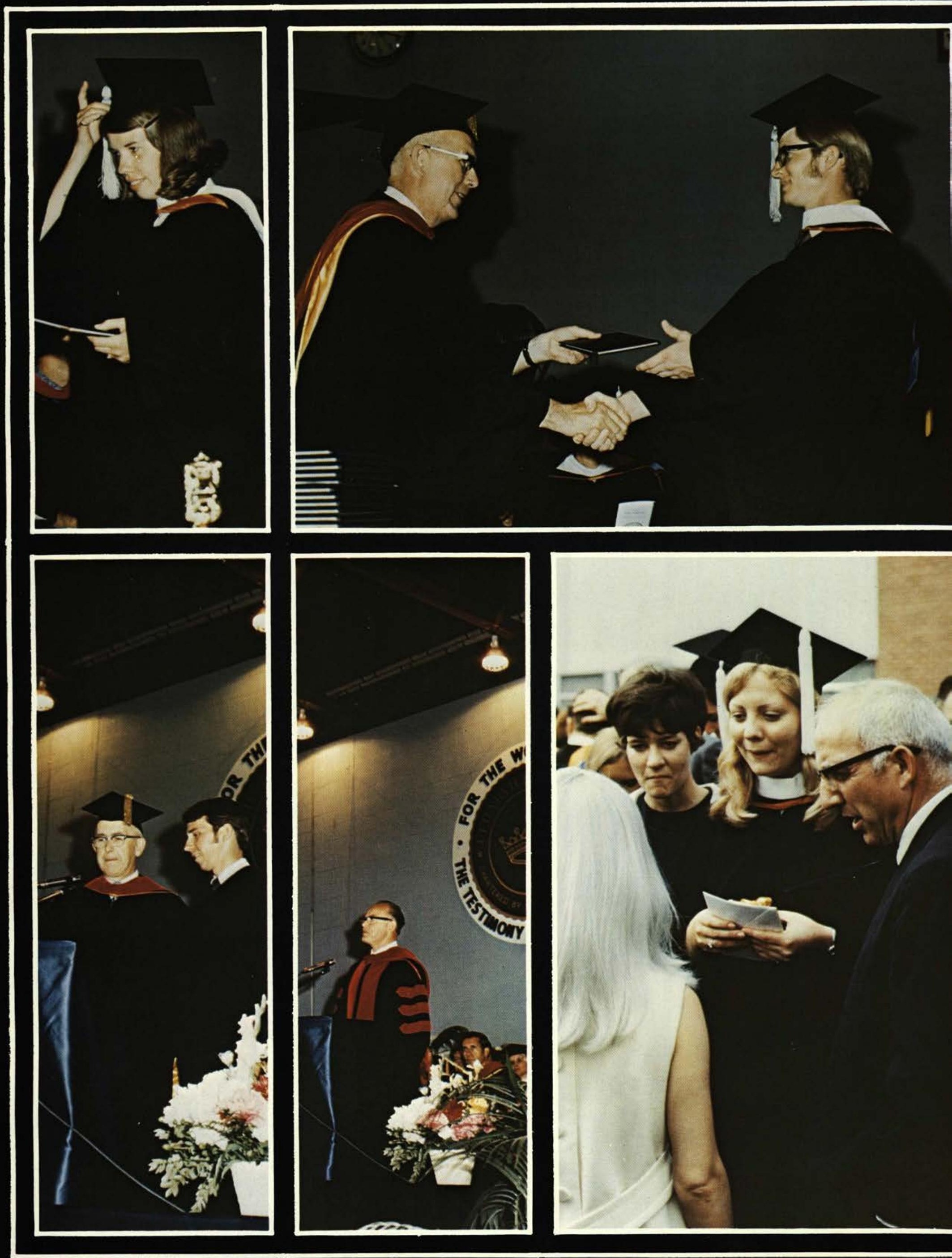




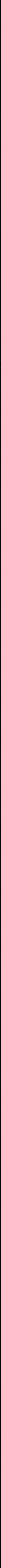




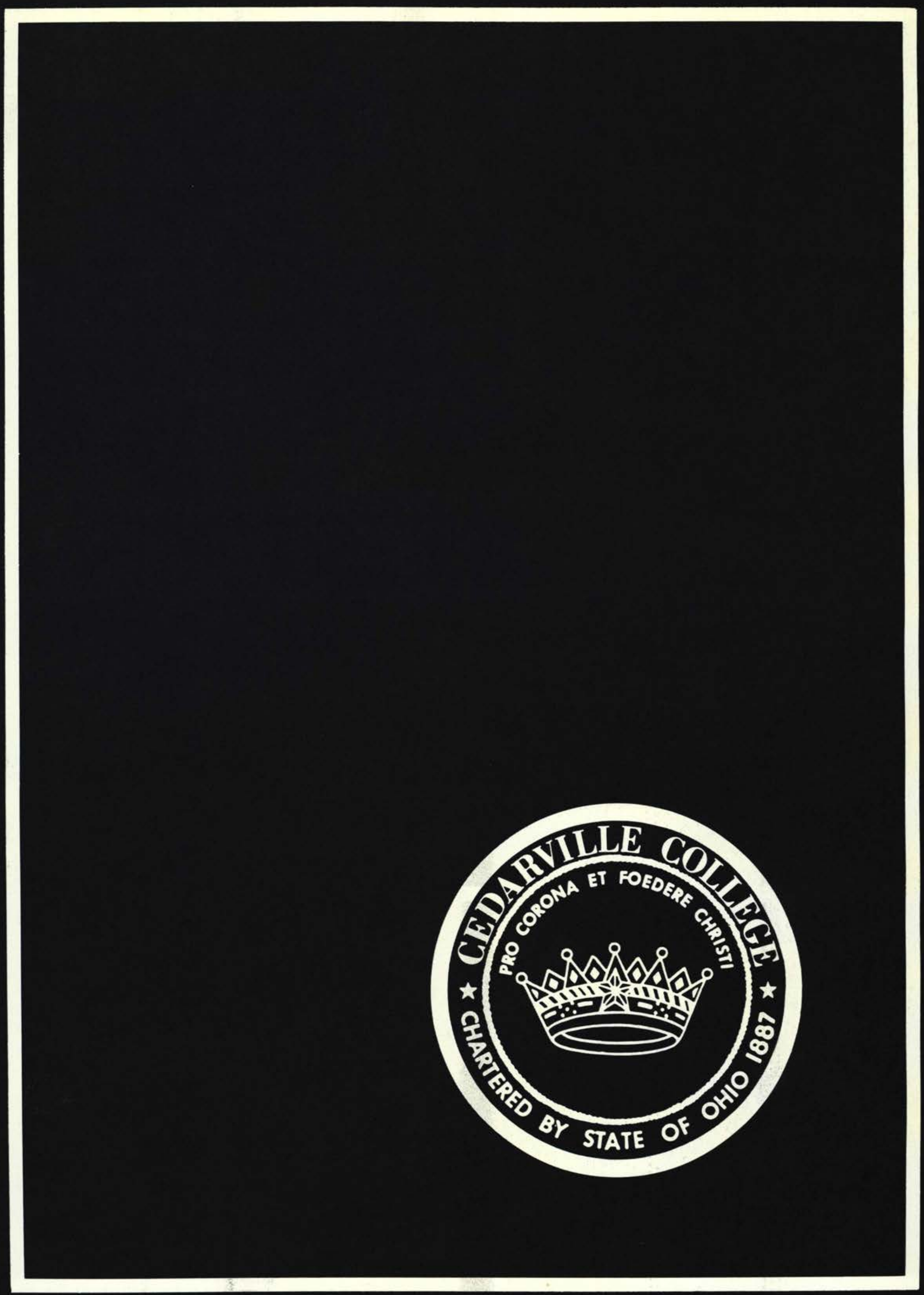




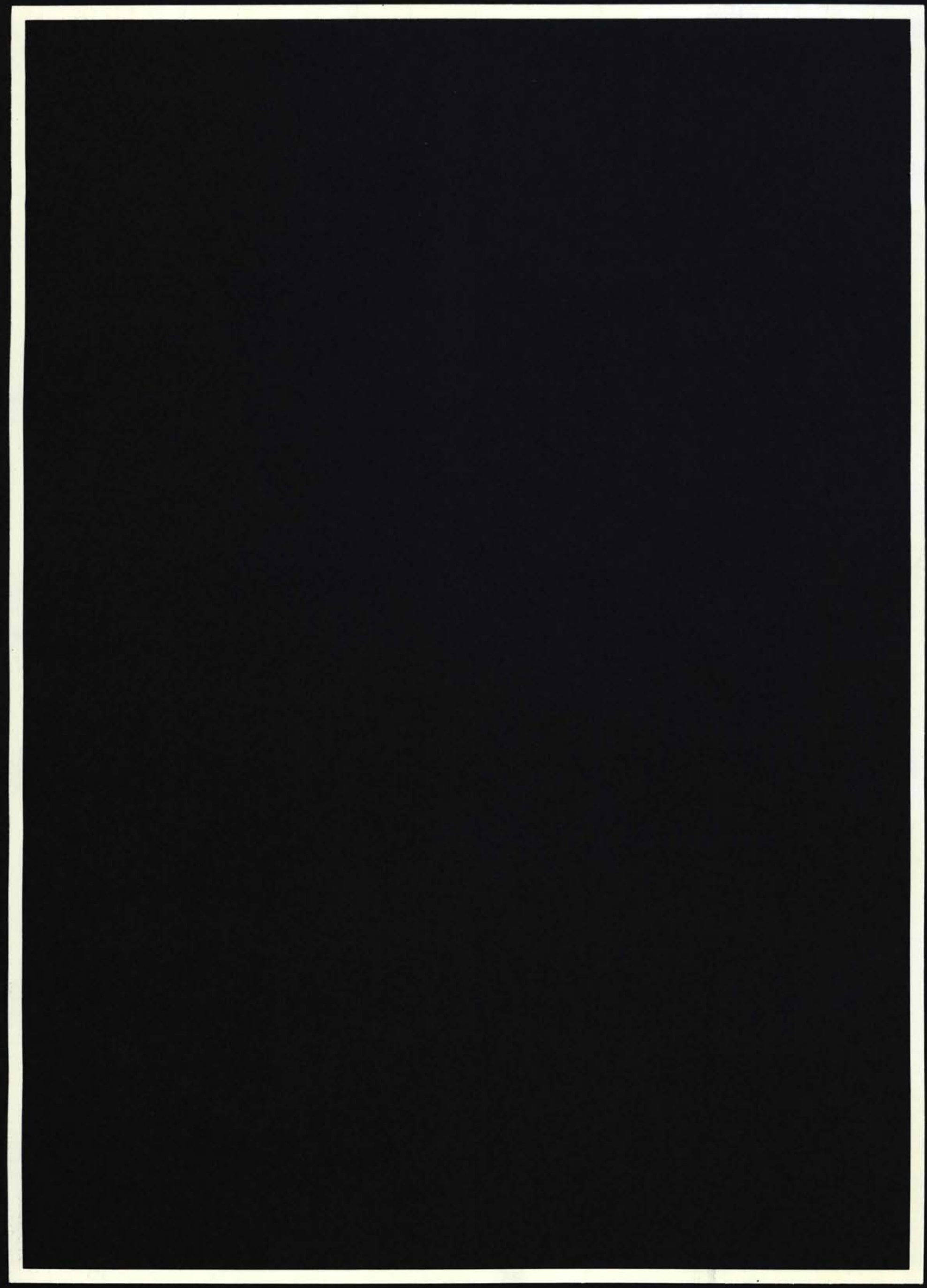




\section{MIRACLE}

\section{Table of Contents}

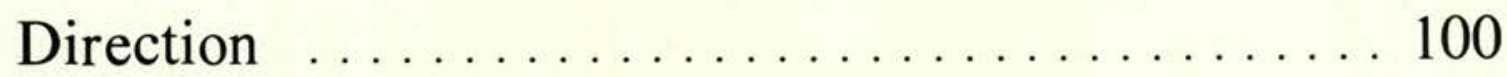

Identification $\ldots \ldots \ldots \ldots \ldots \ldots \ldots \ldots \ldots . \ldots \ldots$

Participation ....................... 172

Competition .......................... 194

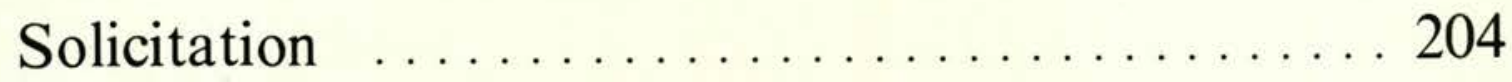


PRESIDENT

ACADEMIC DEAN

BUSINESS OFFICE

BUSINESS MANAGER

DIR. OF PURCH. \& ACCT.

CASHIER

DIR. OF DEVELOPMENT

STUDENT PERSONNEL

DEAN OF STUDENTS

DEAN OF MEN

DEAN OF WOMEN

DEAN OF FINANCIAL AID
DIR. OF GIDLEY
DIR. OF STUDENT ACTIVITIES MR NICHOLS

MR. ST CLAIR

MR. I W RICKARD MRS. SPENCER

MR. TURNER

MR. D W RICKARD

MR. LOPER

MRS. ARMSTRONG

MR. GIDLEY

REGISTRAR \& ADMISSIONS

MR. HURST

ALUMNI DIRECTOR

estate Planning

EDUCATION DIVISION

MISS DODSON

MRS. STOCKWELL

FACULTY OFFICES

301 MRS. HAHNLEN

302 MR. GROSH

303 MISS MACKAY

304 DR. MONROE

305 MR. SPENCER

306 MR. FRANK

307 DR. FLECK

308 DR. SCOTT

ROOM 3

MR. ARMSTRONG

MR. GRIER
MR. BRYANT

MR. PHILLIPS

309 DR. RIGGS

310 MR. BERGEN

311 MR. MURDOCH

312 MR. GREENWOOD

313 MISS FISHER

314 DR. AGER

315 DR. GROMACKI

316 DR. MC DONALD
106

105

101

101

101

103

104

1

1

1

2

3

102

221

221

203
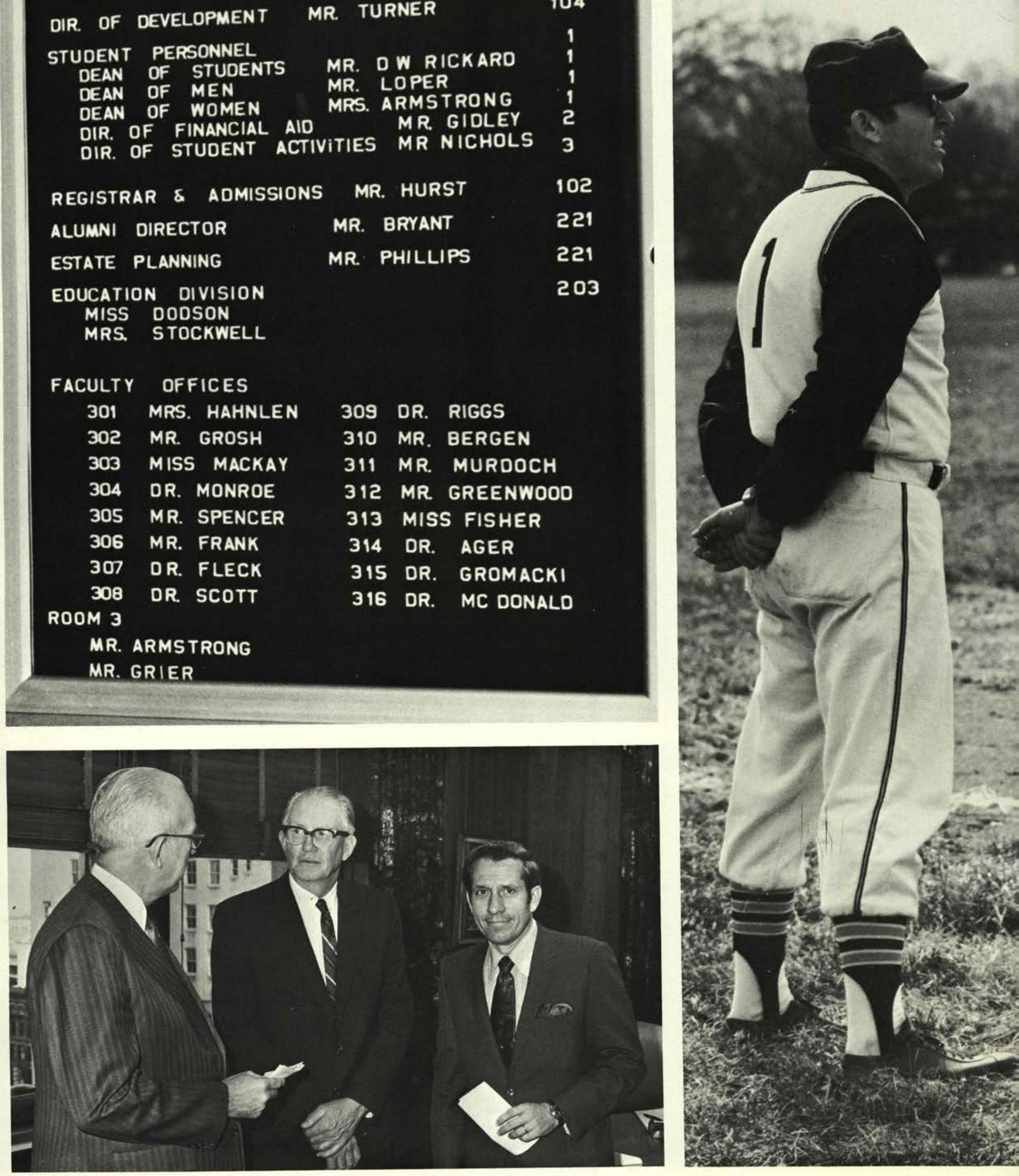


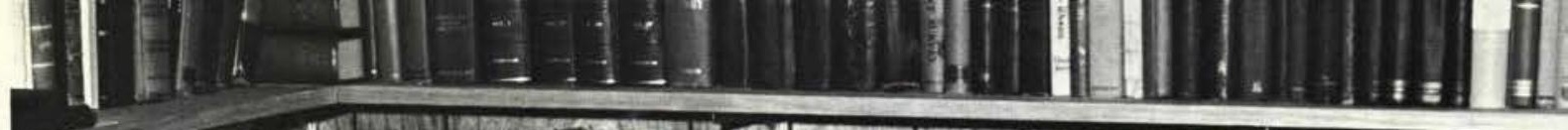
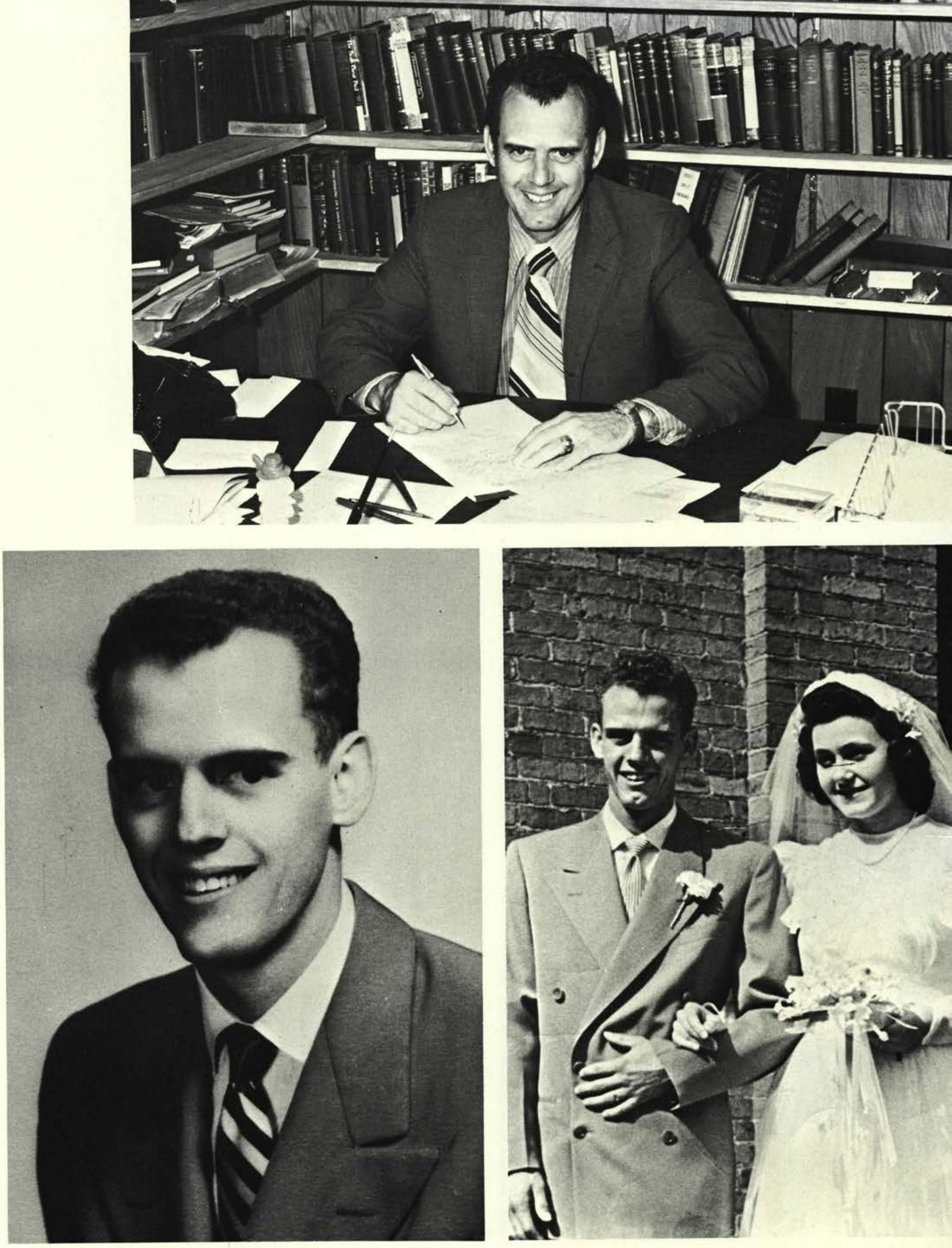


\section{In appreciation for great contributions made to}

the spiritual life of our students - we dedicate this yearbook to Pastor Green.
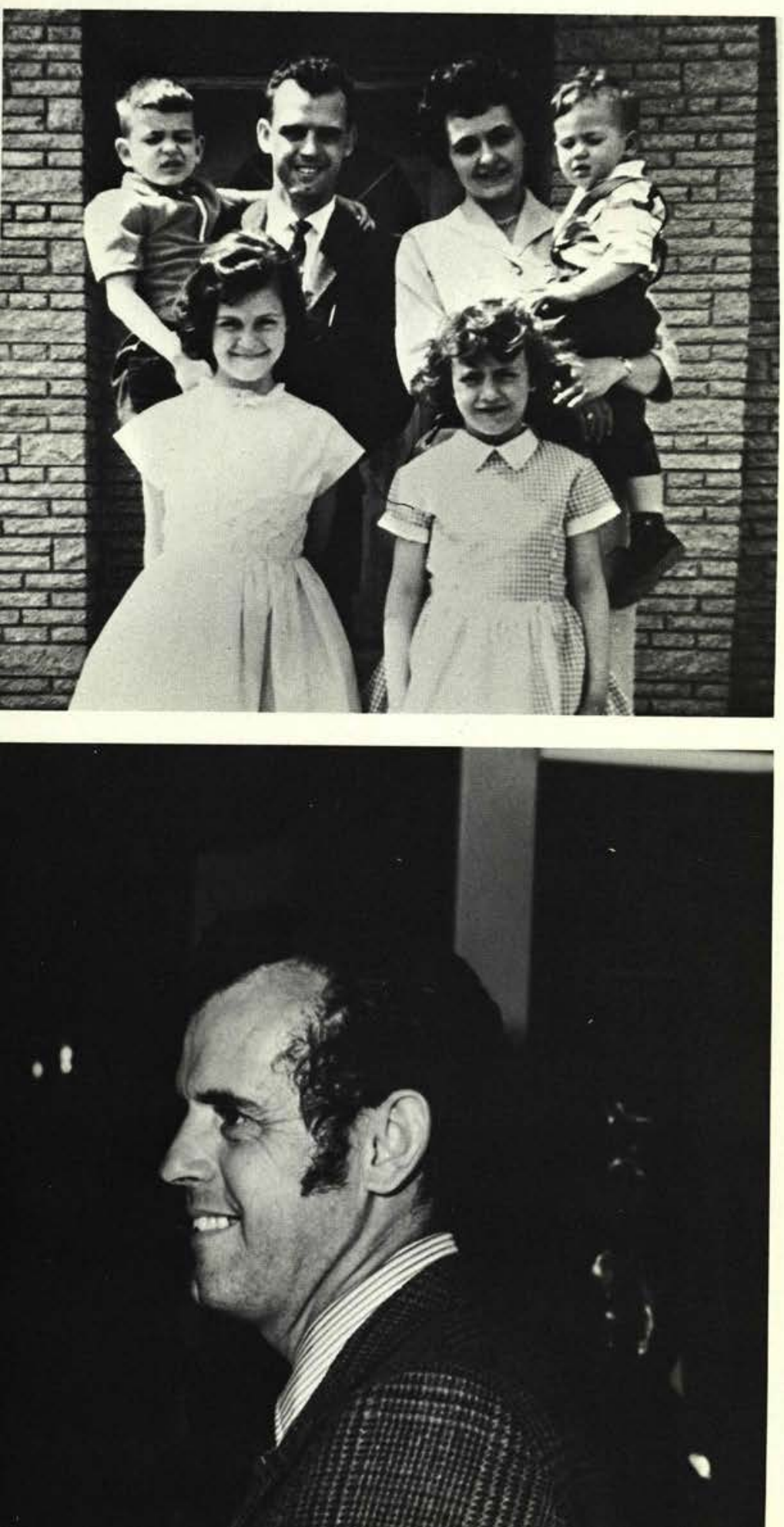

We, the students of Cedarville,

Were lacking -

In the field of Christian service,

In an aura of a godly atmosphere,

Lacking in our own pastor.

"We sought for a man

To stand in the gap,"

And the Lord answered prayer.

From out the halls of

Baptist Bible Seminary,

From out the city of Greenville

From out of a church pastorate,

God sent a real man,

"An example of the believers."

"In word, in conversation:"

He lives this to his four children

As a godly father;

"In charity:"

He proves this to his wife;

A dedication first to God,

Second to family,

Then to others.

Our Christian service department

Turned upside-down for our Lord,

Pastor Green began organizing.

He gave us a challenge!

"In word,"

He preaches from the Scriptures,

He "stomps on toes,"

But many listen,

Through reading,

Exhortation, doctrine.

The Cedarville Baptist Fellowship

Began rolling

Through the employment of his cabinet

Known as the Advisory Twelve.

"In charity,"

Raising support for the Weah family,

Talking with the students

In the dorm, the classroom,

Outside under a tree,

And in his office at his desk

Where he spends much of his time.

Praying, searching the scriptures,

Counselling -

This is our Pastor Green!

Right in with the students

On beach evangelism,

Administering advice for Swordbearer's tour;

Organizing, working on their conference in the fall.

"In spirit,"

Coaching the wrestlers

To physical and spiritual victory;

"In spirit,"

Cheering on the basketball court.

At track meets;

Firing up missionary internees

To service on a foreign field;

Engaging himself in weekend

And summer retreats and camps.

"In faith,"

Believing God will work in servicemen's hearts

At Wright-Patterson Air Force Base;

Believing God will answer each prayer,

Will convict, will speak to students;

"In faith,"

Launching out financially

To build better programs -

And by evidence on the campus,

Pastor Green puts feet to his faith. And "in purity,"

The totality of his character,

The person in word, conversation,

Charity, spirit, and faith.

And if one should be able

To see this man,

In the wee, small hours of morn,

On his knees before God,

One should see the real pastor Green,

The peace of God

Ruling in his heart.

"Mark the perfect man.

For the end of that man is peace." (Ps. 37:37) 


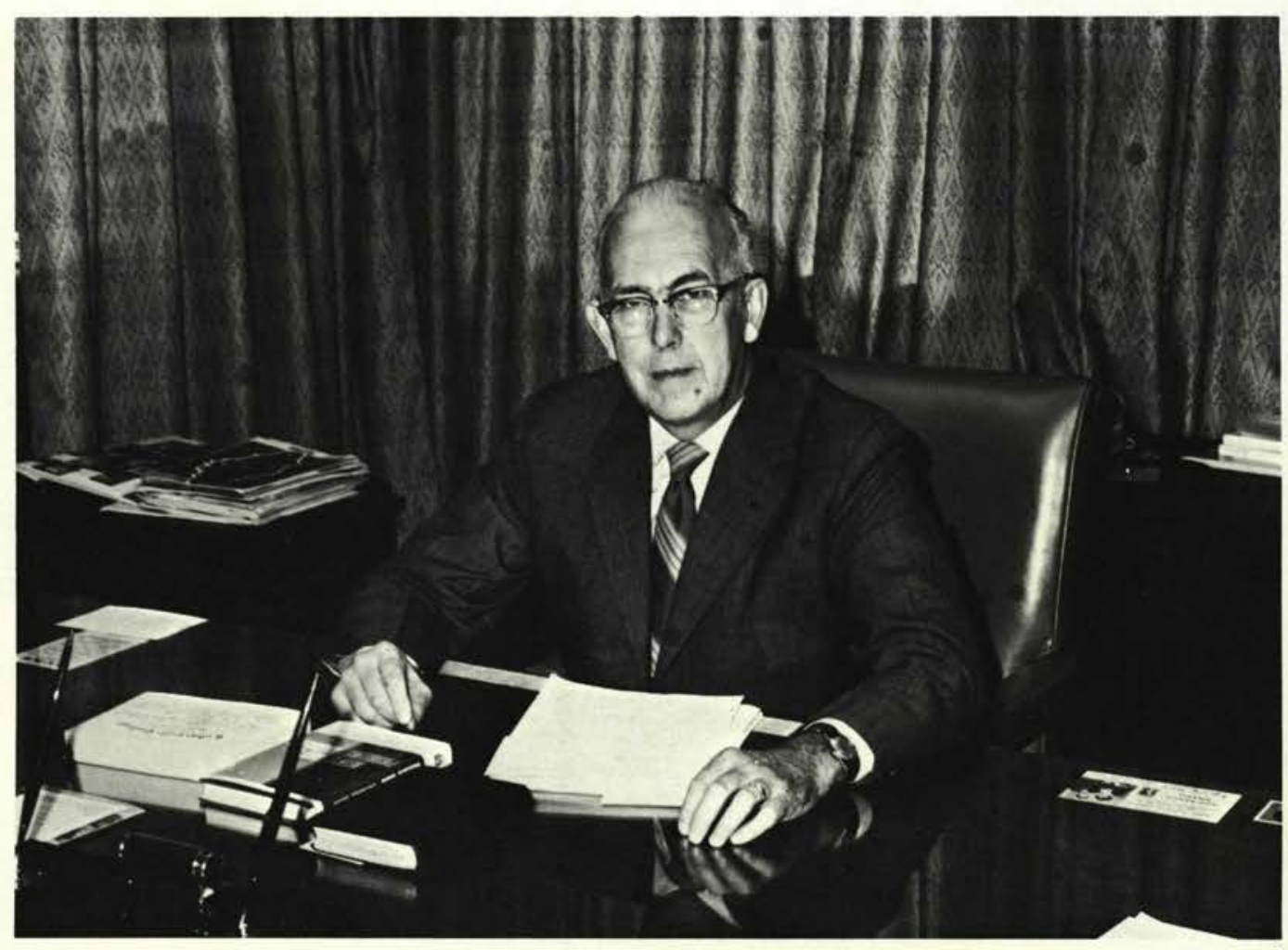

\section{Dr. James T. Jeremiah President}

"For the Word of God and the testimony of Jesus Christ" a faculty and administration is dedicated ... 

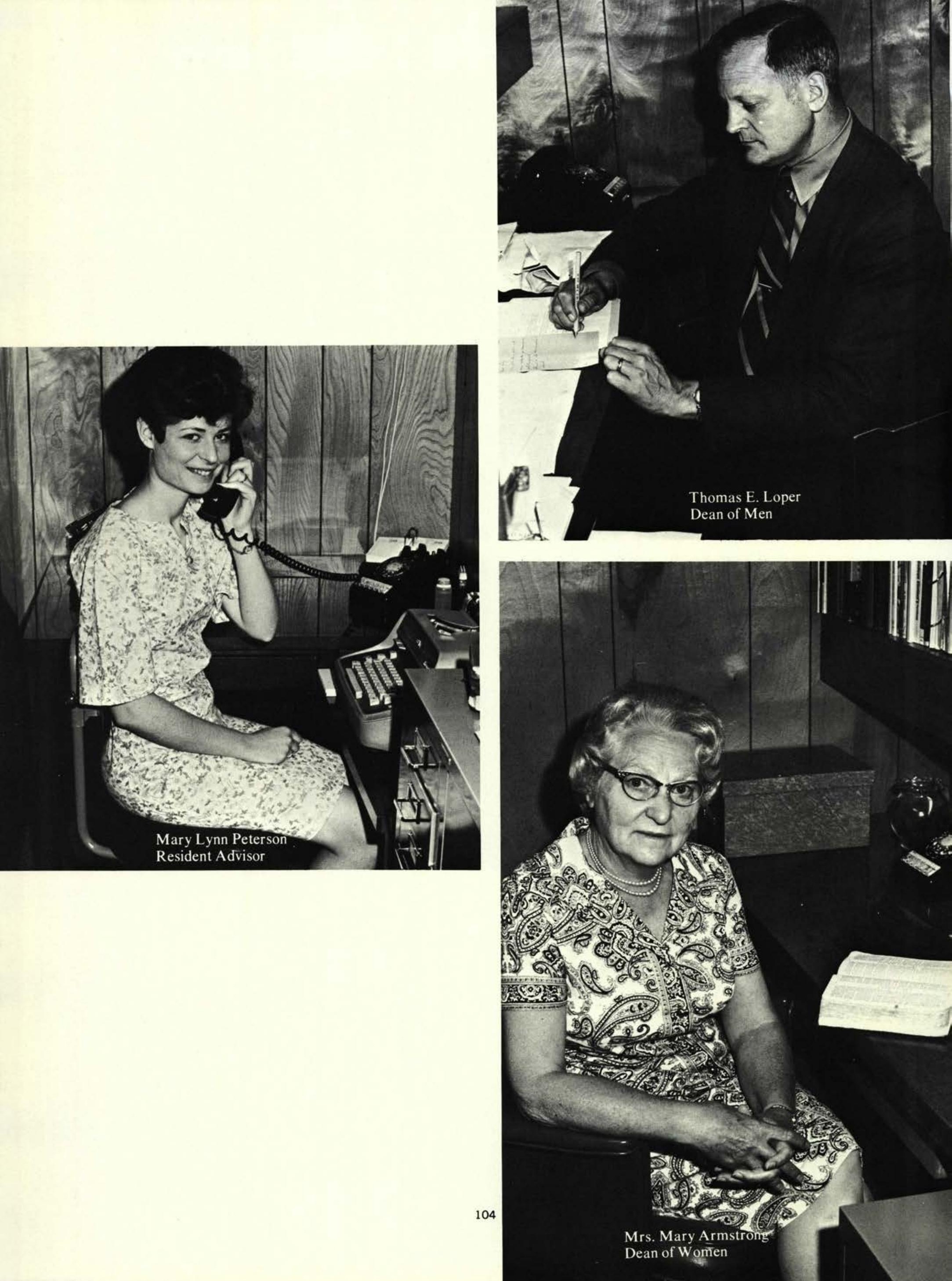

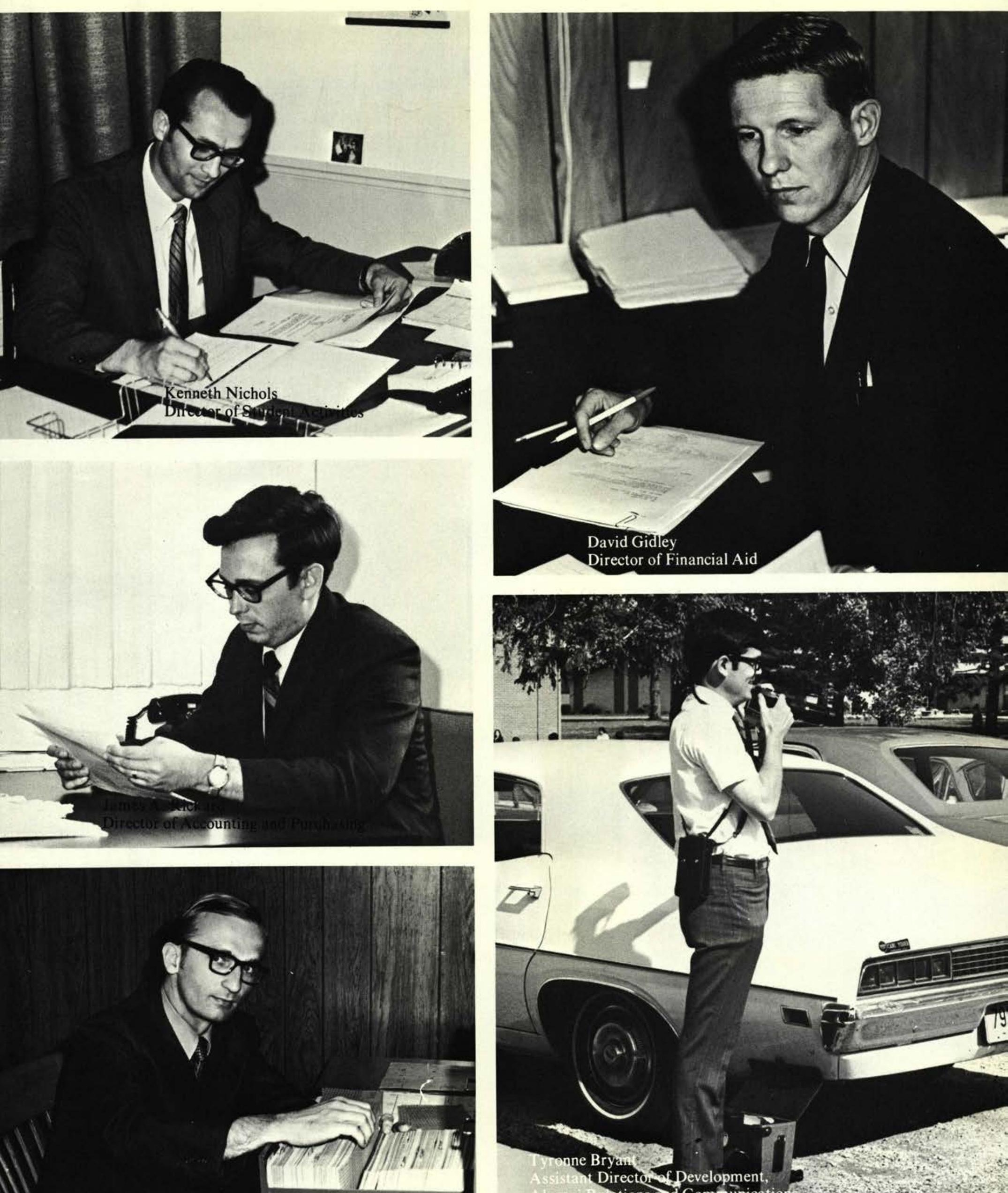


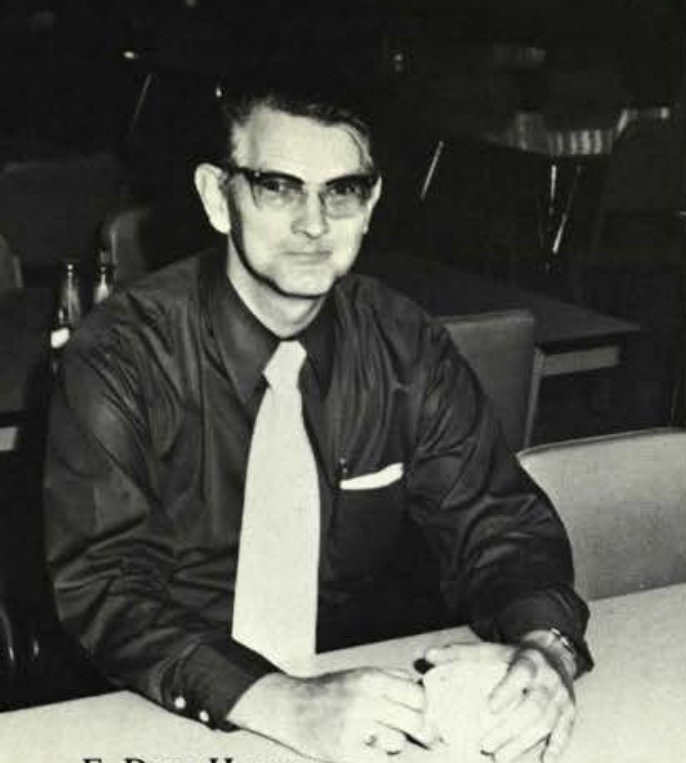

\section{E. Dane Harvey}

Assistant Professor of

Physics and Mathematics
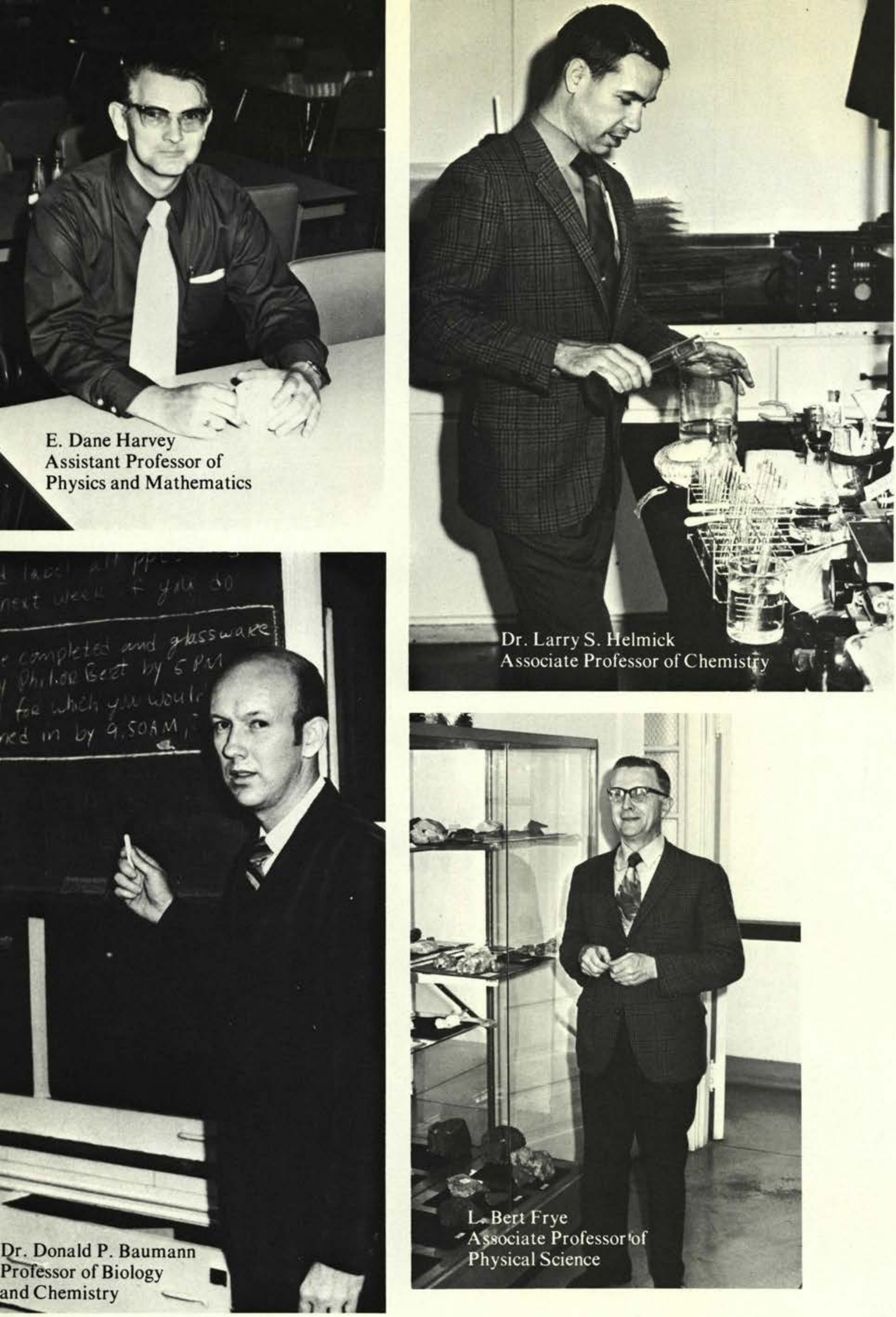

\section{Mathematics}




\section{Health and \\ Physical Education}
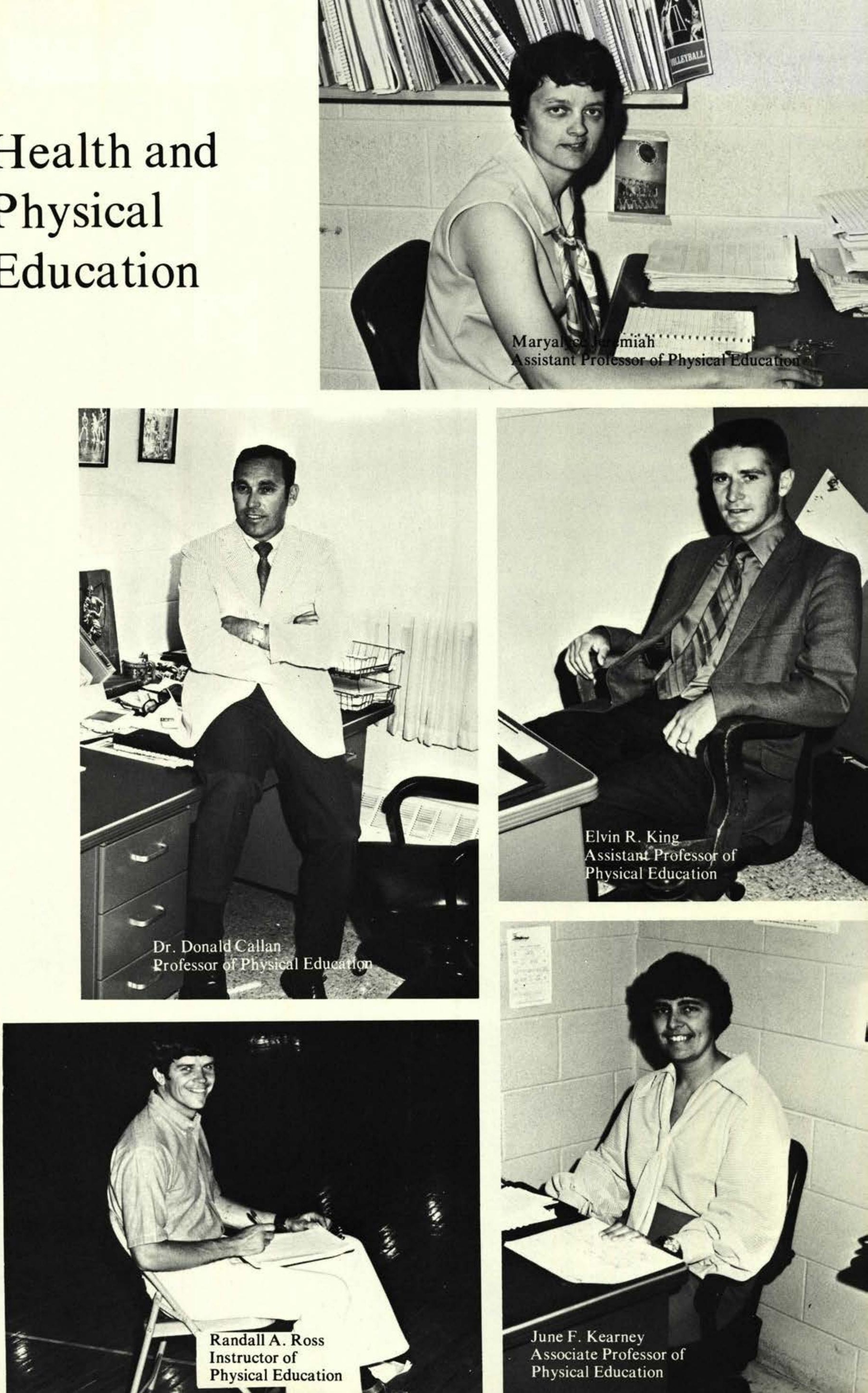

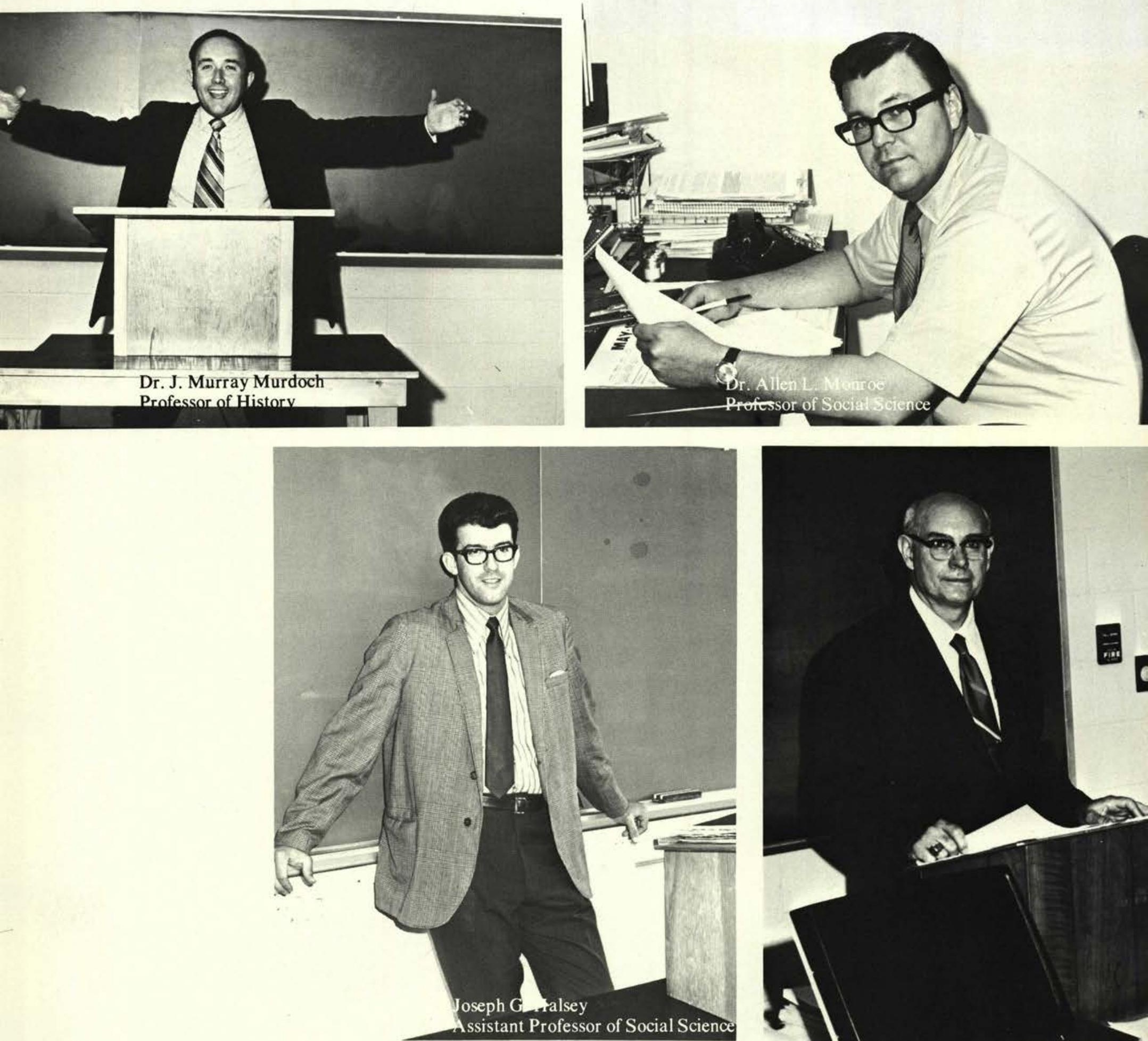

\section{Social Science}

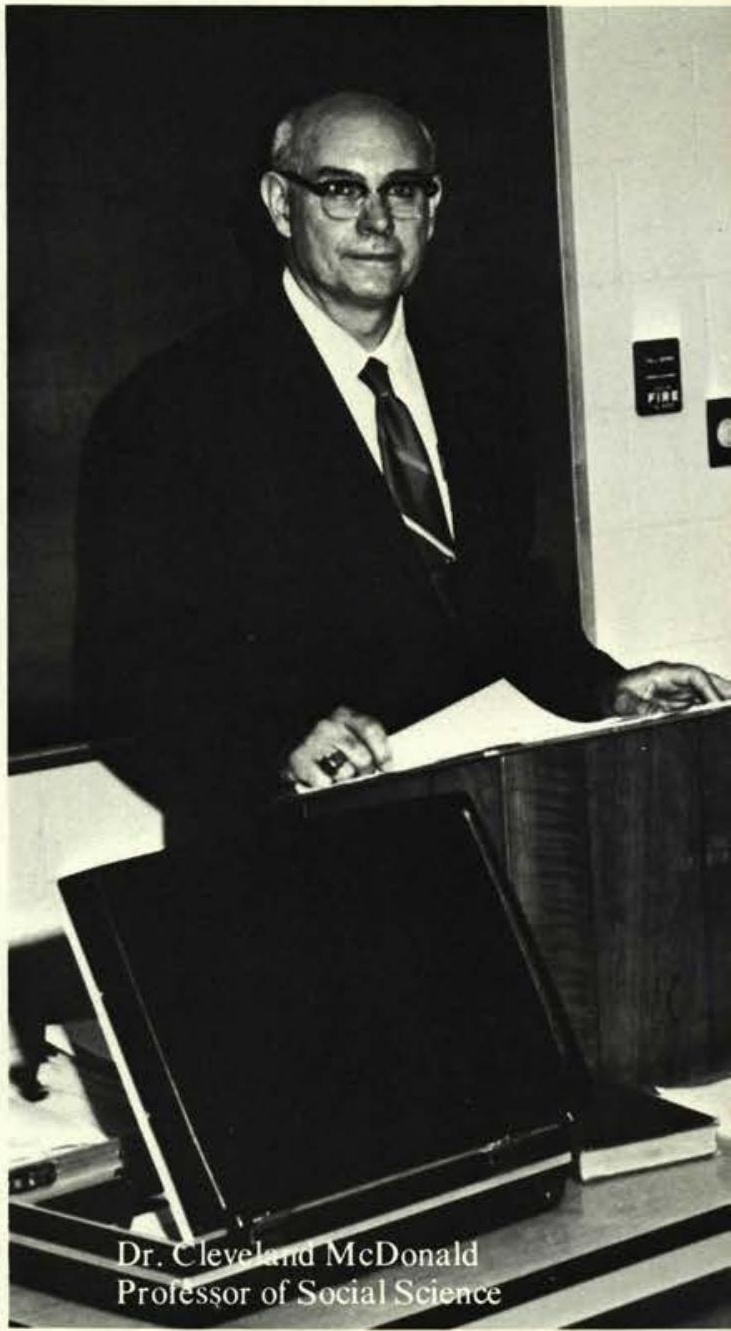



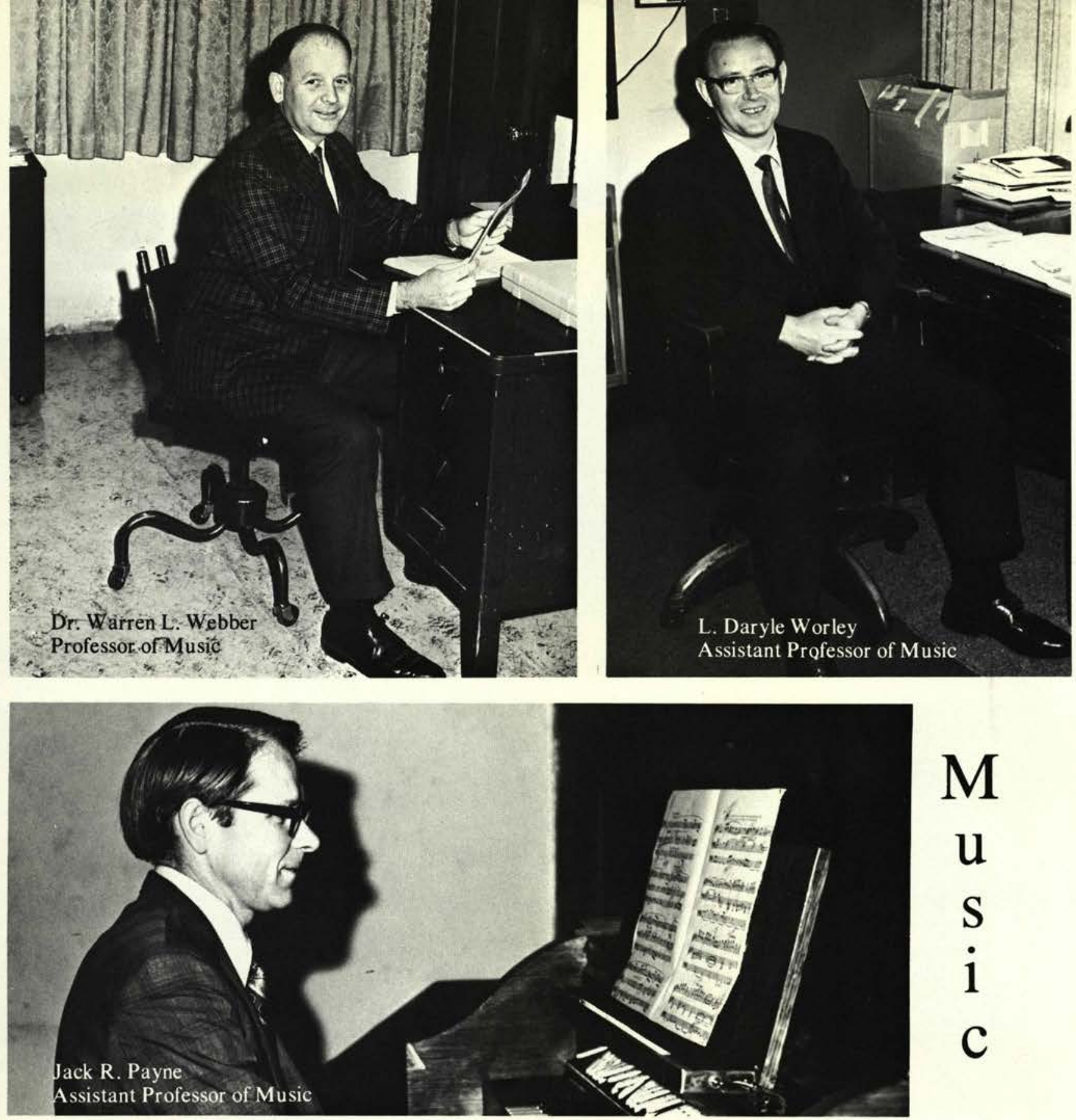

M

u

S

$\mathrm{i}$

c
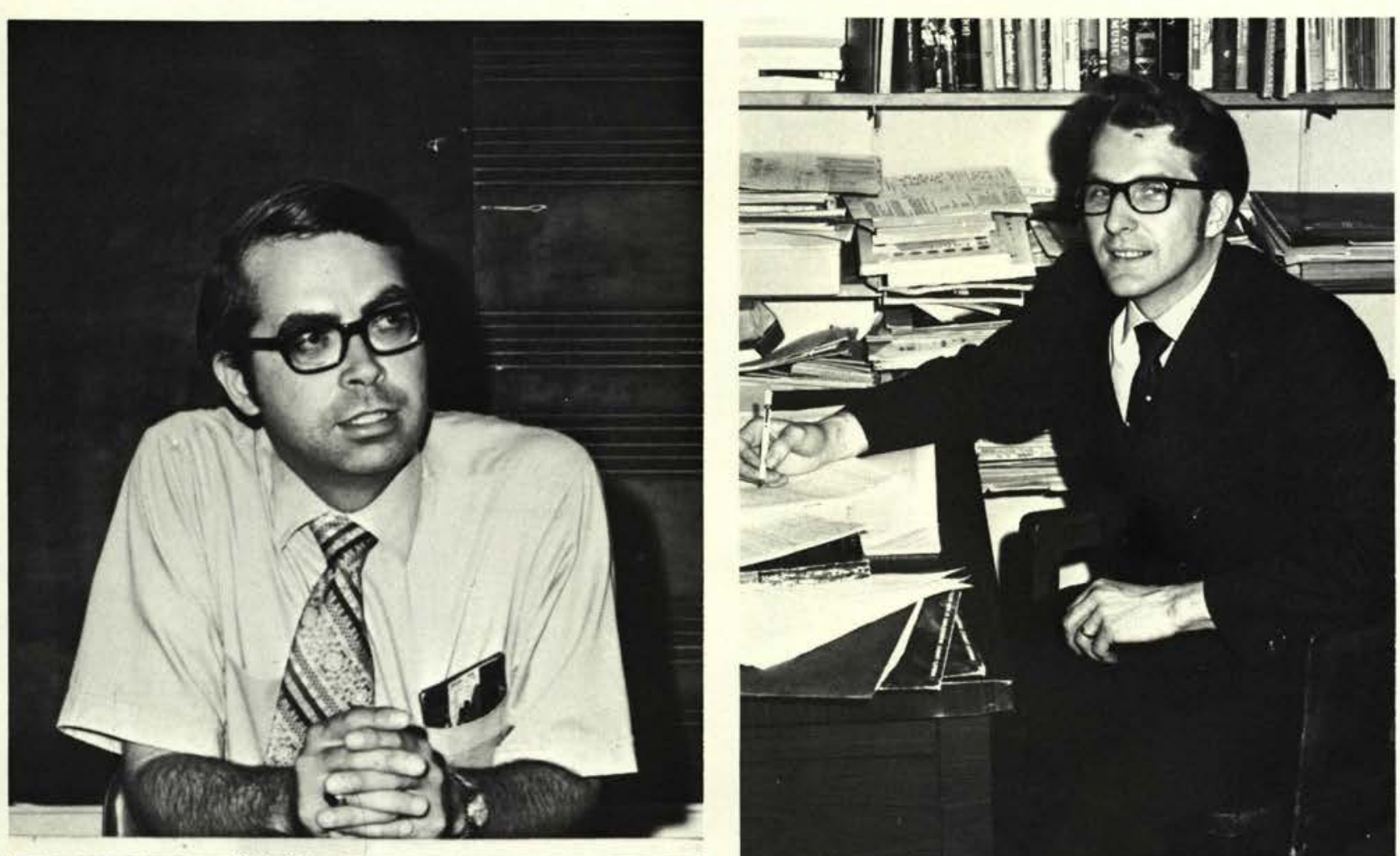

1 Assitant Profes or of Muss

Ralph M. Werner

Assistant Professor of Music 

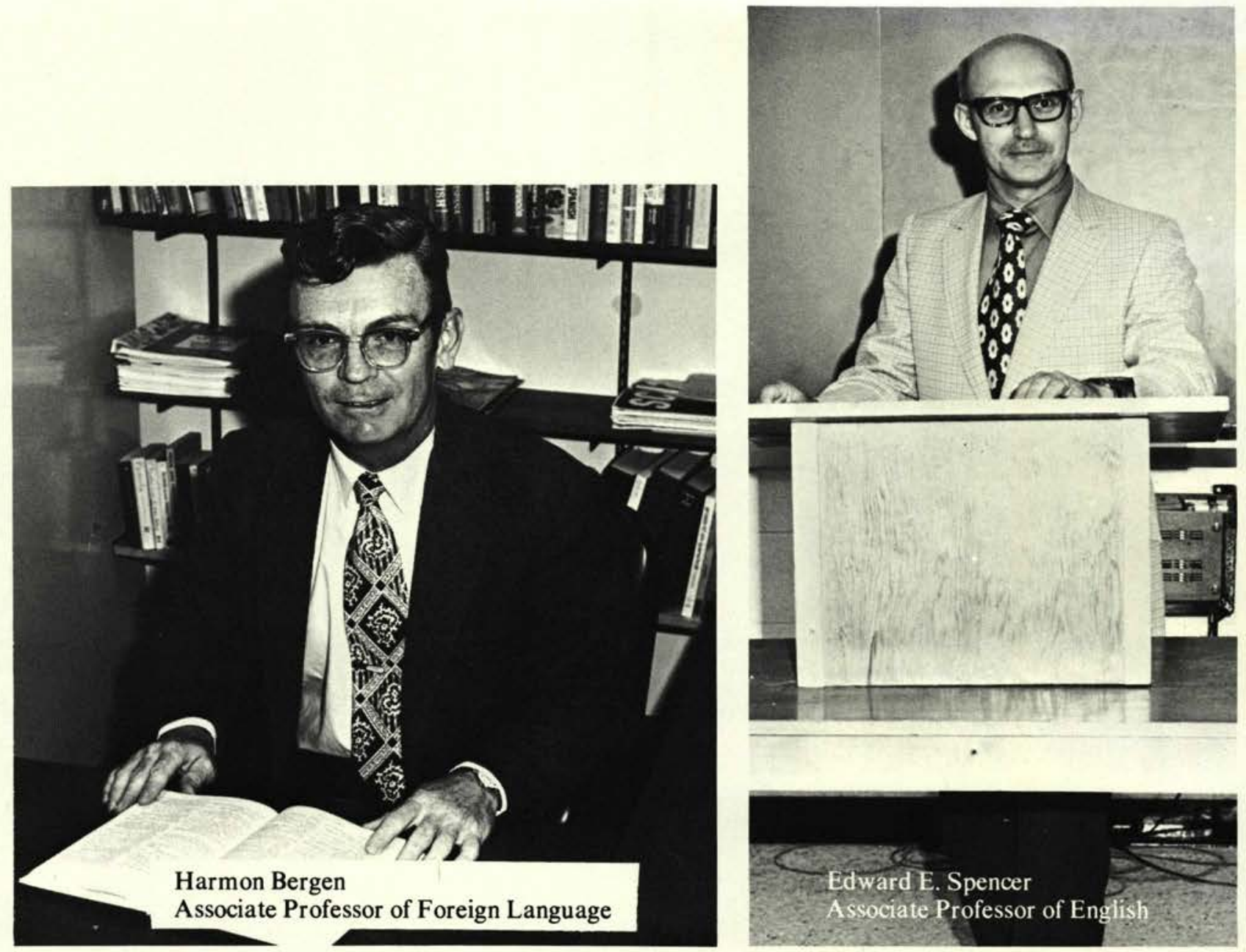

\section{English and}
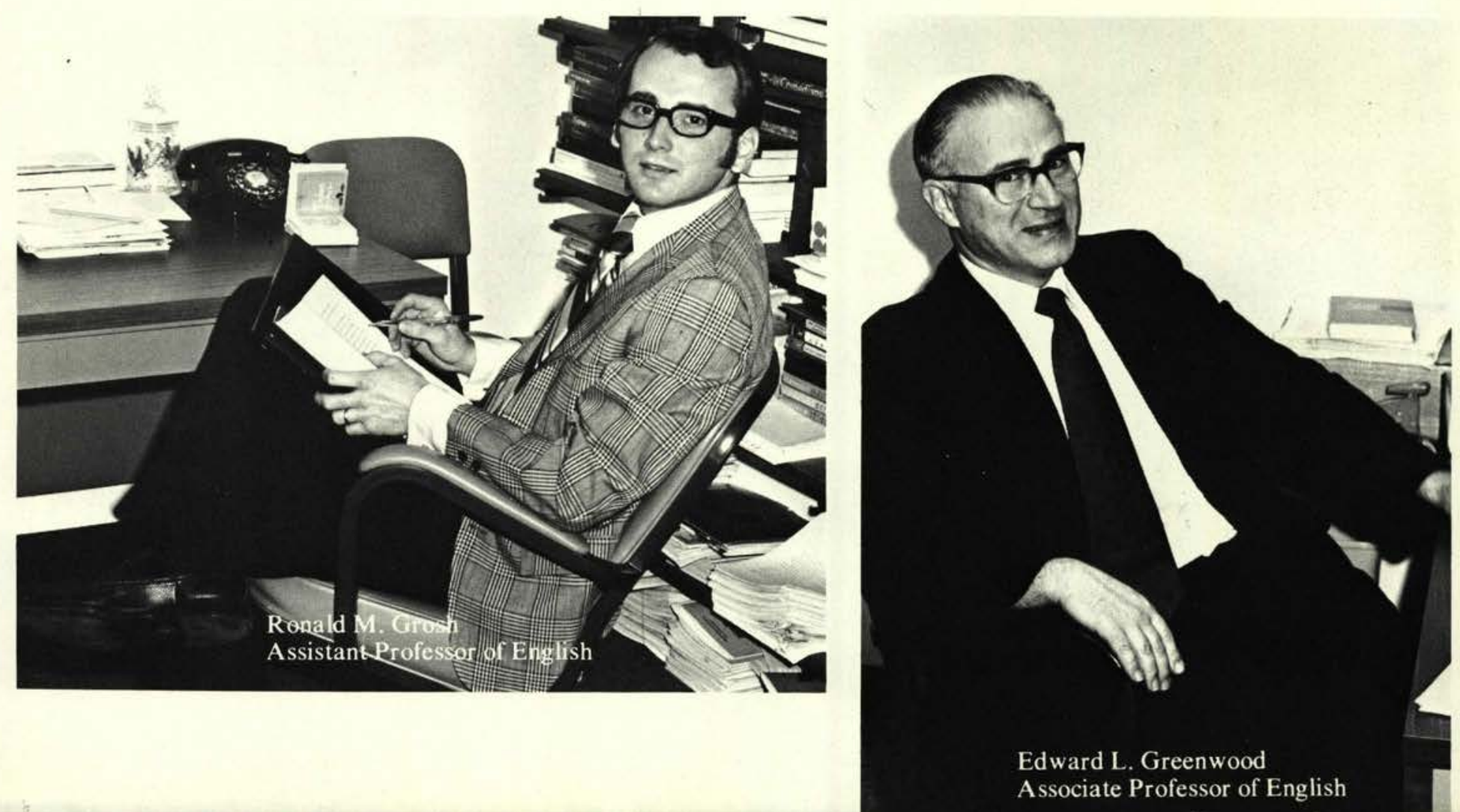

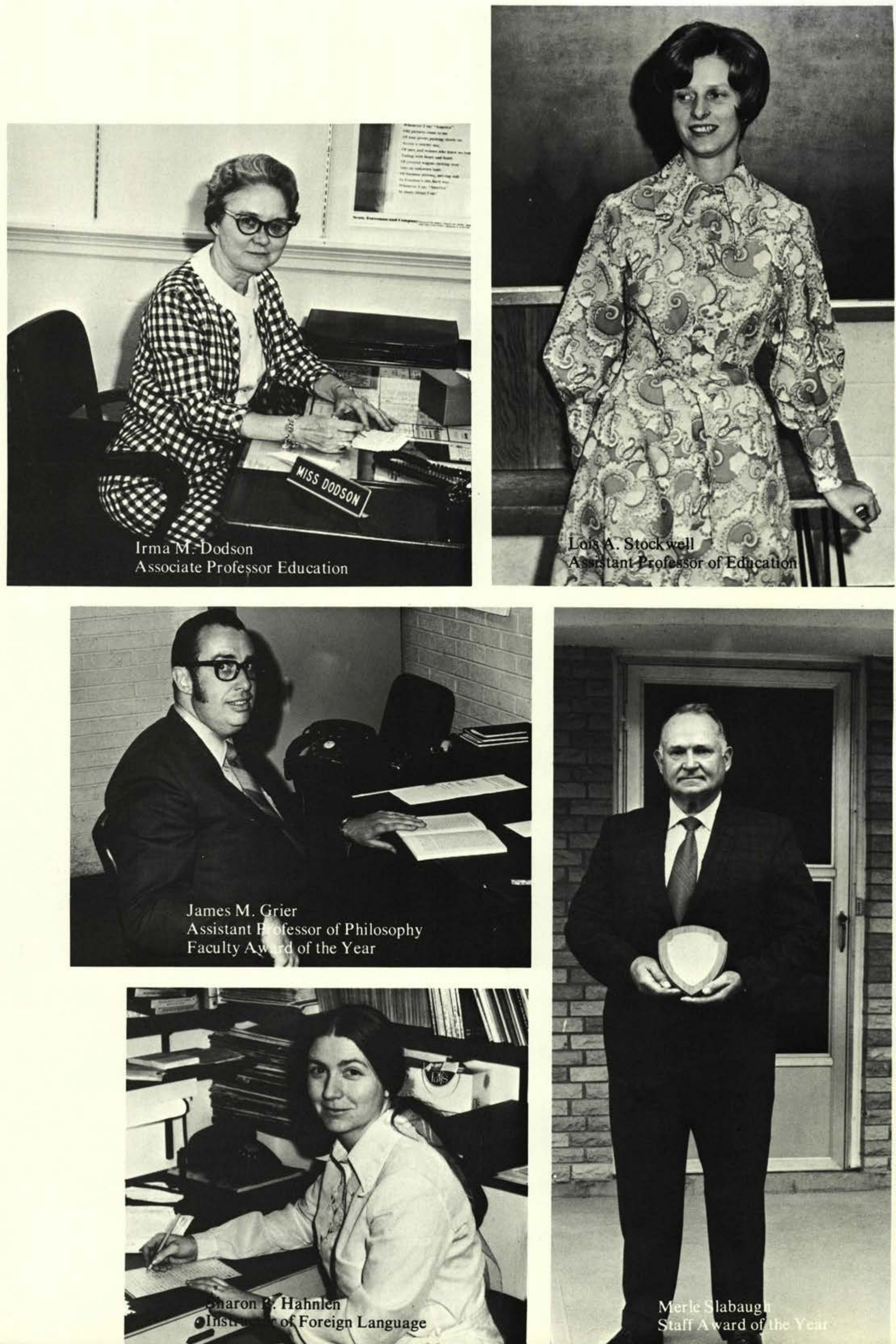

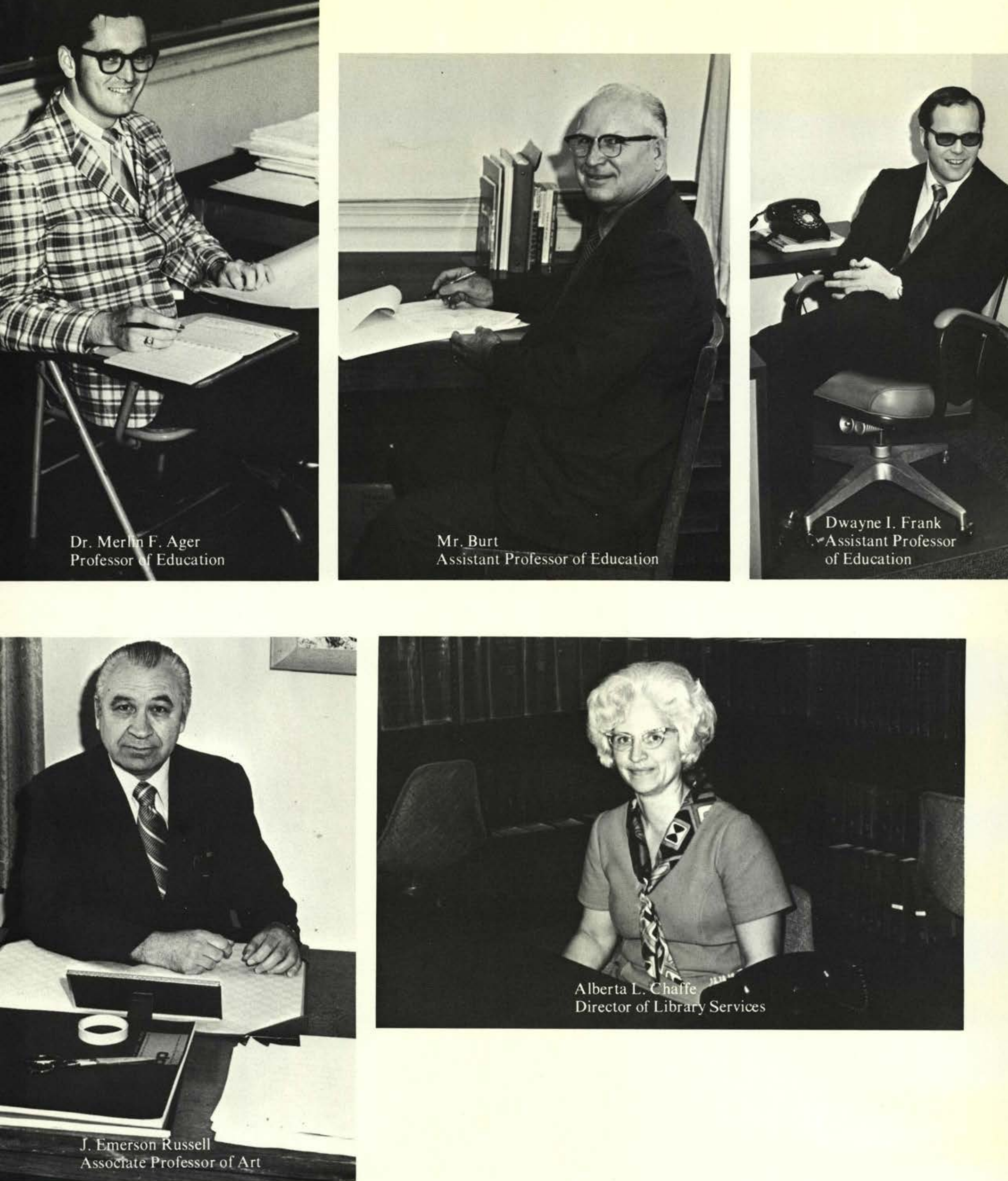


\section{Biblical Education}

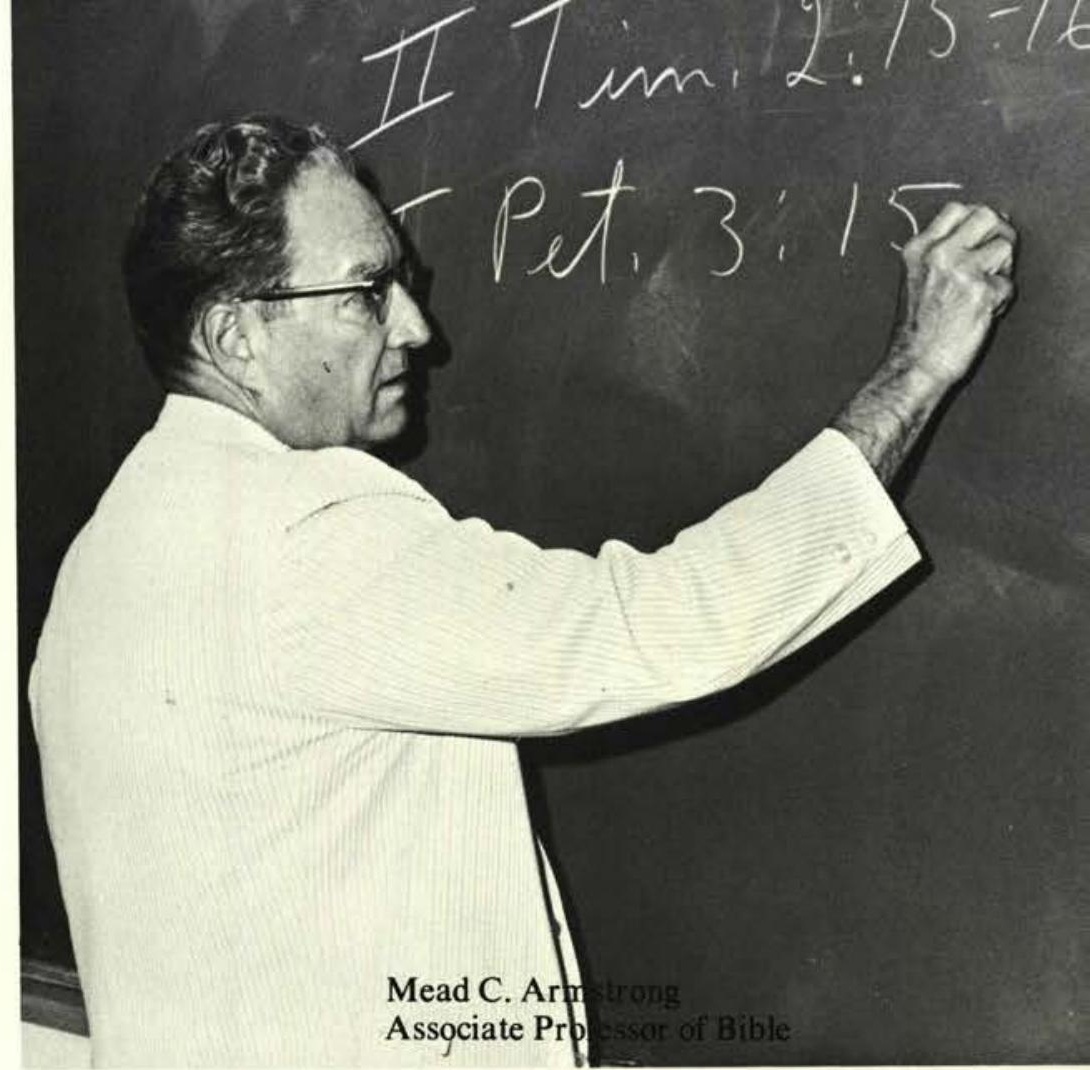



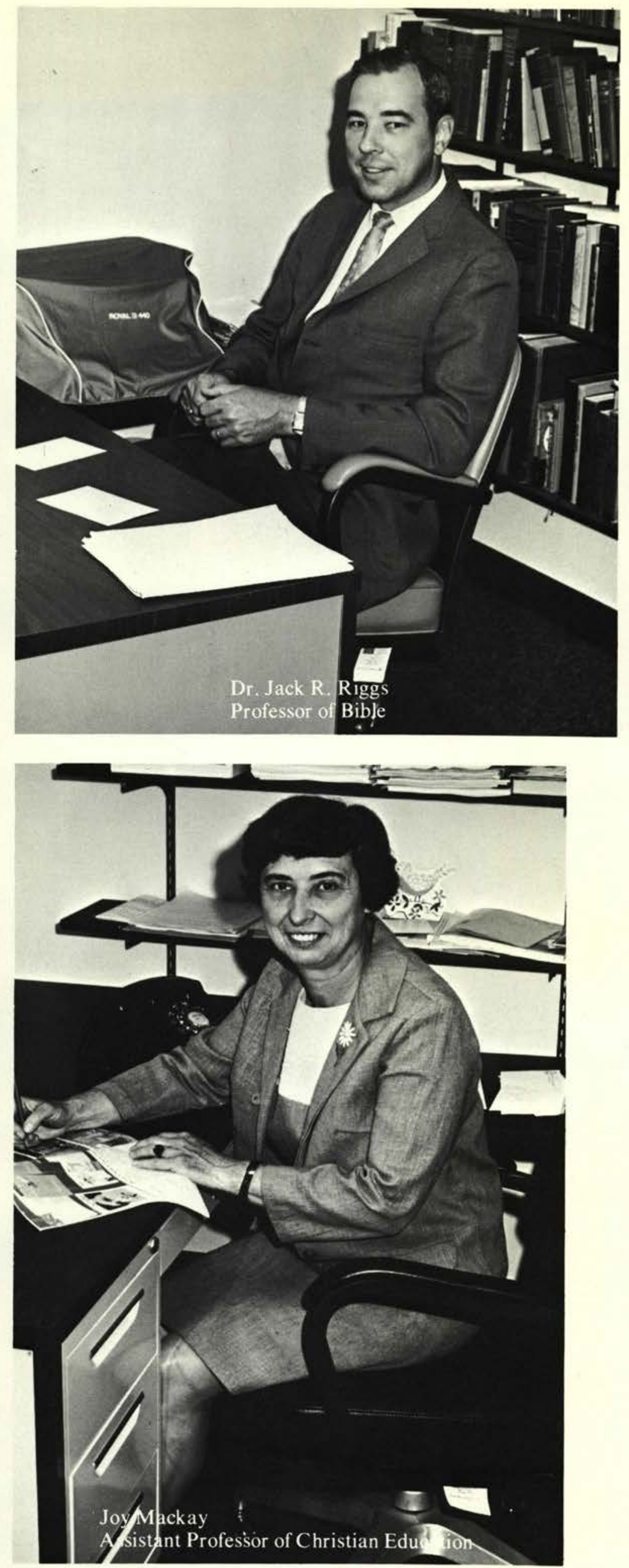

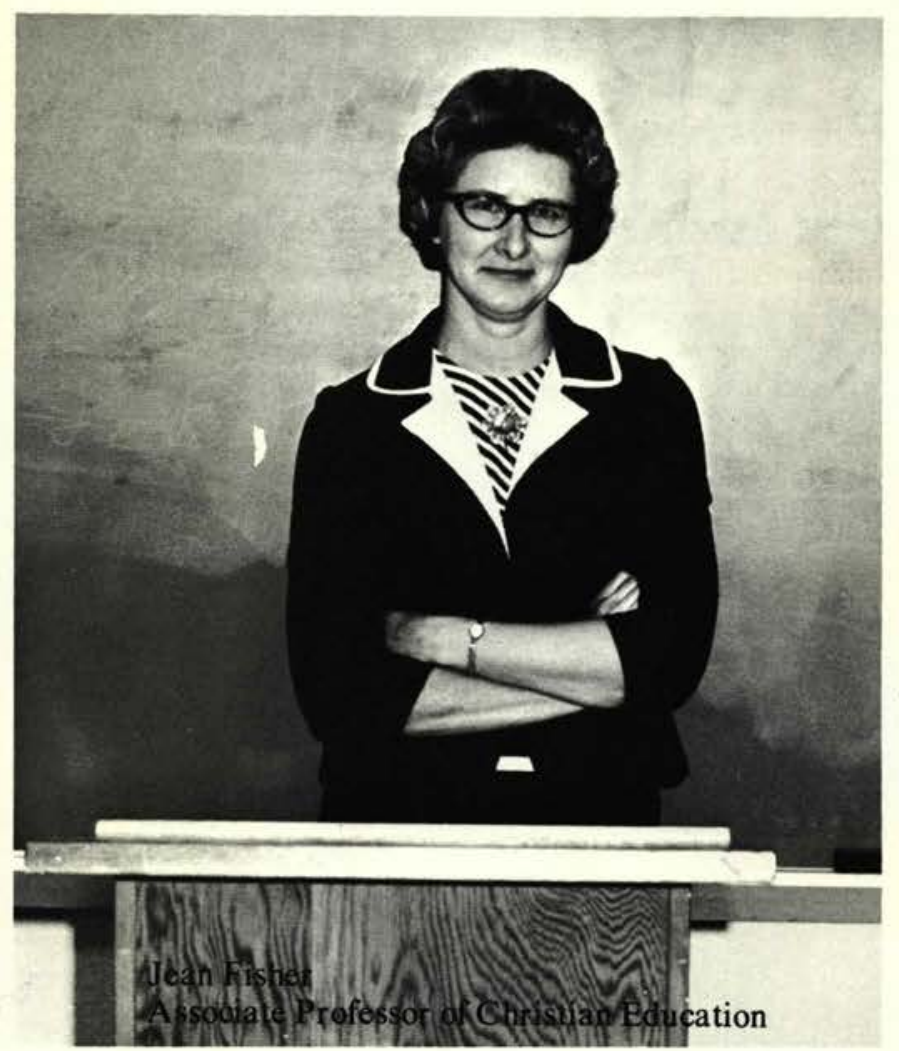

We want to extend a special note of thanks to Miss Mackay for her instruction, her incentive, and her friendship, and wish her the very best that the future holds. 


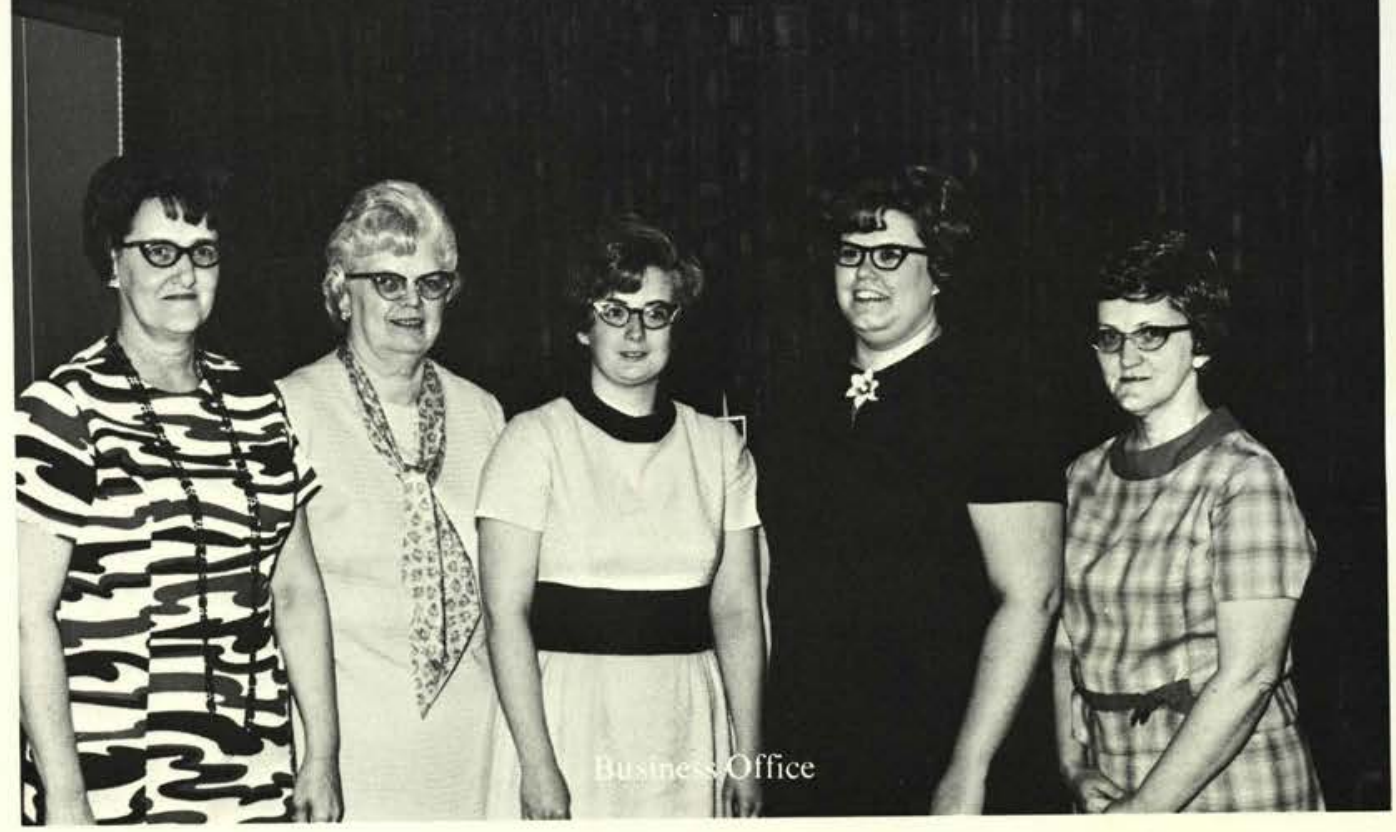

\section{College Staff}
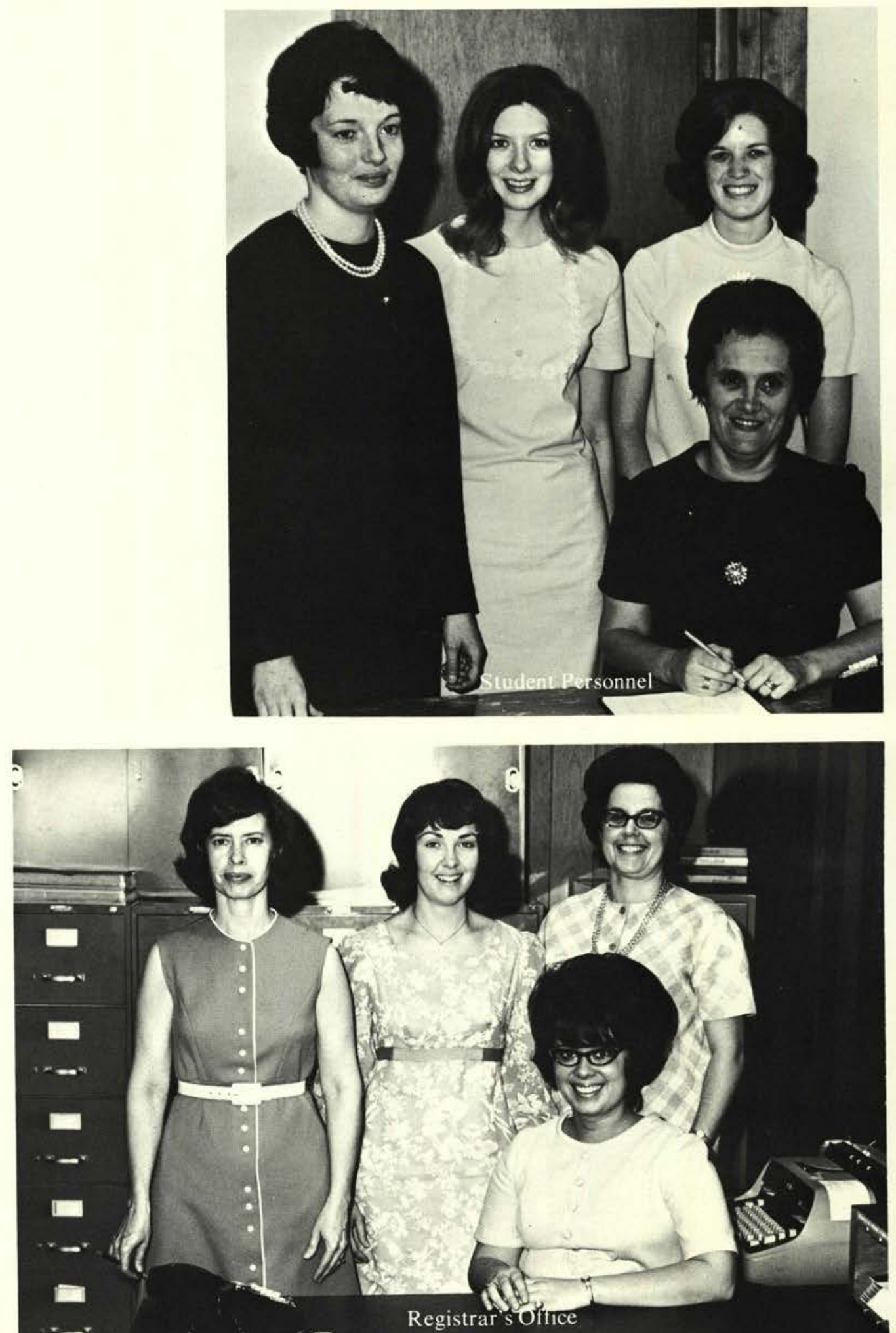



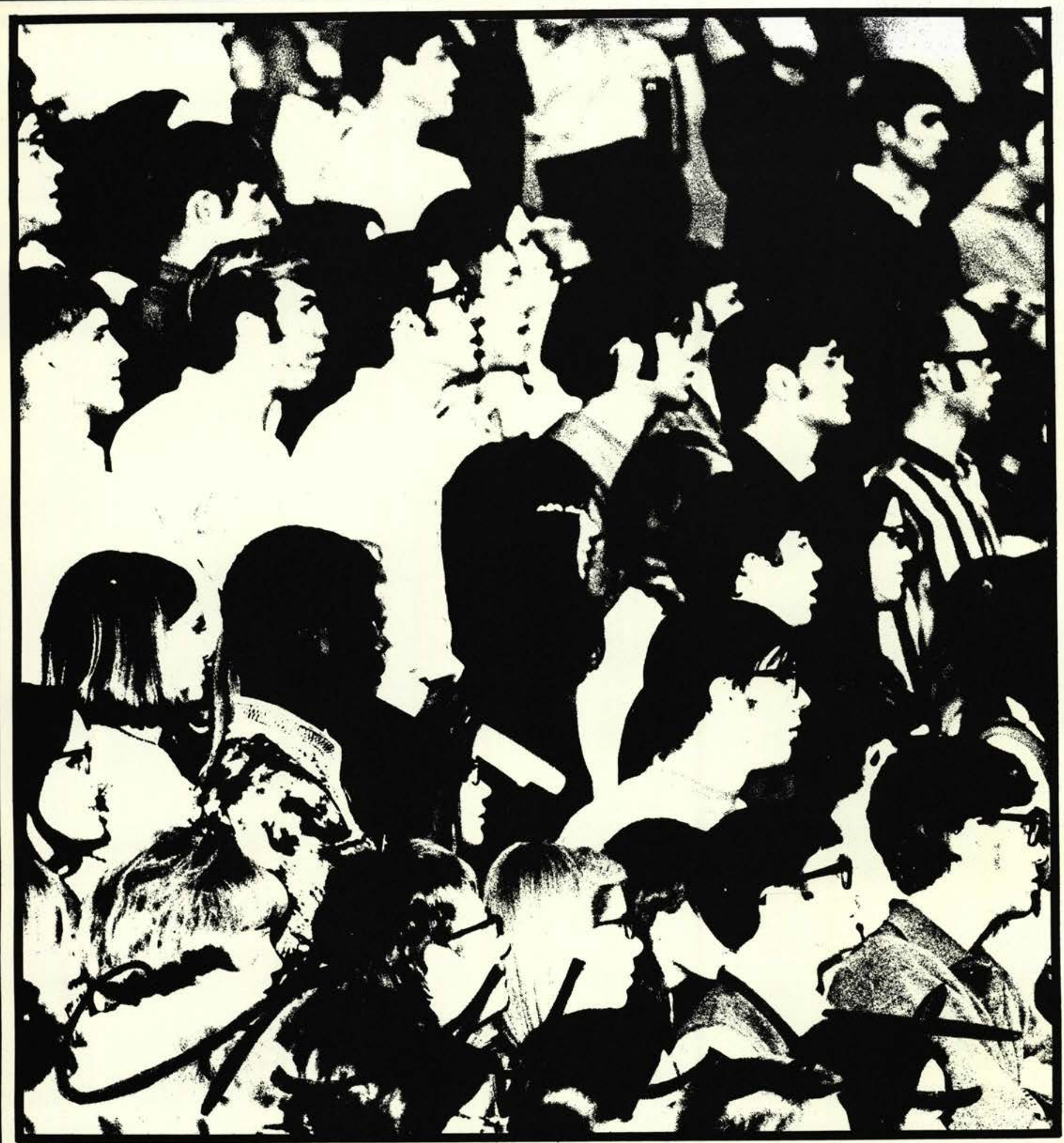

SIGNATURE OF REGISTRAR

THIS CARD IS ISSUED FOR YOUR USE ONLY. REPORT ITS LOSS TO THE REGISTRAR'S OFFICE. IF FOUND, MAIL TO THE REGISTRAR. IF A STUDENT WITHDRAWS FROM THE COLLEGE, THIS CARD MUST BE RETURNED TO THE REGISTRAR. 


\section{CEDARVILLE COLLEGE}

Cedarville, Ohio 45314

Identification Card Issued To:

\section{Charles A. Miller}

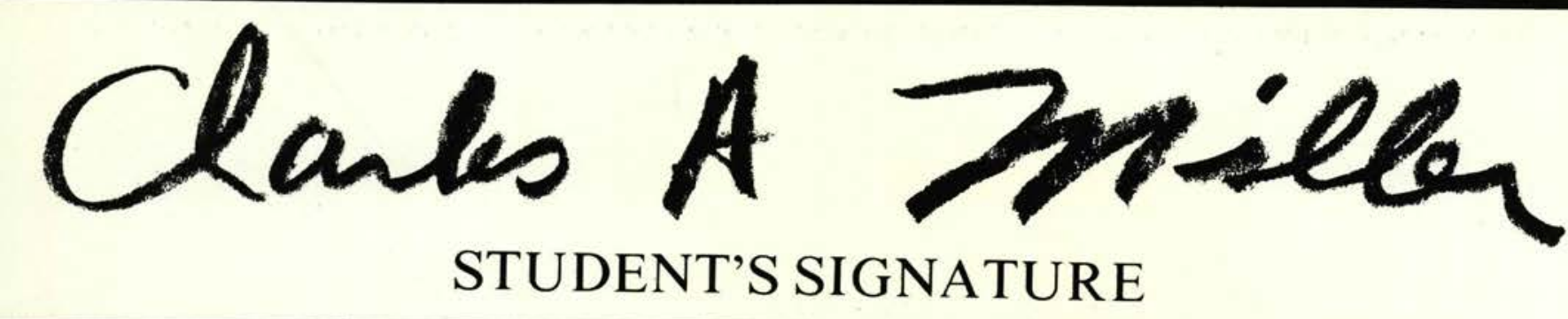

368

I. D. CARD NO.
June 1971

DATE OF EXPIRATION
Senior

CLASS

$$
\text { 9-20-49 }
$$

BIRTH DATE 
Amstutz, William

Cherry Hill, New Jersey

History

Acton, Don

Mentone, Indiana

Social Science

Atherton, Don

Vandalia, Ohio

Physical Education

Ayers, Frank Jr.

Baltimore, Ohio

Business

Barlow, Jacqueline

Villa Park, Illinois

Christian Education

Barr, Candy

Elyria, Ohio

Psychology

Bartlett, Mary

Schiller Park, Illinois

English

Beck, David

Harvey, Illinois

Business Administration

Beesley, Janet

Monroe, Iowa

Psychology

Beitler, Marianne

Medina, Ohio

Christian Education

Beitz, Mary

Cheektowaga, New York

Psychology

Berkheiser, William

Old Saybrook, Connecticut

Business Administration

Boyd, Joan

Millersburg, Ohio

Elementary Education

Boothroyd, Hilda

Chicago, Illinois

English/Psychology

Brookhart, Joyce

Lima, Ohio

Psychology
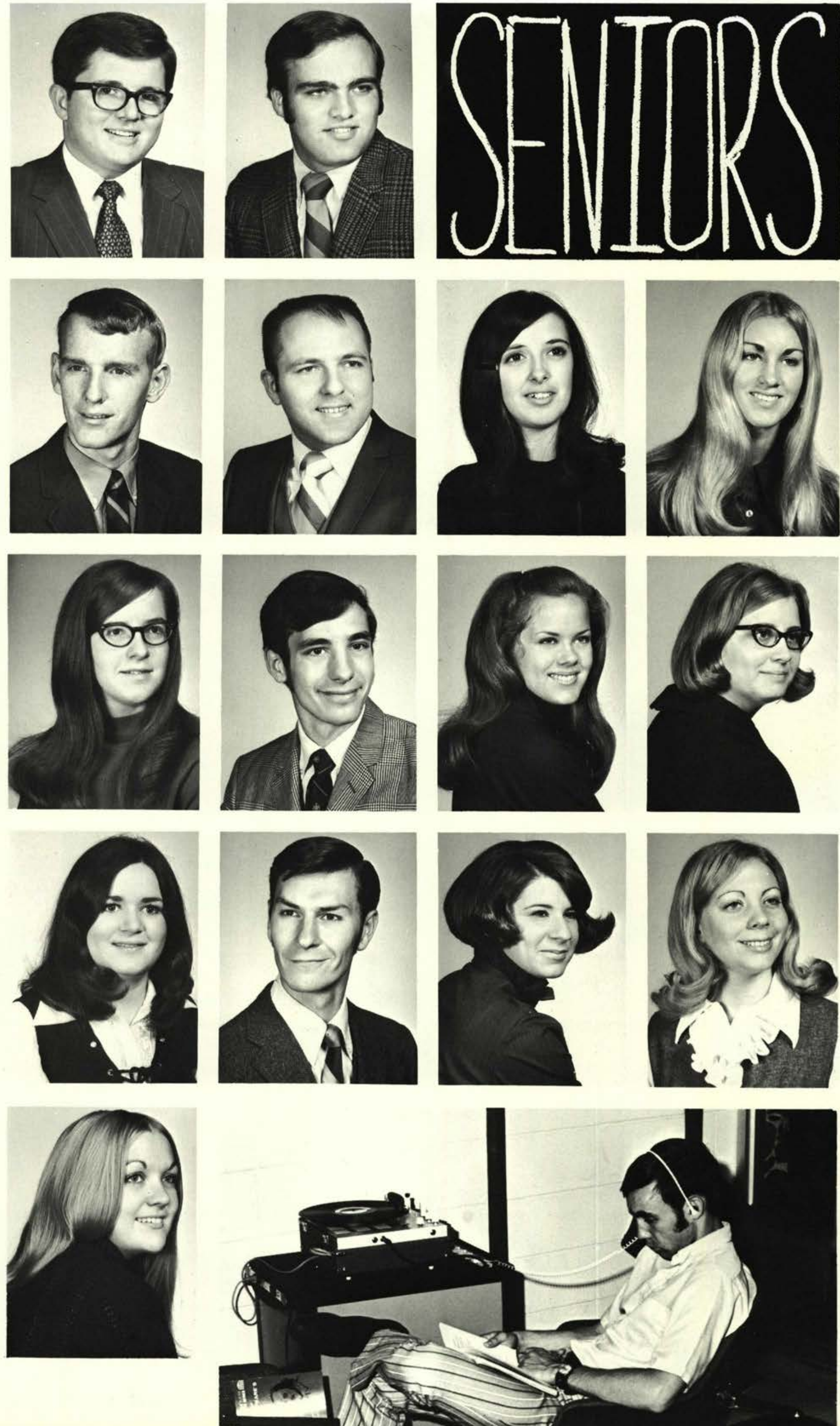


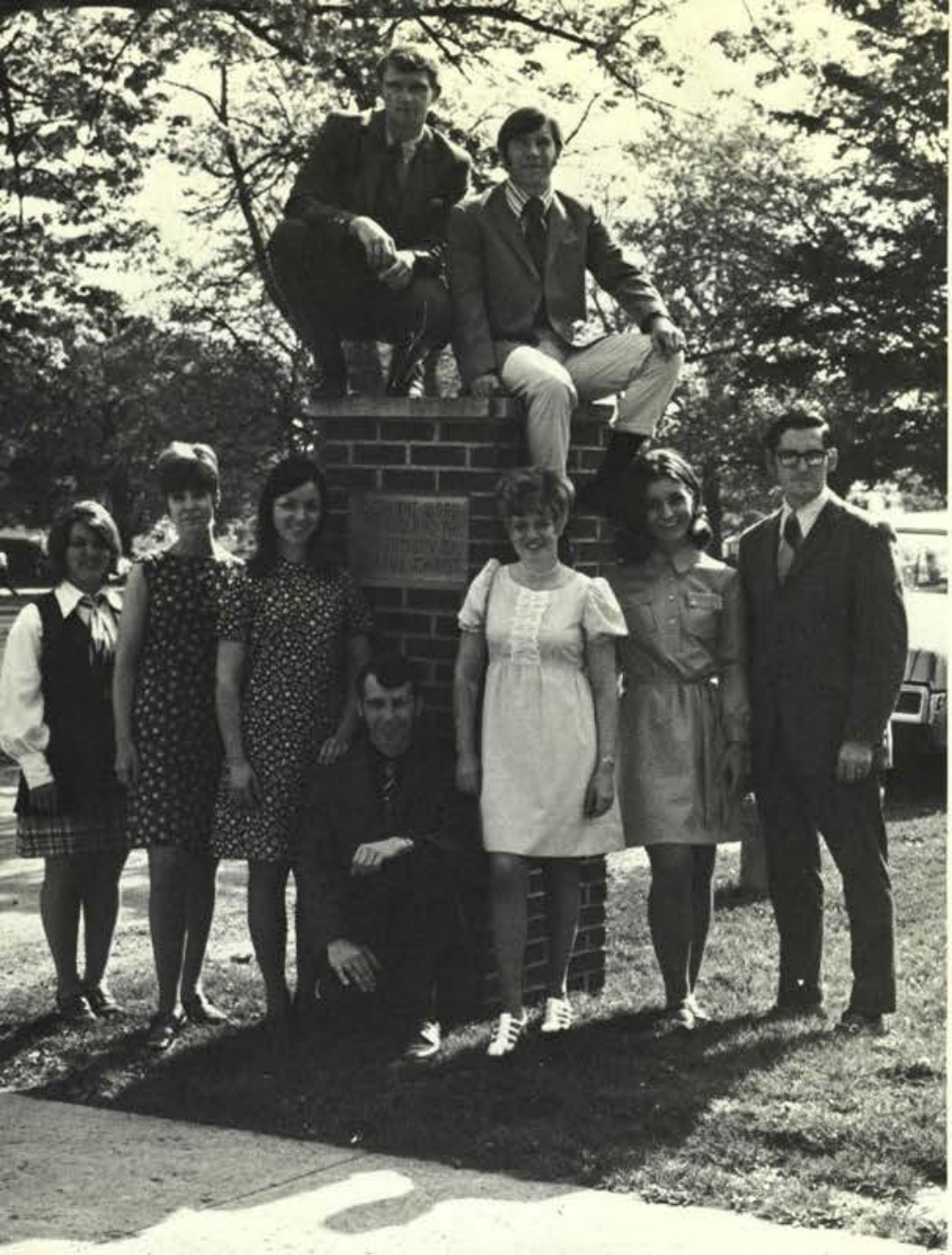

Somewhere the world has a place for you

That is all your own;

Somewhere is work that your hand can do, And yours alone.

Whether afar over land and sea

Or close at your door may the duty be,

It calls for your service full and free!

Take your place!

Somewhere the world has bitter tears

Your smile might dry;

Somewhere the burden of doubts and fears,

The hopeless sigh

There are steps that falter, weary, weak,

For the strong, brave aim they vainly seek -

Will you pass them by on the journey bleak?

Take your place!

Somewhere the world has a desert spot

Your toil might till,

Somewhere a life whose loveless lot

Your love might fill.

If the place that waits be high or low,

Question not, cry not - onward go!!

The world's great battle needs every blow -

TAKE YOUR PLACE!

\section{FRONT ROW}

Jean King, Historian

Connie Busho, Secretary

Rita DeAtley, Treasurer

Randy Patten, President

Fran Chambers, Student Council

(alternate)

Kathy Leeser, Student Council

Dr. Ager, Sponsor

\section{BACK ROW:}

Paul Large, Student Council

Dan Manross, Vice President

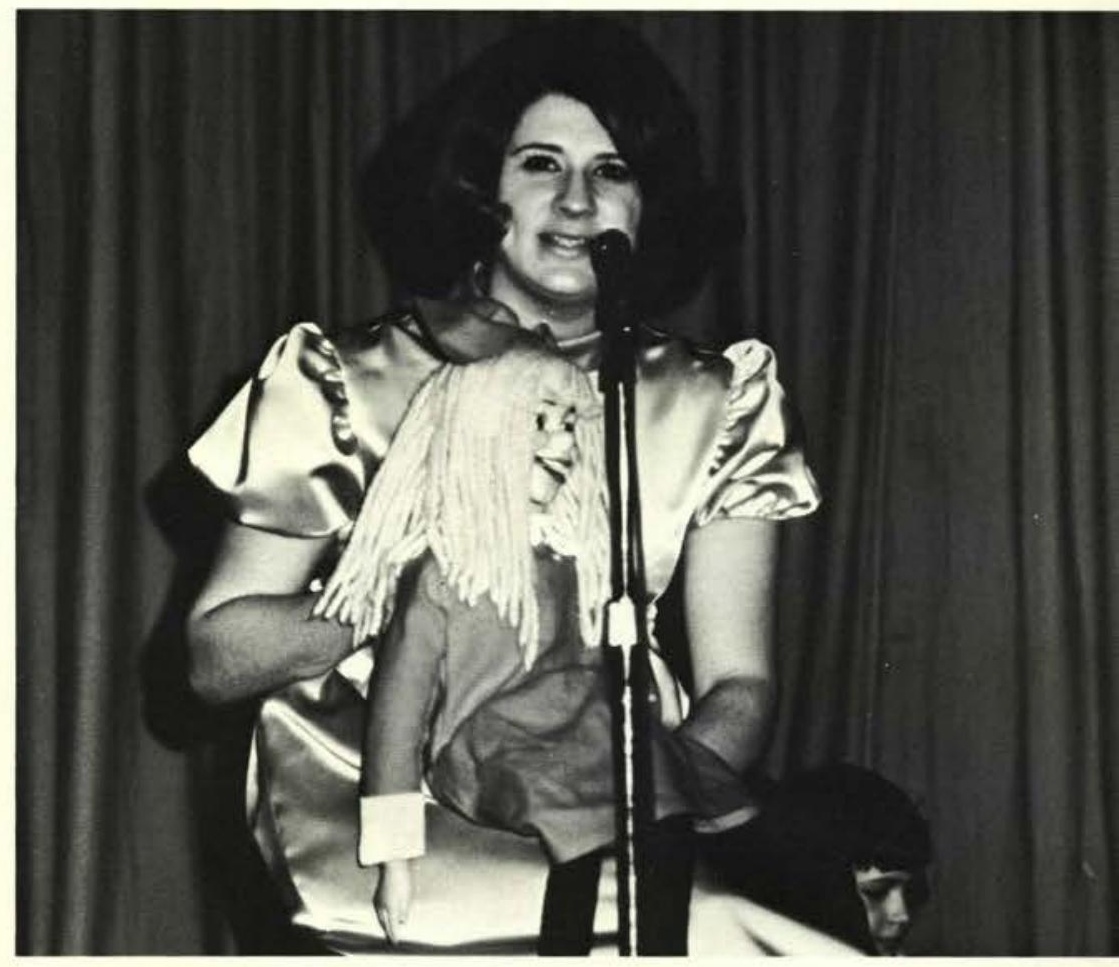


Brown, Kirby

Van Wert, Ohio

Physical Education/English

Brown, Nancy

Taylors, South Carolina

Elementary Education

Brown, Robert

Valley City, Ohio

Speech

Busho, Connie

Austin, Minnesota

Business

Carity, Marcia

Howston, Ohio

Elementary Education

Chambers, Fran

Grand Island, New York

Psychology

Christman, Linda

Cedarville, Ohio

Biology

Clark, Cathy

Hobart, Indiana

Elementary

Education/Speech

Clark, Susan

Clarion, Ohio

Elementary Education

Clay, Joan

Cedarville, Ohio

History/Speech

Cockerill, Diane

Flint, Michigan

Elementary Education

Collison, Ken

Oxon Hill, Maryland

Business

Coombs, Ronald

Cambellsville, Kentuck

Biology

Creviston, Gary

Fort Wayne, Indiana

Physical Education

Cunningham, Jane

Arcanum, Ohio

Physical Education

Davis, Karen

St. Louis, Missouri

Elementary Education
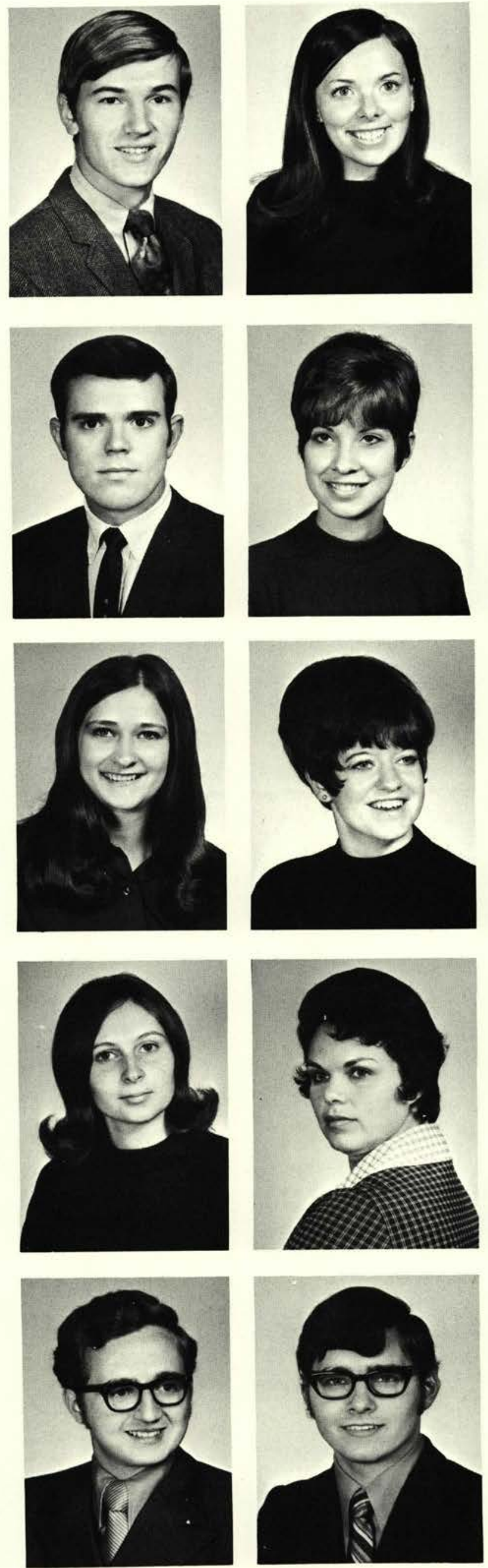
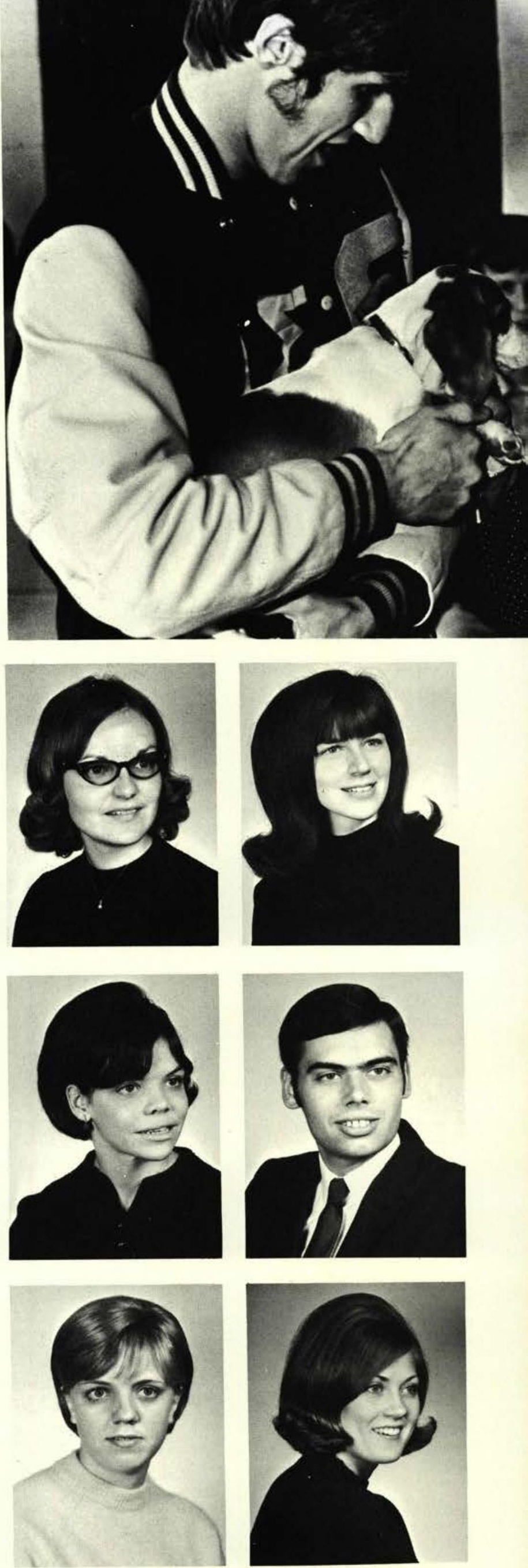

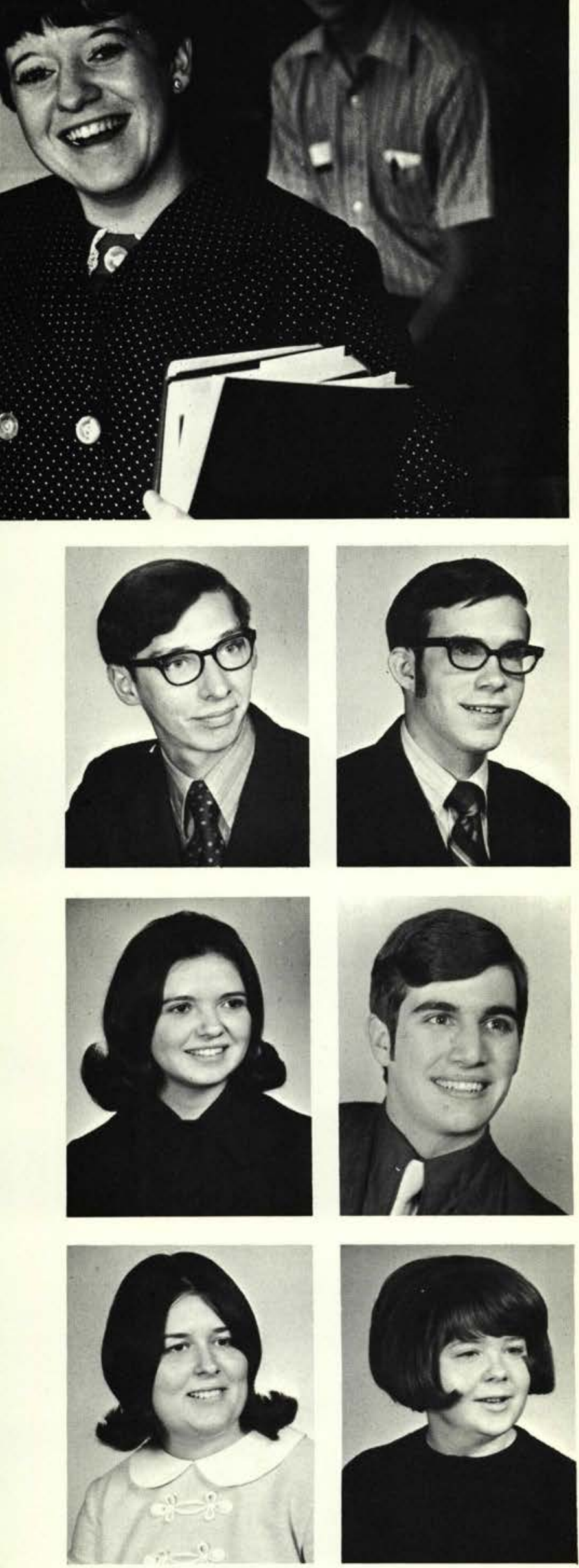
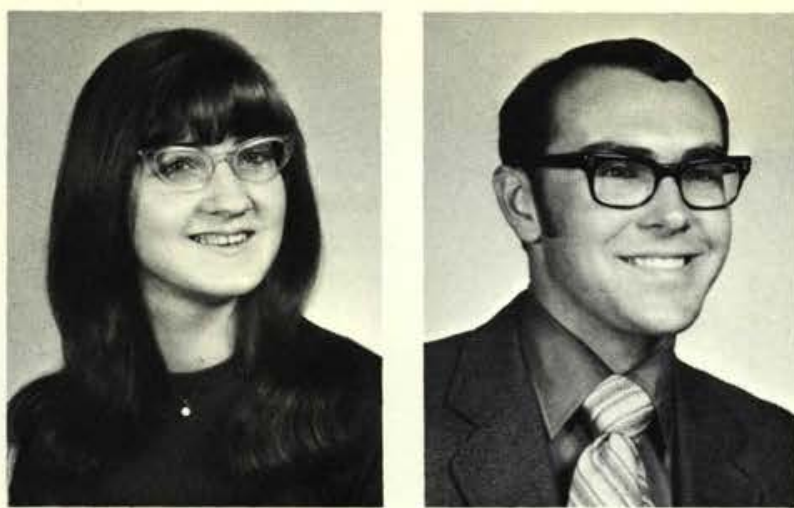

Davis, Lynette

Akron, New York

Biology

Dawley, Bruce

Rochester, Michigan

Biology
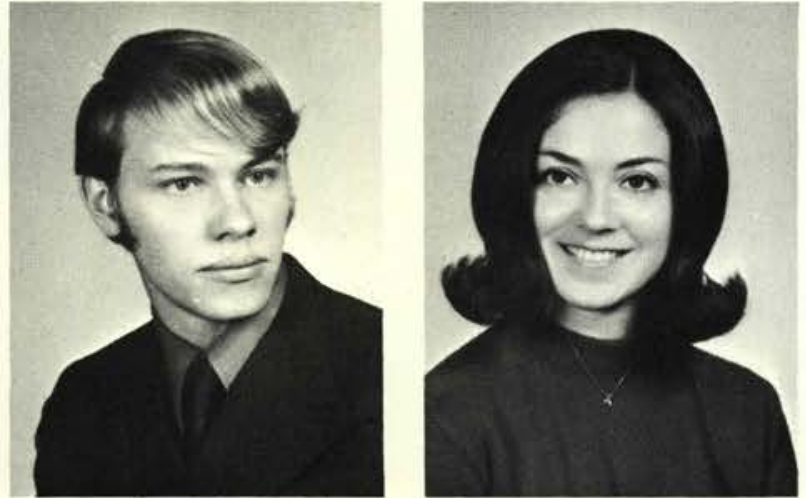

Dawson, Gary

East Moline, Illinois

Social Science

DeAtley, Rita

Minford, Ohio

Speech
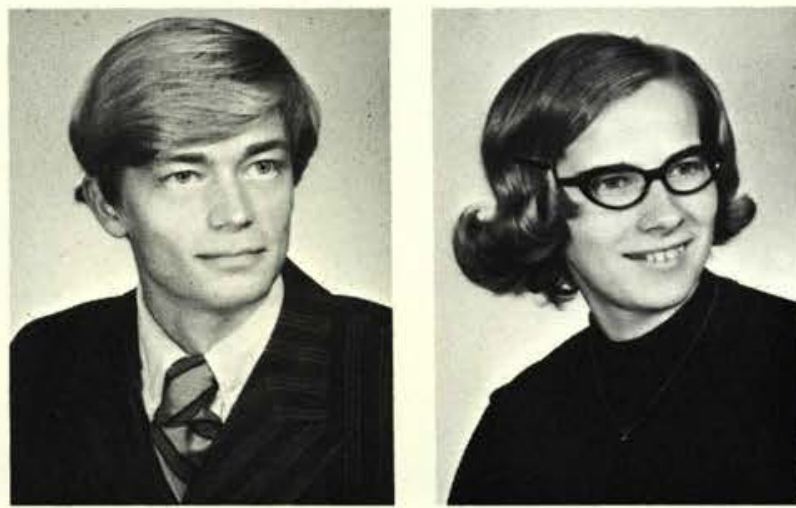

DeBerg, Michael

Reindeck, Iowa

Business Administration

Dieringer, Dennis

St. Mary's, Ohio

Pre-Seminary Bible

Dilks, Harold

Girard, Ohio

Social Science,

Comprehensive

Dixon, Elaine

Spokane, Washington

Business Administration
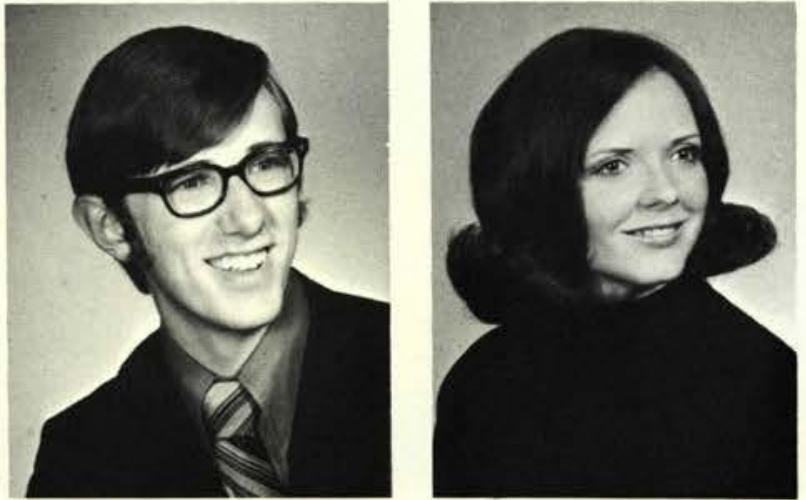

Duell, Regina

Galeton, Pennsylvania

Elementary Education

Durham, David A.

Fairview Park, Ohio

Music Education

\section{Dutton, Chuck}

Lapeer, Michigan

Mathematics

Edmunds, Brenda

Hillsdale, Illinois

English/Mathematics
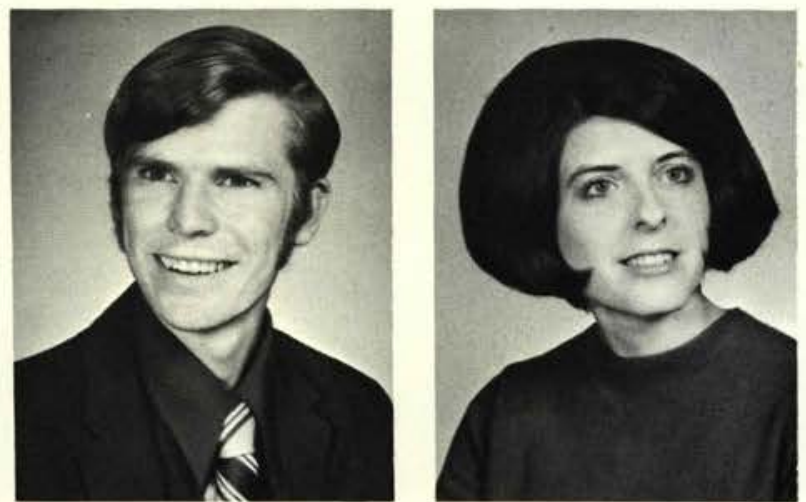

Engle, Carol

Hamburg, New York Business Education

Epps, Jane

Evansville, Indiana

Elementary Education

Evans, Kenneth, Jr.

Plymouth, Michigan

Pre-Seminary Bible

Farley, Susanne

Omaha, Nebraska

Elementary Education 
Farrar, Robert E.

Portsmouth, Ohio

Social Science

Flint, Mark

Cedarville, Ohio

Speech

Frank, Janet

Leavittsburg, Ohio

Elementary Education

Frank, JoAnn

Cedarville, Ohio

Elementary Education

Frields, Mark

Huntingburg, Indiana

Biology

Frye, Jonathan

Xenia, Ohio

History/Government

Gabriel, Jan

Chicago, Illinois

English

Gerber, Lonnie

Berlin, Ohio

Social Science

Goldschmitt, Dick

Pittsburg, Pennsylvania

Pre-Seminary Bible

Gowman, Sandy

Warren, Michigan

Elementary Education

Grover, Judy

Cedarville, Ohio

Music

Haffey, Nancy

Canal Winchester, Ohio

Music Education

Hawley, Marsha E

Tonawanda, New York

Mathematics

Healey, Barbara

Brunswick, Ohio

Psychology

Hirschy, Linda

Evans City, Pennsylvania

Hostetler, Cathy

Kokomo, Indiana

Elementary Education

Hostetler, James

Kokomo, Indiana

Bible

Hubbard, Jdy

Glastonbury, Connecticu

Elementary Education

Huebner, Tanis

Mancelona, Michigan

Elementary Education

Ice, Lawrence R. Jr.

Cedarville, Ohio

History
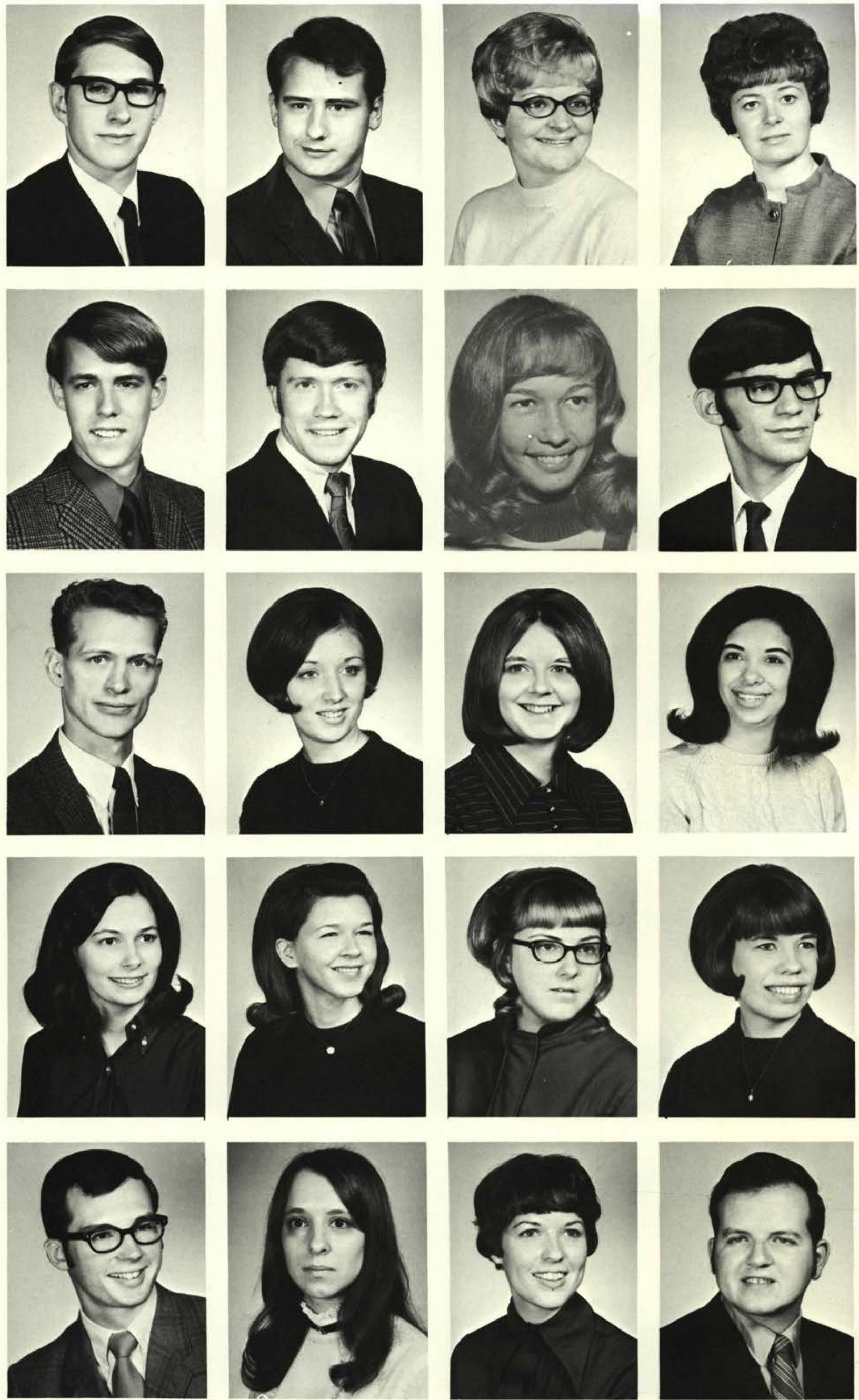

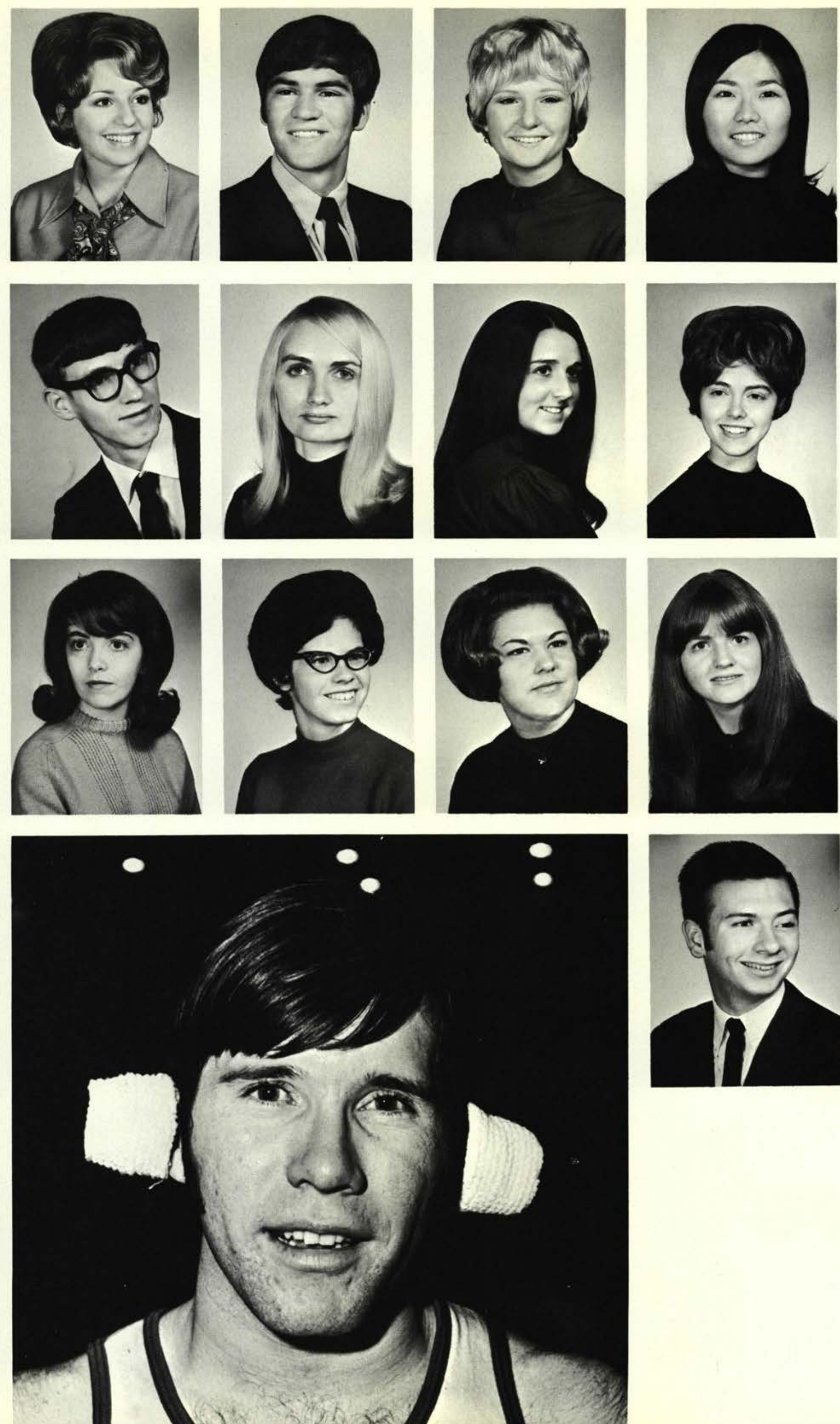

Imbro, Vicki

Carmel, Indiana

Speech

Inghram, Rick

Morning Sun, Iowa

History/Government

Irwin, Patty Jo

Swartz Creek, Michigan

Physical Education

Ishuin, Mitsu

Seabrook, New Jersey

Elementary Education

Jackson, Mike

Bloomfield, Iowa

Mathematics

Jacoby, Elaine

Lina, Ohio

Elementary Education

\section{Jenkins, Patty}

Lundhurst, Ohio

Elementary Education

Johnson, Faye

Eastlake, Ohio

History/English

Jones, Verna

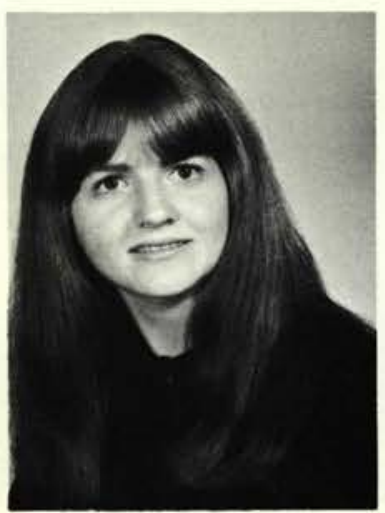

Kingsley, Pennsylvania

Elementary Education

Kemp, Diane

Coal Valley, Illinois

Psychology

King, Jean Ann

Olmsted Falls, Ohio

Elementary Education

Kitting, Deborah Metz

Atlanta, Georgia

Business Administration

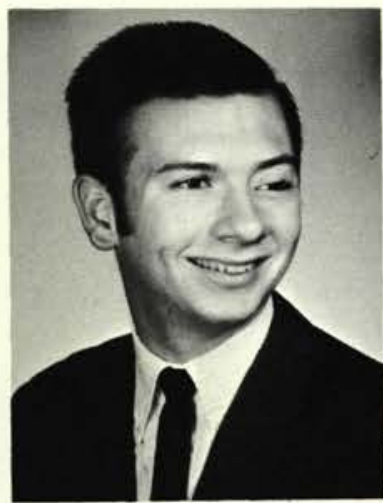

Klamm, Harvey

Cedarville, Ohio

Physical Education 
Klinger, Glenn Allen Grampian, Pennsylvania Business Administration

Krikke, Florence

Greenwich, Ohio

Elementary Education

Krueger, Edwin R.

Richmond Heights, Ohio

Business Administration

Kusumoto, Ann

Seabrook, New Jersey

English

Large, Paul

Iquitos, Peru

Business

Administration/Speech

Leach, Judy

East Northport, New York English

Lear, Pat

Derry, Pennsylvania

Elementary Education

Leeser, Kathy E.

West Palm Beach, Florida

Speech Education

Lewis, Darlene

Maine, New York

Mathematics

Lewis, Judy

Kokomo, Indiana

Elementary Education

Lewright, Barbara

Eagle Grove, Iowa

Elementary Education

Long, Donald

Cedarville, Ohio

Music Education

Lunney, Bob

Port Huron, Michigan

Physical Education/Biology

Madsen, Linda

Hamburg, New York

Elementary Education

Mann, Darlene

Eldora, Iowa

History

Manross, Daniel P.

Akron, Ohio

Social Science

Mason, Lin

Flint, Michigan

Christian Education

Mays, Dee

Chillicothe, Ohio

Physical Education

McDaniel, Chip

Mancelena, Michigan

Psychology

McDowell, Gary

Cedarville, Ohio

Social Studies
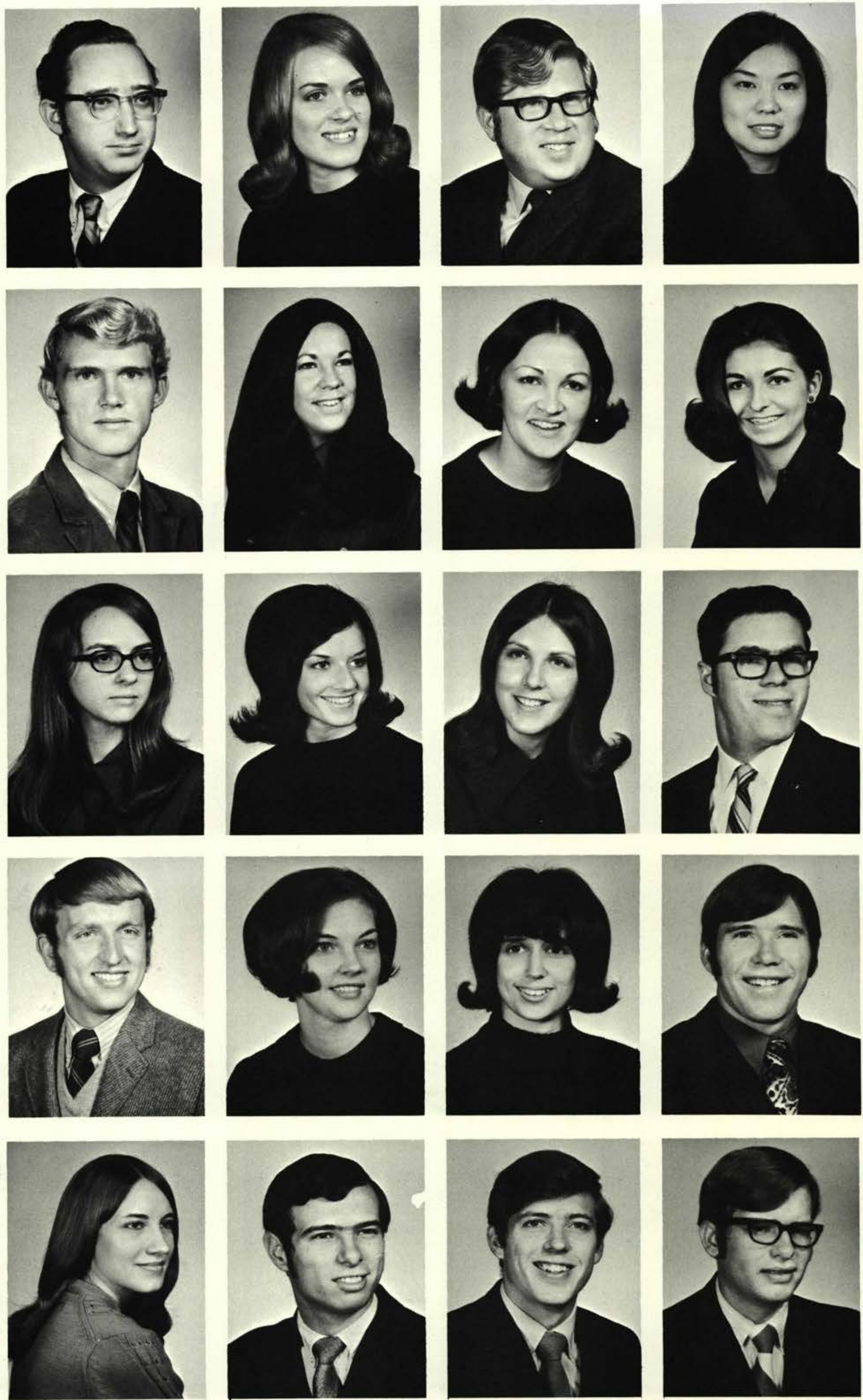

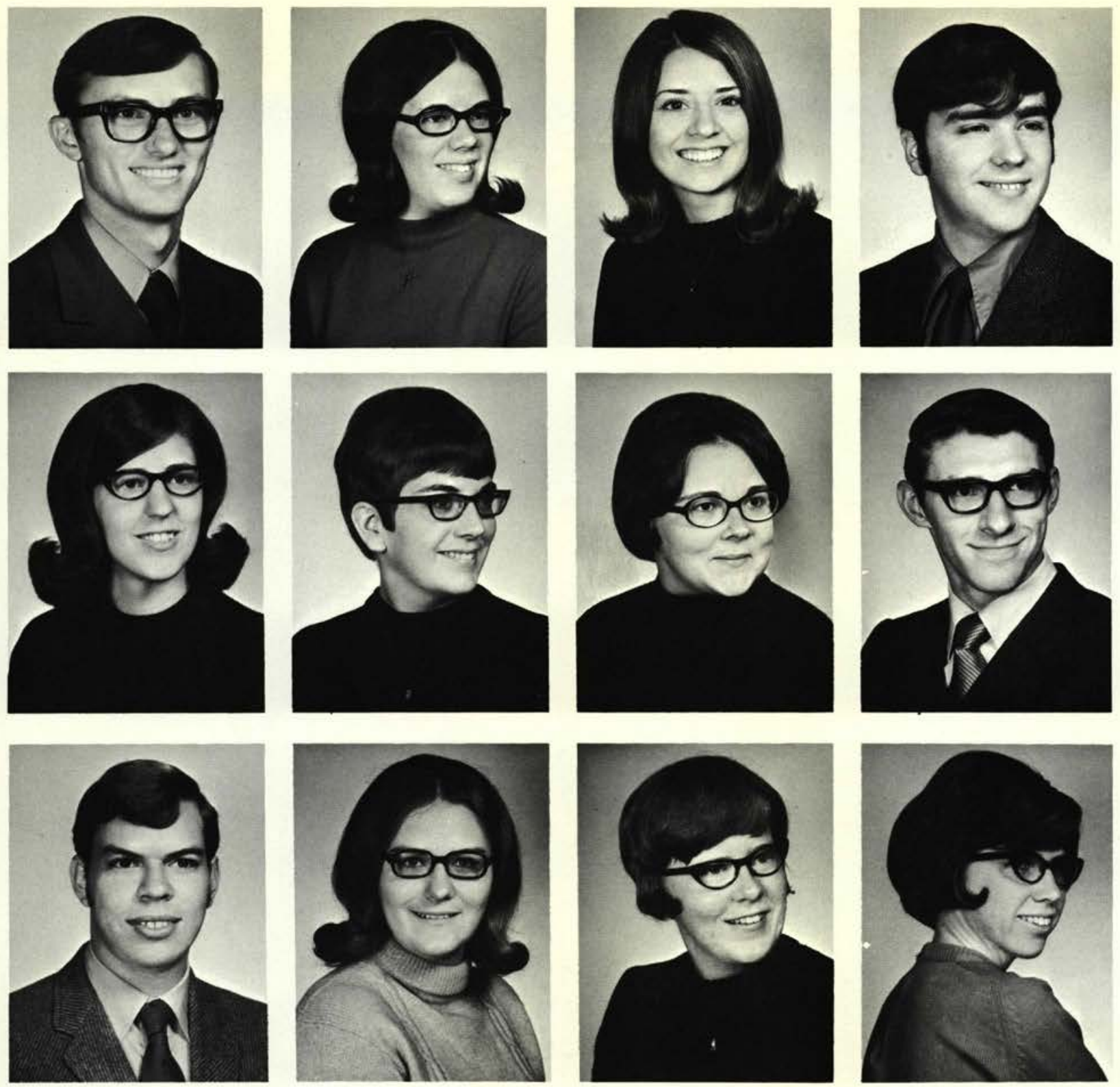

McMillan, Sam

Washington, D.C.

Christian Education

McMurtry, Mary

Pittsburg, Pennsylvania

English

McNeice, Melinda

Valparaiso, Indiana

Speech

Meyers, Ronald

East Moline, Illinois

Chemistry/Mathematics

Miller, Joan E.

Allegan, Michigan

Business/Accounting

Mitchell, Ginny

Moravia, New York

Music/Biological Science

Mix, Bev

Meadsville, Pennsylvania

English

Mohler, Philip H

Litchfield, Ohio

Chemistry

Mooney, Jerry

New Castle, Pennsylvania Business Administration

Moore, Bev

Xenia, Ohio

Elementary Education

Morrow, Coletta M.

Kenton, Ohio

Elementary Education

Morrow, Shirlein

Amherst, Ohio

Elementary Education

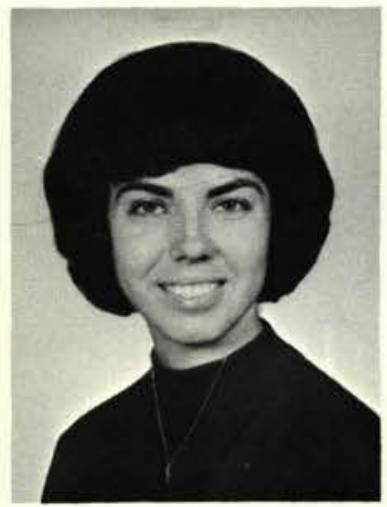

Morse, Carol

Flint, Michigan

Music Education

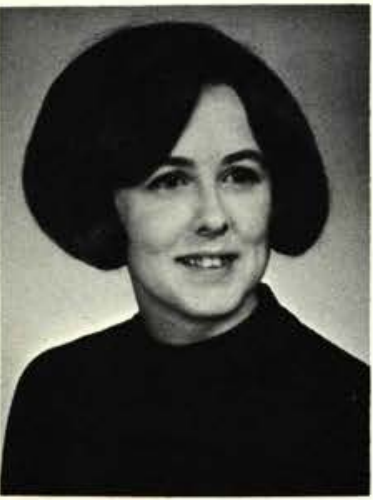

Motts, Joyce

Northfield, Ohio

Music Education

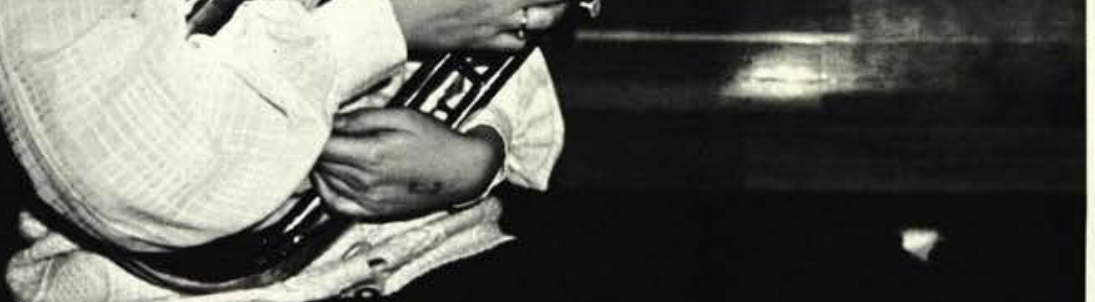



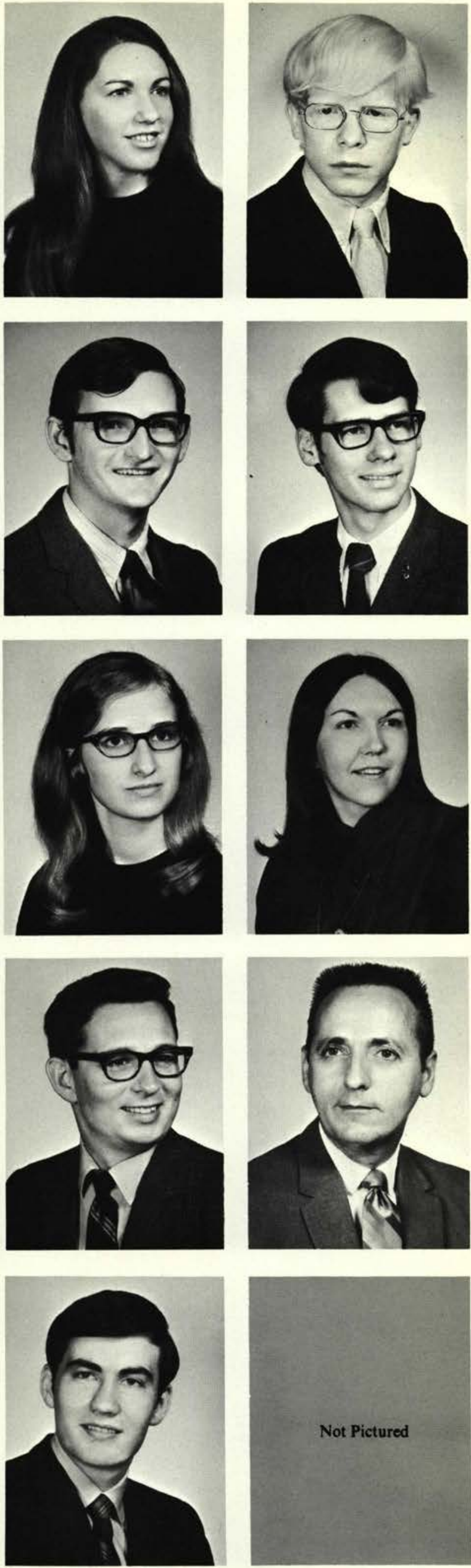
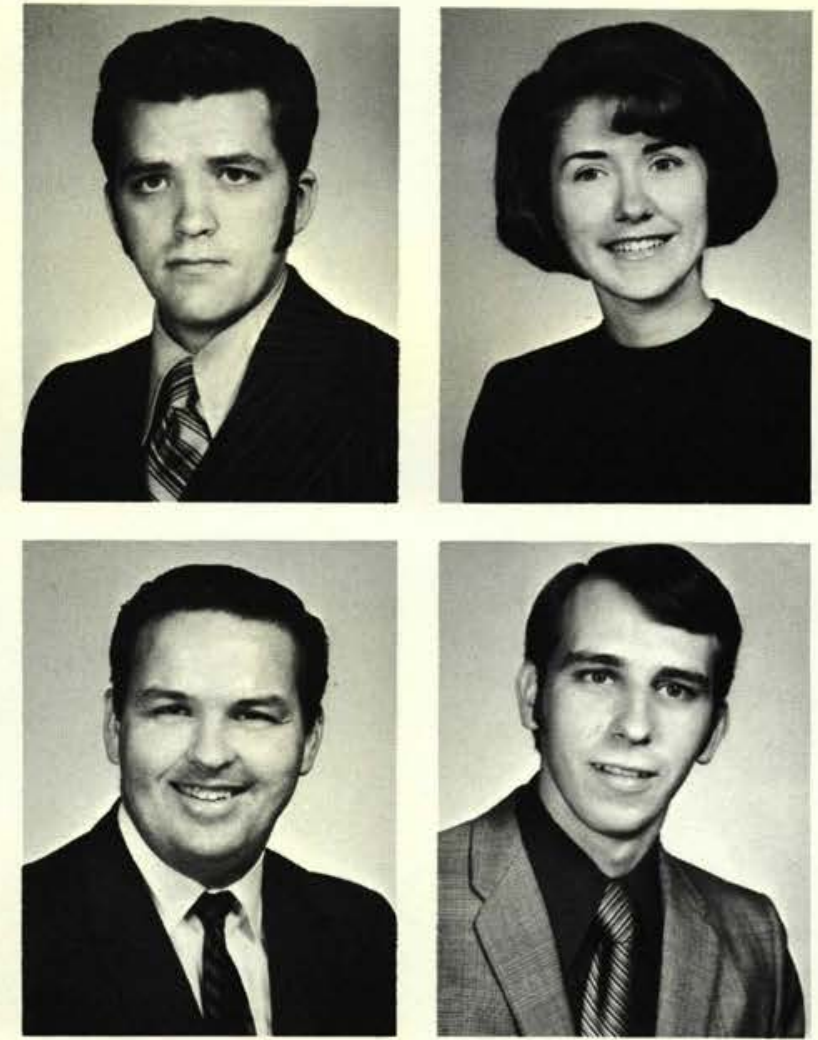
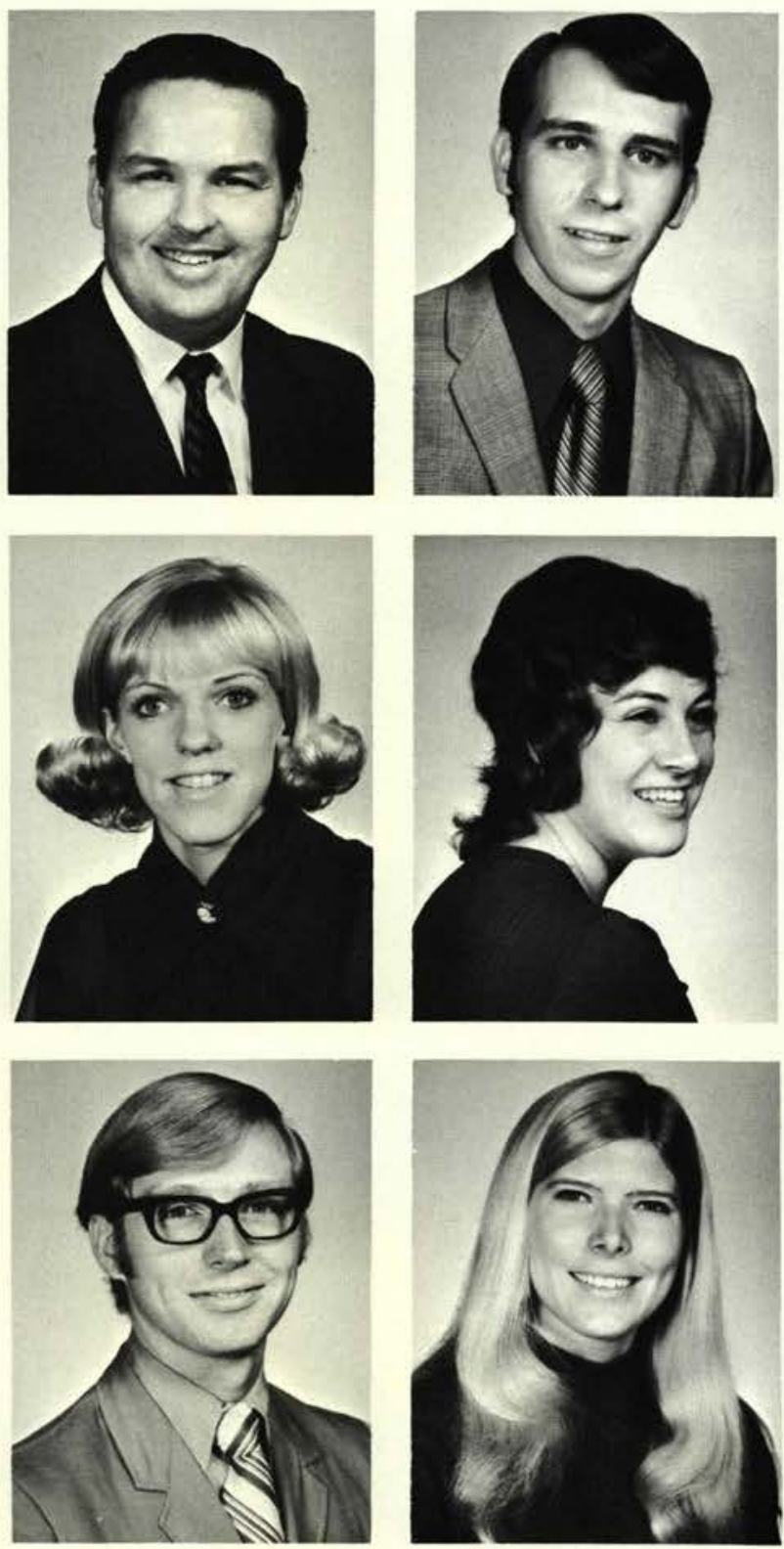

O'Keefe, Carolyn

Wesleyville, Pennsylvania English

Oklak, Mark

Mishawaka, Indiana

Psychology

Olson, Steven B

Cedarville, Ohio

Bible

Osman, Marsha Joy

Jamestown, Ohio

Elementary Education

Palmer, Milton C.

Cedarville, Ohio

Elementary Education

Parcher, George

Cedarville, Ohio

Business Administration

Parkin, Ralph

Royal Oak, Michigan

Bible

Patten, Randy

Caldwell, Ohio

Speech

Paugh, Nancy

Oakland, Maryland

Elementary Education

Peek, Jeanette

Fort Wayne, Indiana

Music Education

Pettitt, Deborah

Cedarville, Ohio

Physical Education/Health

Porterfield, Peggy

Austin, Minnesota

English

Rickard, James

Toledo, Ohio

Business

Administration/Accountino

Ritchie, Wyman

Middle Granville, New

York

Bible

Rohm, Dane

Audubon, New Jersey

Speech

Ross, Joyce

San Diego, California

Social Science

Comprehensive
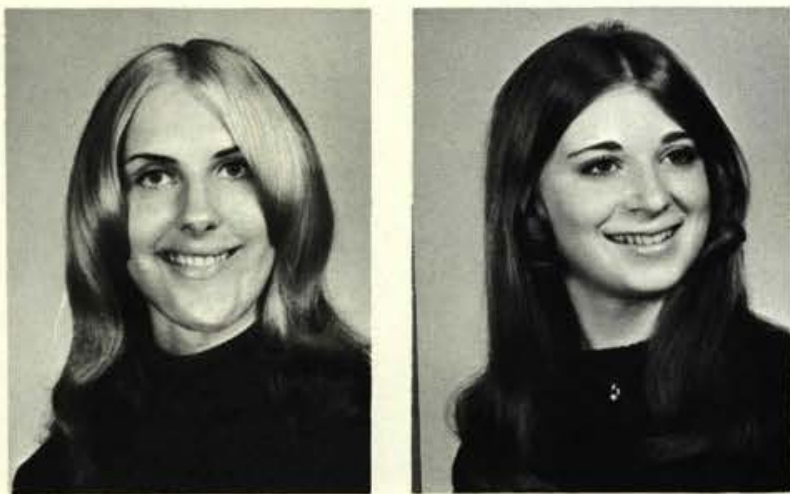

Rowe, Charles

DeRuyter, New York

Pre-Seminary Bible

Schilling, Dale

Novelty, Ohio

Speech

Schneider, Marikay

Hammond, Indian

Elementary Education

Schwarm, Norma

Baberton, Ohio

Elementary Education 
Seeley, Jeff

Oberlin, Ohio

Speech

Schreve, David

Meadville, Pennsylvania

Comprehensive Bible

\section{Silverthorn, Renee}

Center Line, Michigan

Elementary Education

Slates, Linda

St. Louis, Michigan English

Smith, Carol Jean Ashland, Kentucky

Elementary Education

Snider, Nancy

Barberton, Ohio

Elementary Education

Spieth, Linda

Alton, Illinois

Elementary Education

Spieth, Ron

Elyria, Ohio

Music Comprehensive

Stock, Cheryl D.

Lansing, Michigan

English/Secondary

Education

Storey, Mary Lou

Erie, Pennsylvania

Elementary Education

Stowell, Hannah Sue

Chicago, Illinois

Elementary Education

Stutzman, Gary

Berlin, Ohio

Biology

Swanson, Bruce

Port Byron, Illinois

History

Swanson, Roger

Rockford, Illinois

Social Science

Comprehensive

Swiger, Jan

Clarksburg, West Virginia

Christian Education

Tallman, Verna

Creston, Iowa

Psychology
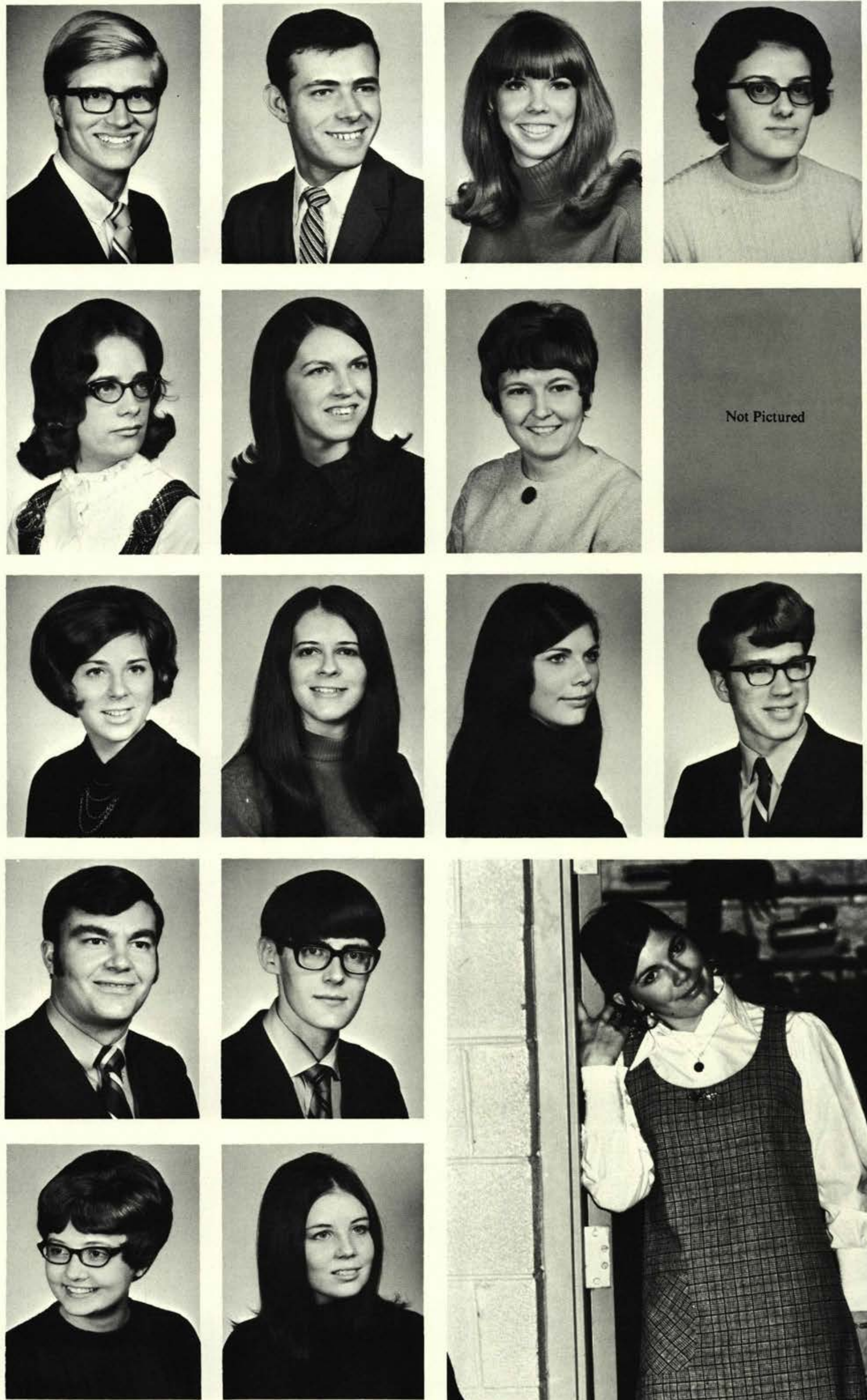

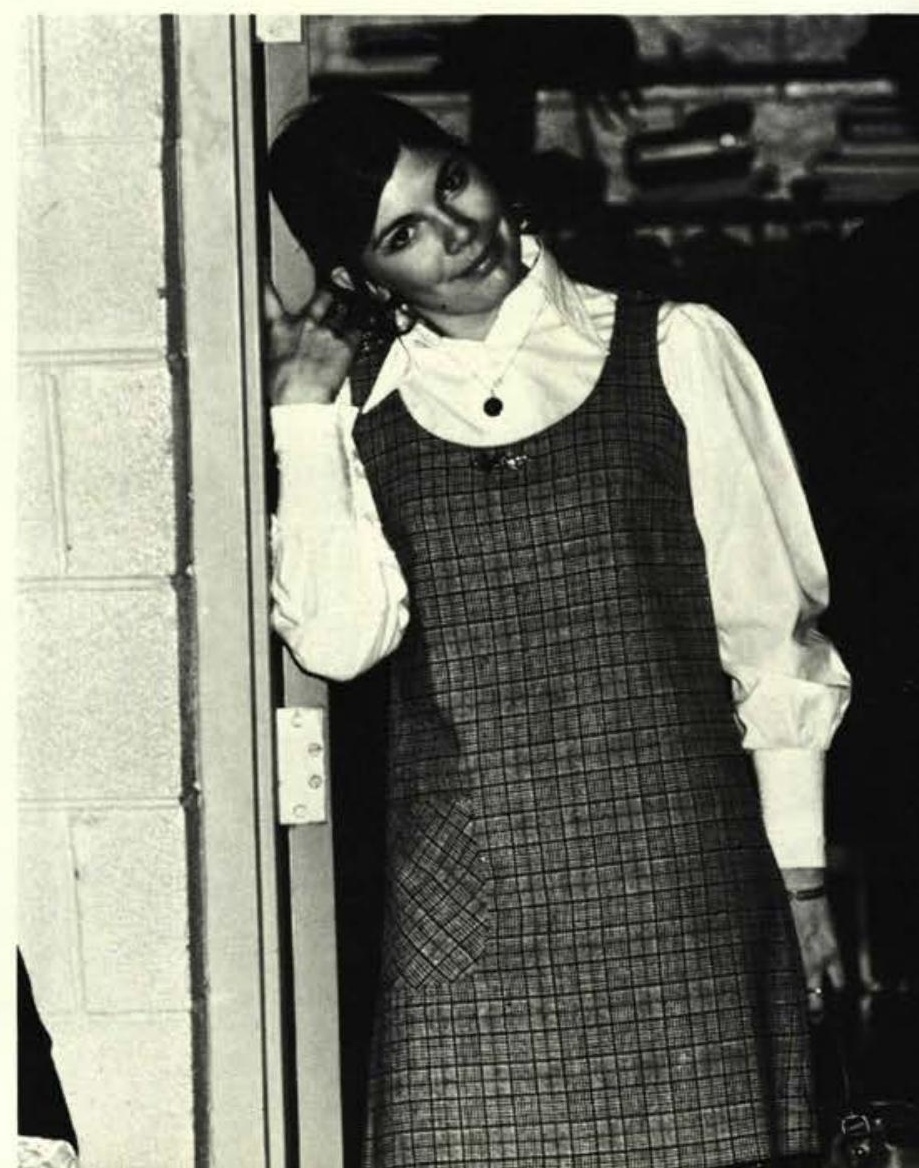



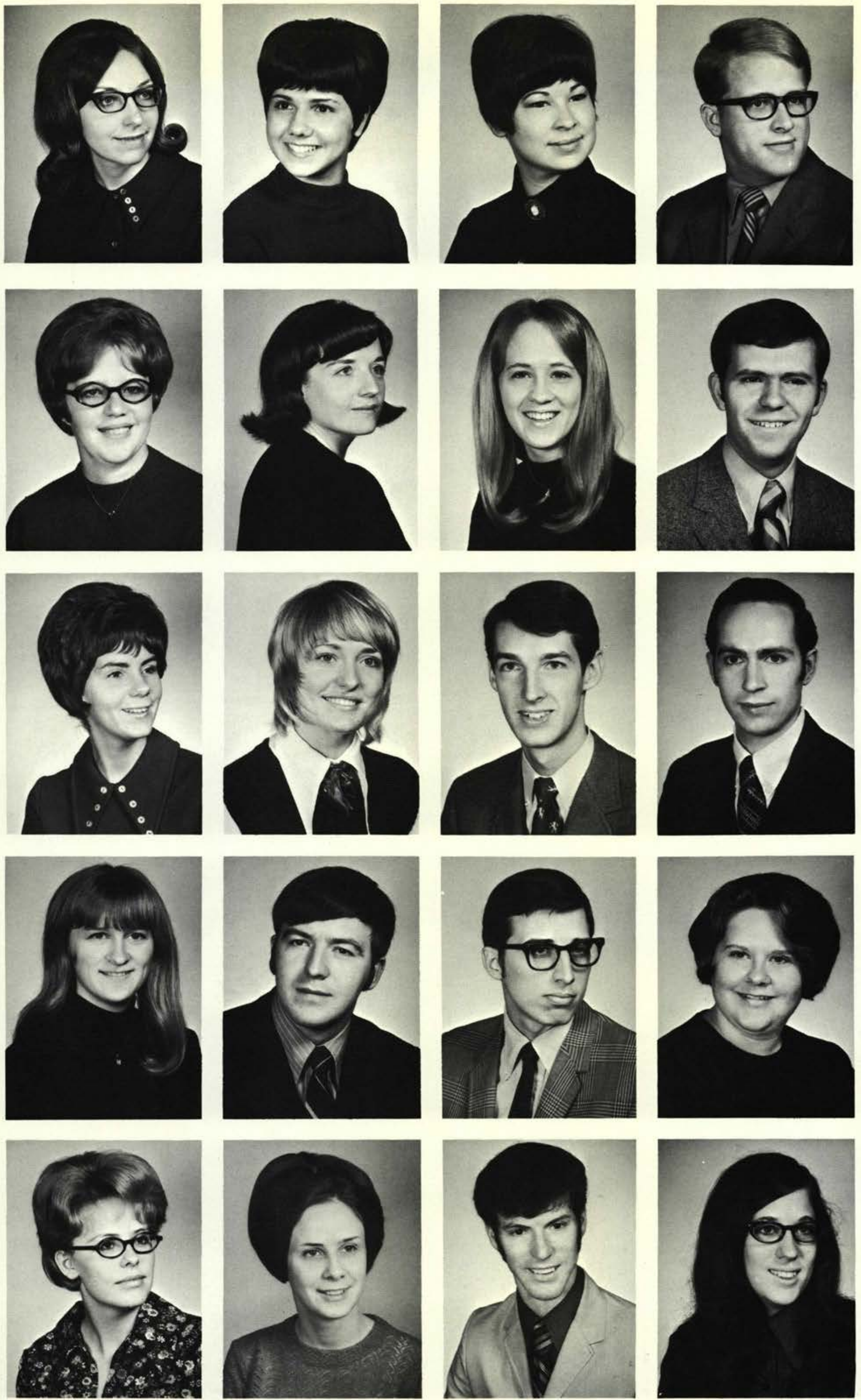
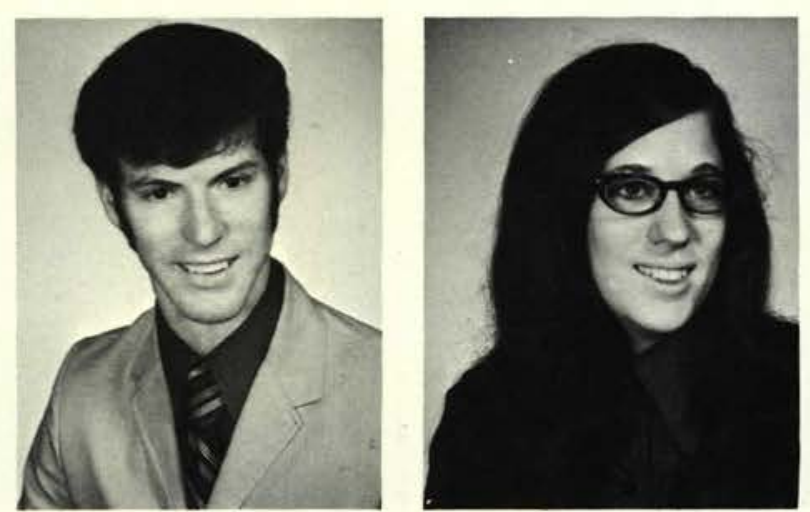

Tennant, Mercy

Bay City, Michigan

Elementary Education

Thomas, Billie

Columbus, Ohio

Physical Education

Tress, Mary

Apollo, Pennsylvania

Elementary Education

Turner, David

Chillicothe, Ohio

Bible

Urban, Carol

Denver, Colorado

Elementary Education

Waggoner, Pat

Coatesville, Indiana

Psychology

Walker, Connie

Kirtland, Ohio

Elementary Education

Walker, Stu

Brownsburg, Indiana

Chemistry

Webb, Susan

Kokomo, Indiana

Elementary Education

Welch, Glenda

Bartonville, Indiana

Speech

Wheeler, Paul

Huntington, West Virginia

Pre-Seminary Bible

Wilhite, Mark

Corwith, Iowa

Christian Education

Williams, Connie

Richmond, Indiana

English

Williams, Joseph

Cuyahoga Falls, Ohio

Business

Wood, Greg

Decatur, Illinois

History

Wood, Rebecca J.

Kokomo, Indiana

Social Science

Wuest, Darlyne

Billings, Montana

English

Yardley, Janet

Troy, Ohio

Elementary Education

Yates, Gary L.

Dayton, Ohio

English

Zwiesler, Cathi

West Alexandria, Ohio

English 
Abernathy, Joy Columbus, Ohio

Adams, JoAnn

Dayton, Ohio

Addleman, Nancy

Hillsdale, Michigan

Alexander, Stephen

Stryker, Ohio

Ashby, Pam

Dayton, Ohio

Austin, Robert

New Milford, New Jersey

Baker, Judy

Schenectady, New York

Beck, Margaret

Oswego, New York

Bernath, Karen

Stryker, Ohio

Betts, Linda

Wellington, Ohio

Billington, Linda

Fostoria, Ohio

Birch, Sandra

Rome, New York

Bittner, Becky

Ruston, Washington

Blum, Linda

Kalamazoo, Michigan

Bodenmiller, Thayne

Homerville, Ohio

Bondorff, Carl

Maywood, New Jersey

Boyce, Michae

Euclid, Ohio

Bradford, David

Coschocton, Ohio

Branning, Pam

Choconut, Pennsylvania

Brooker, Marty

Sebring, Florida

Buschmann, JoAnne

Widefield, Colorado

Cagwin, Raymond $\mathbf{H}$.

Cedarville, Ohio
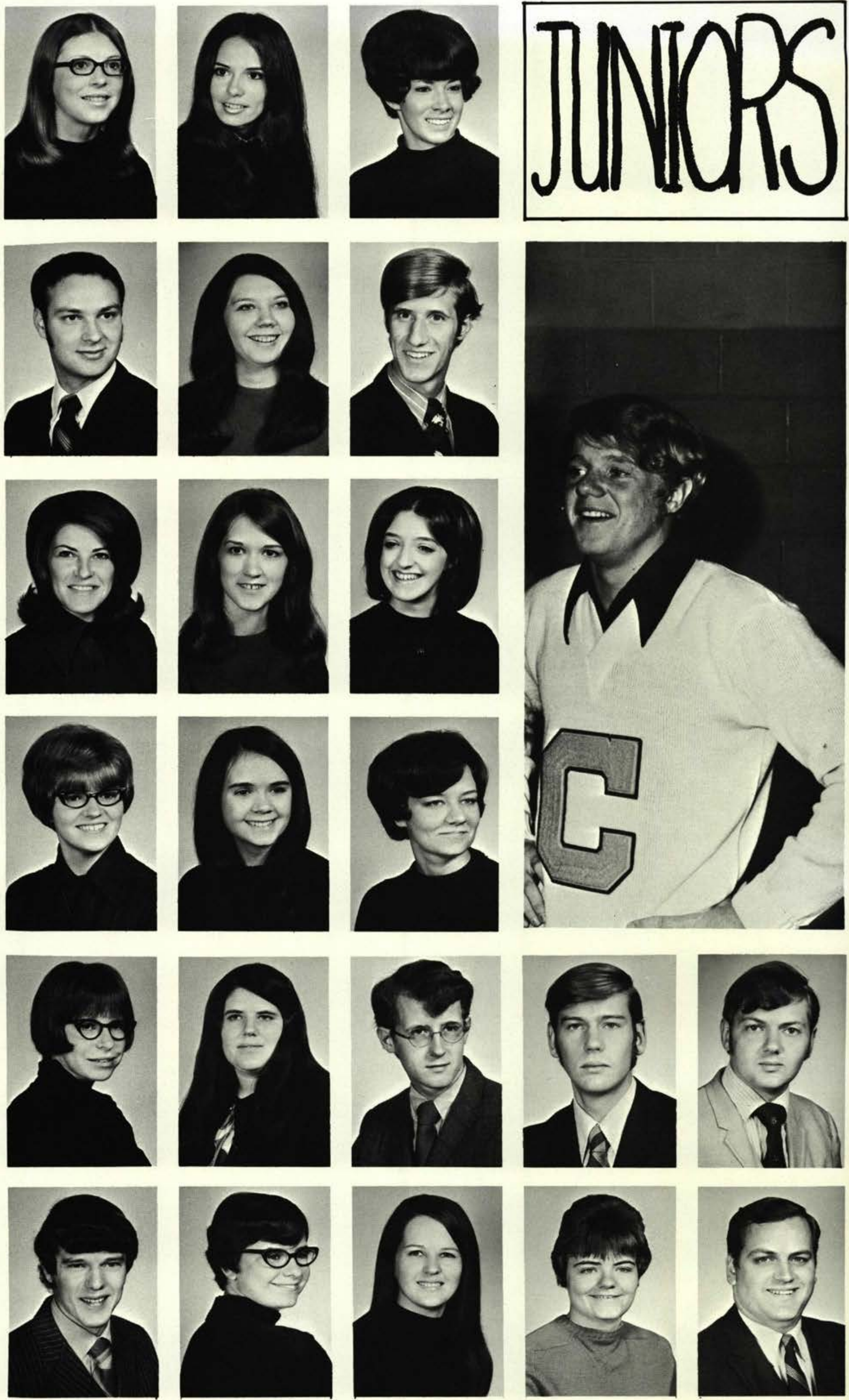

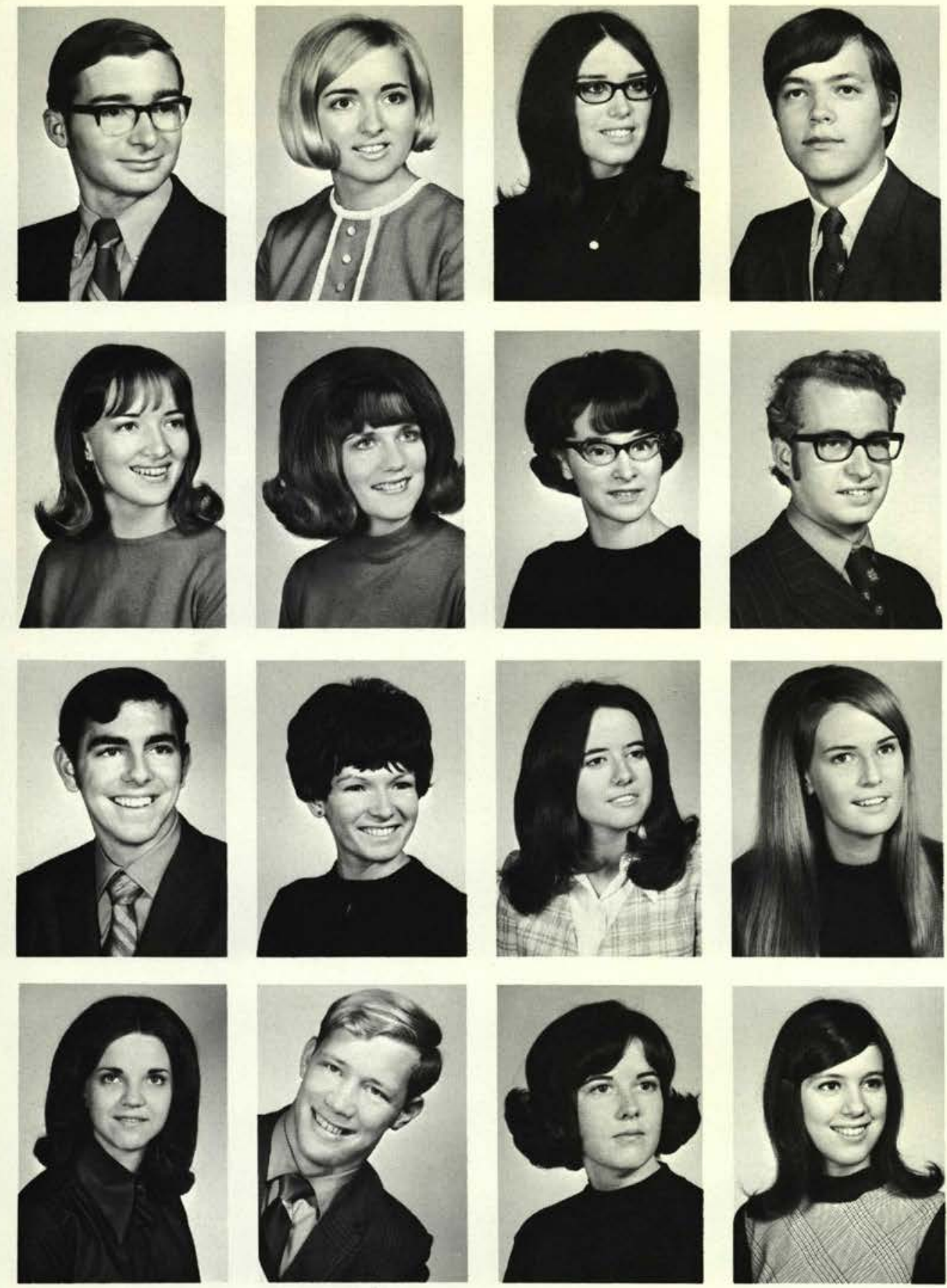

Calvin, Roy

Salem, Ohio

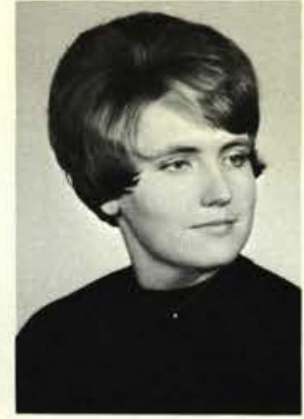

Carr, Diane

Paw Paw, Michigan

Carter, Helen

North Royalton, Ohio

Catteau, John

Parma, Ohio

Christiansen, Linda

Des Moines, Iowa

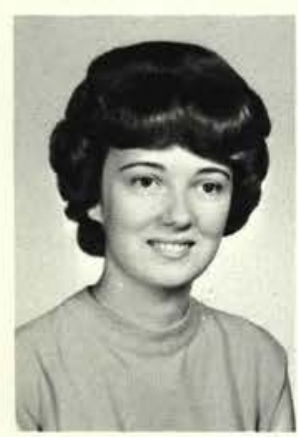

Clark, Annette

Galloway, Ohio

Clark, Connie

Spokane, Washington

Closson, Pam

Berea, Ohio

Conant, John

Iloilo City, Philippines

Crosby, Pat

Vestaburg, Michigan

Curtis, Roger

Newark, New Jersey

Dalton, Glenda

Wakeman, Ohio

Duhon, Corinne

Dayton, Ohio

Edelblute, Meda Sue

Gallipolis, Ohio

Edwards, Marsh $\mathrm{H}$.

Albion, Iowa

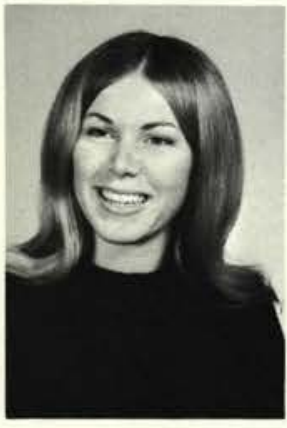

Ellis, Gail

Arlington, Virginia

Emmott, David

Sinclairville, New York

Fiest, Barb

Elyria, Ohio

Flint, Marianne

Cedarville, Ohio

Fowler, Lynda

Crawfordsville, Indiana

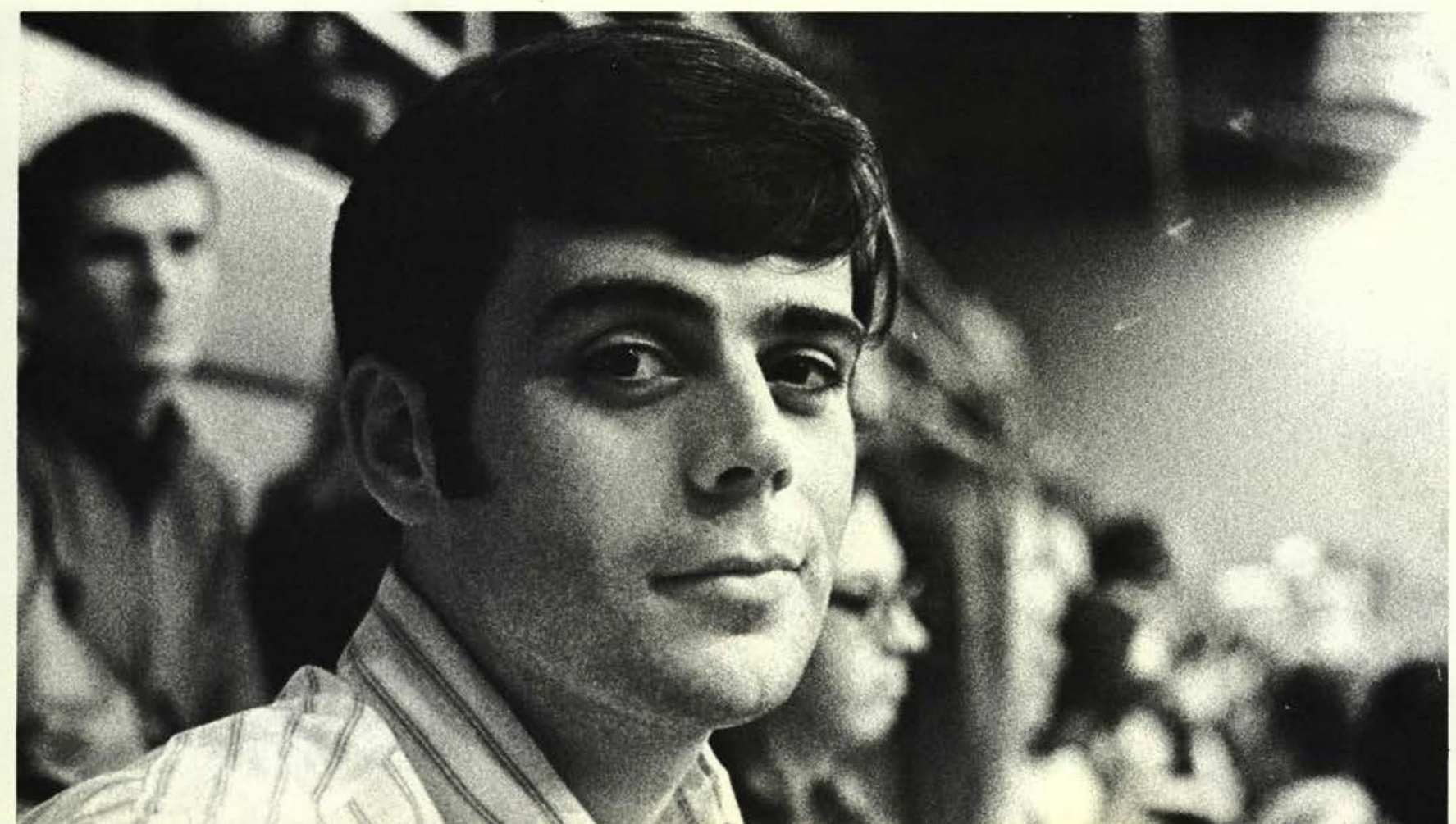


Fulcomer, Darlene Bolwar, Pennsylvania

Geist, Dale

Xenia, Ohio

Glazier, Steven

Troy, Ohio

Good, Debbie

New Castle, Delaware

Grable, Laura Lee

Wheaton, Maryland

Grant, David

New Hyde Park, New York

Gray, Pam

Kansas City, Kansas

Greening, Jim

Johnson City, New York

Gunneson, Bev

Stewartville, Minnesota

Hague, Keith

Cedarville, Ohio

Harold, Susan

Huntington, West Virginia

Harrison, John

New Richmond, Ohio

Hart, Barbara

Des Moines, Iowa

Harvey, Clifford

Columbus, Ohio

Heck, Belinda

Southington, Ohio

Helm, Joan

Allendale, Michigan

Henry, Bill

Painesville, Ohio

Holden, Kevin

Endicott, New York

Holtz, Cheryl

Muscatine, Iowa

Hostetler, Bonnie

Smithville, Ohio

Houchin, Andrea

Kansas City, Kansas

Howdyshell, Harvey

Pickerington, Ohio

Hubble, Paul

Crawfordsville, Indiana

Hull, Rebecca

Ithaca, New York
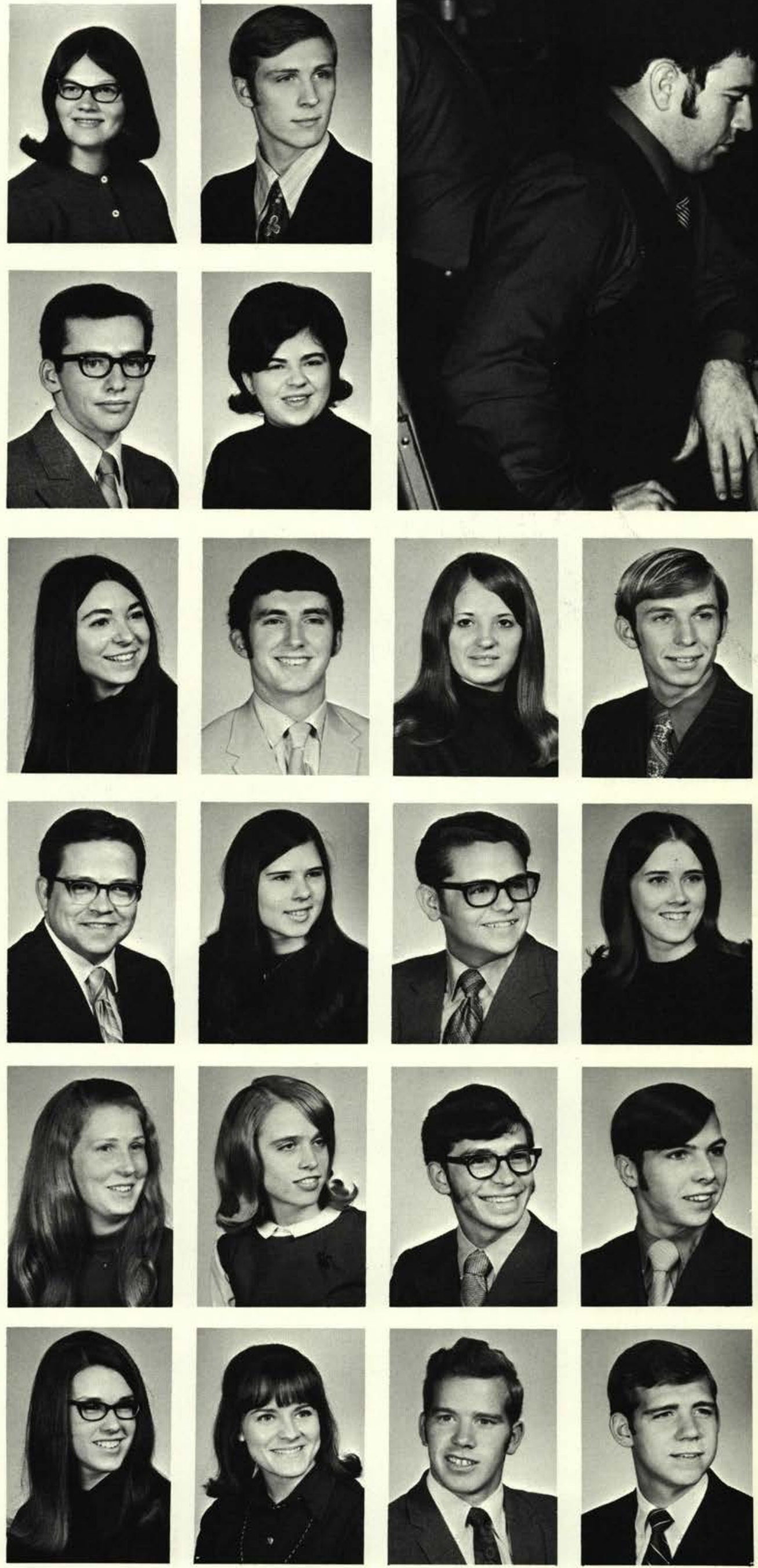
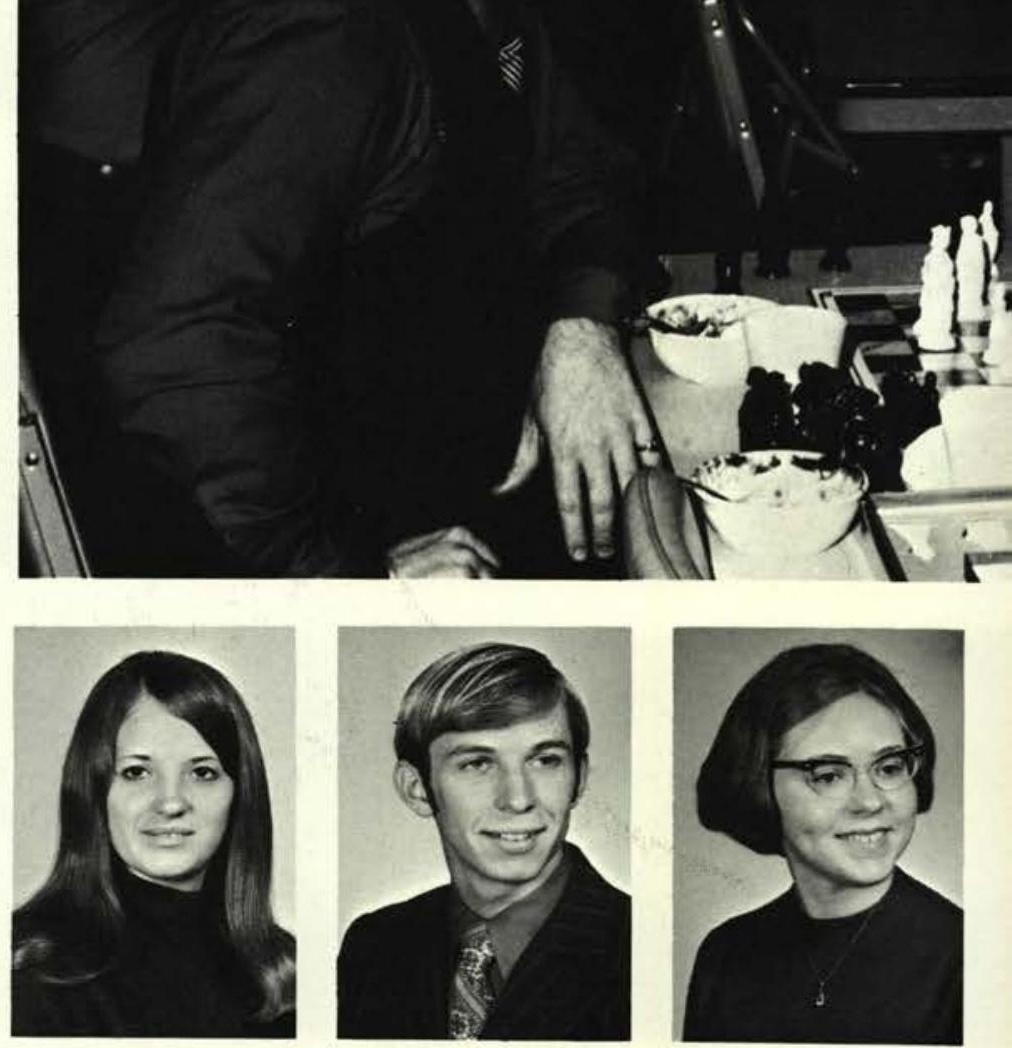
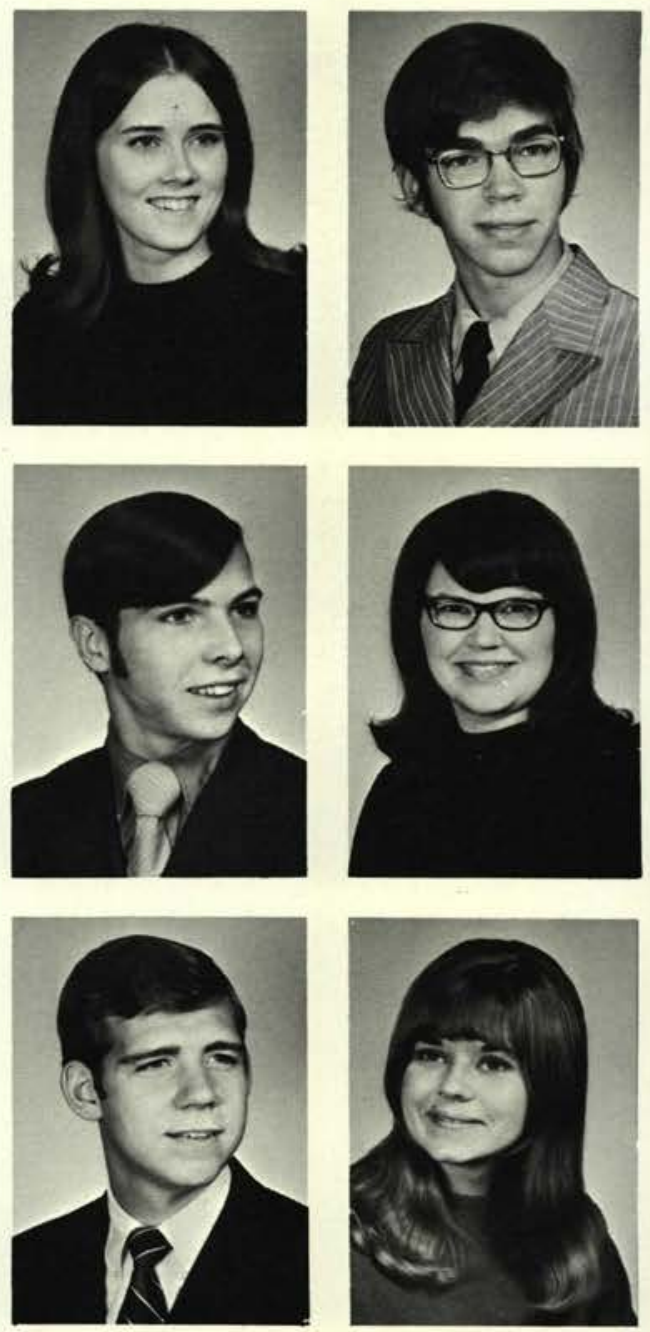

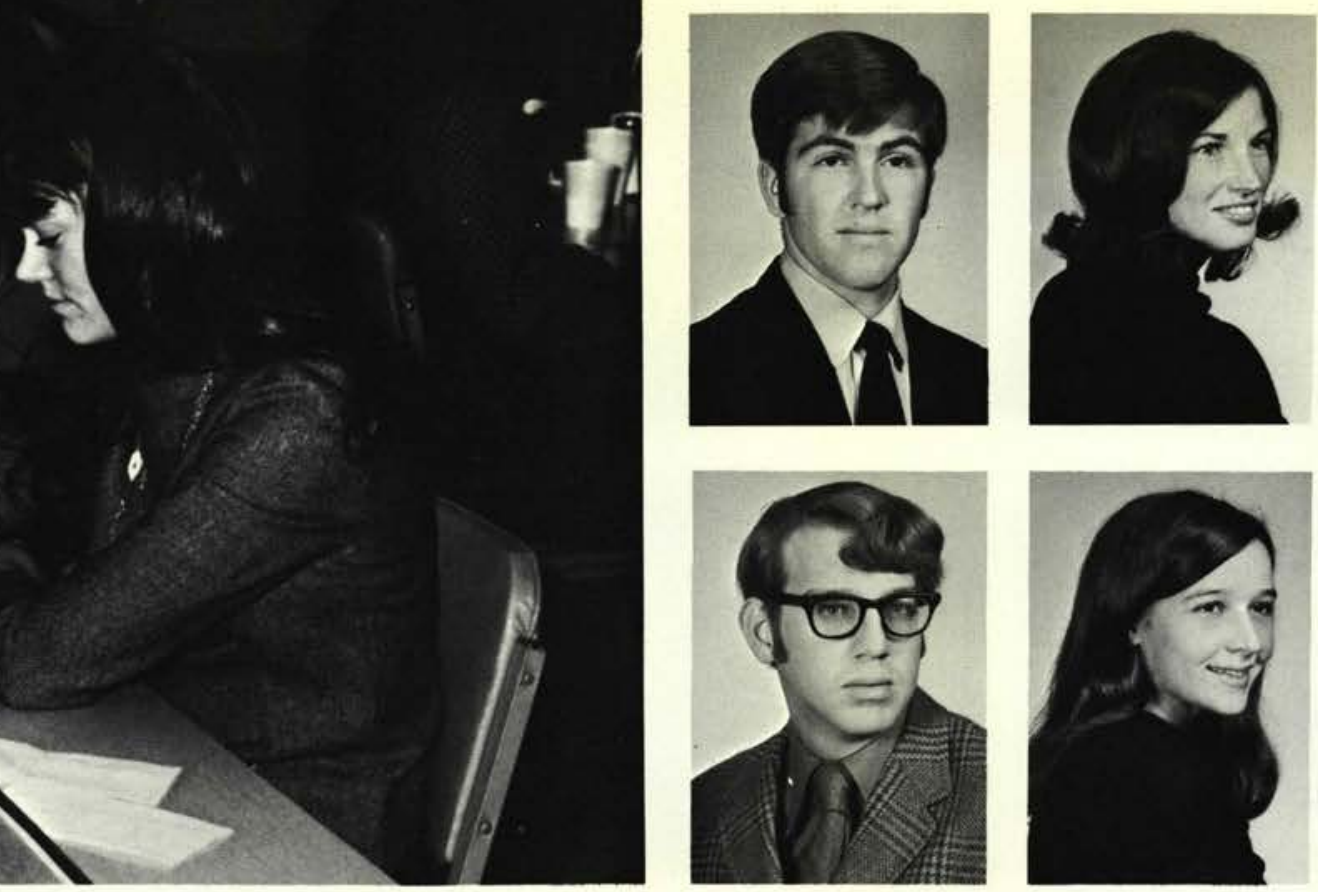

Ingram, Dan

Morning Sun, Iowa

Jacobs, Mary

Vestal, New York
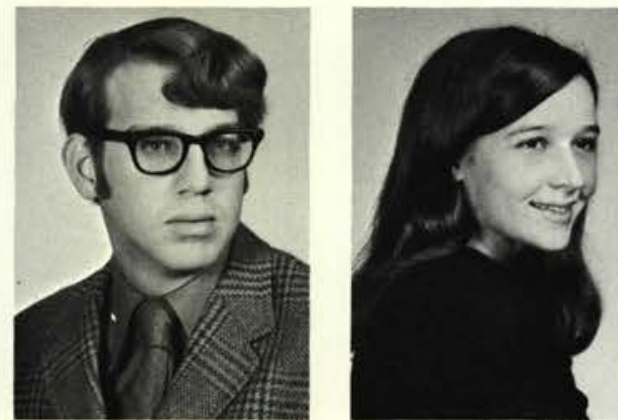

Jarvis, Tim

Norwalk, Ohio

Jeffries, Nancy

Bridgeport, West Virginia
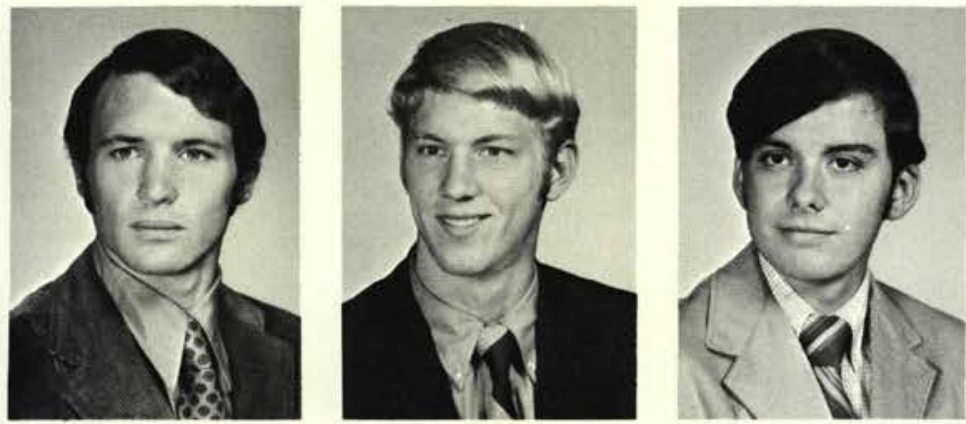

Jenkins, Warren

McLean, Virginia

Jensen, Cliff

Caro, Michigan

Jewell, David

Elyria, Ohio
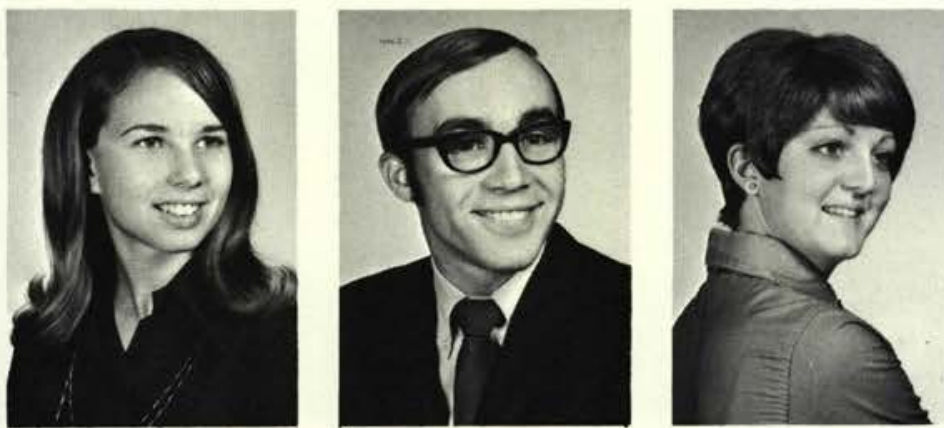

Jones, Laura

Uniontown, Ohio

Jones, Steve

New Palestine, Indiana

Kahler, Donna

Leawood, Kansas
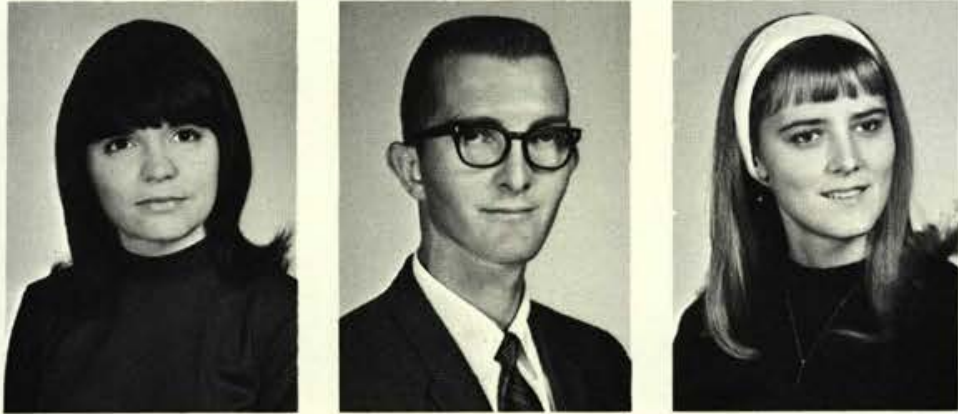

Kendrick, Pam

Shickshinny, Pennsylvania

Kesler, John

Olney, Illinois

Kircher, Ruth

New York City, New York
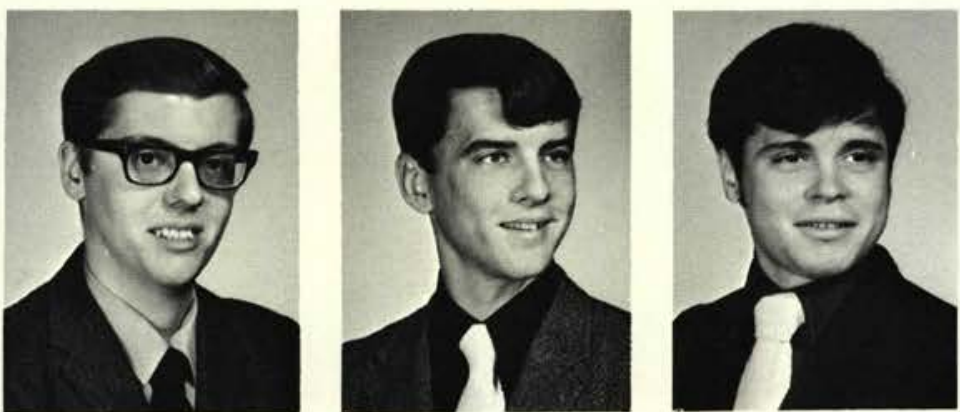

Klemm, Larry

Clarkston, Michigan

Kline, Tim

Greene, Iowa

Laird, Buddy

Carbon Cliff, Illinois 


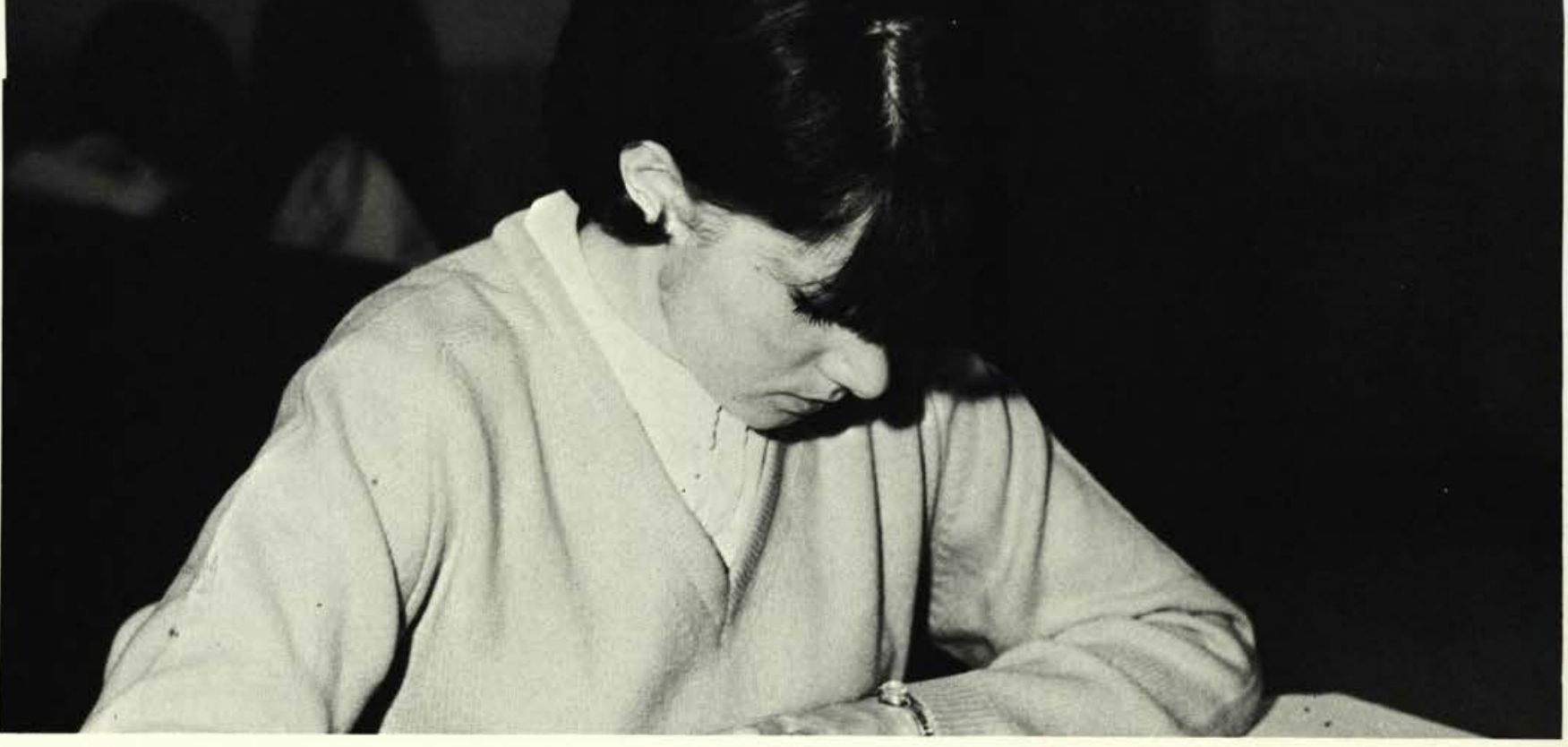

Lakes, Victor

Dayton, Ohio

Lambert, Carolyn

Rittman, Ohio

Lantz, Dawn

Vienna, Virginia

Larson, Curtis M.

Allegan, Michigan

Lathrop, Sue

Downers Grove, Illinois

Leslie, Frank

Meshoppen, Pennsylvania

Linger, Linda

Northfield, Ohio

Lough, Jeff C

Berea, Ohio

Love, Joyce M.

Huntington, West Virginia

Madison, Larry

Austin, Minnesota

Mallinak, Dave

Lakewood, Ohio

Marlowe, Catherine

Oxford, Michigan

Martin, Dan

Crawfordsville, Indiana

Mays, Mike

Chillicothe, Ohio

Mead, Daniel

Fremont, Michigan

Miller, Lyle

St. Charles, Iowa

Miller, Roger

Allegan, Michigan

Mogle, Anita

Xenia, Ohio

Mohr, Becky

East Syracuse, New York
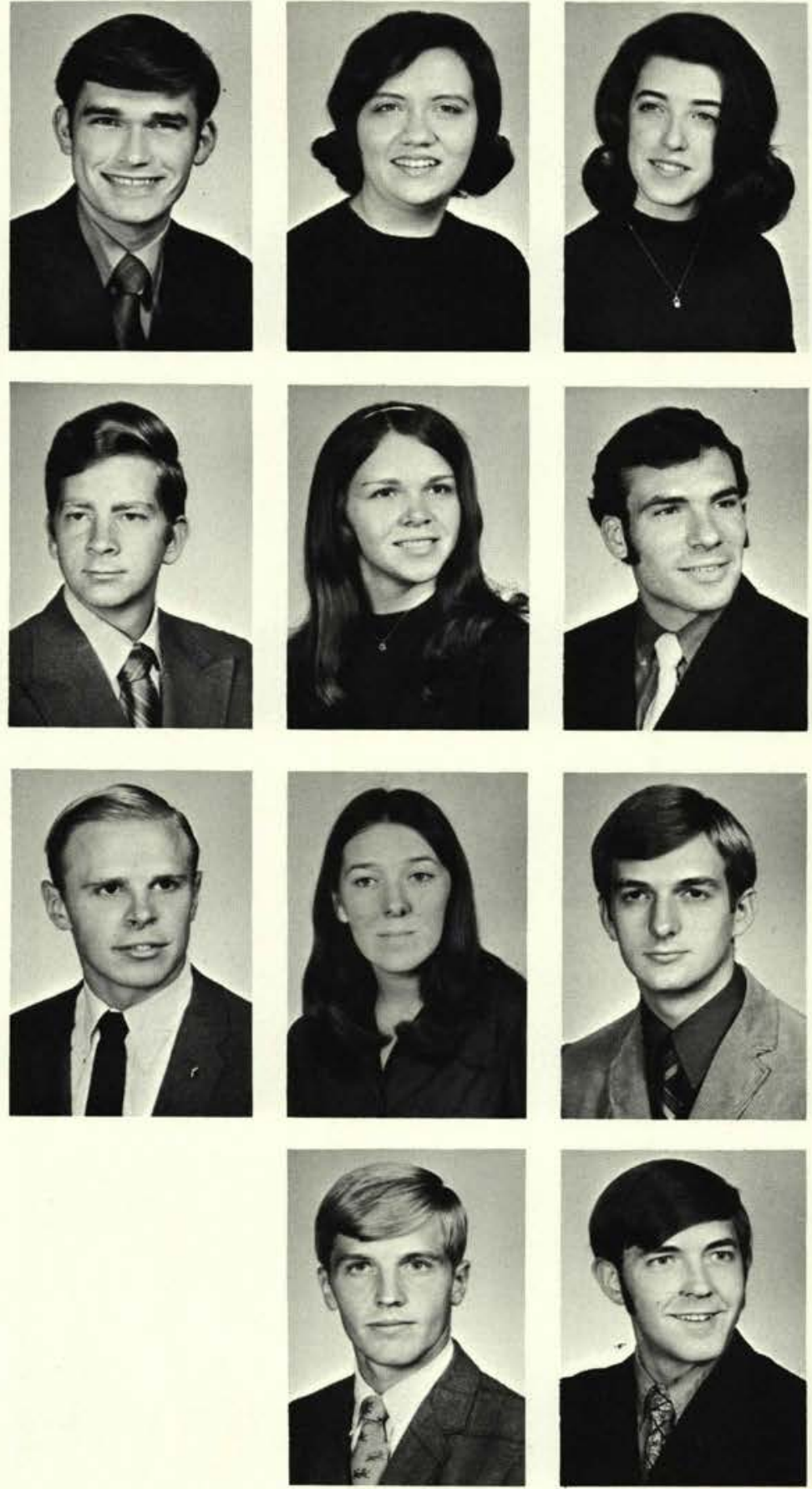
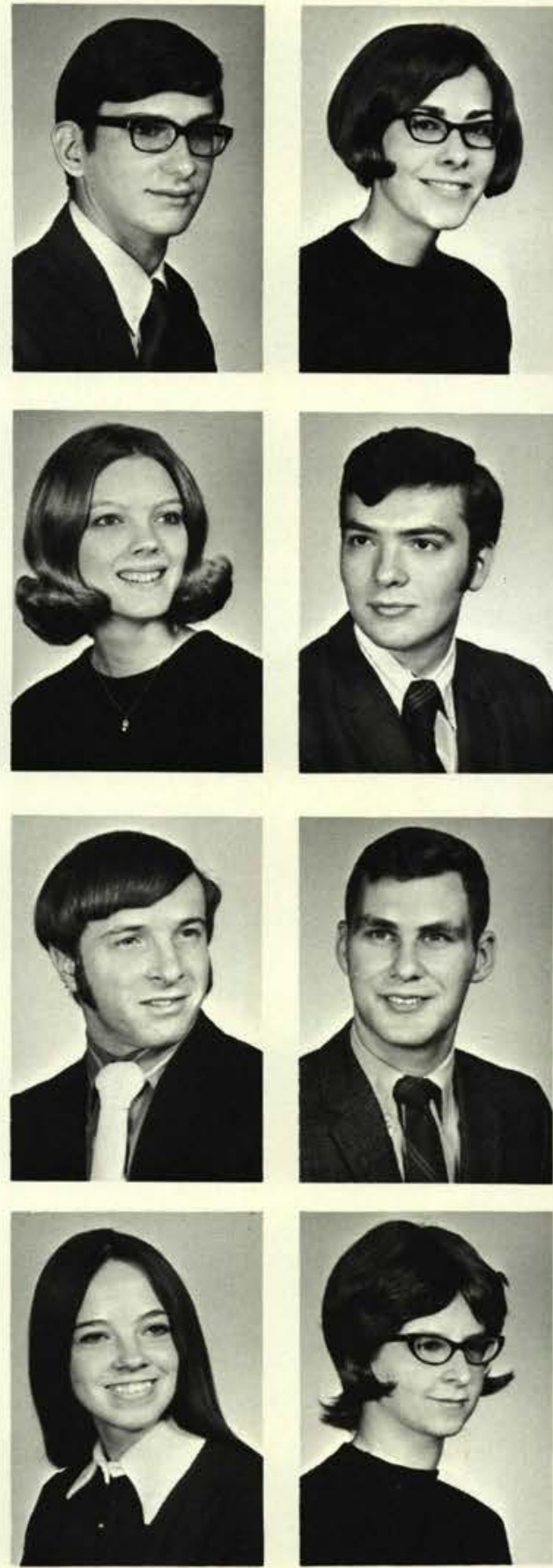

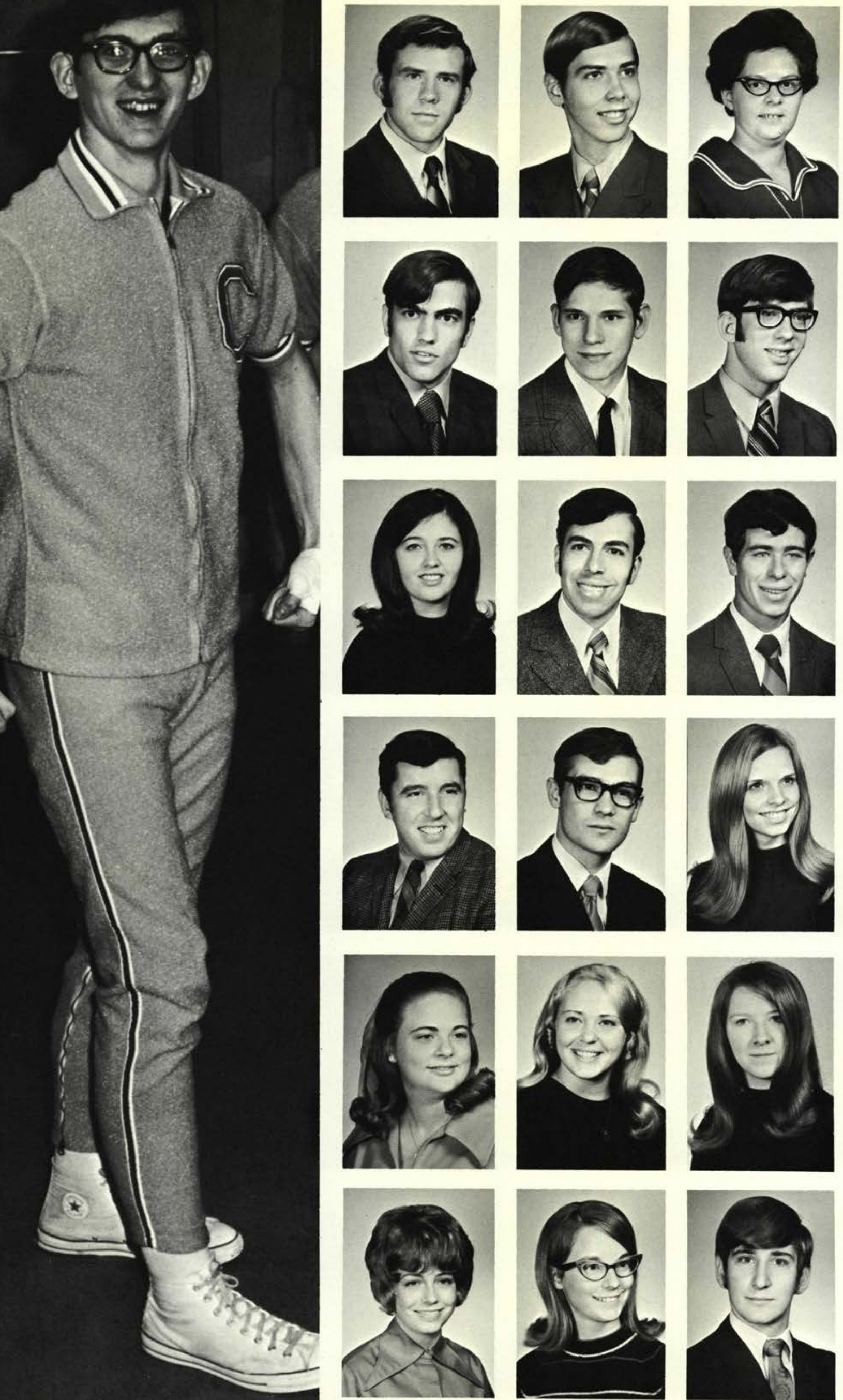

Moon, Philip

Freeport, New York

Nelson, David

Santa Barbara, California

Nolen, Bonnie Louise

Wheelersburg, Ohio
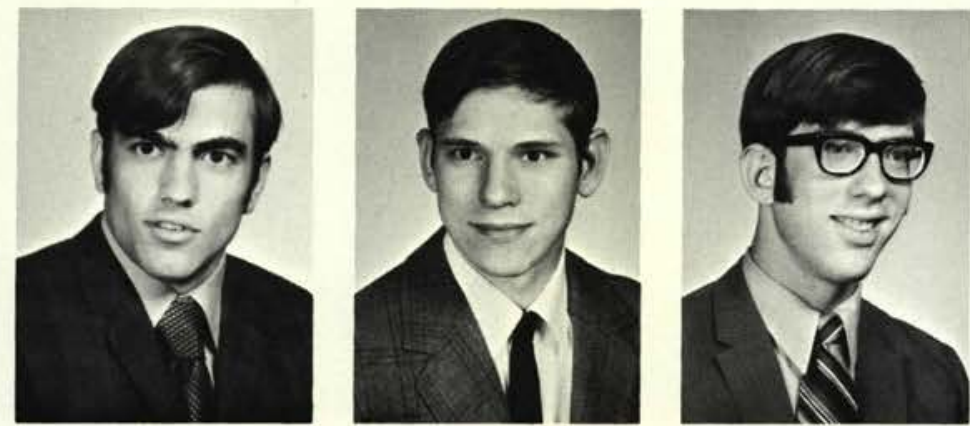

Northey, Ted

Medina, Ohio

Norton, Robert

Whitehall, Michigan

Olson, John

Warren, Pennsylvania
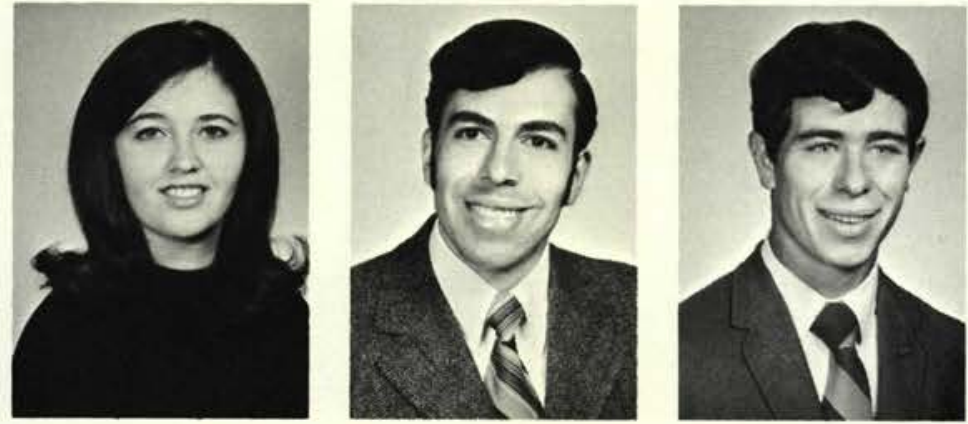

Partlow, Kaye

Indianapolis, Indiana

Pereira, John

Kinos Park, Long Island, New York

Perkins, Ralph

Denver, Colorado
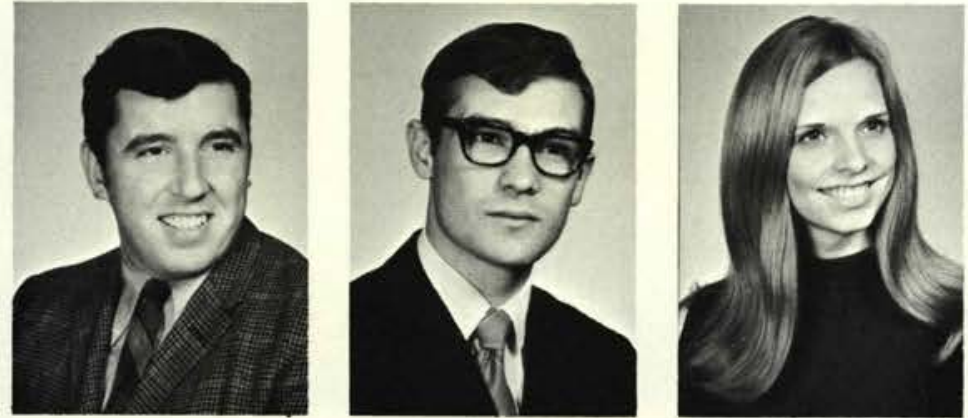

Perry, Bruce

Tiffin, Ohio

Peters, David

Troy, Ohio

Peterson, Karyn

Colona, Illinois
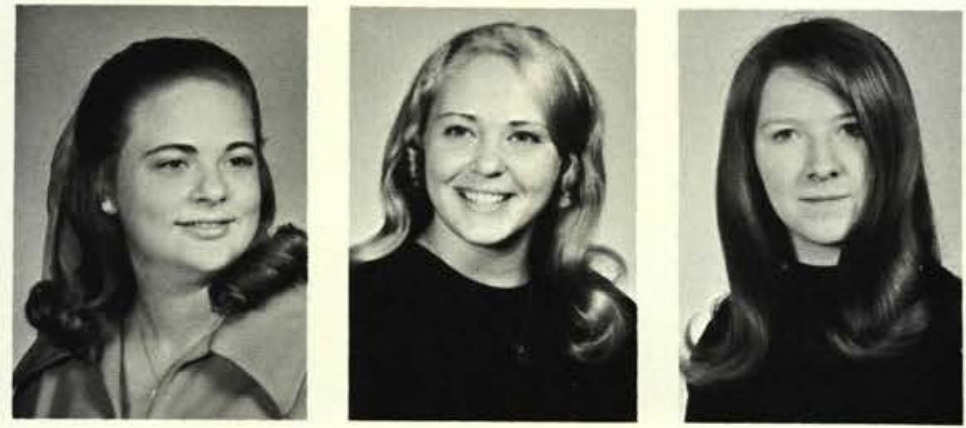

Phelps, Linda

Lorain, Ohio

Poarch, Rebekah

Columbus, Ohio

Porter, Tammy

Onaway, Michigan
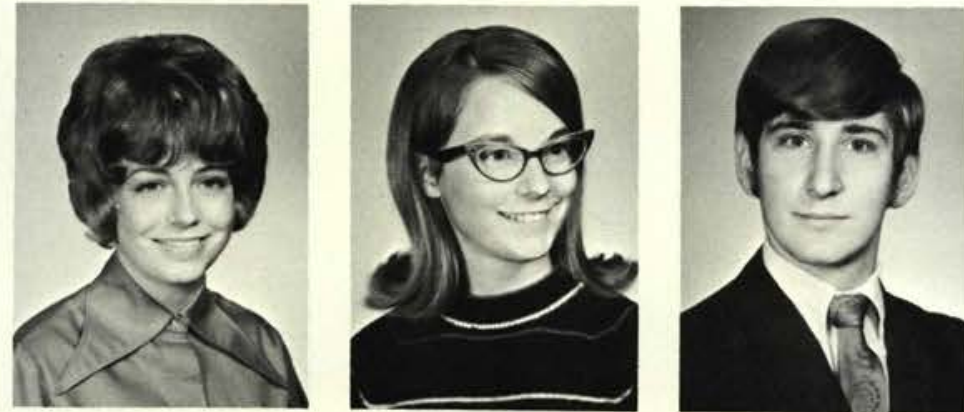

Portz, Gale

Greeneville, Pennsylvania

Powley, Jeanette

Lake Orion, Michigan

Pruden, John

Prudenville, Michigan 
Radcliffe, Paul

Caldwell, Ohio

Railsback, David

Olmstead Falls, Ohio

Ransbottom, Ruth

Pittsburg, Pennsylvania

Reeder, Tom

Youngstown, Ohio

Rice, Dennis

Tamoqua, Pennsylvania

Riley, Patricia

Newark Valley, New Jersey

Rocher, Shirley

Amherst, Ohio

Ruder, Esther

Cedarville, Ohio

Sagraves, Cecil

Plain City, Ohio

Scott, Susan

Cedarville, Ohio

Seidler, Tom

Cincinnati, Ohio

Selfridge, Marcene

Elyria, Ohio

Sickels, Charlene

Barberton, Ohio

Simpkins, Connie

Cambridge City, Indiana

Slavens, Deborah

Crawfordsville, Indiana

Smith, Jonathan

Findlay, Ohio

Smith, Nancy

Sinclairville, New York

Steyer, Ruth Ann

Coshocton, Ohio

Strong, Tom

Painesville, Ohio

Stubrich, Carolyn

Hubbard, Ohio
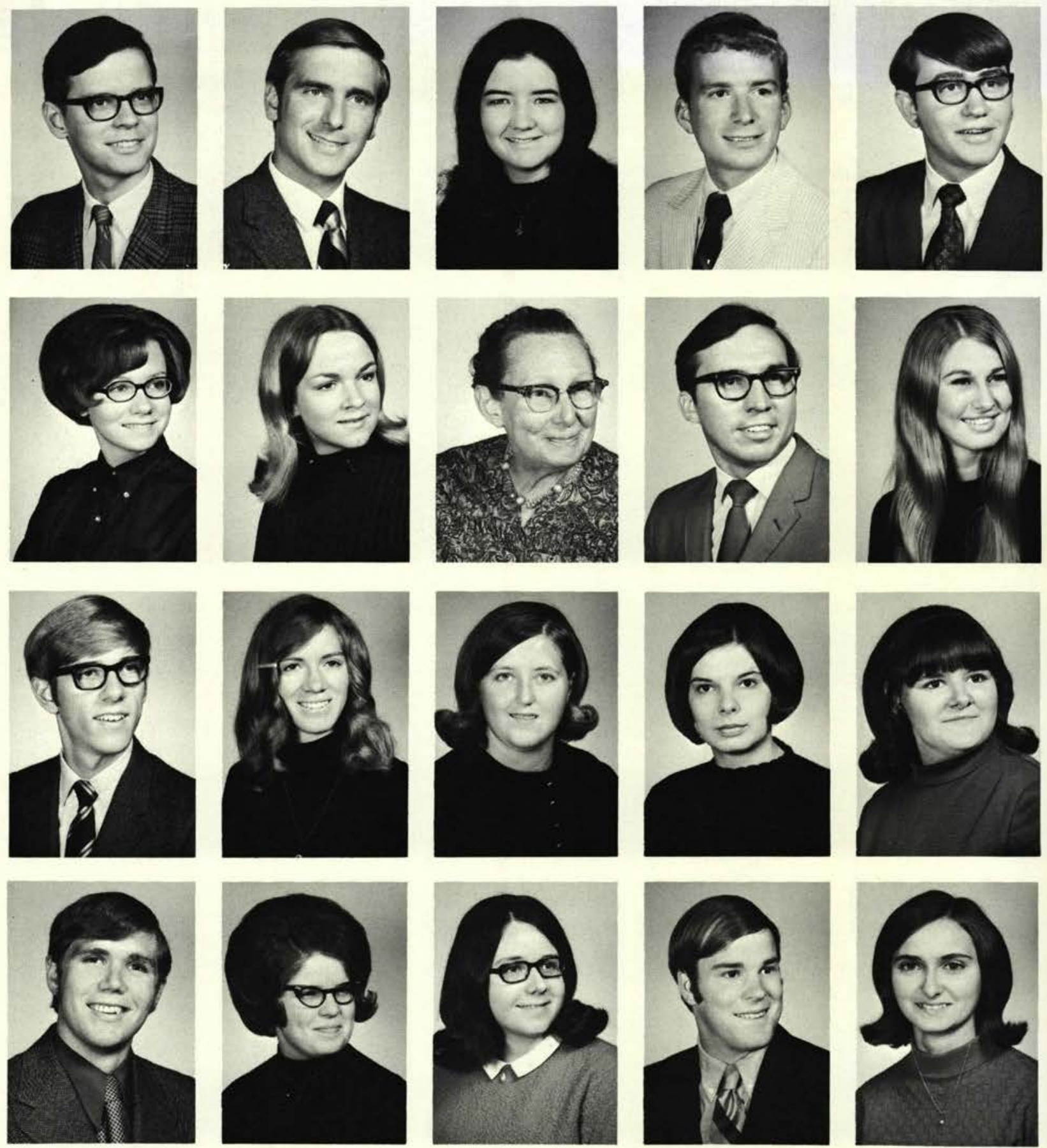

Dave Railsback, President

Ralph Perkins, Student Council

Dan Inghram, Chaplin

Thayne Bodenmiller, Vice President

Sue Scott, Treasurer

Barb Fiest, Secretary

Vicki Tobias, Student Council

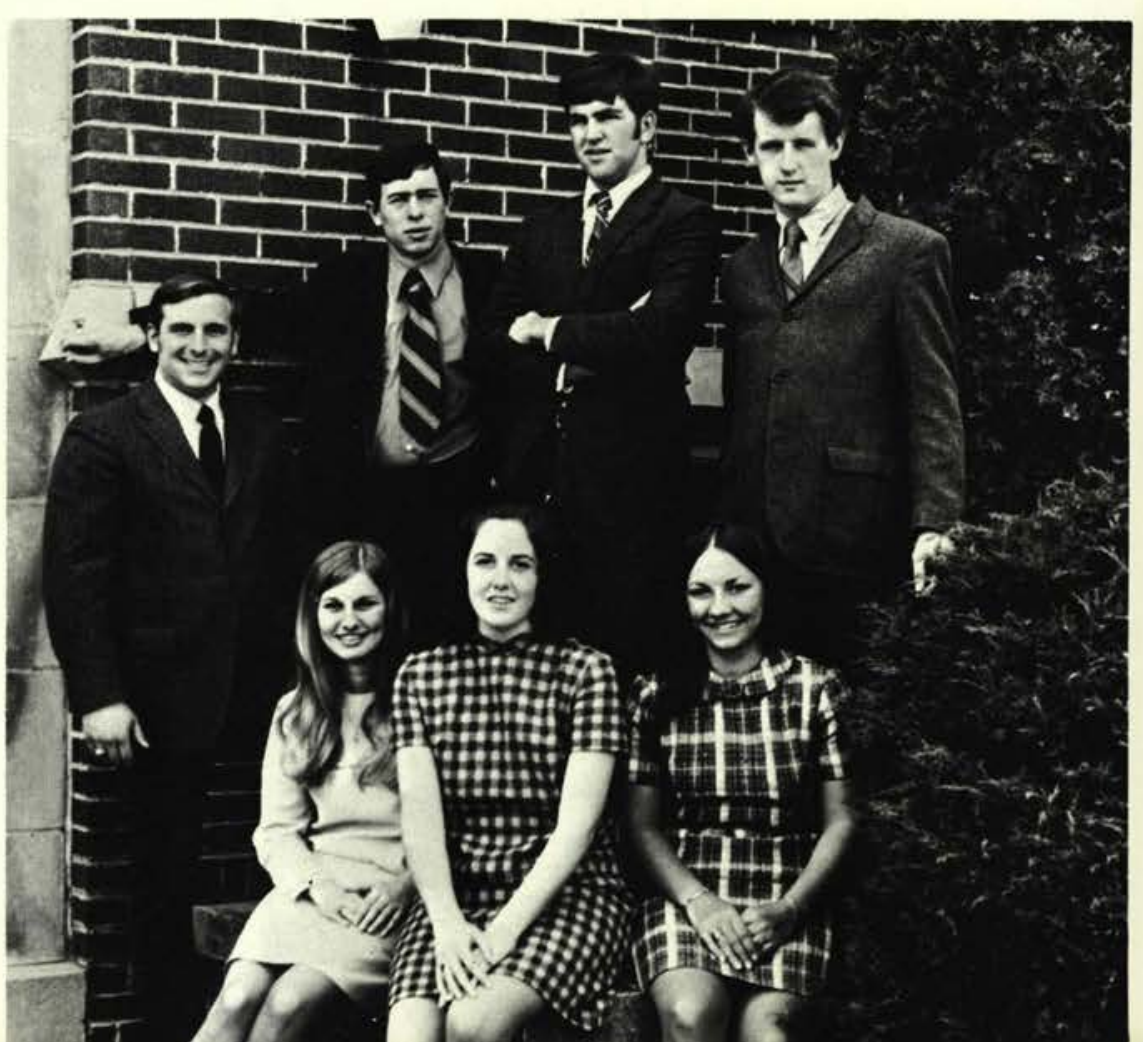




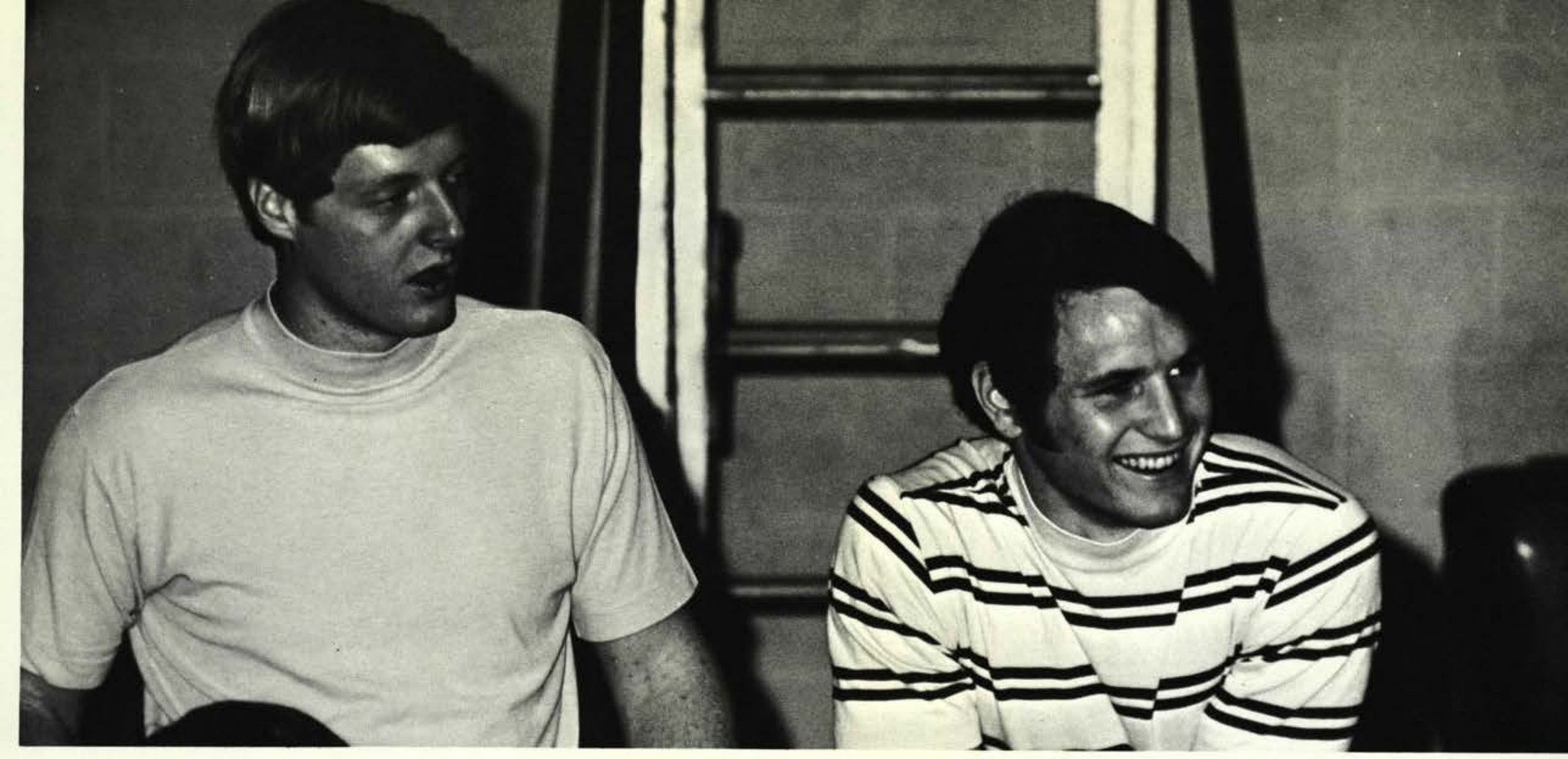

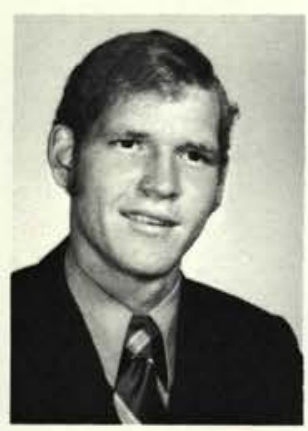
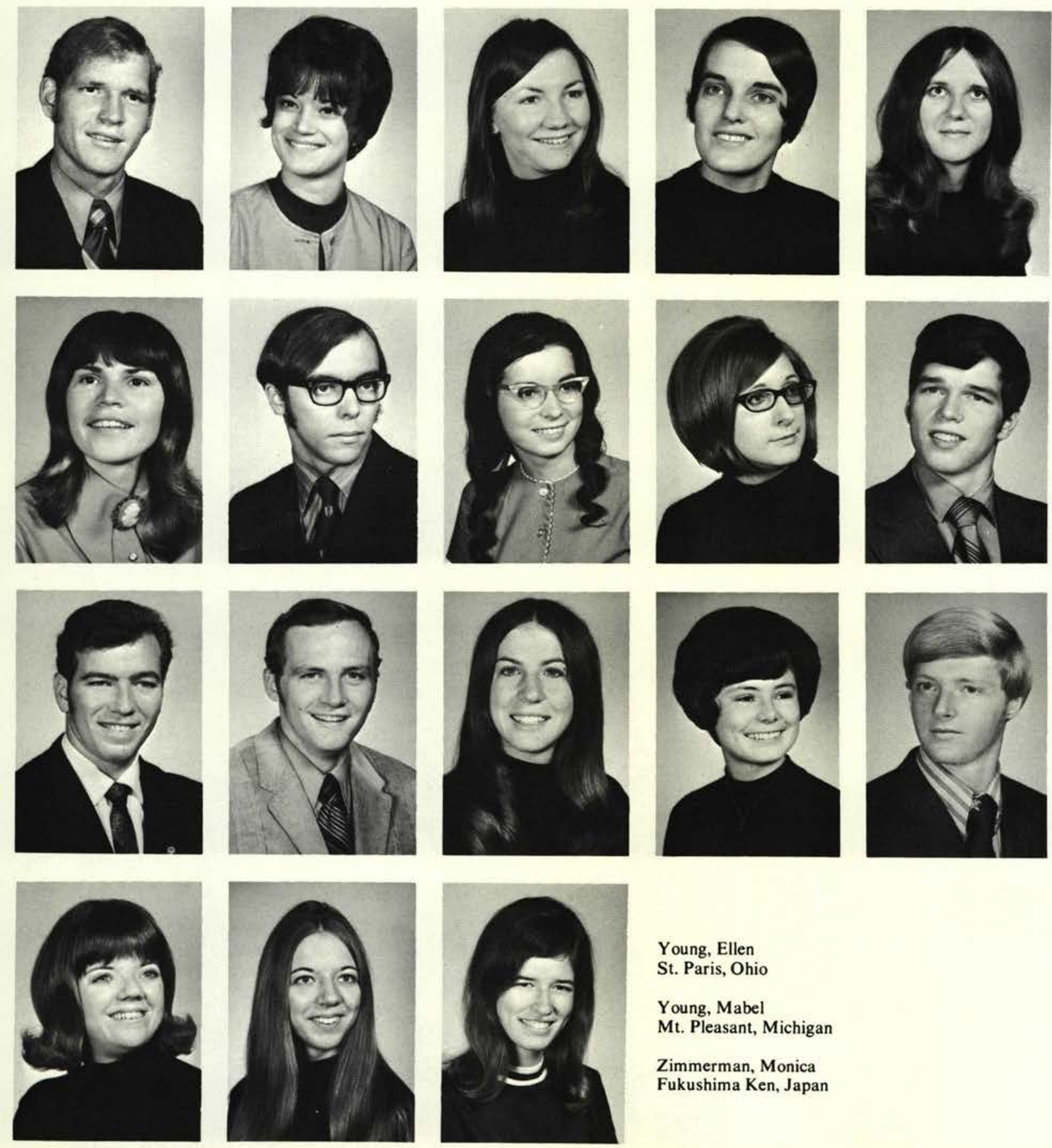

Young, Ellen

St. Paris, Ohio

Young, Mabel

Mt. Pleasant, Michigan

Zimmerman, Monica

Fukushima Ken, Japan
Tewis, Louis

Cedarville, Ohio

Titus, Marlene

Clarion, Iowa

Tobias, Vicki

Port Alleghany, Pennsylvania

Tompkins, Connie

Sherman, New York

Vanderpool, Mary

Tekonsha, Michigan

Vlug, Janice

Wyoming, Michigan

Wall, Tony

Amherst, Ohio

Webber, Carol Lynne

Cedarville, Ohio

Weld, Patricia

Edmore, Michigan

Whims, David W.

Cuyahoga Falls, Ohio

Wilhite, Mike

Clayton, Ohio

Williams, Charles

Nebraska City, Nebraska

Willis, Charlotte

Rochester, Michigan

Wilson, Alice E.

Crook, Colorado

Woodcock, Jeffrey H.

Jamestown, New York 
Acton, Don A.

Mentone, Indiana

Alberding, Donna

Wheatfield, Indiana

Alfrey, Larry

Adrian, Michigan

Allen, Larry

Mattoon, Illinois

Anderson, Kris

Grand Rapids, Michigan

Bacheller, Bil

Summerhill, New York

Bailey, Anne

Endicott, New York

Bailey, Keith

Charleston, West Virginia

Bailey, Marilyn

Bronson, Michigan

Bailey, Randy

Warren, Ohio

Baker, Ilinda

Randolph, New York

Barbely, Katy

Mansfield, Ohio

Bates, Jim

Corry, Pennsylvania

Bayne, Monica

Little Hocking, Ohio

BeDell, Arthur $\mathrm{R}$.

Livingston Manor, New York

Bierbaum, Carol

Bunker Hill, Illinois

Bingham, Donna T.

Goshen, Ohio

Bo, Carol Ann

Rochester, New York

Bobbitt, Wendy

Xenia, Ohio

Bodenmiller, Bonny Berea, Ohio
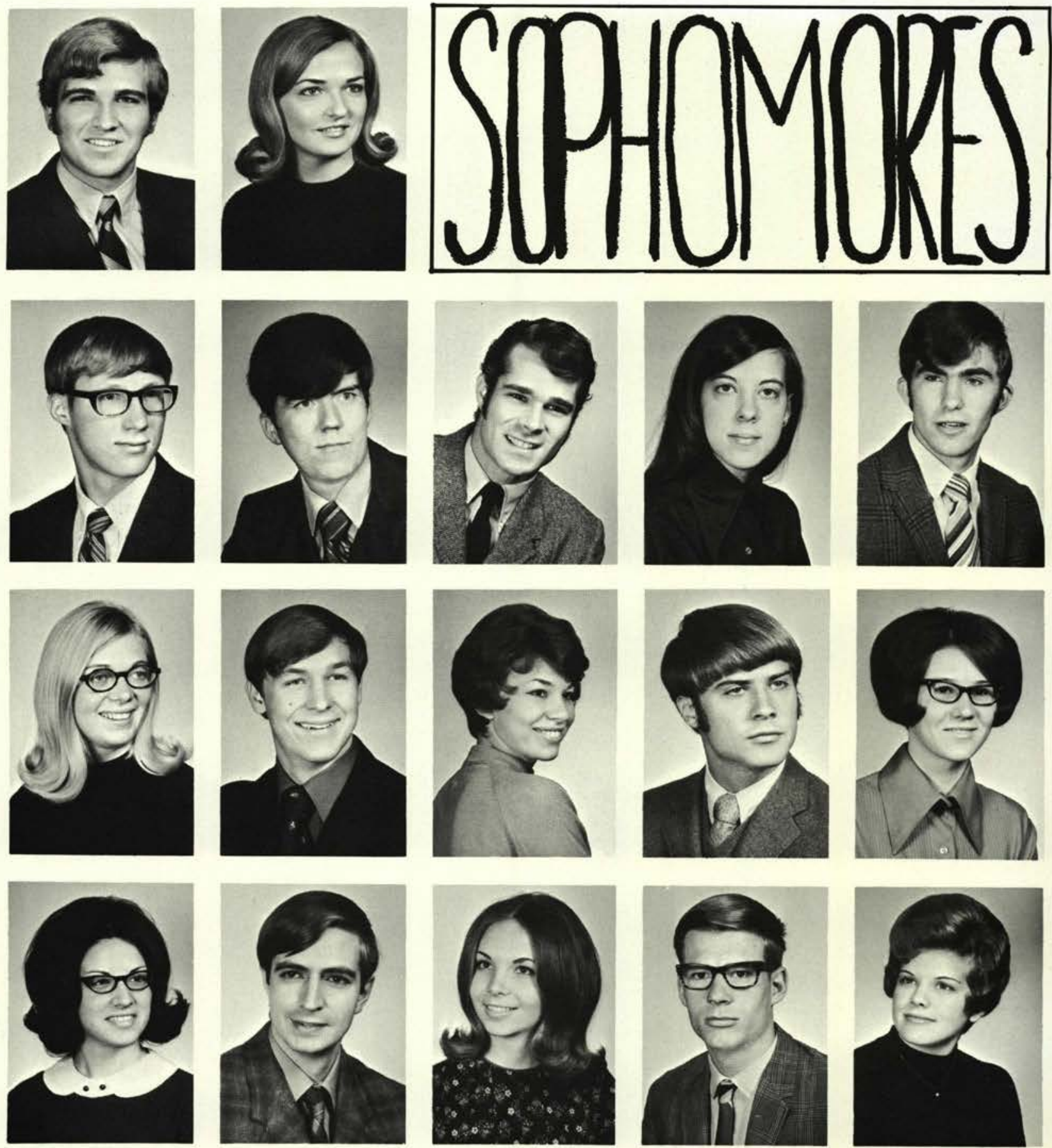
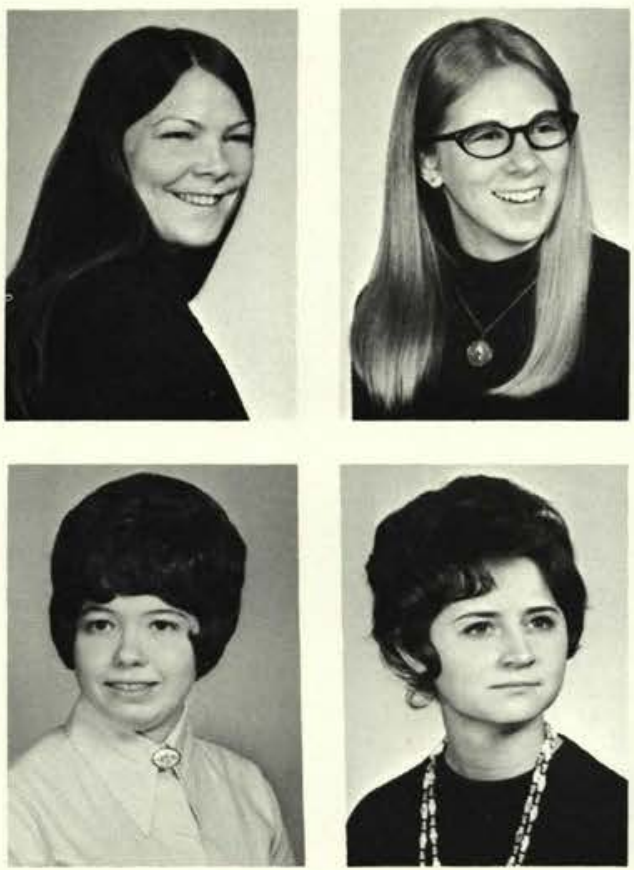
Cason, Janice

Springfield, Ohio

Chambers, Leslie

Grand Island, New York

Chandes, Becki

East Moline, Illinois

Chrestensen, Karen

Berea, Ohio

Chumbley, Rosanna

Monticello, Illinois

Chute, Linda

Dublin, New Hampshire

Clapp, Terry

Meridian, Ohio

Clarke, Ronald

Amelia, Ohio

Clauer, Becky

Springfield, Ohio

Clewell, Dick

Osky, Iowa

Codr, Phyllis

Nampa, Idaho

Cogan, Marjorie

Wauseon, Ohio

Coombs, Ruth Ann

Campbellsville, Kentucky

Cope, Yvonna

Crawfordsville Indiana

Craig, Ronald

Springfield, Ohio

Creviston, Sharon Cedarville, Ohio

Cunningan, Barbara Dayton, Ohio

Davis, Don

Littleton, Colorado
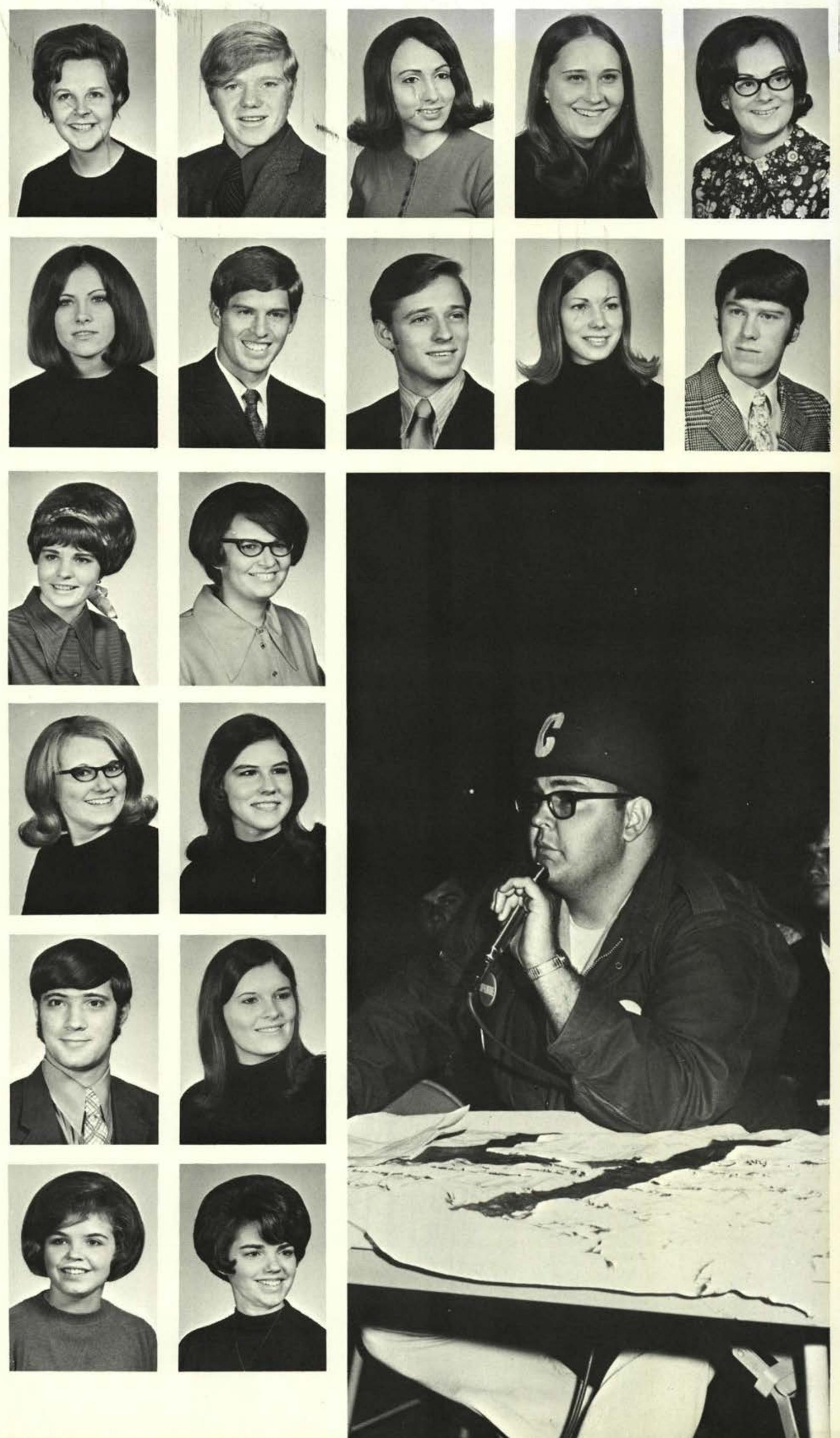
Gast, Rita

Eldora, Iowa

Gerber, Kim

Berlin, Ohio

Gibbons, Ed

Alhambra, Illinois

Gildan, Beth

Elyria, Ohio
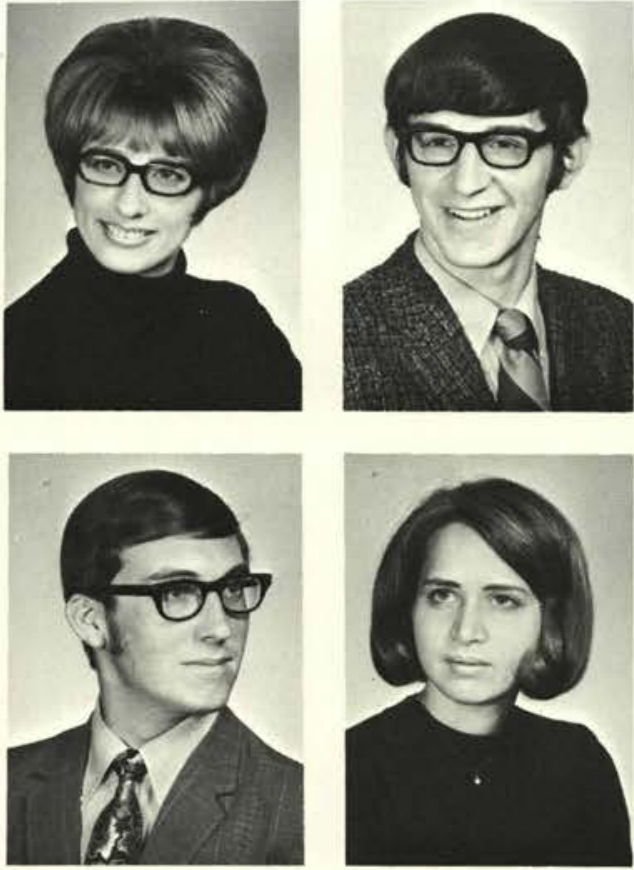

Gillespie, Sandra

Stryker, Ohio

Gillogly, Sharon

Newport, Michigan

Globig, David

Madison, Ohio

Golliher, Carolyn

Richland, Michigan

Good, Leslie A.

Waterville, Ohio

Greenwood, Tim

Cedarville, Ohio

Griffeth, Lee

Houston, Texas

Hall, Susan

Louisville, Kentucky

Hallett, Jan

Conneaut, Ohio

Hansen, Sherlyn

Mt. Pleasant, Michigan

Harmon, Don

Englewood, Colorado

Harmon, Jeanne

Delaware, Ohio

Harris, Jan

Cedar Falls, Iowa

Heal, Virginia

Bunker Hill, Illinois

Hegg, Tim

Spokane, Washington

Hendrickson, Donald

Friendsville, Pennsylvania

Hepworth, Judy

Montague, Michigan

Herman, Stephen

Johnson City, New Jersey

Hess, Douglas

Grundy Center, Iowa

Hillery, Jan

Alton, Illinoi
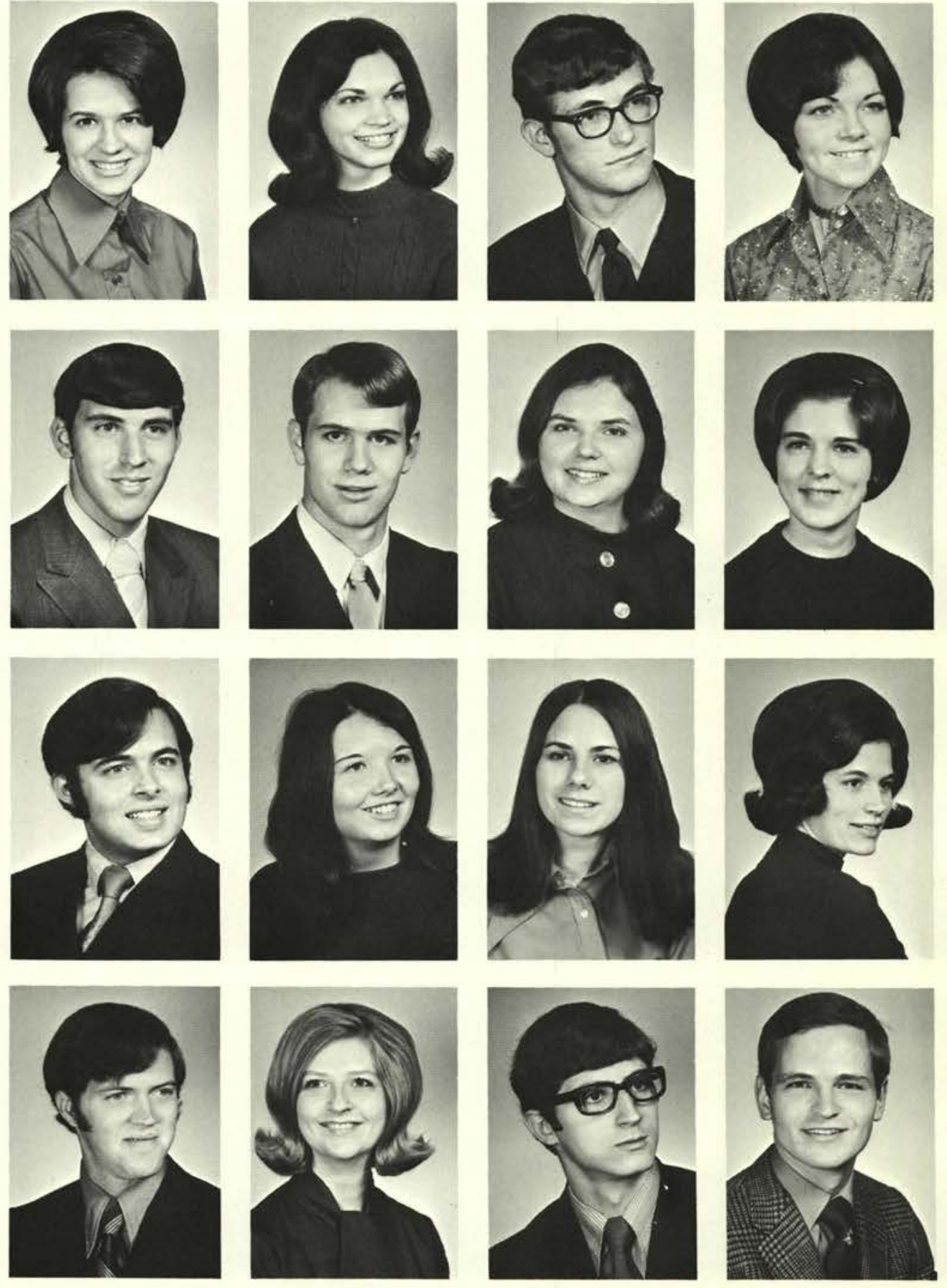
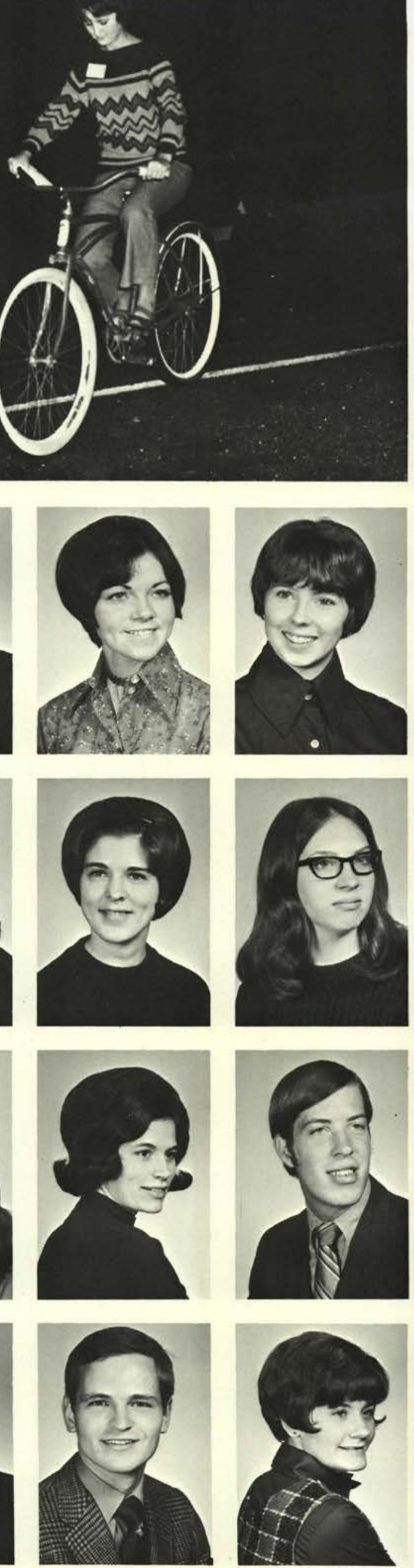
Kainbach, Elaine

Tayette, Ohio

Kauffman, Faun

Webster City, Iowa

Kemp, Beverly

Des Moines, Iowa

Kern, Roy

Waterloo, Iowa

King, Hal

Ke Grafe, Ohio

King, Lois

New London, Ohio

Kinney, Susan

Des Moines, Iowa

Kinter, Marquetta

Vestal, New York

Klimek, Evelyn

North Jackson, Ohio

Klomparens, Cheryl

Grand Rapids, Michigan

Knight, Arlene

Austin, Minnesota

Knowles, Thomas

Milford, Michigan

Kohli, Denver A.

Wauseon, Ohio

Larrick, Dana

Wilmington, Ohio

LeFever, Linda

Rochester, Michigan

Lister, Barbara

St. Charles, Missouri

Love, Diane

Enon, Ohio

Lowery, Landra

Warsaw, Indiana

Lutton, Roberta

Wauseon, Ohio

Mack, Connie

Anderson, California

Martin, Jancie

Scott Depot, West Virginia

McCoy, Barry

Dayton, Ohio

McNeill, Bonita

Charleston, West Virginia

McNiece, Buddy

Valparaiso, Indiana

Meyer, Christine

Batavia, Ohio

Millard, Brenda

Streetsboro, Ohio

Miller, David E.

Springfield, Ohio

Miller, Marilyn

Oil City, Pennsylvania

Miller, Sally

Chesapeake, Ohio

Miller, Yontz

St. Charles, Iowa
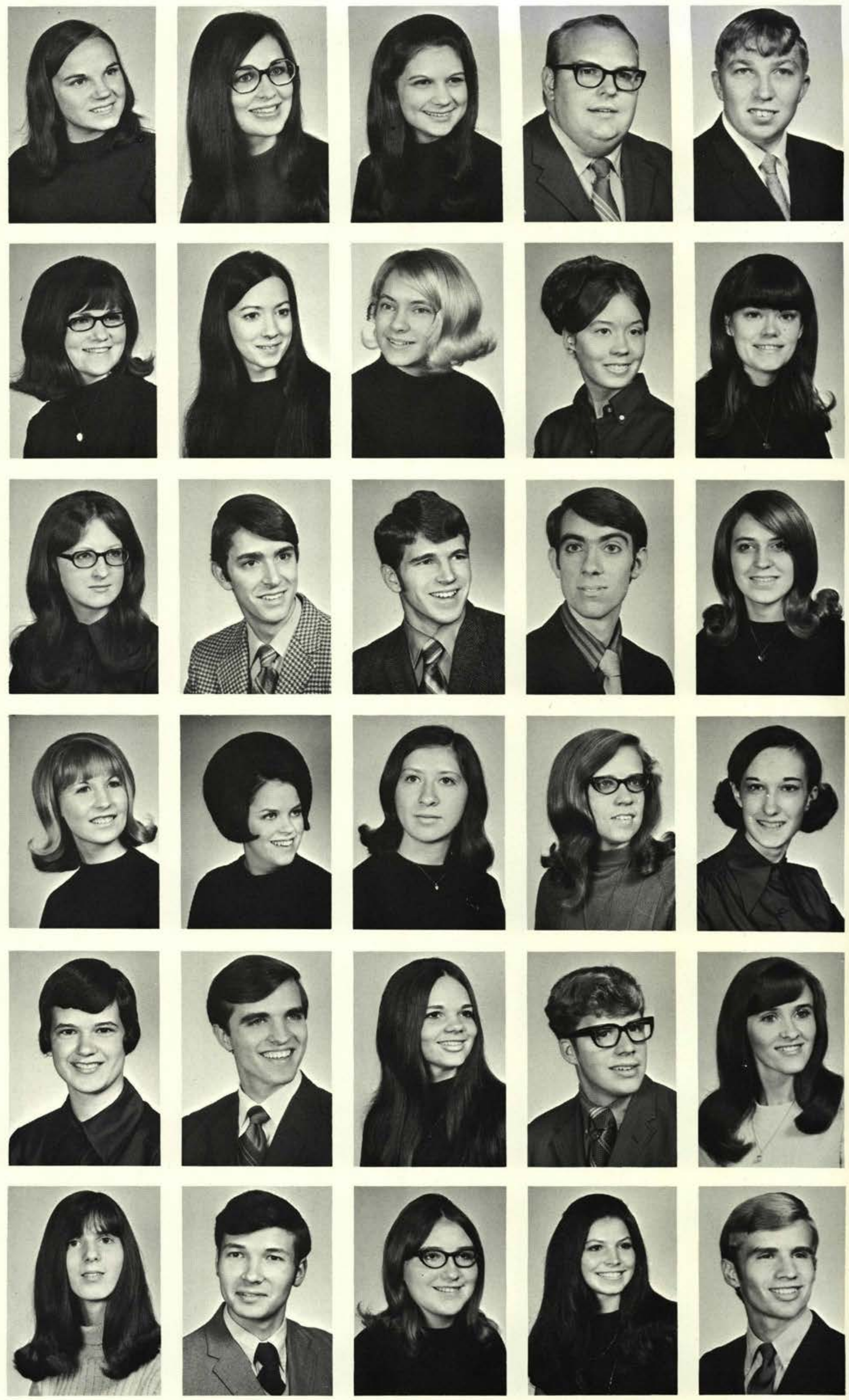
Nelson, Jeanne

St. Louis, Missouri

Odegard, Susan

Flint, Michigan

Olsen, Bruce

Lima, Peru

Peterson, Gene

Cedarville, Ohio

Philips, Sheldon

Portland, Oregon

Post, Rodney

Newark, New York

Prater, Paula

Washington, D.C.

Pruden, Grace

Prudenville, Michigan

Pumpelly, Connie

Newark, Ohio

Ray, Sally

Mancelona, Michigan

Read, Steve

Monticello, Indiana

Reese, Sharon

Lebanon, Ohio

Reynolds, Vicki

New Philadelphia, Ohio

Richardson, Gale

Euclid, Ohio

Riggs, Lynette

Dayton, Ohio

Roberts, Dennis

Bunker Hill, Illinois

Roden, Michael

Dorset, Ohio

Rodgers, Carl E.

Cuyahoga Falls, Ohio

Rodriguez, David

Greenlawn, New York

Rothberg, James A.

Johnson City, New York

Ruby, Candy

Dayton, Ohio

Russell, Priscilla

East Brunswick, New Jersey

St. Denis, Wayne

Anderson, Indiana

Sanderlin, Tim

Portsmouth, Ohio

Sarver, Eldon

Rose Hill, Iowa

Schapmeyer, Lloyd

Cedarville, Ohio

Scharmann, Marion

Schroon Lake, New York

Schlechty, Gretchen

Lakewood, Ohio

Schmidt, Kathy

Detroit, Michigan

Schultz, Esther

Milwaukee, Wisconsin
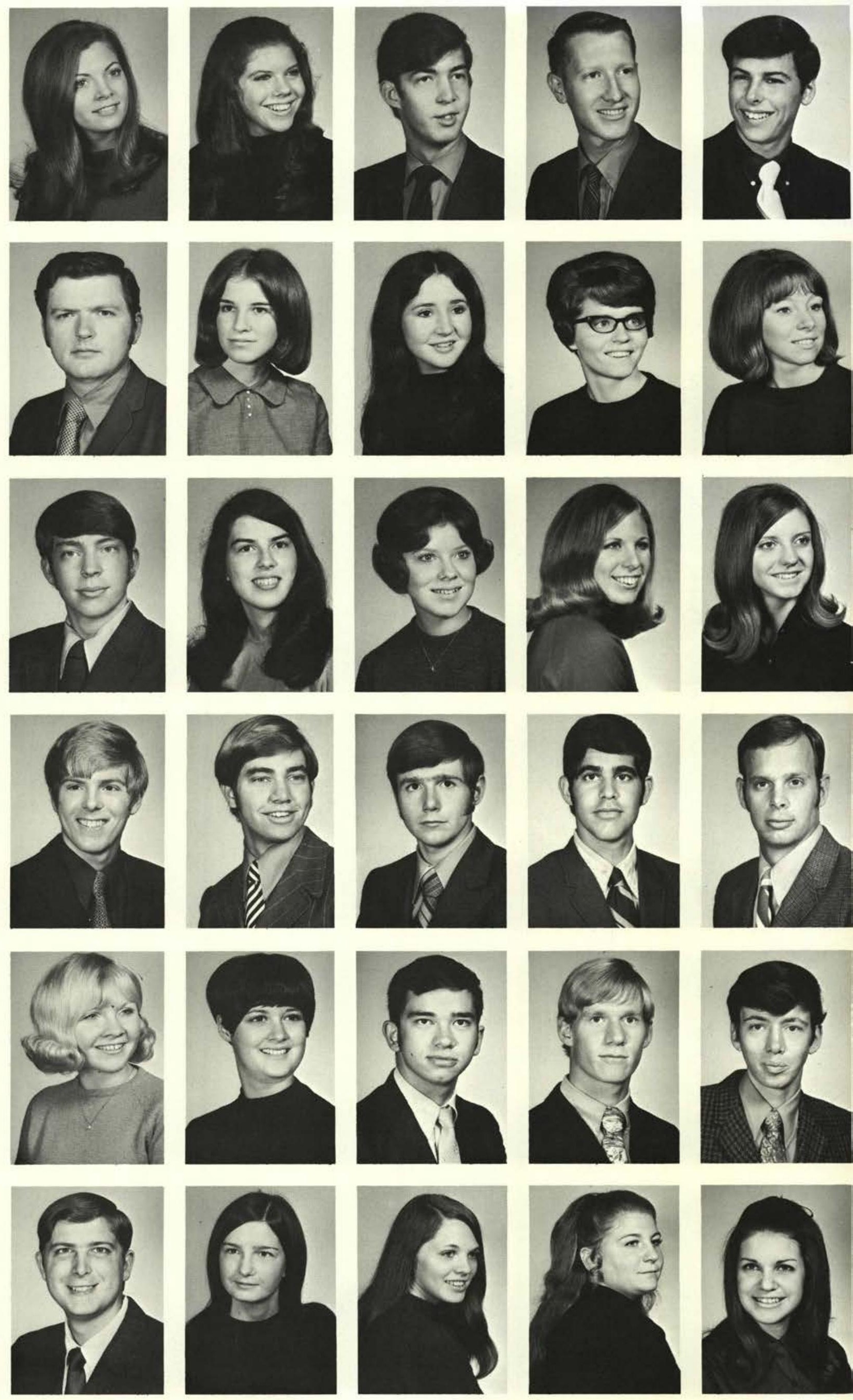
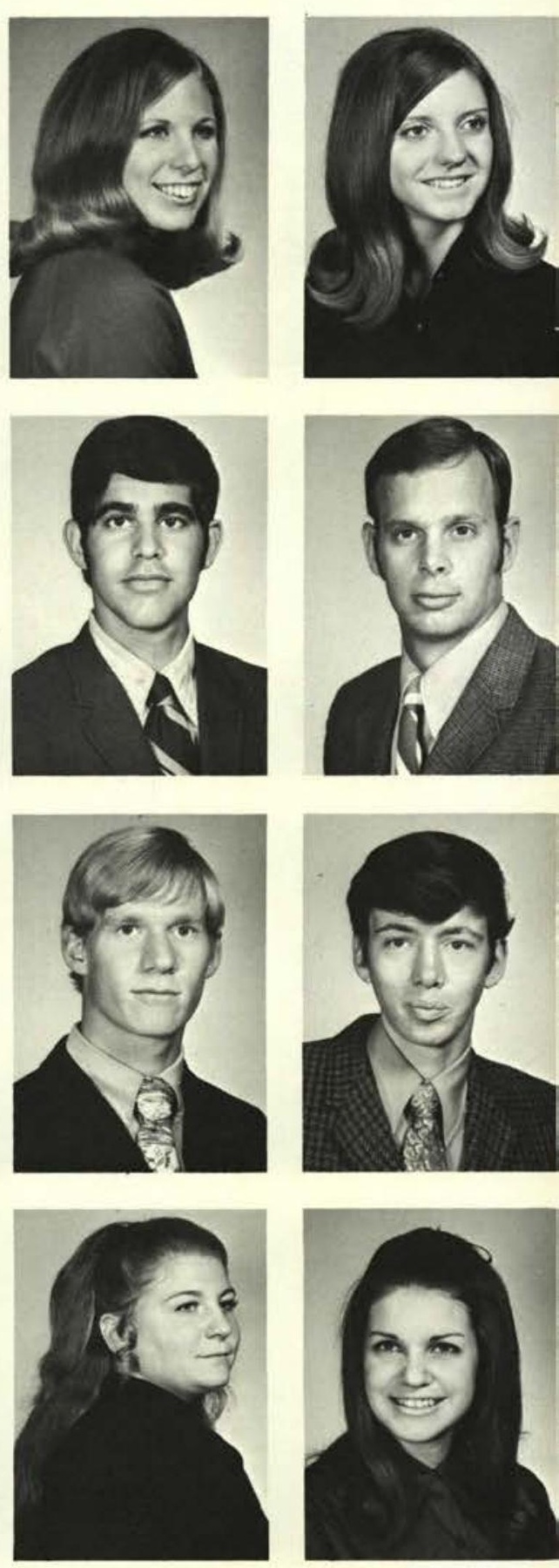

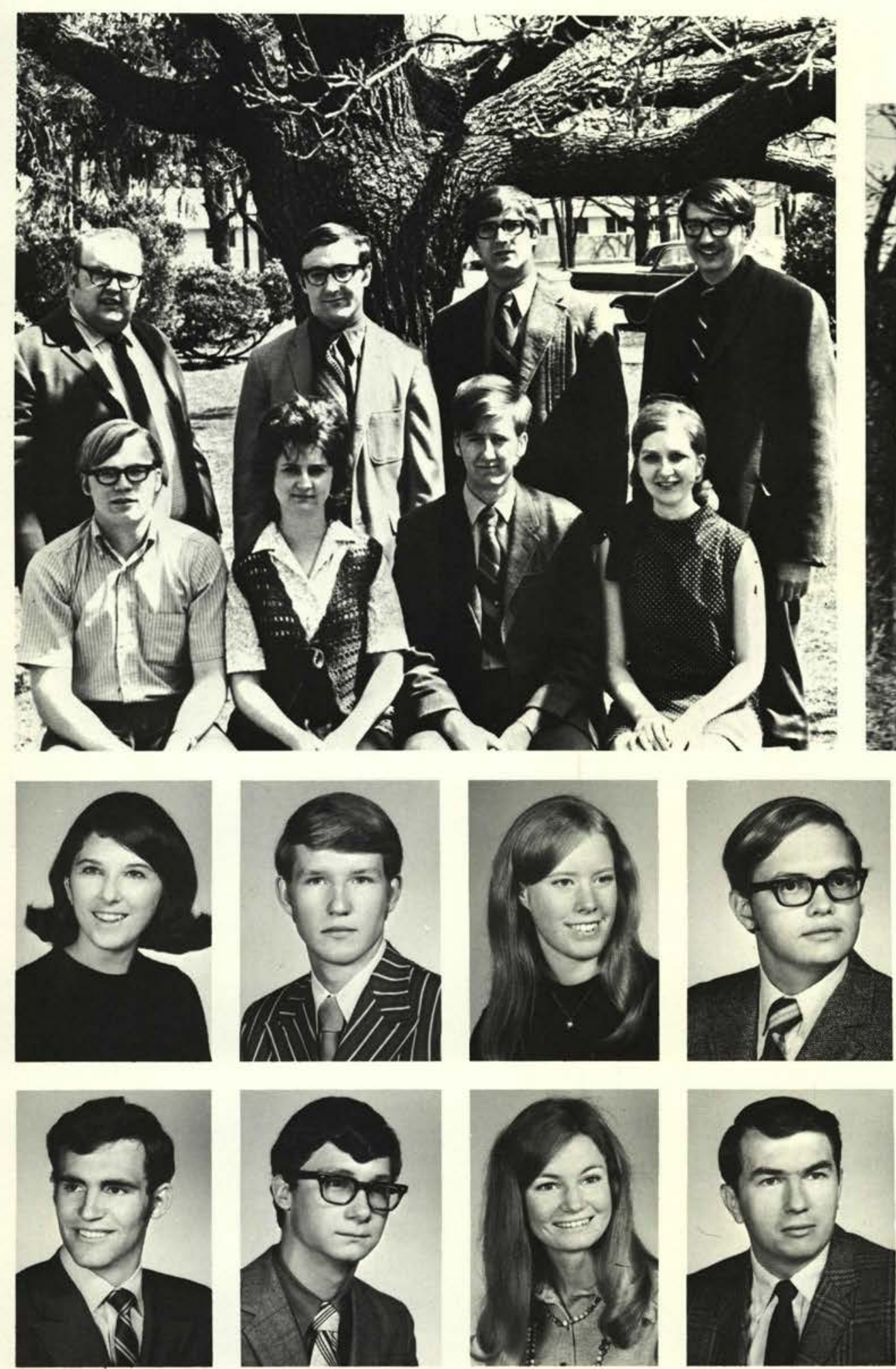
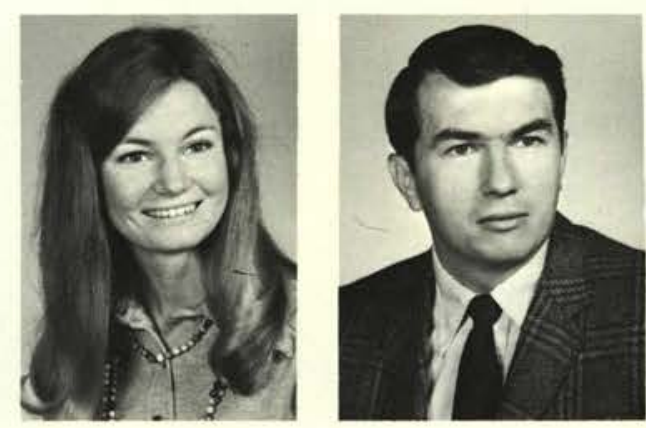

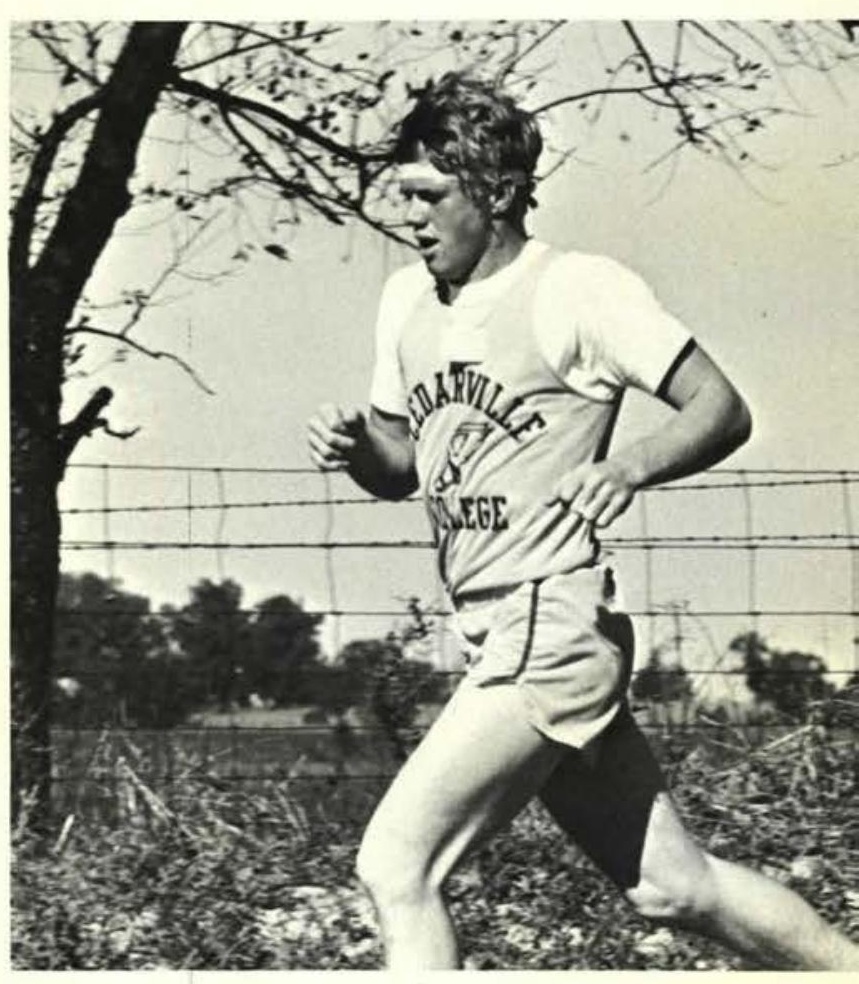

Sell, Nancy Ruth

New Canaan, Connecticut

Shank, Steve

New Madison, Ohio

Shorey, Linda

Westport, Massachusetts

Sloan, Mark

Lima, Ohio

Smith, Becky

Dayton, Ohio

Smith, Bruce

Boise, Idaho

Smith, Dale

Celina, Ohio

Soeder, Adria

Euclid, Ohio

Sowards, Alfred

Portsmouth, Ohio

Spears, Bonnie

Strongsville, Ohio 
Spink, Stephen W. Belfast, New York

Spradlin, Janice Wheaton, Maryland

Stephenson, Mark Grundy Center, Iowa

Stiles, Betty

Flint, Michigan

Stillwell, Craig

Hackensack, New Jersey

Stine, Philip

Brighton, Michigan

Stroupe, Pat

Freedom, Pennsylvania

Strychalski, Paul

Lebanon, Indiana

Sumner, Ron

Indianapolis, Indiana

Suter, Linda

Arlington, Ohio

Swisher, Donita

Griffeth, Indiana

Syphers, Karen

Avon, Ohio

Taylor, Elizabeth

Saddle Brook, New Jersey

Telford, Helen

Goshen, Ohio

Tennant, Jan

Belle Fontaine, Ohio

Thompson, Daniel

South Bend, Indiana

Tingle, Patricia

Rockton, Pennsylvania

Tipton, Jacob

Northfield, Ohio

Tobias, Bill

Port Alleghany, Pennsylvania

Tompkins, Brenda

Sherman, New York

Undermann, Lorraine

Wallace, Patricia A.

Dayton, Ohio

Walls, Linda

Lima, Ohio

Walter, Linda

Westmont, New Jersey

Warfield; Bette

Decatur, Illinois

Warren, Jon

Onaway, Michigan
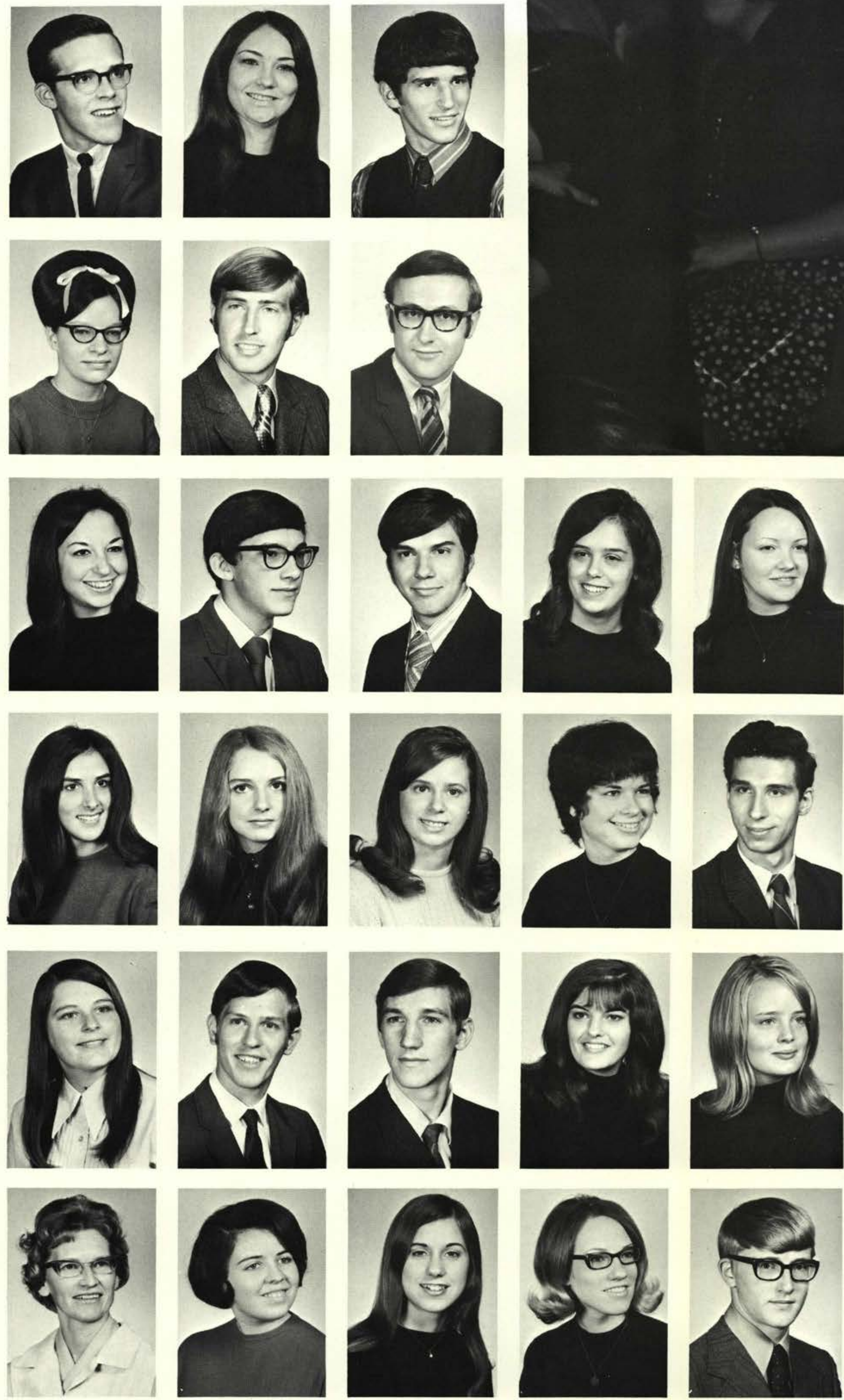

Abbey, Tom C.

Sinclairville, New York

Adamswich, Daniel Buffalo, New York

Albertson, Clayton

Niles, Ohio

Anderson, Becky

Monroe, Iowa

Arthur, Dinah

Norwalk, Ohio

Assey, Paul

Brentwood, New York

Assey, Stephen

Brentwood, New York

A wabdek, Philip

Syria

Axiotis, Georgeanna

Silvis, Illinois

Barb, Bethany

Akron, Ohio

Barker, Tim

Rochester, New York

Barnett, Larry

Royal Oak, Michigan

Barraclough, Jacki

North Tonawanda, New York

Bartemus, Sharon

Pasadena, Maryland

Beacham, James

Elkhart, Indiana

Bechtel, Ronald

Des Moines, Iowa

Bennett, Kelsey

Chagrin Falls, Ohio

Benson, Ernest

Liberia

Bergen, Dan

Waterloo, Iowa

Bernhard, Pat

Toledo, Ohio

Berry, Kathy

Belle Fontaine, Ohio
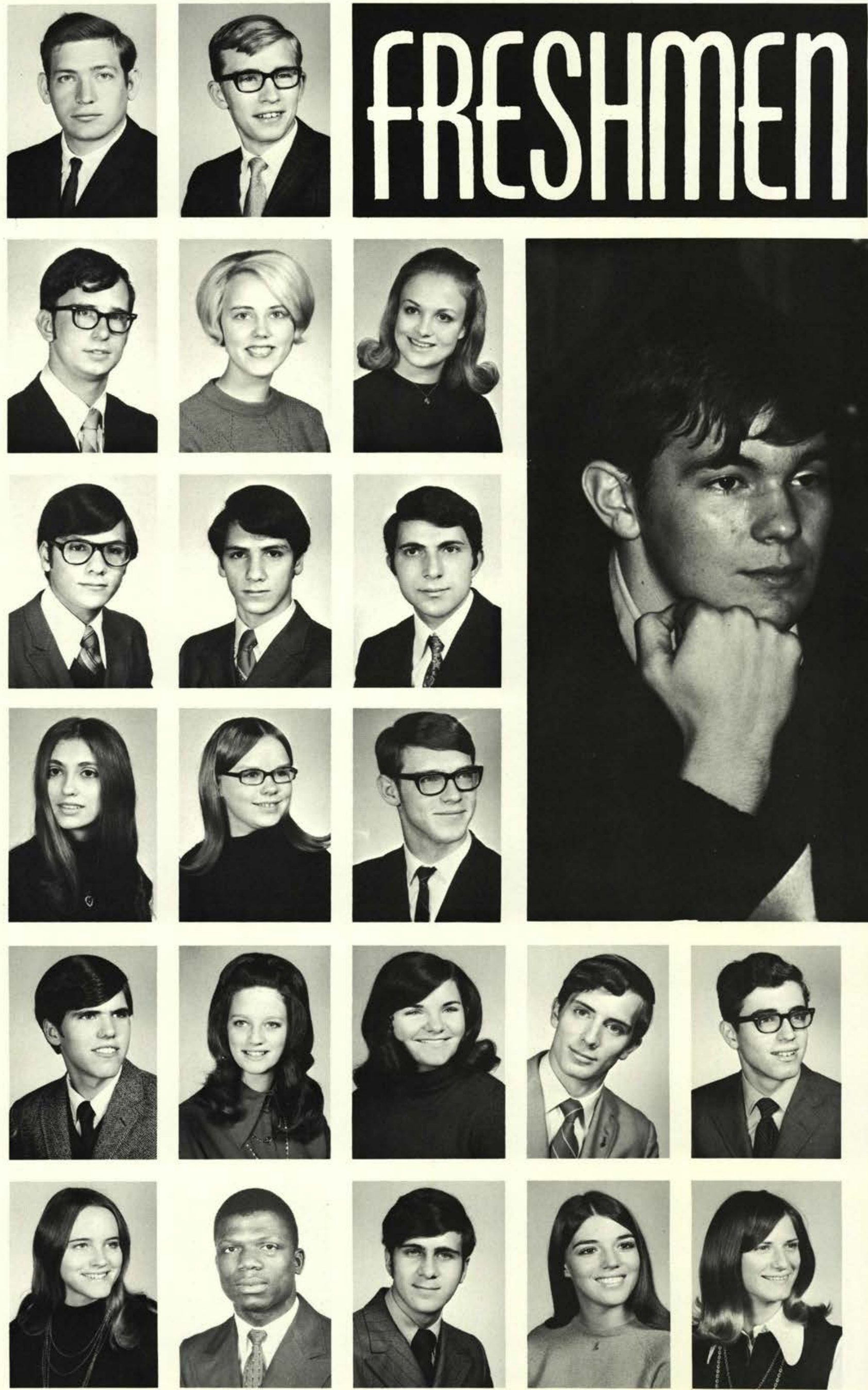

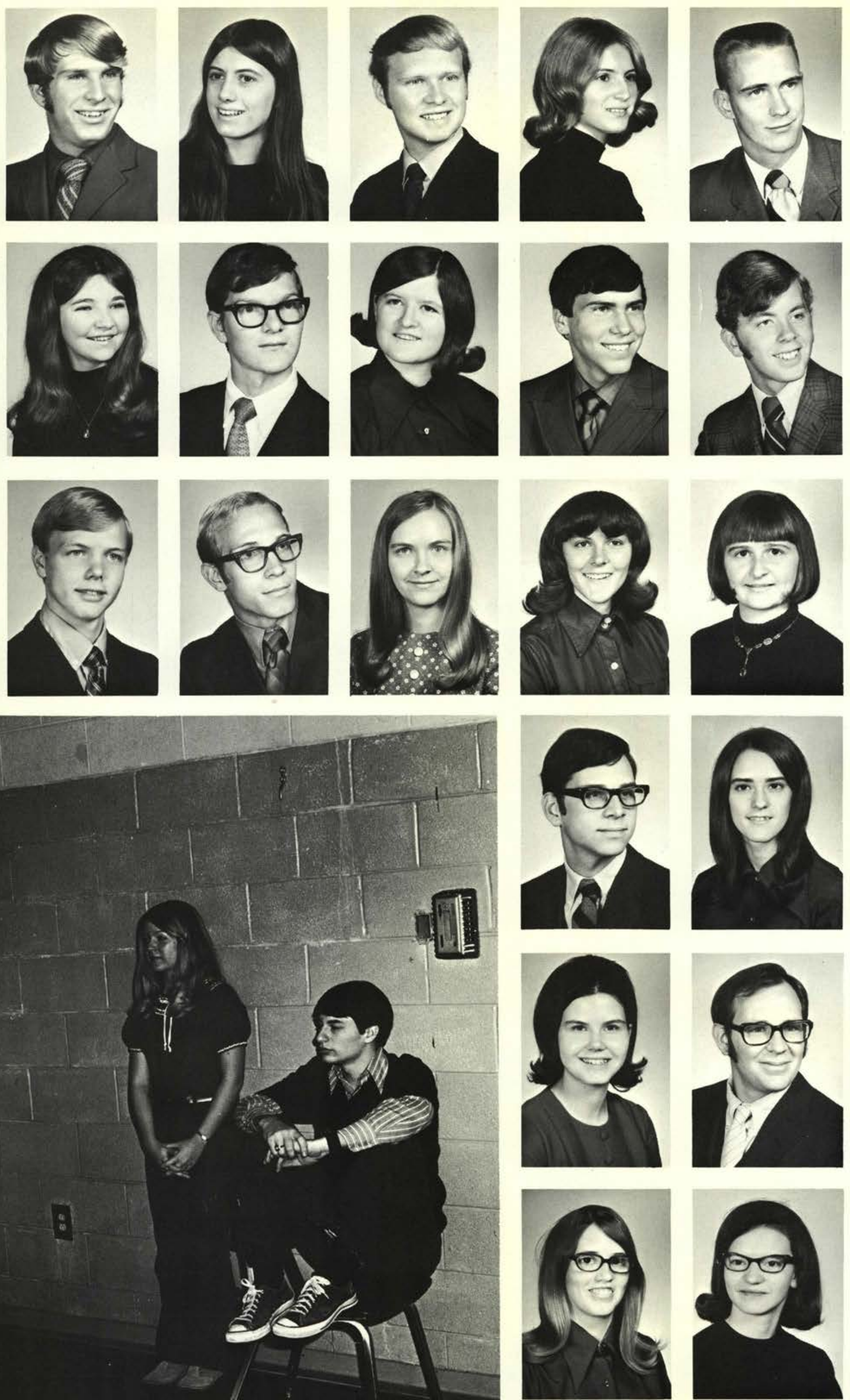
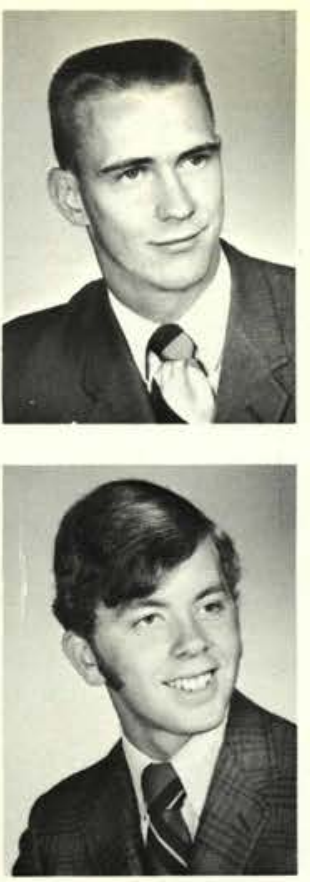

Betts, Glen

Bunker Hill, Illinois

Biggs, Barbara

North Whitefield, Maine

Blackburn, Mark

Wheelersburg, Ohio

Bodenmiller, Wendy

Berea, Ohio

Bohland, Mark

Lexington, Ohio

Bohn, Shirley

Springfield, Ohio

Borders, Larry

Kansas City, Kansas

Bowen, Lenora

New Carrollton, Maryland

Bratcher, John

La Porte, Indiana

Briggs, Fred

Caldwell, Ohio

Brock, Gary

Columbus, Ohio

Busho, Craig

Austin, Minnesota

Byers, Deborah

Boonville, Indiana

Byers, Joleen

Pella, Iowa

Caldwell, Colleen

Adamsville, Pennsylvania

Campbell, Scott

Fairview Park, Ohio

Canine, Becky

Hazel Park, Michigan

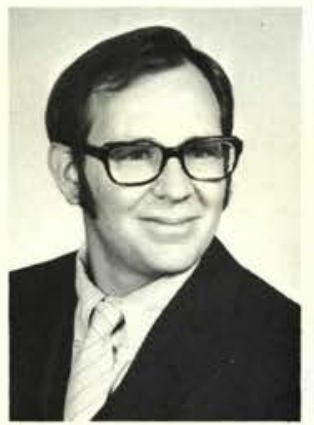

Capps, Diana

Indianapolis, Indiana

Carlton, Kenneth

Harvey, Illinois

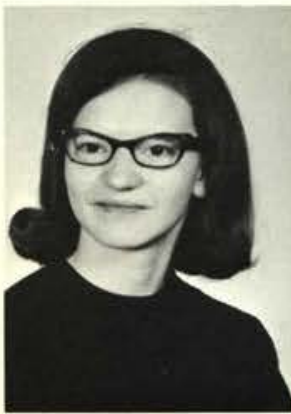

Carter, Bertha

Sheffield Lake, Ohio

Cleaves, Ruth

Leavittsburg, Ohio 
Cline, Jackie

Rockbridge, Ohio

Coldwell, Randall

Cole, Vicki

North Clymer, New York

Cotnoir, David

Quebec, Canada
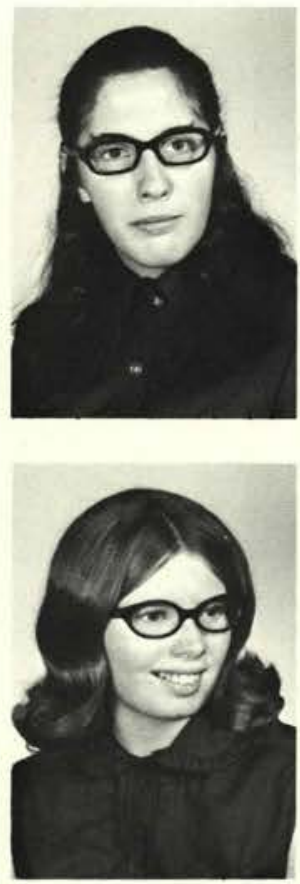

Crankshaw, Bob

Mt. Laurel, New Jersey

Crosby, Janis

Vestaburg, Michigan

Crotty, Melody

Detroit, Michigan

Crouch, Jiles

Sabina, Ohio

Cuenin, Eric

Amherst, Ohio

Culp, Claudia

Fort Wayne, Indiana

Dalton, Kathleen

Wakeman, Ohio

Davis, Donald

Littleton, Colorado

Davison, Bev

Arcanum, Ohio

Dear, Debbie

Saxonburg, Pennyslvania

DeBoer, Debbie

Jamestown, New York

DeBruine, Joyce

Battle Creek, Michigan

DeNardis, Diane

North Royalton, Ohio

Dials, Vivian

Wheelersburg, Ohio

Dillon, Dana

Mansfield, Ohio

Dolph, Chuck

Belle Fontaine, Ohio

Duran, Judy

Winfield, Illinois

Durham, Debbie

Manila, Philippines

Edwards, Denise

Albion, Iowa

Edwards, Mike

Ashland, Kentucky
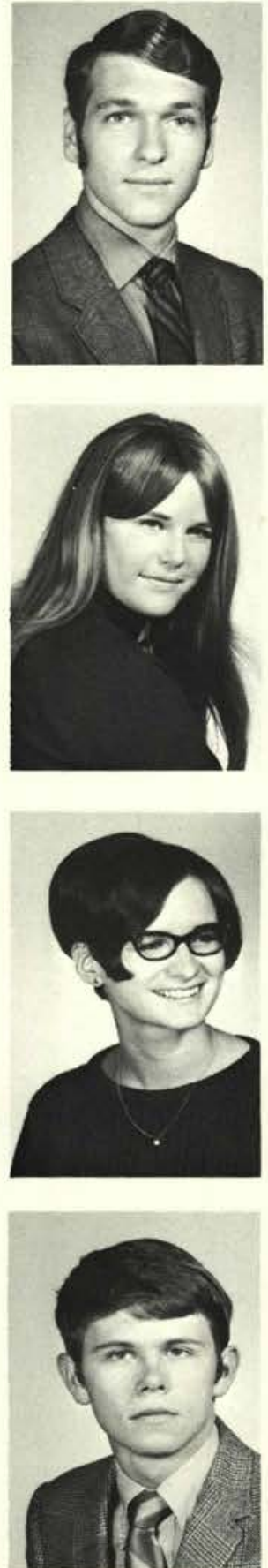
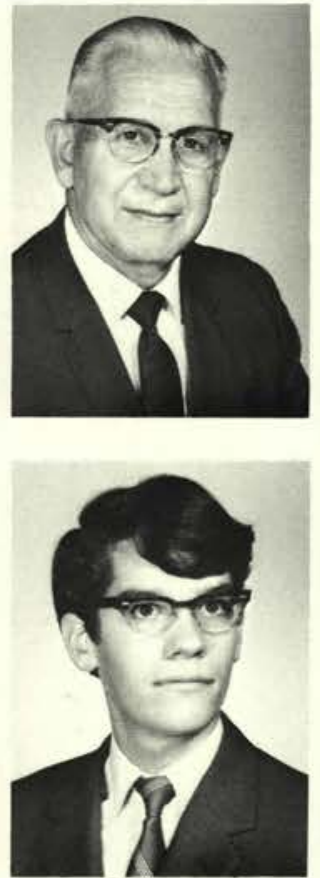
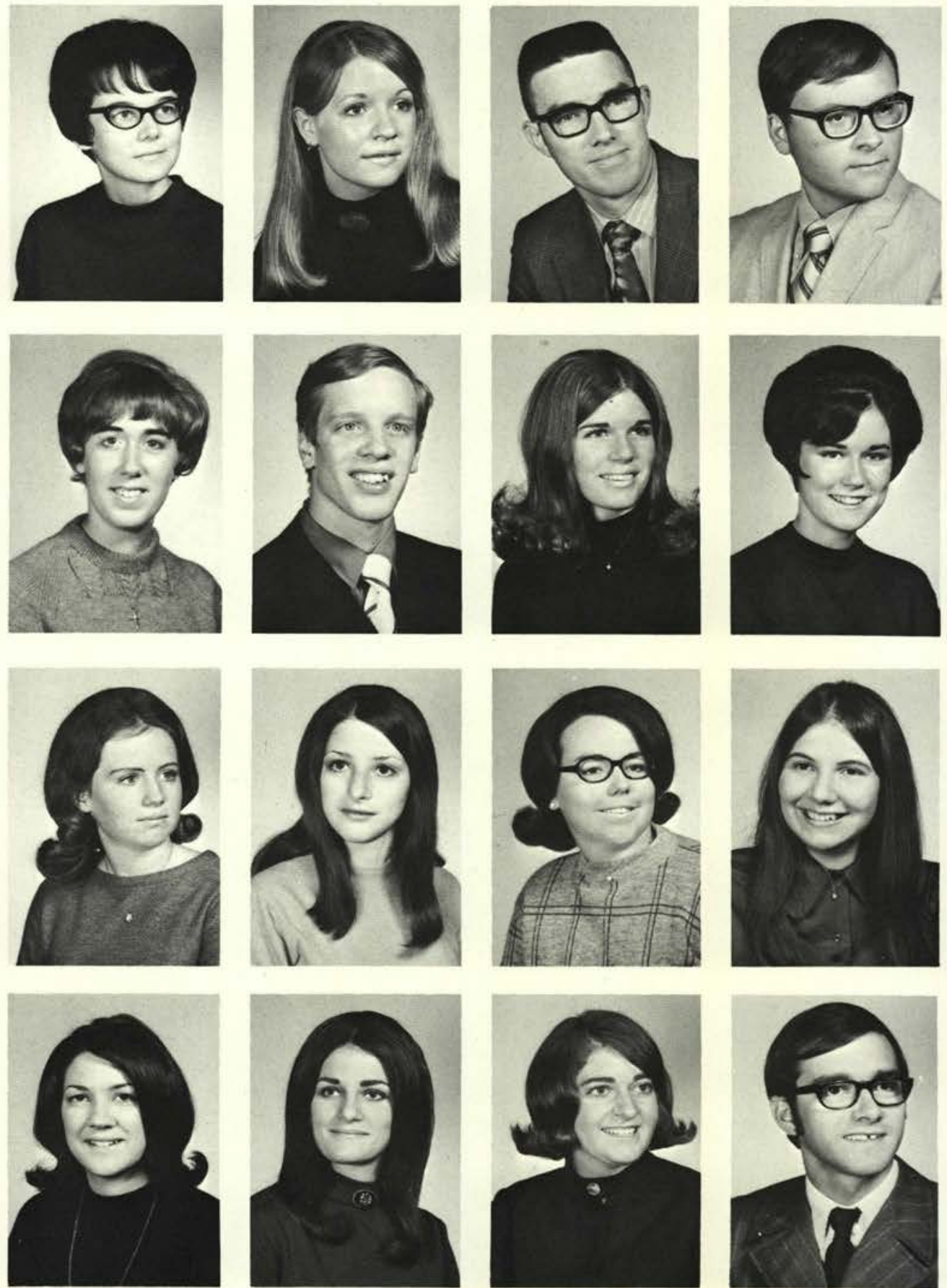

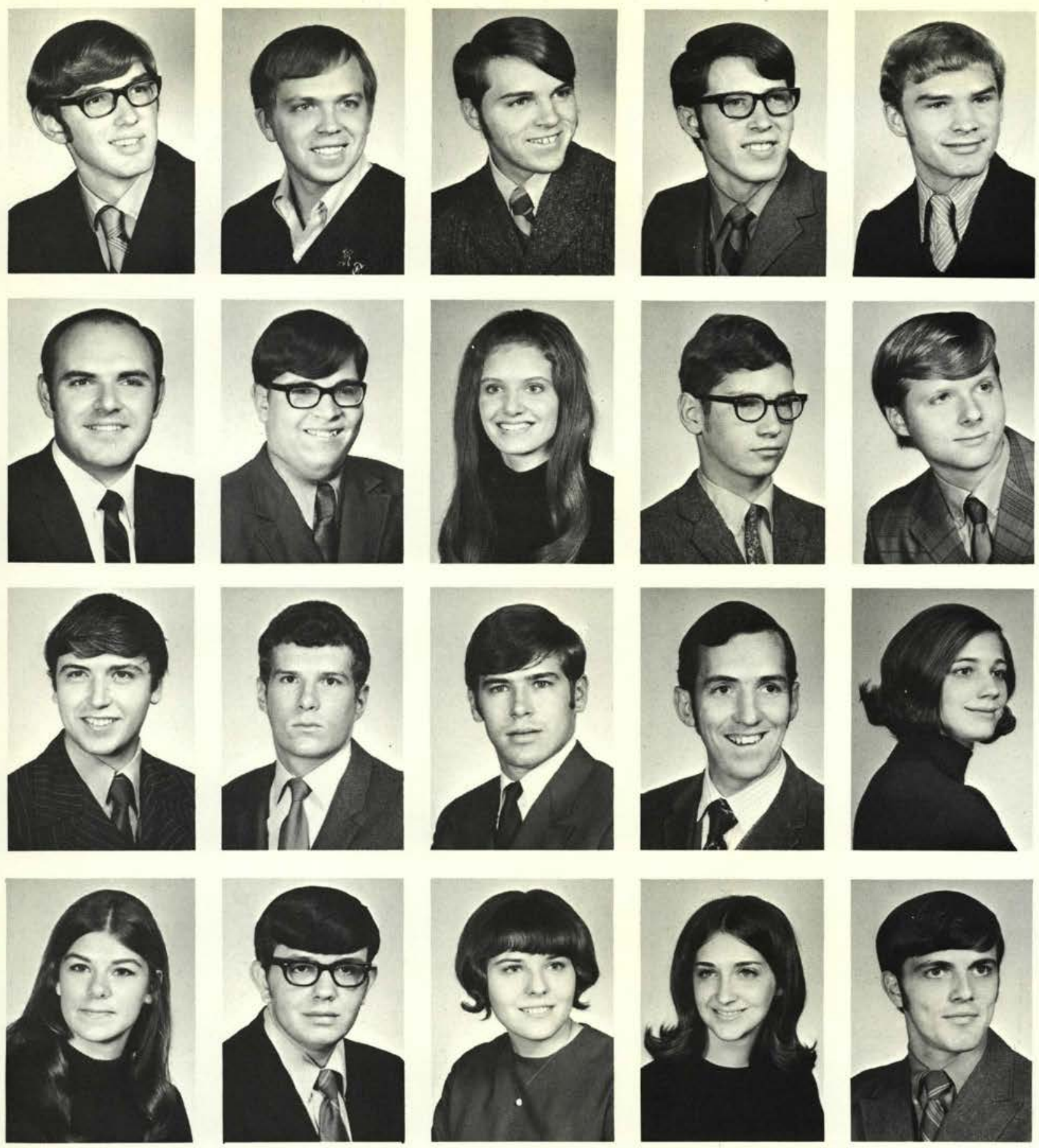

Ehnis, Dan

Brooklyn, Michigan

Elkin, Russell

Painesville, Ohio

Ernsburger, Kenneth

Bremen, Indiana

Estes, Dan

Endicott, New York

Ferguson, Dave

Wheelersburg, Ohio

Filson, Earl

Jamestown, Ohio

Fisher, Floyd

Rittman, Ohio

Flatt, Christine

Ankeny, Iowa

Fogle, Dale

South Bend, Indiana

Forbis, Denis

Waterloo, Iowa

Ford, John

Marion, Ohio

Francis, Steven

Kansas City, Kansas

Frye, Frederick

Holland, Ohio

Gates, Donald

Columbus, Ohio

Gazdik, Sharon

Quebec, Canada

Gifford, Rebecca

Medina, Ohio

Gifford, Ted

West Union, Ohio

Goodrich, Susan L.

Columbus City, Indiana

Gons, Kathy

Fostoria, Ohio

Gray, James

Columbus, Ohio
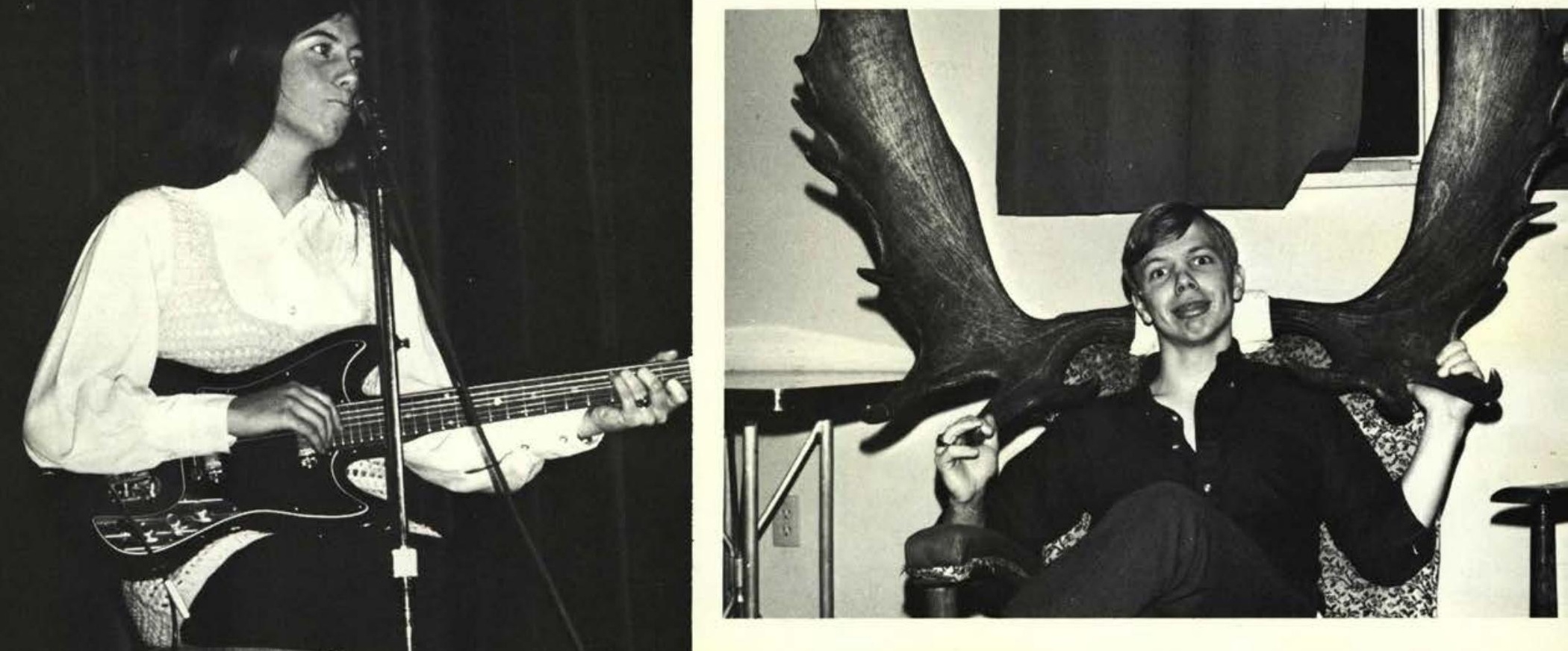
Greene, Judy L.

Charlotte, North Carolina

Griffeth, James

Cedarville, Ohio

Griswold, Jerold

Covington, Indiana

Gruber, Stephen

Greenville, Indiana

Hale, Suzanne

Wood River, Illinois

Hales, Richard

Elyria, Ohio

Hall, Lynda A.

Kearney, Nebraska

Hammach, Merla

Niles, Ohio

Hansen, Rodney

Mt. Pleasant, Michigan

Hargis, Jacqueline
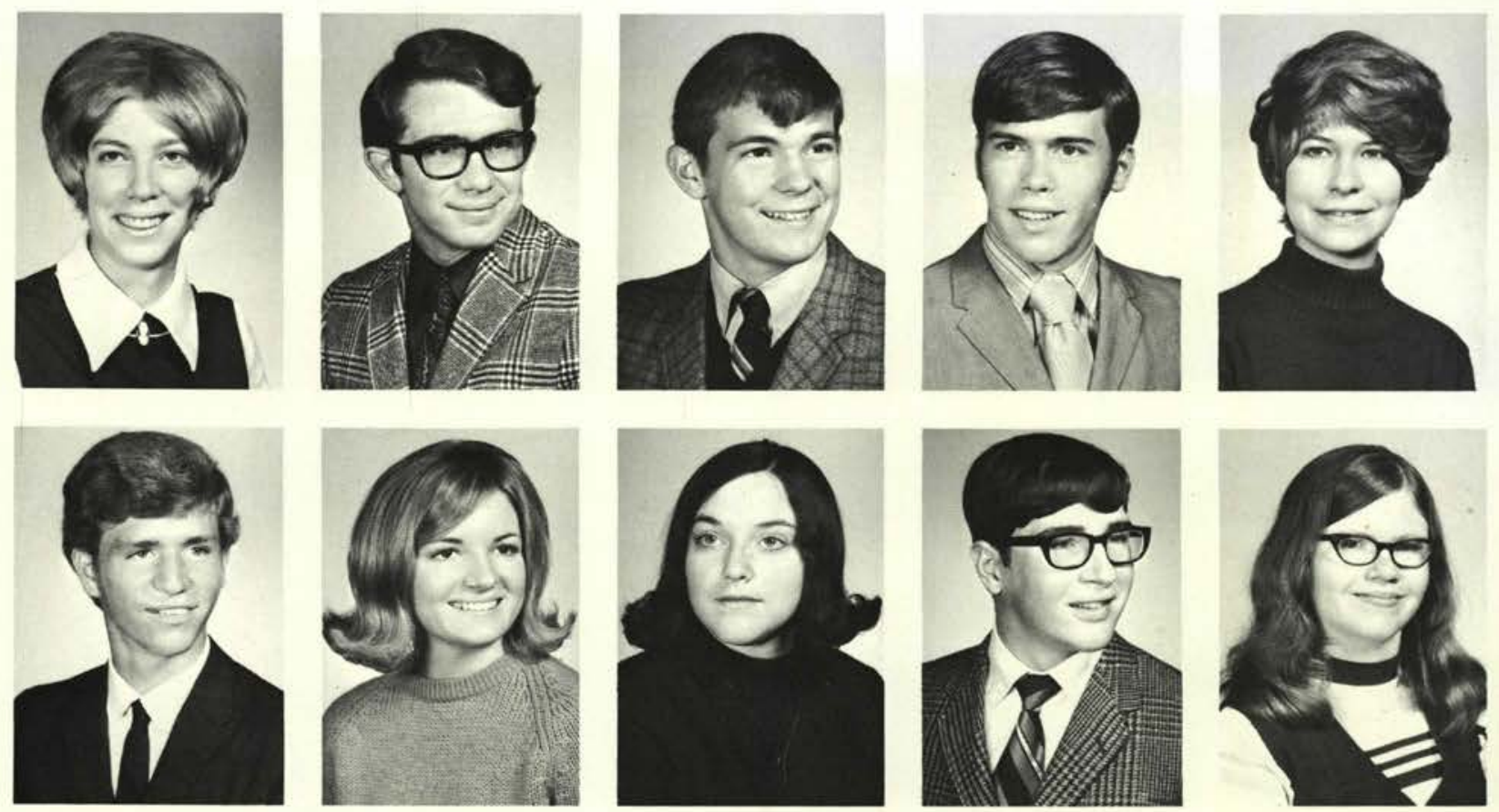

Hart, Ginny

Fort Wayne, Indiana

Haye, Larry

Stryker, Ohio

Henderson, Kathy

Kearney, Nebraska

Henson, Dan

Grand, Rapids, Michigan

Heyl, Beverly

Milford, Ohio

Hickok, Patricia

Tucson, Arizona

Hoerath, Jim

Westerville, Ohio

Holfield, Valerie Jayne Washington, D.C.
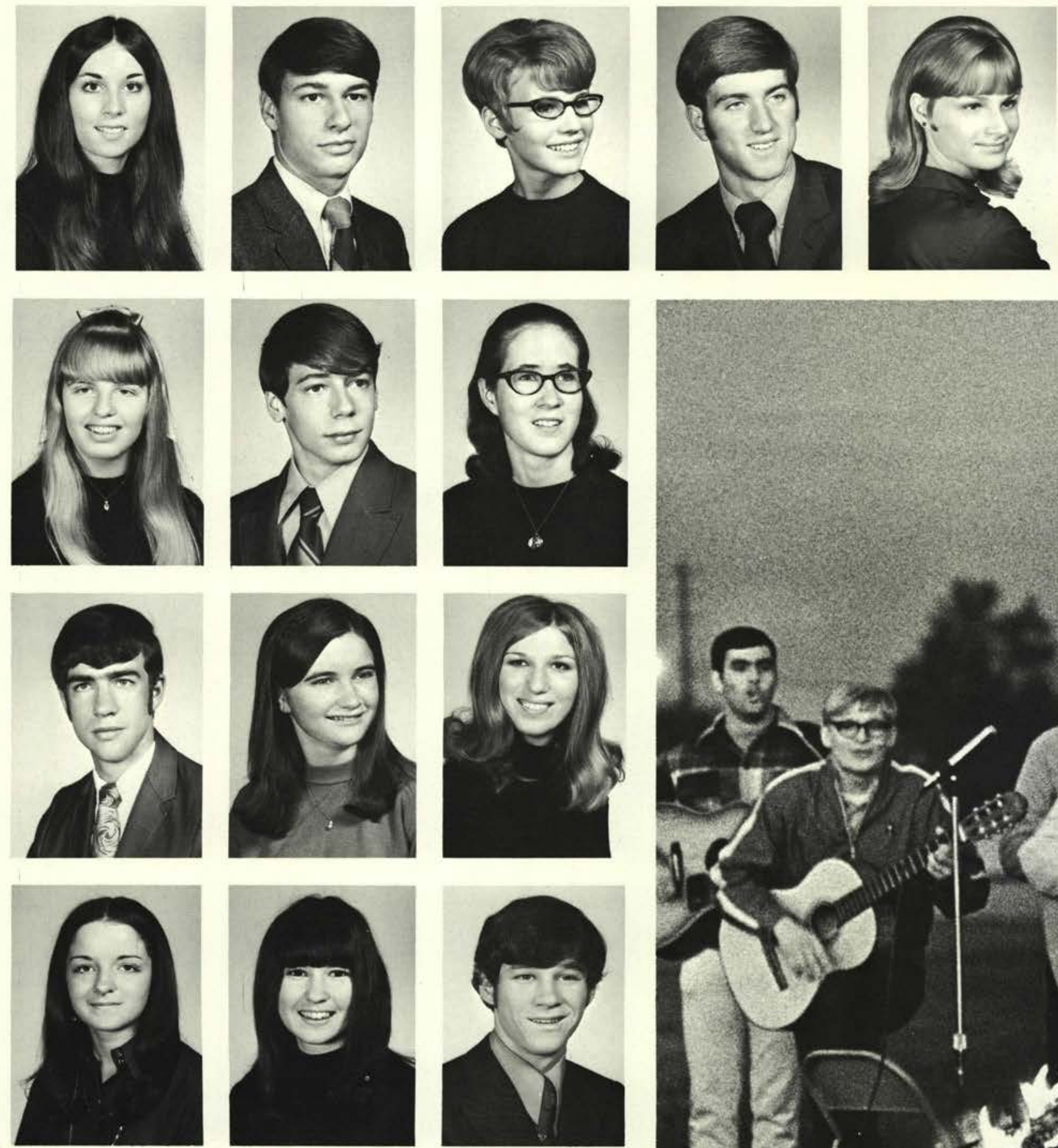

Hooper, Diane

Keene, New Hampshire

Hostetler, Debbie

Smithville, Ohio

Howell, Ed

Pensacola, Florida

Holley, Terry
Twinsburg, Ohio

Holt, Debbie

Holzworth, Holly

Springfield, Ohio 

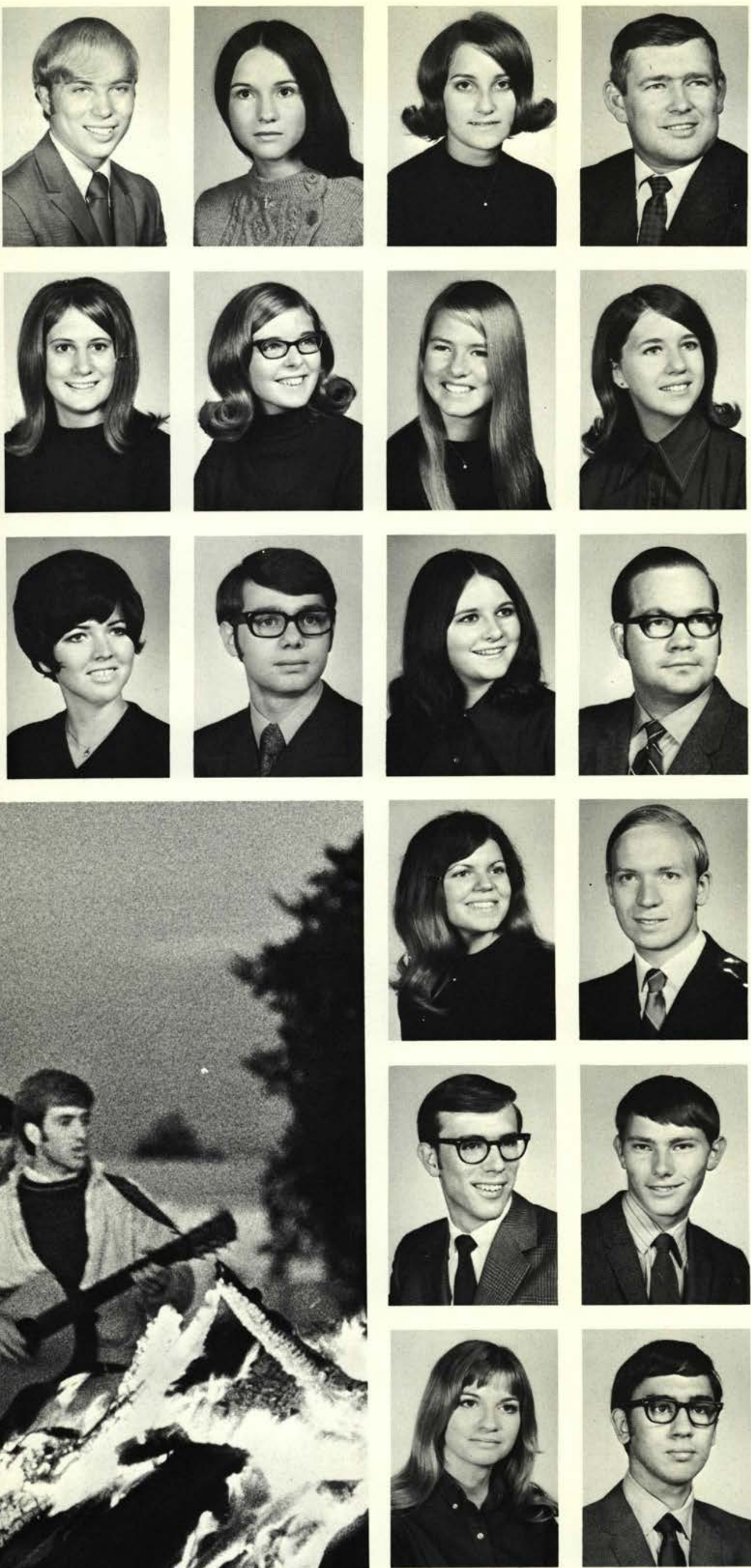
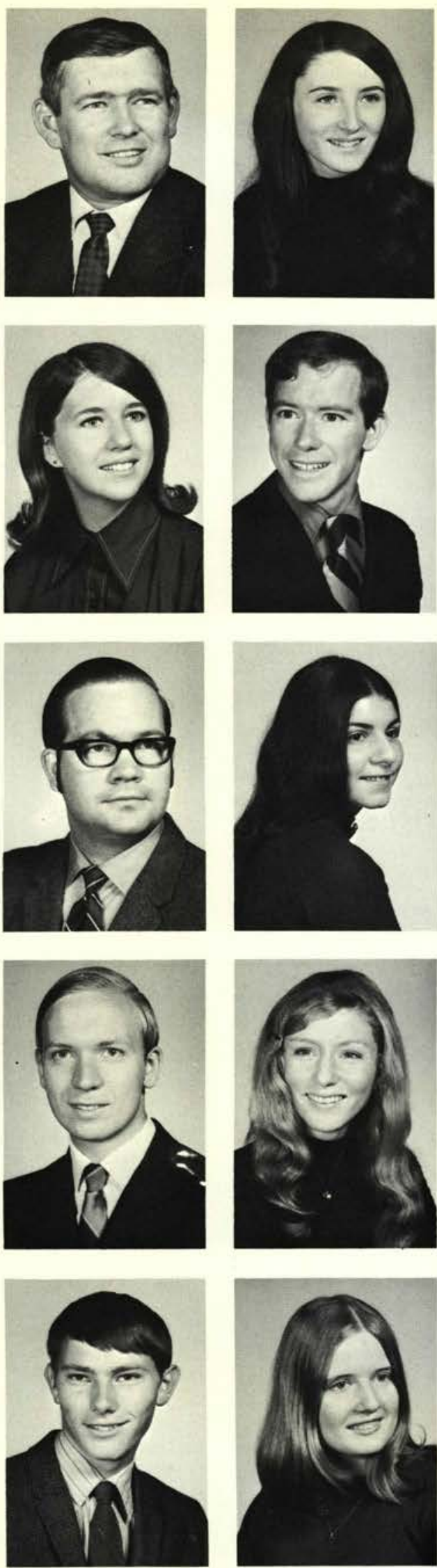

Hudson, Steve

Bemiji, Minnesota

Huff, Chery

Rochester, New York

Humphrey, Natalie

Byesville, Ohio

Humphreys, Delano

Christiansburg, Ohio

Jenkin, Holly

Cleveland, Ohio

John, Laura

Littleton, Colorado

Johnson, Carol

Creston, Iowa

Johnson, Doris

Akron, Ohio

Johnson, Vivian

South Charleston, West Virginia

Jones, David Niles

Jones, Fonda

Gary, Indiana

Jones, Keith

Jones, Pam

Montrosse, Pennsylvania

Jutton, Ron

Fulton, Illinois

Karsian, Kathy

Hackensack, New Jersey

Kemble, Becky

Elyria, Ohio

Kemery, Glen

Columbia City, Indiana

Kenncoy, Karen

Camillus, New York

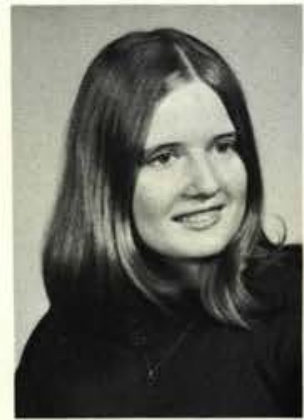

Kistler, Jeff

Haddon Heights, New Jersey

\section{Klett, Bruce}

Boise, Idaho

Knight, Debbie

Clifton, New Jersey
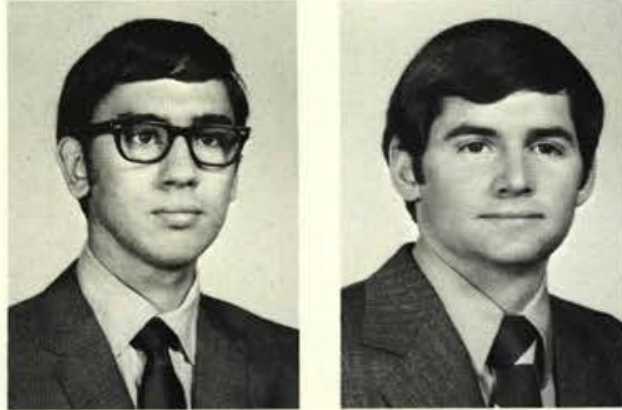

Koncsol, Sally

McDonald, Ohio

Konya, Alex

Maple Heights, Ohio

Kovacik, Thomas

Johnson City, New York 
Kriel, Rita

Cedarville, Ohio

Lantz, Steve

Indianapolis, Indiana

Layne, Nanci

Gallipolis, Ohio

Lee, George

Groton, New York

Lee, Timothy

Trenton, New Jersey

Leemaster, Charles

Xenia, Ohio

Lewis, Brenda

Kansas City, Kansas

Lewis, Janet

Maine, New York

Lewright, John

Eagle Grove, Iowa

Lillback, Pete

Painesville, Ohio

Lippert, Linda

Novi, Michigan

Lippert, Mary Lou

Franklin Grove, Illinois

Long, Janet

Dayton, Ohio

Lowstetter, Dennis

Greensburg, Pennsylvania

Luketic, Barrett M.

Hammond, Indiana

Lysoowan, Boonchuay

Grand Ledge, Michigan

Mahl, Robert

Elyria, Ohio

Malernee, Christine Byesville, Ohio

Marshall, Chris

Allegan, Michigan

Master, Keith

Wauwatosa, Wisconsin

Mattison, Carol

Elyria, Ohio

Mayer, Susan

Bruce, Wisconsin

McCallister, Steve

Columbus, Ohio

McConkey, Rita

Springfield, Ohio
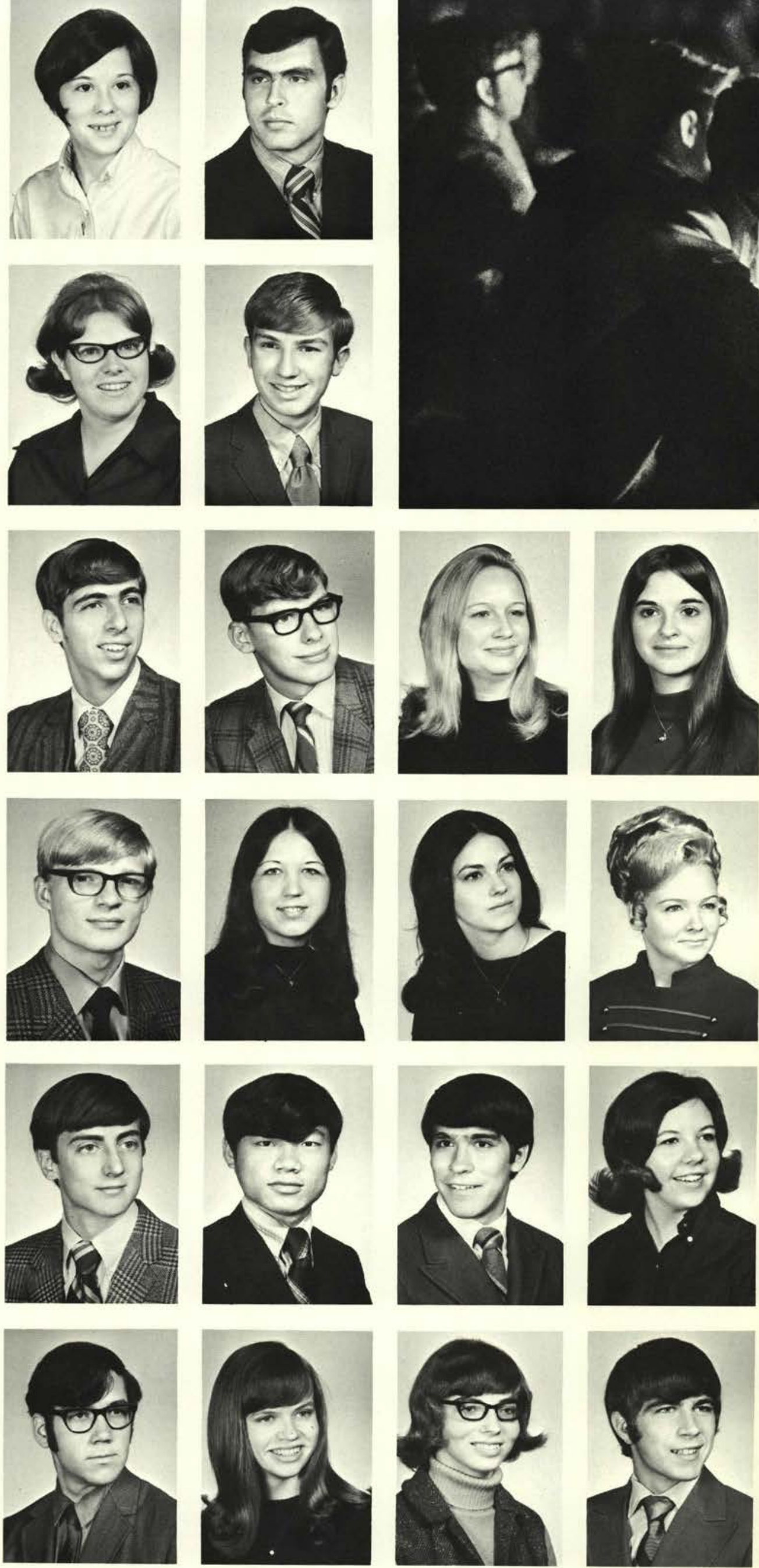
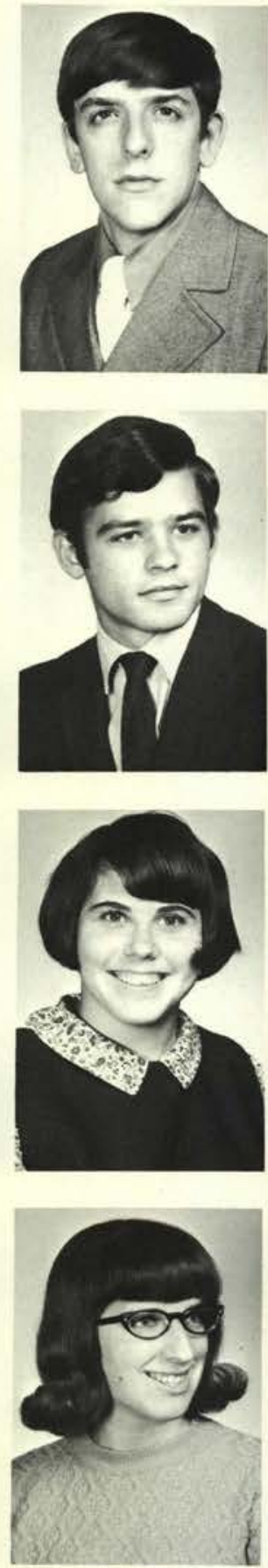

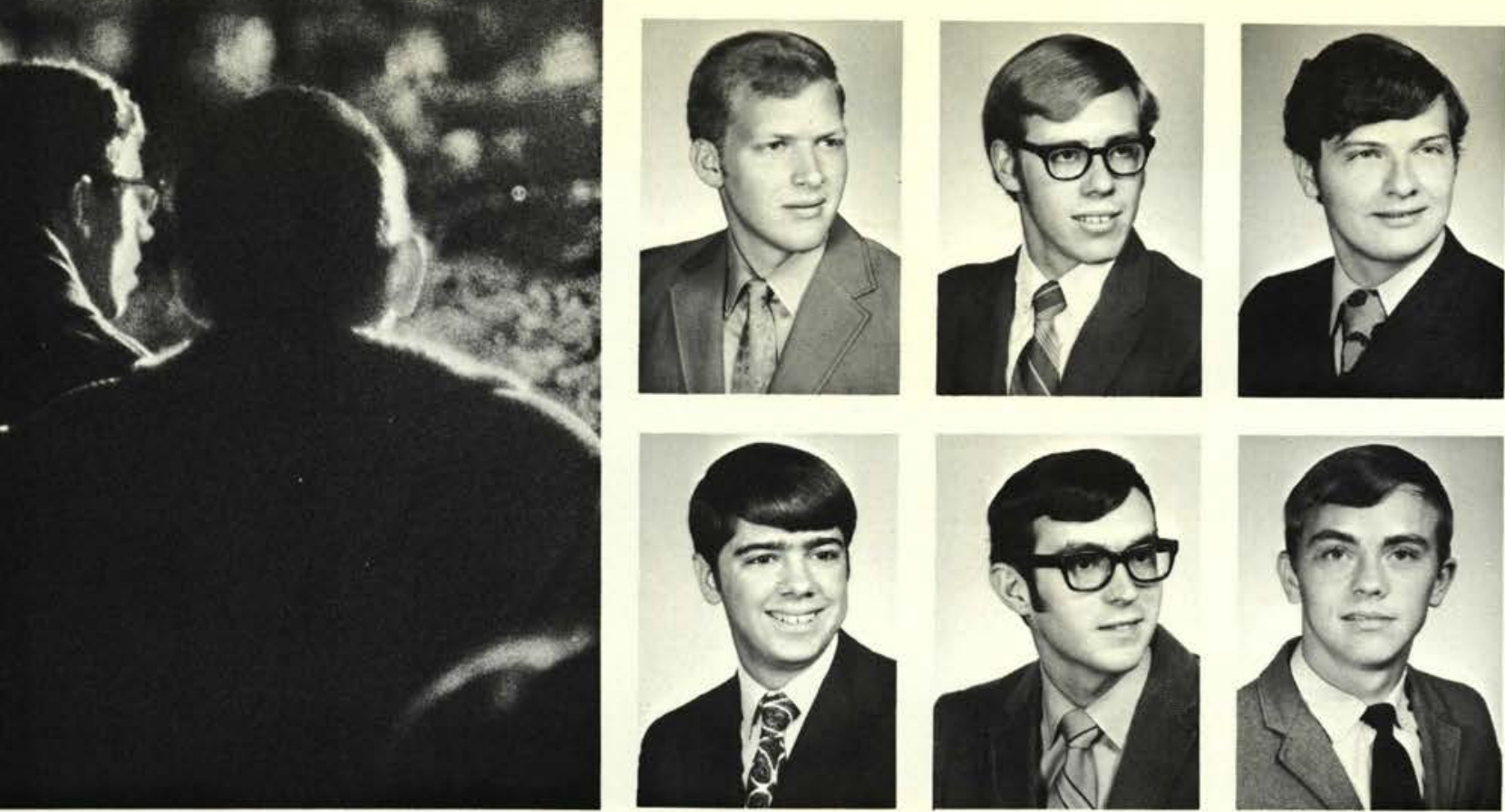

McDaniel, Richard Athens, Ohio

McDonald, Philip

Cedarville, Ohio
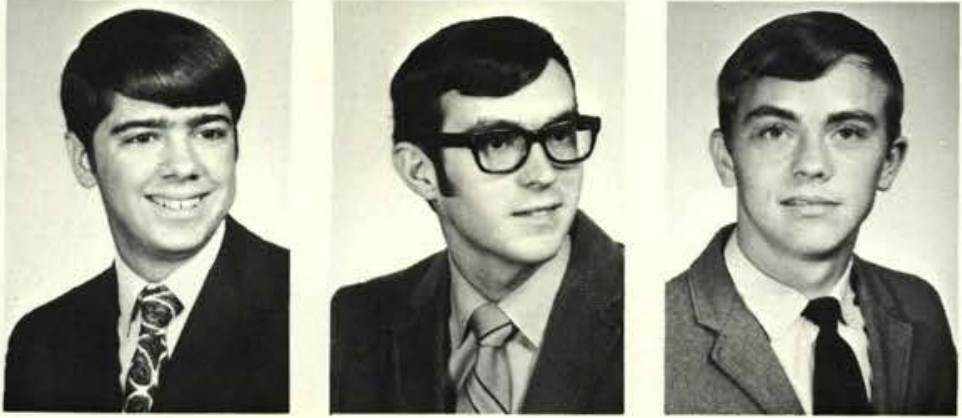

McGhee, David

Altoona, lowa
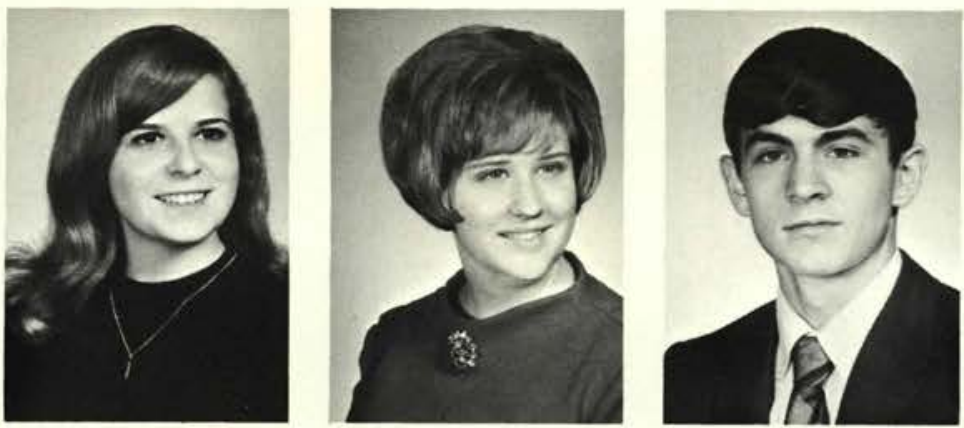

McIntosh, Richard Cedarville, Ohio

McKanna, Don Lima, Ohio

McMillen, Phil Washington, D.C.
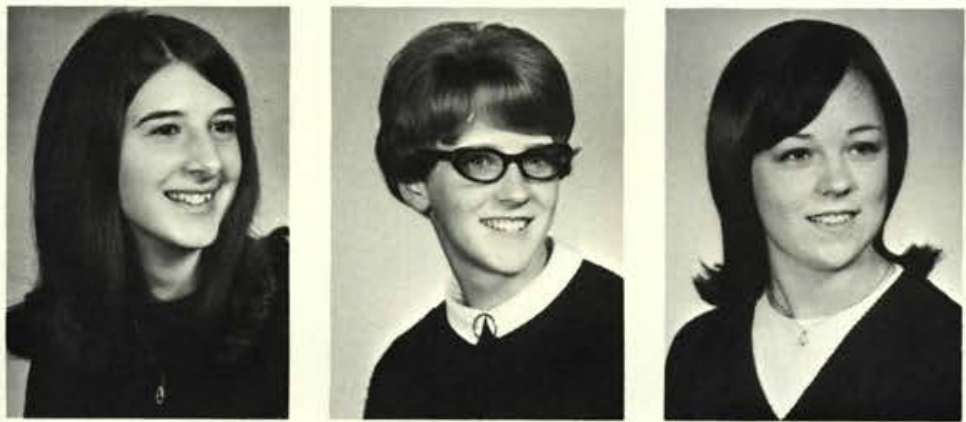

Merz, Meara

Columbus, Ohio

Meyers, Diane

East Moline, Illinois

Millikan, Steven

Bothell, Washington
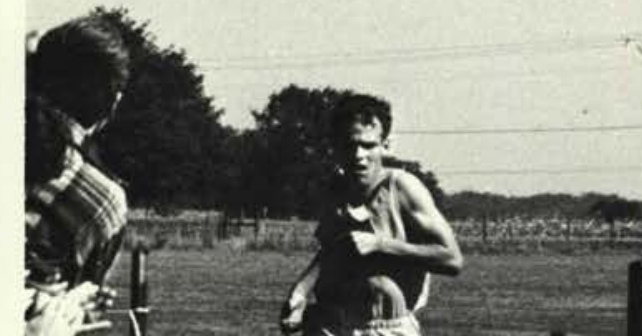
N
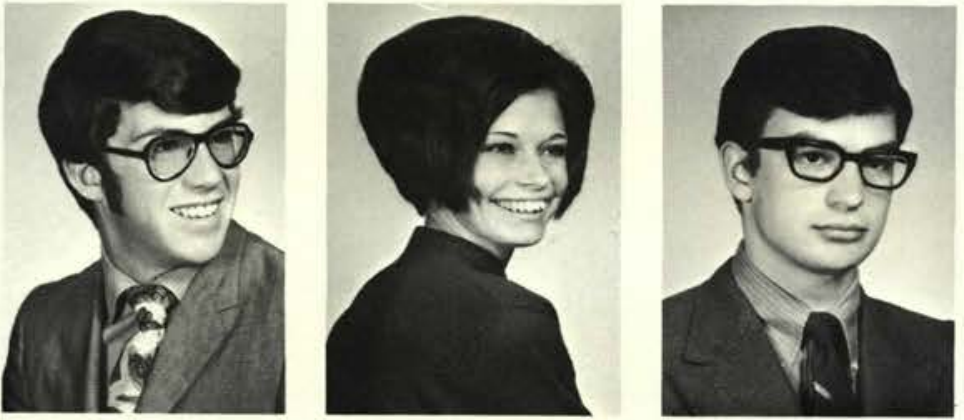

Myers, Brent

Lima, Ohio

Newberry, Linda

Silvis, Illinois

Nicholas, David

Iowa Falls, Iowa
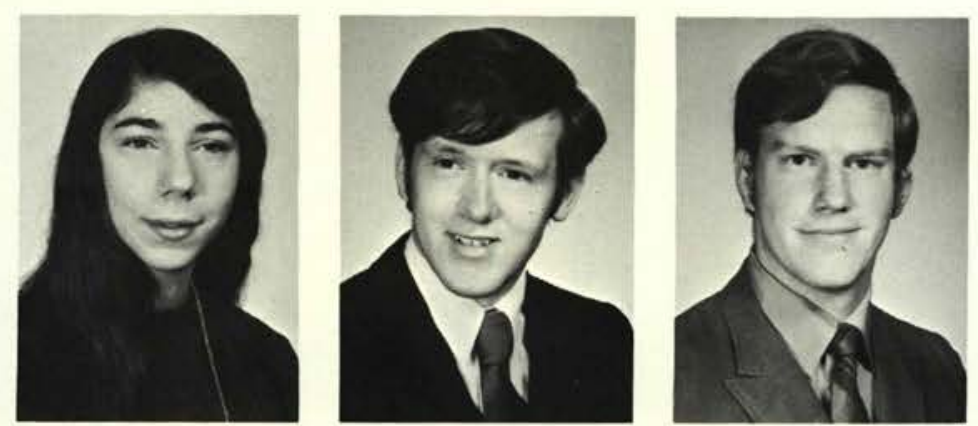

Novkov, Paula

Cuyahoga Falls, Ohio

Otto, Bart F.

Concord, Michigan

Overholt, Steve

Royal Oak, Michigan 
Owen, Ron

Findlay, Ohio

Paasch, Sharon

East Moline, Illinois

Palpant, Pete

Clayton, Michigan

Palser, Ellen

Wilson, Kansas

Parker, Clarence

Portsmouth, Ohio

Parker, Gloria R.

Berea, Ohio

Pauley, Linda

Pana, Illinois

Payne Paulette

Cedarville, Ohio

Pence, Susan

Dayton, Ohio

Penegor, Bernice

Greenland, Michigan

Penfold, Lynn

Eden, New York

Perry, Shirley

Brownsburg, Indiana

Peterson, Jan

Colona, Illinois

Portz, Sharon

Greenville, Pennsylvania

Potter, William

Allegheny, New York

Potts, Linda

Montiose, Pennsylvania
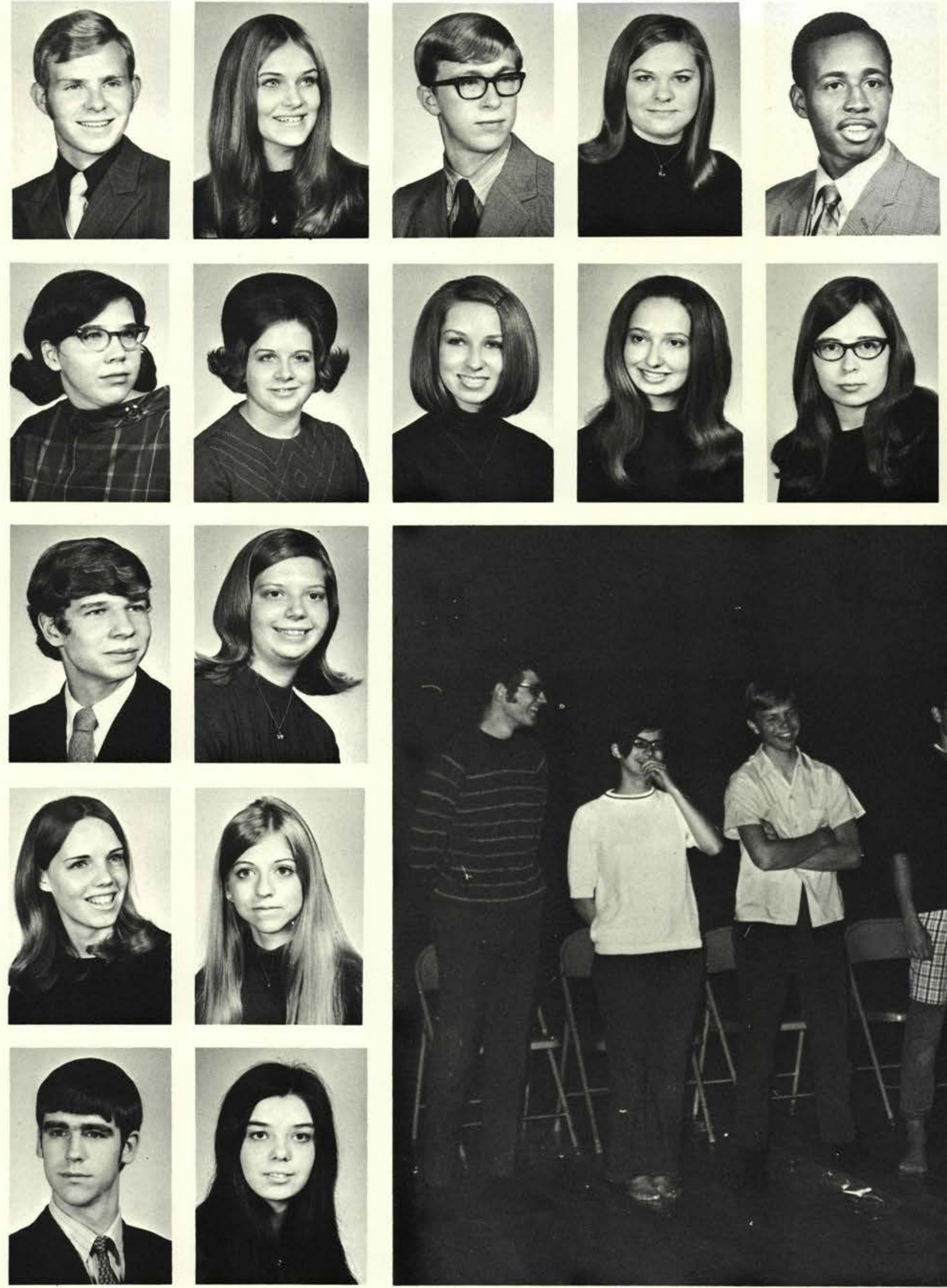

Price, Becky

Anna, Ohio

Price, David J. Jr.

Haddon Heights, New Jersey

Profant, Richard

Parma, Ohio

Prugh, Judy

Loraine, Ohio

Pycraft, Robert

Avon Lake, Ohio
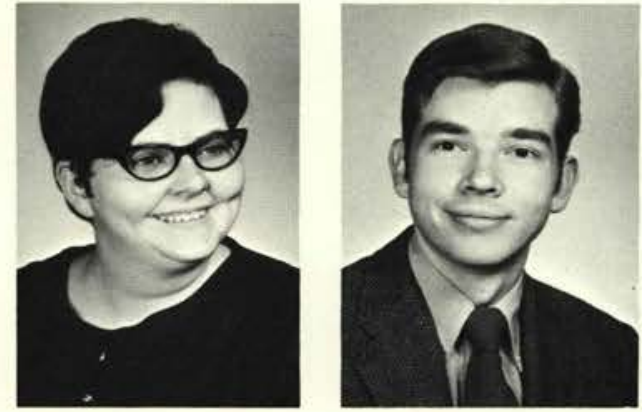
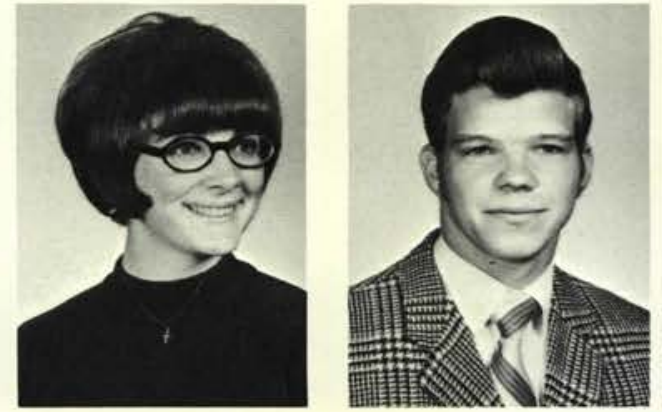
Schwamburger, W. David Portsmouth, Ohio

Seago, Lela

Palson, Montana

Seigneur, Renee

Stryker, Ohio

Seldon, Susan

Nuntsburg, Ohio

Self, Joyce

Flint, Michigan

Severs, Linda

Trenton, New Jersey

Sharp, Cathy

Jonesville, Michigan

Shaw, Richard A

Medina, Ohio

Shepherd, Wayne

Lapeer, Michigan

Sheppard, Charles

Haddon Heights, New Jersey

Simmons, Susan

Belfast, New York

Sims, Kevin

Waterloo, Iowa

Small, Loren

Montrose, Pennsylvania

Smith, Gregory

Teyarkana, Texas

Smith, Larry

Dearborn Heights, Michigan

Smith, Steve

Columbus, Ohio

Sneller, Debbie

Columbus, Ohio

Sorah, Richard

Dayton, Ohio

South, Brett D.

LaPorte, Indiana

Southwell, Dell

Mancelona, Michigan

Spears, Gary

Jeffersonville, Ohio

Spencer, Stephen

Collins, New York

Spieth, Jean

Elyria, Ohio

Spinks, Jacklyn

Sherman, New York
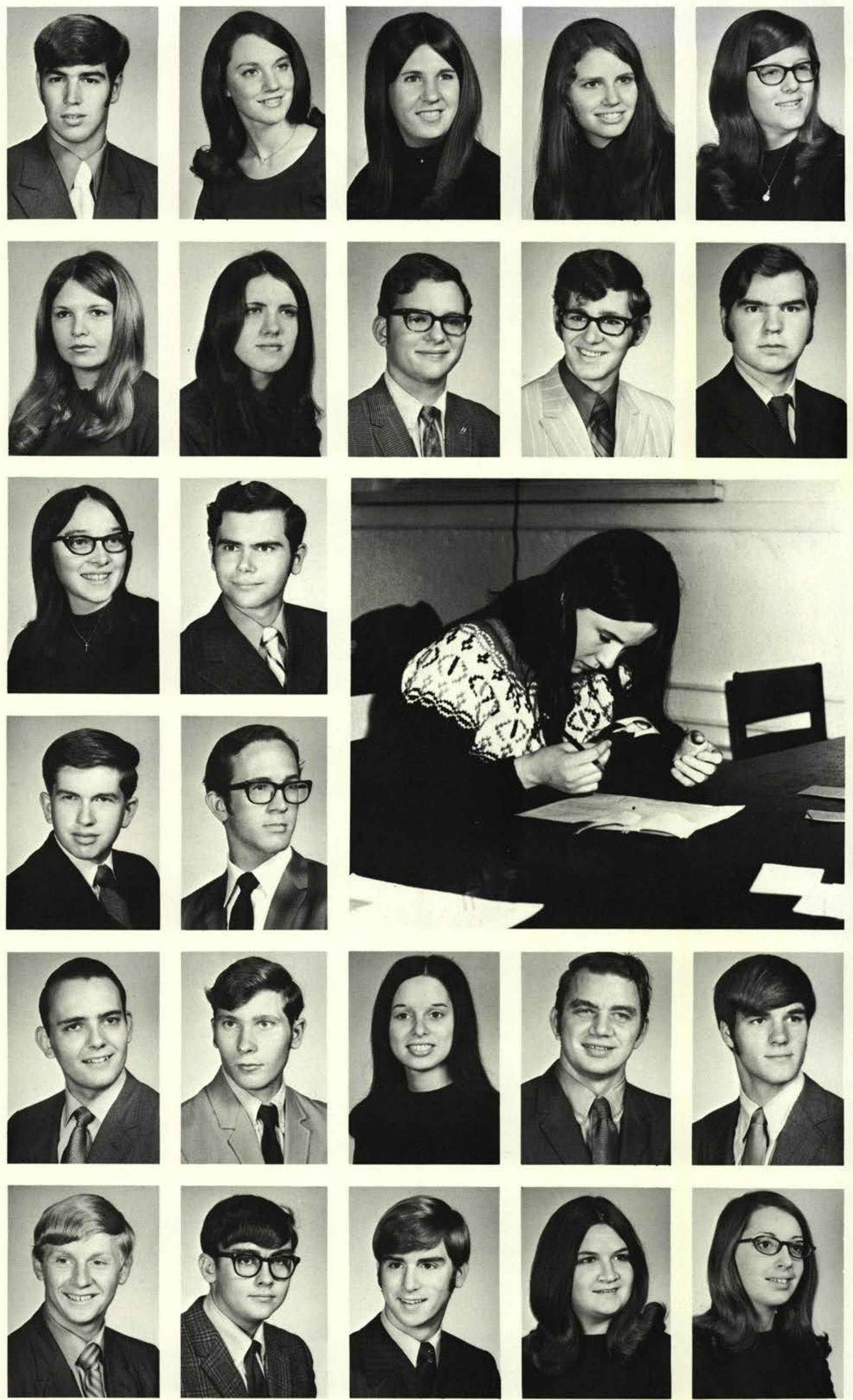

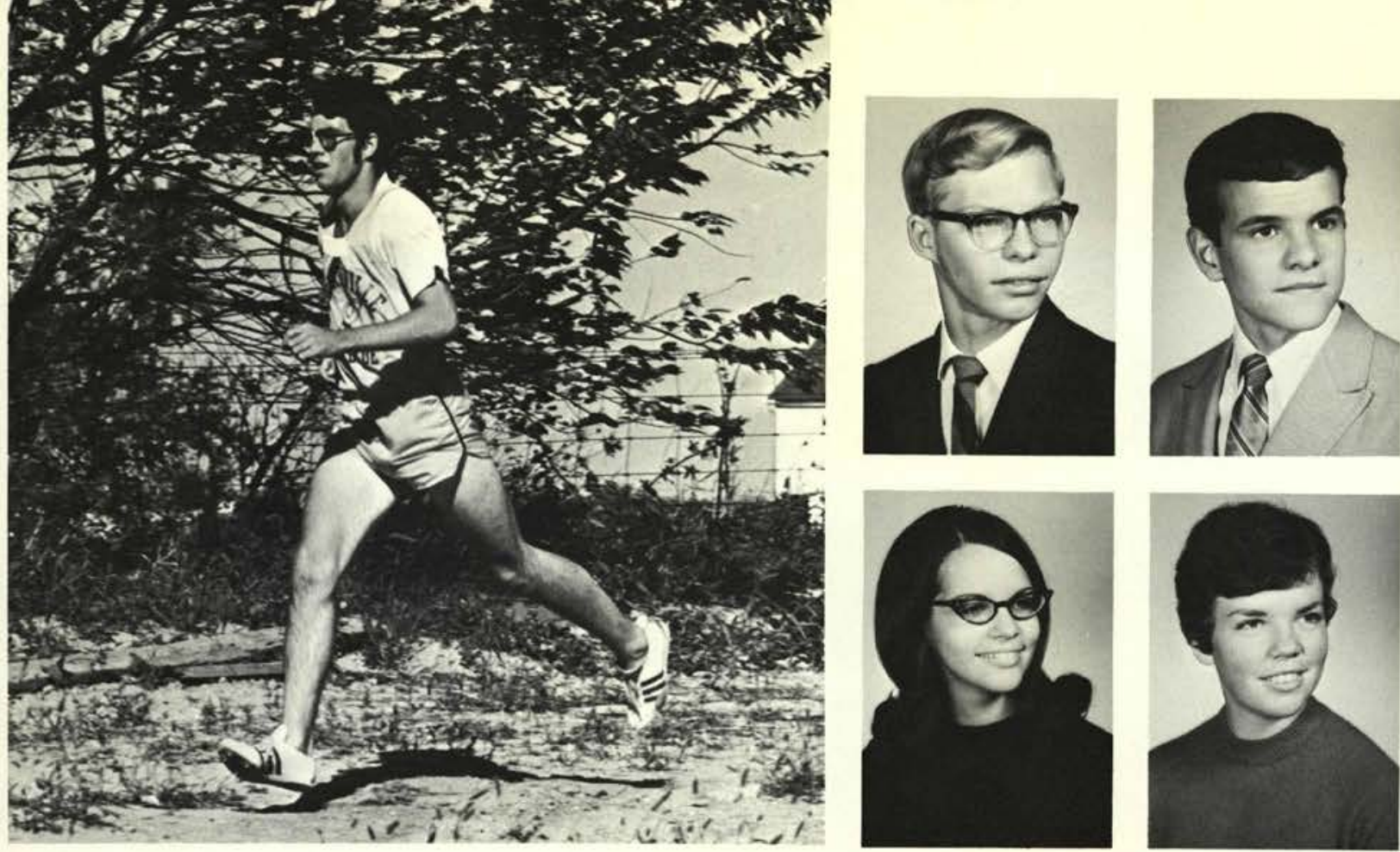

Staley, Mike

Ashland, Ohio

Stemen, Dan

Fort Wayne, Indiana
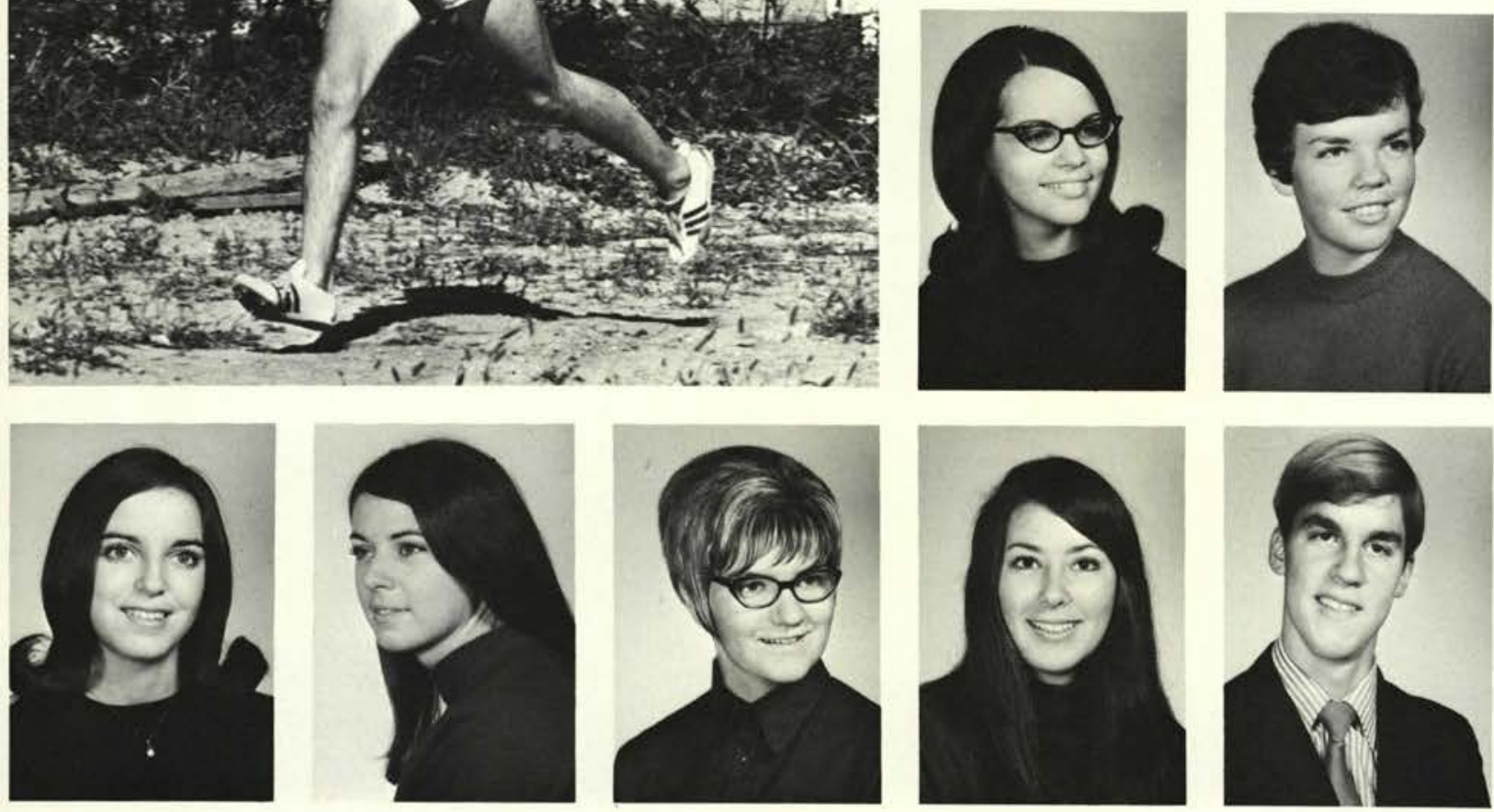

Stevens, Darla

Lindley, New York

Stevenson, Ann

Haddon Heights, New Jersey
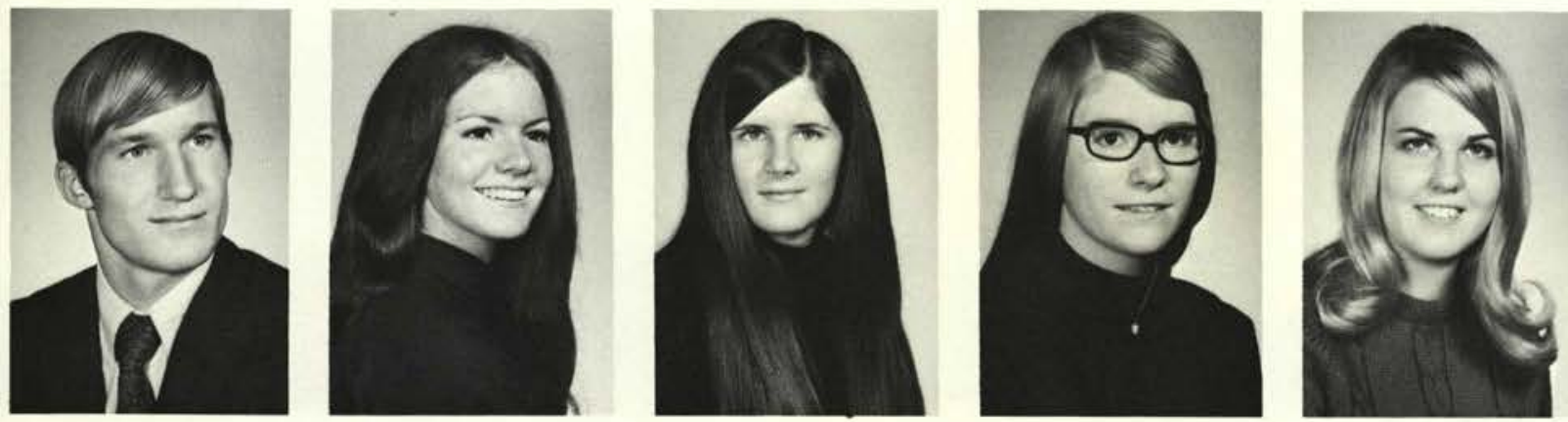

Stone, Sarah Lee

St. Albans, West Virginia

Strawn, Kathy

St. Louis, Missouri

Sullivan, Linda

Huntingburg, Indiana

Summer, Cindy

Troy, Ohio

Summerfield, Tom

Flint, Michigan

Sweazy, Ben

Omaha, Nebraska

Taft, Ellen

Michigan City, Indiana

Tallman, Nancy

Creston, Iowa

Tate, Joy

Alton, Illinois

Taylor, Jean

Steamboat Rock, Iowa
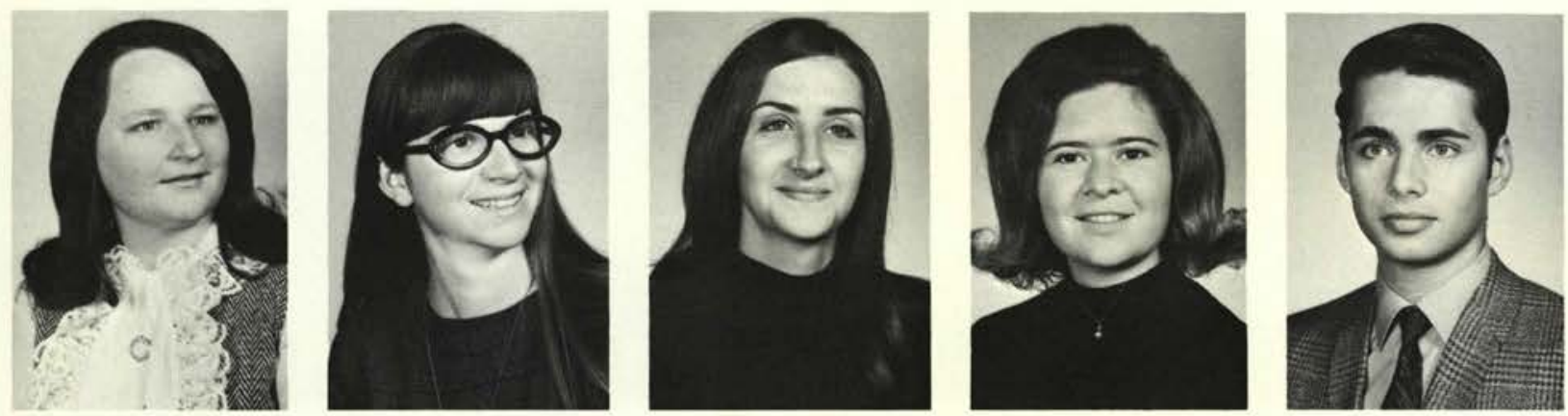

Tingle, Lois

Rockton, Pennsylvania

Tobin, Michaela

Midway, Ohio

Tompkins, Kathy

Des Moines, Iowa

Travis, Rosalie

Creston, Iowa

True, David

La Habra, California

Trueblood, Gaylynn

Griffith, Indiana

Tucker, Michael

Xenia, Ohio

Turner, Cal

Grinnell, Iowa

Tuttle, Sue

Grand Rapids, Michigan

Twigg, Dennis

Indianapolis, Indiana 
Van Duren, Richard Newfield, New York

Wagner, Lois

Climax, Pennsylvania

Walker, Mary Ann

Brownsburg, Indiana

Wall, Joyce

Kettering, Ohio

Watson, Carol

North Jackson, Ohio

Weah, Joseph

Monrovia, Liberia

Webber, David

Cedarville, Ohio

Webster, Greg

Amherst, Ohio

Weld, Sharon

Minneapolis, Minnesota

White, Linda

Springfield, Ohio

White, Raynell

Grafton, Ohio

Will, Brenda

Forestville, New York

Williams, Steve

Friendship, New York

Williams, Wayne

Roseland, New Jersey

Wilson, Janet

Denver, Colorado

Wolfe, Jeffery

East Gary, Indiana

Wolff, Frances

Kansas City, Kansas

Wood, Deborah

Curwensville, Pennsylvania

Work, Ivan

Coudersport, Pennsylvania

Wright, Bonnie

Grafton, Ohio

Wymen, William

Warren, Ohio

Yost, Gwen

Manassas, Virginia

Young, Janet

Waterloo, Iowa

Young, Linda

Brunswick, Ohio

Zent, Susan

Rochester, Indiana
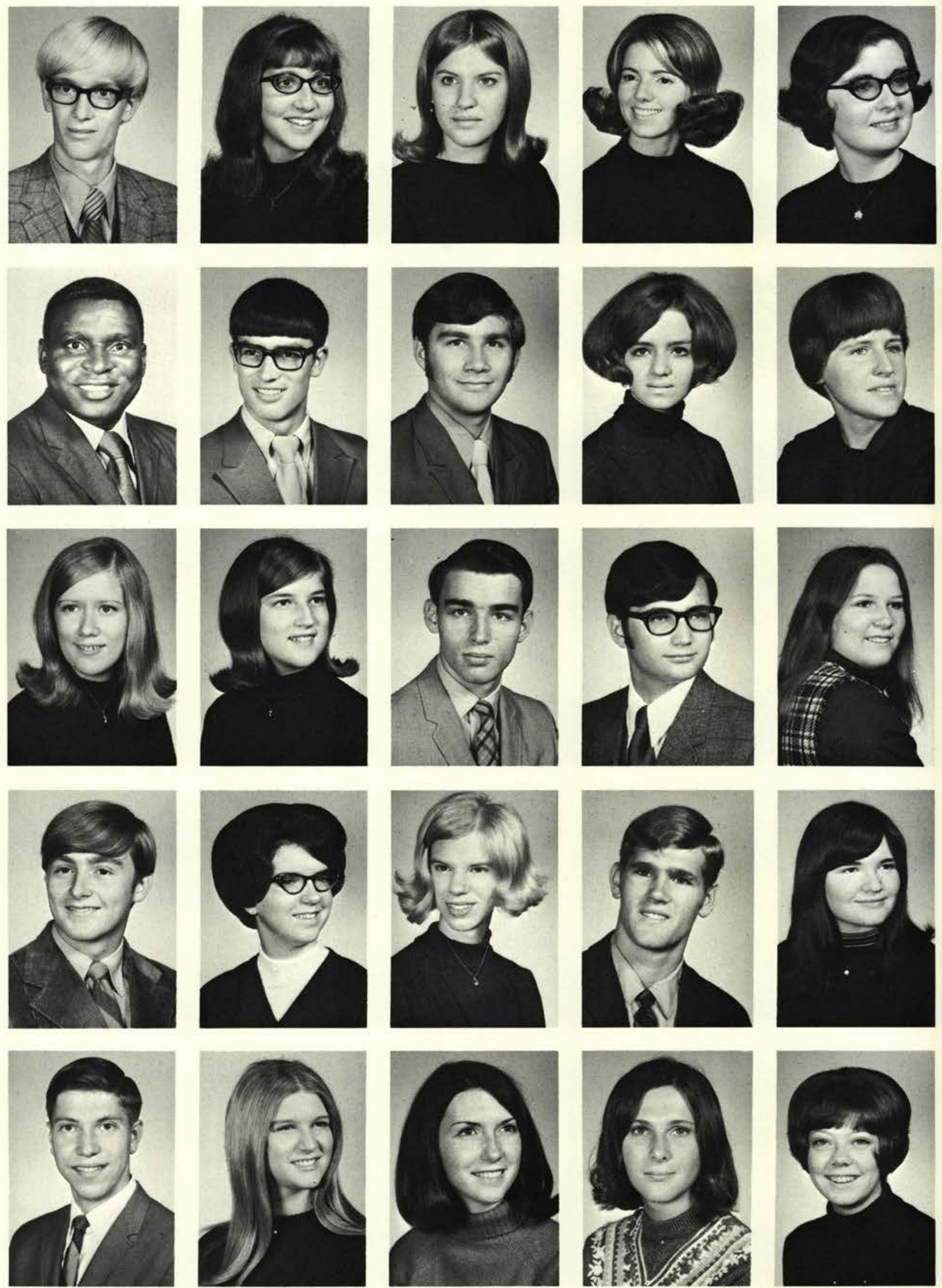


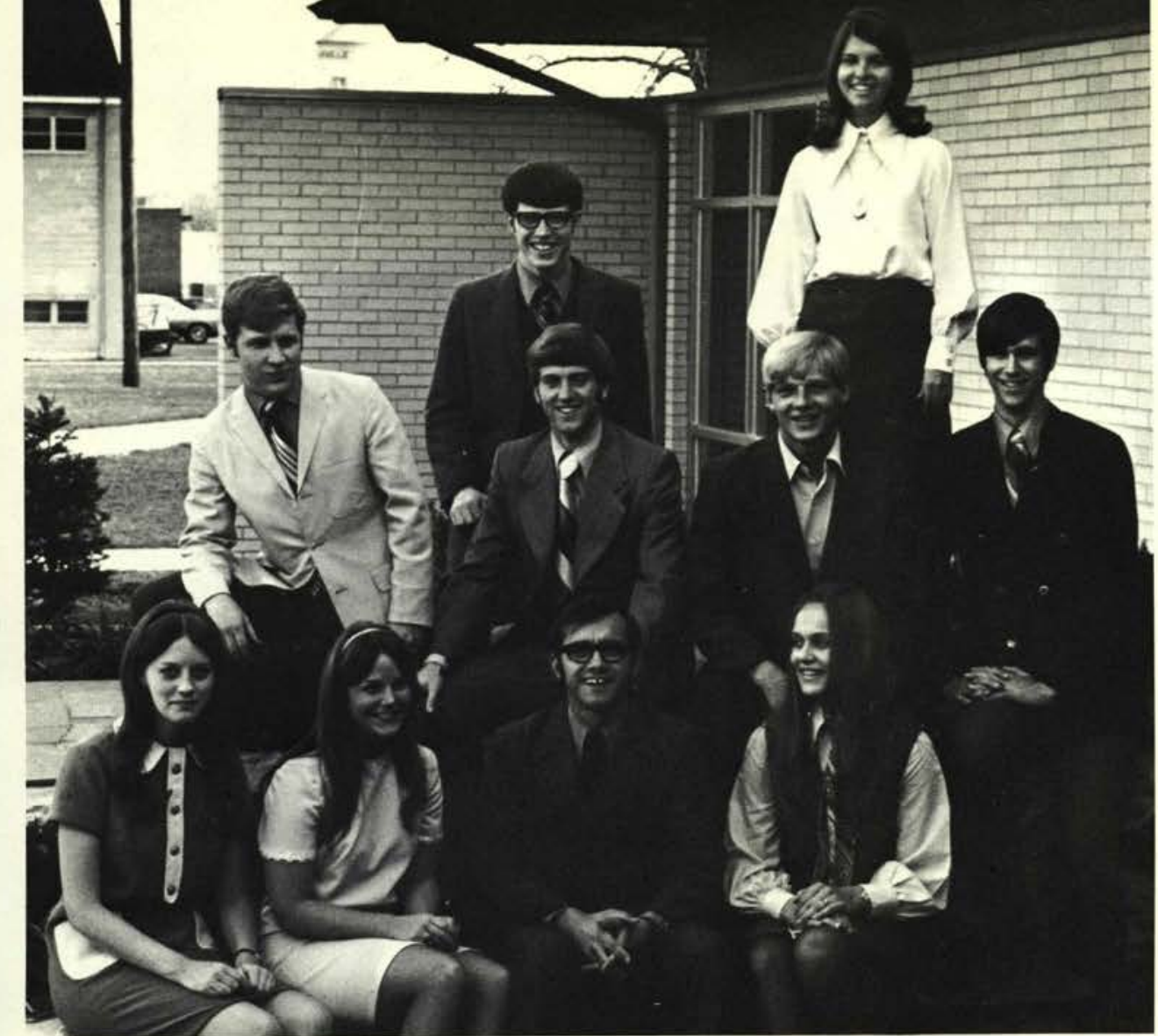

Tune: Ol' McDonald Had a Farm

We love the sophomores bright and keen,

Ei ei o

But we are freshmen dumb and green,

Oink, oink, oink,

With a Be-hog here and a Be-hog there

Ignorant, stupid, just plain naive,

We are freshmen dumb and green,

Oink, oink, oink

(This song must be sung by the frosh upon request from a buttoned sophomore.)
Mr. Jim Phipps, Sponsor

Dan Estes, Student Council

Sue Saunders, Treasurer

Dan Henson, Vice President

Pete Lillback, Chaplain

Bill Potter, Historian

Jackie Barraclough, Secretary

Claudia Culp, Student Council

(Alternate)

Mike Edwards, President

Sharon Paasch, Student Council
The first year of college ...

for many the first opportunity

to be on their own ...

Arriving, unpacking, getting acquainted with new things ...

Registering for fall classes -

O.T. at 8 a.m. ...

Three days of beanies, humiliation, and Kangaroo Court ...

Making the freshman float ...

... a barn party in the hay ...

Second place in the Walk-a-thon ...

The beginning of a new individual ...

The first memory of college life ...

The freshman year. 

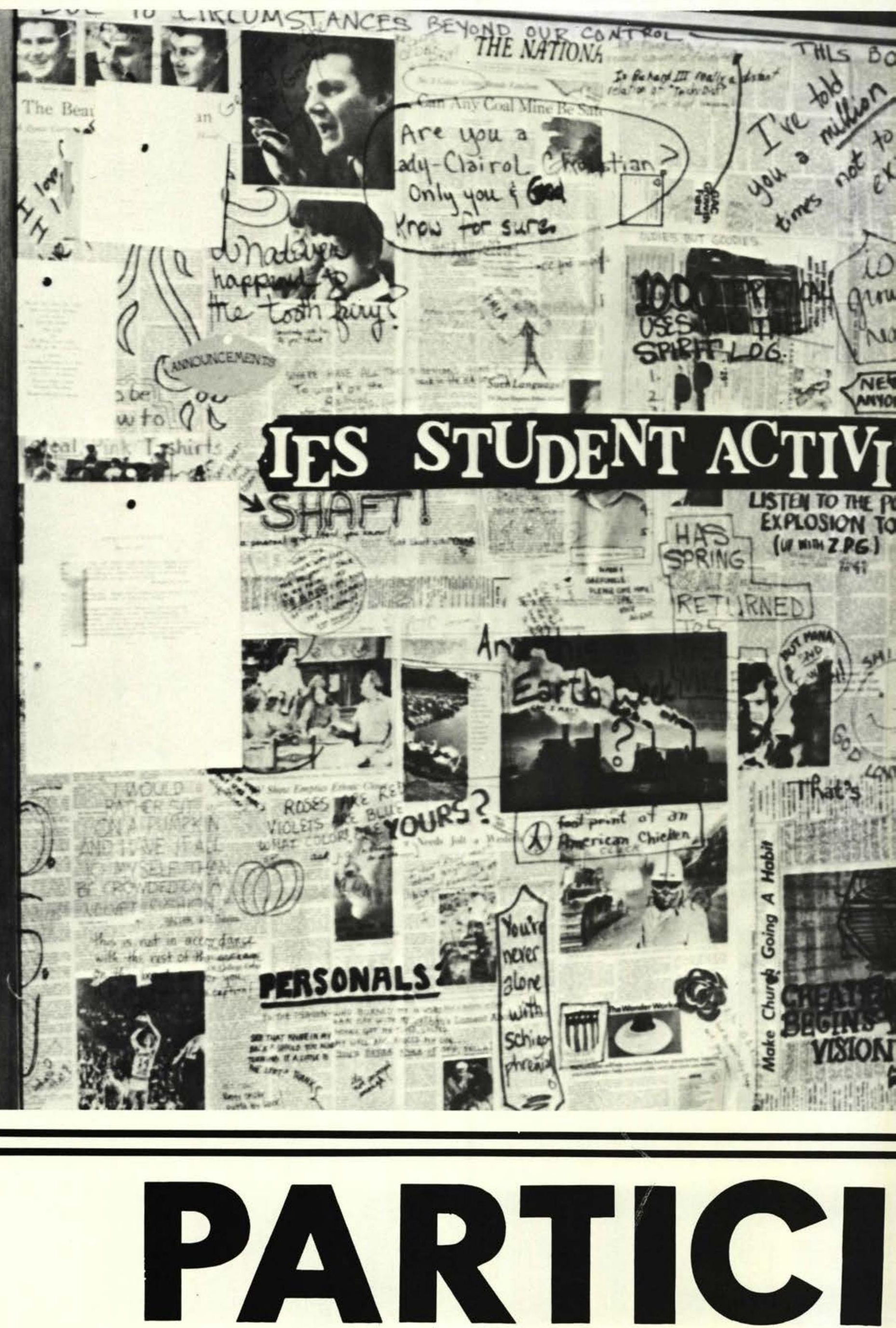


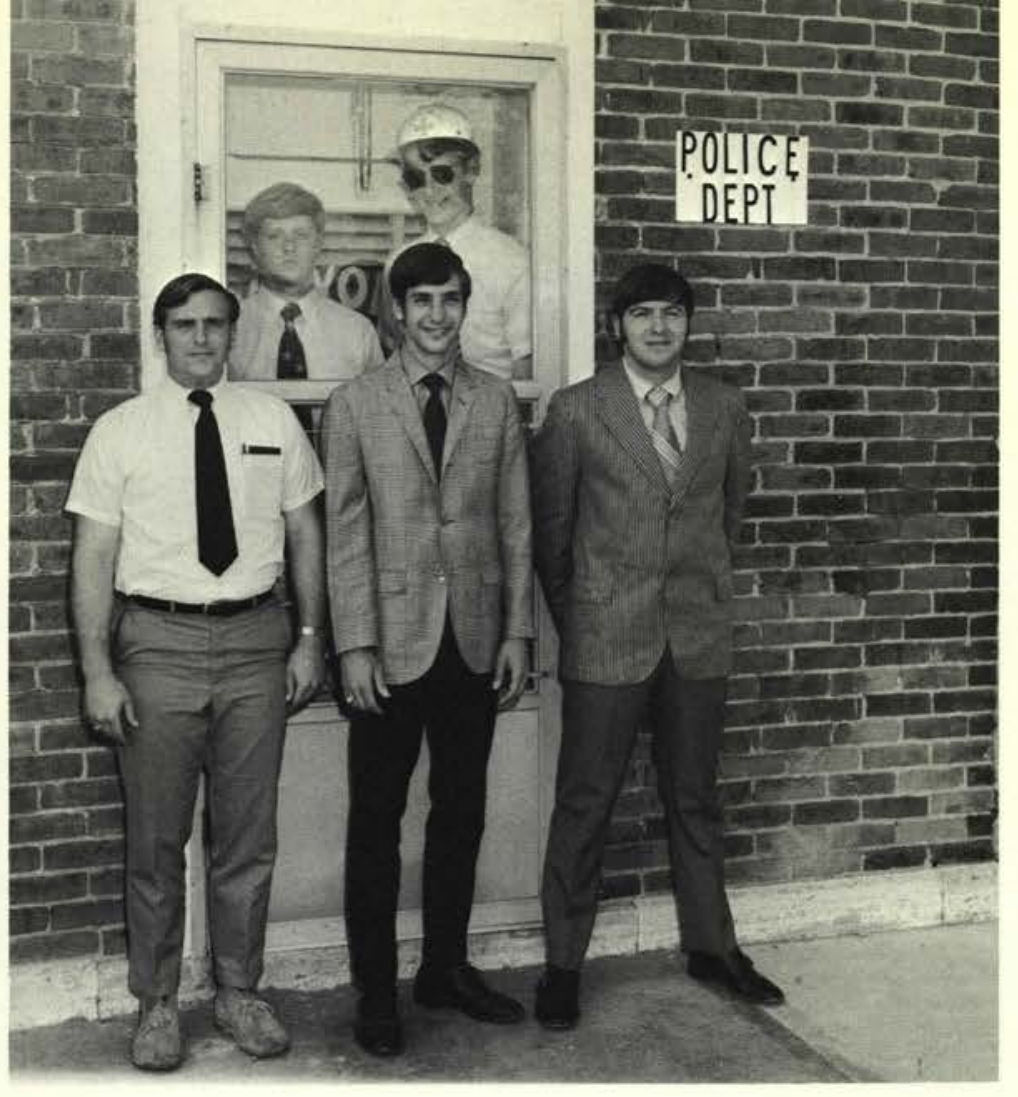

\section{Student Court}

FRONT ROW:

Dave Railsback

Dave Beck

Joe Williams

BACK ROW:

Jeff Woodcock

Don Atherton

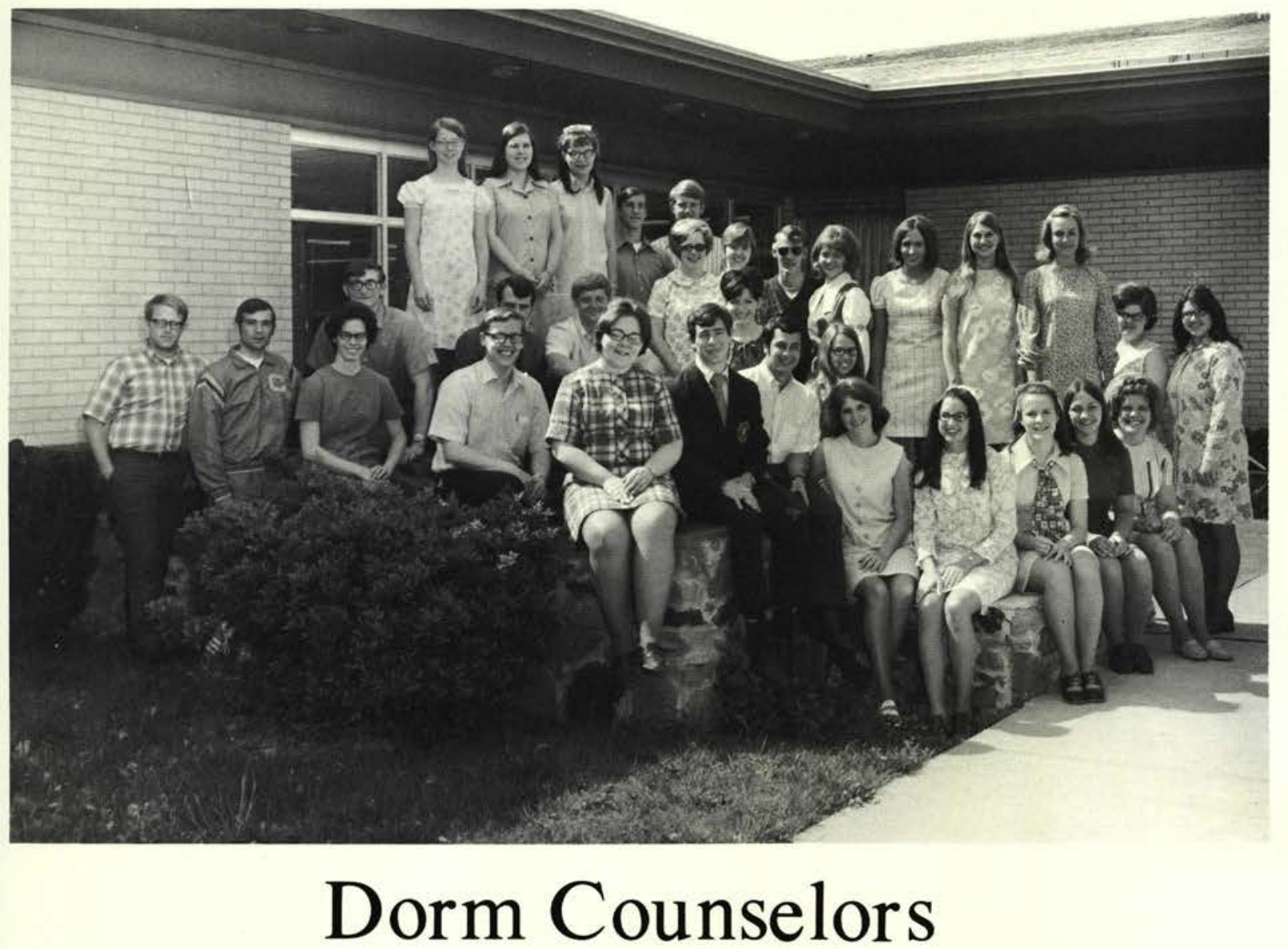

FRONT ROW

Dave Turner

Dave Peters

Dianne Kemp

Dave Globig

Larry Klemm

Randy Patten

Jeff Woodcock

Bev Mix

Ralph Perkins

Glenda Dalton

Randy Brown

Laura Jones

Phyllis Codr

Pat Stroupe

Andrea Houchin

Vicki Tobias

Carol Bierbaum

BACK ROW:

Jeanette Pauley

Sue Harold

Betty Stiles

Bill Tobias

Steve Shank

Carol Urban

Faye Johnson

Don Atherton

Sue Moore

Becki Chandes

Sue Scott

Shirley Rocher

Nancy Smith

Cheryl Holtz

Dorm Counselors 


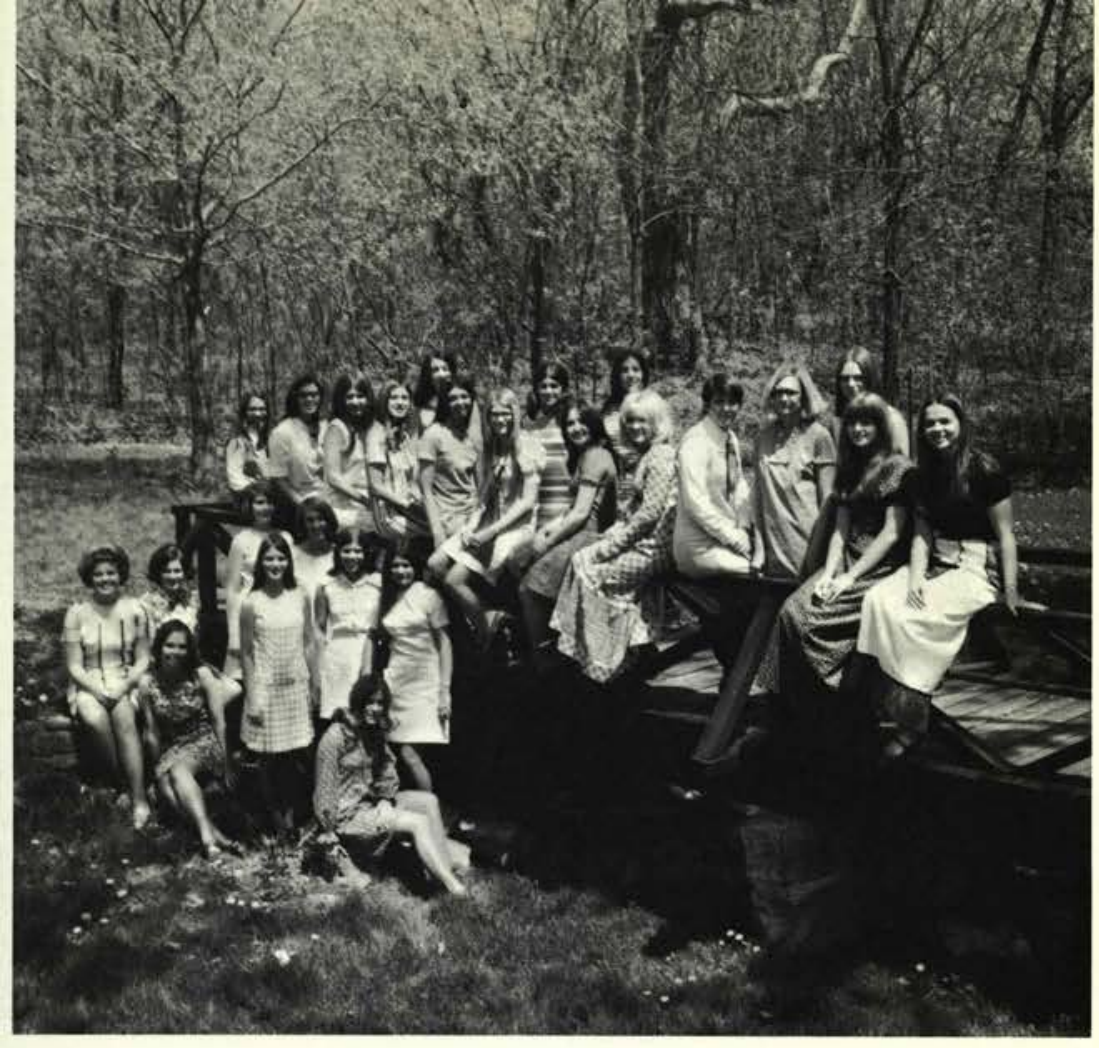

\section{Gamma}

\section{Chi}

FRONT ROW:

Carol Bierbaum Monica Bayne

Jan Hiller

Suzanne Hale

Michele Murry

Julie Jensen

Sue Odegard

Linda Chute

Bev Kemp
BACK ROW:

Laura Jones

Gay Williams

Renee Seigneur

Linda Walter

Dawn Lantz

Lauralee Grable

Joy Tate

Judy Baker

Grace Pruden

Charlotte Willis

Candy Ruby

Becky Decker

Pam Borg

Gretchen Schlechty

Debbie Kitting

Bonnie McNeill

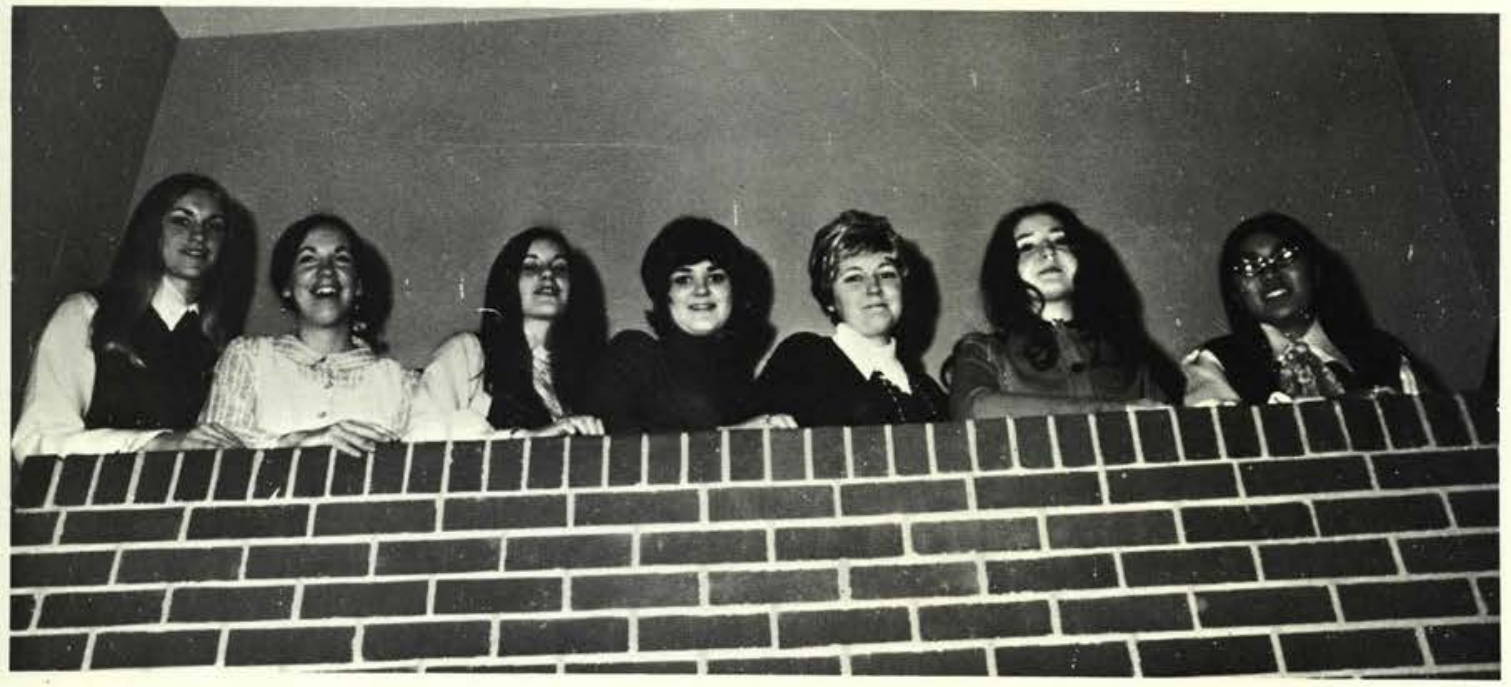

Candy Barr, Treasurer

Judy Leach, Vice President

Linda Walter, Chaplain

Mary Beitz, Secretary

Linda Spieth, Refreshment Chairman

Grace Pruden, Program Chairman

Mitshu Ishuin, President

Mrs. Miriam Maddox, Advisor

(not pictured)

Mrs. Jody Grosh, Assistant Advisor (not pictured)

\section{Rescue}

\section{Squad}

FRONT ROW

Chuck Rowe

George Lee

Jim Jenney

Dave Nelson

John Pereira

BACK ROW:

John Pruden

Ron Wita

Rodger Curtis

Ron Meyers

Bruce Dawley

Dave Grant

Ron Coombs

Mike Dersham 


\section{MENC}

Mr. Ralph Werner, Advisor Carol Lynn Webber

Nancy Haffey

Ron Spieth

Don Harmon

Larry Alfrey

Annette Clark

Dave Durham
Sharon Mummery

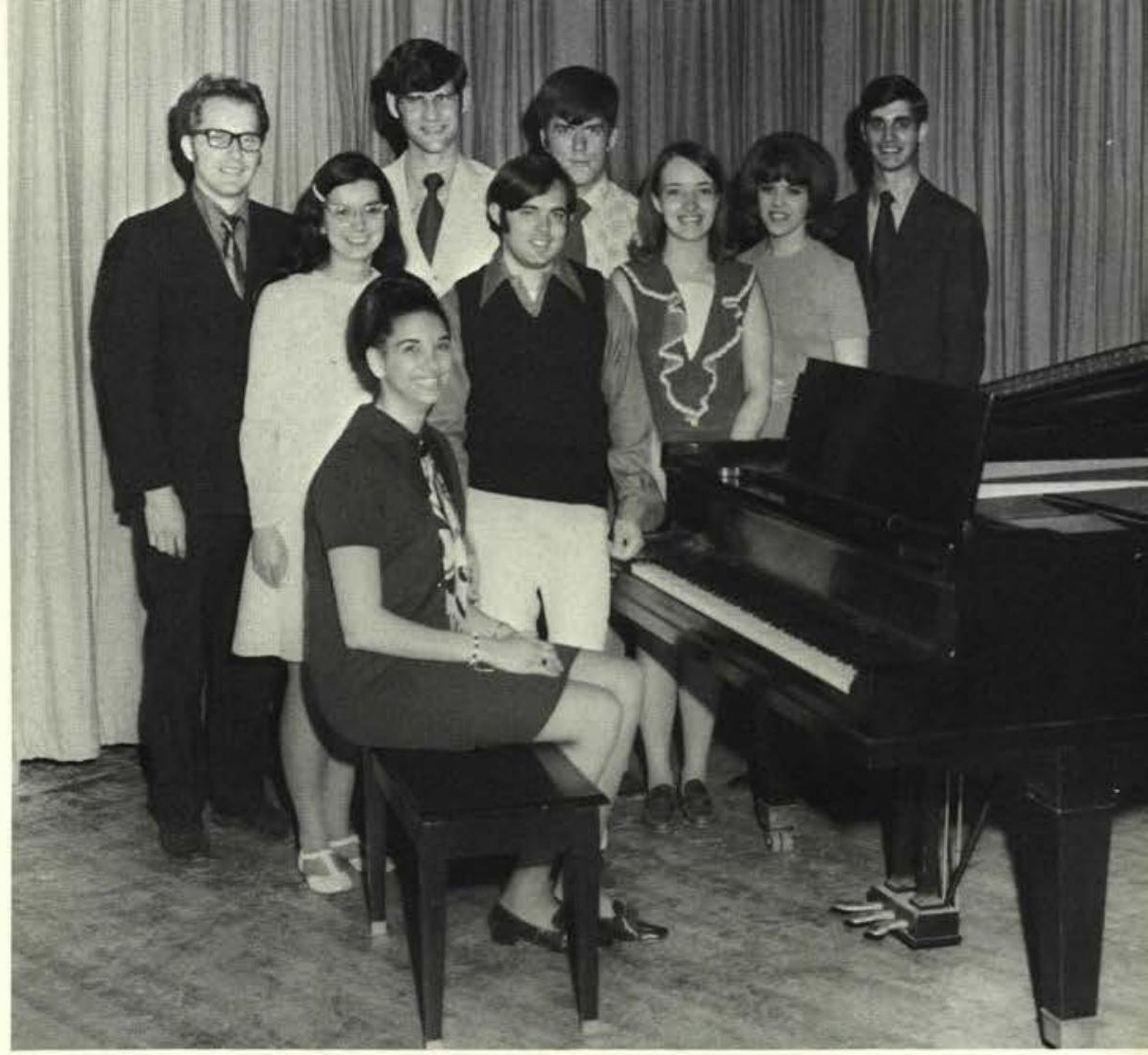

\section{Delta}

Sigma

\section{Alpha}

FRONT ROW:

Becki Chandes

Carole Morse

Mr. Matson, Advisor

Annette Clark

Linda Spieth

BACK ROW:

Larry Alfrey

Jan Beesley

Jim Bucholtz

Nancy Haffey

Ron Spieth

Rita Gast

Dave Durham

Bev Moore

Connie Clark

Carolyn Lambert

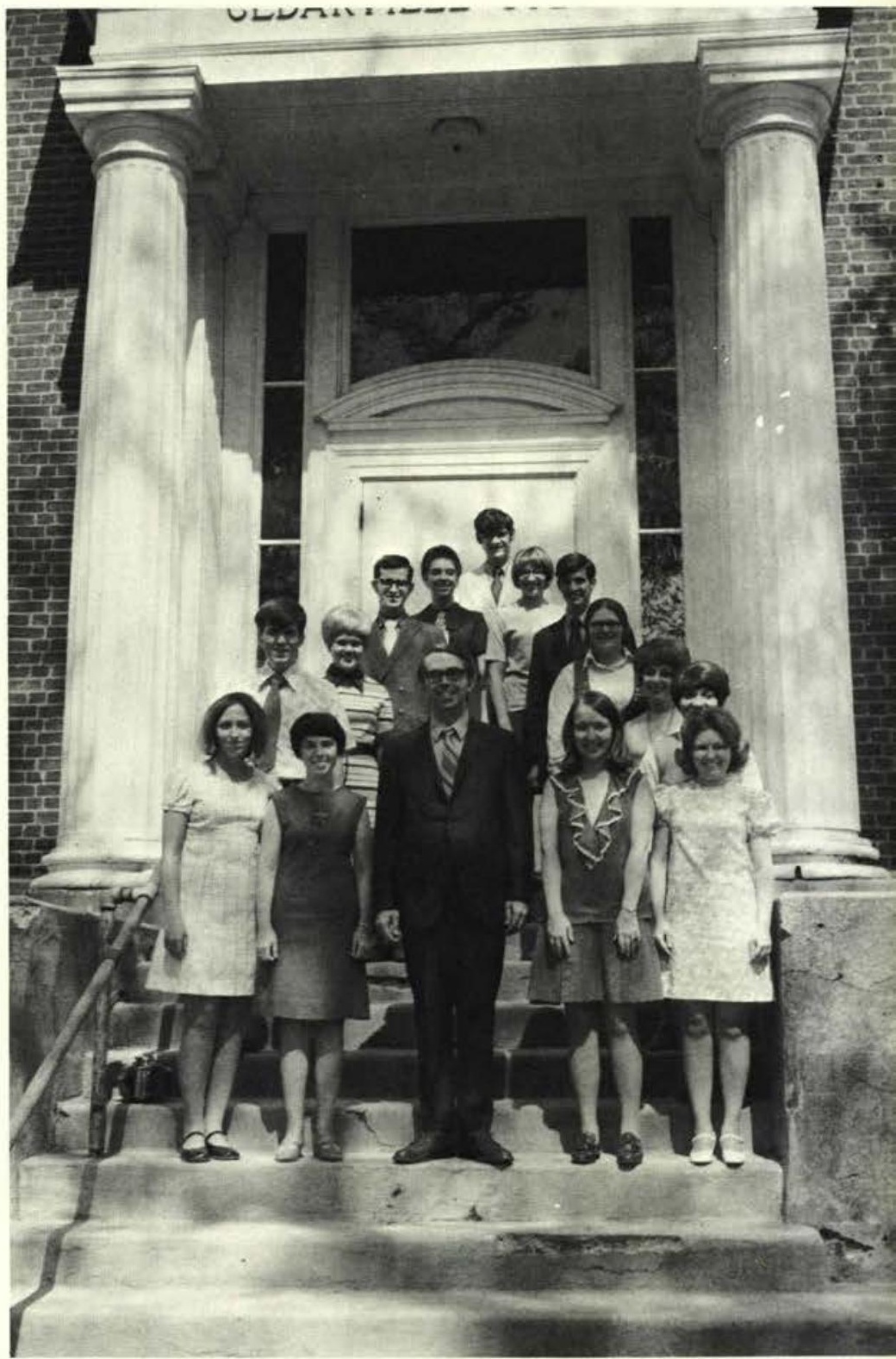




$\begin{array}{cl} & \text { Don Davis } \\ \text { FRONT ROW: } & \text { Rhea Johnson } \\ \text { Keith Hague } & \text { Ty Hartman } \\ \text { Stu Wilker } & \text { Mike DeBerg } \\ \text { Dave True } & \text { Vicki Tobias } \\ \text { Bob Mahl } & \text { Jan Hillery } \\ \text { Don Fleming } & \text { David Butler } \\ \text { Jon Smith } & \text { Vicki Reynolds } \\ \text { Buddy Laird } & \text { Arlene Knight } \\ & \text { Tim Barker } \\ \text { BACK ROW: } & \text { Steve Williams } \\ \text { Russ Elkin } & \text { Virginia Heal } \\ \text { Tom Farlow } & \text { Ron Clarke } \\ \text { Lyle Rodman } & \text { Wayne Sheppard } \\ \text { Becky Decker } & \text { Scott Campbell } \\ \text { Rick McIntosh } & \text { Dave Durham } \\ \text { Dane Rohm } & \text { Carol Bierbaum } \\ \text { Barb Johnson } & \\ \text { Ralph Perkins } & \\ & \end{array}$

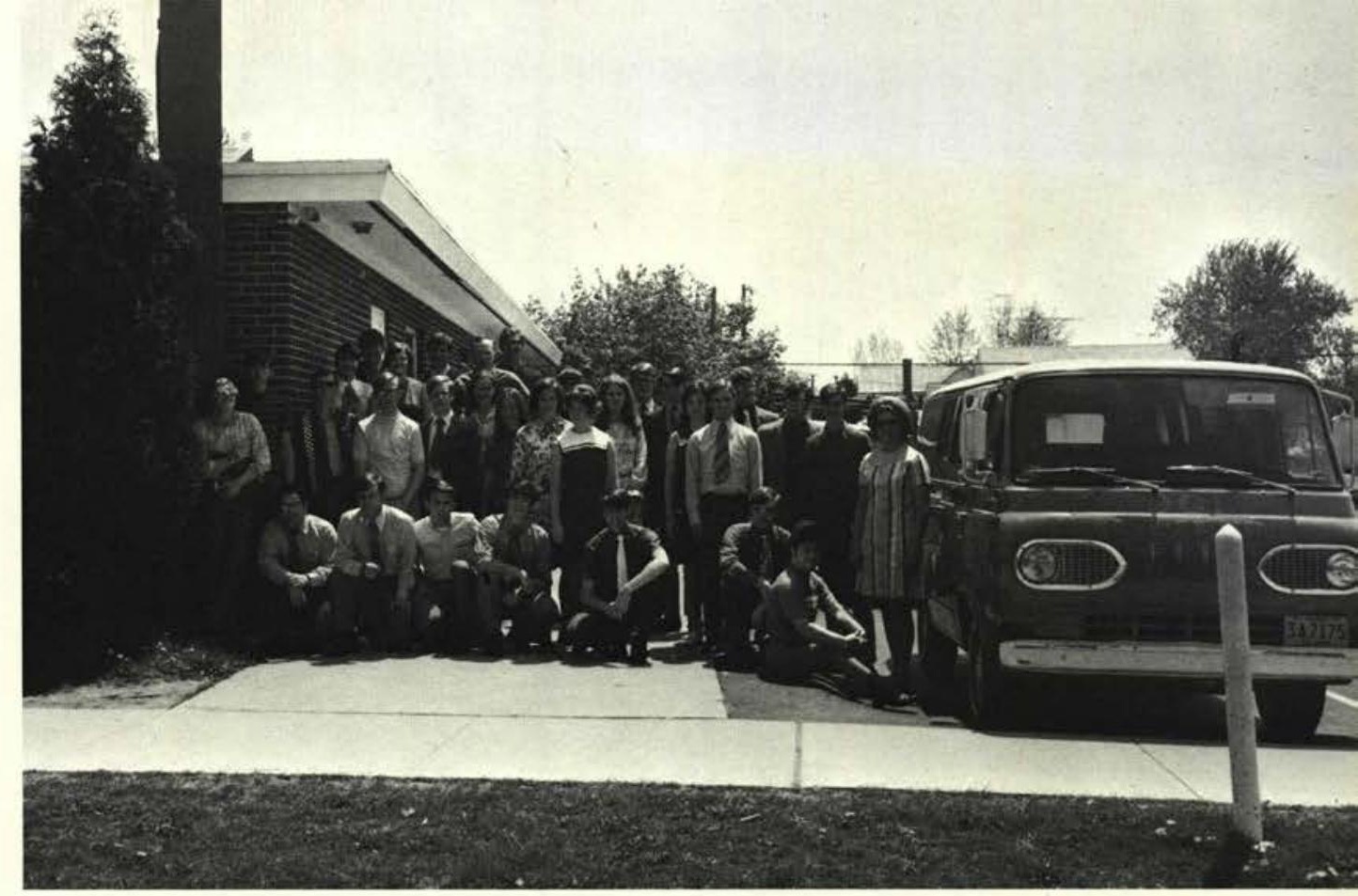

\section{Literati}

FRONT ROW:

Dawn Myers

Nancy Smith

BACK ROW:

Dave Weng

Vicki Tobias

Faye Johnson

Thayne Bodenmiller

Marsha Edwards

\section{Village Players}

Diane Kemp Don Harmon

Dave Railsback Jan Tennant Jerry Grayson Ron Meyers
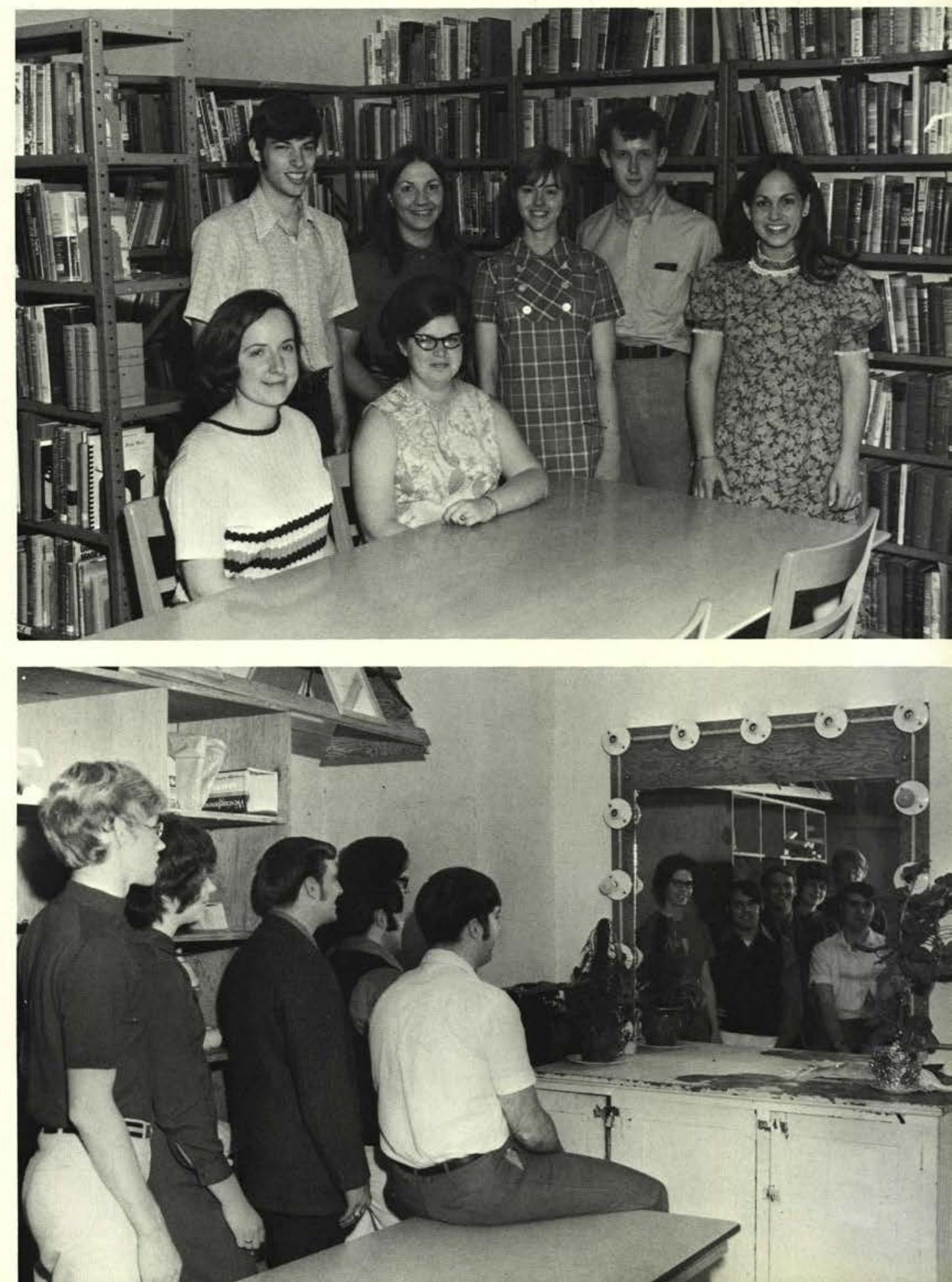


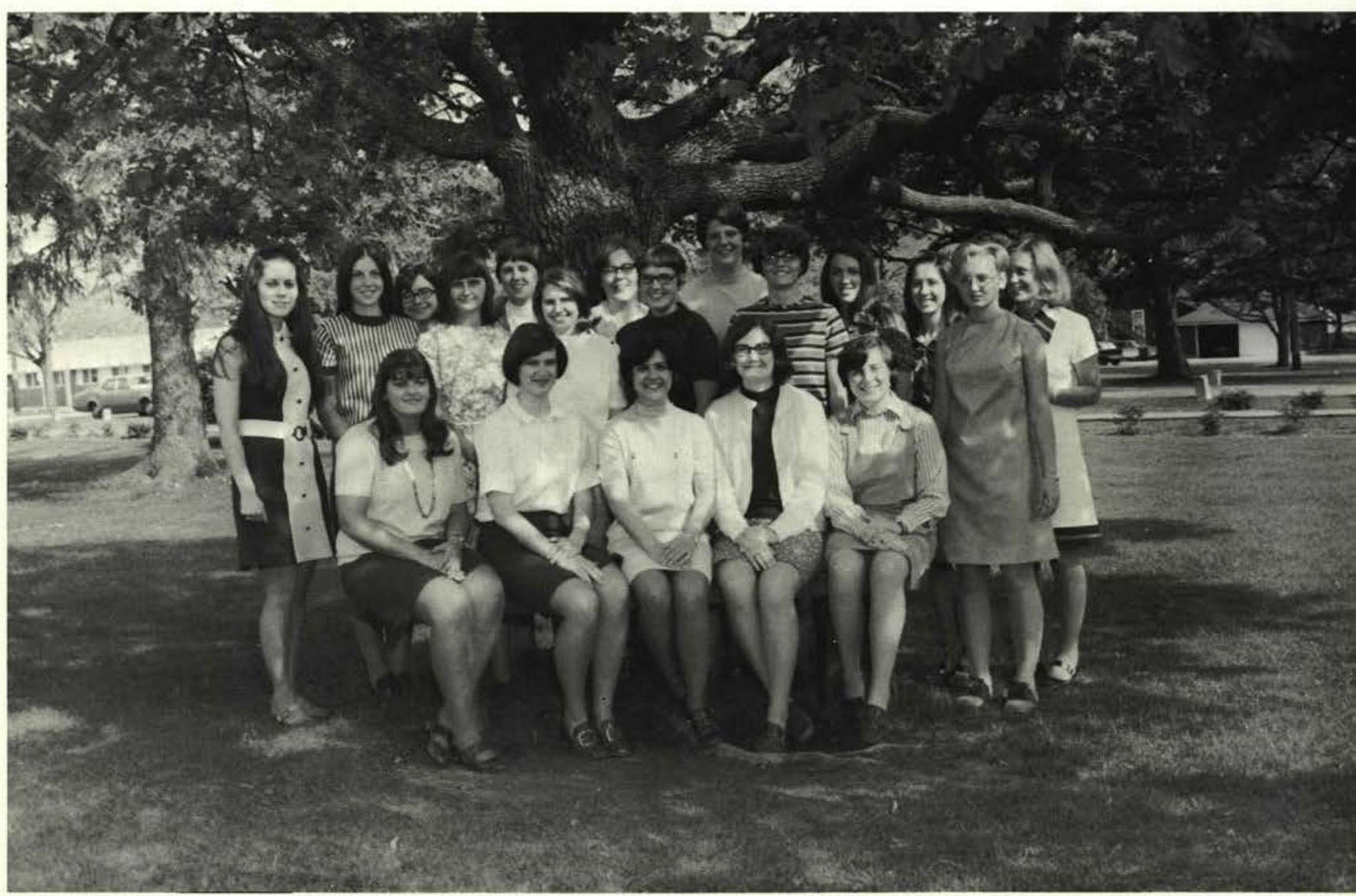

\section{WRA}

FRONT ROW:

Brenda Tompkins

Jan Martin

Billie Thomas

Rae Belle Fisher

Bev Pestel

BACK ROW:

Mary Story

Charlotte Willis

Pam Branning

Colleen Caldwell

Linda White

Sue Selden

Coletta Morrow

Elaine Kalnbach

Karen DeMars

Connie Pumpelly

Carolyn O'Keefe

Landra Lowery

Marsha Sawicki

Roxan Davis 


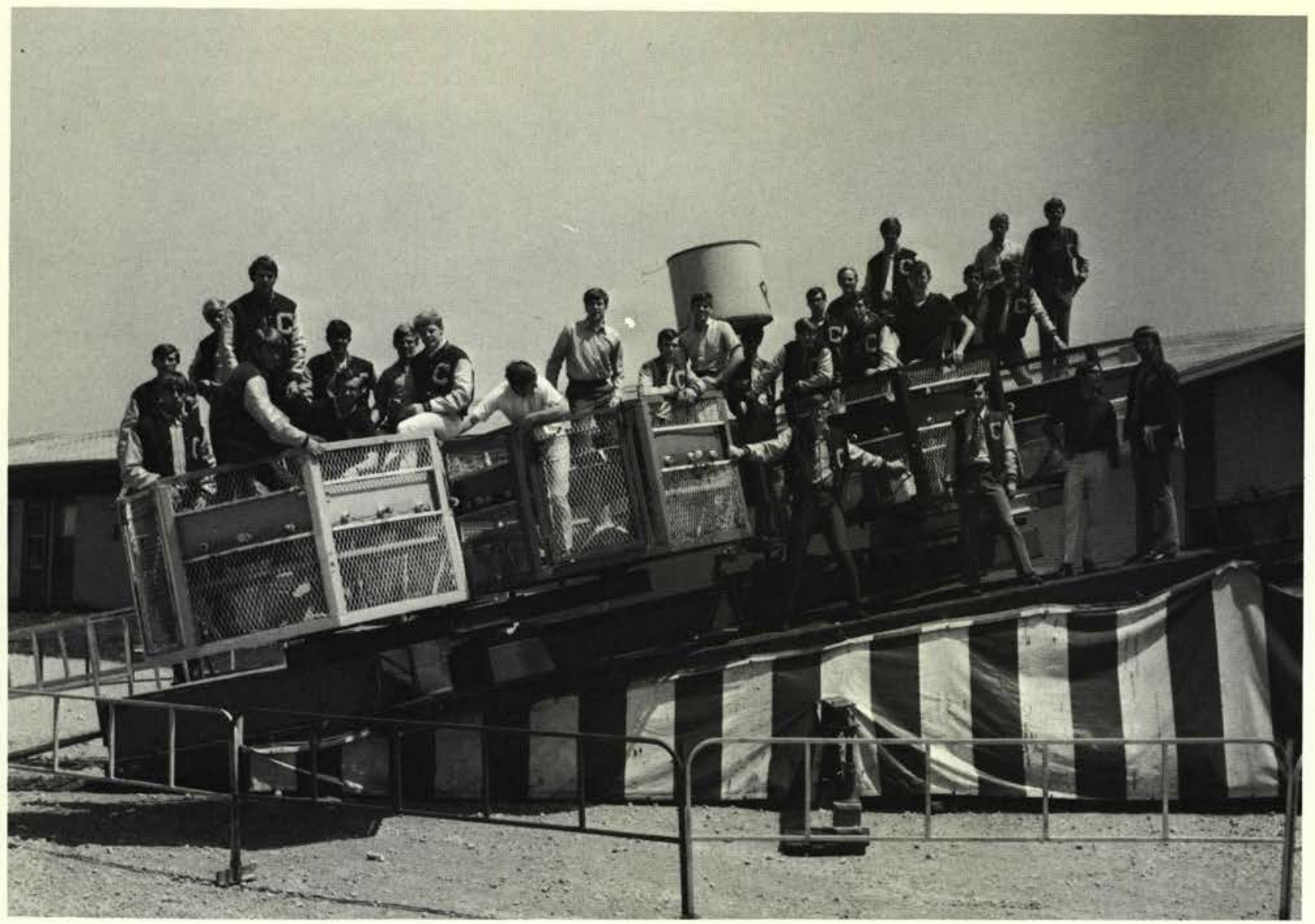

FRONT ROW:

Dave Globig

Stu Walker

Tony Wall

Bob Lunney

BACK ROW:

Mike Coffman

Tom Farlow

Les Chambers

Warren Jenkins

John Rooke

Phil Stine

Dave Rodriguez

Jake Tipton

Jeff Woodcock

Tom Strong

Dan Inghram

Dee Mays

Rick Inghram

Ernie Willborm

Dave Brannon

Dale Smith

Charlie Williams

Bob Wood

Bob Austin

Don Atherton

Dave Jewell

Lynn Howard

Paul Large

Ken Evans

\section{Varsity "C"}

\section{Pi Sigma Nu}

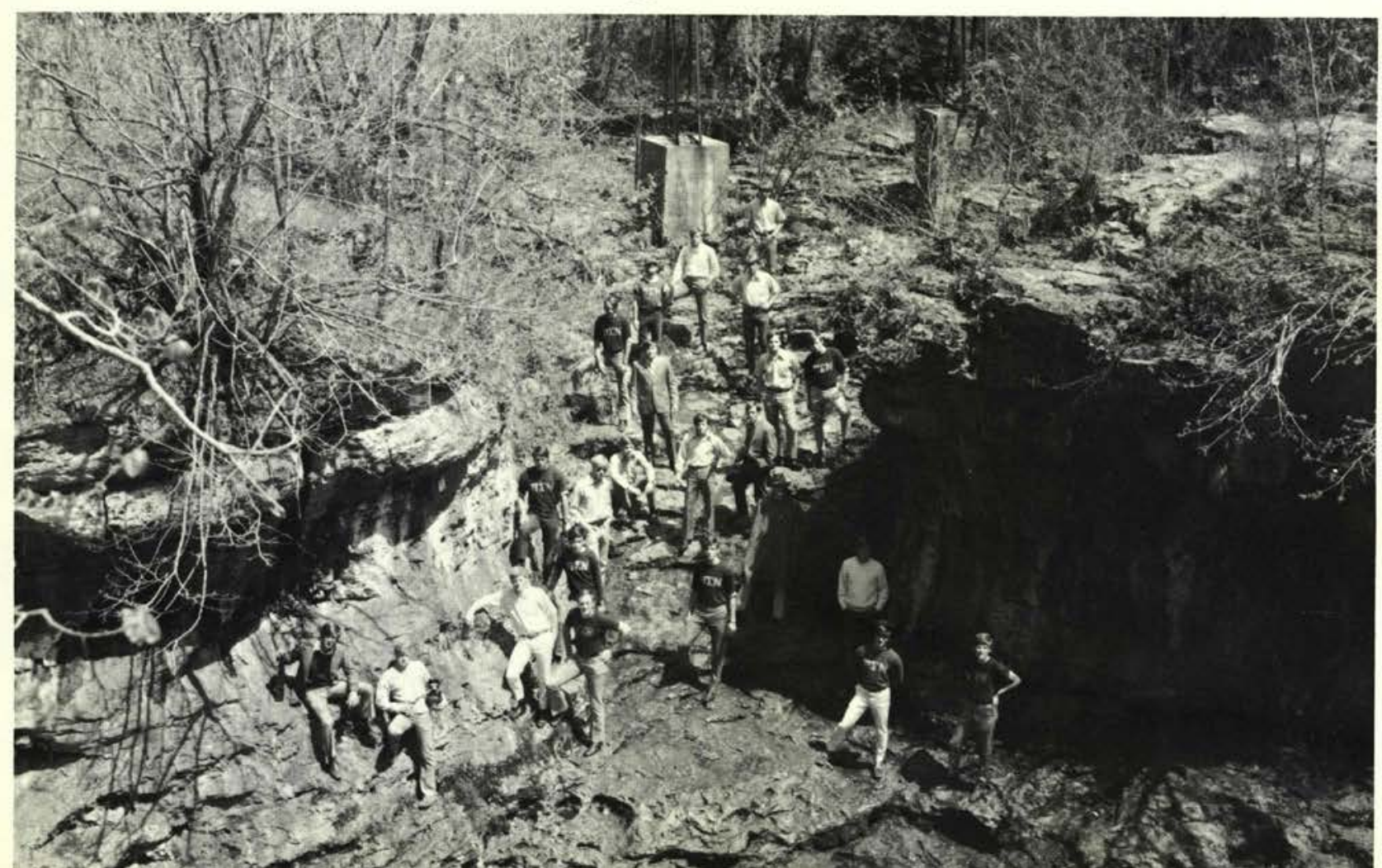




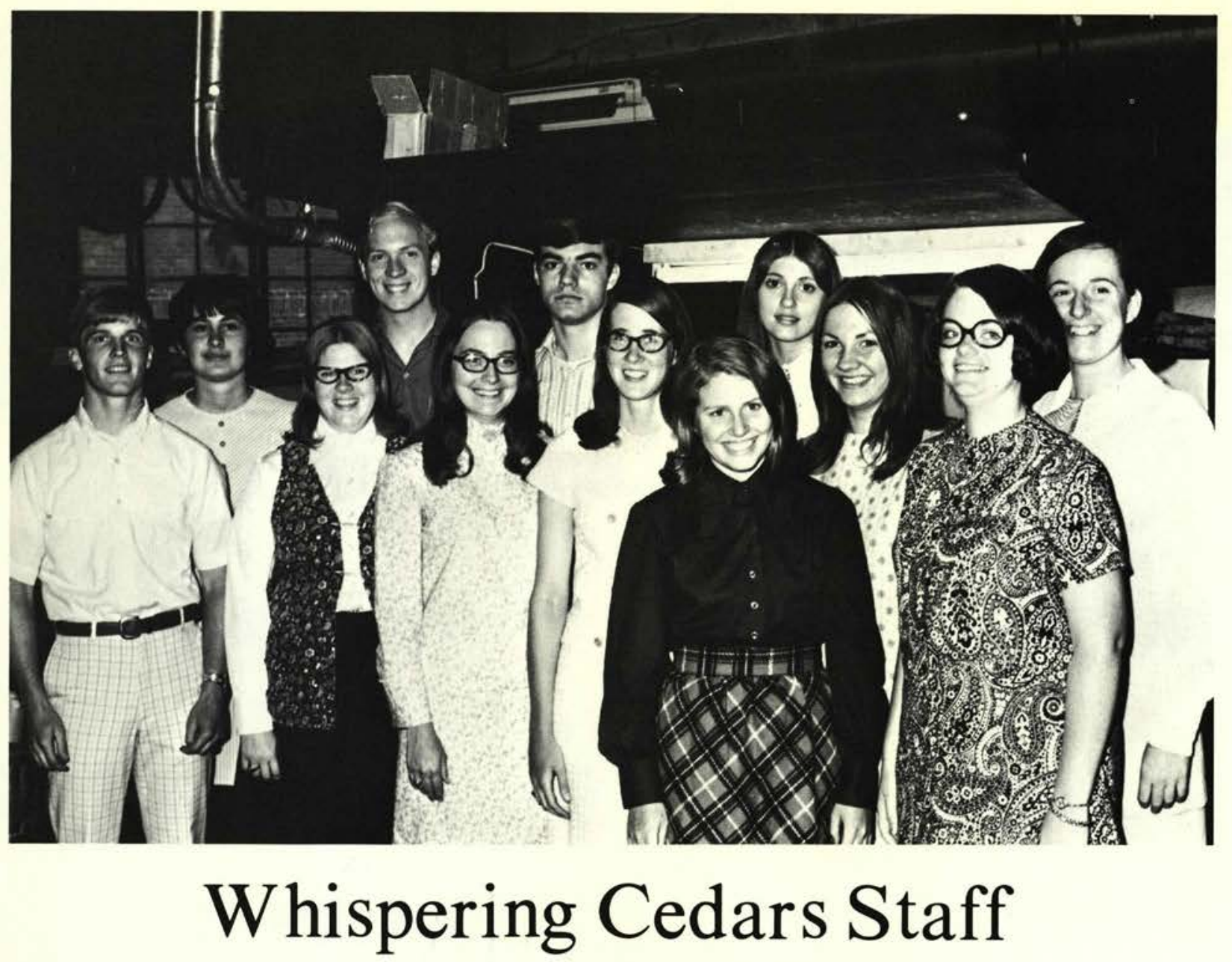

Lyle, Miller

Jayne Eberling

Nanci Layne

Glen

Jane Emerson

John

Valerie Jayne Holfield

Susan Selden

Georgeanna Axiotis

Vicki Tobias

Judy Johnson

Dawn Myers 


\section{Rest Homes}

FRONT ROW:

Sharon Gillogly

Linda Severs

Dianne Kemp

Jan Hallett

Kaye Partlow

BACK ROW:

JoAnne Buschmann

Cathy Sharp

Kathy Strawn

Bernie Horn

Joyce Self

Charlie Williams

Phil McMillen

Dan Thompson

Debbie DeBoer

Dave Romaine

Janet Lewis

Becky Anderson

Sharon Portz

FRONT ROW:

Judy Hepworth

Nancy Addleman

Kathy Berry

Linda Linger

Rae Belle Fisher

BACK ROW:

Grace Pruden

Bonnie Wright

Ron Clarke

John Fiest

Chuck Rowe

Marsha Sawicki

Joyce Mohler
Maryann Butcher
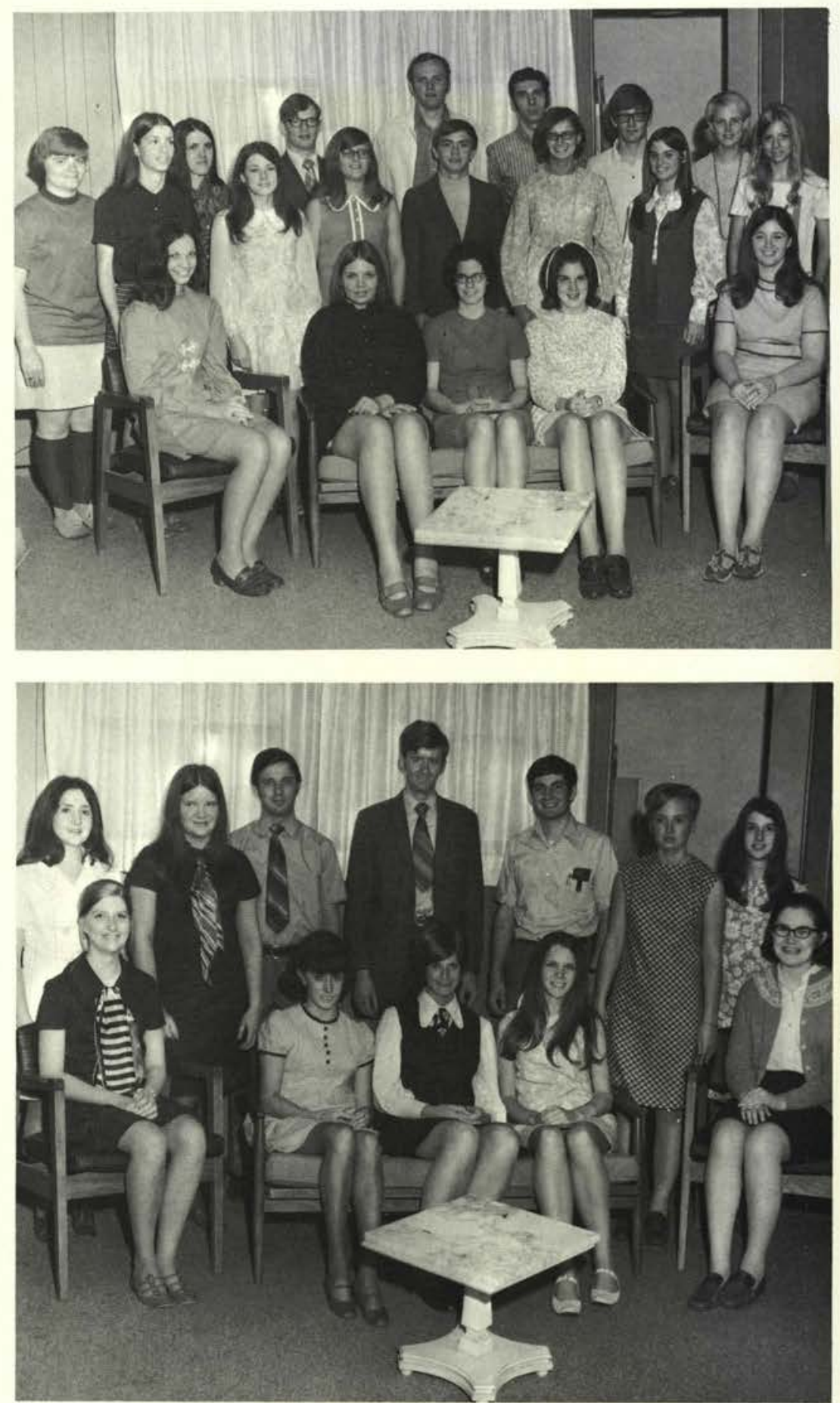

\section{Orphanages}

FRONT ROW:

Sam McMillen

Judy Johnson

Roberta Lutton

Diane DeNardis

Joyce DeBruine

Marsha Sawicki

Bonnie Wright

Becky Williams

BACK ROW:

Shirleen Morrow

Janette Carl

Marjorie Cogan

Julie Jensen

Sue Hall

Debbie Dear

Bill Tobias

Vicki Tobias

Becky Kemble

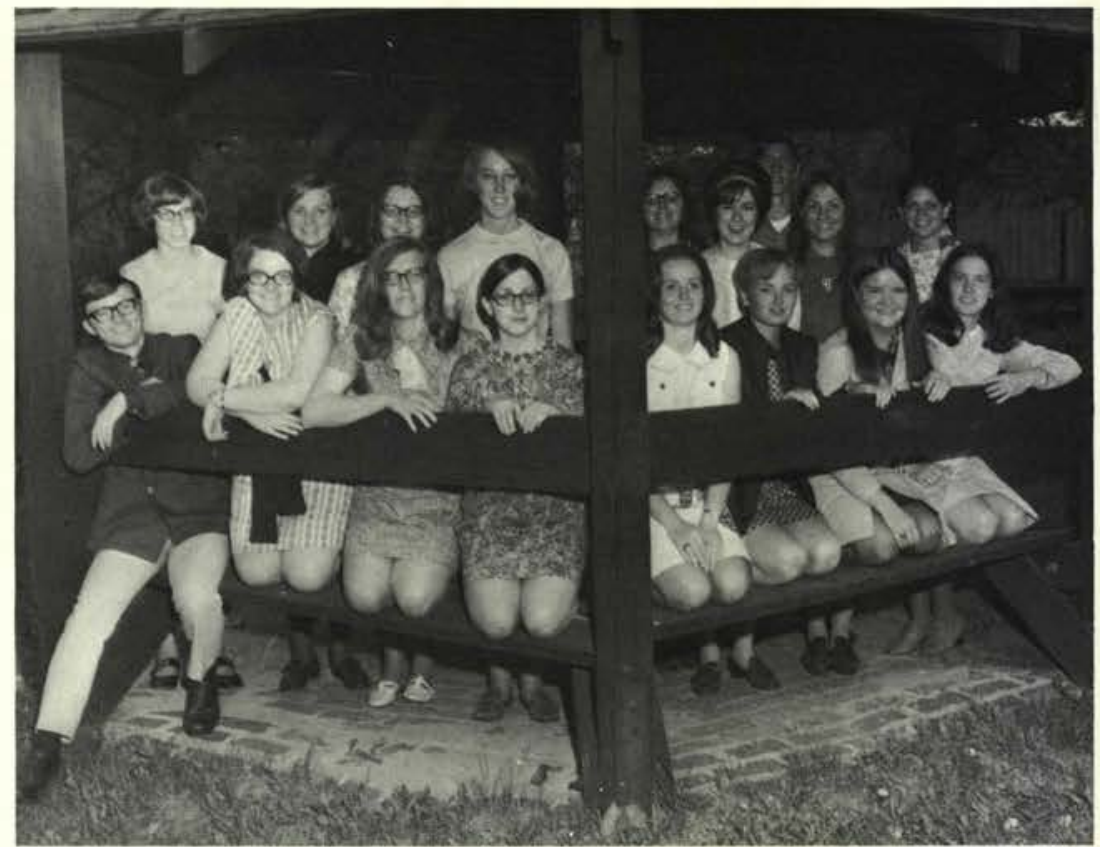




\section{Sunday School and Children's Church}

FRONT ROW:

David Butler

Susan Mayer

Bonnie Hostetler

Mike Wilhite

Cheryl Klomparens

Gale Portz

\section{BACK ROW:}

Roy Kern

Kelsey Bennett

Adria Soeder

Linda Walter

Diane DeNardis

Evelyn Klimek

Darlene Lewis

Ruth Steyer

Shirley Perry

Carol Watson

\section{Advisory 12}

FRONT ROW:

Clancey Parker

Ralph Perkins

Ken Evans

Roy Kern

Pastor Green - advisor

BACK ROW:

Paul Radcliff

Lynn Howard

Al Konya

Buddy McNeice

Bill Amstutz

Dan Henson 


\section{Bible Clubs}
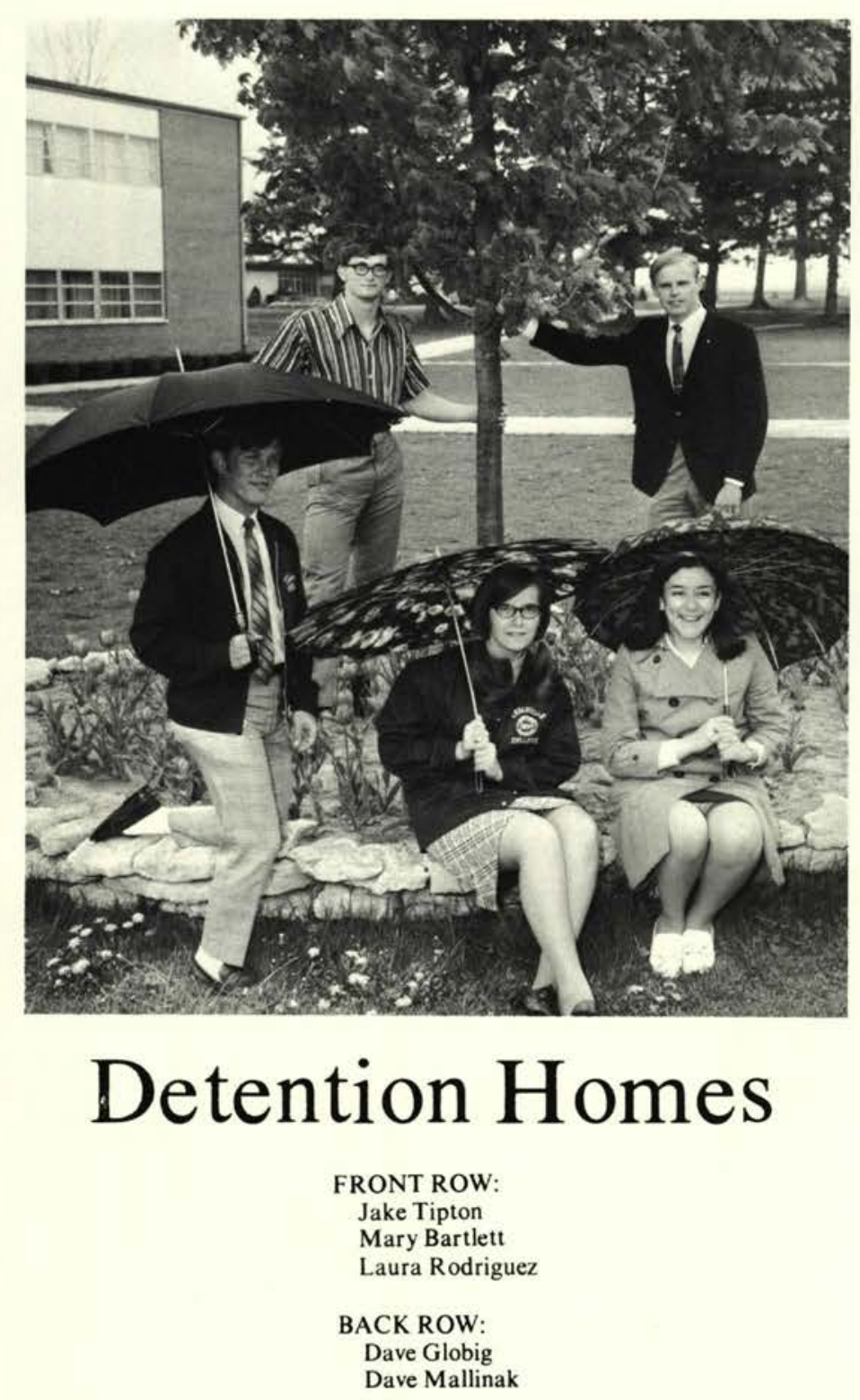

\section{Detention Homes}

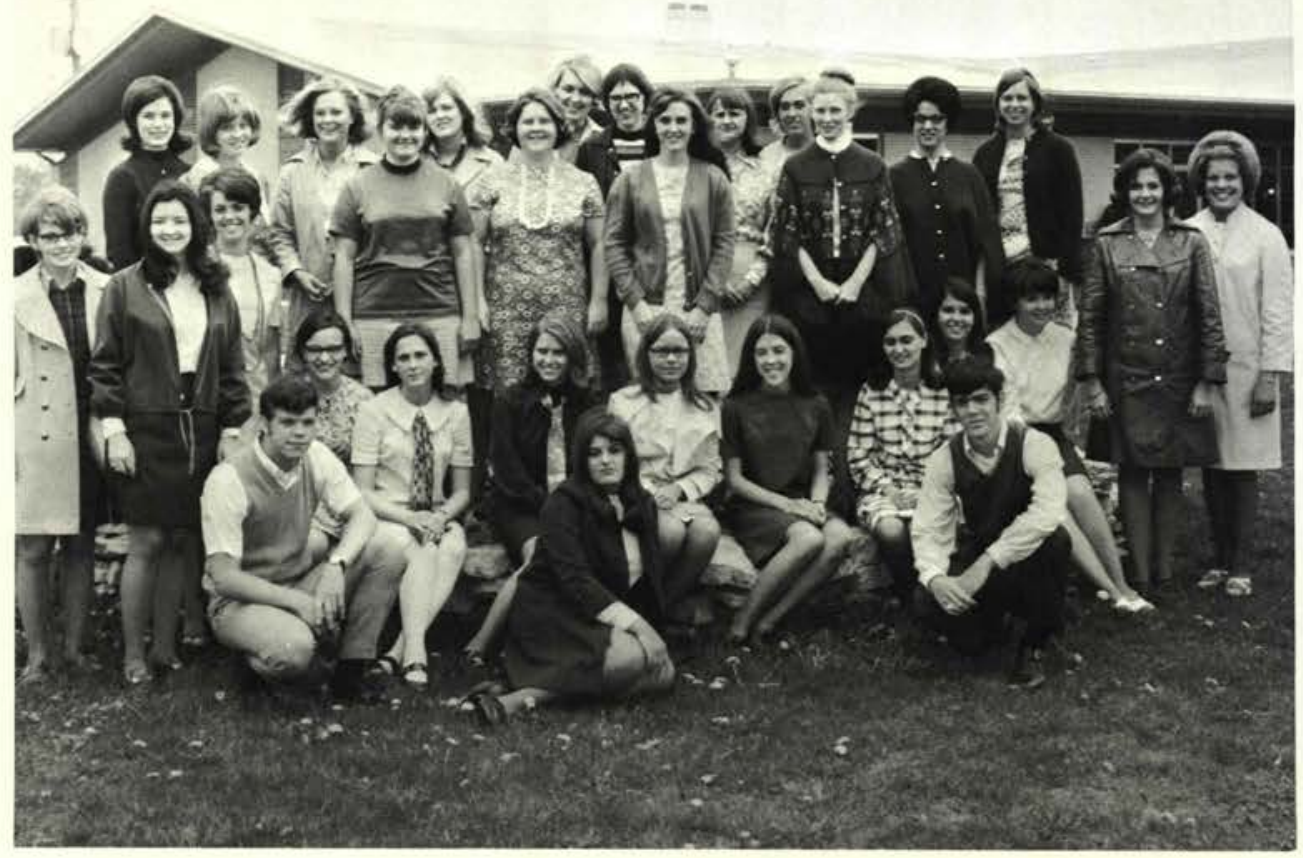

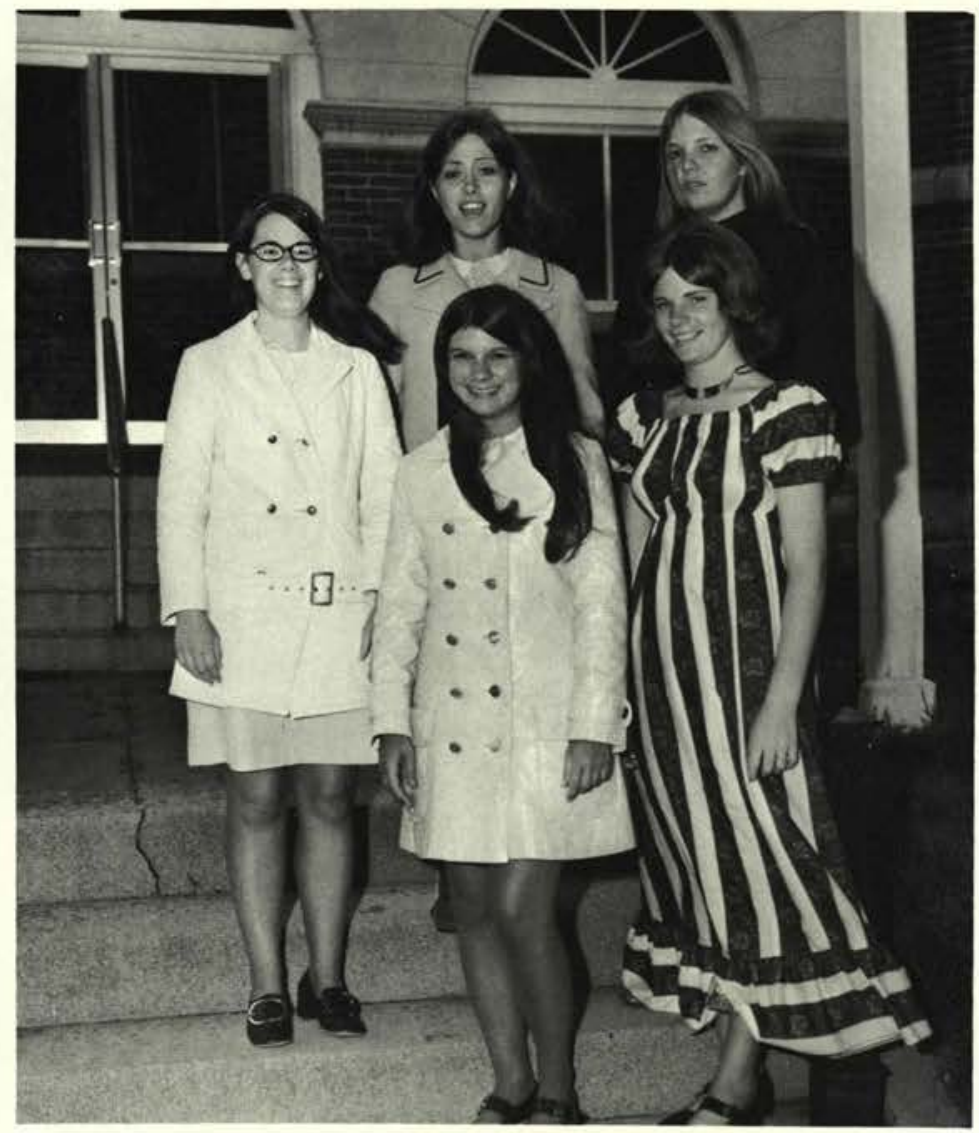

Happy Timers \& Joy Clubs

\author{
FRONT ROW: \\ Bev Kemp \\ Phyllis Codr \\ BACK ROW: \\ Mary McMurtry \\ Linda Chute \\ Linda Severs
}




\section{Military Prayer Band}

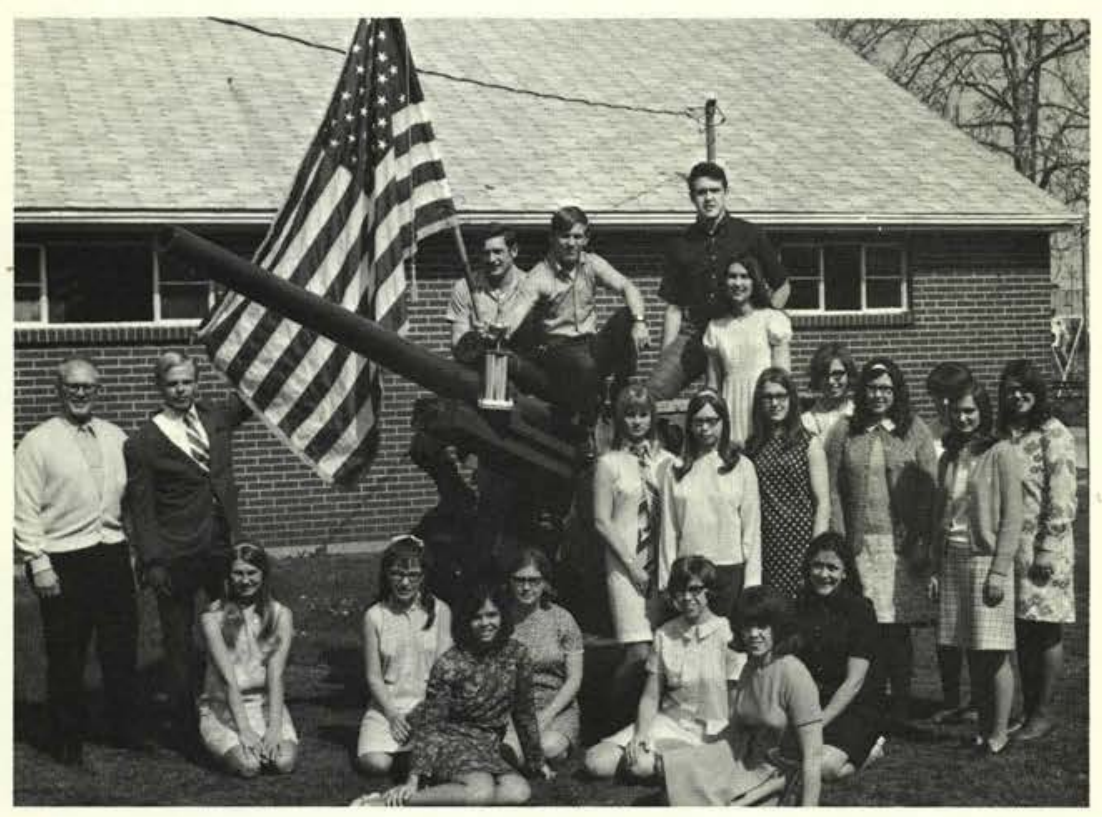

FRONT ROW:

Sue Scott

Betty Stiles

Diane Cockerill

Annie Beitler

Shirleen Morrow

Sharon Mummery

Laura Rodriguez

\section{BACK ROW:}

Dr. Jack Scott, Advisor

Dave Mallinak

Tom Abbey

Jake Tipton

Andy Amos

Debby Hostetler

Bev Heyl

Bernice Penegor

Bonnie Hostetler

Susan Myer

Ruth Steyer

Freda Witt

Carolyn Stubrich

Cheryl Holtz

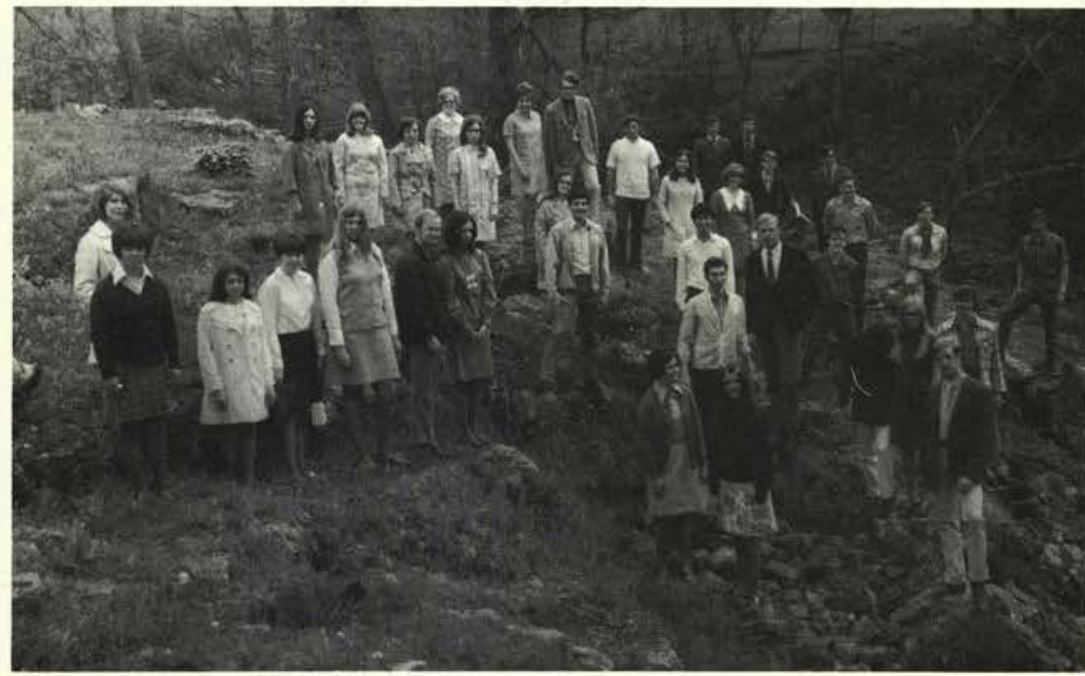

\section{Eva Morse}

Suzanne Hale

Kathy Strawn

Freda Witt

Linda Severs

Mike Gallogly

Sharon Gillogly

Mary Lou Lippert

Ellen Young

Carol Keis

Carol Urban

Bernice Penegor

Vicki Imbro

Jim Griffith

Linda Willms

Chuck Rowe

Jeff Lough

Becky Williams

Alex Konya

Dan Thompson

Cathy Endres

Priscilla Russell

\section{Sword Bearers}

Dave Peters

Ralph Perkins

Sue Moore

Andy Amos

Tom Abbey

Sam McMillen

Carol Anne Bo

Jeff St. Clair

Dave Mallinak

Paul Radcliff

Dave Globig

Dave Price

Don Davis

Don Hendrickson 

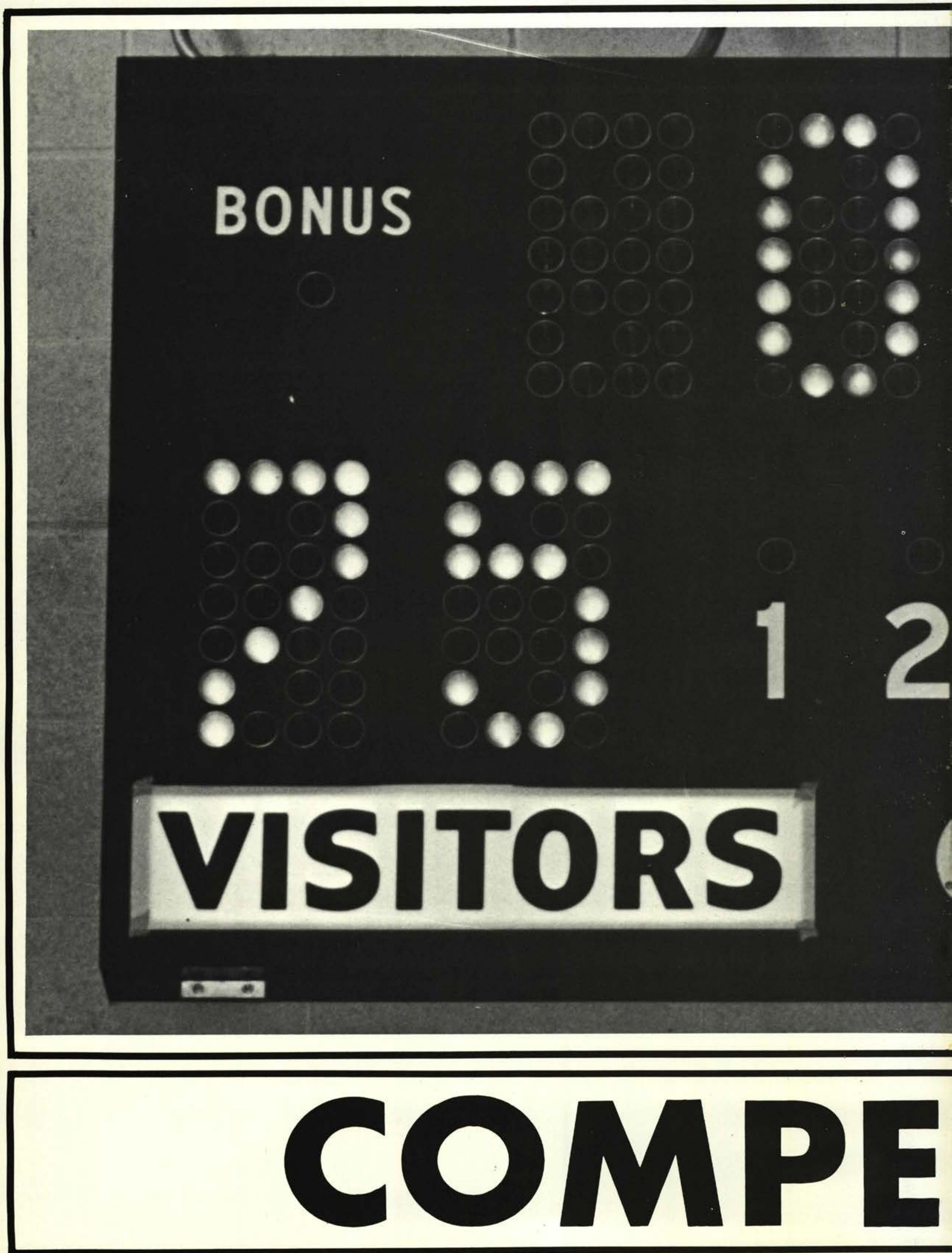


\title{
Field Hockey
}

\author{
FRONT ROW: \\ Elaine Kalnbach \\ BACK ROW: \\ Connie Pumpelly, Manager \\ Brenda Tompkins \\ Linda Y oung \\ Sally Miller \\ Rae Belle Fisher \\ Jan Martin \\ Jan Peterson \\ Diane Kemp \\ Bev Pestel \\ Pam Branning \\ Billie Thomas \\ Miss Kearney, Coach
}

\section{Records}

University of Dayton

Univ. of Cincinnati

Wittenburg

Univ. of Cincinnati

University of Dayton

Captain - Brenda Tompkins, Joy

Trefzger

Coach - Miss Kearney

Manager - Connie Pumpelly

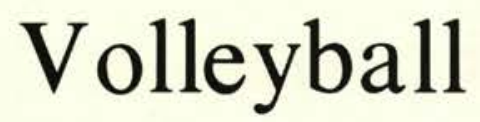

\author{
FRONT ROW: \\ Elaine Kalnbach \\ Bev Pestel \\ Kathy Berry \\ Billie Thomas \\ Pam Branning \\ BACK ROW: \\ Miss Kearney, Coach \\ Jan Martin \\ Karen DeMars \\ Linda White \\ Brenda Tompkins \\ Connie Pumpelly, Manager

\section{Records}

\section{Defiance}

Wilmington

Mt. St. Joseph

University of Cincin.

University of Dayton

Wittenburg

University of Dayton

Mt. Union

Wooster

Kent State

Ohio Northern
Cedarville

3

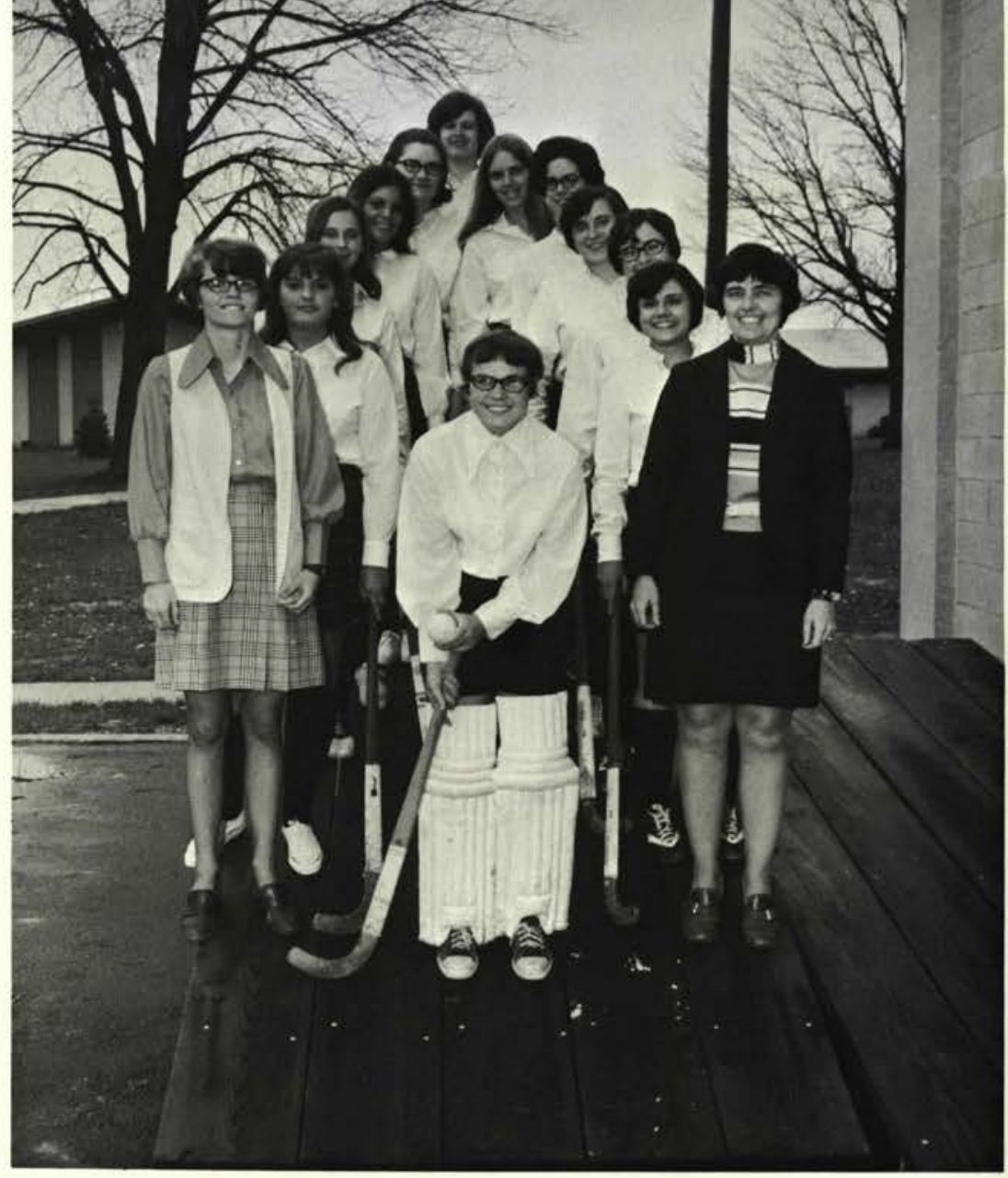

Captain - Jan Martin

Manager - Connie Pumpelly

Coach - Miss Kearney

Loss

Win

Loss

Loss

Win

Win

Loss

Win

Loss

Win

Loss

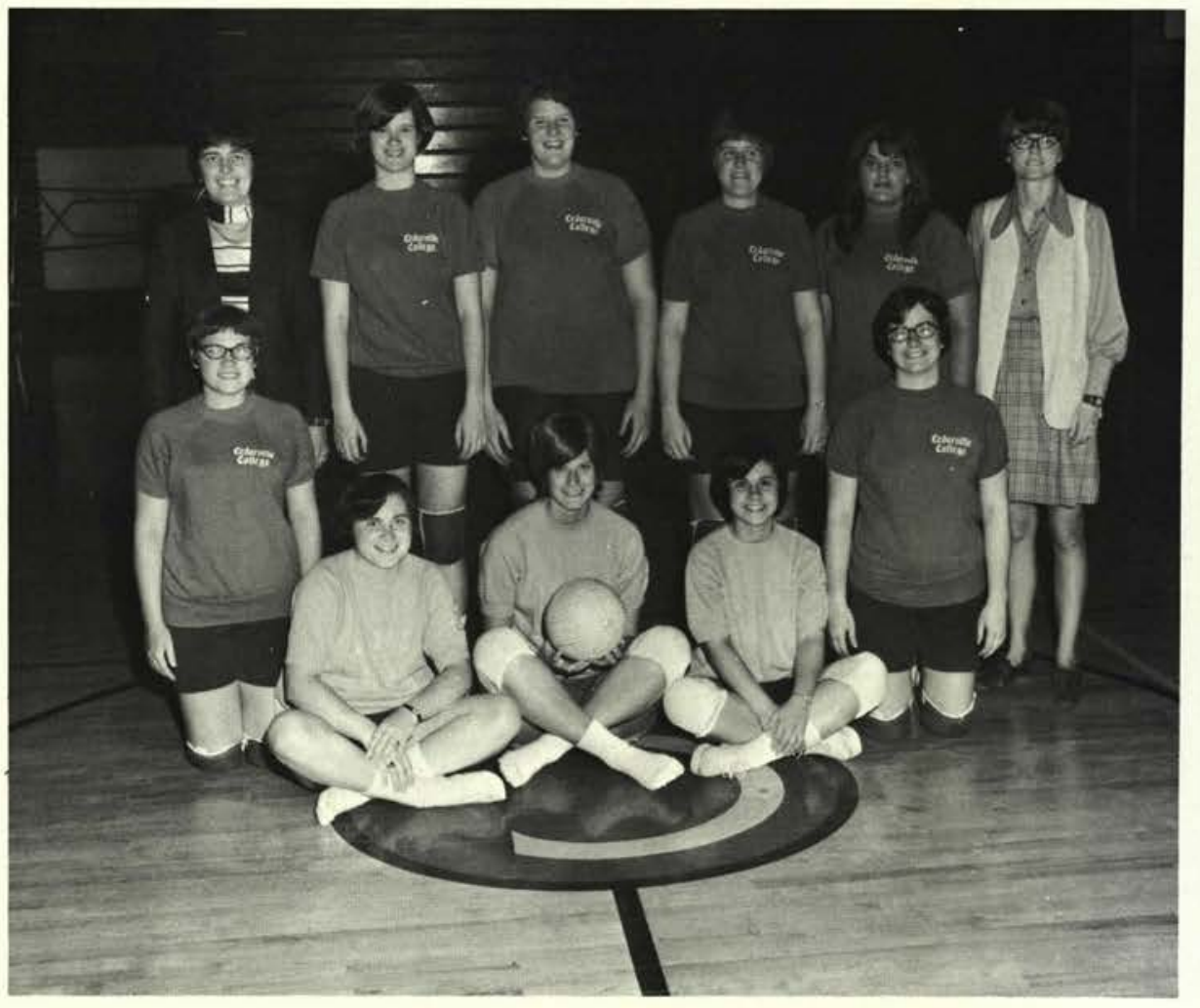



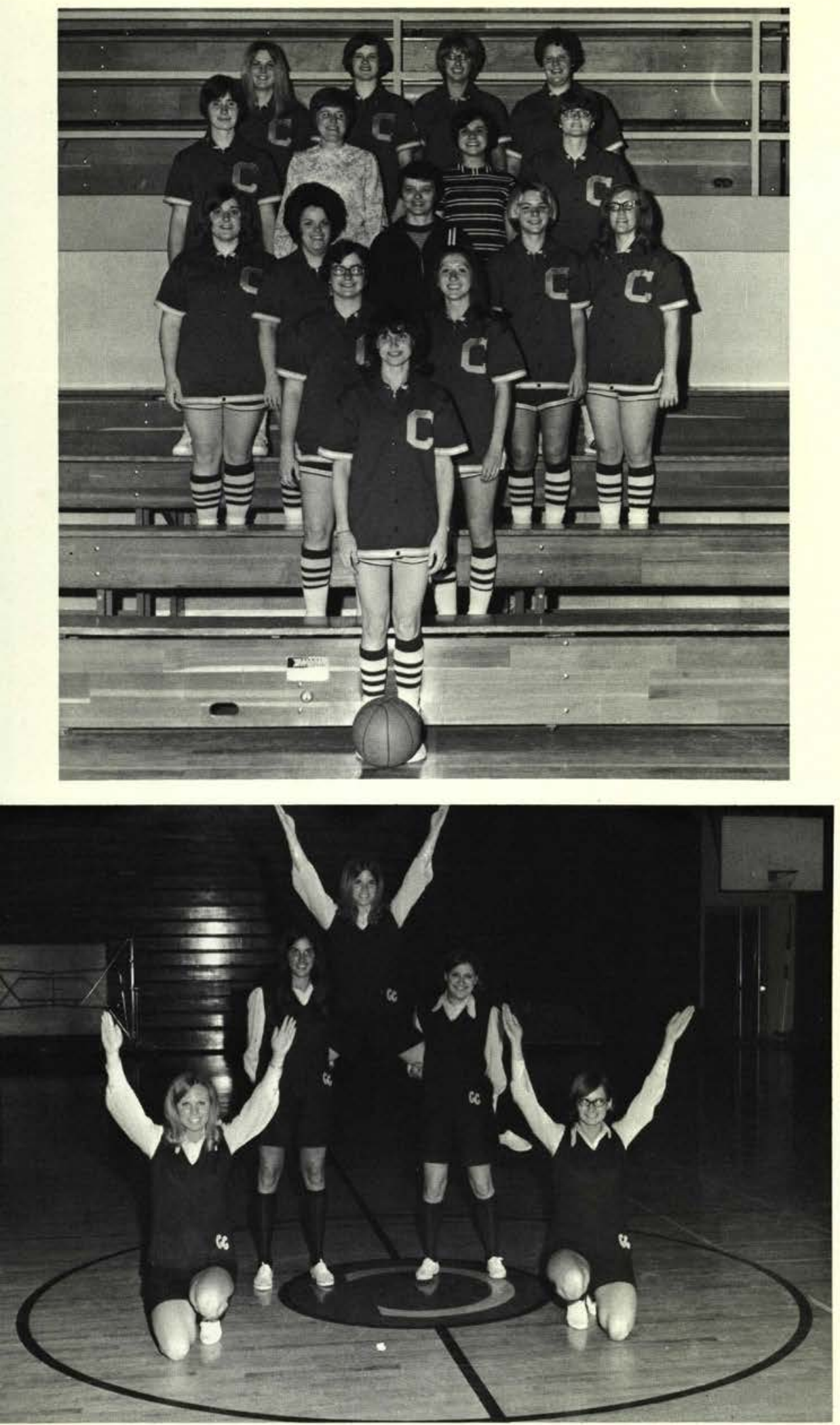

\section{Cheerleaders}

Gail Richardson

Karen Syphers

Bev Davison

Edythe Carity

Debbie DeBoer, Captain

\section{Basketball}

\author{
FRONT ROW: \\ Darlene Mann \\ SECOND ROW: \\ Pam Branning \\ Landra Lowery \\ THIRD ROW: \\ Denise Edwards \\ Diane Love \\ Miss Jeremiah, Coach \\ Sherry Burns \\ Roberta Lutton \\ FOURTH ROW: \\ Linda Osborne \\ Linda White, Manager \\ Billie Thomas, Manager \\ Connie Pumpelly \\ BACK ROW: \\ Jean Taylor \\ Jan Martin \\ Rita Gast \\ Karen DeMars \\ Records
}

University of Cincinnati

Wilmington

Manchester

Mt. St. Joseph

Wilmington

University of Dayton

Ashland

Central State

Mt. St. Joseph

University of Dayton

Alumni
Wittenburg
Cedarville

$36 \quad 34$

$31 \quad 63$

$22 \quad 43$

$51 \quad 48$

$20 \quad 41$

$35 \quad 40$

$45 \quad 30$

$41 \quad 53$

$46 \quad 56$

$25 \quad 35$

$44 \quad 38$

$31 \quad 56$
Managers - Billie Thomas, Linda White Coach - Miss Jeremiah 


\section{Soccer}

BACK ROW:

Randy Ross, Coach

Warren Jenkins

Jim Greening

Bill Henry

Rich Hales

Dan Manross

Yontz Miller

Tom Farlow

Phil McMillen

Dave Beck

Paul Large

Sam McMillen

Randy Bailey

Clif Jensen

Terry Holley

Bobby Austin

FRONT ROW:

Jeff Woodcock

Jim Buzzard

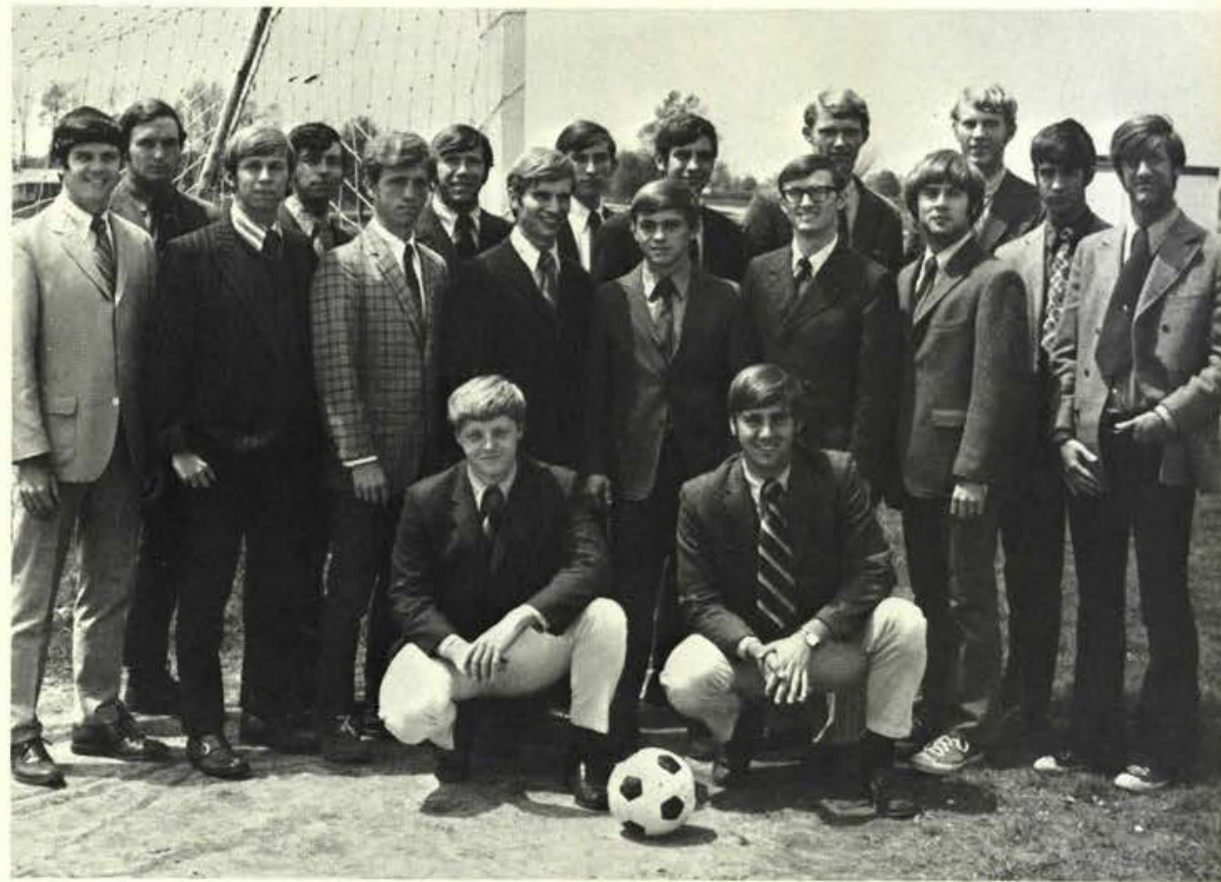

\section{Traveling Soccer Team}

Bobby Austin

Jim Buzzard

Russ Jenkens

Tim Hegg

Dan Acton

Sam McMillen

Craig Stillwell

Jim Greening

Jeff Woodcock

Dan Manross

Clif Jensen

Lee Reno

Louis Tewis

Randy Ross

Don Schill

Warren Jenkins

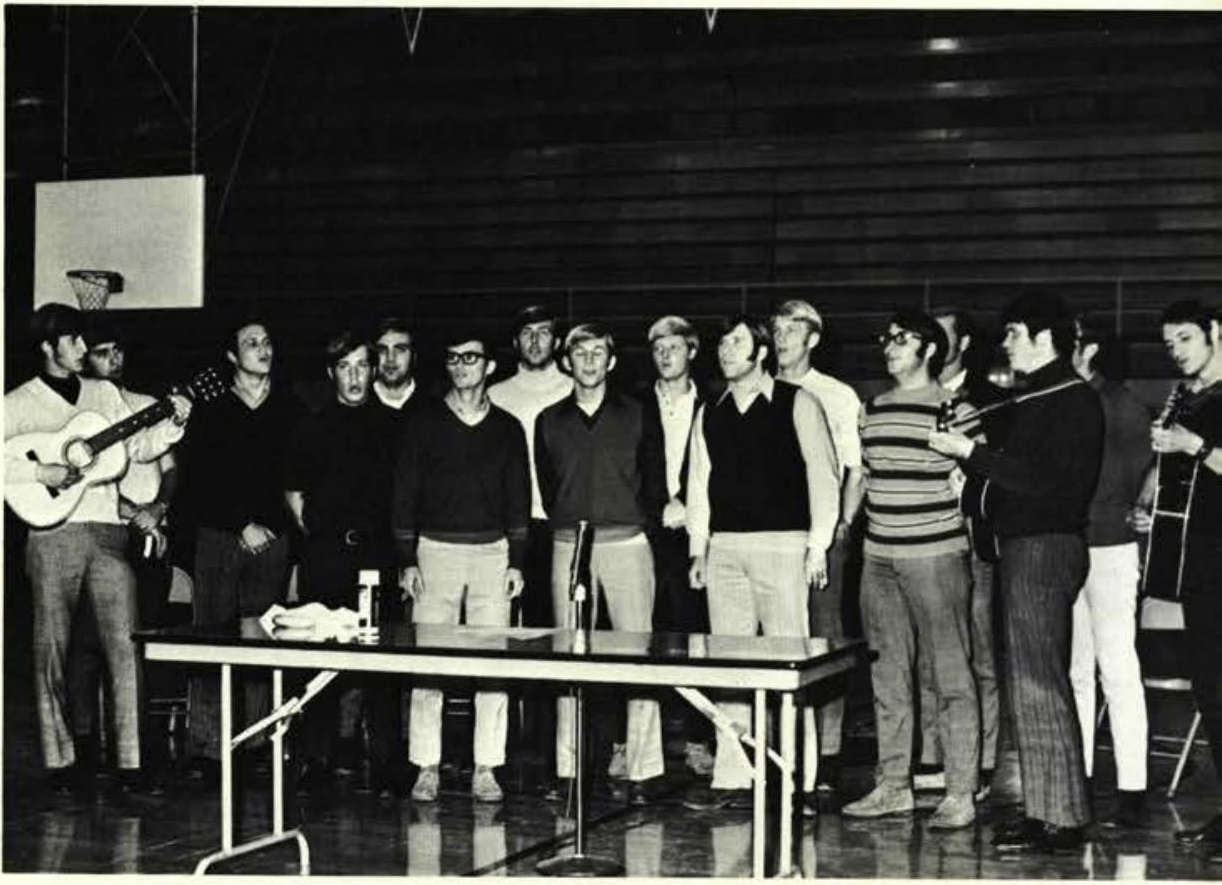




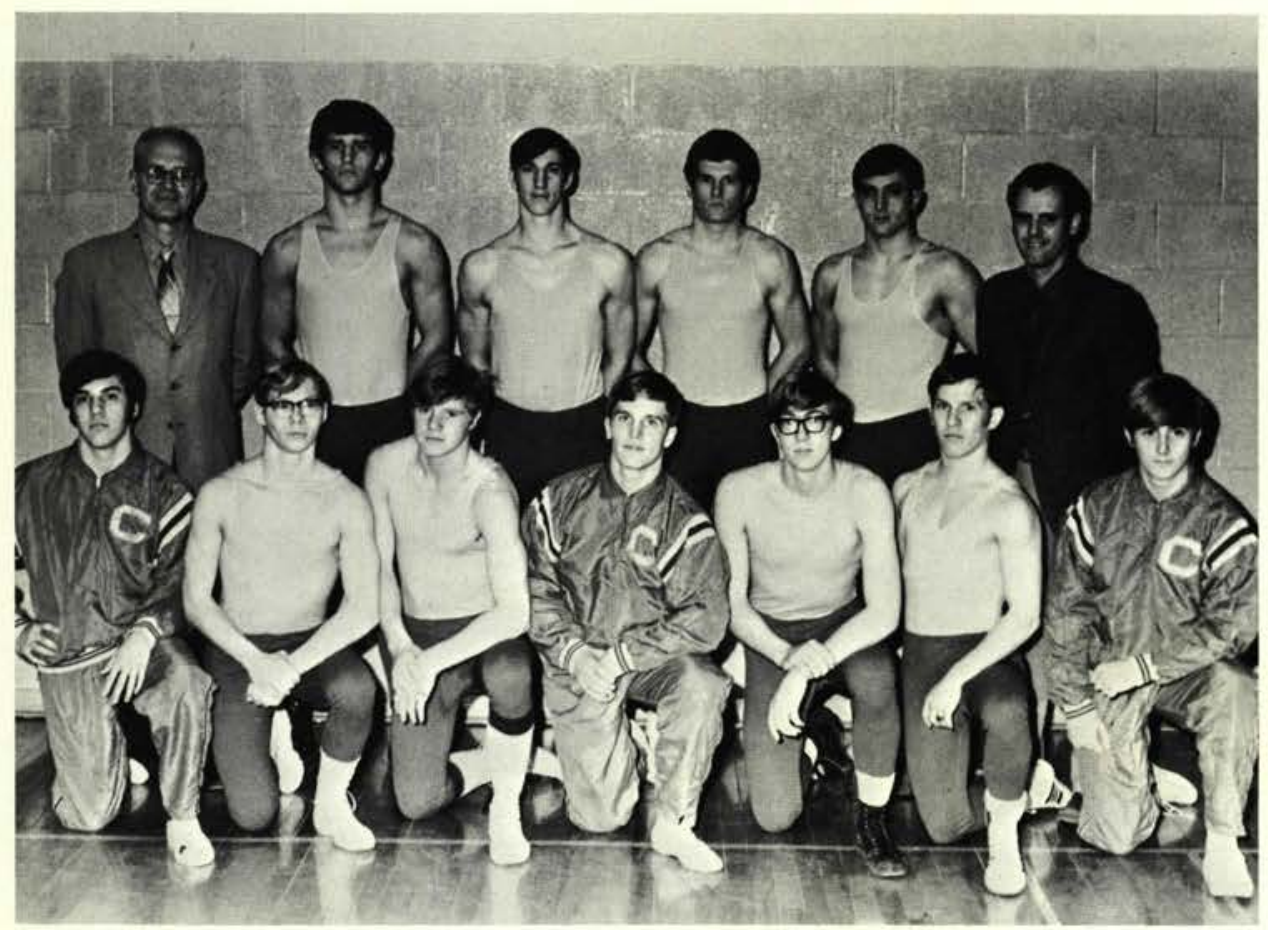

Wrestling

FRONT ROW:

Ron Sumner

Mike Staley

Les Chambers

Lyle Miller

Pete Palpant

Jake Tipton

Jeff Wolfe

BACK ROW:

Dr. Jack Scott, Coach

Mark Stevenson

Bill Tobias

Steve Francis

Tim Northey

Pastor Harold Green, Coach

\section{Soccer Records}

$\begin{array}{llc}\text { Grove City } & 7 & 0 \\ \text { BBS } & 4 & 2 \\ \text { Houghton } & 4 & 2 \\ \text { Dayton } & 7 & 0 \\ \text { Urbana } & 0 & 7 \\ \text { Transylvania } & 3 & 4 \\ \text { Wilberforce } & 0 & 5 \\ \text { Bluffton } & 0 & 1 \\ \text { Wilmington } & 4 & 3 \\ \text { Walsh } & 1 & 1 \\ \text { Berea } & 2 & 1 \\ \text { Kenyon } & 2 & 3 \\ \text { Defiance } & 2 & 4 \\ \text { Malone } & 2 & 4 \\ \text { Wright State } & 6 & 1 \\ \text { Miami } & 1 & 0\end{array}$

\section{Wrestling Records}

$\begin{array}{lcc}\text { Wilberforce } & \begin{array}{c}\text { Home } \\ \text { L }\end{array} & \text { Away } \\ \text { Findlay } & & \text { L } \\ \text { Defiance } & & \text { L } \\ \text { Blufton } & & \text { L } \\ \text { Malone } & & \text { W } \\ \text { Wilberforce } & & \text { L }\end{array}$

$M O C$ at Bluffton, finished fifth.

District 22 at Ohio Northern

University - $134 \mathrm{lb}$. division -

Les Chambers

National at Boone, North Carolina - Les Chambers 


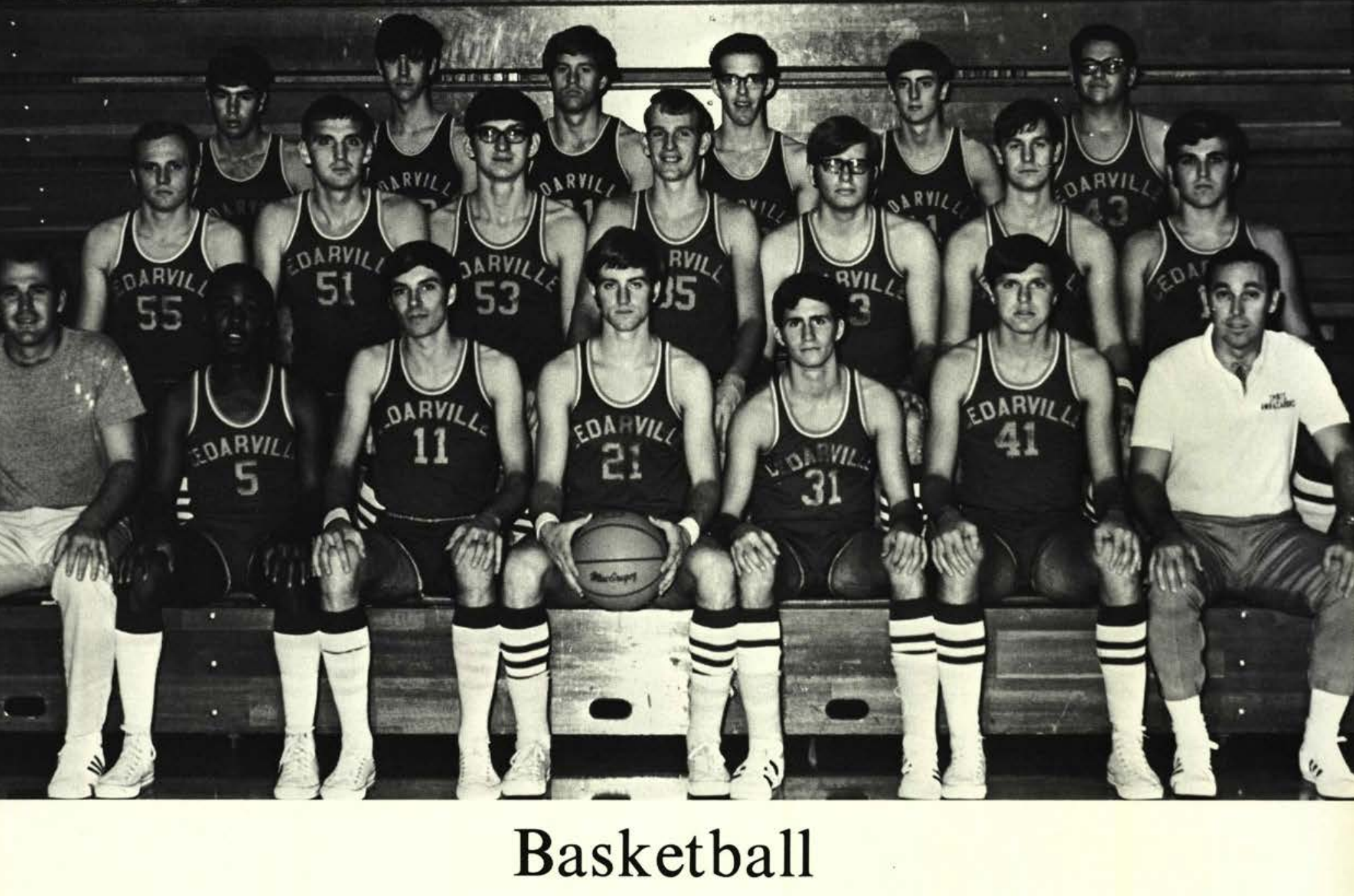

FRONT ROW:

Dick Walker, JV Coach

Clancey Parker

Dave Brannon

Dan Henson

Bill Reno

Lynn Howard

Dr. Callan, Coach

SECOND ROW:

Charlie Williams

Gary Holbrook

Curt Larson

Don Atherton

Gary McDowell

Keith Bailey

Don Acton

BACK ROW:

Dave Schwamburger

Bill Potter

Terry Clapp

Steve Spink

Barrett Luketic

John Harrison 


\section{Baseball}
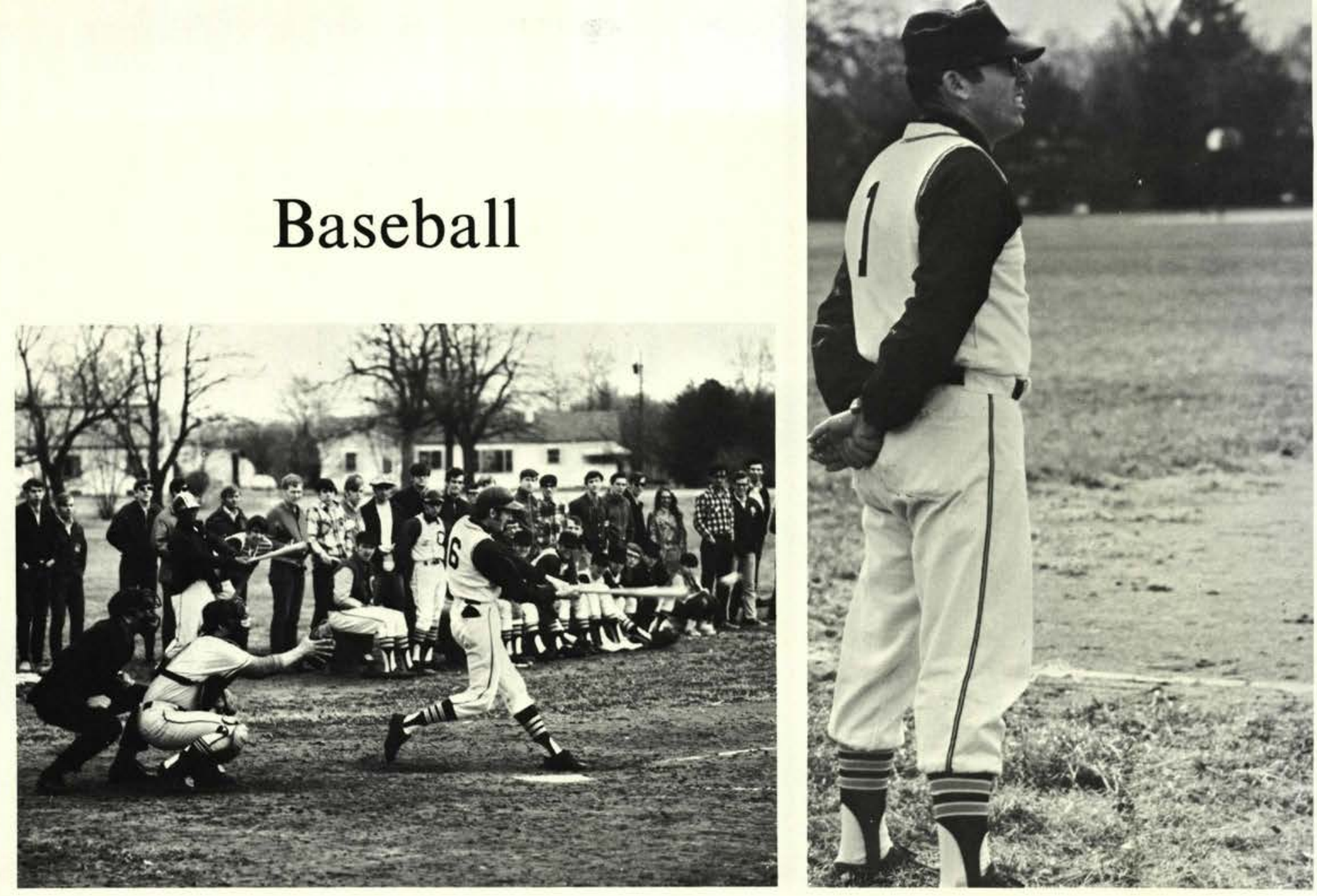

\section{Records}

$\begin{array}{lrr}\text { Rio Grande } & 3 & 4 \\ \text { Rio Grande } & 6 & 7 \\ \text { Dayton } & 8 & 0 \\ \text { Dayton } & 2 & 1 \\ \text { Central State } & 5 & 6 \\ \text { Wright State } & 0 & 5 \\ \text { Wright State } & 3 & 5 \\ \text { Ohio Dominican } & 5 & 3 \\ \text { Ohio Dominican } & 3 & 7 \\ \text { Urbana } & 8 & 7 \\ \text { Malone } & 1 & 2 \\ \text { Malone } & 6 & 5 \\ \text { Earlham } & 6 & 5 \\ \text { Earlham } & 1 & 2 \\ \text { Bluffton } & 2 & 12 \\ \text { Bluffton } & 4 & 2 \\ \text { Mt. Vernon } & 1 & 5 \\ \text { Mt. Vernon } & 16 & 8 \\ \text { Findlay } & 6 & 4 \\ \text { Findlay } & 7 & 4 \\ \text { Urbana } & 10 & 13 \\ \text { Defiance } & 2 & 1 \\ \text { Defiance } & 5 & 6 \\ \text { Central State } & 2 & 7 \\ \text { Central State } & 12 & 15\end{array}$
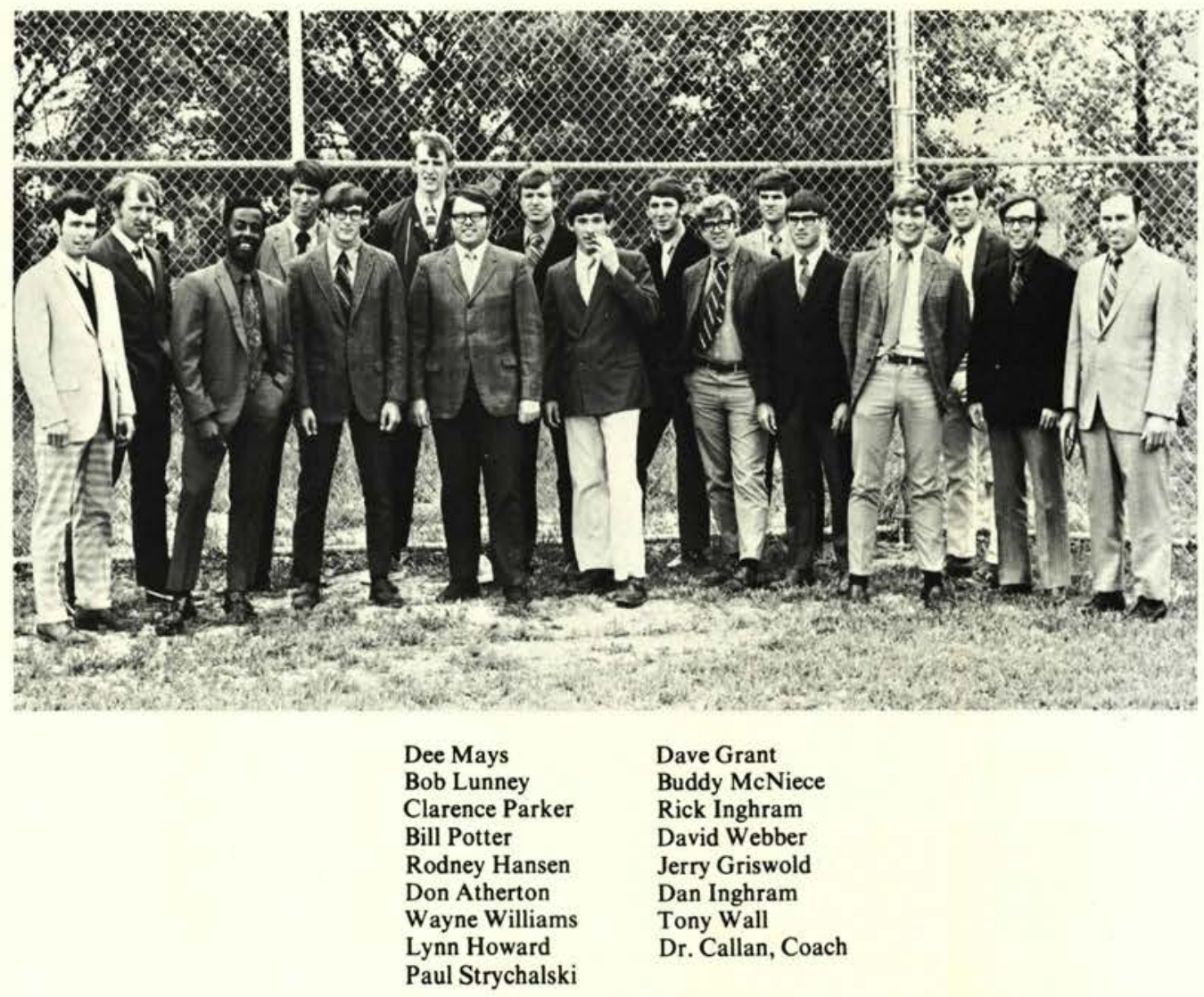

Dave Grant

Buddy McNiece

Rick Inghram

David Webber

Jerry Griswold

Dan Inghram

Tony Wall

Lynn Howard

Dr. Callan, Coach 


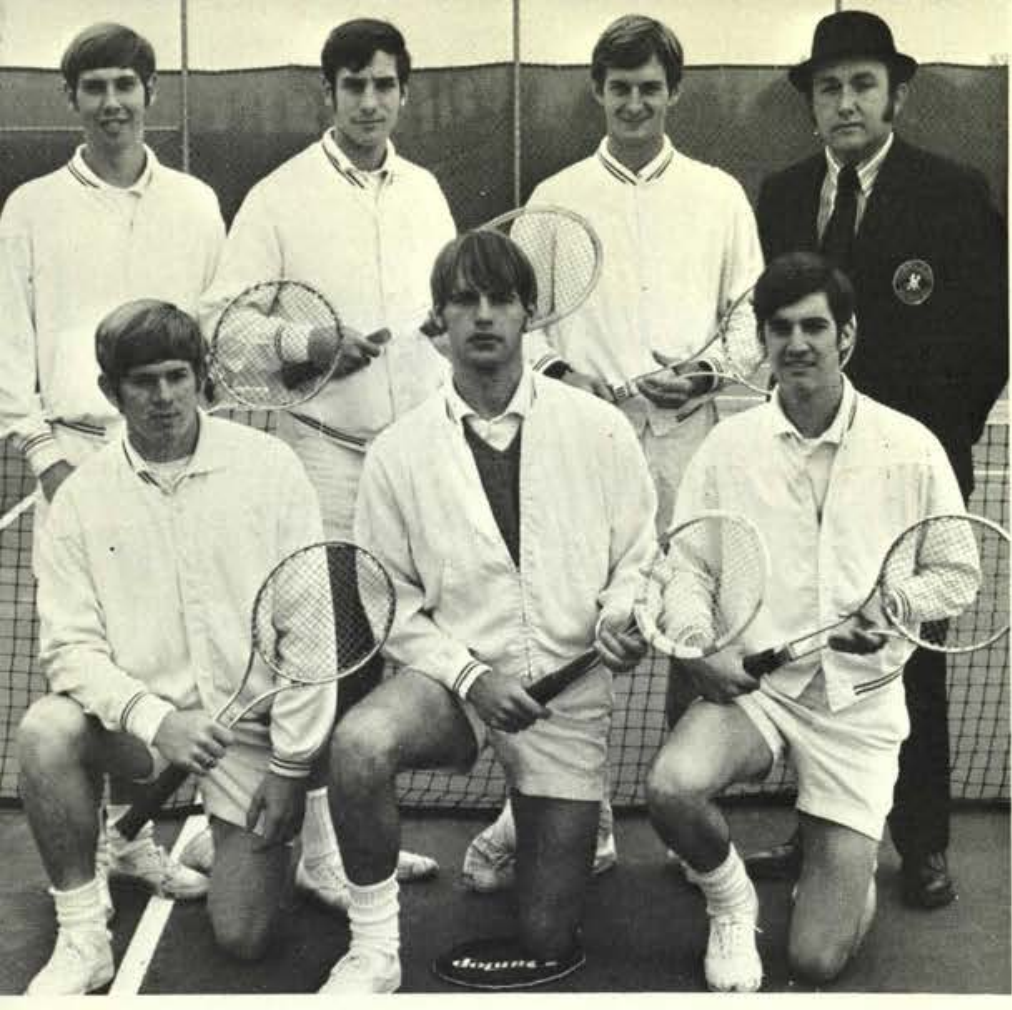

\section{Tennis}

\section{FRONT ROW:}

Dale Wheeler

Mike Coffman

Dave Durham

\section{BACK ROW:}

Dave Nelson

Dave Beck

Dan Martin

Mr. Murdoch, Coach

\section{Records}

\begin{tabular}{lcc} 
Dayton & \multicolumn{3}{c}{ Cedarville } \\
Earlham & 329 & 744 \\
Malone & 7 & 13 \\
Wilmington & $15^{1 / 2}$ & $41 / 2$ \\
Rio Grande & 6 & 4 \\
Defiance & 8 & 2 \\
Central State & 10 & 0 \\
Urbana & 11 & 9 \\
Central State & 19 & 1 \\
Bluffton & 15 & 5 \\
Findlay & 1 & 19 \\
Malone & $41 / 2$ & $151 / 2$ \\
Ohio Northern & 1 & 19 \\
Urbana & $21 / 2$ & $171 / 2$ \\
Walsh & $11 / 2$ & $181 / 2$ \\
Rio Grande & $2^{1 / 2}$ & $17^{1 / 2}$
\end{tabular}

Taylor Invitational - Tenth of twelve teams

Mid-Ohio Conference

Defiance ................... 458

Bluffton ............... 470

Cedarville ................ 476

Findlay $\ldots \ldots \ldots \ldots \ldots \ldots \ldots, 480$

Malone ................484

Wilmington .............. 537

Ohio $\ldots \ldots \ldots \ldots \ldots \ldots .449$

Walsh ................. 451

Defiance .............. 458

Bluffton ................ 470

Cedarville .................. 476

Rio Grande .............. 477

Findlay ............... 480

Malone ................. 484

Wilmington ............... 537

FRONT ROW:

John Myers

Jim Rickard

Dr. Gromacki, Coach

\section{BACK ROW:}

Thom Kukasky

Dave Schwamburger

Stu Walker

Jim Buzzard 


\section{Track and Cross Country}

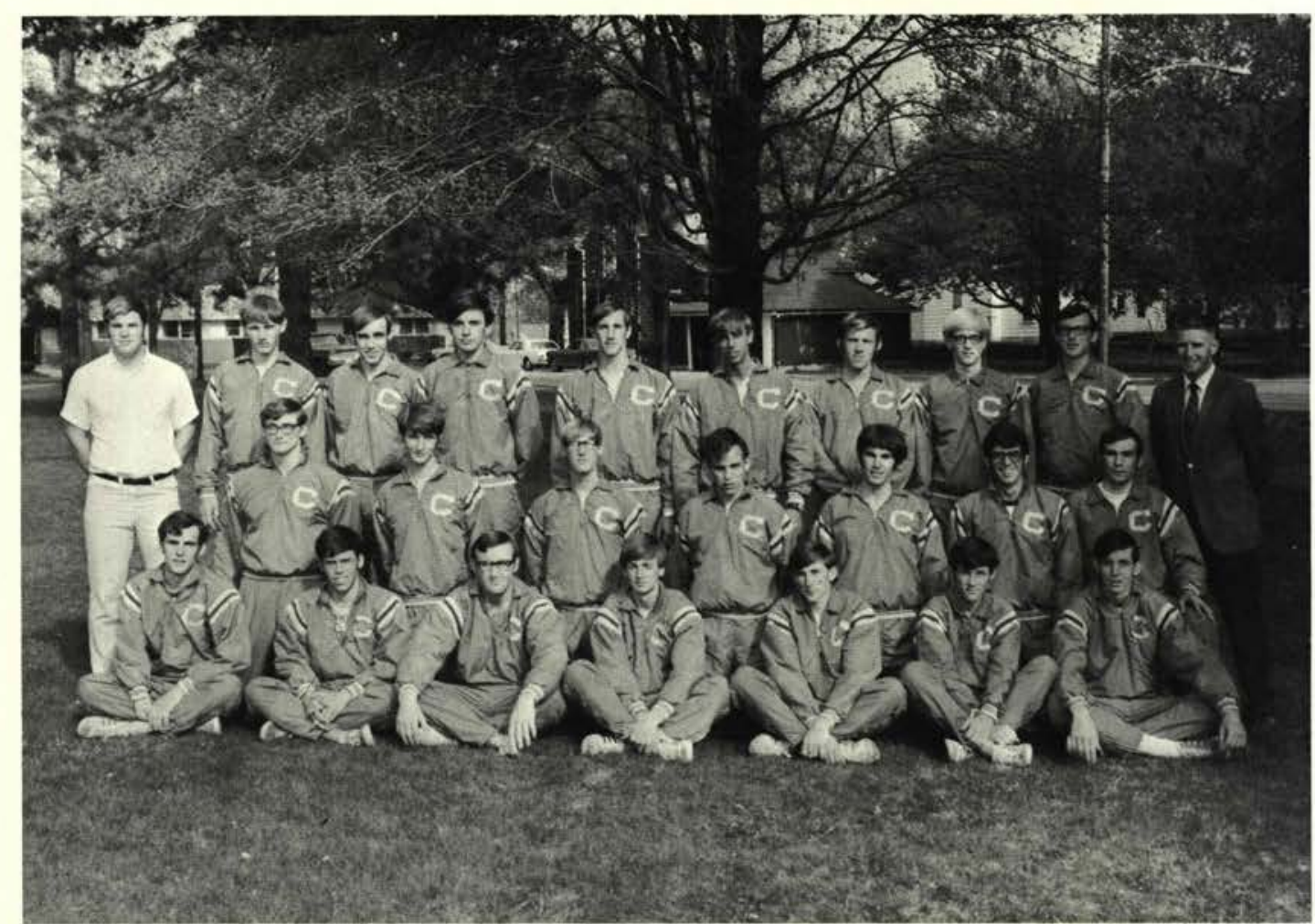

BACK ROW:

Tom Strong

Bruce Klett

Steve Jones

Dave Jewell

Bill Tobias

Tim Lee

Phil Moon

Jim Van Duran

Lyle Rodman

Coach King

MIDDLE ROW:

Dave Globig

Ernie Wellborn

Mark Alexander

Loren Richmond

Bill Bacheller

Brent Myers

Dave Peters

FRONT ROW:

Bruce Smith

Steve Gruber

Phil Stine

Barrett Luketic

Tom Farlow

Ed Howell

Bob Wood 

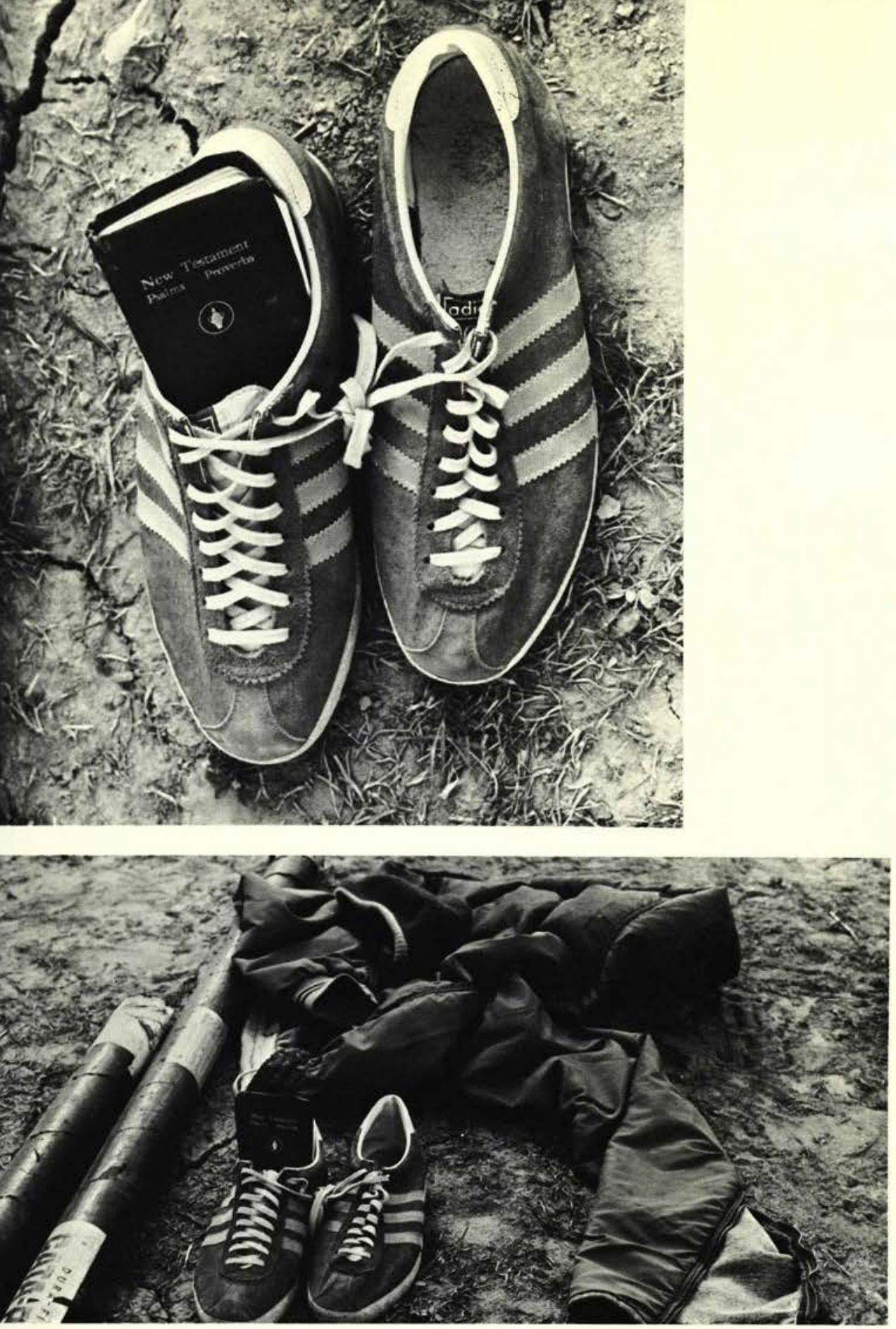

Mt. Vernon

Earlham

Marietta

Wittenberg

Wilmington

Kenyon

Defiance

Wilmington

Ohio Northern

Findlay

Rio Grande

Asburg

District 2 - tied for third MOC - fourth

Track Records 440 Relay

440 Yd Dash

880 Yd Run

440 Yd Int Hurdles

3-mile

Mile Relay

Long jump

\section{Cross Country Records}

$\begin{array}{ll}\text { Bluffton } & 36 \\ \text { Malone } & 27 \\ \text { Central State } & 81 \\ \text { OUTC } & 81 \\ & \\ \text { Mt. Vernon } & 55 \\ \text { Huntington } & 61 \\ & \\ \text { Central State } & 37 \\ \text { Ohio Northern } & 77 \\ \text { Urbana } & 80 \\ \text { Grand Rapids } & 25 \\ \text { Defiance } & 63 \\ \text { Anderson } & 95 \\ & \\ \text { Marion } & 29 \\ \text { Rio Grande } & 30 \\ \text { Rindlay } & 44 \\ \end{array}$

INVITATIONALS

Adrian invitational -9 out of 15

Earlham invitational -6 out of 17

All-Ohio - 10 out of 14

Conference - fourth

NAIA District 22 - fourth

NAIA Nationals at Kansas City -41 in nation
Gruber-Stine

51.2 Welborn

Welborn

5:35.5 Jewell

3:25.2 Luketic-Jones Gruber-Welborn Luketic
Cedarville

23

41

20

32

38

26

16
Cedarville

90

$191 / 3$

72

66

116 
ithin

days

rated

ion to
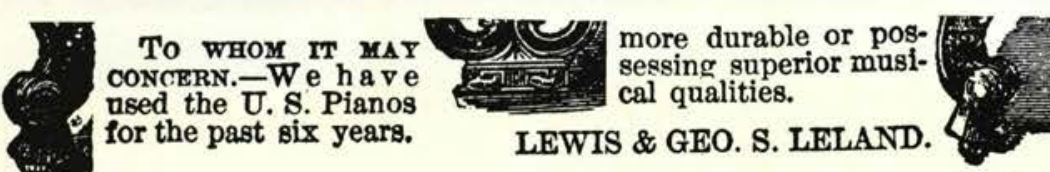

the United States will satisfy

$\mathbf{k}$ in America. We make this

ted Circular containing full particulirs, but also ampany in Piano in the world at any price person. Please state where you saw this notice.

ad States Piano Co., 810 Broadway, Neve York.

\section{WINDING} $F$ ONIT

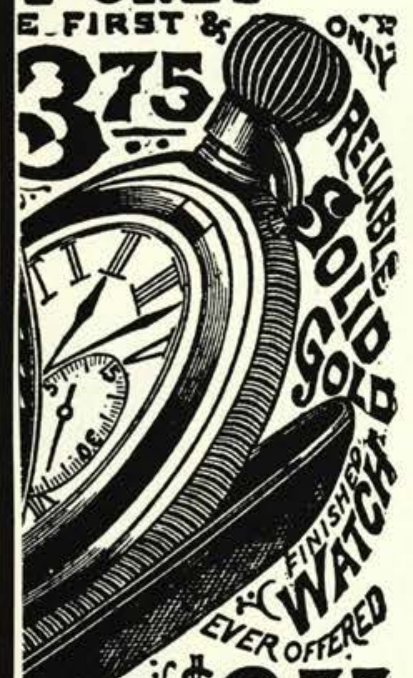

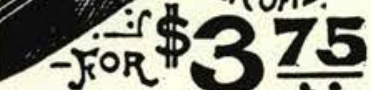

th Consumers at once and Poratur es by registered mall- 0 re $h$ warranted to keep accurate novement hunting case watch of each kind s7 60; oither ngraved cuses or, if you perfer packing. expresichargen, etc. packing. expresscharges, etc. 0 il. Anytime within 60 daya bed movey cheerfully returnwatches soud \$1.0O for each r monoy is sent with order wo C Chr if Cran Had Hede cosis besulfuidesigns and wo will ngnally sold by dealers-for ton asually ading purposes. Ponitivels ang purposes. DWARDs az CO.

er \& Manuracturers. roadway. Now York.

\section{NTHS}

\section{ADIES'}

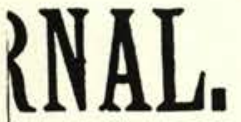

STRATED.

Y PRINTED.

VARIETY.

MESTIC STORIES.

:S' JOURNAL

ndsomest and CheapVOCOLUMNS devoted $\checkmark$ Work Department, in's Exchange, Health F UIY $\mathrm{E}$ IT BTRATIONS.

PFR VFAR

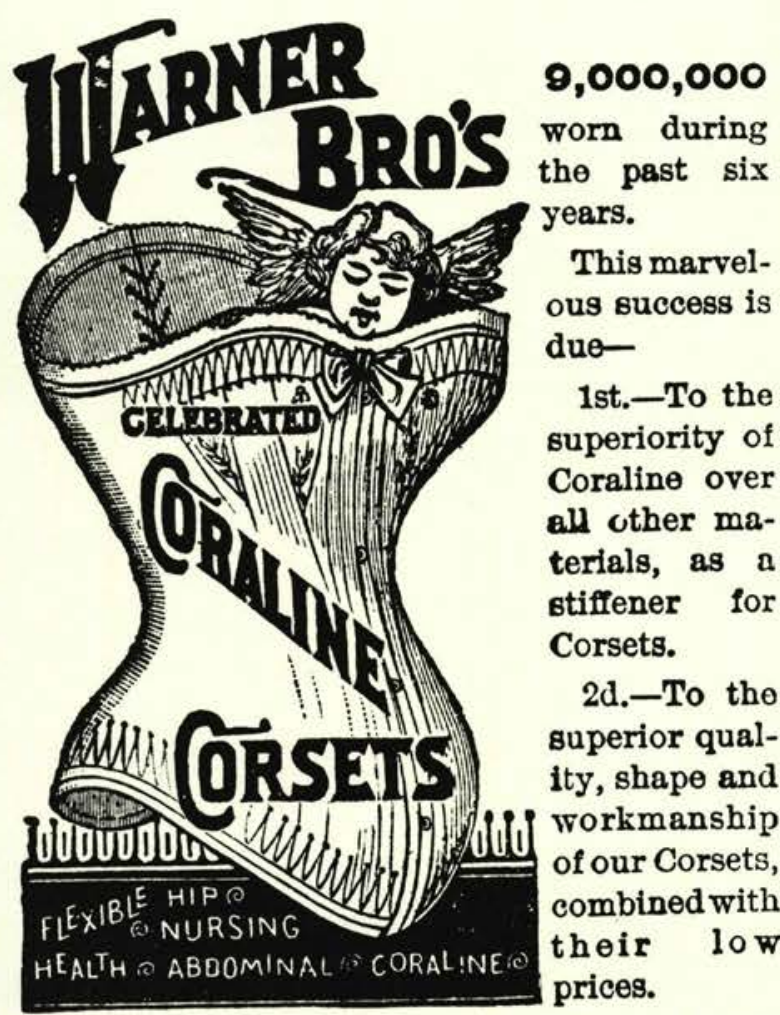

Avoid cheap imitations made of various kinds of cord. None are genuine unless

"DR. WARNER'S CORALINE" is printed on inside of steel cover.

\section{FOR SALE BY ALL LEADING MERCHANTS.}

WARNER BROTHERS,

359 Broadway, Now York Clty.

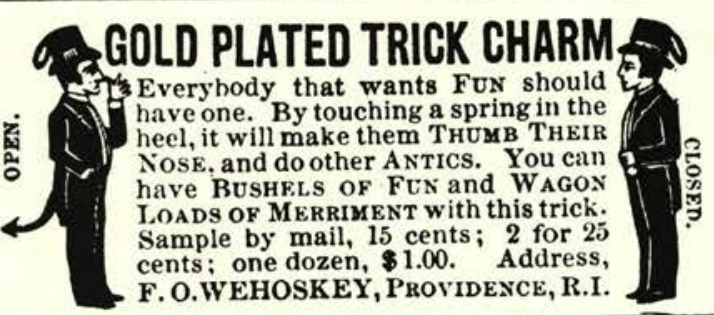

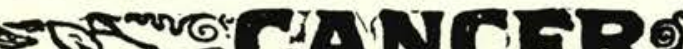
VA ANO SAFELY REMOVED WITHOUT OIII WITHOUT: CHARGE. UNLESS:

SUCCESSFUL. DRW.LEON FLEMING.124W.42nd ST_NEW YORK:

TIIEOIITIA, THE LAND OF FLOWERS.

Send 50 cents, express or money order, and receive by mail, postpaid, 15 large pkts. choice new seeds, growth of 1886 . We will send 35 pkts. for \$1. Pansies, Marigolds, Mignonette, Asters, Phlox Smilax, Cockscomb. Dahlias, single and double: Balsams, Stocks, Hollyhocks, Candytuft, etc. All seeds are grown on our own farm. We challenge the world to grow flower seeds to greater perfection. Satisfaction guaranteed or money refunded. GEO. W. SESSIONS, Seed Farmer \& Nurseryman, San Mateo, San
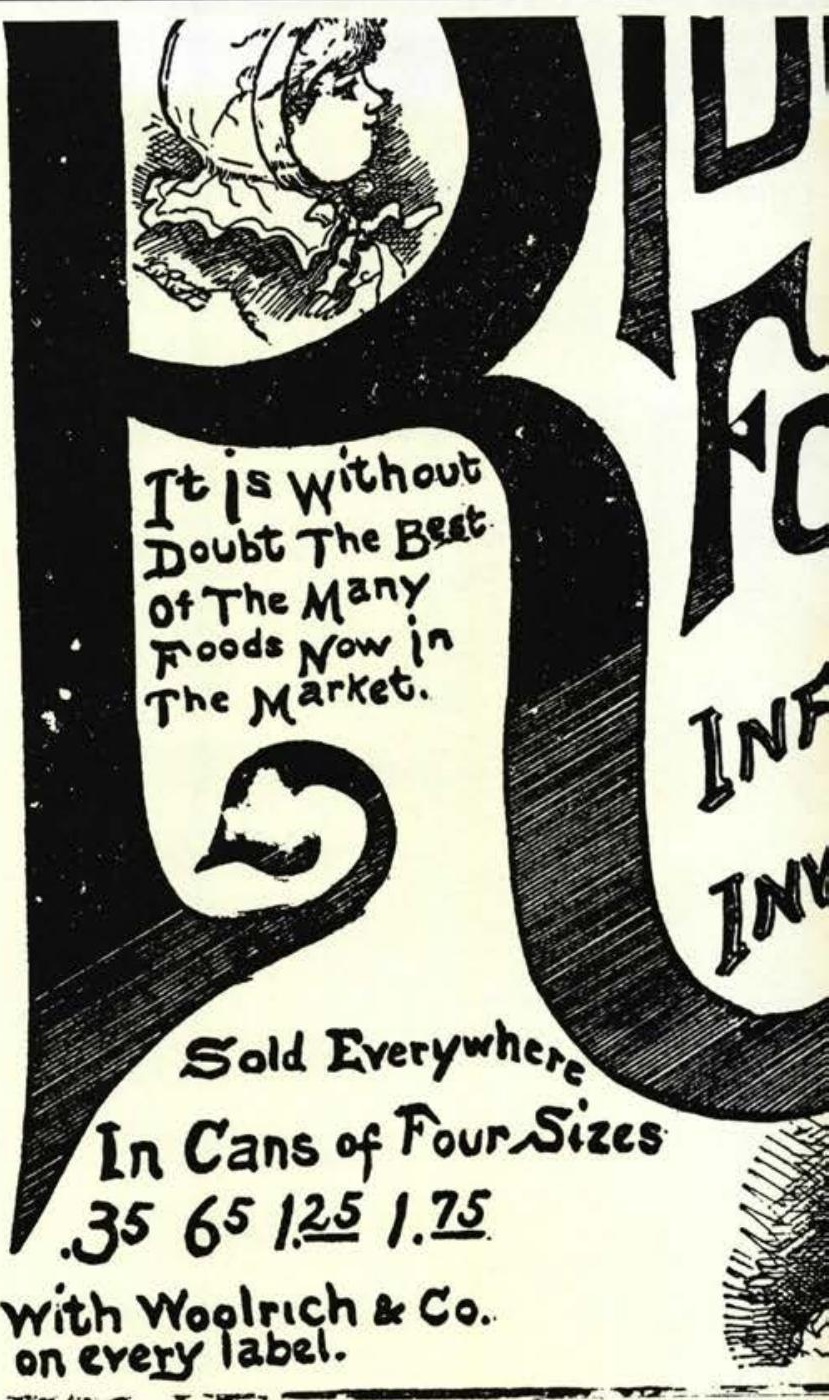

Tt is without Doubt The Best of The Many Foods Now in The Market.
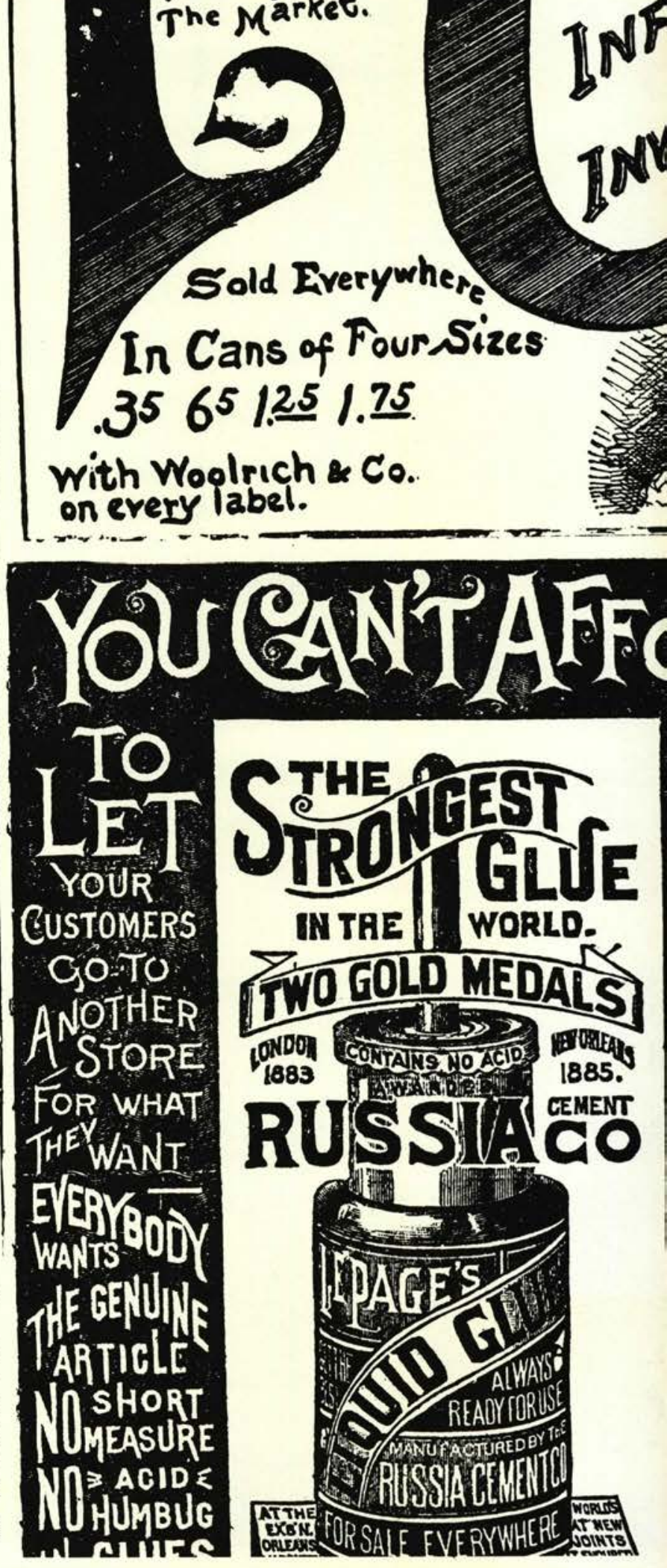


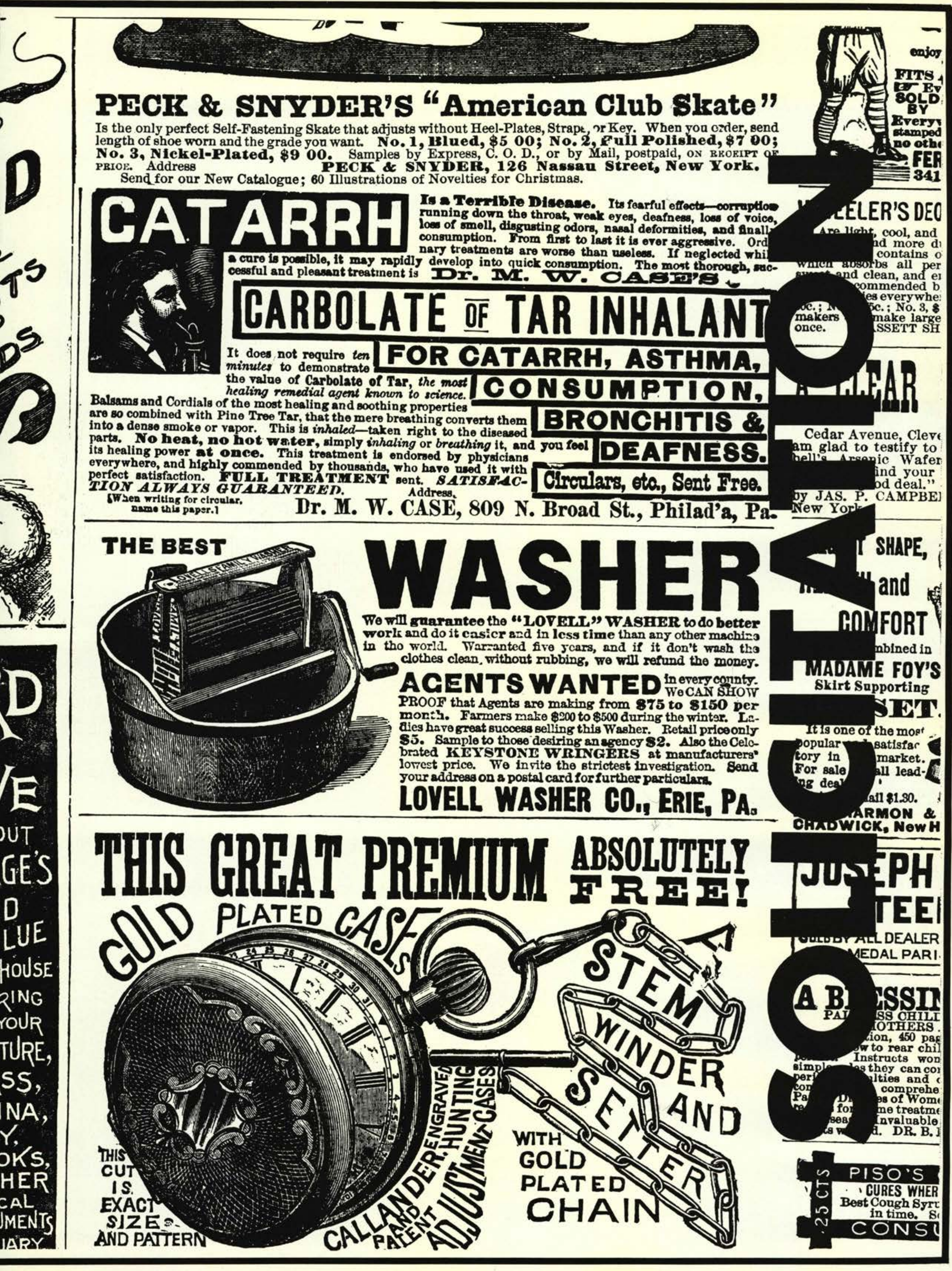




\section{Super Value}

Cedarville

Super Value

\section{Groceries \\ Produce \\ Meats}

Phone: 766-1201

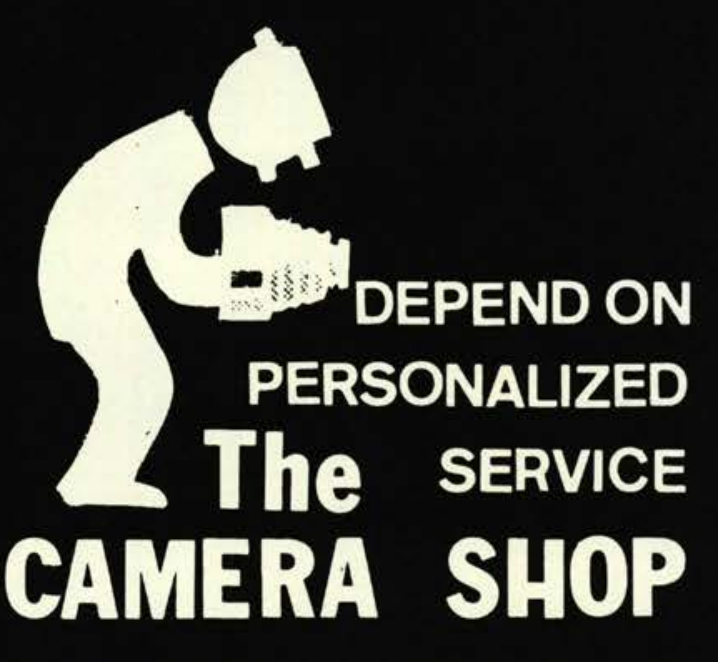

34 W. MAIN XENIA,OHIO

\section{PHONE: 372-1469}

\section{CALVARY BAPTIST CHURCH}

Corner Sixth and Race Auc.

Byesville, Ohio

H. P. House, Pastor

"Presenting the Christ of the Scriptures" 


\section{Chaplin Cleaners \\ Laundry Service}

Cleaning - Shoe Repair

Phone 766-3781

Cedarville, Ohio

\section{"Holding Fast \\ The Word of God"}

West Endicott Baptist Church ENDICOTT, NEW YORK

John E. Trautman, Pastor

\section{PARENT PATRONS}

Rev. and Mrs. Wayne K. Anderson

Monroe, Iowa

Noel Atherton

Vandalia, Ohio

Mr. and Mrs. Robert M

Austin

New Milford, New Jersey

Mr. and Mrs. Virgil Barb

Akron, Ohio

Mr. and Mrs. Steve P. Barbely

Mansfield, Ohio

Mr, and Mrs. Robert H. Barr

Elyria, Ohio

Mr, and Mrs. John J.

Barraclough

North Tonawanda, New York

Mr. and Mrs. James W. Bayne

Little Hocking, Ohio

Mrs. Mary Ellen Beck

Harvey, Illinois

Elmer M. Beitz

Cheek towaga, New York

Norman W. Bergen

Waterloo, Iowa

Mr. and Mrs. Ed K. Bo

Rochester, New York

Ronald Bohn

Springfield, Ohio

Mr. and Mrs. Richard Borders

Kansas City, Kansas

Mr. and Mrs. Dale Boyd

Millersburg, Ohio

Don Bradford

Coshocton, Ohio

Mr. and Mrs. John Branon

Portsmouth, Ohio
Mr. and Mrs. Don Briggs

Caldwell, Ohio

Rodman Brown

Arcanum, Ohio

Milford E. Busho

Austin, Minnesota

Mrs. Lois Byers

Boonville, Indiana

Mr. and Mrs. Carlyle Campbell

Indiana, Pennsylvania

Mr. and Mrs. Robert J.

Catteau, $\mathrm{Sr}$

Parma, Ohio

$\mathrm{Mr}$. and Mrs. George $\mathrm{F}$

Chandes

East Moline, Illinois

Donald C. Chrestensen

Berea, Ohio

Mr. and Mrs. Willard

Chute, Jr.

Dublin, New Hampshire

Mr. and Mrs. Wesley Clapp

Meridian, Idaho

Mr. and Mrs. Edward E. Clark

Spokane, Washington

Mr. and Mrs. Ralph Clarke

Amelia, Ohio

Mr. and Mrs. Kenneth R.

Cleaves

Levittsburg, Ohio

Dr. and Mrs. Jack H. Cline

Rockbridge, Ohio

Mr. and Mrs. Roger Closson

Berea, Ohio

Mr. and Mrs. Emery Cogan

Wauseon, Ohio

Mrs. C. F. Cribbs

Sheffield Lake, Ohio
WHEELERSBURG BAPTIST

CHURCH

Box 224 Wheelersburg, O.

Donald Grollimund, Pastor

STUDENTS AT CEDAR VILLE:

Vivian Dials

David Ferguson

Bonnie Nolen
Fundamental

Independent

Missionary

Premillenial 


\section{WEST CHICAGO BAPTIST CHURCH}

19510 West Chicago Blvd. Detroit, Mich.

Howard K. Miller, Pastor

"The Church with the upward look and

the outward reach"

STUDENTS AT CEDARVILLE:

Larry Smith

Michael Wilkinson

\section{CRITERION \\ XENIA, OHIO \\ MEN'S CLOTHING AND \\ FURNISHINGS}

\author{
VARSITY TOWN - ARROW \\ MAC GREGOR - STETSON \\ PALM BEACH
}

\section{PARENT PATRONS}

Mr. and Mrs. Dudley Bierbaum Bunker Hill, Illinois

Marcel Cotnoir

Ste. Therese, Quebec, Canada

$\mathrm{Mr}$. and Mrs. James Hillery

Alton, Illinois

Mr. and Mrs. Richard Inghram Morning Sun, Iowa

Mr. and Mrs. John Linger

Northfield, Ohio

Mr. and Mrs. Lowell A. Prater

Washington, D.C.

$\mathrm{Mr}$. and Mrs. Richard Selfridge

Elyria, Ohio
Mr. and Mrs, Robert P. Cuenin Amherst, Ohio

Rev. Everett R. Davis

Akron, New York

Floyd A. Davis

Littleton, Colorado

Mr. and Mrs. Raymond Davis

Chautauqua, New Y ork

H. Richard Davison

Arcanum, Ohio

Morgan W. Dawley

Rochester, Michigan

$\mathrm{Mr}$. and Mrs. Ollie De Berg

Reinbeck, Iowa

\section{FIRST BAPTIST CHURCH}

1700 West Oakland Ave.

Austin, Minnesota

David Matthews, Pastor

STUDENTS AT CEDAR VILLE:

\begin{abstract}
Connie Busho
Craig Busho

Larry Madison
\end{abstract}

Carol De Boer

\section{"Earnestly Contending for the Faith",}

\section{PARENT PATRONS}

Clarence De Bruine Battle Creek, Michigan

Mr. and Mrs. Leon De Mars

Columbus, Ohio

Mrs. and Mrs. Emery T. Dials

Wheelersburg, Ohio

Mrs. Betty Dilks

Geraid, Ohio

Mr. and Mrs. Donald Dixon

Spokane, Washington

Maurice Dutton

Lopeer, Michigan

Delos and Maryella Edwards

Albion, Iowa

Mr. and Mrs. LeR oy Ehnis

Brooklyn, Michigan

Mr. and Mrs. I. L. Ellis

Arlington, Virginia

James F. Ely

Lorain, Ohio

Mr. and Mrs. Richard Emerson

Corry, Pennsylvania

Mr. and Mrs. Fred T. Emuson

Richmond Heights, Ohio

Mr. and Mrs. George $\mathrm{O}$.

$$
\text { Endres }
$$

Willingboro, New Jerse

Mr. and Mrs. Jack Erickson

Hobart, Indiana

Mr. and Mrs. Vern L. Farley

Omaha, Nebraska

Mr. and Mrs. Dale Farlow

Camp Point, Illinois

Mrs. Lillie M. Farmer

Mentor, Ohio

Mr. and Mrs. James W. Farrar

Portsmouth. Ohio
Mr. and Mrs. John Fiest

Elyria, Ohio

Michael P. Flood

Lake Orion, Michigan

Rev. and Mrs. F. Lester Fogle

Bangasson, Central African Republic

Edythe and Carl Ford

Marion, Ohio

Mr. and Mrs. Fred Frye

Hollard, Ohio

Mr. Russell Fulcomer

Bolivar, Pennsylvania

James H. Gabriel

Chicago, Illinois

Mr. and Mrs. B. C. Gibbons

Alhambra, Illinois

Mervin Gildan

Elyria, Ohio

Mr. and Mrs. Walter Gons

Fostoria, Ohio

Mr. and Mrs. Kenneth Goodrich

Columbia City, Indiana

Mr. and Mrs. Theodore J. Grable

Silver Spring, Maryland

Mrs. Flora I. Greenine

Johnson City, New York

Mr. and Mrs. Edward L. Greenwood

Cedarville, Ohio

Mr. and Mrs. Walter L. Griffeth

Cedarville, Ohio

Mr. and Mrs. L. W. Griffith

Houston, Texas 


\section{CEDAR VILLE HARDWARE}

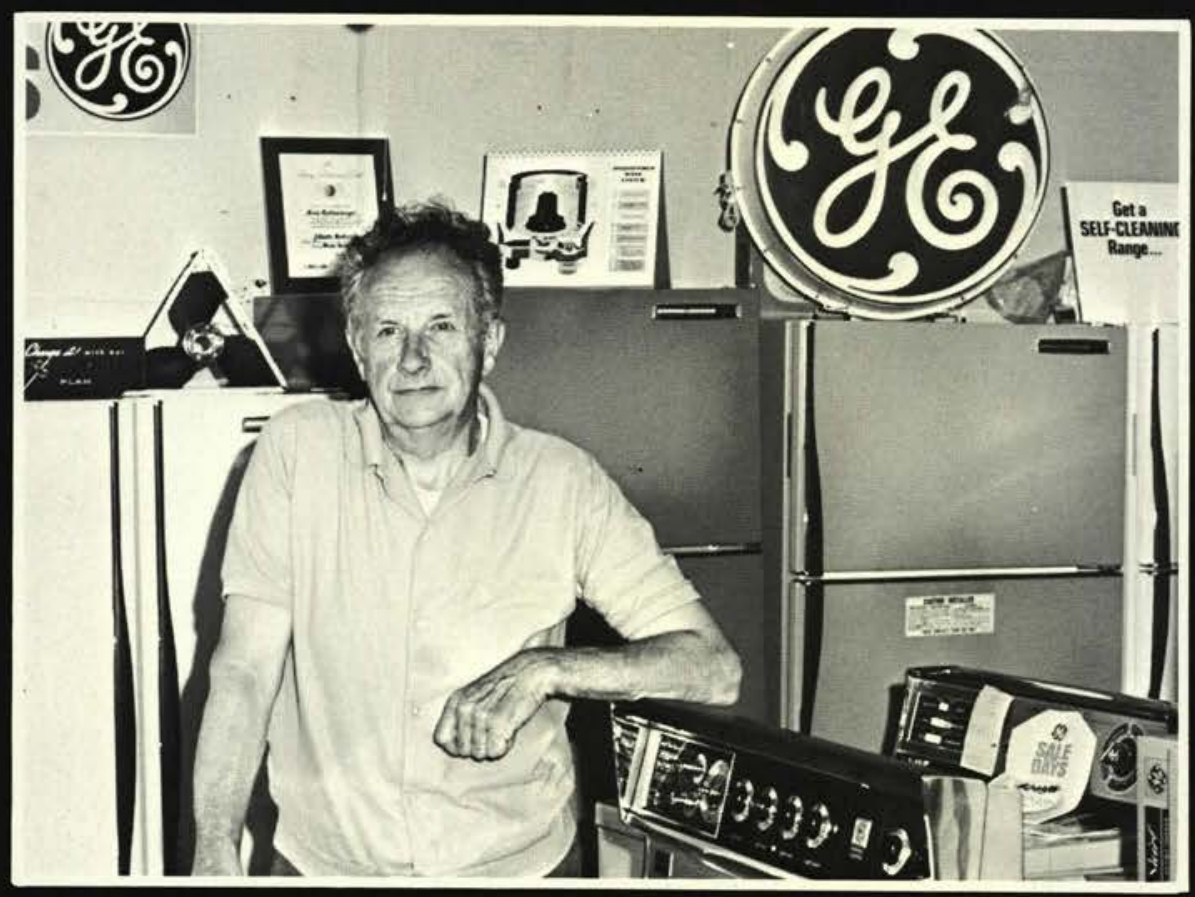

HARDWARE - HOUSEWARES - GIFTS
GE APPLIANCES
"FOR MORE THAN A QUARTER CENTURY"

\section{PARENT PATRONS}

Mr. and Mrs. Ed Gruber Greenville, Ohio

Mr. and Mrs. Harold Haffey

Canal Winchester, Ohio

Homer Hale

East Alton, Illinois

John Hales

Elyria, Ohio

Mr. and Mrs. Monroe Hall

Louisville, Kentucky

Mr. and Mrs. W. M.

Hammack

Niles, Ohio

Mr. and Mrs. Leroy Hansen

Mt. Pleasant, Michigan

Donald H. Harmon, Sr.

Englewood, Colorado

Mr. and Mrs. Donald R. Harold

Huntington, West Virginia

Rev. and Mrs. Charles

Harrison

New Richmond, Ohio

Mr. and Mrs. Howard L. Hart

Des Moines, Iowa

Mr. and Mrs. Wayne Heal

Bunker Hill, Illinois

Mr. Oliver K. Holden

Endicott, New York

Mr. and Mrs. A. W.

Holfield, Jr.

Washington, D. C.

Mr. and Mrs. Thomas Holley

Twinsburg, Ohio

Mrs. Galdys J. Horn

Lima, Ohio

Rev. and Mrs. Leland G

Howard

Oberlin, Ohio

W. C. Howard

Amherst, Ohio
Mr. and Mrs. William J.

Howdyshell

Pickerington, Ohio

Rev. and Mrs. Garland Hudson

Bemidji, Minnesota

Dr. and Mrs. Lloyd Hull

Ithaca, New York

Doanld and Anita Humphrey

Byesville, Ohio

Rev. and Mrs. L. Russell

Ice, Sr.

Iaeger, West Virginia

Mr. and Mrs. Vincent Imbro

Carmel, Indiana

Mr. and Mrs. Richard Inghram

Morning Sun, Iowa

Mr. and Mrs. Morimitser J.

Irwin

Seabrook, New Jersey

W. Sheldon Jackson

Carrall, Ohio

Mrs. Russell W. Jenkins, Jr.

McLean, Virginia

Rev. and Mrs. Oluf C. Jensen

Caro, Michigan

James Jewell

Elyria, Ohio

Mr. and Mrs. Charles Johnson

Akron, Ohio

Dr. and Mrs. Herbert E.

Johnson

South Charleston, West

Virginia

Rev. and Mrs. R. M. Johnson

Eastlake, Ohio

Mr. and Mrs. Thomas Jones

Franklin, Indiana

Mr. and Mrs. Dillon Karsian

Hackensack, New Jersey

Mrs. Myrtle Keithline

John City, New York

\begin{tabular}{|c|}
\hline \\
VILLAGE \\
RESTAURANT \\
"In the Heart \\
of Cedarville" \\
Compelte \\
Food Service \\
Phone - 766-5318
\end{tabular}

CEDAR VILLE FEED AND

\section{GRAIN}

Grain, Feed, Seed,

Coal, Salt, Fert-

ilizer, Builders'

Supplies and

Fencing

CEDAR VILLE, OHIO

Phone 766-2021 


\section{TEMPLE BAPTIST CHURCH 1148 Gallia Street \\ Portsmouth, Ohio}

R. T. Hamilton Pastor •

STUDENTS AT CEDAR VILLE:

FIRST BAPTIST CHURCH

Church St. P.O. Box 3

Maine, New Y ork

Orbin C. Morrison, Pastor

"A BIBLE-BELIEVING CHURCH

IN A VALLEY OF OPPORTUNITY"

\section{BETHEL BAPTIST CHURCH}

RD. \#5 Deerfield Pike

At Love Lane

Bridgeton, New Jersey

\begin{tabular}{|c||c|}
\hline PLEASANT \\
VALLEY \\
BAPTIST \\
CHURCH \\
97 Harvard Ave. \\
Mansfield, Ohio \\
"TO PROCLAT \\
THE TRUTH!" \\
CHURCH \\
530 Meadow Dr. \\
North Tanawanda \\
New York \\
\\
Orlan W. Wilhite, \\
Pastor \\
" A LIVING \\
TESTIMONY \\
FOR \\
A DYING \\
WORLD" \\
\end{tabular}

\section{GRACE GOSPEL CHURCH}

West 12 th St. And Adams Avenue

Huntington, West Va.

Melvin V. Efaw, Pastor

“FOR BY GRACE ARE YE SA VED THROUGH FAITH" — Eph. 2:8 


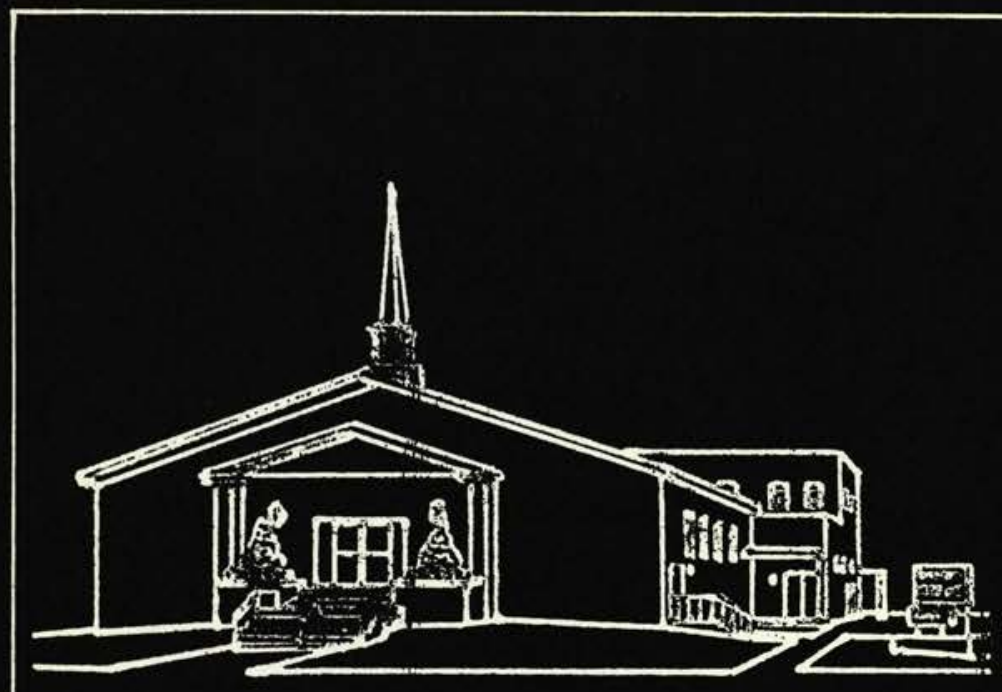

IMMANUEL BAPTIST

\section{CHURCH}

3417 Palmetto Ave.

Columbus, Ohio

William E. Abernathy, Pastor

\section{CALVARY BAPTIST CHURCH}

De Ruyter, N.Y.

George Sanford, Pastor

\section{WEST CORNERS BAPTIST CHURCH}

ENDICOTT, NEW YORK STUDENTS AT CEDAR VILLE

Ann Bailey

Darlene Lewis

Janet Lewis

\title{
SILUIS HEIGHTS BAPTIST CHURCH
}

\author{
900 Middle Road \\ Siluis, Illinois
}

Orville W. Terry, Pastor

STUDENTS AT CEDAR VILLE:

$$
\begin{aligned}
& \text { Becki Chandes } \\
& \text { Gary Dawson } \\
& \text { Brenda Edmunds } \\
& \text { Fred "Bud" Laird } \\
& \text { Diane Meyers }
\end{aligned}
$$

$$
\begin{aligned}
& \text { Ron Meyers } \\
& \text { Linda Newberry } \\
& \text { Sharon Paasch } \\
& \text { Janet Peterson } \\
& \text { Karyn Peterson }
\end{aligned}
$$

“FOR WE PREACH NOT OURSELVES, BUT CHRIST JESUS THE LORD”

II COR. 4:5 


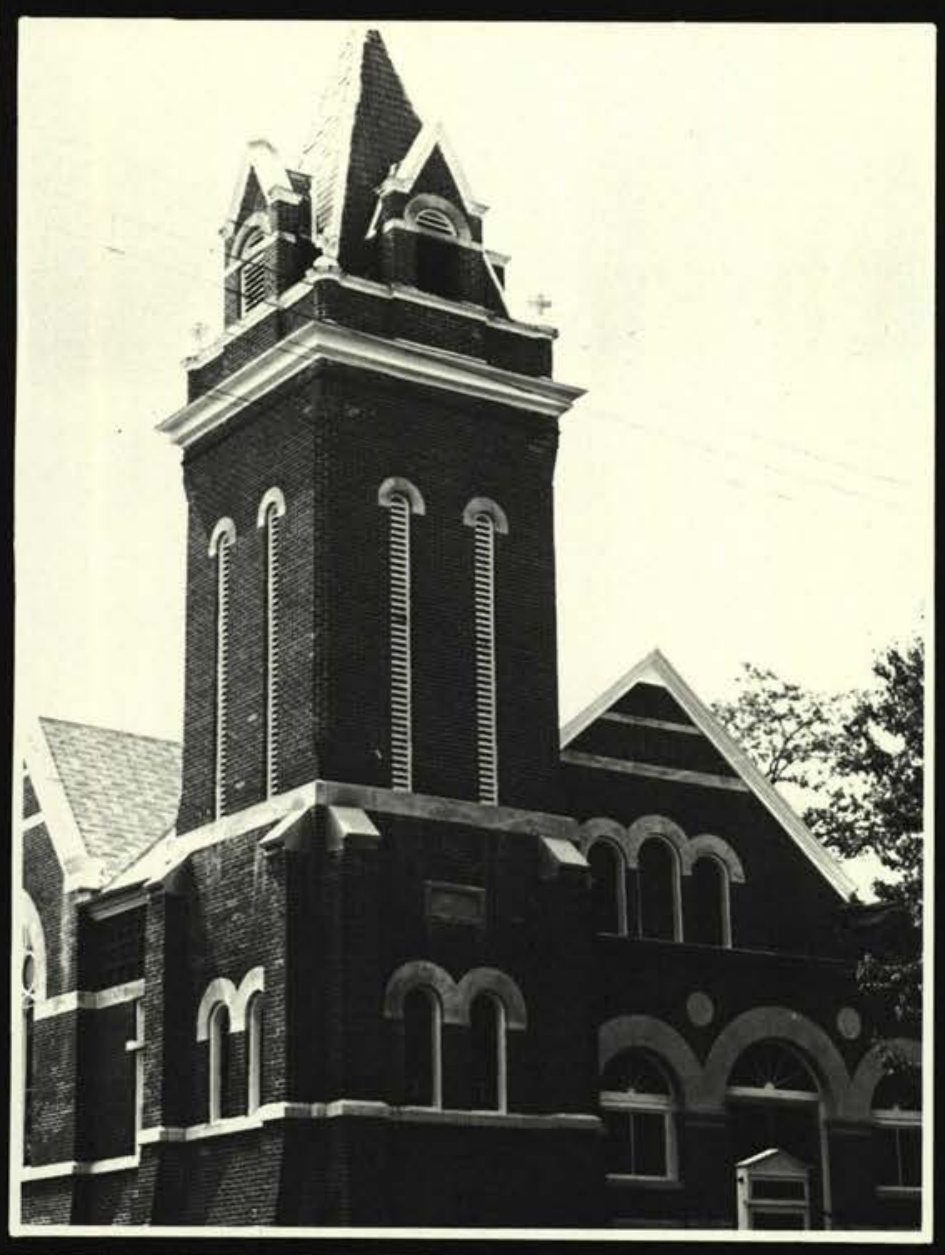

\section{GRACE BAPTIST CHURCH}

Cedarville, Ohio

William Broughton, Pastor

\section{CONGRATULATIONS and \\ BEST WISHES \\ From}

Clintonville Baptist Church 35 Oakand Park Ave. Columbus, Ohio 43214

TEL. 268-4477

GEORGE W. HATTENFIELD, PASTOR

Bible School

Morning Worship

Teen Youth Fellowship

Evening Service

Prayer Meeting, Wed.

\section{SER VICES}

$$
\left.\begin{array}{l}
\text { FOUNDED ON } \\
\text { GROUNDED IN } \\
\text { BOUNDED BY }
\end{array}\right\} \text { THE WORD OF GOD }
$$

9:30 A.M. 10:45 A.M. 6:00 P.M. 7:15 P.M. 7:30 P.M.

\section{FIRST}

BAPTIST

\section{CHURCH}

Pana, Illinois Gerald Thurber Pastor

"Only one life, Twill be soon past,

Only what's done for Christ Will last"

STUDENT AT CEDAR VILLE Linda Pauley
HUNTSBURG BAPTIST CHURCH

Huntsburg, Ohio

STUDENT AT CEDAR VILLE: Susan Sheldon 


\section{HADDON HEIGHTS BAPTIST CHURCH}

Third And Station Avenues

Haddon Heights, New Jersey

Dr. K. W. Masteller, Pastor

STUDENTS AT CEDAR VILLE:

William Amstutz

Jeffery Kistler

Judith Price Olsen
David Price

Dane Rohm

Don Schill
Charles Shepherd

Ann Stevenson

Linda Walter

\section{EASTLAWN CHAPEL}

310 Orchard Avenue

Cuyohoga Falls, Ohio

STUDENT AT CEDAR VILLE:

Paula Norkon

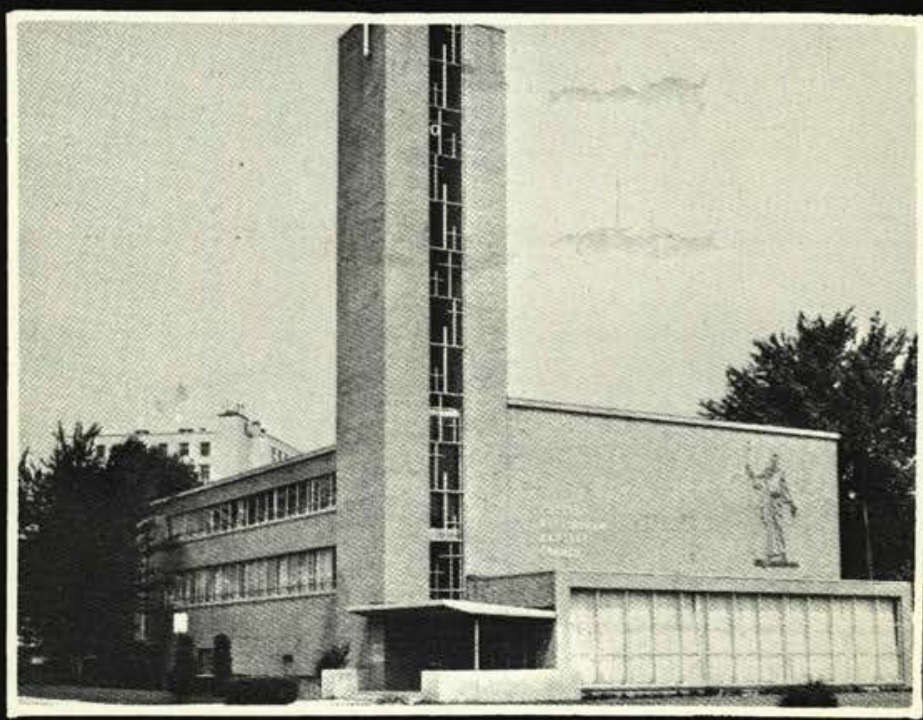

EUCLID-NOTTINGHAM BAPTIST CHURCH 18901 Lake Shore Boulevard Euclid, Ohio 44119

Dr. Wilbur C. Rooke, Pastor Rev. Gary W. Moosey, Associate

Sunday School-9:45 A.M.

Morning Worship_-11:00 A.M.

Evening Worship-7:00 P.M.

Midweek Family Night-7:00 P.M. Wednesday

"Looking Unto Jesus . . ." Hebrews 12:2

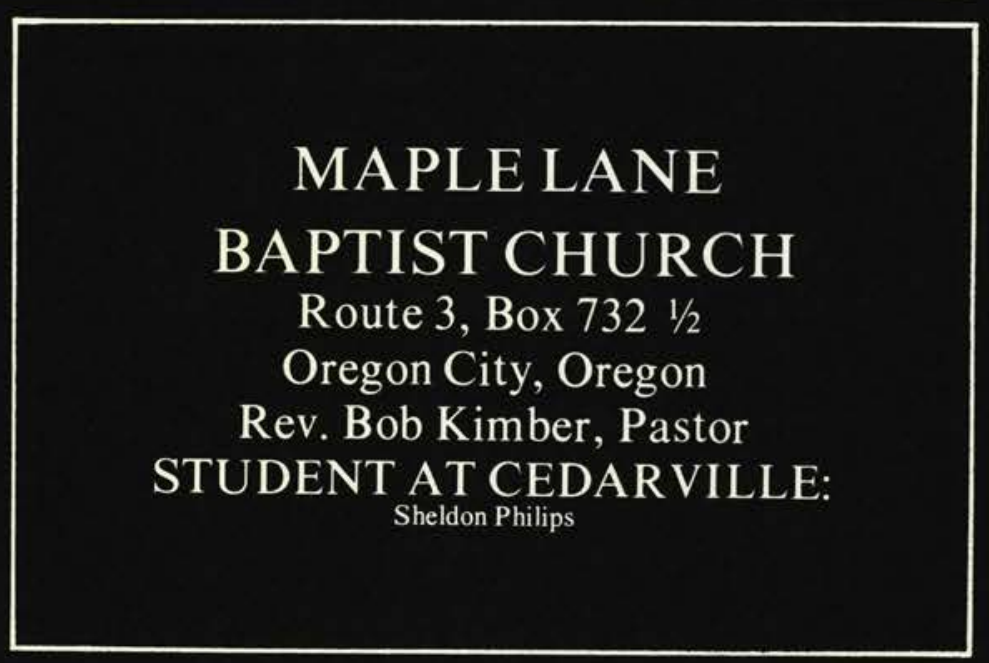

\section{BEREA BAPTIST CHURCH 250 West Street \\ Berea, Ohio \\ Earl V. Willetts, Pastor STUDENTS AT CEDARVILLE:}

Bonnie Bodenmiller Wendy Bodenmiller

Pam Classon

Dave Durham

Debbie Durham

Jean King

Jeff Lough

Gloria Parker

Dave Railsback 


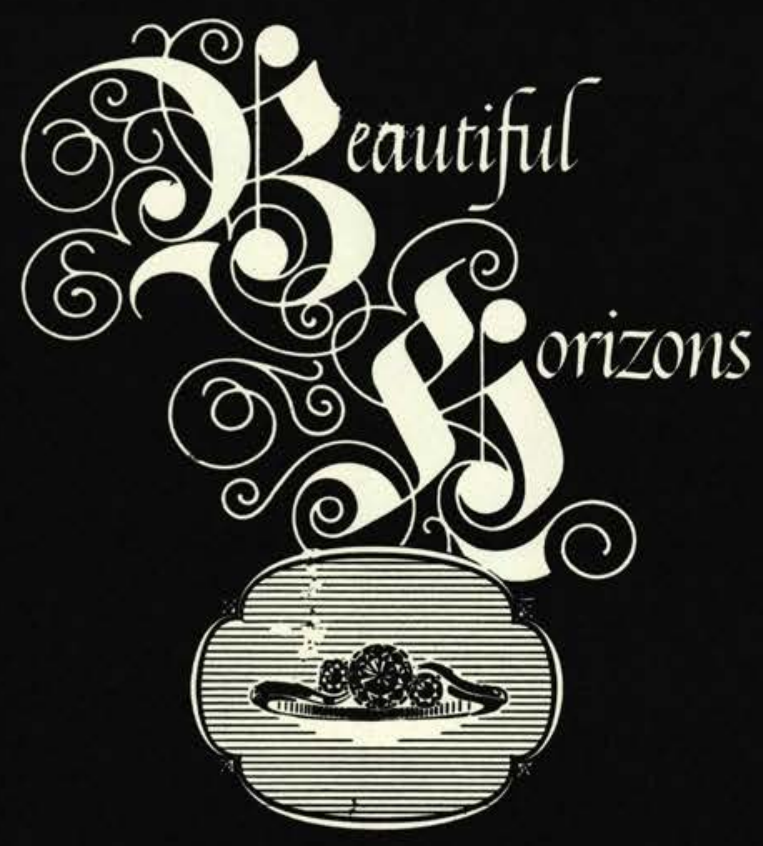

\section{BRAUN'S JEWELERS 74 EAST MAIN XENIA, OHIO}

\section{CONGRATULATIONS:}

\author{
Patricia McLean - 1971 \\ Darlene Folcomen — 1972
}

\section{PARENT}

\section{PATRONS}

Mr. and Mrs. H. A. Kemble Elyria, Ohio

Mr. and Mrs. Neil D. Kennedy

Camillus, New York

$\mathrm{Mr}$, and. Neil Kennedy

Camillus, New York

Mr. and Mrs. Everett Kern

Waterloo, Iowa

William G. Kesler

Olney, Illinois

Oscar, J. Kinney

Des Moines, Iowa

Dr. Robert W. Kitting

Atlanta, Georgia

Mr. and Mrs. H. R. Klett

Boise, Idaho

Mr. and Mrs. Andrew Klimek

Newark, Ohio

Myrtle, A. King

Hew London, Ohio

Howard Klomparens

Grand Rapids, Michigan

Harland Knight

Austin, Minnesota

Mr. and Mrs. Alex W. Konya

Maple Heights, Ohio

$\mathrm{Mr}$, and Mrs. Thomas $\mathrm{P}$.

Kovacik

Johnson City, New York

Mr. and Mrs. Delbert Lakes

Dayton, Ohio

Mr. and Mrs. Delbert L. Lantz

Indianapolis, Indiana

Mr. and Mrs. C. Larson

Allegan, Michigan

\section{PARENT PATRONS}

Mr. and Mrs. Alfred G. Leach, Jr.

East Northport, New Y ork

Howard Lewis

Kokomo, Indiana

H. L. Lewright, Jr.

Eagle Grove, lowa

$\mathrm{Mr}$. and Mrs. John Linger

Northfield, Ohio

Mrs. Frances Lippert

Franklin Grove, Illinois

Harold G. Lowery

Warsaw, Indiana

$\mathrm{Mr}$. and Mrs. James E. Lough

Berea, Ohio

Mr. and Mrs. Nicholas Luketic

Hammond, Indiana

Jacob Mack

Anderson, California

Mrs. Lela Madison

Austin, Minnesota

Mr. and Mrs. Vincent Mahl

La Grange, Ohio

Donald L. Malernee

Byersville, Ohio

Mr. and Mrs. Charles McMurtry

Pittsburg, Pennsylvania

Mr. Clifford Meyer

Batavia, Ohio

Mr. and Mrs. Delbert Miller

Xenia, Ohio

Mr. and Mrs. Earl N. Miller

Allegan, Michigan

\section{FIRST BAPTIST \\ CHURCH OF \\ CHRIST \\ BOLIVAR, PA.}




\section{PARENT PATRONS}

Lloyd Miller

St. Charles, Iowa

Mr. and Mrs. Charles E. Mitchell

Moravia, New York

Mr. Boyce Moore

Jamestown, Ohio

Mr. and Mrs. Emery Moore, Jr.

Elyria, Ohio

Mr. and Mrs. Russell Moore

Freeport, New York

Mr. and Mrs. Max M. Morrow

Kenton, Ohio

Mr. and Mrs. Richard Muntis, Sr

Lima, Ohio

Mr. and Mrs, Leland V. Musser

Marshallville, Ohio

Mr. and Mrs. Leslie Myers

Lima, Ohio

Mr. and Mrs. Frank C. Nabring

Flint, Michigan

Mr. and Mrs. Arnold Nauman

Otselic, New York

Mr. and Mrs. Orville Nolen

Wheelersburg, Ohio

Mr. and Mrs. Joseph Oklak

Mishaviaka, Indiana

Mr. and Mrs. Floyd Otto

Coneord, Michigan

Mr. and Mrs. Harlan R. Palser

Winona Lake, Indiana

Mr. and Mrs. Richard Porterfield

Austin, Minnesota

Mr. and Mrs. Chalres Parker

Portsmouth, Ohio

Mr. and Mrs. A. C. Peek

Fort Wayne, Indiana
Mr. and Mrs. Manuel G. Pereira

Kings Park, New York

Fred and Rosella Petry

Dayton, Ohio

Mr. and Mrs. Hallie Phelps

Lorain, Ohio

Dr. and Mrs. R. W. Phelips

Portland, Oregon

Mr. and Mrs, A. Profant

Parma, Ohio

Joseph A. Pumpelly

Newarks, Ohio

Frank Railsback

Olmsted Falls, Ohio

Mr. and Mrs. John W. Reeder, Sr.

Youngstown, Ohio

John D. Reese

Lebanon, Ohio

Mr. and Mrs. Ausustos Rice

Tamaqua, Pennsylvania

Ralph Richardson

Euclid, Ohio

Mr. and Mrs. O. B. Rodgers

Cuyahoga Falls, Ohio

Rev. and Mrs. Lloyd Rodman

Tower Hill, Illinois

Lad Rodriquez

Gary, Indiana

Mr. and Mrs. Ernest B. Rogers

Byesville, Ohio

$\mathrm{Mr}$. and Mrs. LeR oy Ruby

Dayton, Ohio

Mr. and Mrs. Ralph Rupp

Stryker, Ohio

Harry H. Russell, Jr.

East Brunswick, New Jersey

Mr. and Mrs. Herman C.

Salomon

Hamburg, New York
SASSEN APPLIANCE \& TV

40 S. MAIN STREET CEDARVILLE, OHIO

\section{LAUNDROMAT}

35 East Xenia Ave.

“OPEN 24 HRS. A DAY

7DAYS A WEEK"

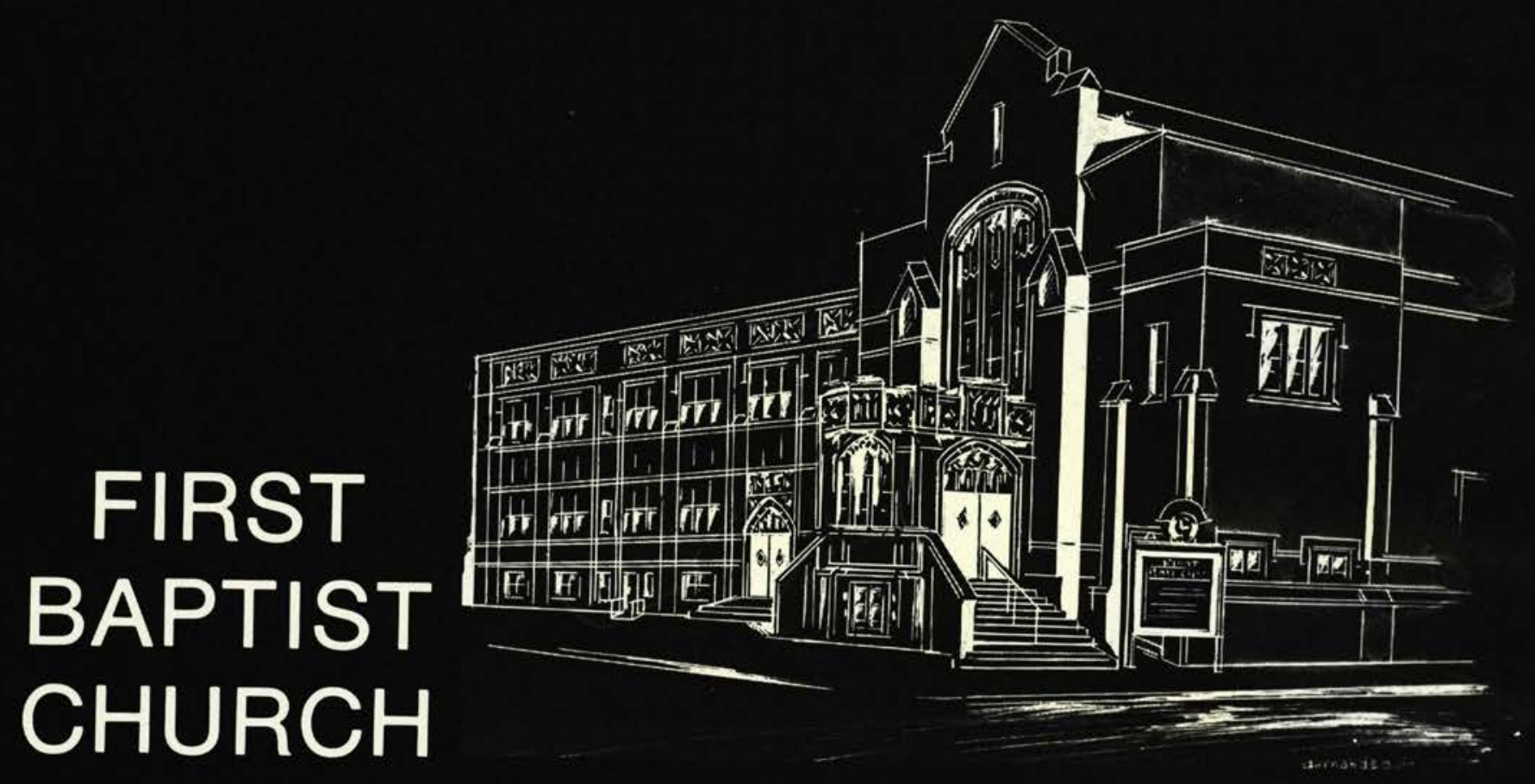

Main at Baldwin Street, Johnson City, N.Y. "Home of Baptist Bible Seminary for 35 Years"

Rev. Lawrence E. Bowland Pastor

"Speaking THE TRUTH

in love" Eph. 4:15 


\section{FIRST BAPTIST CHURCH \\ Union St. At Anderson Park \\ Hackensack, New Jersey \\ Robert C. Gage, Pastor \\ STUDENTS AT CEDARVILLE:}

The MIRACLE staff wants

to thank all its many

supporters for helping

to make the 1971 yearbook a reality,

... and heaves a sigh of relief.

A special thanks to Dr.

Baumann and Mr. Miller for their patience and assistance.

\section{FIRST BAPTIST}

CHURCH

123 West Liberty St.

Medina, Ohio

R. Kenneth Smelser, Pastor

STUDENTS AT CEDARVILLE:

$$
\begin{aligned}
& \text { Marianne Beitler } \\
& \text { Rebecca Gifford } \\
& \text { Tim Northey } \\
& \text { Richard Shaw }
\end{aligned}
$$

"The Lord Jesus Christ Saves Believers"

\section{MOUNT ENON MISSIONARY}

\section{BAPTIST CHURCH}

501 West Third St. at Euclid Ave.

Dayton, Ohio

Thomas David Howard, Pastor 


\section{STURTVANT CHAPEL}

Washington St. and Washington

Ave.

Keeng, N.J.

Ken Betchelder,

\section{Pastor}

"The Church with the heart in the heart of New England"

\section{BEREAN}

BAPTIST

\section{CHURCH}

Bunker Hill, Ill.

Rev. Lee Russell,

Pastor

\section{STUDENTS AT}

CEDARVILLE:

\section{Glenn Betts}

Carol Bierbaum

Virginia Heal

Dennis Roberts

\section{FAITH BAPTIST CHURCH}

Russ Road

Greenville, Ohio

45331

Joseph Godwin, Pastor

"To Know Christ And

To Make Him Known"

STUDENTS AT CEDAR VILLE:

Randy Brown

Sherry Burns

Jane Cunningham

Steve Gruber

Paul Large

Steve Shank 


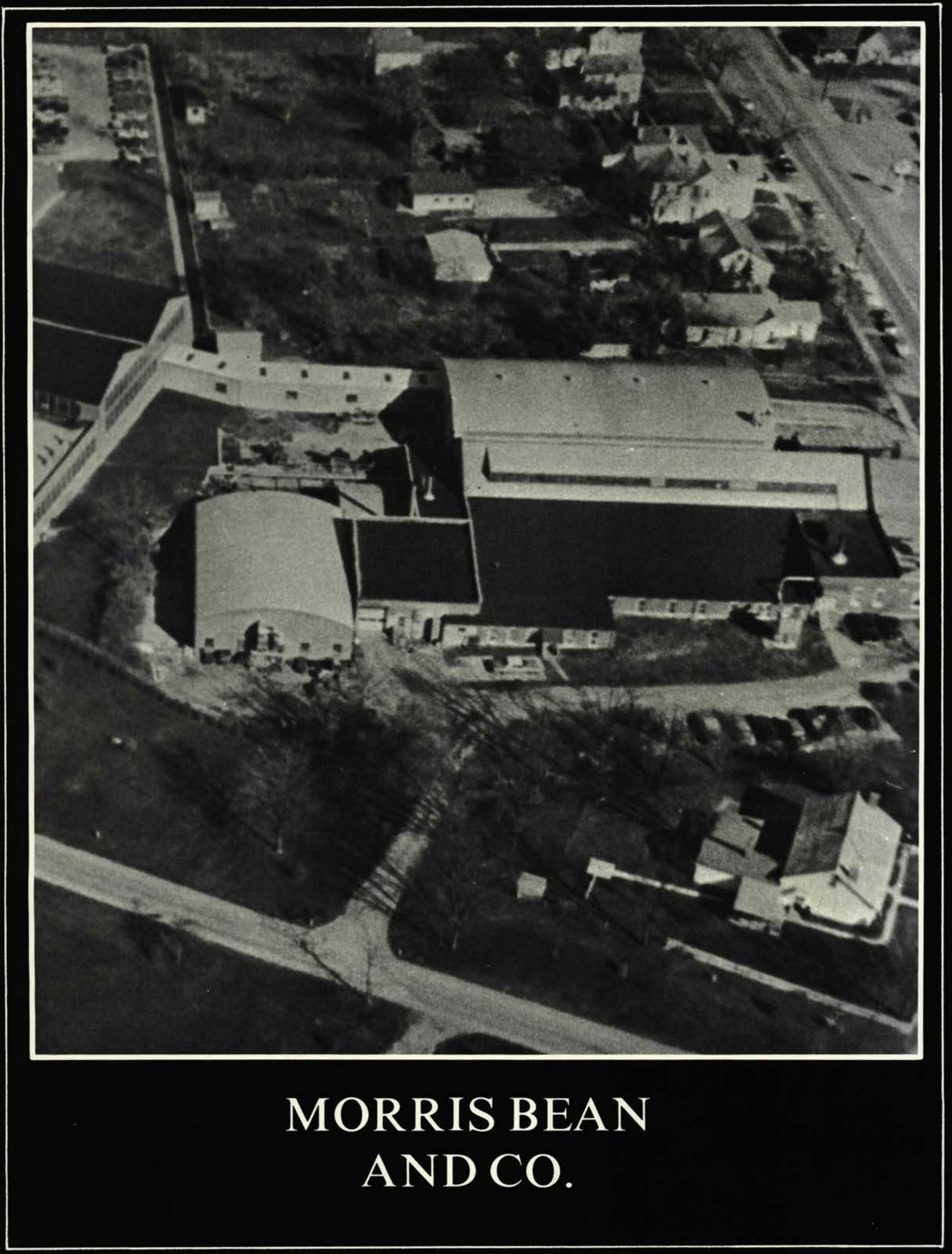




\section{FIRST BAPTIST CHURCH}

724 North Maine St.

Mijhawaka, Indiana

R. G. Hamman D.D., Pastor

"The Bible As It Is For Men

As They Are"

\section{Congratulations}

Sophomores

For

S.M.P.

Jet Plane

Race!

\section{PARENT PATRONS}

James Trueblood

Griffith, Indiana

Donald J. Turner

Grennell, Iowa

Mr. and Mrs. Ralph Tuttle

Grand Rapids, Michigan

Mr. and Mrs. Peter H. Vlug

Wyoming, Michigan

Mr. and Mrs. Merle Wagner

Climax, Pennsylvania

Mr. and Mrs. Hugh Walker

Kirtland, Ohio

Mr. Hiram J. Walter

Westmont, New Jersey

Mr. and Mrs. Ray W. Warren

Onaway, Michigan

Mr. and Mrs. Everett Welborn

Mentone, Indiana

Mr. and Mrs. Eugene Weng

Dayton, Ohio

Mr. and Mrs. Paul L. Wheeler

Huntington, West Virginia

Gail E. White

Springfield, Ohio

Paul A. White

Grafton, Ohio

Mr. and Mrs. Charles W.

Williams

Nebraska City, Nebraska

Henry and Glenna Williams

Flint, Michigan

Mr. Donald L. Wolfe

East Gary, Indiana

Mr. and Mrs. Gordon Wood

Curwensville, Pennsylvania

Charles M. Wright

Fairfax, Virginia
COLLEGE

HILL

IGA

Serving

Cedarville

Monday Thru

Saturday

Route 72, M. St

Cedarville, $\mathbf{O}$. 
Abbey, Tom 156, 190

Abernathy, Joy 136

Acton, Daniel 14, 15, 196

Acton, Donald 124, 144, 198

Adamowich, Daniel 156

Adams, Jo Anna 14, 136

Adams, Richard

Adams, Tim

Addleman, Nancy 136, 140, 186

Alberding, Donna 144

Albertson, Clay 156, 182

Alderson, Dan

Alexander, Mark 144, 174, 202

Alexander, Stephen 136, 174

Alfrey, Larry 144, 172, 176

Allen, Larry 144

Allen, Donald

Amos, Andy 188, 190

Amstutz, William 124, 187

Anderson, Becky 23, 35, 156, 186

Anderson, Kris 36, 144, 188

Arthur, Dinah 67, 156, 188

Aschbrenner, Marcia

Ashby, Pam 136

Assey, Paul 156

Assey, Stephen E. 156

Atherton, Don 25, 124, 173, 174, 175, 181, 198, 200

Austin, Robert 14, 48, 126, 136, 181, 196

A wabdeh, Phillip

Axiotis, Georgeanna 21, 58, 68, 156, 183

A yers, Frank 124

Babbett, Wendy

Bacheller, William 67, 144, 174, 190, 202

Bailey, Anne 67, 144

Bailey, Keith 24, 144, 198

Bailey, Marilyn 89,144

Bailey, Randy 13, 14, 15, 144, 196, 181

Baker, Linda 144

Baker, Judy 136, 175

Baker, Rodney

Barb, Bethany 156,188

Barbely, Katy 144, 188

Barker, Timothy N. 156, 178

Barlow, Jacqueline 36, 124

Barnett, Larry 156

Barr, Candace 12, 124, 175

Barraclough, Jacalyn 68, 156, 169

Bartemus, Sharon 156

Bartlett, Mary 188

Bates, Basil

Bates, James E. 144

Bates, Carolyn

Bayne, Monica 67, 144, 175

Beacham, Jeb 156

Beaujean, William

Bechtel, Ronald 68, 156, 162, 164

Beck, David 13, 85, 124, 173, 175, 196, 200

Beck, Margaret 136

BeDell, Arthur 144

Beesley, Janet 14, 68, 85, 90, 124, 176

Beitler, Mareanne 124, 190

Beitz, Mary 124, 175

Bennett, Kelsey 156, 186, 188

Benson, Ernest 65, 156

Bergen, Daniel 68, 82, 156

Berkheiser, William 124

Bernath, Karen 136

Bernhard. Patricia 156

Berry, Kathy 156, 186, 194

Betts, Glen $61,89,157$

Betts, Linda 68, 136

Bierbaum, Carol 144, 172, 173, 175, 177, 178, 182,188

Biggs, Barbara 157,159

Billington, Linda 136

Bingham, Donna T. 36, 144

Birch, Sandra 136

Bittner, Becky 15, 136

Black, Timothy

Blackburn, Mark 157

Blum, Linda 136

Bo, Carol Anne 89, 144, 190

Bobbitt, Wendy 144

Bodenmiller, Bonny 7, 144, 148

Bodenmiller. Thayne 136, 142, 178

Bodenmiller, Wendy 157

Bohland, Mark 157, 188

Bohn, Shirley 157

Bondorff, Carl 136
Boothe, Ken

Boothroyd, Hilda 124

Borders, Larry 157

Borg, Pam 144, 175

Bosh, Donald

Bosworth, Tim 132

Bowen, Lenora 157

Boyce, Michael 136

Boyd, Joan 124

Bradford, David 136

Branning, Pamela 136, 180, 194

Branon, Dave 24, 145, 181, 198

Bratcher, John 157, 190

Briggs, Fred 7, 157

Briggs, Pamela 145

Briggs, Don

Brock, Gary 7, 157, 159, 162, 164

Brooker, Marty 136

Brookhart, Joyce 124

Brown, Kirby 126

Brown, Marilyn 68, 145

Brown, Nancy 126

Brown, Randy 39, 145, 173, 174

Brown, Robert 126

Browning, Asa G., Jr. 145

Bruder, Carol

Bucholtz, Jim 145, 176, 190

Buck, Cheryl 145

Burget, Ginger 8, 145, 147

Burkhard, Kathy 68, 145

Burkhart, Lillian

Burlingame, Lois 145, 188

Burns, Sherry 89, 145, 194

Buschmann, JoAnne 136, 186, 188, 190

Busho, Connie 52, 125, 126

Busho, Craig 157

Butcher, Maryann 145, 186

Butler, David 145, 149, 178, 186

Buzzard, Jim 11, 13, 14, 26, 196, 200

Byers, Deborah 157

Byers, Joleen 157

Cagwin, Daniel J. 145

Cagwin, Raymond H., Jr. 136

Caldwell, Colleen 157, 180, 188

Caldwell, Randall 158

Calvin, Roy 137

Campbell, Hope 145

Campbell, Jack

Campbell, Scott 157, 178

Canine, Becky 157, 188, 190

Capps, Diana 157

Carey, Phyllis 145

Carity, Edythe 145, 194

Carity, Marcia 126

Carl, Jan 145, 186

Carlton, Kenneth 157

Carpenter, Diane 145

Carpenter, James 137

Carr, Diane

Carter, Bertha 157

Carter, Helen 137

Cason, Janice 146

Catteau, Jack 137

Cavey, James

Chambers, Fran 125, 126

Chambers, Leslie 43, 67, 146, 131, 153, 181, 196

Chandes, Becki 68, 146, 173, 176

Chrestensen, Karen 7, 145

Christiansen, Linda 137, 188

Christman, Linda 126

Chumbley, Rosanna 146

Chute, Linda 50, 146, 175, 188

Clapp, Terry 24, 28, 67, 146, 188, 190, 198

Clark, Annette 137, 176

Clark, Cathy 126

Clark, Connie 68, 136, 176

Clark, Susan 126

Clarke, Ronald 89, 146, 178, 186

Clauer, Becky 146

Clauer, John

Clay, Joan 91, 126

Cleaves, Ruth 157

Clewell, Dick 146

Cline, Jackie 158

Cline, Kathy

Closson, Pamela 137

Cochenour, Don

Cockerill, Diane 36, 126, 190

Codr, Phyllis 73, 146, 188

Coffman, Michael 172, 181, 200
Cogan, Marjorie 146, 186

Cole, Vicki 158

Collison, Ken 126

Conant, John 137

Cooley, Beth

Coombs, Ronald 55, 68, 126, 175

Coombs, Ruth 68, 82, 146

Cooper, Dolores

Cope, Yvonna 146

Cotnoir, David 158

Craig, Ronald 146

Crankshaw, Robert 158

Creviston, Gary 126

Creviston, Sharon 146

Crosby, Janis 158

Crosby, Pat 137

Crotty, Melody 7, 50, 67, 157, 158

Crouch, Jiles 158

Cuenin, Eric 61, 158

Culp, Claudia 158, 164, 169

Cunnagin, Barbara 146

Cunningham, Jane 126

Curtis, Roger 137, 174, 175

Cyrus, Bobby

Dalton, Glenda 137, 173

Dalton, Kathleen 158, 188

Davis, Deborah 146, 188

Davis, Don 158, 178, 190

Davis, Karen 126, 188

Davis, Lynette 127

Davis, Roxane 147, 180, 188

Davis, William

Davison, Beverly 14, 158, 194

Davison, Pamela

Dawley, Bruce 77, 127, 174, 175

Dawson, Gary 127

Dear, Debbie 158, 186, 190

DeAtley, Rita 125, 127

DeBerg, Michael 127, 178, 190

DeBoer, Debbie 158, 164, 186, 194

DeBruine, Joyce 158, 186

Decker, Ed

Decker, Rebecca 147, 175, 178

DeMars, Karen 147, 180, 194

Demos, Carol 147, 190

DeNardis, Diane 158, 186

Dersham, Michael 137, 175

Dials, Vivian 158

Dibble, Kenn 147

Dieringer, Dennis 127, 188, 190

Dilks, Harold 127

Dillon, Dana 158, 188

Dixon, Elaine 127

Dolph, Chuck 158

Duell, Regina 127

Duhon, Corinne 137, 190

Duran, Judy

Durham, David A. 68, 127, 176, 178, 200

Durham, Debbie 68, 158, 190

Dutton, Charles 88, 127, 174

Eadie, Jan 147

Eagleston, Sally 147

Eberling, Jayne 183, 190

Edelblute, Meda Sue 59, 137, 174

Edmunds, Brenda 14, 127

Edwards, Denise 158, 194

Edwards, Marsha 57, 137, 172, 178

Edwards, Michael 59, 158, 169, 174, 188

Ehnis, Daniel 159

Elkin, Russell T. 45, 159, 178

Ellis, Janet Gail 137

Ellis, Richard

Ellis, Shirley

Elsner, James

Ely, Bob 147, 151

Emerson, Edmund

Emerson, Jane 147, 183

Emerson, Patricia 67

Emmott, David N. 137, 174

Endres, Cathy 36, 147, 190

Engle, Carol 127

Entenman, Laura

Epps, Janet 127

Erickson, Randall 181

Ernsburger, Kenneth 61, 159

Erskin, Fred 147

Ertle, Virgil 88

Estes, Daniel 24, 68, 159, 169

Evans, Kenneth 14, 89, 127, 181, 187

Eveland, Jean 
Farley, Susanne 127

Farlow, Tom 147, 178, 181, 196, 202

Farrar, Bob 128

Fenstermaker, Merle

Ferguson, Dave 159

Fiest, Barbara 137, 142

Fiest, John 147, 155, 186, 190

Fillinger, Thomas C. 147

Filson, Earl 159

Fischer, Cynthia

Fisher, Floyd 77, 159, 190

Fisher, RaeBelle 147, 180, 186, 194

Fissel, Nancy

Fitzgerald, Daria 147

Flack, Lavena (Beanie) 28, 190

Flatt, Christine 159

Fleming, Don 147, 178

Flint, Marianne

Flint, Mark 128, 137

Flood, Harold 147, 174

Fogle, Dale 64, 67, 159

Folkmann, Walter

Forbis, Dennis 159

Ford, John 159

Fowler, Lynda 137

Francis, Steve 159, 174, 196

Frank, Janet 128

Frank, JoAnn 128

Franklin, Dennis 147

Frauenknecht, Marianne

French, Gar

Frields, Kathie 26, 147

Frields, Mark 128

Fringer, Vicky 147

Frye, Frederick 128, 159

Frye, Jonathan

Fulcomer, Darlene 38, 138

Fultz, Dennis

Gabriel, Janet 128

Galbraith, Constance

Gallogly, Michael 147, 174, 190

Gast, Rita 16, 50, 58, 68, 148, 176, 184, 194

Gates, Donald L. 159

Gazdik, Sharon 159

Geist, Dale 138

Gerber, Kim 148, 149, 174

Gerber, Lonnie 128

Gibbons, Ed 148, 174

Gidley, David

Gifford, Rebecca 159

Gifford, Ted 159, 190

Gilbert, Stephen

Gildan, Beth 147, 148

Gillespie, Sandra 148

Gillogly, Sharon 13, 148, 186, 190

Glazier, Steve 138

Globig, David 18, 148, 173, 181, 188, 190, 202

Godby, Louis

Goldschmitt, Richard 128

Golliher, Carolyn 148

Good, Debbie 138

Good, Leslie Ann 148

Gons, Kathy 159

Goodrich, Susan 159

Gowman, Sandra 128

Grable, Lauralee 20, 21, 37, 138, 175

Grant, David 138, 174, 175, 200

Graves, Christine

Gray, Pam 138

Grayson, Jerry 68, 82, 178

Greene, Judy L. 160, 182

Greening, James 14, 138, 196

Greenwood, Timothy 148, 174

Greenwood, Thomas

Griffeth, James 160, 190

Griffith, Lee 148

Griswold, Jerry $61,156,160,200$

Grover, Judy 15, 128, 131

Gruber, Stephen 79, 160, 202

Gunneson, Beverly 138

Haffey, Nancy 128, 176

Hague, Keith 138, 178

Hale, Suzanne 160, 175, 190

Hales, Richard 67, 160, 196

Hall, Lynda A. 160

Hall, Susan 148, 188

Hallett, Jan 65, 148, 186, 190

Hammack, Merla 20, 21, 160

Hansen, Rodney 160, 200

Hansen, Sherlyn 148, 185, 190
Harer, Merrilee

Hargis, Jacqueline 160

Harmon, Don 7, 20, 21, 68, 82, 83, 148, 176, 178

Harmon, Jeanne 148

Harold, Susan 138, 173

Harriman, Stephen 190

Harris, Jan 148

Harrison, John 51, 138, 198

Hart, Barbara 138, 177

Hart, Ginny 160

Hartman, Tyler 178

Harvey, Clifford 138

Harvey, Nelson 128

Hawley, Marsha E.

Haye, Larry 157, 160

Hayes, Bruce

Heal, Virginia 148, 177, 178, 188, 190

Healey, Barbara 35, 128

Heater, Linda

Heck, Belinda 138, 188, 190

Hegg, Tim 56, 68, 148, 181, 184, 196

Helm, Joan 138

Henderson, Kathy 160

Hendrickson, Donald 7, 38, 41, 147, 148, 190

Henry, Bill 138, 196

Henson, Dan 24, 27, 68, 160, 164, 169, 187, 198

Hepworth, Judy $148,185,186$

Herman, Stephen 148, 15

Hess, Douglas 14, 148, 18

Heubner, Tanis 57

Heyl, Beverly 160,190

Hickok, Patricia 160

Hillery, Jan 148, 175, 177, 178, 182, 188

Hinton, Mark

Hirschy, Linda 128

Hochstetler, Gregory

Hodgin, Steven

Hoerath, James 160

Hofert, Cynthia 149

Holbrook, Gary 198

Holden, Kevin 138

Holfield, Valerie Jayne 160, 182, 183

Holley, Terry 160, 196

Hollingsworth, Rosemary

Holt, Debbie 160

Holtz, Cheryl 138, 173, 184, 190

Holzworth, Holly 160

Hook, Leslie

Hooper, Diane 160

Horn, Bernard 38, 45, 76, 148, 172, 186, 190

Hostetler, Bonnie 36, 138, 188, 190

Hostetler, Cathy 128

Hostetler, Debbie 160, 190

Hostetler, James 128, 190

Hothem, Sharon 149

Houchin, Andrea 138, 173, 177

Howard, Lynn 14, 78, 149, 181, 187, 198, 200

Howard, Pat 149, 151

Howard, Rebecca 4, 149

Howard, Rosalie 64, 91, 149, 184, 190

Howdyshell, Harvey 138,174

Howell, Ed 89, 160, 202

Hubbard, Judy 128

Hubble, Paul 138

Hudson, Steve 161

Huff, Cheryl 161, 166

Hull, Rebecca 19, 68, 138

Humphrey, Nonnie 16

Humphreys, Delano R. 161

Hyatt, Bill

Hyatt, Janet

Ice, Lawrence R., Jr. 128

Imbro, Vicki 39, 82, 129, 190

Inghram, Dan 55, 84, 129, 139, 142, 181, 200

Inghram, Rick 55, 84, 181, 200

Irwin, Patti 129

Ishuin, Mitsu 129, 175

Jackson, Cheryl

Jackson, Michael 129

Jackson, Rebecca 149, 177

Jacobs, Mary 139

Jacoby, Elaine 129

Jarvis, Timothy 139

Jatton, Roberta

Jefferies, Nancy 139

Jenkin, Holly 161

Jenkin, Patricia 129

Jenkins, Russell 14, 149, 151, 196

Jenkins, Warren 139, 143, 181, 196

Jenney, James 89. 149, 174, 175
Jenney, Jonathan

Jensen, Clifton 12, 139, 172, 174, 196

Jensen, Julianne $149,172,175,186$

Jewell, Dave 139, 181, 202

John, Laura 161

Johnson, Barbara 22, 84, 149, 178

Johnson, Carol 161

Johnson, Doris 10,161

Johnson, Eunice

Johnson, Judy 141, 183, 186

Johnson, L. Faye 129, 172, 173, 178

Johnson, R hea 149, 178, 188

Johnson, Vivian 161

Jones, David 9, 16

Jones, Fonda 161

Jones, Keith 7, 161

Jones, Laura 139, 173, 175

Jones, Jerry

Jones, Pamela 161

Jones, Steven 139, 202

Jones, Verna 129

Jutton, Ronald 161, 190

Kahler, Donna 139

Kalnbach, Elaine 150, 180, 194

Kambla, Rebecca

Karolyi, Robert

Karsian, Kathryn 161

Kauffman, Faun 57, 150

Kemble, Becky 67, 161, 177, 185, 186, 188, 190

Kemery, Glen 161

Kemp, Beverly 67, 150, 175, 188

Kemp, Diane 52, 84, 129, 172, 173, 178, 186, 194

Kendrick, Pamela 139

Kennedy, Karen 161

Kern, Roy 7, 8, 9, 29, 38, 66, 147, 150, 174, 182 , 187, 188

Kesler, John 139

Kies, Carole 190

King, Hal 150

King, Jean 58, 125, 129

King, Karen

King, Lois 150, 190

Kinney, Susan 150

Kinniburgh, Jerry

Kintner, Marquetta 36, 150

Kircher, Ruth 139

Kistler, Jeff 161

Kitting, Deborah 37, 129, 175

Klamm, Harvey 129

Klemm, Larry 61, 139, 173, 174, 190

Klett, Bruce 161, 188, 190, 202

Klimek, Evelyn 150, 188

Klimek, Ken

Kline, Tim 139, 172

Klinger, Glenn Allen 130

Klomparens, Cheryl 150, 177, 186

Knight, Arlene 14, 56, 150, 172, 178

Knight, Deborah 161

Knowles, Thomas 150

Kohli, Denver A. 150

Konssol, Sally 56, 161

Konya, Alex 161, 187, 190

Kovacik, Thomas 161

Kriel, Rita 162

Krikke, Florence 130

Krueger, Edwin 130

Kukasky, Thom 200

Kusumoto, Ann 130

Laird, Fred 139, 174, 178

Lakes, Victor 16, 68, 140

Lambert, Carolyn 68, 140, 176

Lantz, Dawn 140, 175

Lantz, Steve 12, 27, 68, 160, 162

Large, Paul 125, 130, 172, 181, 196

Larrick, Dana 150

Larson, Curtis M. 24, 140, 198

Lathrop, Sue 36, 140

Layne, Nanci 162,183

Leach, Judith 10, 130, 175

Lear, Patricia 130

Lee, George 162, 174

Lee, Timothy 162, 202

Leeser, Kathy 125, 130

LeFever, Linda 150

Lemaster, Charles 162

Leonard, Susan

Leslie, Frank 140

Lewis, Brenda 162

Lewis, Darlene 130, 186

Lewis, Janet 47, 130, 162, 186, 188 
Lewis, Joseph

Lewis, Judy

Lewright, Barb 130

Lewright, John 162

L'Hommedieu, Terry

Lillback, Peter 27, 67, 160, 162, 169, 188, 190

Linderman, Lorraine

Linger, Linda 140, 186

Lippert, Linda 162

Lippert, Mary Lou 162, 190

Lister, Barbara 150

Long, Donald 14, 130

Long, Diane

Long, Janet $12,14,82,83,162,164,190$

Losier, Richard

Lough, Jeff C. $61,140,190$

Love, Diane 150, 194

Love, Joyce 140

Lowery, Landra 150, 180, 194

Lowes, Effie

Lowstettler, Dennis 162

Luckett, John

Luketic, Barrett 24, 162, 198, 202

Lunney, Robert 130, 181, 200

Lutton, Roberta 150,186, 194

Lyon, Gene

Lysoowan, Boonchuay 162

Mack, Connie 150

Madison, Larry D. 140

Madsen, Linda 130

Mahl, Bob 162, 178, 182

Malernee, Christine 162

Mallinak, Dave 76, 140, 188, 190

Mann, Darlene 130, 194

Manross, Dan 15, 85, 125, 129, 130, 196

Marlowe, Catherine 140

Mare, Bob

Marshall, Christine G. 162

Martin, Dan 78, 80, 140, 200

Martin, Janice 150, 180, 194

Mason, Lin 55, 130

Master, Keith 20, 21, 162

Mattison, Carol 162

Mauk, Joseph

Mayer, Susan 162, 186, 190

Mays, Dee 130, 132, 181, 200

Mays, Mike 140

McCall, Alan

McCall, Lind

McCallister, Steve 68, 88, 162

McConkey, Rita 162

McCoy, Barry 59, 150

McCulloch, James

McDaniel, Chip 130

McDaniel, Richard G.

McDonald, Phil 163

McDowell, Gary 130, 198

McGhee, David E. 163

McIntosh, Richard 15, 163, 174, 178

McKanna, Don 163

McKanna, Jon

McMillen, Philip 19, 163, 174, 186, 190, 196

McMillen, Sam 15, 53, 89, 131, 172, 177, 186 190,196

McMurtry, Mary 36, 131, 188

McNeil, Terry

McNeill, Bonita 150, 175

McNiece, Melinda 131, 187

McNiece, Buddy $18,40,68,88,150,200$

Mead, Daniel 140

Mellish, Ronald

Merz, Meara 163

Meyer, Christine 150, 188

Meyer, John 78, 200

Mevers, Diane 163

Meyers, Ronald 55, 68, 131, 175, 178

Miesse, Rickey

Millard, Brenda 150

Miller, Darryl 190

Miller, David 150

Miller, Joan E. 131

Miller, Lyle 140, 183, 196

Miller, Marilyn 36, 150

Miller, Roger 140

Miller, Sally $90,136,150,188,194$

Miller, Yontz 8, 9, 59, 61, 68, 147, 150, 196

Millikan, Steve 163

Mitchell, Lynn

Mitchell, Virginia 131

Mix, Beverly 131, 173
Mogle, Anita 140

Mohler, Joyce 163, 186

Mohler, Philip H. 55, 131

Mohr, Janene 163

Mohr, Rebecca 140

Moon, Philip 140, 202

Mooney, Jerry 131

Moore, Beverly 68, 131, 172, 176

Moore, Becki 151

Moore, Susan 151, 173, 188, 190

Morris, Bonnie 12, 14, 15, 15

Morrow, Coletta 131, 180

Morrow, Shirleen 131, 186, 190

Morse, Carole 68, 131, 176

Morse, Eva 163, 190

Motts, Joyce 181, 190

Moulder, Steve 26, 132

Mudder, Thomas 56, 147, 151, 155

Mummery, Sharon 151, 176, 190

Muntis, Richard 151

Murphy, Glennie 151

Murphy, Phyllis 132

Murry, Michele 151, 175

Musser, Edith 132

Myers, Brent 163, 167, 202

Myers, Dawn 36, 132, 178, 183

Myers, John

Nabring, Ranelle

Nauman, Susan

Neeley, Sharon

Nelson, Brenda 132

Nelson, David 19, 85, 88, 140, 175, 200

Nelson, Jeanne 151

Nelson, Linda Ann 132

Nelson, Linda D. 12, 14, 132

Newberry, Linda 8,163

Nicholas, David 163

Nolen, Bonnie Louise 140

Northey, Ted 140

Northey, Tim 43, 132, 196

Norton, Daniel Carl 132

Norton, Robert 140

Novkov, Paula, 163

Odegard, Susan 152, 175

O'Keefe, Carolyn 133, 180

Oklak, Mark 133, 172, 179

Olsen, Bruce 152

Olson, John Jr.

Olson, Steven B., 79, 133, 140

Orr, Danny 190

Osborne, Linda 194

Osman, Marsha Joy 133

Otto, Bart 163

Overholt, Steve 163, 190

Owen, Ron 164

Paasch, Sharon 164, 169

Palmer, Milton C. 25, 133

Palpant, Pete 43, 164, 196

Palser, Ellen 164

Parcher, George 133, 174

Parker, Clarence M. 24, 57, 59, 164, 187, 198, 200

Parker, Gloria 164

Parkin, Ralph E. 133

Partlow, Kaye 140, 184, 186

Patten, Randy 53, 59, 84, 85, 125, 133, 173

Paugh, Nancy 133

Pauley, Linda 164,173

Payne, Paulette 7, 8, 164, 190

Peek, Jeanette 133, 190

Pence, Susan 164

Penegor, Bernice 164, 190

Penfold, Lynn 164

Perdue, Carol

Pereira, Carol

Pereira, John 140, 175, 188

Perkins, Ralph 57, 140, 142, 172, 173, 174, 178, 187,190

Perry, Bruce 140, 164

Perry, Shirley 186, 190

Pestel, Beverly 180, 194

Peters, David 78, 140, 173, 174, 190, 202

Peterson, Gene 152

Peterson, Jan 164, 194

Peterson, Karyn 140

Pettitt, Deborah 133

Phelps, Linda 140

Philips, Sheldon 10, 76, 152

Phillips, Marcheta 182

Poarch, Rebekah 140
Porter, Tammy 140

Portz, Gale 140, 186

Portz, Sharon 47, 164, 186

Post, Mary

Post, Rodney 152

Potter, William 59, 164, 169, 174, 198, 200

Potts, Linda 164

Powley, Jeanette 36, 140

Powley, Robert

Prater, Paula 50, 68, 82, 152, 185

Price, James

Price, Dave 164, 190

Price, Becky 164

Profant, Richard 164

Pruden, Grace 152, 175, 186

Pruden, John 140, 174, 175

Prugh, Judy 164

Pumpelly, Connie 152, 180, 194

Pycraft, Robert 67, 164, 188

Radcliff, Paul 142, 172, 187, 190

Raike, Jody 4, 165

Railsback, David 20, 21, 54, 68, 82, 83, 84, 142 , 173,178

Ransbottom, Ruth 142,188

Rathbun, Cynthia 165

Ray, Sally 152, 155

Read, Steve 152

Reeder, Thomas 142

Reese, Sharon 152

Reno, Lee 14

Reno, William 165, 196, 198

Reynolds, Vicki 152, 178

Rice, Dennis 142

Rice, Gerald

Richardson, Gale 24, 152, 194

Richmond, Loren 163, 165, 202

Rickard, James 133, 200

Rigg, Lynette 152

Riley, Patricia 142

Ritchie, Wyman 133

Robb, Luann 165

Roberts, Christine

Roberts, Dennis 152, 174, 182

Roberts, Craig

Rocher, Shirley 67, 90, 142, 173

Roden, Michael 152

Rodgers, Carl 152, 181

Rodman, Lyle 68, 88, 178, 202

Rodriguez, David 152, 181

Rodriguez, Laura 165, 188, 190

Rogers, Carl 48, 65

Rogers, Rex 165

Rogers, Sheila 68, 165, 185

Rohm, Dane 54, 133, 178

Romaine, David 7, 165, 186, 190

Romanow, Sally 165

Rooke, Connie

Rooke, John 181

Rose, Richard 165

Ross, Joyce 133

Rothberg, James 152

Rowe, Charles 133, 174, 175, 186, 190

Ruby, Candy 152

Ruder, Esther 142

Rudnik, Donna 165

Rupp, Jack 165

Russell, Priscilla 152, 190

Sagendorf, Sue 165

Sagraves, Cecil 5, 142

St. Clair, Jeff 165, 190

St. Denis, Wayne 152

Salomon, Fay 165

Samson, Deborah

Samuelson, James

Sanderlin, Tim 152, 181

Sarver, Eldon 152, 172

Saunders, Susan 19, 82, 165, 169, 180, 188

Sawicki, Marsha 165, 180, 186

Scharmann, Marion 152

Schill, Don 50, 165, 196

Schilling, Dale 133

Schlechty, Gretchen 152, 175

Schmidt, Kathy 152

Schneider, Anita 165

Schneider, Marikay 68, 133

Schopmeyer, Lloyd 152

Schulz, Esther 152

Schutte, Steve 165, 190

Schwamburger, Dave 79, 166, 198, 200

Schwarm, Norma 133 
Scott, Susan 68, 142, 173, 184, 190 Seago, Lela 166

Seeley, Jeff 53, 82, 83, 84, 85, 134, 172

Seidler, Thomas 10, 142

Seigneur, Renee 166, 175

Selden, Susan 166, 183, 188

Self, Joyce 166, 185, 186, 188, 190

Selfridge, Marcene 142

Sell, Nancy Ruth 153

Severs, Linda 166, 182, 186, 188, 190

Shank, Steve 153, 173, 174

Sharp, Cathy 166, 186

Shaw, Cathy

Shaw, Richard 166

Shawn, Kathy

Shepherd, Wayne 7, 84, 164, 166, 178

Sheppard, Charles 28, 68, 88, 166, 174

Shorey, Linda 153

Shreve, David 134

Sickels, Charlene 142

Silverthorn, Renee 134

Simmons, Susan 166

Simpkins, Connie 142

Sims, Kevin 56, 166, 184

Slates, Linda 134

Slavens, Deborah 142

Sloan, Mark 48, 56, 153, 184

Small, Loren 56, 166, 184

Smith, Becky 153, 190

Smith, Bonnie

Smith, Bruce 15, 153, 202

Smith, Carol 134

Smith, Dale 153, 181

Smith, Gregory 166

Smith, Jonathan 142, 178

Smith, Larry 166

Smith, Nancy 142, 173, 178

Smith, Steve 166

Sneller, Debbie 166

Snider, Nancy 36,134

Soeder, Adria 153, 186

Sorah, Richard 166

South, Brett 166

Southwell, Del 166

Sowards, Alfred 153

Spaulding, James

Spears, Bonnie 153

Spears, Gary 166

Spencer, Steve 20, 21, 67, 166

Spieth, Jean 166

Spieth, Linda 134, 175, 176

Spieth, Ron 134, 176

Spink, Stephen W. 154, 198

Spinks, Jacklyn 166

Spradlin, Janice 154

Stacey, Carol E

Staley, Mike 167, 196

Stemen, Dan 167, 174

Stevens, Darla 167, 185

Stevenson, Ann 167

Stevenson, Mark 48, 154, 181, 196

Steyer, Ruth Ann 142, 186, 190

Stiles, Betty 154, 173, 190

Stillwell, Craig 154, 196

Stine, Philip 48, 49, 80, 89, 154, 172, 181, 202

Stock, Cheryl 134

Stone, Sarah 15, 167

Storey, Mary Lou 134, 180

Stoudt, Shirley

Stowell, Hannah Sue 12, 37, 134

Strawn, Kathy $167,186,190$

Strong, Tom 18, 68, 69, 142, 181, 202

Stroupe, Pat 65, 154, 173

Strychalski, Charles

Strychalski, Paul 154, 200

Stubrich, Carolyn 142, 151, 188, 190

Sturdevant, Pat 36

Stutzman, Gary 134, 188

Sullivan, Linda 167

Summer, Cindy 67, 167

Summerfield, Tom 167

Sumner, Ron 14, 147, 154, 181, 196

Suter, Linda 154

Swanson, Bruce 134

Swanson, Roger 134

Sweazy, Ben 34, 167

Swisher, Donita 154

Swiger, Jan 134

Syphers, Karen 154, 194

Taft, Ellen 65, 167
Tallmon, Nancy $167,184,190$

Tallmon, Verna 4, 179, 84, 134, 172

Tanner, Faith

Tate, Joy 15, 167, 175, 182

Taylor, Elizabeth 154

Taylor, Jean 68, 167, 185, 194

Telford, Helen 154, 188

Temple, Allan 188

Tennant, Jan 154, 172, 178

Tennant, Mercy 135

Tewis, Louis 14, 143, 181, 196

Thomas, Billie 135, 180, 194

Thomas, Martin

Thompson, Daniel 154, 186, 190

Timpe, Janice 68

Tingle, Lois 167

Tingle, Patricia 154

Tipton, Jacob 43, 67, 147, 154, 174, 181, 188, 190 196

Titus, Marlene 143

Tobias, Vicki 36, 67, 142, 143, 172, 173, 177, 178, 183,186

Tobias, William A. 147, 154, 172, 173, 186, 190, 196, 202

Tobin, Michael 167

Tompkins, Brenda 154, 180, 188, 194

Tompkins, Connie J. 143

Tompkins, Kathy 167

Travis, Rosalie 167

Trefzger, Joy

Tress, Mary 135

True, David, L. 68, 69, 167, 178

Trueblood, Gaylynn 167

Tucker, Michael 167

Turner, Cal 167

Turner, David 135, 173, 190

Turner, Mary

Tuttle, Sue 167

Twigg, Dennis 167, 188

Urban, Carol 135, 173, 190

Vanderpool, Mary 36, 143

VanDuren, Raymond 168, 190, 202

VanDuren, Richard

Vansickle, Melvin

Vinson, Paul

Vlug, Janice 143

Waggoner, Pat 135

Wagner, Lois 168

Wainscott, Lynda

Walker, Connie 54, 135

Walker, Mary 168

Walker, Richard 89

Walker, Stuart 54, 84, 135, 172, 178, 181, 200

Wall, Darlene

Wall, Joyce 8, 168, 185, 190

Wall, Tony $18,143,181,200$

Wallace, Patricia A. 154

Walls, Elaine

Walls, Linda 154

Walter, Linda 154, 175, 186

Warfield, Bette 154

Warren, Jon 154

Watson, Carol 168, 186

Weah, Joseph S. 81, 168

Webb, Susan 135

Webber, Carol Lynne 143, 176

Webber, David 14, 168, 184, 190, 200

Webster, Greg 168

Weddle, Kenneth 155

Weddle, Laura 155

Weiss, John 155

Welborn, Ernie 12, 15, 155, 181, 202

Welch, Glenda 135

Weld, Patricia 143

Weld, Sharon 168

Weng, David 155, 178

Wenner, Holly $67,147,155$

Werner, Patricia

Wessel, Lynda

Wheeler, Dale 200

Wheeler, Paul 52, 84, 135, 179, 190

Whims, David 143

Whitaker, Pat

White, Linda 168, 180, 194

White, Raynell 168

White, Sandy 155

Wilcoxon, Barbara

Wilhite, Mark 91, 135, 143, 174

Wilhite, Mike 172, 177, 186

Wilkinson. Michael 155
Will, Brenda 168

Williams, Becky 155, 182, 186, 190

Williams, Charles 24, 64, 143,181, 186, 198

Williams, Connie 135

Witliams, Gay 155, 175, 184, 190

Williams, Joseph 52, 84, 135, 175, 181

Williams, Russell 155

Williams, Steve $68,160,168,178$

Williams, Wayne $168,174,202$

Willis, Charlotte 143, 175, 180

Willms, Linda 155, 190

Wilson, Alice 143

Wilson, Janet 168

Wilson, Janet 168

Wineberg, Thomas

Wita, Ronald 12, 155, 174, 175

Witt, Ardell 149, 155

Witt, Freda 155, 188, 190

Wolfe, Jeffery 168, 174, 196

Wolfe, Ron 155, 168

Wolff, Frances

Wolff, Howard 155, 174

Wood, Deborah 168

Wood, Greg 135

Wood, Rebecca 135, 188

Wood, Robert 13, 155, 181, 202

Wood, William

Woodcock, Jeffrey 14, 23, 143, 172, 173, 181, 196

Work, Ivan 168

Worm, Fuzzy 29

Wright, Becky 155

Wright, Bonnie 168, 185, 186

Wright, Deborah 155

Wuest, Darlyne 135

Wymer, William 168

Yardlay, Janet 135

Yates, Gary 135

Yoder, Ronald 155, 174

Yost, Gwen 168

Young, Alma 188, 194

Young, Ellen 143, 188, 190

Young, Janet 67, 168

Young, Mabel 143

Younger, Brenda 155

Zent, Susan 168

Zimmerman, Monica 143

Zook, James

Zwiesler, Cathi 135 
A miracle

Not that another year ended,

Not that success had been ours

Not that another goal was achieved.

A miracle -

That Christ cared for us,

That $\mathrm{He}$ would walk this earth,

That He loved us so much

That He should die on the cross.

Another year -

Striving, seeking, working for a place in the world,

Yet always by His side.

Seeing others yearning for peace,

And knowing within us the only real peace there is

Working for a purpose -

Not the glory of man,

But the glory of God.

New responsibilities and challenges,

Indecision in our minds,

Seeking God's will on our knees,

Knowing true joy when doing His work.

A year of growth,

A year of attainment,

A year for God ...

... the year of our Lord 1971

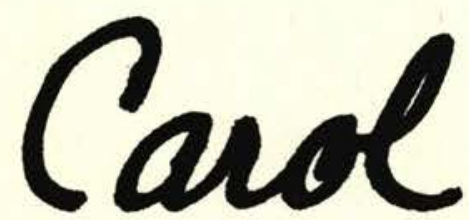

I Peter 5:6-10

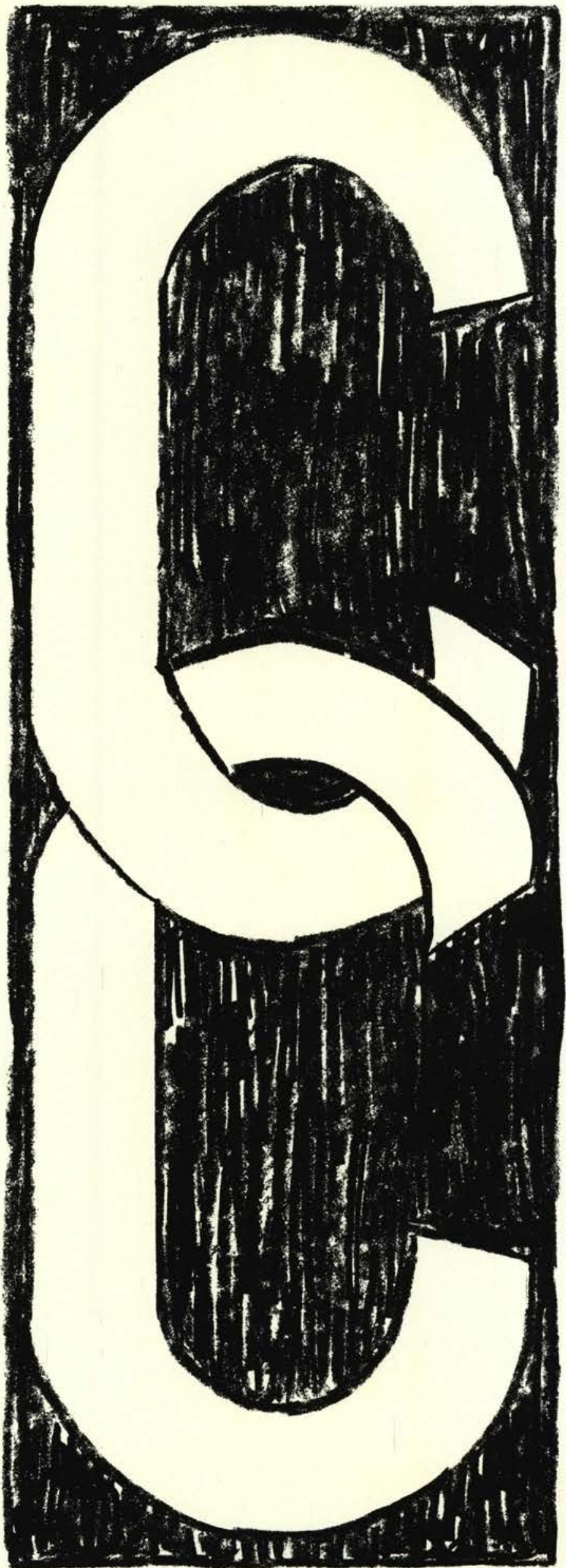




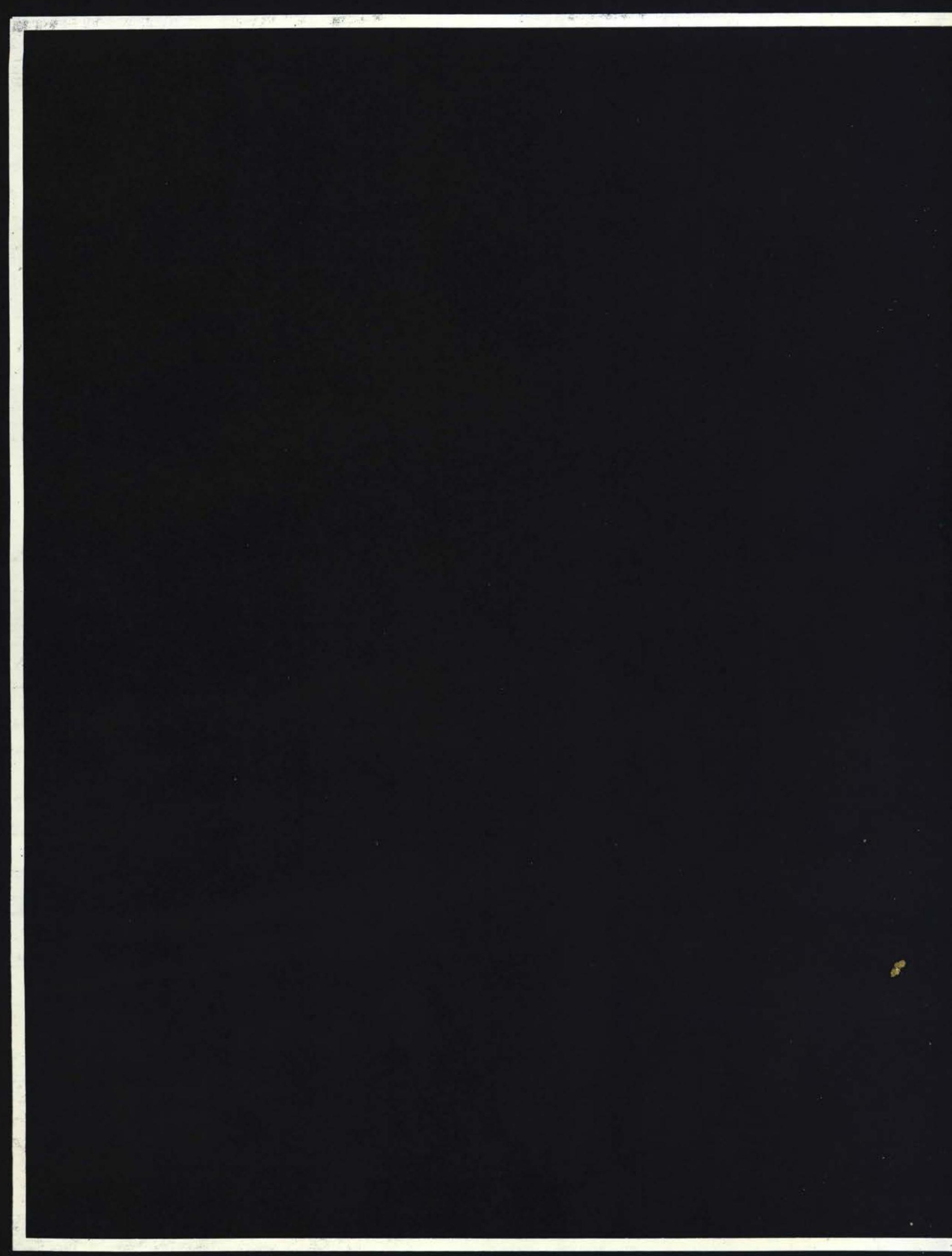




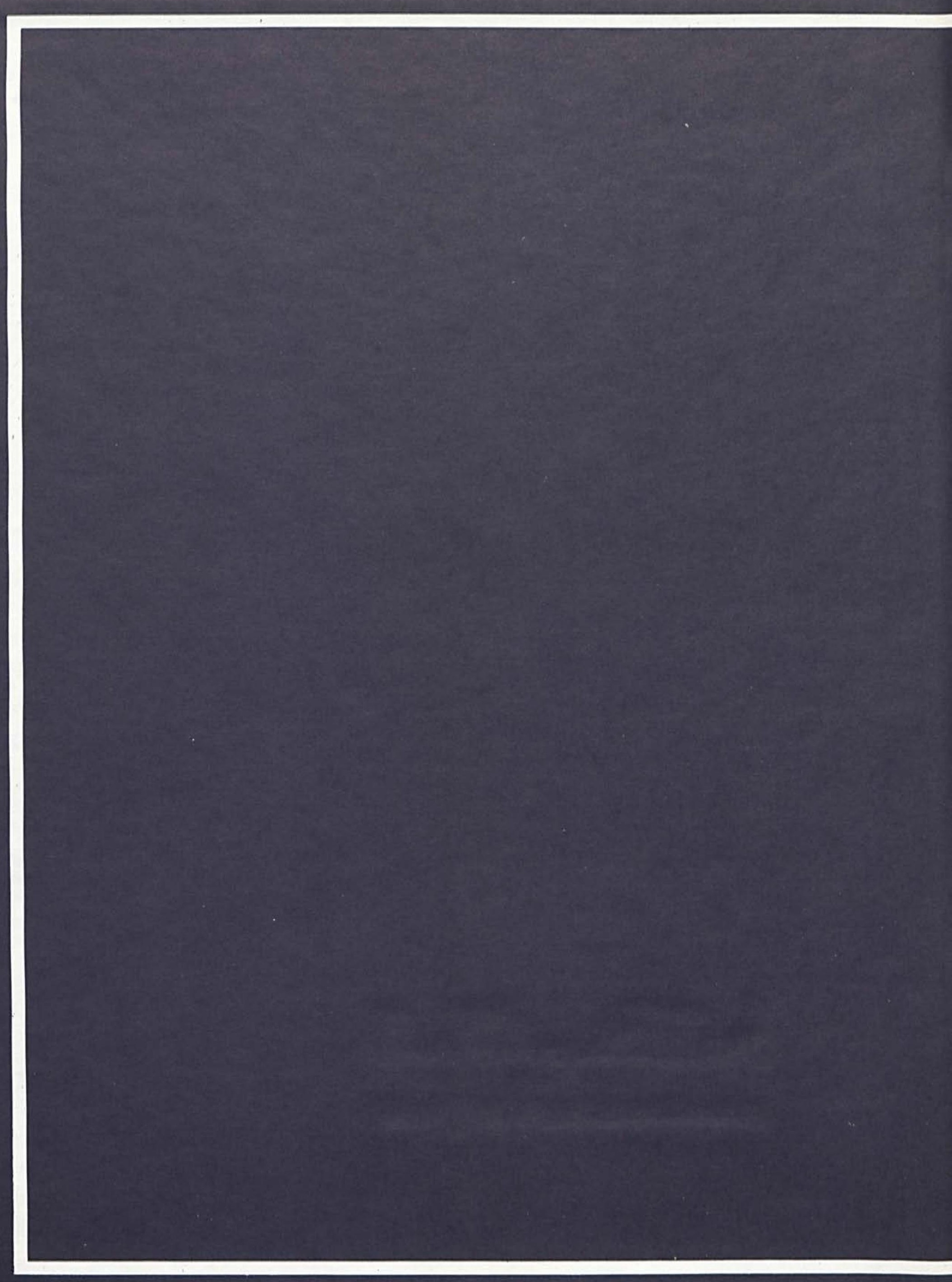




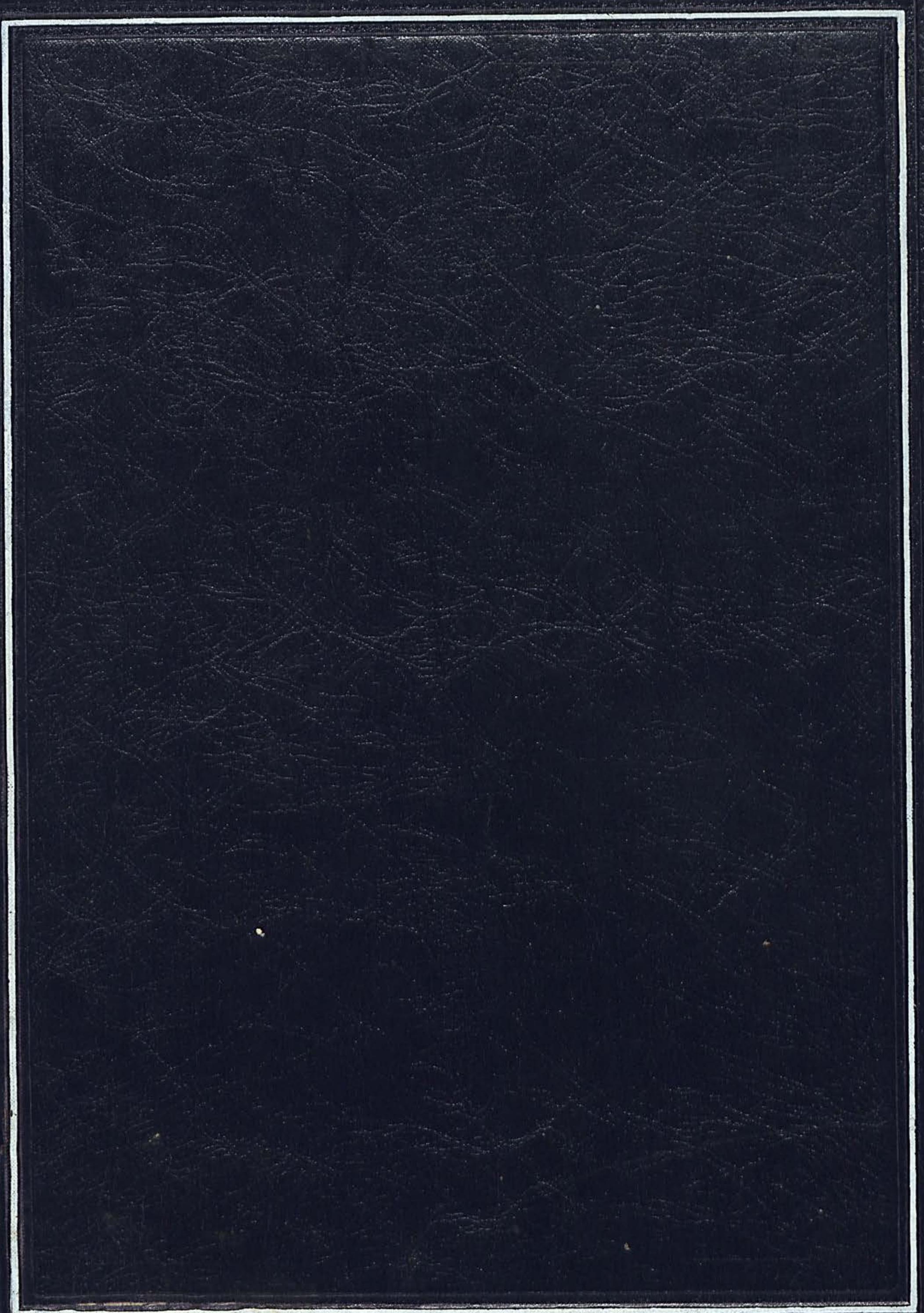

